\title{
Diastereodivergent Synthesis of $\alpha$-Chiral Tertiary Azides through Catalytic Asymmetric Michael Addition
}

\author{
Pei-Gang Ding, ${ }^{\dagger}$ Xiao-Si Hu, ${ }^{\dagger}$ Jin-Sheng Yu, $, *, \dagger,+$ and Jian Zhou ${ }^{*}, \dagger, \S$ \\ † Shanghai Engineering Research Center of Molecular Therapeutics and New Drug Development, and Shanghai Key \\ Laboratory of Green Chemistry and Chemical Processes, East China Normal University, Shanghai 200062, P. R. China \\ ${ }^{*}$ Key Laboratory of Tropical Medicinal Resource Chemistry of Ministry of Education, Hainan Normal University, Haikou \\ 571158, China. \\ $\S$ State Key Laboratory of Organometallic Chemistry, Shanghai Institute of Organic Chemistry, Shanghai 200032, P. R. \\ China \\ E-mail:jsyu@chem.ecnu.edu.cn; jzhou@chem.ecnu.edu.cn
}

\section{Supporting Information}

\begin{tabular}{|l|c|}
\hline \multicolumn{1}{|c|}{ Table of Contents } & Page \\
\hline 1. General information & S1 \\
\hline 2. The synthesis of catalysts C4 & S2-S3 \\
\hline 3. The synthesis of $\alpha$-azido ketones $\mathbf{1}$ & S4-S5 \\
\hline 4. Selected condition optimization & S6 \\
\hline 5. The preparation of racemic Michael adducts 3 and unsuccessful examples & S7 \\
\hline 6. General procedure for syn-selective Michael addition & S8-S16 \\
\hline 7. General procedure for anti-selective Michael addition & S17-S25 \\
\hline 8. Gram-scale synthesis and synthetic elaboration of 3m & S26-S28 \\
\hline 9. X-ray crystallographic data of syn-3i and 4b & S29-S47 \\
\hline 10. ${ }^{1}$ H, ${ }^{13}$ C, ${ }^{31}$ P and ${ }^{19}$ F NMR spectra & S48-S158 \\
\hline 11. HPLC spectra & S159-S200 \\
\hline 12. Typical references for catalytic asymmetric diastereodivergent reactions & S201-S202 \\
\hline
\end{tabular}




\section{General information}

Unless otherwise noted, all reactions were run in air, and monitored by thin layer chromatography (TLC) using UV light to visualize the course of reaction. Purification of reaction products was carried out by flash chromatography on silica gel. Chemical yields referred to pure isolated substances. The infrared (IR) spectra were obtained using a Bruker tensor 27 infrared spectrometer. Chiral HPLC analysis was performed on a Shimadzu LC-20AD instrument using Daicel Chiral columns at room temperature and a mixture of HPLC-grade hexane and isopropanol as eluent. Optical rotation was measured using a JASCOP-1030 Polarimeter equipped with a sodium vapor lamp at $589 \mathrm{~nm}$. The HRMS spectra were measured on Waters Synapt TOF G2-S mass spectrometer or Bruker maXis impact spectrometer using electron spray ionization (ESI) method. ${ }^{1} \mathrm{H},{ }^{13} \mathrm{C},{ }^{19} \mathrm{~F}$ and ${ }^{31} \mathrm{P}$ NMR spectra were obtained using Bruker DPX-500, Bruker DPX-400 or Bruker DPX-300 spectrometer. Chemical shifts were reported in ppm from $\mathrm{CDCl}_{3}$ with the solvent resonance as the internal standard. The following abbreviations were used to designate chemical shift multiplicities: $\mathrm{s}=$ singlet, $\mathrm{d}=$ doublet, $\mathrm{t}$ $=$ triplet, $\mathrm{q}=$ quartet, $\mathrm{h}=$ heptet, $\mathrm{m}=$ multiplet, $\mathrm{br}=$ broad.

Anhydrous $\mathrm{CH}_{2} \mathrm{Cl}_{2}$ and $\mathrm{CH}_{3} \mathrm{CN}$ were prepared by first distillation over $\mathrm{P}_{2} \mathrm{O}_{5}$ and then from $\mathrm{CaH}_{2}$. Anhydrous $\mathrm{Et}_{2} \mathrm{O}$, THF and toluene were prepared by distillation over sodium-benzophenone ketyl prior to use. Catalysts $\mathbf{C 5}^{1}$ and $\mathbf{C} \mathbf{6}^{2}$ were prepared according to the corresponding literature method.

\section{List of abbreviation:}

\begin{tabular}{ccc}
\hline Entry & Chemical name & Abbreviation \\
\hline 1 & Petroleum ether & PE \\
2 & Ethyl acetate & EtOAc \\
3 & Tetrahydrofuran & $\mathrm{THF}$ \\
4 & Dichloromethane & $\mathrm{CH}_{2} \mathrm{Cl}_{2}$ \\
5 & Acetonitrile & $\mathrm{CH}_{3} \mathrm{CN}$ \\
\hline
\end{tabular}

1 Yang, W.; Du, D.-M. Org. Lett. 2010, 12, 5450-5453.

2 Massolo, E.; Benaglia, M.; Genoni, A.; Annunziata, R.; Celentanob, G.; Gaggero, N. Org. Biomol. Chem. 2015, 13, 5591-5596. 


\section{The synthesis of catalysts $\mathrm{C4}$}

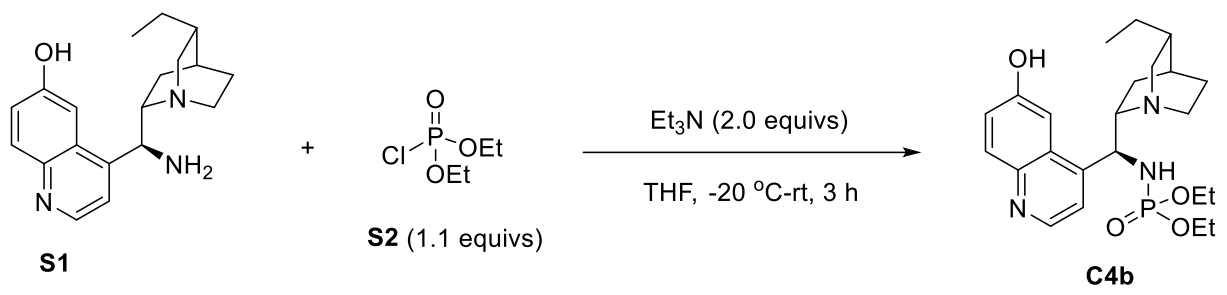

General procedure: Under an atmosphere of $\mathrm{N}_{2}$, to a flame-dried $50 \mathrm{~mL}$ three-necked flask were added chiral primary amine $\mathbf{S 1}^{3}(0.31 \mathrm{~g}, 1.0 \mathrm{mmol})$ and anhydrous THF $(10 \mathrm{~mL})$, followed by the dropwise addition of $\mathrm{Et}_{3} \mathrm{~N}(0.28 \mathrm{~mL}, 2.0 \mathrm{mmol})$ and a solution of freshly distilled diethyl chlorophosphate S2 $(0.19 \mathrm{~g}, 1.1 \mathrm{mmol})$ in anhydrous THF $(4.0 \mathrm{~mL})$ at $-20{ }^{\circ} \mathrm{C}$, successively. The resulting mixture was stirred at room temperature until full consumption of $\mathbf{S 1}$ by TLC analysis (about $3 \mathrm{~h})$. After adding $15 \mathrm{~mL}$ of $\mathrm{H}_{2} \mathrm{O}$, the reaction mixture was exacted with EtOAc $(15 \mathrm{~mL} \times 3)$. The combined organic layers were washed with brine, dried over $\mathrm{Na}_{2} \mathrm{SO}_{4}$, and concentrated in vacuo to give the residue, which was purified by silica gel column chromatography using $\mathrm{CH}_{2} \mathrm{Cl}_{2}: \mathrm{MeOH}: \mathrm{NH}_{3} \cdot \mathrm{H}_{2} \mathrm{O}$ (200:4:1 to $200: 6: 1$, v/v/v) as eluent to afford catalyst $\mathbf{C 4 b}$ as a foamy solid $(0.17 \mathrm{~g}, 37 \%$ yield $)$, m.p. $=101-104{ }^{\circ} \mathrm{C} .[\alpha]_{\mathrm{D}}{ }^{20}=+25.0\left(c=0.11, \mathrm{CHCl}_{3}\right)$. (Due to the distinct presence of rotameric isomers, the ${ }^{1} \mathrm{H} \mathrm{NMR},{ }^{13} \mathrm{C}$ NMR, and ${ }^{31} \mathrm{P}$ NMR spectra contained extra peaks and seemed complex, so we did not designate the data, and attached the spectrum behind). ${ }^{1} \mathrm{H}$ NMR (400 $\left.\mathrm{MHz}, \mathrm{CDCl}_{3}\right)$ : see below; ${ }^{13} \mathrm{C} \mathrm{NMR}\left(100 \mathrm{MHz}, \mathrm{CDCl}_{3}\right)$ : see below; ${ }^{31} \mathrm{P} \mathrm{NMR}(161.7 \mathrm{MHz}$, $\mathrm{CDCl}_{3}$ ): see below; IR (neat): 1734, 1697, 1620, 1514, 1472, 1395, 1261, 1211, 1103, 1020, $797 \mathrm{~cm}^{-1}$; HRMS (ESI) $m / z:[\mathrm{M}+\mathrm{H}]^{+}$Calcd for $\mathrm{C}_{23} \mathrm{H}_{35} \mathrm{~N}_{3} \mathrm{O}_{4} \mathrm{P}$ 448.2360, Found: 448.2360. Other catalysts C4a, and $\mathbf{C 4 c - C 4 e}$ were synthesized from the corresponding chiral primary amine or chlorophosphate by using the above same procedure.

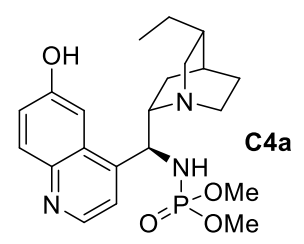

Catalyst C4a was obtained as foamy solid (0.05 g, 40\% yield), m.p. $=215-217{ }^{\circ} \mathrm{C}$. $[\alpha]_{\mathrm{D}}{ }^{20}=+25.4\left(c=0.17, \mathrm{CHCl}_{3}\right) .{ }^{1} \mathrm{H}$ NMR $\left(400 \mathrm{MHz}, \mathrm{CDCl}_{3}\right)$ : see below; ${ }^{13} \mathrm{C}$ NMR (100 MHz, $\left.\mathrm{CDCl}_{3}\right)$ : see below; ${ }^{31} \mathrm{P} \mathrm{NMR}\left(161.7 \mathrm{MHz}, \mathrm{CDCl}_{3}\right.$ ): see below; IR (neat): 1694, 1516, 1470, 1389, 1339, 1246, 1184, 1028, $756 \mathrm{~cm}^{-1}$; HRMS (ESI) $m / z:[\mathrm{M}+\mathrm{H}]^{+}$Calcd for $\mathrm{C}_{21} \mathrm{H}_{31} \mathrm{~N}_{3} \mathrm{O}_{4} \mathrm{P} 420.2047$, Found: 420.2050 .

${ }^{3}$ S1 was prepared according to the reported procedure: Cassanil, C.; Martín-Rapún, R.; Arceo, E.; Bravo, F.; Melchiorre, P. Nature Protocols 2013, 8, 325-344. 


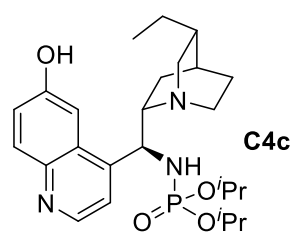

Catalyst $\mathbf{C 4 c}$ was obtained as foamy solid $(0.15 \mathrm{~g}, 61 \%$ yield $)$, m.p. $=98-99^{\circ} \mathrm{C}$. $[\alpha]_{\mathrm{D}}{ }^{20}=+45.1\left(c=0.11, \mathrm{CHCl}_{3}\right) .{ }^{1} \mathrm{H}$ NMR $\left(400 \mathrm{MHz}, \mathrm{CDCl}_{3}\right):$ see below; ${ }^{13} \mathrm{C}$ NMR (100 MHz, $\left.\mathrm{CDCl}_{3}\right)$ : see below; ${ }^{31} \mathrm{P}$ NMR $\left(161.7 \mathrm{MHz}, \mathrm{CDCl}_{3}\right)$ : see below; IR (neat): 1707, 1620, 1514, 1470, 1221, 1096, 989, 854, 775, $644 \mathrm{~cm}^{-1}$; HRMS (ESI) $m / z:[\mathrm{M}+\mathrm{H}]^{+}$Calcd for $\mathrm{C}_{25} \mathrm{H}_{39} \mathrm{~N}_{3} \mathrm{O}_{4} \mathrm{P} 476.2673$, Found: 476.2680 .

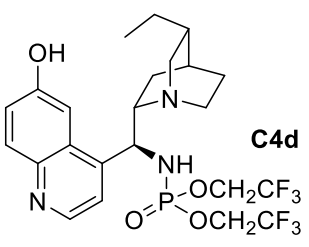

Catalyst C4d was obtained as foamy solid (0.14 g, 49\% yield), m.p. $=97-98{ }^{\circ} \mathrm{C}$. $[\alpha]_{\mathrm{D}}{ }^{20}=-146.0\left(c=0.1, \mathrm{CHCl}_{3}\right) .{ }^{1} \mathrm{H}$ NMR $\left(400 \mathrm{MHz}, \mathrm{CDCl}_{3}\right)$ : see below; ${ }^{13} \mathrm{C}$ NMR (125 MHz, $\left.\mathrm{CDCl}_{3}\right)$ : see below; ${ }^{19} \mathrm{~F}$ NMR $\left(376 \mathrm{MHz}, \mathrm{CDCl}_{3}\right)$ : see below; ${ }^{31} \mathrm{P}$ NMR (161.7 MHz, $\left.\mathrm{CDCl}_{3}\right)$ : see below; IR (neat): 1694, 1516, 1472, 1389, 1290, 1165, 1084, 962, 860, $689 \mathrm{~cm}^{-1}$; HRMS (ESI) $\mathrm{m} / z:[\mathrm{M}+\mathrm{H}]^{+}$Calcd for $\mathrm{C}_{23} \mathrm{H}_{29} \mathrm{~F}_{6} \mathrm{~N}_{3} \mathrm{O}_{4} \mathrm{P} 556.1794$, Found: 556.1801.

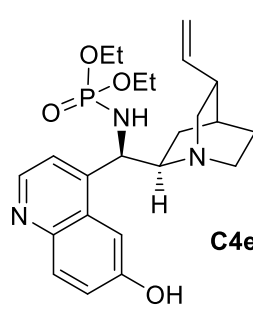

Catalyst C4e was obtained as foamy solid $(0.14 \mathrm{~g}, 32 \%$ yield $)$, m.p. $=91-92{ }^{\circ} \mathrm{C}$. $[\alpha]_{\mathrm{D}}{ }^{20}=+6.4\left(c=0.17, \mathrm{CHCl}_{3}\right) .{ }^{1} \mathrm{H}$ NMR $\left(400 \mathrm{MHz}, \mathrm{CDCl}_{3}\right)$ : see below; ${ }^{13} \mathrm{C}$ NMR (100 MHz, $\left.\mathrm{CDCl}_{3}\right)$ : see below; ${ }^{31} \mathrm{P} \mathrm{NMR}\left(161.7 \mathrm{MHz}, \mathrm{CDCl}_{3}\right)$ : see below; c4e IR (neat): 1620, 1514, 1395, 1281, 1215, 1022, 970, 853, 723, $627 \mathrm{~cm}^{-1}$; HRMS (ESI) $m / z:[\mathrm{M}+\mathrm{H}]^{+}$Calcd for $\mathrm{C}_{23} \mathrm{H}_{33} \mathrm{~N}_{3} \mathrm{O}_{4} \mathrm{P} 446.2203$, Found: 446.2203 . 


\section{The synthesis of $\alpha$-azido ketones 1}

All known $\alpha$-azido ketones $\mathbf{1 a}, \mathbf{1 b}, \mathbf{1 d}, \mathbf{1 g}$ and $\mathbf{1 h}$ were prepared according to the literature report, ${ }^{4}$ Several unknown $\alpha$-azido ketones 1c, 1e, and 1f were prepared following a similar procedure, as shown below:

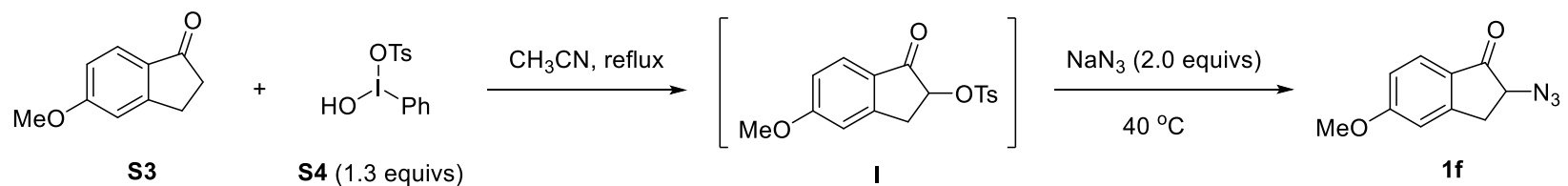

To a $50 \mathrm{~mL}$ three-necked flask were added 5-methoxyl-1-indanone $\mathbf{S 3}(0.81 \mathrm{~g}, 5.0 \mathrm{mmol})$ and $\mathrm{CH}_{3} \mathrm{CN}(50 \mathrm{~mL})$, followed by the addition of $\mathbf{S 4}^{5}(2.55 \mathrm{~g}, 6.5 \mathrm{mmol})$ at room temperature. The reaction was refluxed (oil bath) for $5 \mathrm{~h}$ until full consumption of $\mathbf{S 3}$ by TLC analysis. After cooling to $40{ }^{\circ} \mathrm{C}, \mathrm{NaN}_{3}\left(0.65 \mathrm{~g}, 10 \mathrm{mmol}, 2.0\right.$ equivs) was added. The resulting mixture was kept stirring at $40{ }^{\circ} \mathrm{C}$ (oil bath) until full consumption of intermediate I by TLC analysis (about $8 \mathrm{~h}$ ). After evaporating solvent, the residue was dissolved in $\mathrm{CH}_{2} \mathrm{Cl}_{2}$, and washed with water $(20 \mathrm{~mL} \times 3)$. The organic layer was dried over $\mathrm{Na}_{2} \mathrm{SO}_{4}$, and concentrated in vacuo to give the crude product, which was purified by silica gel column chromatography using PE:EtOAc $(10: 1, \mathrm{v} / \mathrm{v})$ as eluent to furnish the desired product If as a white solid in $57 \%$ yield $(0.58 \mathrm{~g})$, m.p. $=73-75{ }^{\circ} \mathrm{C} .{ }^{1} \mathrm{H} \mathrm{NMR}\left(400 \mathrm{MHz}, \mathrm{CDCl}_{3}\right): \delta 7.73-7.70$ (m, 1H), 6.95-6.92 (m, 1H), 6.87-6.86 (m, 1H), 4.31-4.27 (m, 1H), $3.90(\mathrm{~s}, 3 \mathrm{H}), 3.44$ (ABd, $J=17.2$ $\mathrm{Hz}, J=8.0 \mathrm{~Hz}, 1 \mathrm{H}), 2.87(\mathrm{ABd}, J=17.4 \mathrm{~Hz}, J=4.4 \mathrm{~Hz}, 1 \mathrm{H}) ;{ }^{13} \mathrm{C} \mathrm{NMR}\left(100 \mathrm{MHz}, \mathrm{CDCl}_{3}\right): \delta 199.6$, 166.3, 154.3, 127.3, 126.4, 116.2, 109.6, 62.0, 55.7, 33.0. HRMS (ESI) $m / z:[\mathrm{M}+\mathrm{Na}]^{+}$Calcd for $\mathrm{C}_{10} \mathrm{H}_{9} \mathrm{~N}_{3} \mathrm{NaO}_{2} 226.0587$, Found: 226.0585.

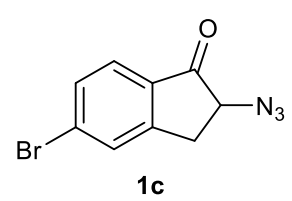

Substrate 1c was prepared in $25 \%$ yield $(0.14 \mathrm{~g})$ by using above synthetic procedure, white solid, m.p. $=93-95{ }^{\circ} \mathrm{C} .{ }^{1} \mathrm{H}$ NMR $\left(300 \mathrm{MHz}, \mathrm{CDCl}_{3}\right): \delta 7.67-7.64$ (m, 2H), 7.59-7.56 (m, 1H), 4.32 (dd, $J=8.2 \mathrm{~Hz}, J=4.5 \mathrm{~Hz}, 1 \mathrm{H}), 3.48$ (ABd, $J=$ $17.2 \mathrm{~Hz}, J=8.4 \mathrm{~Hz}, 1 \mathrm{H}), 2.92(\mathrm{ABd}, J=17.4 \mathrm{~Hz}, J=4.5 \mathrm{~Hz}, 1 \mathrm{H}) ;{ }^{13} \mathrm{C} \mathrm{NMR}\left(100 \mathrm{MHz}, \mathrm{CDCl}_{3}\right): \delta$ 200.6, 152.7, 133.0, 131.9, 131.5, 129.9, 125.8, 61.7, 32.7. HRMS (DART) $m / z:[\mathrm{M}+\mathrm{H}]^{+}$Calcd for $\mathrm{C}_{9} \mathrm{H}_{7} \mathrm{BrN}_{3} \mathrm{O} 251.9767$; Found: 251.9766.

\footnotetext{
${ }^{4}$ (a) Ding, P.-G.; Zhou, F.; Wang, X.; Zhao, Q.-H.; Yu, J.-S.; Zhou, J. Chem. Sci., 2020, 11, 3852-3861. (b) Lee, J. C.; Kim, S.; Shin, W. C. Synth. Comm. 2000, 30, 4271-4275.

5 S4 was prepared according to the literature method: Koser, G. F.; Wettach, R. H. J. Org. Chem. 1977, 42, 1476-1478.
} 


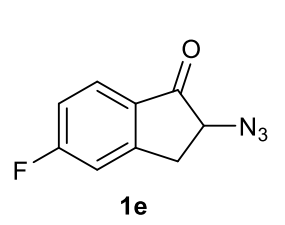

Substrate 1e was prepared in $43 \%$ yield $(0.41 \mathrm{~g})$ by using above synthetic procedure, white solid, m.p. $=50-52{ }^{\circ} \mathrm{C} .{ }^{1} \mathrm{H}$ NMR $\left(300 \mathrm{MHz}, \mathrm{CDCl}_{3}\right): \delta 7.84-7.79$ (m, 1H), 7.16-7.09 (m, 2H), $4.34(\mathrm{dd}, J=8.1 \mathrm{~Hz}, J=4.5 \mathrm{~Hz}, 1 \mathrm{H}), 3.50(\mathrm{ABd}, J=$ $17.4 \mathrm{~Hz}, J=8.1 \mathrm{~Hz}, 1 \mathrm{H}), 2.92(\mathrm{ABd}, J=17.4 \mathrm{~Hz}, J=4.5 \mathrm{~Hz}, 1 \mathrm{H}) ;{ }^{13} \mathrm{C}$ NMR $(100$ $\left.\mathrm{MHz}, \mathrm{CDCl}_{3}\right): \delta 199.7,167.9\left(\mathrm{~d}, J_{\mathrm{C}-\mathrm{F}}=257.1 \mathrm{~Hz}, 1 \mathrm{C}\right), 154.2\left(\mathrm{~d}, J_{\mathrm{C}-\mathrm{F}}=10.4 \mathrm{~Hz}, 1 \mathrm{C}\right), 130.6\left(\mathrm{~d}, J_{\mathrm{C}-\mathrm{F}}=\right.$ $2.0 \mathrm{~Hz}, 1 \mathrm{C}), 127.2\left(\mathrm{~d}, J_{\mathrm{C}-\mathrm{F}}=10.6 \mathrm{~Hz}, 1 \mathrm{C}\right), 116.7\left(\mathrm{~d}, J_{\mathrm{C}-\mathrm{F}}=23.7 \mathrm{~Hz}, 1 \mathrm{C}\right), 113.4\left(\mathrm{~d}, J_{\mathrm{C}-\mathrm{F}}=22.5 \mathrm{~Hz}, 1 \mathrm{C}\right)$, 61.9, $33.0\left(\mathrm{~d}, J_{\mathrm{C}-\mathrm{F}}=2.1 \mathrm{~Hz}, 1 \mathrm{C}\right) ;{ }^{19} \mathrm{~F}$ NMR $\left(282 \mathrm{MHz}, \mathrm{CDCl}_{3}\right): \delta-99.85(\mathrm{~s}, 1 \mathrm{~F})$. HRMS (ESI) $m / z$ : $[\mathrm{M}+\mathrm{Na}]^{+}$Calcd for $\mathrm{C}_{9} \mathrm{H}_{6} \mathrm{FN}_{3} \mathrm{NaO} 214.0387$; Found: 214.0389 . 


\section{Selected condition optimization}

Given that only moderate yield and stereoselectivity of the target was obtained under the catalysis of our new synthesized phosphoramide C4b (entry 1), a variety of additives were evaluated to improve the reaction outcomes, in the presence of $10 \mathrm{~mol} \% \mathbf{C 4 b}$ in $\mathrm{CH}_{2} \mathrm{Cl}_{2}$ at $0{ }^{\circ} \mathrm{C}$, as shown in Table S1. It was found that the addition of either $5 \AA$ molecular sieve (MS) or $\mathrm{LiCl}$ could greatly improve the reactivity and dr value, but no improvement for the ee value of major isomer of product (entries 2-3 vs entry 1). Subsequently, phenols and fluoroalcohols were examined as the additives (entries 4-6). Intriguingly, the reversal of diastereoselectivity was observed in these cases, and $\left(\mathrm{CF}_{3}\right)_{2} \mathrm{CHOH}$ (HFIP) proves to be best one, affording syn-3a with 1.7:1 syn/anti and 98\% ee (syn) (entry 6). The diastereoselectivity of the reaction could be improved by increasing the amount of HFIP (entries 7-9), and the use of $80 \mathrm{~mol} \%$ of HFIP delivered syn-3a with $91 \%$ yield, 3.5:1 dr and 99\% ee (entry 8). Decreasing the reaction temperature to $-40{ }^{\circ} \mathrm{C}$, the ratio of syn/anti and ee value of product 3a could further improved to $7.6: 1$ and $99 \%$, albeit with prolonging the reaction time.

Table S1. The evaluation of fluoroalcohols for syn-selective Michael addition.

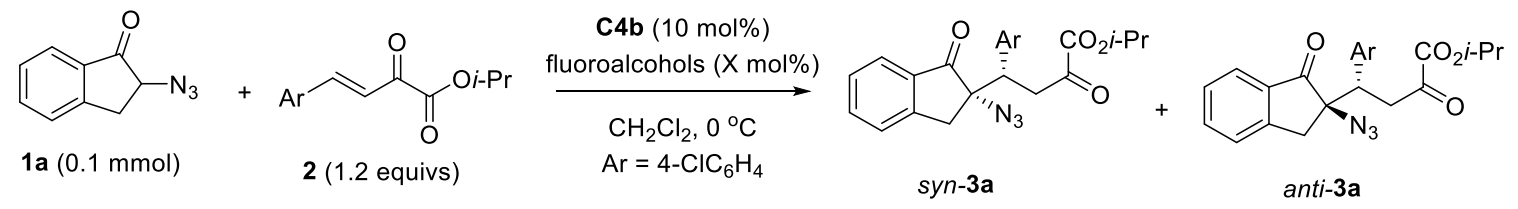

\begin{tabular}{ccccccc}
\hline Entry & Additive & $\mathrm{X}(\mathrm{mol} \%)$ & Time (day) & ${\text { Yield }(\%)^{a}}^{a}$ & syn:anti $^{b}$ & Ee $(\%)^{c}$ \\
\hline 1 & - & & 4 & 54 & $1: 2.2$ & $41 / 64$ \\
2 & $5 \AA \mathrm{MS}$ & $50 \mathrm{mg}$ & 2 & 74 & $1: 4.0$ & $81 / 64$ \\
3 & $\mathrm{LiCl}$ & 10 & 2 & 99 & $1: 4.3$ & $2 / 2$ \\
4 & $4-\mathrm{CF}_{3} \mathrm{C}_{6} \mathrm{H}_{4} \mathrm{OH}$ & 10 & 2 & 97 & $1.2: 1$ & $96 / 66$ \\
5 & $\mathrm{CF}_{3} \mathrm{CH}_{2} \mathrm{OH}$ & 10 & 2 & 93 & $1.1: 1$ & $97 / 68$ \\
6 & $\left(\mathrm{CF}_{3}\right)_{2} \mathrm{CHOH}$ & 10 & 1 & 91 & $1.7: 1$ & $98 / 74$ \\
7 & $\left(\mathrm{CF}_{3}\right)_{2} \mathrm{CHOH}$ & 60 & 2 & 98 & $2.3: 1$ & $91 / 54$ \\
8 & $\left(\mathrm{CF}_{3}\right)_{2} \mathrm{CHOH}$ & 80 & 2 & 91 & $3.5: 1$ & $99 / 77$ \\
9 & $\left(\mathrm{CF}_{3}\right)_{2} \mathrm{CHOH}$ & 100 & 2 & 93 & $3.1: 1$ & $98 / 75$ \\
$10^{d}$ & $\left(\mathrm{CF}_{3}\right)_{2} \mathrm{CHOH}$ & 80 & 6 & 99 & $7.6: 1$ & $99 /-$ \\
\hline
\end{tabular}

\footnotetext{
${ }^{a}$ Isolated yield of the mixture of diastereoisomers are shown. ${ }^{b}$ Determined by ${ }^{1} \mathrm{H}$ NMR analysis of the crude reaction mixtures. ${ }^{c}$ Determined by chiral HPLC analysis, for major diastereomer. ${ }^{d}$ At $-40{ }^{\circ} \mathrm{C}$.
} 


\section{The preparation of racemic Michael adducts 3 and unsuccessful examples}

\subsection{General procedure for the synthesis of racemic product $\mathrm{rac}-3 \mathrm{~m}$}

The synthesis of racemic products was obtained by using $20 \mathrm{~mol} \%$ of bifunctional tertiary amine-thiourea Cat. $\mathbf{I}^{6}$ as the catalyst in $\mathrm{CH}_{2} \mathrm{Cl}_{2}$ at $0{ }^{\circ} \mathrm{C}$ to room temperature. In the following, we provided the procedure for the synthesis of $r a c-3 m$ as an example, and only 1:1.6 $\mathrm{dr}$ for $r a c-3 \mathbf{m}$ was obtained. The poor $\mathrm{dr}$ suggested trivial substrate bias in the diastereoselectivity of this reaction. ${ }^{7}$

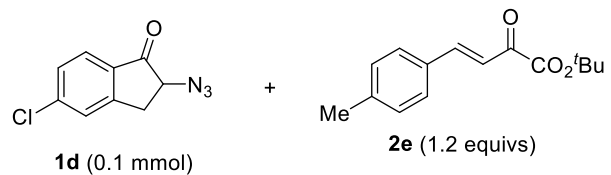

$1 \mathrm{~d}(0.1 \mathrm{mmol})$

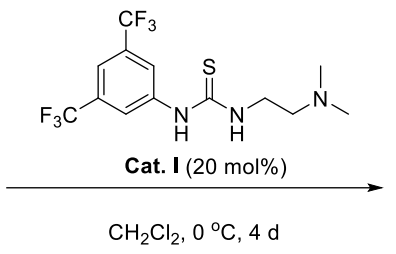

$\mathrm{CH}_{2} \mathrm{Cl}_{2}, 0^{\circ} \mathrm{C}, 4 \mathrm{~d}$

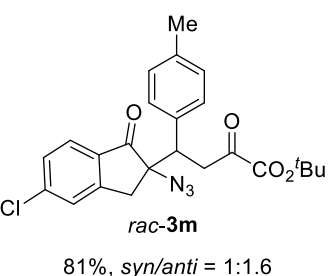

$81 \%$, syn/anti $=1: 1.6$

General procedure: To a $5.0 \mathrm{~mL}$ vial were added tertiary amine-thiourea catalyst Cat. I $(7.2 \mathrm{mg}$, $0.02 \mathrm{mmol}, 20 \mathrm{~mol} \%), \alpha$-azido indanones $1 \mathbf{d}(0.1 \mathrm{mmol}, 1.0$ equiv) and $\beta, \gamma$-unsaturated $\alpha$-ketoesters $2 \mathrm{e}\left(0.12 \mathrm{mmol}, 1.2\right.$ equivs), followed by the addition of anhydrous $\mathrm{CH}_{2} \mathrm{Cl}_{2}(1.0 \mathrm{~mL})$. The reaction mixture was stirred at $0{ }^{\circ} \mathrm{C}$. After full consumption of $\alpha$-azido ketones $1 \mathrm{~d}$ by TLC analysis, the reaction mixture was concentrated under vacuo. The obtained residue was then purified by silica gel column chromatography using PE: $\mathrm{Et}_{2} \mathrm{O}(10: 1, \mathrm{v} / \mathrm{v})$ as the eluent to afford the desired products rac-3m in $81 \%$ yield. The ${ }^{1} \mathrm{H}$ NMR analysis of the crude mixture revealed that the dr value was 1:1.6 syn/anti.

\subsection{Unsuccessful examples for other $\alpha$-azido ketones}

Several different types of $\alpha$-azido ketones were also examind, unfortunately, no desired targets were obtained in all cases, under the action of either Condition A or Condition B.

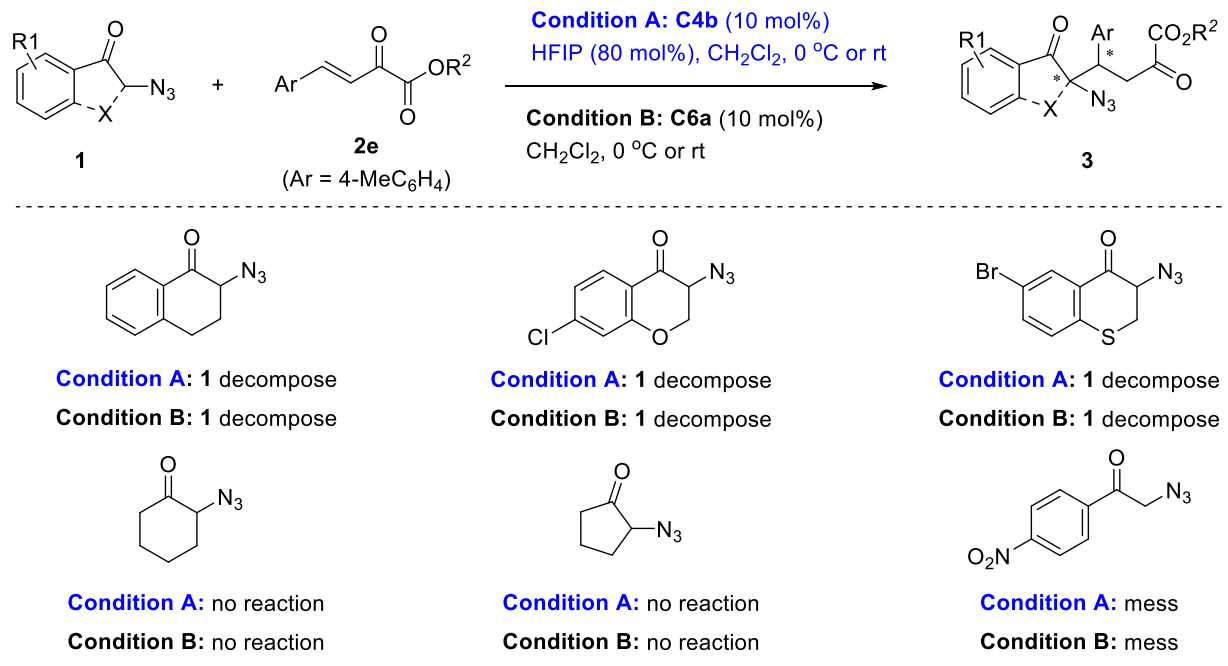

${ }^{6}$ Cat. I was prepared according to the reported procedure: Opalka, S. M.; Steinbacher, J. L.; Lambiris, B. A.; McQuade, D. T. J. Org. Chem. 2011, 76, 6503-6517.

7 Masamune, S.; Choy, W.; Petersen, J. S.; Sita, L. R. Angew. Chem. Int. Ed. 1985, 24, 1-30. 


\section{General procedure for syn-selective Michael addition.}

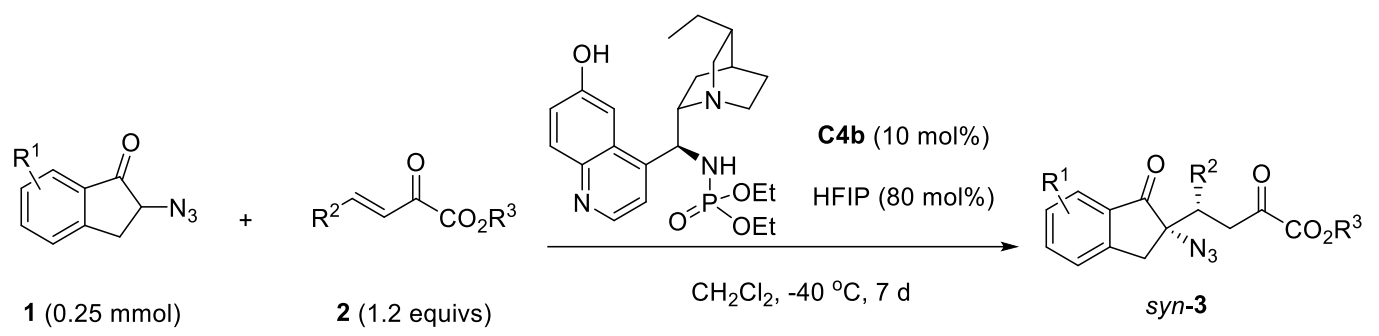

To a $5.0 \mathrm{~mL}$ vial were added tertiary amine-phosphoramide catalyst $\mathbf{C 4 b}(11.2 \mathrm{mg}, 0.025 \mathrm{mmol}$, $10 \mathrm{~mol} \%), \alpha$-azido indanones 1 (0.25 mmol, 1.0 equiv) and HFIP (22.5 $\mu \mathrm{L}, 80 \mathrm{~mol} \%)$, followed by the addition of anhydrous $\mathrm{CH}_{2} \mathrm{Cl}_{2}(2.5 \mathrm{~mL})$ except as noted. The reaction mixture was stirred at $-40{ }^{\circ} \mathrm{C}$ for $30 \mathrm{~min}$, and $\beta, \gamma$-unsaturated $\alpha$-ketoesters 2 (0.30 mmol, 1.2 equivs) was added. After full consumption of $\alpha$-azido ketones $\mathbf{1}$ as indicated by TLC analysis, the reaction mixture was rapidly passed through a short pad of silica gel, and washed with the mixed solvent of PE and EtOAc (4:1, $\mathrm{v} / \mathrm{v})$. The obtained organic solution was concentrated in vacuo to give the crude product. To determine the diastereoselectivity of the product, the residue was first dissolved in $\mathrm{CDCl}_{3}$, and took some samples for ${ }^{1} \mathrm{H}$ NMR analysis. Then the sample for analysis and the rest of crude residue were recombined and purified by silica gel column chromatography using the indicated eluent to afford the desired products syn-3. [Note: all the yields shown here for syn-product $\mathbf{3}$ refer to the isolated yields of the mixture of diastereoisomers, unless otherwise noted.]

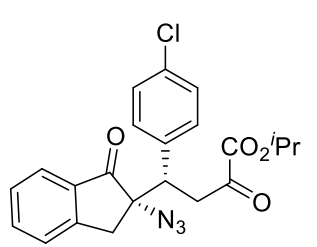

syn-3a

Column chromatography with $\mathrm{PE} / \mathrm{Et}_{2} \mathrm{O}(10: 1$, v/v) afforded product syn-3a in $99 \%$ yield (106.3 mg) as light yellow liquid. ${ }^{1} \mathrm{H}$ NMR analysis of the crude product revealed that the dr value was 8:1. HPLC analysis [Chiralcel OZ-H, 3\% ${ }^{i} \mathrm{PrOH} / \mathrm{h}$ exane, $1.0 \mathrm{~mL} / \mathrm{min}, 230 \mathrm{~nm}$; major diastereomer: $\mathrm{t}_{\mathrm{r}}$ (major) $=19.32 \mathrm{~min}$, $\left.\mathrm{t}_{\mathrm{r}}(\operatorname{minor})=17.00 \mathrm{~min}\right]$ gave the isomeric composition of major diastereomer: $98 \%$ ee; $[\alpha]_{\mathrm{D}}^{20}=+91.3\left(c=0.9, \mathrm{CHCl}_{3}\right) .{ }^{1} \mathrm{H} \mathrm{NMR}\left(300 \mathrm{MHz}, \mathrm{CDCl}_{3}\right): \delta 7.79(\mathrm{~d}, J=7.5 \mathrm{~Hz}, 1 \mathrm{H})$, 7.65-7.61 (m, 1H), 7.44-7.36 (m, 2H), $7.23(\mathrm{~s}, 4 \mathrm{H}), 5.08-5.00(\mathrm{~m}, 1 \mathrm{H}), 3.96(\mathrm{dd}, J=10.5 \mathrm{~Hz}, J=3.6$ $\mathrm{Hz}, 1 \mathrm{H}), 3.49(\mathrm{ABd}, J=18.1 \mathrm{~Hz}, J=10.8 \mathrm{~Hz}, 1 \mathrm{H}), 3.44(\mathrm{AB}, J=17.4 \mathrm{~Hz}, 1 \mathrm{H}), 3.10(\mathrm{ABd}, J=18.0$ $\mathrm{Hz}, J=3.6 \mathrm{~Hz}, 1 \mathrm{H}), 3.00(\mathrm{AB}, J=17.4 \mathrm{~Hz}, 1 \mathrm{H}), 1.28(\mathrm{~d}, J=1.5 \mathrm{~Hz}, 3 \mathrm{H}), 1.26(\mathrm{~d}, J=1.5 \mathrm{~Hz}, 3 \mathrm{H})$; ${ }^{13} \mathrm{C}$ NMR (100 MHz, $\left.\mathrm{CDCl}_{3}\right): \delta 200.7,191.4,160.1,150.9,136.5,136.1,134.6,133.7,130.2,128.8$, 128.5, 126.5, 125.0, 71.4, 71.1, 44.0, 39.8, 36.6, 21.5, 21.5; IR (neat): 2099, 1717, 1605, 1493, 1369, 1221, 1094, 910, 739, $559 \mathrm{~cm}^{-1}$; HRMS (ESI) $\mathrm{m} / z$ : $[\mathrm{M}+\mathrm{Na}]^{+}$Calcd for $\mathrm{C}_{22} \mathrm{H}_{20} \mathrm{ClN}_{3} \mathrm{NaO}_{4} 448.1035$; Found: 448.1037. 


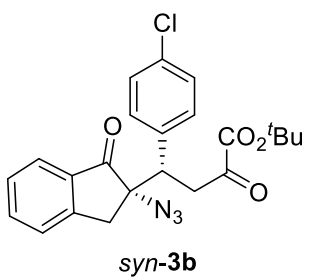

syn-3b

Column chromatography with $\mathrm{PE} / \mathrm{Et}_{2} \mathrm{O}(10: 1, \mathrm{v} / \mathrm{v})$ afforded product syn-3b in $97 \%$ yield $(106.7 \mathrm{mg})$ as light yellow liquid. ${ }^{1} \mathrm{H}$ NMR analysis of the crude product revealed that the dr value was 8:1. HPLC analysis [Chiralcel OZ-H, 3\% ${ }^{i} \mathrm{PrOH} / \mathrm{hexane}, 1.0 \mathrm{~mL} / \mathrm{min}, 230 \mathrm{~nm}$; major diastereomer: $\mathrm{t}_{\mathrm{r}}$ (major) $=15.78 \mathrm{~min}$, $\mathrm{t}_{\mathrm{r}}($ minor $)=14.63$ min, minor diastereomer: $\mathrm{t}_{\mathrm{r}}($ major $)=19.04$ min, $\mathrm{t}_{\mathrm{r}}($ minor $)=$ $22.01 \mathrm{~min}]$ gave the isomeric composition of major diastereomer: $99 \%$ ee; $[\alpha]_{\mathrm{D}}{ }^{20}=+98.9(c=0.9$, $\left.\mathrm{CHCl}_{3}\right) .{ }^{1} \mathrm{H} \mathrm{NMR}\left(400 \mathrm{MHz}, \mathrm{CDCl}_{3}\right): \delta 7.79(\mathrm{~d}, J=7.6 \mathrm{~Hz}, 1 \mathrm{H}), 7.64-7.60(\mathrm{~m}, 1 \mathrm{H}), 7.43-7.37(\mathrm{~m}$, 2H), $7.24(\mathrm{~s}, 4 \mathrm{H}), 3.96(\mathrm{dd}, J=10.8 \mathrm{~Hz}, J=3.6 \mathrm{~Hz}, 1 \mathrm{H}), 3.48-3.41(\mathrm{~m}, 2 \mathrm{H}), 3.08-3.03(\mathrm{~m}, 1 \mathrm{H}), 2.99$ (d, $J=17.2 \mathrm{~Hz}, 1 \mathrm{H}), 1.47$ (s, 9H); ${ }^{13} \mathrm{C}$ NMR $\left(100 \mathrm{MHz}, \mathrm{CDCl}_{3}\right): \delta 200.7,192.0,159.9,151.0,136.5$, 136.2, 134.6, 133. 7, 130.2, 128.7, 128.5, 126.5, 124.9, 84.4, 71.4, 44.1, 39.6, 36.5, 27.7; IR (neat): 2099, 1713, 1605, 1492, 1369, 1155, 1012, 835, 743, $662 \mathrm{~cm}^{-1}$; HRMS (ESI) $m / z:[\mathrm{M}+\mathrm{Na}]^{+} \mathrm{Calcd}$ for $\mathrm{C}_{23} \mathrm{H}_{22} \mathrm{ClN}_{3} \mathrm{NaO}_{4} 462.1191$; Found: 462.1192.

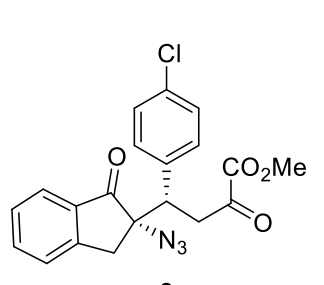

syn-3c

Column chromatography with $\mathrm{PE} / \mathrm{Et}_{2} \mathrm{O}(10: 1, \mathrm{v} / \mathrm{v})$ afforded product syn-3c in $98 \%$ yield (97.2 $\mathrm{mg}$ ) as light yellow liquid. ${ }^{1} \mathrm{H}$ NMR analysis of the crude product revealed that the dr value was 9:1. HPLC analysis [Chiralcel OZ-H, 3\% ${ }^{i} \mathrm{PrOH} / \mathrm{h}$ exane, $1.0 \mathrm{~mL} / \mathrm{min}, 230 \mathrm{~nm}$; major diastereomer: $\mathrm{t}_{\mathrm{r}}$ (major) $=39.88 \mathrm{~min}$, $\left.\mathrm{t}_{\mathrm{r}}(\operatorname{minor})=28.80 \mathrm{~min}\right]$ gave the isomeric composition of major diastereomer: $99 \%$ ee; $[\alpha]_{\mathrm{D}}^{20}=+79.8\left(c=0.8, \mathrm{CHCl}_{3}\right) .{ }^{1} \mathrm{H} \mathrm{NMR}\left(300 \mathrm{MHz}, \mathrm{CDCl}_{3}\right): \delta 7.79(\mathrm{~d}, J=7.8 \mathrm{~Hz}, 1 \mathrm{H})$, 7.65-7.61 (m, 1H), 7.44-7.36 (m, 2H), 7.22 (s, 4H), $3.96(\mathrm{dd}, J=10.5 \mathrm{~Hz}, J=3.3 \mathrm{~Hz}, 1 \mathrm{H}), 3.80(\mathrm{~s}$, $3 \mathrm{H}), 3.53$ (ABd, $J=18.3 \mathrm{~Hz}, J=10.5 \mathrm{~Hz}, 1 \mathrm{H}), 3.43(\mathrm{AB}, J=17.4 \mathrm{~Hz}, 1 \mathrm{H}), 3.15(\mathrm{ABd}, J=18.3 \mathrm{~Hz}, J$ $=3.6 \mathrm{~Hz}, 1 \mathrm{H}), 3.02(\mathrm{AB}, J=17.4 \mathrm{~Hz}, 1 \mathrm{H}) ;{ }^{13} \mathrm{C} \mathrm{NMR}\left(100 \mathrm{MHz}, \mathrm{CDCl}_{3}\right): \delta 200.7,190.6,160.8,150.9$, 136.5, 136.0, 134.6, 133.8, 130.1, 128.8, 128.5, 126.4, 125.0, 71.4, 53.2, 44.0, 40.0, 36.6; IR (neat): 2099, 1715, 1605, 1493, 1364, 1223, 1013, 831, $737 \mathrm{~cm}^{-1}$; HRMS (ESI) $\mathrm{m} / z:[\mathrm{M}+\mathrm{Na}]^{+}$Calcd for $\mathrm{C}_{20} \mathrm{H}_{16} \mathrm{ClN}_{3} \mathrm{NaO}_{4} 420.0722$; Found: 420.0731.

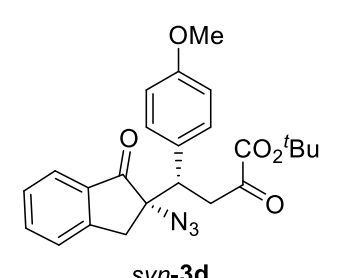

syn-3d

Column chromatography with $\mathrm{PE} / \mathrm{Et}_{2} \mathrm{O}(10: 1, \mathrm{v} / \mathrm{v})$ afforded product syn-3d in $99 \%$ yield (107.8 mg) as light yellow liquid. ${ }^{1} \mathrm{H}$ NMR analysis of the crude product revealed that the dr value was 15:1. HPLC analysis [Chiralcel OZ-H, 3\% ${ }^{i} \mathrm{PrOH} /$ hexane, $1.0 \mathrm{~mL} / \mathrm{min}, 230 \mathrm{~nm}$; major diastereomer: $\mathrm{t}_{\mathrm{r}}$ (major) $=25.63 \mathrm{~min}$, $\mathrm{t}_{\mathrm{r}}$ (minor) $\left.=24.51 \mathrm{~min}\right]$ gave the isomeric composition of major diastereomer: $>99 \%$ ee; $[\alpha]_{\mathrm{D}}{ }^{20}=+109.3\left(c=1.0, \mathrm{CHCl}_{3}\right) .{ }^{1} \mathrm{H}$ NMR $\left(400 \mathrm{MHz}, \mathrm{CDCl}_{3}\right): \delta 7.80-7.77$ 
$(\mathrm{m}, 1 \mathrm{H}), 7.62-7.58(\mathrm{~m}, 1 \mathrm{H}), 7.41-7.35(\mathrm{~m}, 2 \mathrm{H}), 7.21-7.19(\mathrm{~m}, 2 \mathrm{H}), 6.79-6.77(\mathrm{~m}, 2 \mathrm{H}), 3.94(\mathrm{dd}, J=$ $10.6 \mathrm{~Hz}, J=4.0 \mathrm{~Hz}, 1 \mathrm{H}), 3.74(\mathrm{~s}, 3 \mathrm{H}), 3.47(\mathrm{AB}, J=17.6 \mathrm{~Hz}, 1 \mathrm{H}), 3.41(\mathrm{ABd}, J=17.4 \mathrm{~Hz}, J=10.8$ $\mathrm{Hz}, 1 \mathrm{H}), 3.04(\mathrm{ABd}, J=17.6 \mathrm{~Hz}, J=3.6 \mathrm{~Hz}, 1 \mathrm{H}), 2.97(\mathrm{AB}, J=17.6 \mathrm{~Hz}, 1 \mathrm{H}), 1.47(\mathrm{~s}, 9 \mathrm{H}) ;{ }^{13} \mathrm{C} \mathrm{NMR}$ $\left(100 \mathrm{MHz}, \mathrm{CDCl}_{3}\right): \delta 201.2,192.4,160.0,159.1,151.2,136.2,134.8,129.9,129.4,128.3,126.4$, 124.8, 114.0, 84.1, 71.7, 55.2, 44.0, 39.8, 36.5, 27.7; IR (neat): 2995, 2104, 1743, 1609, 1495, 1155, 1090, 839, $758 \mathrm{~cm}^{-1}$; HRMS (ESI) $m / z$ : $[\mathrm{M}+\mathrm{Na}]^{+}$Calcd for $\mathrm{C}_{24} \mathrm{H}_{25} \mathrm{~N}_{3} \mathrm{NaO}_{5}$ 458.1686; Found: 458.1692 .

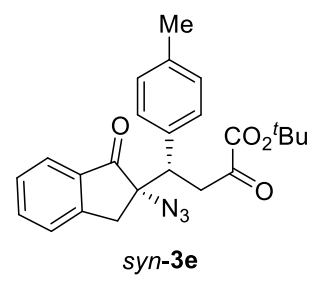

Column chromatography with $\mathrm{PE} / \mathrm{Et}_{2} \mathrm{O}(10: 1, \mathrm{v} / \mathrm{v})$ afforded product syn-3e in $97 \%$ yield (101.9 mg) as light yellow liquid (8 days). ${ }^{1} \mathrm{H}$ NMR analysis of the crude product revealed that the dr value was 15:1. HPLC analysis [Chiralpak $\mathrm{AS}-\mathrm{H}, 3 \%{ }^{i} \mathrm{PrOH} /$ hexane, $1.0 \mathrm{~mL} / \mathrm{min}, 230 \mathrm{~nm}$; major diastereomer: $\mathrm{t}_{\mathrm{r}}$ (major) $=$ $30.22 \mathrm{~min}, \mathrm{t}_{\mathrm{r}}($ minor $)=23.46 \mathrm{~min}$, minor diastereomer: $\mathrm{t}_{\mathrm{r}}($ major $)=15.31 \mathrm{~min}, \mathrm{t}_{\mathrm{r}}$ $($ minor $)=16.45 \mathrm{~min}]$ gave the isomeric composition of major diastereomer: $>99 \%$ ee; $[\alpha]_{\mathrm{D}}{ }^{20}=+100.8$ $\left(c=0.9, \mathrm{CHCl}_{3}\right) .{ }^{1} \mathrm{H} \mathrm{NMR}\left(400 \mathrm{MHz}, \mathrm{CDCl}_{3}\right): \delta 7.79(\mathrm{~d}, J=7.6 \mathrm{~Hz}, 1 \mathrm{H}), 7.62-7.58(\mathrm{~m}, 1 \mathrm{H})$, 7.41-7.36 (m, 2H), $7.18(\mathrm{~d}, J=8.0 \mathrm{~Hz}, 2 \mathrm{H}), 7.06$ (d, $J=7.6 \mathrm{~Hz}, 2 \mathrm{H}), 3.98(\mathrm{dd}, J=10.8 \mathrm{~Hz}, J=3.6 \mathrm{~Hz}$, $1 \mathrm{H}), 3.50(\mathrm{AB}, J=17.2 \mathrm{~Hz}, 1 \mathrm{H}), 3.42(\mathrm{ABd}, J=17.6 \mathrm{~Hz}, J=10.8 \mathrm{~Hz}, 1 \mathrm{H}), 3.01(\mathrm{ABd}, J=17.6 \mathrm{~Hz}, J$ $=3.6 \mathrm{~Hz}, 1 \mathrm{H}), 2.95(\mathrm{AB}, J=17.6 \mathrm{~Hz}, 1 \mathrm{H}), 2.27(\mathrm{~s}, 3 \mathrm{H}), 1.46(\mathrm{~s}, 9 \mathrm{H}) ;{ }^{13} \mathrm{C} \mathrm{NMR}\left(100 \mathrm{MHz}, \mathrm{CDCl}_{3}\right): \delta$ 201.2, 192.3, 160.0, 151.3, 137.5, 136.2, 134.7, 134.5, 129.3, 128.7, 128.3, 126.5, 124.9, 84.1, 71.6, 44.2, 39.8, 36.4, 27.7, 21.0; IR (neat): 2099, 1717, 1611, 1468, 1369, 1252, 1155, 1078, 835, $638 \mathrm{~cm}^{-1}$; HRMS (ESI) $m / z:[\mathrm{M}+\mathrm{Na}]^{+}$Calcd for $\mathrm{C}_{24} \mathrm{H}_{25} \mathrm{~N}_{3} \mathrm{NaO}_{4} 442.1737$; Found: 442.1736.

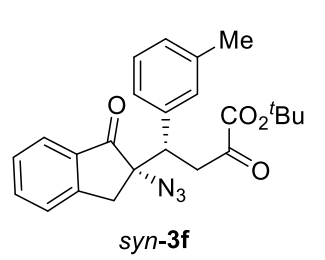

Column chromatography with $\mathrm{PE} / \mathrm{Et}_{2} \mathrm{O}(10: 1, \mathrm{v} / \mathrm{v})$ afforded product syn-3f in $99 \%$ yield (104.0 mg) as light yellow liquid. ${ }^{1} \mathrm{H}$ NMR analysis of the crude product revealed that the dr value was 9:1. HPLC analysis [Chiralcel OZ-H, 3\% ${ }^{i} \mathrm{PrOH} /$ hexane, $1.0 \mathrm{~mL} / \mathrm{min}, 230 \mathrm{~nm}$; major diastereomer: $\mathrm{t}_{\mathrm{r}}$ (major) = $13.94 \mathrm{~min}$, $\mathrm{t}_{\mathrm{r}}($ minor $)=11.66 \mathrm{~min}$, minor diastereomer: $\mathrm{t}_{\mathrm{r}}($ major $)=15.19 \mathrm{~min}, \mathrm{t}_{\mathrm{r}}($ minor $\left.)=12.40 \mathrm{~min}\right]$ gave the isomeric composition of major diastereomer: $99 \%$ ee; $[\alpha]_{\mathrm{D}}{ }^{20}=+107.1\left(c=0.9, \mathrm{CHCl}_{3}\right) .{ }^{1} \mathrm{H} \mathrm{NMR}(400$ $\left.\mathrm{MHz}, \mathrm{CDCl}_{3}\right): \delta 7.80-7.78(\mathrm{~m}, 1 \mathrm{H}), 7.62-7.58(\mathrm{~m}, 1 \mathrm{H}), 7.41-7.34(\mathrm{~m}, 2 \mathrm{H}), 7.15-7.11(\mathrm{~m}, 1 \mathrm{H})$, 7.08-7.06 (m, 2H), 7.02-7.00 (m, 1H), $3.96(\mathrm{dd}, J=10.4 \mathrm{~Hz}, J=4.0 \mathrm{~Hz}, 1 \mathrm{H}), 3.51-3.39(\mathrm{~m}, 2 \mathrm{H})$, 3.08-3.03 (m, 1H), $2.98(\mathrm{~d}, J=17.6 \mathrm{~Hz}, 1 \mathrm{H}), 2.28(\mathrm{~s}, 3 \mathrm{H}), 1.47(\mathrm{~s}, 9 \mathrm{H}) ;{ }^{13} \mathrm{C} \mathrm{NMR}\left(100 \mathrm{MHz}, \mathrm{CDCl}_{3}\right)$ : 
$\delta 201.1,192.3,156.0,151.2,138.1,137.4,136.2,134.8,129.7,128.6,128.4,128.2,126.4,125.8$, 124.8, 84.1, 71.6, 44.7, 39.7, 36.5, 27.7, 21.4; IR (neat): 2097, 1713, 1607, 1369, 1281, 1155, 1034, $748 \mathrm{~cm}^{-1}$; HRMS (ESI) $m / z:[\mathrm{M}+\mathrm{Na}]^{+}$Calcd for $\mathrm{C}_{24} \mathrm{H}_{25} \mathrm{~N}_{3} \mathrm{NaO}_{4} 442.1737$; Found: 442.1738 .

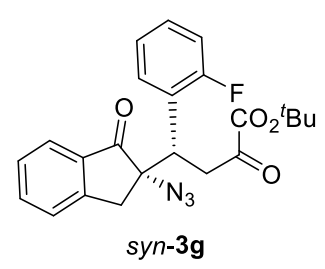

Column chromatography with $\mathrm{PE} / \mathrm{Et}_{2} \mathrm{O}(10: 1, \mathrm{v} / \mathrm{v})$ afforded product $s y n-3 \mathrm{~g}$ in $94 \%$ yield $\left(99.9 \mathrm{mg}\right.$ ) as light yellow liquid. ${ }^{1} \mathrm{H}$ NMR analysis of the crude product revealed that the dr value was 4:1. HPLC analysis [Chiralpak AS-H, 5\% ${ }^{i} \mathrm{PrOH} / \mathrm{hexane}, 1.0 \mathrm{~mL} / \mathrm{min}, 230 \mathrm{~nm}$; major diastereomer: $\mathrm{t}_{\mathrm{r}}$ (major) $=15.94 \mathrm{~min}$, $\left.t_{r}(\operatorname{minor})=12.74 \mathrm{~min}\right]$ gave the isomeric composition of major diastereomer: $86 \%$ ee; $[\alpha]_{D}{ }^{20}=-56.8$ $\left(c=0.6, \mathrm{CHCl}_{3}\right) .{ }^{1} \mathrm{H}$ NMR $\left(300 \mathrm{MHz}, \mathrm{CDCl}_{3}\right): \delta 7.72(\mathrm{~d}, J=7.8 \mathrm{~Hz}, 1 \mathrm{H}), 7.60(\mathrm{t}, J=7.8 \mathrm{~Hz}, 1 \mathrm{H})$, 7.44-7.35 (m, 3H), 7.19-7.15 (m, 1H), $7.06(\mathrm{t}, J=7.5 \mathrm{~Hz}, 1 \mathrm{H}), 6.98-6.92(\mathrm{~m}, 1 \mathrm{H}), 4.10(\mathrm{dd}, J=8.7$ $\mathrm{Hz}, J=5.1 \mathrm{~Hz}, 1 \mathrm{H}), 3.91-3.84(\mathrm{~m}, 1 \mathrm{H}), 3.50-3.35(\mathrm{~m}, 2 \mathrm{H}), 2.99(\mathrm{~d}, J=17.4 \mathrm{~Hz}, 1 \mathrm{H}), 1.52(\mathrm{~s}, 9 \mathrm{H})$; ${ }^{13} \mathrm{C}$ NMR $\left(125 \mathrm{MHz}, \mathrm{CDCl}_{3}\right): \delta 200.5,192.2,161.3\left(\mathrm{~d}, J_{\mathrm{C}-\mathrm{F}}=244.5 \mathrm{~Hz}, 1 \mathrm{C}\right), 159.8,151.1,136.3$, $134.5,129.9\left(\mathrm{~d}, J_{\mathrm{C}-\mathrm{F}}=3.6 \mathrm{~Hz}, 1 \mathrm{C}\right), 129.4\left(\mathrm{~d}, J_{\mathrm{C}-\mathrm{F}}=8.6 \mathrm{~Hz}, 1 \mathrm{C}\right), 128.3,126.4,125.0,124.5\left(\mathrm{~d}, J_{\mathrm{C}-\mathrm{F}}=\right.$ $13.6 \mathrm{~Hz}, 1 \mathrm{C}), 124.2\left(\mathrm{~d}, J_{\mathrm{C}-\mathrm{F}}=3.5 \mathrm{~Hz}, 1 \mathrm{C}\right), 115.8\left(\mathrm{~d}, J_{\mathrm{C}-\mathrm{F}}=23.3 \mathrm{~Hz}, 1 \mathrm{C}\right), 84.4,71.8,39.1\left(\mathrm{~d}, J_{\mathrm{C}-\mathrm{F}}=1.8\right.$ $\mathrm{Hz}, 1 \mathrm{C}), 38.1\left(\mathrm{~d}, J_{\mathrm{C}-\mathrm{F}}=1.8 \mathrm{~Hz}, 1 \mathrm{C}\right), 36.9\left(\mathrm{~d}, J_{\mathrm{C}-\mathrm{F}}=3.1 \mathrm{~Hz}, 1 \mathrm{C}\right), 27.7 ;{ }^{19} \mathrm{~F} \mathrm{NMR}\left(282 \mathrm{MHz}, \mathrm{CDCl}_{3}\right): \delta$ -114.30 -114.38 (m, 1F); IR (neat): 2100, 1715, 1609, 1456, 1252, 1155, 1014, $756 \mathrm{~cm}^{-1}$; HRMS (ESI) $m / z:[\mathrm{M}+\mathrm{Na}]^{+} \mathrm{Calcd}$ for $\mathrm{C}_{23} \mathrm{H}_{22} \mathrm{FN}_{3} \mathrm{NaO}_{4} 446.1487$; Found: 446.1487 .

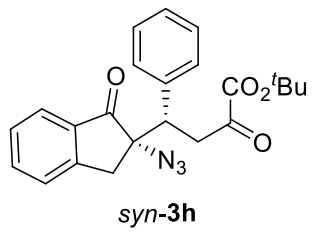

Column chromatography with $\mathrm{PE} / \mathrm{Et}_{2} \mathrm{O}(10: 1, \mathrm{v} / \mathrm{v})$ afforded product syn-3h in 99\% yield (100.6 mg) as light yellow liquid. ${ }^{1} \mathrm{H}$ NMR analysis of the crude product revealed that the dr value was 16:1. HPLC analysis [Chiralcel OZ-H, 3\% ${ }^{i} \mathrm{PrOH} /$ hexane, $1.0 \mathrm{~mL} / \mathrm{min}, 230 \mathrm{~nm}$; major diastereomer: $\mathrm{t}_{\mathrm{r}}$ (major) $=16.07$ $\left.\min , t_{r}(\operatorname{minor})=13.80 \mathrm{~min}\right]$ gave the isomeric composition of major diastereomer: $99 \%$ ee; $[\alpha]_{\mathrm{D}}{ }^{20}=+118.0\left(c=1.0, \mathrm{CHCl}_{3}\right) .{ }^{1} \mathrm{H}$ NMR $\left(400 \mathrm{MHz}, \mathrm{CDCl}_{3}\right): \delta 7.78(\mathrm{~d}, J=$ 7.6 Hz, 1H), 7.61-7.57 (m, 1H), 7.40-7.34 (m, 2H), 7.30-7.18 (m, 5H), $4.00(\mathrm{dd}, J=10.4 \mathrm{~Hz}, J=3.6$ $\mathrm{Hz}, 1 \mathrm{H}), 3.50(\mathrm{AB}, J=17.6 \mathrm{~Hz}, 1 \mathrm{H}), 3.45(\mathrm{ABd}, J=17.6 \mathrm{~Hz}, J=10.4 \mathrm{~Hz}, 1 \mathrm{H}), 3.08(\mathrm{ABd}, J=17.6$ $\mathrm{Hz}, J=4.0 \mathrm{~Hz}, 1 \mathrm{H}), 2.98(\mathrm{AB}, J=17.6 \mathrm{~Hz}, 1 \mathrm{H}), 1.46(\mathrm{~s}, 9 \mathrm{H}) ;{ }^{13} \mathrm{C} \mathrm{NMR}\left(100 \mathrm{MHz}, \mathrm{CDCl}_{3}\right): \delta 201.0$, 192.3, 159.9, 151.2, 137.5, 136.3, 134.7, 128.8, 128.6, 128.3, 127.8, 126.4, 124.9, 84.2, 71.6, 44.7, 39. 7, 36.5, 27.7; IR (neat): 2986, 2095, 1721, 1364, 1157, 1086, 839, $764 \mathrm{~cm}^{-1}$; HRMS (ESI) $\mathrm{m} / z$ : $[\mathrm{M}+\mathrm{Na}]^{+}$Calcd for $\mathrm{C}_{23} \mathrm{H}_{23} \mathrm{~N}_{3} \mathrm{NaO}_{4} 428.1581$; Found: 428.1583 . 


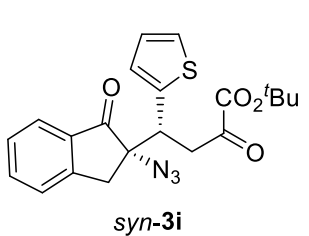

Column chromatography with $\mathrm{PE} / \mathrm{Et}_{2} \mathrm{O}(10: 1, \mathrm{v} / \mathrm{v})$ afforded product $s y n-3 \mathbf{i}$ in 99\% yield (102.7 mg) as white solid, m.p. $=82-85{ }^{\circ} \mathrm{C} .{ }^{1} \mathrm{H}$ NMR analysis of the crude product revealed that the dr value was 14:1. HPLC analysis [Chiralpak AS-H, 3\% ${ }^{i} \mathrm{PrOH} / \mathrm{hexane}, 1.0 \mathrm{~mL} / \mathrm{min}, 230 \mathrm{~nm}$; major diastereomer: $\mathrm{t}_{\mathrm{r}}$ (major) $=$ $25.58 \mathrm{~min}, \mathrm{t}_{\mathrm{r}}($ minor $)=21.27 \mathrm{~min}$, minor diastereomer: $\mathrm{t}_{\mathrm{r}}($ major $\left.)=16.36 \mathrm{~min}, \mathrm{t}_{\mathrm{r}}(\operatorname{minor})=17.69 \mathrm{~min}\right]$ gave the isomeric composition of major diastereomer: $>99 \%$ ee; $[\alpha]_{\mathrm{D}}{ }^{20}=+121.8\left(c=0.7, \mathrm{CHCl}_{3}\right) .{ }^{1} \mathrm{H}$ NMR (400 MHz, $\left.\mathrm{CDCl}_{3}\right): \delta 7.81(\mathrm{~d}, J=7.6 \mathrm{~Hz}, 1 \mathrm{H}), 7.67-7.63(\mathrm{~m}, 1 \mathrm{H}), 7.45-7.40(\mathrm{~m}, 2 \mathrm{H}), 7.19-7.18$ $(\mathrm{m}, 1 \mathrm{H}), 7.03-7.02(\mathrm{~m}, 1 \mathrm{H}), 6.93-6.91(\mathrm{~m}, 1 \mathrm{H}), 4.38(\mathrm{dd}, J=10.6 \mathrm{~Hz}, J=3.6 \mathrm{~Hz}, 1 \mathrm{H}), 3.52(\mathrm{AB}, J=$ $18.0 \mathrm{~Hz}, 1 \mathrm{H}), 3.36(\mathrm{ABd}, J=17.4 \mathrm{~Hz}, J=10.4 \mathrm{~Hz}, 1 \mathrm{H}), 3.02(\mathrm{AB}, J=17.6 \mathrm{~Hz}, 1 \mathrm{H}), 2.91(\mathrm{ABd}, J=$ $17.4 \mathrm{~Hz}, J=3.2 \mathrm{~Hz}, 1 \mathrm{H}), 1.48(\mathrm{~s}, 9 \mathrm{H}) ;{ }^{13} \mathrm{C} \mathrm{NMR}\left(100 \mathrm{MHz}, \mathrm{CDCl}_{3}\right): \delta 200.9191 .4,159.8,151.3$, 140.3, 136.4, 134.6, 128.4, 127.4, 126.8, 126.6, 125.0, 125.0, 84.4, 71.1, 41.1, 39.9, 36.5, 27.7; IR (neat): 2089, 1703, 1474, 1284, 1163, 1053, 835, $664 \mathrm{~cm}^{-1}$; HRMS (ESI) $\mathrm{m} / z:[\mathrm{M}+\mathrm{Na}]^{+} \mathrm{Calcd}$ for $\mathrm{C}_{21} \mathrm{H}_{21} \mathrm{~N}_{3} \mathrm{NaO}_{4} \mathrm{~S}$ 434.1145; Found: 434.1151.

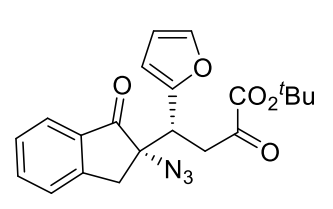

Column chromatography with $\mathrm{PE} / \mathrm{Et}_{2} \mathrm{O}(10: 1, \mathrm{v} / \mathrm{v})$ afforded product syn-3j in $96 \%$ yield $\left(94.9 \mathrm{mg}\right.$ ) as light yellow liquid. ${ }^{1} \mathrm{H}$ NMR analysis of the crude product revealed that the dr value was 6:1. HPLC analysis [Chiralcel OZ-H, 3\% ${ }^{i} \mathrm{PrOH} / \mathrm{hexane}, 1.0 \mathrm{~mL} / \mathrm{min}, 230 \mathrm{~nm}$; major diastereomer: $\mathrm{t}_{\mathrm{r}}$ (major) $=16.33 \mathrm{~min}$, $\mathrm{t}_{\mathrm{r}}($ minor $)=15.02 \mathrm{~min}$, minor diastereomer: $\mathrm{t}_{\mathrm{r}}($ major $)=20.67 \mathrm{~min}, \mathrm{t}_{\mathrm{r}}($ minor $\left.)=15.64 \mathrm{~min}\right]$ gave the isomeric composition of major diastereomer: $99 \%$ ee; $[\alpha]_{D}{ }^{20}=+155.8\left(c=1.0, \mathrm{CHCl}_{3}\right) .{ }^{1} \mathrm{H} \mathrm{NMR}(400$ $\left.\mathrm{MHz}, \mathrm{CDCl}_{3}\right): \delta 7.79(\mathrm{~d}, J=7.6 \mathrm{~Hz}, 1 \mathrm{H}), 7.66-7.62(\mathrm{~m}, 1 \mathrm{H}), 7.44-7.39(\mathrm{~m}, 2 \mathrm{H}), 7.28-7.27(\mathrm{~m}, 1 \mathrm{H})$, 6.30-6.25 (m, 2H), $4.15(\mathrm{dd}, J=10.6 \mathrm{~Hz}, J=3.2 \mathrm{~Hz}, 1 \mathrm{H}), 3.58(\mathrm{AB}, J=18.0 \mathrm{~Hz}, 1 \mathrm{H}), 3.36(\mathrm{ABd}, J=$ $17.8 \mathrm{~Hz}, J=10.4 \mathrm{~Hz}, 1 \mathrm{H}), 3.01(\mathrm{AB}, J=18.0 \mathrm{~Hz}, 1 \mathrm{H}), 2.83(\mathrm{ABd}, J=17.6 \mathrm{~Hz}, J=3.6 \mathrm{~Hz}, 1 \mathrm{H}), 1.49$ (s, 9H); ${ }^{13} \mathrm{C}$ NMR $\left(100 \mathrm{MHz}, \mathrm{CDCl}_{3}\right): \delta 200.9,191.6,159.7,151.5,151.1,142.3,136.4,134.3,128.3$, 126.5, 124.9, 110.4, 109.2, 84.3, 70.4, 38.1, 38.0, 36.6, 27.7; IR (neat): 2099, 1713, 1283, 1155, 1034, 835, $598 \mathrm{~cm}^{-1}$; HRMS (ESI) $\mathrm{m} / z$ : [M+Na] $]^{+}$Calcd for $\mathrm{C}_{21} \mathrm{H}_{21} \mathrm{~N}_{3} \mathrm{NaO}_{5} 418.1373$; Found: 418.1374 .

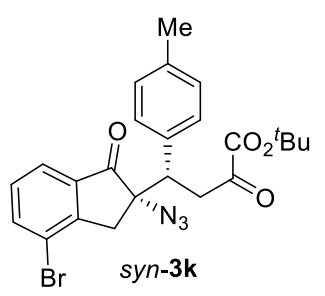

Column chromatography with $\mathrm{PE} / \mathrm{Et}_{2} \mathrm{O}(10: 1, \mathrm{v} / \mathrm{v})$ afforded product syn-3k in $99 \%$ yield $(123.4 \mathrm{mg})$ as light yellow liquid (4 days). ${ }^{1} \mathrm{H}$ NMR analysis of the crude product revealed that the dr value was 14:1. HPLC analysis [Chiralcel OZ-H, 3\% ${ }^{i} \mathrm{PrOH} / \mathrm{hexane}, 1.0 \mathrm{~mL} / \mathrm{min}, 230 \mathrm{~nm}$; major diastereomer: $\mathrm{t}_{\mathrm{r}}$ (major) $=$ 
$16.95 \mathrm{~min}, \mathrm{t}_{\mathrm{r}}($ minor $)=12.53 \mathrm{~min}$, minor diastereomer: $\mathrm{t}_{\mathrm{r}}($ major $)=14.35 \mathrm{~min}, \mathrm{t}_{\mathrm{r}}($ minor $\left.)=13.64 \mathrm{~min}\right]$ gave the isomeric composition of major diastereomer: $>99 \%$ ee; $[\alpha]_{\mathrm{D}}{ }^{20}=+100.8\left(c=0.9, \mathrm{CHCl}_{3}\right) .{ }^{1} \mathrm{H}$ NMR (400 MHz, $\left.\mathrm{CDCl}_{3}\right): \delta 7.77-7.72(\mathrm{~m}, 2 \mathrm{H}), 7.32-7.28(\mathrm{~m}, 1 \mathrm{H}), 7.19-7.17(\mathrm{~m}, 2 \mathrm{H}), 7.08(\mathrm{~d}, J=8.0$ $\mathrm{Hz}, 2 \mathrm{H}), 3.98(\mathrm{dd}, J=10.4 \mathrm{~Hz}, J=3.6 \mathrm{~Hz}, 1 \mathrm{H}), 3.44(\mathrm{ABd}, J=17.4 \mathrm{~Hz}, J=10.4 \mathrm{~Hz}, 1 \mathrm{H}), 3.40(\mathrm{AB}$, $J=18.0 \mathrm{~Hz}, 1 \mathrm{H}), 3.02(\mathrm{ABd}, J=17.6 \mathrm{~Hz}, J=3.6 \mathrm{~Hz}, 1 \mathrm{H}), 2.91(\mathrm{AB}, J=18.0 \mathrm{~Hz}, 1 \mathrm{H}), 2.27(\mathrm{~s}, 3 \mathrm{H})$, 1.47 (s, 9H); ${ }^{13} \mathrm{C}$ NMR (100 MHz, $\left.\mathrm{CDCl}_{3}\right): \delta 200.6,192.1,159.9,151.0,138.8,137.6,136.6,134.1$, 130.0, 129.4, 128.6, 123.6, 121.9, 84.2, 71.6, 44.2, 39.6, 37.6, 27.7, 21.0; IR (neat): 2974, 2118, 1708, 1608, 1431, 1362, 1217, 1105, 1020, $764 \mathrm{~cm}^{-1}$; HRMS (ESI) m/z: [M+Na] Calcd for $\mathrm{C}_{24} \mathrm{H}_{24} \mathrm{BrN}_{3} \mathrm{NaO}_{4}$ 520.0842; Found: 520.0844 .

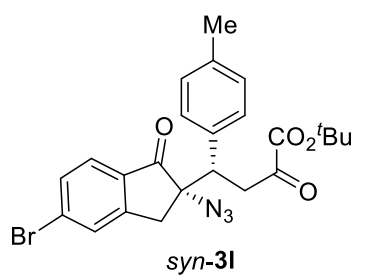

Column chromatography with $\mathrm{PE} / \mathrm{Et}_{2} \mathrm{O}(10: 1, \mathrm{v} / \mathrm{v})$ afforded product syn-3I in $95 \%$ yield (117.9 mg) as light yellow liquid (4 days). ${ }^{1} \mathrm{H}$ NMR analysis of the crude product revealed that the dr value was 8:1. HPLC analysis [Chiralcel $\mathrm{OZ}-\mathrm{H}, 3 \%{ }^{i} \mathrm{PrOH} /$ hexane, $1.0 \mathrm{~mL} / \mathrm{min}, 230 \mathrm{~nm}$; major diastereomer: $\mathrm{t}_{\mathrm{r}}$ (major) $=17.84 \mathrm{~min}, \mathrm{t}_{\mathrm{r}}($ minor $)=13.12 \mathrm{~min}$ ] gave the isomeric composition of major diastereomer: $99 \%$ ee; $[\alpha]_{\mathrm{D}}{ }^{20}=+100.3\left(c=1.0, \mathrm{CHCl}_{3}\right) .{ }^{1} \mathrm{H} \mathrm{NMR}\left(400 \mathrm{MHz}, \mathrm{CDCl}_{3}\right): \delta 7.57-7.55(\mathrm{~m}, 1 \mathrm{H}), 7.46-7.44(\mathrm{~m}$, 2H), 7.07-7.06 (m, 2H), 6.99-6.97 (m, 2H), $3.86(\mathrm{dd}, J=10.6 \mathrm{~Hz}, J=3.6 \mathrm{~Hz}, 1 \mathrm{H}), 3.40-3.31(\mathrm{~m}, 2 \mathrm{H})$, 3.00-2.84 (m, 2H), 2.19 (s, 3H), 1.39 (s, 9H); ${ }^{13} \mathrm{C}$ NMR (100 MHz, $\left.\mathrm{CDCl}_{3}\right): \delta 200.0,192.2,159.9$, 152.7, 137.6, 134.2, 133.6, 132.0, 131. 8, 129.8, 129.4, 128.6, 126.0, 84.2, 71.6, 44.4, 39.7, 36.3, 27.7, 21.0; IR (neat): 3021, 1792, 1705, 1609, 1420, 1225, 1157, $905 \mathrm{~cm}^{-1}$; HRMS (ESI) $m / z:[\mathrm{M}+\mathrm{Na}]^{+}$ Calcd for $\mathrm{C}_{24} \mathrm{H}_{24} \mathrm{BrN}_{3} \mathrm{NaO}_{4}$ 520.0842; Found: 520.0846 .

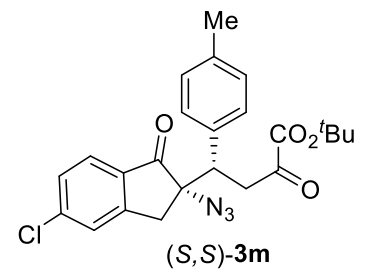

Column chromatography with $\mathrm{PE} / \mathrm{Et}_{2} \mathrm{O}(10: 1$, v/v) afforded product $(S, S)-\mathbf{3 m}$ in $95 \%$ yield (108.1 mg) as light yellow liquid (4.5 days). ${ }^{1} \mathrm{H}$ NMR analysis of the crude product revealed that the dr value was 10:1. HPLC analysis [Chiralcel OZ-H, 3\% ${ }^{i} \mathrm{PrOH} /$ hexane, $1.0 \mathrm{~mL} / \mathrm{min}, 230 \mathrm{~nm}$; major diastereomer: $\mathrm{t}_{\mathrm{r}}$ (major) $=16.77 \min , \mathrm{t}_{\mathrm{r}}($ minor $)=12.48 \mathrm{~min} ;$ major diastereomer: $\mathrm{t}_{\mathrm{r}}($ major $)=14.98 \mathrm{~min}, \mathrm{t}_{\mathrm{r}}($ minor $)=14.18$ $\mathrm{min}]$ gave the isomeric composition of major diastereomer: $98 \%$ ee; $[\alpha]_{\mathrm{D}}^{20}=+112.1\left(c=1.0, \mathrm{CHCl}_{3}\right)$. ${ }^{1} \mathrm{H}$ NMR $\left(400 \mathrm{MHz}, \mathrm{CDCl}_{3}\right): \delta 7.71(\mathrm{~d}, J=8.4 \mathrm{~Hz}, 1 \mathrm{H}), 7.38-7.35(\mathrm{~m}, 2 \mathrm{H}), 7.15(\mathrm{~d}, J=8.0 \mathrm{~Hz}, 2 \mathrm{H})$, $7.06(\mathrm{~d}, J=7.6 \mathrm{~Hz}, 2 \mathrm{H}), 3.94(\mathrm{dd}, J=10.4 \mathrm{~Hz}, J=4.0 \mathrm{~Hz}, 1 \mathrm{H}), 3.48-3.40(\mathrm{~m}, 2 \mathrm{H}), 3.08-3.02(\mathrm{~m}, 1 \mathrm{H})$, $2.94(\mathrm{~d}, J=17.6 \mathrm{~Hz}, 1 \mathrm{H}), 2.27(\mathrm{~s}, 3 \mathrm{H}), 1.47(\mathrm{~s}, 9 \mathrm{H}) ;{ }^{13} \mathrm{C} \mathrm{NMR}\left(100 \mathrm{MHz}, \mathrm{CDCl}_{3}\right): \delta 199.8,192.2$, 
159.9, 152.6, 142.8, 137.6, 134.2, 133.2, 129.4, 129.1, 128.6, 126. 7, 125.9, 84.2, 71.7, 44.3, 39.7, 36.3, 27.7, 21.0; IR (neat): 2099, 1715, 1599, 1369, 1261, 1157, 1055, 907, 820, 745, $604 \mathrm{~cm}^{-1}$; HRMS (ESI) $m / z:[\mathrm{M}+\mathrm{Na}]^{+} \mathrm{Calcd}$ for $\mathrm{C}_{24} \mathrm{H}_{24} \mathrm{ClN}_{3} \mathrm{NaO}_{4} 476.1348$; Found: 476.1350 .

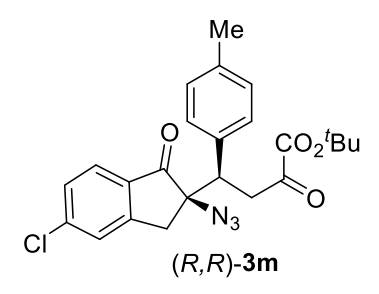

min, $t_{r}($ minor $\left.)=16.23 \mathrm{~min}\right]$ gave the isomeric composition of major diastereomer: $>99 \%$ ee; $[\alpha]_{\mathrm{D}}{ }^{20}=$ $-119.8\left(c=0.3, \mathrm{CHCl}_{3}\right) .{ }^{1} \mathrm{H}$ NMR $\left(400 \mathrm{MHz}, \mathrm{CDCl}_{3}\right): \delta 7.73-7.71(\mathrm{~m}, 1 \mathrm{H}), 7.39-7.37(\mathrm{~m}, 1 \mathrm{H})$, 7.35-7.34 (m, 1H), 7.16-7.13 (m, 2H), 7.08-7.06 (m, 2H), $3.94(\mathrm{dd}, J=10.4 \mathrm{~Hz}, J=3.6 \mathrm{~Hz}, 1 \mathrm{H}), 3.45$ $(\mathrm{AB}, J=17.6 \mathrm{~Hz}, 1 \mathrm{H}), 3.43(\mathrm{ABd}, J=17.6 \mathrm{~Hz}, J=10.4 \mathrm{~Hz}, 1 \mathrm{H}), 3.04(\mathrm{ABd}, J=17.6 \mathrm{~Hz}, J=3.6 \mathrm{~Hz}$, $1 \mathrm{H}), 2.94(\mathrm{AB}, J=17.6 \mathrm{~Hz}, 1 \mathrm{H}), 2.28(\mathrm{~s}, 3 \mathrm{H}), 1.47(\mathrm{~s}, 9 \mathrm{H}) ;{ }^{13} \mathrm{C}$ NMR $\left(100 \mathrm{MHz}, \mathrm{CDCl}_{3}\right): \delta 199.8$, 192.2, 159.9, 152.6, 142.9, 137.7, 134.2, 133.2, 129.4, 129.2, 128.6, 126.7, 126.0, 84.2, 71.7, 44.3, 39.7, 36.3, 27.7, 21.0; IR (neat): 2100, 1715, 1599, 1516, 1420, 1321, 1221, 1072, 745, $604 \mathrm{~cm}^{-1}$; HRMS (ESI) $m / z:[\mathrm{M}+\mathrm{Na}]^{+} \mathrm{Calcd}$ for $\mathrm{C}_{24} \mathrm{H}_{24} \mathrm{ClN}_{3} \mathrm{NaO}_{4} 476.1348$; Found: 476.1340 .

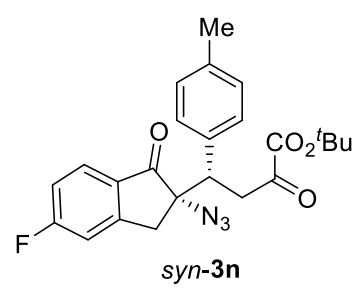

Column chromatography with $\mathrm{PE} / \mathrm{Et}_{2} \mathrm{O}(10: 1, \mathrm{v} / \mathrm{v})$ afforded product syn-3n in $99 \%$ yield (108.8 mg) as light yellow liquid. ${ }^{1} \mathrm{H}$ NMR analysis of the crude product revealed that the dr value was 18:1. HPLC analysis [Chiralcel OZ-H, $3 \%{ }^{i} \mathrm{PrOH} / \mathrm{hexane}, 1.0 \mathrm{~mL} / \mathrm{min}, 230 \mathrm{~nm}$; major diastereomer: $\mathrm{t}_{\mathrm{r}}$ (major) $=$ $19.17 \mathrm{~min}, \mathrm{t}_{\mathrm{r}}($ minor $\left.)=14.58 \mathrm{~min}\right]$ gave the isomeric composition of major diastereomer: $>99 \%$ ee; $[\alpha]_{\mathrm{D}}{ }^{20}=+104.0\left(c=0.9, \mathrm{CHCl}_{3}\right) .{ }^{1} \mathrm{H} \mathrm{NMR}\left(300 \mathrm{MHz}, \mathrm{CDCl}_{3}\right): \delta 7.82-7.78$ (m, 1H), 7.17-7.15 (m, 2H), 7.12-7.00 (m, 4H), $3.95(\mathrm{dd}, J=10.5 \mathrm{~Hz}, J=3.9 \mathrm{~Hz}, 1 \mathrm{H}), 3.51-3.39(\mathrm{~m}$, 2H), 3.09-3.02 (m, 1H), 2.95 (d, $J=17.7 \mathrm{~Hz}, 1 \mathrm{H}), 2.27$ (s, 3H), 1.47 (s, 9H); ${ }^{13} \mathrm{C}$ NMR (100 MHz, $\left.\mathrm{CDCl}_{3}\right): \delta 199.3,192.2,167.9\left(\mathrm{~d}, J_{\mathrm{C}-\mathrm{F}}=257.6 \mathrm{~Hz}, 1 \mathrm{C}\right), 159.9,154.2\left(\mathrm{~d}, J_{\mathrm{C}-\mathrm{F}}=10.6 \mathrm{~Hz}, 1 \mathrm{C}\right), 137.6$, $134.2,131.2\left(\mathrm{~d}, J_{\mathrm{C}-\mathrm{F}}=1.6 \mathrm{~Hz}, 1 \mathrm{C}\right), 129.3,128.6,127.3\left(\mathrm{~d}, J_{\mathrm{C}-\mathrm{F}}=10.7 \mathrm{~Hz}, 1 \mathrm{C}\right), 116.7\left(\mathrm{~d}, J_{\mathrm{C}-\mathrm{F}}=23.8\right.$ $\mathrm{Hz}, 1 \mathrm{C}), 113.2\left(\mathrm{~d}, J_{\mathrm{C}-\mathrm{F}}=22.6 \mathrm{~Hz}, 1 \mathrm{C}\right), 84.2,71.8,44.4,39.7,36.5,27.7,21.0 ;{ }^{19} \mathrm{~F}$ NMR $(282 \mathrm{MHz}$, $\mathrm{CDCl}_{3}$ ): $\delta-99.26 \sim 99.33$ (m, 1F); IR (neat): 2992, 2100, 1746, 1570, 1435, 1260, 1157, 1063, 758 $\mathrm{cm}^{-1}$; GC-MS: 362 (M+1 0.2$), 144$ (100), 129 (39), 116 (22), 105 (7), 89 (17), 63 (4); HRMS (ESI) m/z: $[\mathrm{M}+\mathrm{Na}]^{+} \mathrm{Calcd}$ for $\mathrm{C}_{24} \mathrm{H}_{24} \mathrm{FN}_{3} \mathrm{NaO}_{4} 460.1643$; found: 460.1653 . 


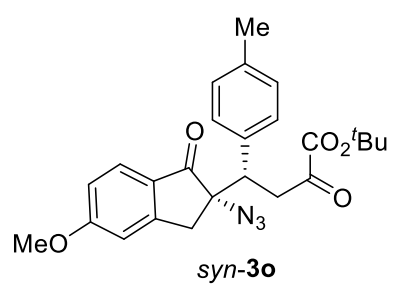

The reaction was conducted with $20 \mathrm{~mol} \% \mathbf{C 4 b}$ and $160 \mathrm{~mol} \%$ HFIP. Column chromatography with $\mathrm{PE} / \mathrm{Et}_{2} \mathrm{O}(10: 1, \mathrm{v} / \mathrm{v})$ afforded product syn-3o in $70 \%$ yield $\left(78.3 \mathrm{mg}\right.$ ) as light yellow liquid. ${ }^{1} \mathrm{H}$ NMR analysis of the crude product revealed that the dr value was 8:1. HPLC analysis [Chiralcel OZ-H, $10 \%{ }^{i} \mathrm{PrOH} /$ hexane, $1.0 \mathrm{~mL} / \mathrm{min}, 230 \mathrm{~nm}$; major diastereomer: $\mathrm{t}_{\mathrm{r}}$ (major) $=$ $14.02 \mathrm{~min}, \mathrm{t}_{\mathrm{r}}(\operatorname{minor})=10.73 \mathrm{~min}$ ] gave the isomeric composition of major diastereomer: $98 \%$ ee; $[\alpha]_{\mathrm{D}}^{20}=+136.9\left(c=0.9, \mathrm{CHCl}_{3}\right) .{ }^{1} \mathrm{H} \mathrm{NMR}\left(400 \mathrm{MHz}, \mathrm{CDCl}_{3}\right): \delta 7.72(\mathrm{~d}, J=8.8 \mathrm{~Hz}, 1 \mathrm{H}), 7.20-7.17$ (m, 2H), $7.07(\mathrm{~d}, J=8.0 \mathrm{~Hz}, 2 \mathrm{H}), 6.92(\mathrm{dd}, J=8.6 \mathrm{~Hz}, J=2.4 \mathrm{~Hz}, 1 \mathrm{H}), 6.78(\mathrm{~d}, J=2.4 \mathrm{~Hz}, 1 \mathrm{H}), 3.96$ (dd, $J=10.8 \mathrm{~Hz}, J=3.6 \mathrm{~Hz}, 1 \mathrm{H}), 3.86(\mathrm{~s}, 3 \mathrm{H}), 3.43(\mathrm{AB}, J=17.6 \mathrm{~Hz}, 1 \mathrm{H}), 3.41(\mathrm{ABd}, J=17.4 \mathrm{~Hz}, J$ $=10.4 \mathrm{~Hz}, 1 \mathrm{H}), 3.00(\mathrm{ABd}, J=17.2 \mathrm{~Hz}, J=3.6 \mathrm{~Hz}, 1 \mathrm{H}), 2.88(\mathrm{AB}, J=18.0 \mathrm{~Hz}, 1 \mathrm{H}), 2.28(\mathrm{~s}, 3 \mathrm{H})$, 1.46 (s, 9H); ${ }^{13} \mathrm{C}$ NMR (100 MHz, $\left.\mathrm{CDCl}_{3}\right): \delta 199.1,192.4,166.5,160.0,154.4,137.4,134.7,129.3$, 128.7, 127.8, 126.7, 116.4, 109.6, 84.1, 71.8, 55.8, 44.1, 39.8, 36.4, 27.7, 21.0; IR (neat): 2095, 1713, 1597, 1491, 1369, 1258, 1152, 1022, 837, $650 \mathrm{~cm}^{-1}$; HRMS (ESI) $\mathrm{m} / z:[\mathrm{M}+\mathrm{Na}]^{+}$Calcd for $\mathrm{C}_{25} \mathrm{H}_{27} \mathrm{~N}_{3} \mathrm{NaO}_{5} 472.1843$; Found: 472.1850 .

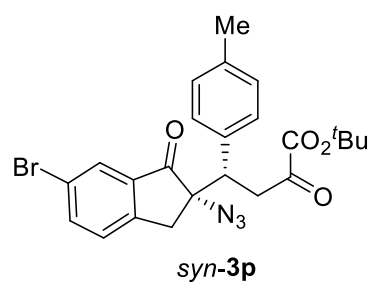

Column chromatography with $\mathrm{PE} / \mathrm{Et}_{2} \mathrm{O}(10: 1, \mathrm{v} / \mathrm{v})$ afforded product $\operatorname{syn}-\mathbf{3 p}$ in $97 \%$ yield (120.7 mg) as light yellow liquid (4 days). ${ }^{1} \mathrm{H}$ NMR analysis of the crude product revealed that the dr value was >20:1. HPLC analysis [Chiralpak AS-H, 5\% ${ }^{i} \mathrm{PrOH} / \mathrm{hexane}, 1.0 \mathrm{~mL} / \mathrm{min}, 230 \mathrm{~nm}$; major diastereomer: $\mathrm{t}_{\mathrm{r}}$ (major) $=22.42 \mathrm{~min}, \mathrm{t}_{\mathrm{r}}($ minor $\left.)=13.96 \mathrm{~min}\right]$ gave the isomeric composition of major diastereomer: $99 \%$ ee; $[\alpha]_{\mathrm{D}}{ }^{20}=+109.1\left(c=1.1, \mathrm{CHCl}_{3}\right) .{ }^{1} \mathrm{H}$ NMR $\left(400 \mathrm{MHz}, \mathrm{CDCl}_{3}\right): \delta 7.90(\mathrm{~d}, J=$ $2.0 \mathrm{~Hz}, 1 \mathrm{H}), 7.69(\mathrm{dd}, J=8.2 \mathrm{~Hz}, J=2.0 \mathrm{~Hz}, 1 \mathrm{H}), 7.26-7.23(\mathrm{~m}, 1 \mathrm{H}), 7.15-7.13(\mathrm{~m}, 2 \mathrm{H}), 7.06(\mathrm{~d}, J=$ $8.0 \mathrm{~Hz}, 2 \mathrm{H}), 3.93(\mathrm{dd}, J=10.4 \mathrm{~Hz}, J=3.6 \mathrm{~Hz}, 1 \mathrm{H}), 3.425(\mathrm{ABd}, J=17.6 \mathrm{~Hz}, J=10.4 \mathrm{~Hz}, 1 \mathrm{H}), 3.424$ $(\mathrm{AB}, J=17.6 \mathrm{~Hz}, 1 \mathrm{H}), 3.05(\mathrm{ABd}, J=17.6 \mathrm{~Hz}, J=3.6 \mathrm{~Hz}, 1 \mathrm{H}), 2.91(\mathrm{AB}, J=17.6 \mathrm{~Hz}, 1 \mathrm{H}), 2.27(\mathrm{~s}$, 3H), 1.47 (s, 9H); ${ }^{13} \mathrm{C}$ NMR (100 MHz, $\left.\mathrm{CDCl}_{3}\right): \delta$ 199. 9, 192.2, 159.9, 149.8, 138.9, 137.6, 136.5, 134.2, 129.4, 128.6, 128.0, 127.7, 122.4, 84.2, 72.0, 44.4, 39.7, 36.3, 27.7, 21.0; IR (neat): 2099, 1715, 1599, 1417, 1256, 1157, 1034, 835, $683 \mathrm{~cm}^{-1}$; HRMS (ESI) $\mathrm{m} / \mathrm{z}:[\mathrm{M}+\mathrm{Na}]^{+} \mathrm{Calcd}$ for $\mathrm{C}_{24} \mathrm{H}_{24} \mathrm{BrN}_{3} \mathrm{NaO}_{4}$ 520.0842; Found: 520.0849. 


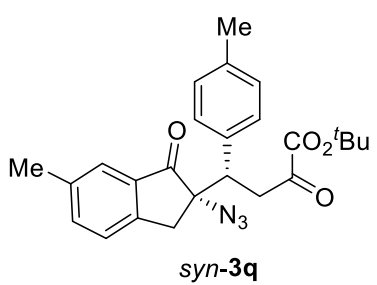

syn-3q

Column chromatography with $\mathrm{PE} / \mathrm{Et}_{2} \mathrm{O}(10: 1, \mathrm{v} / \mathrm{v})$ afforded product syn-3q in 94\% yield (102.2 $\mathrm{mg}$ ) as light yellow liquid. ${ }^{1} \mathrm{H}$ NMR analysis of the crude product revealed that the dr value was >20:1. HPLC analysis [Chiralcel OZ-H, $5 \%{ }^{i} \mathrm{PrOH} /$ hexane, $1.0 \mathrm{~mL} / \mathrm{min}, 230 \mathrm{~nm}$; major diastereomer: $\mathrm{t}_{\mathrm{r}}$ (major) $=$ $29.31 \mathrm{~min}, \mathrm{t}_{\mathrm{r}}($ minor $)=24.21 \mathrm{~min}$, minor diastereomer: $\mathrm{t}_{\mathrm{r}}($ major $)=13.53$ min, $t_{r}($ minor $\left.)=15.75 \mathrm{~min}\right]$ gave the isomeric composition of major diastereomer: $>99 \%$ ee; $[\alpha]_{\mathrm{D}}^{20}=$ $+114.2\left(c=1.0, \mathrm{CHCl}_{3}\right) .{ }^{1} \mathrm{H}$ NMR $\left(400 \mathrm{MHz}, \mathrm{CDCl}_{3}\right): \delta 7.59-7.58(\mathrm{~m}, 1 \mathrm{H}), 7.43-7.41(\mathrm{~m}, 1 \mathrm{H})$, 7.27-7.25 (m, 1H), 7.20-7.18 (m, 2H), 7.07 (d, $J=8.0 \mathrm{~Hz}, 2 \mathrm{H}), 3.97$ (dd, $J=10.8 \mathrm{~Hz}, J=3.6 \mathrm{~Hz}, 1 \mathrm{H})$, $3.45(\mathrm{AB}, J=17.6 \mathrm{~Hz}, 1 \mathrm{H}), 3.41(\mathrm{ABd}, J=17.4 \mathrm{~Hz}, J=10.4 \mathrm{~Hz}, 1 \mathrm{H}), 2.98(\mathrm{ABd}, J=17.6 \mathrm{~Hz}, J=$ $3.6 \mathrm{~Hz}, 1 \mathrm{H}), 2.89(\mathrm{AB}, J=17.6 \mathrm{~Hz}, 1 \mathrm{H}), 2.39(\mathrm{~s}, 3 \mathrm{H}), 2.27(\mathrm{~s}, 3 \mathrm{H}), 1.46(\mathrm{~s}, 9 \mathrm{H}) ;{ }^{13} \mathrm{C} \mathrm{NMR}(100 \mathrm{MHz}$, $\left.\mathrm{CDCl}_{3}\right): \delta 201.2,192.3,160.0,148.7,138.4,137.6,137.4,134.8,134.6,129.3,128.7,126.2,124.7$, 84.1, 71.9, 44.1, 39.8, 36.0, 27.7, 21.1, 21.0; IR (neat): 3007, 2986, 2102, 1709, 1609, 1420, 1157, 1040, $764 \mathrm{~cm}^{-1}$; HRMS (ESI) $\mathrm{m} / z:[\mathrm{M}+\mathrm{Na}]^{+} \mathrm{Calcd}$ for $\mathrm{C}_{25} \mathrm{H}_{27} \mathrm{~N}_{3} \mathrm{NaO}_{4}[\mathrm{M}+\mathrm{Na}]^{+}:$456.1894; Found: 456.1897 .

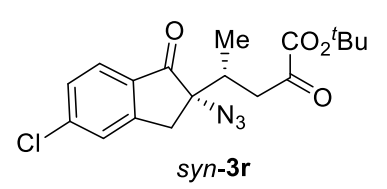

The reaction was conducted at $-20{ }^{\circ} \mathrm{C}$. Column chromatography with $\mathrm{PE} / \mathrm{Et}_{2} \mathrm{O}$ $(10: 1, \mathrm{v} / \mathrm{v})$ afforded product $s y n-3 \mathbf{r}$ in $60 \%$ yield $(57.1 \mathrm{mg})$ as light yellow liquid. ${ }^{1} \mathrm{H}$ NMR analysis of the crude product revealed that the dr value was 3:1. HPLC analysis [Chiralcel OZ-H, 3\% ${ }^{i} \mathrm{PrOH} / \mathrm{h}$ exane, $1.0 \mathrm{~mL} / \mathrm{min}, 230 \mathrm{~nm}$; major diastereomer: $\mathrm{t}_{\mathrm{r}}$ $($ major $)=11.16 \mathrm{~min}, \mathrm{t}_{\mathrm{r}}($ minor $)=9.58 \mathrm{~min}$, minor diastereomer: $\mathrm{t}_{\mathrm{r}}($ major $)=25.60 \mathrm{~min}, \mathrm{t}_{\mathrm{r}}($ minor $)=$ $11.76 \mathrm{~min}]$ gave the isomeric composition of major diastereomer: $69 \%$ ee; $[\alpha]_{\mathrm{D}}{ }^{20}=+209.8(c=1.0$, $\left.\mathrm{CHCl}_{3}\right) .{ }^{1} \mathrm{H}$ NMR $\left(400 \mathrm{MHz}, \mathrm{CDCl}_{3}\right): \delta 7.76-7.73(\mathrm{~m}, 1 \mathrm{H}), 7.46(\mathrm{~d}, J=1.6 \mathrm{~Hz}, 1 \mathrm{H}), 7.43-7.41(\mathrm{~m}$, $1 \mathrm{H}), 3.33-3.18(\mathrm{~m}, 1 \mathrm{H}), 3.00-2.96(\mathrm{~m}, 1 \mathrm{H}), 2.91-2.81(\mathrm{~m}, 1 \mathrm{H}), 2.71-2.58(\mathrm{~m}, 2 \mathrm{H}), 1.57(\mathrm{~s}, 1.9 \mathrm{H})$ (minor), $1.53(\mathrm{~s}, 7.5 \mathrm{H})$ (major), $1.10(\mathrm{~d}, J=6.8 \mathrm{~Hz}, 2.6 \mathrm{H})$ (major), $0.82(\mathrm{~d}, J=6.8 \mathrm{~Hz}, 0.6 \mathrm{H})$ (minor) ${ }^{13} \mathrm{C}$ NMR (100 MHz, $\mathrm{CDCl}_{3}$ ): $\delta$ 200.4, 193.2 (minor), 192.9 (major), 160.3 (minor), 160.2 (major), 152.9 (minor), 152.7 (major), 143.0 (major), 133.2 (major), 133.1 (minor), 129.25 (major), 129.17 (minor), 126.8 (major), 126.7 (minor), 126.05 (minor), 126.00 (major), 84.4 (major), 84.3 (minor), 71.6 (major), 71.1 (minor), 40.82 (minor), 40.77 (major), 35.7 (major), 34.5 (minor), 33.7 (minor), 32.8 (major), 27.8 (minor), 27.7 (major), 15.4 (minor), 14.8 (major); IR (neat): 2980, 2099, $1715,1599,1369,1256,1069,897,611 \mathrm{~cm}^{-1}$; HRMS (ESI) $\mathrm{m} / \mathrm{z}:[\mathrm{M}+\mathrm{Na}]^{+} \mathrm{Calcd}$ for $\mathrm{C}_{18} \mathrm{H}_{20} \mathrm{ClN}_{3} \mathrm{NaO}_{4}$ 400.1035; Found: 400.1040. 


\section{General procedure for anti-selective Michael addition.}

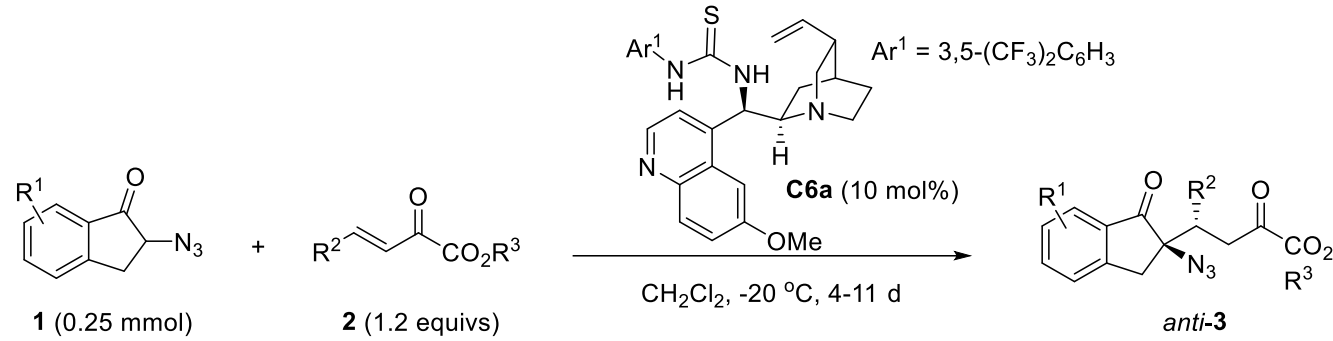

To a $5.0 \mathrm{~mL}$ vial were added chiral tertiary amine-thiourea bifunctional catalyst C6a (14.9 mg, $0.025 \mathrm{mmol}, 10 \mathrm{~mol} \%)$ and $\alpha$-azido indanones 1 ( $0.25 \mathrm{mmol}, 1.0$ equiv), followed by the addition of anhydrous $\mathrm{CH}_{2} \mathrm{Cl}_{2}(2.5 \mathrm{~mL})$ except as noted. The resulting mixture was stirred at $-20{ }^{\circ} \mathrm{C}$ for $30 \mathrm{~min}$, and $\beta, \gamma$-unsaturated $\alpha$-ketoesters $2(0.30 \mathrm{mmol}, 1.2$ equivs) was added. After full consumption of $\alpha$-azido ketones as indicated by TLC analysis, the reaction mixture was rapidly passed through a short pad of silica gel, and washed with the mixed solvent of PE and EtOAc (4:1, v/v). The obtained organic solution was concentrated in vасио to give the crude product. To determine the diastereoselectivity of the product, the residue was first dissolved in $\mathrm{CDCl}_{3}$, and took some samples for ${ }^{1} \mathrm{H}$ NMR analysis. Then the sample for analysis and the rest of crude residue were recombined and purified by silica gel column chromatography using the indicated eluent to afford the desired products anti-3. [Note: all the yields shown here for anti-product 3 refer to the isolated yields of the mixture of diastereoisomers, unless otherwise noted.]

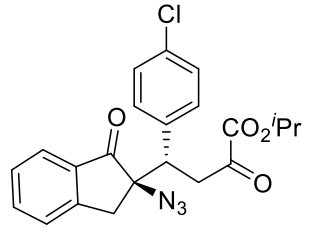

anti-3a

Column chromatography with $\mathrm{PE} / \mathrm{Et}_{2} \mathrm{O}(10: 1, \mathrm{v} / \mathrm{v})$ afforded product anti-3a in $79 \%$ yield (83.8 $\mathrm{mg}$ ) as light yellow liquid (7 days). ${ }^{1} \mathrm{H}$ NMR analysis of the crude product revealed that the dr value was 5:1. HPLC analysis [Chiralcel OZ-H, $3 \%{ }^{i} \mathrm{PrOH} /$ hexane, $1.0 \mathrm{~mL} / \mathrm{min}, 230 \mathrm{~nm}$; major diastereomer: $\mathrm{t}_{\mathrm{r}}$ (major) $=24.36$ $\min , \mathrm{t}_{\mathrm{r}}($ minor $\left.)=22.47 \mathrm{~min}\right]$ gave the isomeric composition of major diastereomer: $86 \%$ ee; $[\alpha]_{\mathrm{D}}^{20}=-155.6\left(c=0.6, \mathrm{CHCl}_{3}\right) .{ }^{1} \mathrm{H}$ NMR $\left(300 \mathrm{MHz}, \mathrm{CDCl}_{3}\right): \delta 7.68(\mathrm{~d}, J=7.8 \mathrm{~Hz}, 1 \mathrm{H})$, 7.60-7.55 (m, 1H), 7.37-7.30 (m, 2H), 7.23-7.15 (m, 4H), 5.16-5.08 (m, 1H), 3.93-3.79 (m, 2H), 3.40-3.29 (m, 2H), $2.96(\mathrm{~d}, J=17.7 \mathrm{~Hz}, 1 \mathrm{H}), 1.34(\mathrm{~d}, J=1.5 \mathrm{~Hz}, 3 \mathrm{H}), 1.32(\mathrm{~d}, J=1.5 \mathrm{~Hz}, 3 \mathrm{H}) ;{ }^{13} \mathrm{C}$ NMR (100 MHz, $\left.\mathrm{CDCl}_{3}\right): \delta 200.8,191.6,160.2,150.5,136.3,135.9,134.0,133.7,130.6,128.7$, 128.3, 126.2, 125.1, 71.4, 71.0, 44.0, 39.4, 36.1, 21.6; IR (neat): 2100, 1717, 1607, 1493, 1184, 1015, 910, 799, 673, $557 \mathrm{~cm}^{-1}$; HRMS (ESI) $\mathrm{m} / z$ : $[\mathrm{M}+\mathrm{Na}]^{+}$Calcd for $\mathrm{C}_{22} \mathrm{H}_{20} \mathrm{ClN}_{3} \mathrm{NaO}_{4}$ 448.1035; Found: 448.1036. 


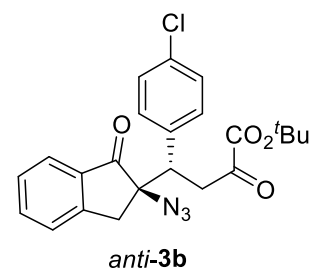

$86 \%$ ee; $[\alpha]_{\mathrm{D}}^{20}=-157.9\left(c=1.00, \mathrm{CHCl}_{3}\right) .{ }^{1} \mathrm{H} \mathrm{NMR}\left(400 \mathrm{MHz}, \mathrm{CDCl}_{3}\right): \delta 7.67(\mathrm{~d}, J=7.6 \mathrm{~Hz}, 1 \mathrm{H})$, 7.59-7.54 (m, 1H), 7.33-7.31 (m, 2H), 7.23-7.20 (m, 2H), 7.17-7.14 (m, 2H), 3.91 (dd, $J=8.6 \mathrm{~Hz}, J=$ $5.6 \mathrm{~Hz}, 1 \mathrm{H}), 3.80-3.74(\mathrm{~m}, 1 \mathrm{H}), 3.39-3.30(\mathrm{~m}, 2 \mathrm{H}), 2.96(\mathrm{~d}, J=17.6 \mathrm{~Hz}, 1 \mathrm{H}), 1.53(\mathrm{~s}, 9 \mathrm{H}) ;{ }^{13} \mathrm{C} \mathrm{NMR}$ $\left(100 \mathrm{MHz}, \mathrm{CDCl}_{3}\right): \delta 200.8,192.3,160.0,150.6,136.3,135.9,134.0,133.6,130.6,128.7,128.3$, 126.2, 125.0, 84.3, 71.4, 44.0, 39.2, 36.1, 27.8; IR (neat): 2997, 2338, 2093, 1719, 1609, 1424, 1310, 1157, 1055, $750 \mathrm{~cm}^{-1}$; HRMS (ESI) $\mathrm{m} / z$ : $[\mathrm{M}+\mathrm{Na}]^{+}$Calcd for $\mathrm{C}_{23} \mathrm{H}_{22} \mathrm{ClN}_{3} \mathrm{NaO}_{4}$ 462.1191; Found: 462.1198.

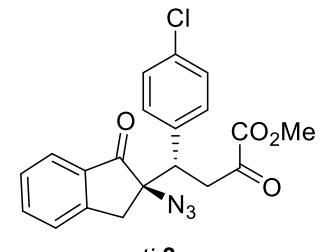

Column chromatography with $\mathrm{PE} / \mathrm{Et}_{2} \mathrm{O}(10: 1$, v/v) afforded product anti-3c in $90 \%$ yield (89.8 mg) as light yellow liquid (7 days). ${ }^{1} \mathrm{H}$ NMR analysis of the crude product revealed that the dr value was 3:1. HPLC analysis [Chiralcel OZ-H, $10 \%{ }^{i} \mathrm{PrOH} / \mathrm{hexane}, 1.0 \mathrm{~mL} / \mathrm{min}, 230 \mathrm{~nm}$; major diastereomer: $\mathrm{t}_{\mathrm{r}}$ (major) $=17.93$ $\min , \mathrm{t}_{\mathrm{r}}($ minor $\left.)=15.31 \mathrm{~min}\right]$ gave the isomeric composition of major diastereomer: $89 \%$ ee; $[\alpha]_{\mathrm{D}}{ }^{20}=-149.5\left(c=0.55, \mathrm{CHCl}_{3}\right) .{ }^{1} \mathrm{H} \mathrm{NMR}\left(300 \mathrm{MHz}, \mathrm{CDCl}_{3}\right): \delta 7.69(\mathrm{~d}, J=7.5 \mathrm{~Hz}, 1 \mathrm{H})$, $7.58(\mathrm{t}, J=7.5 \mathrm{~Hz}, 1 \mathrm{H}), 7.38-7.31(\mathrm{~m}, 2 \mathrm{H}), 7.23-7.16(\mathrm{~m}, 4 \mathrm{H}), 3.93-3.83(\mathrm{~m}, 5 \mathrm{H}), 3.41-3.28(\mathrm{~m}, 2 \mathrm{H})$, $2.96(\mathrm{~d}, J=17.7 \mathrm{~Hz}, 1 \mathrm{H}) ;{ }^{13} \mathrm{C}$ NMR (100 MHz, $\left.\mathrm{CDCl}_{3}\right): \delta 200.8,190.7,161.0,150.5,136.3,135.9$, 134.0, 133.7, 130.5, 128.8, 128.4, 126.2, 125.1, 71.4, 53.2, 43.8, 39. 6, 36.2; IR (neat): 2100, 1713, 1607, 1492, 1364, 1242, 1015, 908, 799, 733, $646 \mathrm{~cm}^{-1}$; HRMS (ESI) m/z: [M+Na] Calcd for $\mathrm{C}_{20} \mathrm{H}_{16} \mathrm{ClN}_{3} \mathrm{NaO}_{4} 420.0722$; Found: 420.0717.

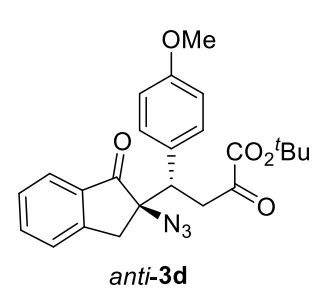

Column chromatography with $\mathrm{PE} / \mathrm{Et}_{2} \mathrm{O}(10: 1, \mathrm{v} / \mathrm{v})$ afforded product anti-3d in $66 \%$ yield (72.4 mg) as light yellow liquid (11 days). ${ }^{1} \mathrm{H}$ NMR analysis of the crude product revealed that the dr value was 9:1. HPLC analysis [Chiralcel OZ-H, $10 \%{ }^{i} \mathrm{PrOH} /$ hexane, $1.0 \mathrm{~mL} / \mathrm{min}, 230 \mathrm{~nm}$; major diastereomer: $\mathrm{t}_{\mathrm{r}}$ (major) $=13.62$ $\min , \mathrm{t}_{\mathrm{r}}($ minor $\left.)=12.96 \mathrm{~min}\right]$ gave the isomeric composition of major diastereomer: $92 \%$ ee; $[\alpha]_{\mathrm{D}}{ }^{20}=-204.6\left(c=0.94, \mathrm{CHCl}_{3}\right) .{ }^{1} \mathrm{H}$ NMR $\left(400 \mathrm{MHz}, \mathrm{CDCl}_{3}\right): \delta 7.65(\mathrm{~d}, J=7.6 \mathrm{~Hz}, 1 \mathrm{H})$, 7.56-7.51 (m, 1H), 7.32-7.27 (m, 2H), 7.17-7.15 (m, 2H), 6.72-6.68 (m, 2H), $3.92(\mathrm{dd}, J=8.6 \mathrm{~Hz}, J=$ 
5.6 Hz, 1H), 3.75-3.69 (m, 1H), $3.68(\mathrm{~s}, 3 \mathrm{H}), 3.40-3.29(\mathrm{~m}, 2 \mathrm{H}), 2.93(\mathrm{~d}, J=17.6 \mathrm{~Hz}, 1 \mathrm{H}), 1.53(\mathrm{~s}$, 9H); ${ }^{13} \mathrm{C}$ NMR (100 MHz, $\left.\mathrm{CDCl}_{3}\right): \delta 201.2,192.6,160.2,158.9,150.8,136.0,134.2,130.3,129.0$, 128.1, 126.2, 124.9, 113.9, 84.1, 71.6, 55.1, 43.9, 39.4, 35.9, 27.8; IR (neat): 2994, 2359, 2102, 1719, 1609, 1493, 1356, 1223, 1159, 1094, $766 \mathrm{~cm}^{-1}$; HRMS (ESI) m/z: $\left[\mathrm{M}+\mathrm{NH}_{4}\right]^{+}$Calcd for $\mathrm{C}_{24} \mathrm{H}_{25} \mathrm{~N}_{3} \mathrm{NaO}_{5} 458.1686$; Found: 458.1686.

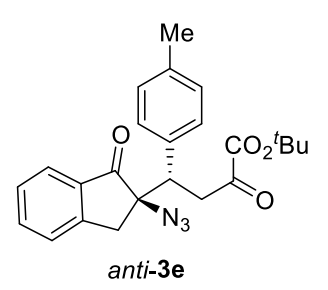

Column chromatography with $\mathrm{PE} / \mathrm{Et}_{2} \mathrm{O}(10: 1, \mathrm{v} / \mathrm{v})$ afforded product anti-3e in $72 \%$ yield $(75.8 \mathrm{mg})$ as light yellow liquid (10 days). ${ }^{1} \mathrm{H}$ NMR analysis of the crude product revealed that the dr value was 12:1. HPLC analysis [Chiralpak AS-H, 3\% ${ }^{i} \mathrm{PrOH} /$ hexane, $1.0 \mathrm{~mL} / \mathrm{min}, 230 \mathrm{~nm}$; major diastereomer: $\mathrm{t}_{\mathrm{r}}($ major $)=$ $15.25 \mathrm{~min}, \mathrm{t}_{\mathrm{r}}($ minor $)=16.41 \mathrm{~min}$, minor diastereomer: $\mathrm{t}_{\mathrm{r}}($ major $)=30.61 \mathrm{~min}, \mathrm{t}_{\mathrm{r}}$ $($ minor $)=23.37 \mathrm{~min}]$ gave the isomeric composition of major diastereomer: $93 \%$ ee; $[\alpha]_{\mathrm{D}}{ }^{20}=-192.0$ $\left(c=1.00, \mathrm{CHCl}_{3}\right) .{ }^{1} \mathrm{H}$ NMR $\left(400 \mathrm{MHz}, \mathrm{CDCl}_{3}\right): \delta 7.65(\mathrm{~d}, J=7.6 \mathrm{~Hz}, 1 \mathrm{H}), 7.55-7.51(\mathrm{~m}, 1 \mathrm{H})$, 7.31-7.26 (m, 2H), $7.13(\mathrm{~d}, J=8.0 \mathrm{~Hz}, 2 \mathrm{H}), 6.97(\mathrm{~d}, J=8.0 \mathrm{~Hz}, 2 \mathrm{H}), 3.92(\mathrm{dd}, J=8.6 \mathrm{~Hz}, J=6.0 \mathrm{~Hz}$, $1 \mathrm{H}), 3.78-3.73(\mathrm{~m}, 1 \mathrm{H}), 3.42-3.31(\mathrm{~m}, 2 \mathrm{H}), 2.93(\mathrm{~d}, J=17.6 \mathrm{~Hz}, 1 \mathrm{H}), 2.19(\mathrm{~s}, 3 \mathrm{H}), 1.52(\mathrm{~s}, 9 \mathrm{H}) ;{ }^{13} \mathrm{C}$ NMR (100 MHz, $\left.\mathrm{CDCl}_{3}\right): \delta 201.1,192.6,160.2,150.8,137.3,136.0,134.2,134.2,129.2,129.1$, 128.1, 126.2, 124.9, 84.0, 71.6, 44.2, 39.4, 36.0, 27.8, 20.9; IR (neat): 3007, 2097, 1717, 1609, 1423, 1312, 1157, 1042, $758 \mathrm{~cm}^{-1}$; HRMS (ESI) $\mathrm{m} / z:[\mathrm{M}+\mathrm{Na}]^{+}$Calcd for $\mathrm{C}_{24} \mathrm{H}_{25} \mathrm{~N}_{3} \mathrm{NaO}_{4} 442.1737$; Found: 442.1743 .

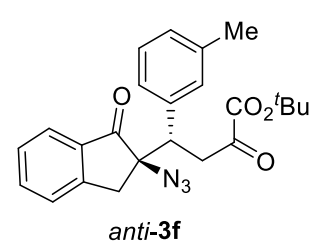

Column chromatography with $\mathrm{PE} / \mathrm{Et}_{2} \mathrm{O}(10: 1, \mathrm{v} / \mathrm{v})$ afforded product anti-3f in $60 \%$ yield $(62.5 \mathrm{mg})$ as light yellow liquid (9 days). ${ }^{1} \mathrm{H}$ NMR analysis of the crude product revealed that the $\mathrm{dr}$ value was 6:1. HPLC analysis [Chiralcel OZ-H, 3\% ${ }^{i} \mathrm{PrOH} /$ hexane, $1.0 \mathrm{~mL} / \mathrm{min}, 230 \mathrm{~nm}$; major diastereomer: $\mathrm{t}_{\mathrm{r}}$ (major) $=$ $14.81 \mathrm{~min}, \mathrm{t}_{\mathrm{r}}($ minor $)=12.18 \mathrm{~min}$ ] gave the isomeric composition of major diastereomer: $90 \%$ ee; $[\alpha]_{\mathrm{D}}{ }^{20}=-200.3\left(c=0.95, \mathrm{CHCl}_{3}\right) .{ }^{1} \mathrm{H}$ NMR $\left(400 \mathrm{MHz}, \mathrm{CDCl}_{3}\right): \delta 7.66(\mathrm{~d}, J=7.2 \mathrm{~Hz}, 1 \mathrm{H}), 7.56-7.51$ (m, 1H), 7.33-7.29 (m, 2H), 7.08-7.04 (m, 3H), 6.94-6.91 (m, 1H), 3.89 (dd, $J=8.2 \mathrm{~Hz}, J=6.0 \mathrm{~Hz}$, $1 \mathrm{H}), 3.79-3.73(\mathrm{~m}, 1 \mathrm{H}), 3.41-3.32(\mathrm{~m}, 2 \mathrm{H}), 2.93(\mathrm{~d}, J=17.6 \mathrm{~Hz}, 1 \mathrm{H}), 2.23(\mathrm{~s}, 3 \mathrm{H}), 1.53(\mathrm{~s}, 9 \mathrm{H}) ;{ }^{13} \mathrm{C}$ NMR (100 MHz, $\left.\mathrm{CDCl}_{3}\right): \delta 201.2,192.6,160.1,150.7,138.0,137.3,136.0,134.2,130.1,128.4$, 128.3, 128.1, 126.1, 124.9, 84.0, 71.7, 44.6, 39.3, 36.1, 27.8, 21.4; IR (neat): 2980, 2099, 1713, 1607, 1466, 1369, 1254, 1155, 1016, 783, $646 \mathrm{~cm}^{-1}$; HRMS (ESI) $m / z:[\mathrm{M}+\mathrm{Na}]^{+} \mathrm{Calcd}$ for $\mathrm{C}_{24} \mathrm{H}_{25} \mathrm{~N}_{3} \mathrm{NaO}_{4}$ 442.1737; Found: 442.1741. 


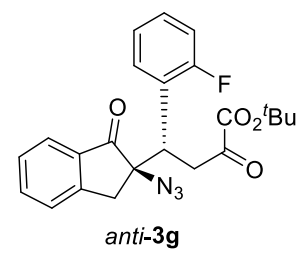

The reaction was conducted with 20 mol\% of C6a. Column chromatography with $\mathrm{PE} / \mathrm{Et}_{2} \mathrm{O}(10: 1, \mathrm{v} / \mathrm{v})$ afforded product anti-3g in $81 \%$ yield $(85.4 \mathrm{mg})$ as light yellow liquid (11 days). ${ }^{1} \mathrm{H}$ NMR analysis of the crude product revealed that the $\mathrm{dr}$ value was 3:1. HPLC analysis [Chiralpak AS-H, $3 \%{ }^{i} \mathrm{PrOH} / \mathrm{hexane}, 1.0 \mathrm{~mL} / \mathrm{min}$, $230 \mathrm{~nm}$; major diastereomer: $\mathrm{t}_{\mathrm{r}}($ major $)=14.82 \mathrm{~min}, \mathrm{t}_{\mathrm{r}}($ minor $\left.)=15.82 \mathrm{~min}\right]$ gave the isomeric composition of major diastereomer: $74 \%$ ee; $[\alpha]_{\mathrm{D}}{ }^{20}=-96.7\left(c=0.96, \mathrm{CHCl}_{3}\right) .{ }^{1} \mathrm{H} \mathrm{NMR}(300 \mathrm{MHz}$, $\left.\mathrm{CDCl}_{3}\right): \delta 7.74-7.71(\mathrm{~m}, 1 \mathrm{H})$, 7.64-7.59 $(\mathrm{m}, 1 \mathrm{H})$, 7.46-7.35 (m, 3H), 7.23-7.7.15 (m, 1H), 7.09-7.04 (m, 1H), 7.00-6.93 (m, 1H), $4.12(\mathrm{dd}, J=8.7 \mathrm{~Hz}, J=5.1 \mathrm{~Hz}, 1 \mathrm{H}), 3.94-3.85(\mathrm{~m}, 1 \mathrm{H}), 3.52-3.37(\mathrm{~m}$, 2H), $3.00(\mathrm{~d}, J=17.7 \mathrm{~Hz}, 1 \mathrm{H}), 1.54(\mathrm{~s}, 9 \mathrm{H}) ;{ }^{13} \mathrm{C} \mathrm{NMR}\left(100 \mathrm{MHz}, \mathrm{CDCl}_{3}\right): \delta 200.8,192.5,160.8(\mathrm{~d}$, $\left.J_{\mathrm{C}-\mathrm{F}}=244.8 \mathrm{~Hz}, 1 \mathrm{C}\right), 159.9,150.4,136.2,134.0,130.7\left(\mathrm{~d}, J_{\mathrm{C}-\mathrm{F}}=3.7 \mathrm{~Hz}, 1 \mathrm{C}\right), 129.4,129.4,128.3$, 126.2, $125.2\left(\mathrm{~d}, J_{\mathrm{C}-\mathrm{F}}=13.4 \mathrm{~Hz}, 1 \mathrm{C}\right), 125.0,124.4\left(\mathrm{~d}, J_{\mathrm{C}-\mathrm{F}}=3.5 \mathrm{~Hz}, 1 \mathrm{C}\right), 115.7\left(\mathrm{~d}, J_{\mathrm{C}-\mathrm{F}}=23.4 \mathrm{~Hz}, 1 \mathrm{C}\right)$, 84.1, 71.9, $39.1\left(\mathrm{~d}, J_{\mathrm{C}-\mathrm{F}}=2.6 \mathrm{~Hz}, 1 \mathrm{C}\right), 38.2,37.3\left(\mathrm{~d}, J_{\mathrm{C}-\mathrm{F}}=3.2 \mathrm{~Hz}, 1 \mathrm{C}\right), 27.8 ;{ }^{19} \mathrm{~F}$ NMR $(282 \mathrm{MHz}$, $\mathrm{CDCl}_{3}$ ): $\delta$-114.29 -114.37 (M, 1F); IR (neat): 2093, 1709, 1609, 1491, 1221, 1094, $756 \mathrm{~cm}^{-1}$; HRMS (ESI) $m / z:[\mathrm{M}+\mathrm{Na}]^{+} \mathrm{Calcd}$ for $\mathrm{C}_{23} \mathrm{H}_{22} \mathrm{FN}_{3} \mathrm{NaO}_{4}$ 446.1487; Found: 446.1485.

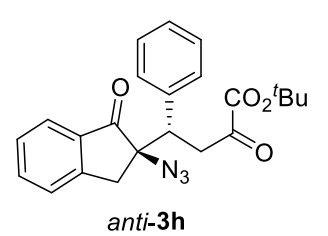

Column chromatography with $\mathrm{PE} / \mathrm{Et}_{2} \mathrm{O}(10: 1, \mathrm{v} / \mathrm{v})$ afforded product anti-3h in $59 \%$ yield $(59.1 \mathrm{mg})$ as light yellow liquid (9 days). ${ }^{1} \mathrm{H}$ NMR analysis of the crude product revealed that the dr value was 11:1. HPLC analysis [Chiralpak AS-H, 3\% ${ }^{i} \mathrm{PrOH} /$ hexane, $1.0 \mathrm{~mL} / \mathrm{min}, 230 \mathrm{~nm}$; major diastereomer: $\mathrm{t}_{\mathrm{r}}$ (major) $=$ $13.71 \mathrm{~min}, \mathrm{t}_{\mathrm{r}}($ minor $\left.)=15.07 \mathrm{~min}\right]$ gave the isomeric composition of major diastereomer: $92 \%$ ee; $[\alpha]_{\mathrm{D}}{ }^{20}=-211.0\left(c=0.89, \mathrm{CHCl}_{3}\right) .{ }^{1} \mathrm{H} \mathrm{NMR}\left(400 \mathrm{MHz}, \mathrm{CDCl}_{3}\right): \delta 7.58(\mathrm{~d}, J=7.6 \mathrm{~Hz}, 1 \mathrm{H}), 7.48-7.44$ (m, 1H), 7.25-7.16 (m, 4H), 7.12-7.02 (m, 3H), $3.87(\mathrm{dd}, J=8.2 \mathrm{~Hz}, J=5.6 \mathrm{~Hz}, 1 \mathrm{H}), 3.70(\mathrm{ABd}, J=$ $17.2 \mathrm{~Hz}, J=5.6 \mathrm{~Hz}, 1 \mathrm{H}), 3.31(\mathrm{AB}, J=17.6 \mathrm{~Hz}, 1 \mathrm{H}), 3.29(\mathrm{ABd}, J=17.6 \mathrm{~Hz}, J=8.4 \mathrm{~Hz}, 1 \mathrm{H}), 2.87$ $(\mathrm{AB}, J=17.6 \mathrm{~Hz}, 1 \mathrm{H}), 1.45(\mathrm{~s}, 9 \mathrm{H}) ;{ }^{13} \mathrm{C} \mathrm{NMR}\left(100 \mathrm{MHz}, \mathrm{CDCl}_{3}\right): \delta 201.1,192.5160 .1,150.7,137.3$ 136.0, 134.2 129.2, 128.5 128.1, 127.7 126.1, 124.9, 84.1, 71.6 44.7, 39.3 36.0, 27.8; IR (neat): 2984, 2338, 2106, 1715, 1609, 1491, 1157, 1059, $764 \mathrm{~cm}^{-1}$; HRMS (ESI) $\mathrm{m} / z:[\mathrm{M}+\mathrm{Na}]^{+}$Calcd for $\mathrm{C}_{23} \mathrm{H}_{23} \mathrm{~N}_{3} \mathrm{NaO}_{4} 428.1581$; Found: 428.1578. 


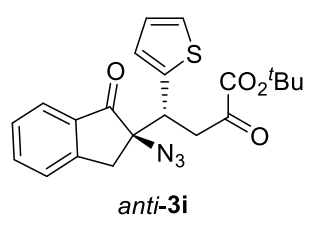

$\mathrm{mL} / \mathrm{min}, 230 \mathrm{~nm}$; major diastereomer: $\mathrm{t}_{\mathrm{r}}$ (major) $=16.22 \mathrm{~min}, \mathrm{t}_{\mathrm{r}}$ (minor) $=17.65 \mathrm{~min}$, minor diastereomer: $\mathrm{t}_{\mathrm{r}}($ major $)=26.11 \mathrm{~min}, \mathrm{t}_{\mathrm{r}}($ minor $\left.)=21.26 \mathrm{~min}\right]$ gave the isomeric composition of major diastereomer: $93 \%$ ee; $[\alpha]_{\mathrm{D}}{ }^{20}=-247.2\left(c=1.00, \mathrm{CHCl}_{3}\right) .{ }^{1} \mathrm{H} \mathrm{NMR}\left(400 \mathrm{MHz}, \mathrm{CDCl}_{3}\right): \delta 7.71-7.69(\mathrm{~m}$, 1H), 7.59-7.55 (m, 1H), 7.36-7.31 (m, 2H), 7.05-7.04 (m, 1H), 6.89-6.88 (m, 1H), 6.79-6.77 (m, 1H), $4.33(\mathrm{dd}, J=8.6 \mathrm{~Hz}, J=5.2 \mathrm{~Hz}, 1 \mathrm{H}), 3.72(\mathrm{ABd}, J=17.4 \mathrm{~Hz}, J=5.6 \mathrm{~Hz}, 1 \mathrm{H}), 3.48(\mathrm{AB}, J=17.6 \mathrm{~Hz}$, $1 \mathrm{H}), 3.35(\mathrm{ABd}, J=17.2 \mathrm{~Hz}, J=8.4 \mathrm{~Hz}, 1 \mathrm{H}), 3.00(\mathrm{AB}, J=17.6 \mathrm{~Hz}, 1 \mathrm{H}), 1.54(\mathrm{~s}, 9 \mathrm{H}) ;{ }^{13} \mathrm{C} \mathrm{NMR}$ $\left(100 \mathrm{MHz}, \mathrm{CDCl}_{3}\right): \delta 200.8,191.9,159.9,151.0,139.8,136.1,134.2,128.2,127.7,126.7,126.3$, 125.1, 125.0, 84.3, 71.1, 40.8, 40.6, 36.2, 27.8; IR (neat): 2980, 2100, 1713, 1607, 1466, 1369, 1258, 1155, 1009, 835, $700 \mathrm{~cm}^{-1}$; HRMS (ESI) $\mathrm{m} / z$ : $[\mathrm{M}+\mathrm{Na}]^{+}$Calcd for $\mathrm{C}_{21} \mathrm{H}_{21} \mathrm{~N}_{3} \mathrm{NaO}_{4} \mathrm{~S}$ 434.1145; Found: 434.1148.

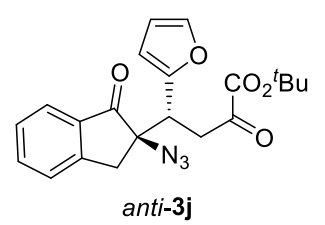

The reaction was conducted with 20 mol\% C6a. Column chromatography with $\mathrm{PE} / \mathrm{Et}_{2} \mathrm{O}(10: 1, \mathrm{v} / \mathrm{v})$ afforded product anti-3j in $72 \%$ yield $(71.5 \mathrm{mg})$ as light yellow liquid (11 days). ${ }^{1} \mathrm{H}$ NMR analysis of the crude product revealed that the dr value was 7:1. HPLC analysis [Chiralcel OZ-H, 3\% ${ }^{i} \mathrm{PrOH} / \mathrm{hexane}, 1.0 \mathrm{~mL} / \mathrm{min}$, $230 \mathrm{~nm}$; major diastereomer: $\mathrm{t}_{\mathrm{r}}($ major $)=20.28 \mathrm{~min}, \mathrm{t}_{\mathrm{r}}($ minor $)=15.64 \mathrm{~min}$ ] gave the isomeric composition of major diastereomer: $88 \%$ ee; $[\alpha]_{\mathrm{D}}{ }^{20}=-393.1\left(c=0.93, \mathrm{CHCl}_{3}\right) .{ }^{1} \mathrm{H} \mathrm{NMR}(400 \mathrm{MHz}$, $\left.\mathrm{CDCl}_{3}\right): \delta 7.71(\mathrm{~d}, J=8.0 \mathrm{~Hz}, 1 \mathrm{H}), 7.58-7.54(\mathrm{~m}, 1 \mathrm{H}), 7.36-7.32(\mathrm{~m}, 2 \mathrm{H}), 7.08-7.07(\mathrm{~m}, 1 \mathrm{H})$, 6.08-6.06 (m, 2H), $4.20(\mathrm{dd}, J=8.8 \mathrm{~Hz}, J=5.2 \mathrm{~Hz}, 1 \mathrm{H}), 3.58(\mathrm{ABd}, J=17.4 \mathrm{~Hz}, J=5.6 \mathrm{~Hz}, 1 \mathrm{H})$, $3.46(\mathrm{AB}, J=17.2 \mathrm{~Hz}, 1 \mathrm{H}), 3.41(\mathrm{ABd}, J=17.4 \mathrm{~Hz}, J=8.8 \mathrm{~Hz}, 1 \mathrm{H}), 2.95(\mathrm{AB}, J=17.6 \mathrm{~Hz}, 1 \mathrm{H})$, 1.56 (s, 9H); ${ }^{13} \mathrm{C}$ NMR (100 MHz, $\left.\mathrm{CDCl}_{3}\right): \delta 200.9$, 192.1, 160.0, 151.2, 150.4, 142.1, 136.0, 134.0, 128.0, 126.3, 124.9, 110.4, 109.1, 84.3, 70.3, 38.6, 37.5, 35.8, 27.8; IR (neat): 2099, 1713, 1607, 1466, 1369, 1155, 1011, 835, $746 \mathrm{~cm}^{-1}$; HRMS (ESI) $\mathrm{m} / z$ : $[\mathrm{M}+\mathrm{Na}]^{+}$Calcd for $\mathrm{C}_{21} \mathrm{H}_{21} \mathrm{~N}_{3} \mathrm{NaO}_{5} 418.1373$; Found: 418.1384. 


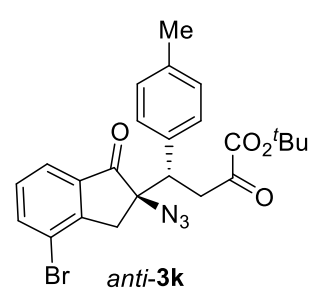

Column chromatography with $\mathrm{PE} / \mathrm{Et}_{2} \mathrm{O}(10: 1$, v/v) afforded product anti-3k in $76 \%$ yield $\left(94.5 \mathrm{mg}\right.$ ) as light yellow liquid (6 days). ${ }^{1} \mathrm{H}$ NMR analysis of the crude product revealed that the dr value was 8:1. HPLC analysis [Chiralcel OZ-H, $3 \%{ }^{i} \mathrm{PrOH} / \mathrm{hexane}, 1.0 \mathrm{~mL} / \mathrm{min}, 230 \mathrm{~nm}$; major diastereomer: $\mathrm{t}_{\mathrm{r}}$ (major) $=13.66$ $\min , \mathrm{t}_{\mathrm{r}}($ minor $\left.)=12.81 \mathrm{~min}\right]$ gave the isomeric composition of major diastereomer: $90 \%$ ee; $[\alpha]_{\mathrm{D}}{ }^{20}=-149.2\left(c=0.95, \mathrm{CHCl}_{3}\right) .{ }^{1} \mathrm{H} \mathrm{NMR}\left(400 \mathrm{MHz}, \mathrm{CDCl}_{3}\right): \delta 7.70-7.67(\mathrm{~m}, 1 \mathrm{H})$, 7.60-7.58 (m, 1H), 7.22-7.17 (m, 1H), 7.15-7.11 (m, 2H), 6.99-6.97 (m, 2H), 3.99 (dd, $J=8.4 \mathrm{~Hz}, J=$ $5.6 \mathrm{~Hz}, 1 \mathrm{H}), 3.74(\mathrm{ABd}, J=17.4 \mathrm{~Hz}, J=6.0 \mathrm{~Hz}, 1 \mathrm{H}), 3.38(\mathrm{ABd}, J=17.2 \mathrm{~Hz}, J=8.4 \mathrm{~Hz}, 1 \mathrm{H}), 3.36$ $(\mathrm{AB}, J=18.0 \mathrm{~Hz}, 1 \mathrm{H}), 2.87(\mathrm{AB}, J=18.0 \mathrm{~Hz}, 1 \mathrm{H}), 2.19(\mathrm{~s}, 3 \mathrm{H}), 1.55(\mathrm{~s}, 9 \mathrm{H}) ;{ }^{13} \mathrm{C} \mathrm{NMR}(100 \mathrm{MHz}$, $\left.\mathrm{CDCl}_{3}\right): \delta 200.7,192.4,160.1,150.6,138.5,137.5,136.2,133.5,129.7,129.3,128.9,123.6,121.5$, 84.2, 71.6, 44.2, 39.2, 36.8, 27.8, 20.9; IR (neat): 3001, 2359, 2102, 1713, 1574, 1435, 1358, 1221, 1117, 1092, 905, $718 \mathrm{~cm}^{-1}$; HRMS (ESI) $\mathrm{m} / z:[\mathrm{M}+\mathrm{Na}]^{+} \mathrm{Calcd}$ for $\mathrm{C}_{24} \mathrm{H}_{24} \mathrm{BrN}_{3} \mathrm{NaO}_{4}$ 520.0842; Found: 520.0840 .

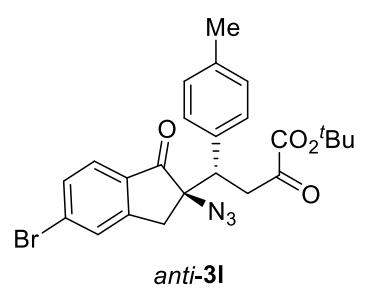

Column chromatography with $\mathrm{PE} / \mathrm{Et}_{2} \mathrm{O}(10: 1$, v/v) afforded product anti-3l in $91 \%$ yield (113.2 $\mathrm{mg}$ ) as light yellow liquid (6 days). ${ }^{1} \mathrm{H}$ NMR analysis of the crude product revealed that the dr value was 12:1. HPLC analysis [Chiralpak AS-H, 3\% ${ }^{i} \mathrm{PrOH} /$ hexane, $1.0 \mathrm{~mL} / \mathrm{min}, 230 \mathrm{~nm}$; major diastereomer: $\mathrm{t}_{\mathrm{r}}$ (major) $=11.10 \mathrm{~min}, \mathrm{t}_{\mathrm{r}}($ minor $\left.)=12.01 \mathrm{~min}\right]$ gave the isomeric composition of major diastereomer: $71 \%$ ee; $[\alpha]_{\mathrm{D}}{ }^{20}=-181.5\left(c=1.00, \mathrm{CHCl}_{3}\right) .{ }^{1} \mathrm{H}$ NMR $\left(400 \mathrm{MHz}, \mathrm{CDCl}_{3}\right): \delta 7.51-7.42(\mathrm{~m}$, $3 \mathrm{H}), 7.11(\mathrm{~d}, J=8.0 \mathrm{~Hz}, 2 \mathrm{H}), 6.98(\mathrm{~d}, J=7.6 \mathrm{~Hz}, 2 \mathrm{H}), 3.96-3.92(\mathrm{~m}, 1 \mathrm{H}), 3.75-3.69(\mathrm{~m}, 1 \mathrm{H})$, 3.40-3.29 (m, 2H), $2.90(\mathrm{~d}, J=17.6 \mathrm{~Hz}, 1 \mathrm{H}), 2.20(\mathrm{~s}, 3 \mathrm{H}), 1.53(\mathrm{~s}, 9 \mathrm{H}) ;{ }^{13} \mathrm{C} \mathrm{NMR}\left(100 \mathrm{MHz}, \mathrm{CDCl}_{3}\right)$ : $\delta 200.0192 .4,160.1,152.3,137.5,133.8$ 133.0, 131.8, 131.5, 129.5, 129.3, 129.0, 126.1, 84.1, 71.5, 44.1, 39.2, 35.5, 27.8, 20.9; IR (neat): 2100, 1715, 1595, 1369, 1209, 1057, 891, $733 \mathrm{~cm}^{-1}$; HRMS (ESI) $m / z:[\mathrm{M}+\mathrm{Na}]^{+}$Calcd for $\mathrm{C}_{24} \mathrm{H}_{24} \mathrm{BrN}_{3} \mathrm{NaO}_{4}$ 520.0842; Found: 520.0848 .

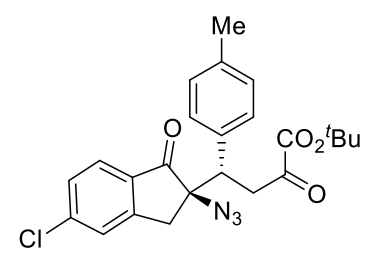

$(R, S)-3 m$

Column chromatography with $\mathrm{PE} / \mathrm{Et}_{2} \mathrm{O}(10: 1$, v/v) afforded product $(R, S)-\mathbf{3 m}$ in $67 \%$ yield $\left(75.9 \mathrm{mg}\right.$ ) as light yellow liquid (9 days). ${ }^{1} \mathrm{H}$ NMR analysis of the crude product revealed that the dr value was 11:1. HPLC analysis [Chiralcel $\mathrm{OZ}-\mathrm{H}, 3 \%{ }^{i} \mathrm{PrOH} /$ hexane, $1.0 \mathrm{~mL} / \mathrm{min}, 230 \mathrm{~nm}$; major diastereomer: $\mathrm{t}_{\mathrm{r}}$ (major) $=13.14 \mathrm{~min}, \mathrm{t}_{\mathrm{r}}($ minor $\left.)=12.02 \mathrm{~min}\right]$ gave the isomeric composition of major diastereomer: $90 \%$ ee; $[\alpha]_{\mathrm{D}}{ }^{20}=-218.4\left(c=1.00, \mathrm{CHCl}_{3}\right) .{ }^{1} \mathrm{H} \mathrm{NMR}\left(400 \mathrm{MHz}, \mathrm{CDCl}_{3}\right): \delta 7.57(\mathrm{~d}, J=$ 
8.0 Hz, 1H), 7.28-7.25 (m, 2H), $7.11(\mathrm{~d}, J=8.0 \mathrm{~Hz}, 2 \mathrm{H}), 6.98(\mathrm{~d}, J=8.0 \mathrm{~Hz}, 2 \mathrm{H}), 3.95$ (dd, $J=8.4 \mathrm{~Hz}$, $J=5.6 \mathrm{~Hz}, 1 \mathrm{H}), 3.76-3.70(\mathrm{~m}, 1 \mathrm{H}), 3.40-3.29$ (m, 2H), 2.90 (d, $J=17.6 \mathrm{~Hz}, 1 \mathrm{H}), 2.19$ (s, 3H), 1.53 (s, $9 \mathrm{H}) ;{ }^{13} \mathrm{C}$ NMR $\left(100 \mathrm{MHz}, \mathrm{CDCl}_{3}\right): \delta 199.8,192.4,160.1,152.3,142.5,137.5,133.8,132.7,129.3$, 129.0, 128.9, 126.4, 126.0, 84.1, 71.6, 44.2, 39.2, 35.6, 27.8, 20.9; IR (neat): 2100, 1715, 1599, 1369, 1263, 1159, 1069, 899, $598 \mathrm{~cm}^{-1}$; HRMS (ESI) $\mathrm{m} / \mathrm{z}:[\mathrm{M}+\mathrm{Na}]^{+}$Calcd for $\mathrm{C}_{24} \mathrm{H}_{24} \mathrm{ClN}_{3} \mathrm{NaO}_{4} 476.1348$; Found: 476.1347.

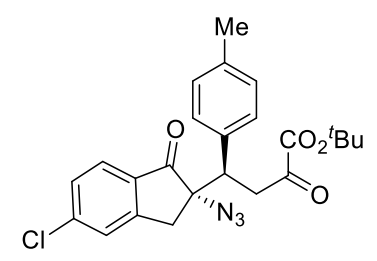

$(S, R)-\mathbf{3 m}$

Column chromatography with $\mathrm{PE} / \mathrm{Et}_{2} \mathrm{O}(10: 1$, v/v) afforded product $(S, R)-3 \mathbf{m}$ in 99\% yield (112.4 mg) as light yellow liquid (7 days) by replacing C6a with C6b under other same conditions. ${ }^{1} \mathrm{H}$ NMR analysis of the crude product revealed that the dr value was 7:1. HPLC analysis [Chiralcel OZ-H, 5\% ${ }^{i} \mathrm{PrOH} /$ hexane, $1.0 \mathrm{~mL} / \mathrm{min}, 230 \mathrm{~nm}$; major diastereomer: $\mathrm{t}_{\mathrm{r}}$ (major) $=11.97$ $\min , \mathrm{t}_{\mathrm{r}}($ minor $)=13.16 \mathrm{~min}$, minor diastereomer: $\mathrm{t}_{\mathrm{r}}($ major $)=10.88 \mathrm{~min}, \mathrm{t}_{\mathrm{r}}($ minor $\left.)=13.63 \mathrm{~min}\right]$ gave the isomeric composition of major diastereomer: $77 \%$ ee; $[\alpha]_{\mathrm{D}}{ }^{20}=166.18\left(c=1.00, \mathrm{CHCl}_{3}\right) .{ }^{1} \mathrm{H} \mathrm{NMR}$ (400 MHz, $\left.\mathrm{CDCl}_{3}\right): \delta 7.60-7.58(\mathrm{~m}, 1 \mathrm{H}), 7.30-7.27(\mathrm{~m}, 2 \mathrm{H}), 7.12-7.10(\mathrm{~m}, 2 \mathrm{H}), 6.99(\mathrm{~d}, J=8.0 \mathrm{~Hz}$, 2H), $3.93(\mathrm{dd}, J=8.4 \mathrm{~Hz}, J=6.0 \mathrm{~Hz}, 1 \mathrm{H}), 3.72(\mathrm{ABd}, J=17.2 \mathrm{~Hz}, J=6.0 \mathrm{~Hz}, 1 \mathrm{H}), 3.37(\mathrm{AB}, J=$ $18.0 \mathrm{~Hz}, 1 \mathrm{H}), 3.32(\mathrm{ABd}, J=17.2 \mathrm{~Hz}, J=8.4 \mathrm{~Hz}, 1 \mathrm{H}), 2.89(\mathrm{AB}, J=17.6 \mathrm{~Hz}, 1 \mathrm{H}), 2.21(\mathrm{~s}, 3 \mathrm{H}), 1.53$ (s, 9H); ${ }^{13} \mathrm{C}$ NMR (125 MHz, $\left.\mathrm{CDCl}_{3}\right): \delta 199.8,192.4,160.1,152.3,1426,137.5,133.8,132.6,129.3$, 129.0, 129.0, 126.4, 126.0, 84.1, 71.6, 44.1, 39.2, 35.6, 27.76, 20.9; IR (neat): 2100, 1715, 1599, 1369, 1221, 1087, 899, 734, $600 \mathrm{~cm}^{-1}$; HRMS (ESI) $\mathrm{m} / z$ : $[\mathrm{M}+\mathrm{Na}]^{+}$Calcd for $\mathrm{C}_{24} \mathrm{H}_{24} \mathrm{ClN}_{3} \mathrm{NaO}_{4} 476.1348$; Found: 476.1353 .

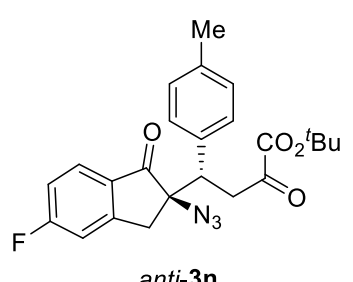

anti-3n

Column chromatography with $\mathrm{PE} / \mathrm{Et}_{2} \mathrm{O}(10: 1$, v/v) afforded product anti-3n in $78 \%$ yield $\left(85.1 \mathrm{mg}\right.$ ) as light yellow liquid (9 days). ${ }^{1} \mathrm{H}$ NMR analysis of the crude product revealed that the dr value was 8:1. HPLC analysis [Chiralcel OZ-H, 3\% ${ }^{i} \mathrm{PrOH} / \mathrm{h}$ exane, $1.0 \mathrm{~mL} / \mathrm{min}, 230 \mathrm{~nm}$; major diastereomer: $\mathrm{t}_{\mathrm{r}}$ (major) = $15.73 \mathrm{~min}, \mathrm{t}_{\mathrm{r}}($ minor $\left.)=14.46 \mathrm{~min}\right]$ gave the isomeric composition of major diastereomer: $90 \%$ ee; $[\alpha]_{\mathrm{D}}{ }^{20}=-187.3\left(c=1.07, \mathrm{CHCl}_{3}\right) .{ }^{1} \mathrm{H} \mathrm{NMR}\left(300 \mathrm{MHz}, \mathrm{CDCl}_{3}\right): \delta 7.70-7.66(\mathrm{~m}$, 1H), 7.15-7.12 (m, 2H), 7.04-6.97 (m, 4H), 3.95 (dd, $J=8.2 \mathrm{~Hz}, J=6.0 \mathrm{~Hz}, 1 \mathrm{H}), 3.75(\mathrm{ABd}, J=17.1$ $\mathrm{Hz}, J=5.7 \mathrm{~Hz}, 1 \mathrm{H}), 3.40(\mathrm{AB}, J=18.0 \mathrm{~Hz}, 1 \mathrm{H}), 3.34(\mathrm{ABd}, J=17.1 \mathrm{~Hz}, J=8.4 \mathrm{~Hz}, 1 \mathrm{H}), 2.92(\mathrm{AB}$, $J=18.0 \mathrm{~Hz}, 1 \mathrm{H}), 2.22(\mathrm{~s}, 3 \mathrm{H}), 1.54(\mathrm{~s}, 9 \mathrm{H}) ;{ }^{13} \mathrm{C} \mathrm{NMR}\left(100 \mathrm{MHz}, \mathrm{CDCl}_{3}\right): \delta 199.3,192.5,167.7$ (d, $\left.J_{\mathrm{C}-\mathrm{F}}=257.2 \mathrm{~Hz}, 1 \mathrm{C}\right), 160.1,153.8\left(\mathrm{~d}, J_{\mathrm{C}-\mathrm{F}}=10.4 \mathrm{~Hz}, 1 \mathrm{C}\right), 137.5,133.9,130.6\left(\mathrm{~d}, J_{\mathrm{C}-\mathrm{F}}=1.8 \mathrm{~Hz}, 1 \mathrm{C}\right)$, 
129.3, 129.0, $127.4\left(\mathrm{~d}, J_{\mathrm{C}-\mathrm{F}}=10.6 \mathrm{~Hz}, 1 \mathrm{C}\right), 116.5\left(\mathrm{~d}, J_{\mathrm{C}-\mathrm{F}}=23.7 \mathrm{~Hz}, 1 \mathrm{C}\right), 113.0\left(\mathrm{~d}, J_{\mathrm{C}-\mathrm{F}}=22.6 \mathrm{~Hz}, 1 \mathrm{C}\right)$, 84.1, 71.7, 44.2, 39.2, $35.8\left(\mathrm{~d}, J_{\mathrm{C}-\mathrm{F}}=1.8 \mathrm{~Hz}, 1 \mathrm{C}\right), 27.8,20.9 ;{ }^{19} \mathrm{~F}$ NMR $\left(282 \mathrm{MHz}, \mathrm{CDCl}_{3}\right): \delta$ -99.71 -99.78 (m, 1F); IR (neat): 3011, 2079, 1719, 1609, 1433, 1302, 1215, 1105, $1063 \mathrm{~cm}^{-1}$; HRMS (ESI) $m / z:[\mathrm{M}+\mathrm{Na}]^{+}$Calcd for $\mathrm{C}_{24} \mathrm{H}_{24} \mathrm{FN}_{3} \mathrm{NaO}_{4} 460.1643$; Found: 460.1650 .

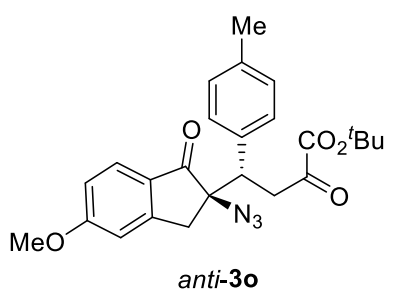

$\min , \mathrm{t}_{\mathrm{r}}($ minor $\left.)=11.71 \mathrm{~min}\right]$ gave the isomeric composition of major diastereomer: $89 \%$ ee; $[\alpha]_{\mathrm{D}}{ }^{20}=$ -148.1 $\left(c=0.50, \mathrm{CHCl}_{3}\right) .{ }^{1} \mathrm{H} \mathrm{NMR}\left(300 \mathrm{MHz}, \mathrm{CDCl}_{3}\right): \delta 7.61(\mathrm{~d}, J=8.7 \mathrm{~Hz}, 1 \mathrm{H}), 7.14(\mathrm{~d}, J=8.1 \mathrm{~Hz}$, 2H), $6.99(\mathrm{~d}, J=7.8 \mathrm{~Hz}, 2 \mathrm{H}), 6.85-6.82(\mathrm{~m}, 1 \mathrm{H}), 6.72(\mathrm{~d}, J=2.4 \mathrm{~Hz}, 1 \mathrm{H}), 3.89(\mathrm{dd}, J=8.6 \mathrm{~Hz}, J=$ $6.0 \mathrm{~Hz}, 1 \mathrm{H}), 3.84(\mathrm{~s}, 3 \mathrm{H}), 3.76(\mathrm{ABd}, J=17.2 \mathrm{~Hz}, J=5.6 \mathrm{~Hz}, 1 \mathrm{H}), 3.35-3.28(\mathrm{~m}, 2 \mathrm{H}), 2.87$ (d, $J=$ $18.0 \mathrm{~Hz}, 1 \mathrm{H}), 2.22(\mathrm{~s}, 3 \mathrm{H}), 1.52(\mathrm{~s}, 9 \mathrm{H}) ;{ }^{13} \mathrm{C} \mathrm{NMR}\left(125 \mathrm{MHz}, \mathrm{CDCl}_{3}\right): \delta 198.9,192.6,166.2,160.2$, 153.8, 137.2, 134.4, 129.1, 129.1, 127.3, 126.8, 116.1, 109.4, 84.0, 71.8, 55.7, 44.3, 39.4, 36.0, 27.7, 20.9; IR (neat): 2099, 1717, 1612, 1472, 1371, 1223, 1088, $754 \mathrm{~cm}^{-1}$; HRMS (ESI) $m / z:[\mathrm{M}+\mathrm{Na}]^{+}$ Calcd for $\mathrm{C}_{25} \mathrm{H}_{27} \mathrm{~N}_{3} \mathrm{NaO}_{5} 472.1843$; Found: 472.1848 .

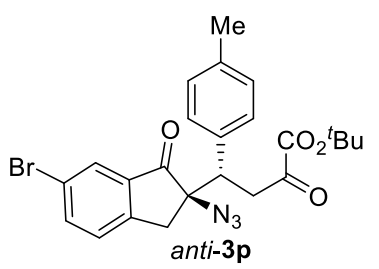

Column chromatography with $\mathrm{PE} / \mathrm{Et}_{2} \mathrm{O}(10: 1$, v/v) afforded product anti-3p in $89 \%$ yield (110.9 mg) as light yellow liquid (7 days). ${ }^{1} \mathrm{H}$ NMR analysis of the crude product revealed that the dr value was 5:1. HPLC analysis [Chiralcel $\mathrm{OZ}-\mathrm{H}, 3 \%{ }^{i} \mathrm{PrOH} /$ hexane, $1.0 \mathrm{~mL} / \mathrm{min}, 230 \mathrm{~nm}$; major diastereomer: $\mathrm{t}_{\mathrm{r}}$ (major) $=16.97 \mathrm{~min}, \mathrm{t}_{\mathrm{r}}($ minor $)=14.48 \mathrm{~min}$, minor diastereomer: $\mathrm{t}_{\mathrm{r}}($ major $)=14.11 \mathrm{~min}, \mathrm{t}_{\mathrm{r}}($ minor $)=12.21$ $\min ]$ gave the isomeric composition of major diastereomer: $85 \%$ ee; $[\alpha]_{\mathrm{D}}^{20}=-173.0\left(c=0.96, \mathrm{CHCl}_{3}\right)$. ${ }^{1} \mathrm{H}$ NMR (400 MHz, $\left.\mathrm{CDCl}_{3}\right): \delta 7.77(\mathrm{~d}, J=2.0 \mathrm{~Hz}, 1 \mathrm{H}), 7.64-7.62(\mathrm{~m}, 1 \mathrm{H}), 7.20-7.17(\mathrm{~m}, 1 \mathrm{H})$, 7.11-7.09 (m, 2H), 6.98 (d, $J=8.0 \mathrm{~Hz}, 2 \mathrm{H}), 3.93(\mathrm{dd}, J=8.4 \mathrm{~Hz}, J=6.0 \mathrm{~Hz}, 1 \mathrm{H}), 3.70(\mathrm{ABd}, J=17.2$ $\mathrm{Hz}, J=6.0 \mathrm{~Hz}, 1 \mathrm{H}), 3.33(\mathrm{AB}, J=17.6 \mathrm{~Hz}, 1 \mathrm{H}), 3.31(\mathrm{ABd}, J=17.4 \mathrm{~Hz}, J=8.4 \mathrm{~Hz}, 1 \mathrm{H}), 2.86(\mathrm{AB}$, $J=18.0 \mathrm{~Hz}, 1 \mathrm{H}), 2.21(\mathrm{~s}, 3 \mathrm{H}), 1.52(\mathrm{~s}, 9 \mathrm{H}) ;{ }^{13} \mathrm{C} \mathrm{NMR}\left(100 \mathrm{MHz}, \mathrm{CDCl}_{3}\right): \delta 199.8,192.4,160.1$, 149.3, 138.7, 137.5, 136.0, 133.8, 129.3, 129.0, 127.8, 127.7, 122.2, 84.1, 71.9, 44.1, 39.2, 35.6, 27.8, 20.9; IR (neat): 2999, 2359, 2093, 1719, 1570, 1420, 1362, 1252, 1157, 1092, $708 \mathrm{~cm}^{-1}$; HRMS (ESI) $m / z:[\mathrm{M}+\mathrm{Na}]^{+}$Calcd for $\mathrm{C}_{24} \mathrm{H}_{24} \mathrm{BrN}_{3} \mathrm{NaO}_{4}$ 520.0842; Found: 520.0849 . 


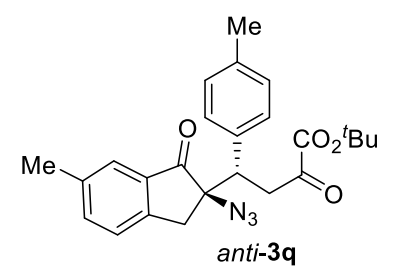

The reaction was conducted with $20 \mathrm{~mol} \%$ C6a. Column chromatography with $\mathrm{PE} / \mathrm{Et}_{2} \mathrm{O}(10: 1, \mathrm{v} / \mathrm{v})$ afforded product anti-3q in $64 \%$ yield $(69.9 \mathrm{mg})$ as light yellow liquid (9 days). ${ }^{1} \mathrm{H}$ NMR analysis of the crude product revealed that the dr value was 6:1. HPLC analysis [Chiralpak AS-H, $3 \%{ }^{i} \mathrm{PrOH} / \mathrm{h}$ exane, $1.0 \mathrm{~mL} / \mathrm{min}, 230 \mathrm{~nm}$; major diastereomer: $\mathrm{t}_{\mathrm{r}}($ major $)=12.50 \mathrm{~min}, \mathrm{t}_{\mathrm{r}}($ minor $)=$ $13.63 \mathrm{~min}]$ gave the isomeric composition of major diastereomer: $88 \%$ ee; $[\alpha]_{\mathrm{D}}{ }^{20}=-209.9(c=0.98$, $\mathrm{CHCl}_{3}$ ). ${ }^{1} \mathrm{H}$ NMR (400 MHz, $\left.\mathrm{CDCl}_{3}\right): \delta 7.46$ (s, 1H), 7.37-7.35 (m, 1H), 7.20-7.12 (m, 3H), 6.99 (d, $J$ $=8.0 \mathrm{~Hz}, 2 \mathrm{H}), 3.89(\mathrm{dd}, J=8.4 \mathrm{~Hz}, J=6.0 \mathrm{~Hz}, 1 \mathrm{H}), 3.74(\mathrm{ABd}, J=17.2 \mathrm{~Hz}, J=5.6 \mathrm{~Hz}, 1 \mathrm{H})$, 3.36-3.29 (m, 2H), $2.88(\mathrm{~d}, J=17.2 \mathrm{~Hz}, 1 \mathrm{H}), 2.33(\mathrm{~s}, 3 \mathrm{H}), 2.21$ (s, 3H), $1.52(\mathrm{~s}, 9 \mathrm{H}) ;{ }^{13} \mathrm{C}$ NMR $(100$ $\left.\mathrm{MHz}, \mathrm{CDCl}_{3}\right): \delta 201.0,192.7,160.2,148.2,138.1,137.3,137.3,134.4,134.3,129.2,129.1,125.9$, 124.9, 84.0, 72.0, 44.2, 39.4, 35.7, 27.8, 21.0, 20.9; IR (neat): 2988, 2110, 1707, 1609, 1491, 1366, 1225, 1103, 1024, 839, $750 \mathrm{~cm}^{-1}$; HRMS (ESI) $\mathrm{m} / z$ : $[\mathrm{M}+\mathrm{Na}]^{+} \mathrm{Calcd}$ for $\mathrm{C}_{25} \mathrm{H}_{27} \mathrm{~N}_{3} \mathrm{NaO}_{4} 456.1894$; Found: 456.1893.

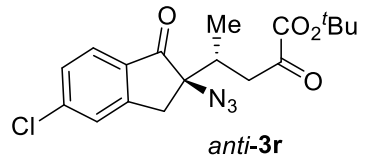

The reaction was conducted at $0{ }^{\circ} \mathrm{C}$. Column chromatography with $\mathrm{PE} / \mathrm{Et}_{2} \mathrm{O}$ $(10: 1, \mathrm{v} / \mathrm{v})$ afforded product anti-3r in $56 \%$ yield $(52.9 \mathrm{mg})$ as light yellow liquid (6 days). ${ }^{1} \mathrm{H}$ NMR analysis of the crude product revealed that the $\mathrm{dr}$ value was 6:1. HPLC analysis [Chiralcel $\mathrm{OZ}-\mathrm{H}, 3 \%{ }^{i} \mathrm{PrOH} / \mathrm{h}$ exane, $1.0 \mathrm{~mL} / \mathrm{min}, 230 \mathrm{~nm}$; major diastereomer: $\mathrm{t}_{\mathrm{r}}($ major $)=24.10 \mathrm{~min}, \mathrm{t}_{\mathrm{r}}($ minor $)=12.04 \mathrm{~min}$, minor diastereomer: $\mathrm{t}_{\mathrm{r}}($ major $)=10.31$ $\min , t_{r}($ minor $\left.)=11.62 \mathrm{~min}\right]$ gave the isomeric composition of major diastereomer: $81 \%$ ee; $[\alpha]_{\mathrm{D}}{ }^{20}=$ -232.5 $\left(c=0.88, \mathrm{CHCl}_{3}\right) .{ }^{1} \mathrm{H}$ NMR $\left(400 \mathrm{MHz}, \mathrm{CDCl}_{3}\right): \delta 7.76-7.73(\mathrm{~m}, 1 \mathrm{H}), 7.46(\mathrm{~s}, 1 \mathrm{H}), 7.43-7.40$ (m, 1H), 3.33-3.20 (m, 2H), 2.94-2.85 (m, 2H), 2.69-2.63 (m, 1H), 1.57 (s, 7.3H) (major), 1.53 (s, $1.7 \mathrm{H})$ (minor), $1.10(\mathrm{~d}, J=6.8 \mathrm{~Hz}, 0.5 \mathrm{H})$ (minor), $0.82(\mathrm{~d}, J=6.8 \mathrm{~Hz}, 2.5 \mathrm{H})$ (major); ${ }^{13} \mathrm{C}$ NMR $(100$ $\mathrm{MHz}, \mathrm{CDCl}_{3}$ ): $\delta 200.3$ (major), 193.2 (major), 192.9 (minor), 160.3 (major), 152.9 (major), 152.7 (minor), 142.9 (major), 133.1 (major), 129.3 (minor), 129.2 (major), 126. 8 (minor), 126.7 (major), 126.1 (major), 126.0 (minor), 84.4 (minor), 84.3 (major), 71.6 (minor), 71.1 (major), 40.8 (major), 40.8 (minor), 35.7 (minor), 34.5 (major), 33.7 (major), 32.8 (minor), 27.8 (major), 27.7 (minor), 15.4 (major), 14.8 (minor); IR (neat): 2980, 2099, 1715, 1599, 1369, 1157, 1057, 897, $611 \mathrm{~cm}^{-1}$; HRMS (ESI) $m / z:[\mathrm{M}+\mathrm{Na}]^{+} \mathrm{Calcd}$ for $\mathrm{C}_{18} \mathrm{H}_{20} \mathrm{ClN}_{3} \mathrm{NaO}_{4} 400.1035$; Found: 400.1039. 


\section{Gram-scale synthesis and synthetic elaboration of $3 \mathrm{~m}$}

\subsection{Gram-scale synthesis of $\mathbf{3 m}$}

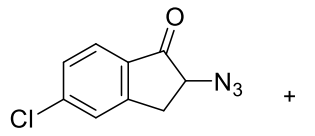

$1 d(2.2 \mathrm{mmol})$

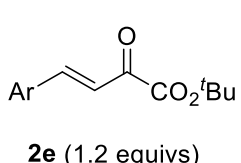

2e (1.2 equivs)

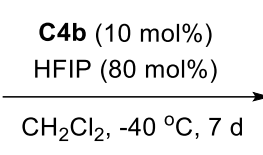

$\mathrm{Ar}=4-\mathrm{MeC}_{6} \mathrm{H}_{4}$

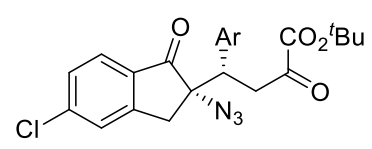

$(S, S)-3 m$

To a $100 \mathrm{~mL}$ Schlenk flask were added tertiary amine-phosphoramide catalyst $\mathbf{C 4 b}$ (98.6 mg, 0.22 mmol, 10 mol\%), $\alpha$-azido indanones $1 d$ (0.457 g, $2.2 \mathrm{mmol}, 1.0$ equiv) and HFIP (198 $\mu \mathrm{L}, 80 \mathrm{~mol} \%)$, followed by the addition of anhydrous $\mathrm{CH}_{2} \mathrm{Cl}_{2}(22 \mathrm{~mL})$ except as noted. The reaction mixture was stirred at $-40{ }^{\circ} \mathrm{C}$ for $30 \mathrm{~min}$, and $\beta, \gamma$-unsaturated $\alpha$-ketoester $2 \mathrm{e}(0.65 \mathrm{~g}, 0.30 \mathrm{mmol}, 1.2$ equivs $)$ was added. After full consumption of $\alpha$-azido ketones $\mathbf{1 d}$ as indicated by TLC analysis, ${ }^{1} \mathrm{H}$ NMR analysis of the crude product revealed that the dr value was 8:1. The crude product was purified by silica gel column chromatography using PE: $\mathrm{Et}_{2} \mathrm{O}(10: 1, \mathrm{v} / \mathrm{v})$ as the eluent to afford the desired $(S, S)-\mathbf{3 m}$ in $91 \%$ yield $(0.91 \mathrm{~g})$. HPLC analysis [Chiralcel OZ-H, 3\% ${ }^{i} \mathrm{PrOH} / \mathrm{hexane}, 1.0 \mathrm{~mL} / \mathrm{min}, 230 \mathrm{~nm}$; $\mathrm{t}_{\mathrm{r}}$ (major) = $\left.15.21 \mathrm{~min}, \mathrm{t}_{\mathrm{r}}(\operatorname{minor})=11.378 \mathrm{~min}\right]$ gave the isomeric composition of the product: $98 \%$ ee.

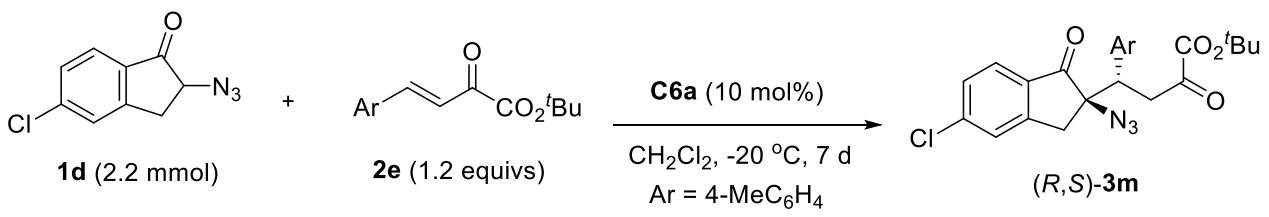

To a $100 \mathrm{~mL}$ Schlenk flask were added tertiary amine-phosphoramide catalyst C6a (131 mg, 0.22 mmol, 10 mol\%), $\alpha$-azido indanones $1 d(0.457$ g, $2.2 \mathrm{mmol}, 1.0$ equiv) and HFIP (198 $\mu \mathrm{L}, 80 \mathrm{~mol} \%)$, followed by the addition of anhydrous $\mathrm{CH}_{2} \mathrm{Cl}_{2}(22 \mathrm{~mL})$ except as noted. The reaction mixture was stirred at $-20^{\circ} \mathrm{C}$ for $30 \mathrm{~min}$, and $\beta, \gamma$-unsaturated $\alpha$-ketoester $2 \mathrm{e}(0.65 \mathrm{~g}, 0.30 \mathrm{mmol}, 1.2$ equivs) was added. After full consumption of $\alpha$-azido ketones $\mathbf{1 d}$ as indicated by TLC analysis, ${ }^{1} \mathrm{H}$ NMR analysis of the crude product revealed that the dr value was 9:1. The crude product was purified by silica gel column chromatography using $\mathrm{PE}: \mathrm{Et}_{2} \mathrm{O}(10: 1, \mathrm{v} / \mathrm{v})$ as the eluent to afford the desired $(S, S)-\mathbf{3 m}$ in $70 \%$ yield (0.70 g). HPLC analysis [Chiralcel OZ-H, 3\% ${ }^{i} \mathrm{PrOH} / \mathrm{hexane}, 1.0 \mathrm{~mL} / \mathrm{min}, 230 \mathrm{~nm}$; $\mathrm{t}_{\mathrm{r}}$ (major) = $14.69 \mathrm{~min}, \mathrm{t}_{\mathrm{r}}($ minor $\left.)=13.53 \mathrm{~min}\right]$ gave the isomeric composition of the product: $89 \%$ ee. 


\subsection{Synthetic elaboration of $3 \mathrm{~m}$}

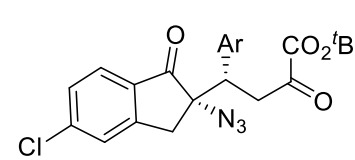

$(S, S)-3 m, 98 \%$ ee

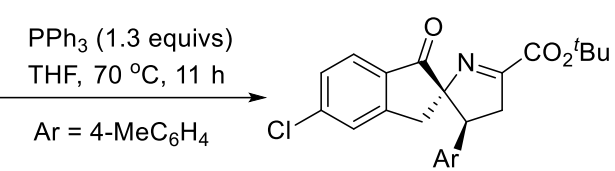

4a, $75 \%, 96 \%$ ee

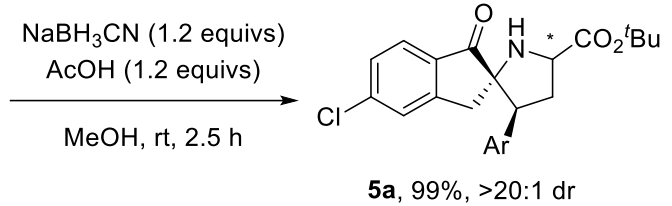

$99 \%$ ee

Under an atmosphere of $\mathrm{N}_{2}$, to a solution of $(S, S)-3 \mathrm{~m}(86.2 \mathrm{mg}, 0.19 \mathrm{mmol})$ in THF $(2.5 \mathrm{~mL})$ was added $\mathrm{PPh}_{3}(64.8 \mathrm{mg}, 0.25 \mathrm{mmol})$. The reaction mixture was stirred at $70{ }^{\circ} \mathrm{C}$ (oil bath) until full consumption of $\mathbf{3 m}$ by TLC analysis (about $11 \mathrm{~h}$ ). After cooling to room temperature (rt), the resulting solution was concentrated under vacuo, and purified by silica gel column chorography (PE/EtOAc, $8: 1, \mathrm{v} / \mathrm{v})$ to furnish spiro-dihydropyrrole derivative $\mathbf{4 a}$ in $75 \%$ yield $(58.4 \mathrm{mg})$ as a white solid, $\mathrm{m} . \mathrm{p} .=$ 89-91 ${ }^{\circ} \mathrm{C}$. HPLC analysis [Chiralcel OZ-H, 10\% ${ }^{i} \mathrm{PrOH} / \mathrm{hexane}, 1.0 \mathrm{~mL} / \mathrm{min}, 230 \mathrm{~nm}$; $\mathrm{t}_{\mathrm{r}}$ (major) = $16.18 \mathrm{~min}, \mathrm{t}_{\mathrm{r}}($ minor $\left.)=18.60 \mathrm{~min}\right]$ gave the isomeric composition of the product: $96 \%$ ee. $[\alpha]_{\mathrm{D}}{ }^{20}=$ +318.2 $\left(c=0.88, \mathrm{CHCl}_{3}\right) .{ }^{1} \mathrm{H} \mathrm{NMR}\left(300 \mathrm{MHz} \mathrm{CDCl}_{3}\right): \delta 7.38-7.36(\mathrm{~m}, 2 \mathrm{H}), 7.16(\mathrm{~d}, J=8.4 \mathrm{~Hz}, 1 \mathrm{H})$, $7.06(\mathrm{~d}, J=7.6 \mathrm{~Hz}, 2 \mathrm{H}), 6.93(\mathrm{~d}, J=8.0 \mathrm{~Hz}, 2 \mathrm{H}), 3.95(\mathrm{AB}, J=17.6 \mathrm{~Hz}, 1 \mathrm{H}), 3.73-3.59$ (m, 2H), 3.40 $(\mathrm{AB}, J=17.6 \mathrm{~Hz}, 1 \mathrm{H}), 3.23-3.17(\mathrm{~m}, 1 \mathrm{H}), 2.18(\mathrm{~s}, 3 \mathrm{H}), 1.59(\mathrm{~s}, 9 \mathrm{H}) ;{ }^{13} \mathrm{C} \mathrm{NMR}\left(100 \mathrm{MHz}, \mathrm{CDCl}_{3}\right): \delta$ 200.3, 172.6, 161.6, 153.8, 141.8, 137.1, 133.9, 132.1, 129.1, 128.4, 127.9, 126.2, 125.4, 88.2, 83.3, 54.5, 41.4, 39.8, 28.0, 20.9; IR (neat): 3005, 2359, 2097, 1709, 1533, 1362, 1219, 1111, 1030, 895, $716 \mathrm{~cm}^{-1}$; HRMS (ESI) m/z: [M+Na] ${ }^{+}$Calcd for $\mathrm{C}_{24} \mathrm{H}_{24} \mathrm{ClNNaO}_{3} 432.1337$; Found: 432.1341.

To a solution of $4 \mathbf{a}(40.9 \mathrm{mg}, 0.1 \mathrm{mmol})$ in $\mathrm{MeOH}(1.0 \mathrm{~mL})$ was added $\mathrm{NaBH}_{3} \mathrm{CN}(7.5 \mathrm{mg}, 0.12$ $\mathrm{mmol})$ and $\mathrm{AcOH}(7.2 \mathrm{mg}, 0.12 \mathrm{mmol})$. The reaction mixture was stirred at $\mathrm{rt}$ for $2.5 \mathrm{~h}$. The crude residue was purified by silica gel column chorography (PE/EtOAc, 8:1, v/v) to give the desired spirocyclic proline derivative 5a in $99 \%$ yield $(40.8 \mathrm{mg})$ as a colorless liquid. ${ }^{1} \mathrm{H}$ NMR analysis of the crude product revealed that the $\mathrm{dr}$ value was >20:1. HPLC analysis [Chiralcel OZ-H, 25\% ${ }^{i} \mathrm{PrOH} /$ hexane, $1.0 \mathrm{~mL} / \mathrm{min}, 230 \mathrm{~nm}$; $\mathrm{t}_{\mathrm{r}}($ major $)=9.39 \mathrm{~min}, \mathrm{t}_{\mathrm{r}}($ minor $\left.)=7.65 \mathrm{~min}\right]$ gave the isomeric composition of the product: $99 \%$ ee. $[\alpha]_{\mathrm{D}}{ }^{20}=91.0\left(c=0.7, \mathrm{CHCl}_{3}\right) .{ }^{1} \mathrm{H} \mathrm{NMR}\left(400 \mathrm{MHz}, \mathrm{CDCl}_{3}\right): \delta$ $7.30(\mathrm{~d}, J=8.0 \mathrm{~Hz}, 1 \mathrm{H}), 7.23(\mathrm{~s}, 1 \mathrm{H}), 7.12-7.09(\mathrm{~m}, 1 \mathrm{H}), 6.99-6.97(\mathrm{~m}, 2 \mathrm{H}), 6.86(\mathrm{~d}, J=7.6 \mathrm{~Hz}, 2 \mathrm{H})$, $4.06(\mathrm{dd}, J=9.0 \mathrm{~Hz}, J=7.6 \mathrm{~Hz}, 1 \mathrm{H}), 3.48(\mathrm{dd}, J=12.6 \mathrm{~Hz}, J=6.4 \mathrm{~Hz}, 1 \mathrm{H}), 3.36(\mathrm{AB}, J=17.2 \mathrm{~Hz}$, $1 \mathrm{H}), 3.25(\mathrm{AB}, J=17.2 \mathrm{~Hz}, 1 \mathrm{H}), 2.88-2.80(\mathrm{~m}, 1 \mathrm{H}), 2.50-2.43(\mathrm{~m}, 1 \mathrm{H}), 2.16(\mathrm{~s}, 3 \mathrm{H}), 1.56(\mathrm{~s}, 9 \mathrm{H}) ;{ }^{13} \mathrm{C}$ NMR (100 MHz, $\left.\mathrm{CDCl}_{3}\right): \delta 207.3$ 172.7, 152.5, 141.2, 136.9, 134.1, 133.1, 128.9, 128.0, 127.8, 125.9, 124.7, 81.6, 74.9, 59.2, 55.6, 40.7, 34.6, 28.2, 20.9; IR (neat): 1717, 1601, 1479, 1319, 1225, 816, 754, $665 \mathrm{~cm}^{-1}$; HRMS (ESI) m/z: [M+H] ${ }^{+}$Calcd for $\mathrm{C}_{24} \mathrm{H}_{27} \mathrm{ClNO}_{3}$ 412.1674; Found: 412.1667. 


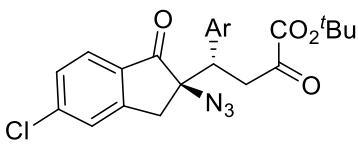

$(R, S)-3 \mathbf{m}, 90 \%$ ee

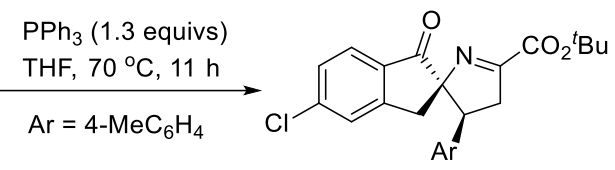

4b, $79 \%, 90 \%$ ee

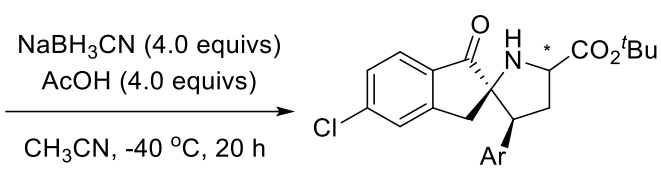

5b, $61 \%$ $18: 1 \mathrm{dr}, 88 \%$ ee

Under an atmosphere of $\mathrm{N}_{2}$, to a solution of $(R, S)-3 \mathrm{~m}(90.8 \mathrm{mg}, 0.2 \mathrm{mmol})$ in THF $(2.5 \mathrm{~mL})$ was added $\mathrm{PPh}_{3}\left(68.2 \mathrm{mg}, 0.26 \mathrm{mmol}\right.$ ). The reaction mixture was stirring at $70{ }^{\circ} \mathrm{C}$ (oil bath) until full consumption of $\mathbf{3 m}$ by TLC analysis (about $10 \mathrm{~h}$ ). After cooling to room temperature (rt), the resulting solution was concentrated under vacuo, and purified by silica gel column chorography (PE/EtOAc, $8: 1, \mathrm{v} / \mathrm{v})$ to furnish spiro-dihydropyrrole derivative $\mathbf{4 b}$ in $79 \%$ yield $(64.5 \mathrm{mg})$ as white solid, m.p. $=$ 136-138 ${ }^{\circ} \mathrm{C}$. HPLC analysis [Chiralcel OZ-H, 25\% ${ }^{i} \mathrm{PrOH} / \mathrm{hexane}, 1.0 \mathrm{~mL} / \mathrm{min}, 230 \mathrm{~nm}$; $\mathrm{t}_{\mathrm{r}}$ (major) = $15.59 \mathrm{~min}, \mathrm{t}_{\mathrm{r}}($ minor $\left.)=6.83 \mathrm{~min}\right]$ gave the isomeric composition of the product: $90 \%$ ee. $[\alpha]_{\mathrm{D}}{ }^{20}=$ -203.2 $\left(c=0.94, \mathrm{CHCl}_{3}\right) .{ }^{1} \mathrm{H}$ NMR $\left(300 \mathrm{MHz}, \mathrm{CDCl}_{3}\right): \delta 7.71(\mathrm{~d}, J=8.1 \mathrm{~Hz}, 1 \mathrm{H}), 7.35-7.30(\mathrm{~m}, 2 \mathrm{H})$, $7.24(\mathrm{~s}, 1 \mathrm{H}), 7.07$ (d, $J=7.8 \mathrm{~Hz}, 2 \mathrm{H}), 6.90-6.87(\mathrm{~m}, 2 \mathrm{H}), 3.90(\mathrm{dd}, J=9.0 \mathrm{~Hz}, J=5.4 \mathrm{~Hz}, 1 \mathrm{H}), 3.63$ $(\mathrm{ABd}, J=18.4 \mathrm{~Hz}, J=9.0 \mathrm{~Hz}, 1 \mathrm{H}), 3.32(\mathrm{ABd}, J=18.3 \mathrm{~Hz}, J=5.4 \mathrm{~Hz}, 1 \mathrm{H}), 3.16(\mathrm{AB}, J=17.7 \mathrm{~Hz}$, 1H), 2.89 (AB, $J=17.7 \mathrm{~Hz}, 1 \mathrm{H}), 2.30(\mathrm{~s}, 3 \mathrm{H}), 1.58(\mathrm{~s}, 9 \mathrm{H}) ;{ }^{13} \mathrm{C} \mathrm{NMR}\left(100 \mathrm{MHz}, \mathrm{CDCl}_{3}\right): \delta 199.8$, $192.4,160.2$, 152.3, 142.6, 137.5, 133.8, 132.7, 129.3, 129.0, 128.9, 126.4, 126.0, 84.1, 71.6, 44.2, 39.2, 35.6, 27.8, 20.9; IR (neat): 3007, 2102, 1728, 1599, 1435, 1354, 1244, 1157, 1065, 903, 718 $\mathrm{cm}^{-1}$; HRMS (ESI) $m / z$ : [M+Na] ${ }^{+}$Calcd for $\mathrm{C}_{24} \mathrm{H}_{24} \mathrm{ClNNaO}_{3} 432.1337$; Found: 432.1343.

To a solution of $\mathbf{4 b}(40.9 \mathrm{mg}, 0.1 \mathrm{mmol})$ in $\mathrm{CH}_{3} \mathrm{CN}(2.0 \mathrm{~mL})$ was added $\mathrm{NaBH}_{3} \mathrm{CN}(25 \mathrm{mg}, 0.4$ $\mathrm{mmol}$ ) and $\mathrm{AcOH}(24 \mathrm{mg}, 0.4 \mathrm{mmol})$. The resulting reaction mixture was stirred at $-40{ }^{\circ} \mathrm{C}$ for $20 \mathrm{~h}$. The crude residue was purified by fast column chorography (PE/EtOAc, 6:1, v/v) to give the desired spirocyclic proline derivative $\mathbf{5 b}$ in $61 \%$ yield $(25.1 \mathrm{mg})$ as a colorless liquid. ${ }^{1} \mathrm{H}$ NMR analysis of the crude product revealed that the dr value was 18:1. HPLC analysis [Chiralcel OD-H, $3 \%{ }^{i} \mathrm{PrOH} / \mathrm{hexane}$, $0.5 \mathrm{~mL} / \mathrm{min}, 230 \mathrm{~nm} ; \mathrm{t}_{\mathrm{r}}$ (major) $=21.89 \mathrm{~min}, \mathrm{t}_{\mathrm{r}}($ minor $)=30.25 \mathrm{~min}$ ] gave the isomeric composition of the product: $88 \%$ ee. $[\alpha]_{\mathrm{D}}{ }^{20}=-78.4\left(c=0.1, \mathrm{CHCl}_{3}\right) .{ }^{1} \mathrm{H} \mathrm{NMR}\left(400 \mathrm{MHz}, \mathrm{CDCl}_{3}\right): \delta 7.64(\mathrm{~d}, J=8.0$ $\mathrm{Hz}, 1 \mathrm{H}), 7.26-7.24(\mathrm{~m}, 1 \mathrm{H}), 7.06(\mathrm{~d}, J=1.6 \mathrm{~Hz}, 1 \mathrm{H}), 6.94-6.87(\mathrm{~m}, 4 \mathrm{H}), 4.16(\mathrm{dd}, J=9.2 \mathrm{~Hz}, J=7.6$ $\mathrm{Hz}, 1 \mathrm{H}), 3.61(\mathrm{dd}, J=11.6 \mathrm{~Hz}, J=6.8 \mathrm{~Hz}, 1 \mathrm{H}), 2.97(\mathrm{AB}, J=17.6 \mathrm{~Hz}, 1 \mathrm{H}), 2.92(\mathrm{AB}, J=17.6 \mathrm{~Hz}$, $1 \mathrm{H}), 2.70-2.63(\mathrm{~m}, 1 \mathrm{H}), 2.40-2.31(\mathrm{~m}, 1 \mathrm{H}), 2.22(\mathrm{~s}, 3 \mathrm{H}), 1.52(\mathrm{~s}, 9 \mathrm{H}) ;{ }^{13} \mathrm{C} \mathrm{NMR}\left(100 \mathrm{MHz}, \mathrm{CDCl}_{3}\right): \delta$ 206.7, 173.9, 153.9, 141.7, 136.7, 134.6, 134.1, 128.9, 128.1, 127.7126 .3125 .181 .4 74.9, 59.3 52.6, 38.2, 35.4 28.1, 20.9; IR (neat): 1717, 1601, 1514, 1366, 1223, 1157, 1070, $723 \mathrm{~cm}^{-1}$; HRMS (ESI) $m / z:[\mathrm{M}+\mathrm{H}]^{+}$Calcd for $\mathrm{C}_{24} \mathrm{H}_{27} \mathrm{ClNO}_{3} 412.1674$, Found: 412.1667 . 


\section{X-ray crystallographic data of syn-3i and $4 \mathrm{~b}$}

Single crystals of syn-3i were obtained by slow diffusion of the solution of $\operatorname{syn}-3 \mathbf{i}$ in $\mathrm{PE} / \mathrm{CH}_{2} \mathrm{Cl}_{2}$ at RT. Data intensity of $s y n-\mathbf{3 i}^{8}$ was collected using a 'Bruker APEX-II CCD' diffractometer at 293(2) K. Data collection and reduction were done by using Olex2 and the structure was solved with the ShelXS structure solution program using direct methods and refined by full-matrix least-squares on $F^{2}$ with anisotropic displacement parameters for non-H atoms using SHELX-97. Hydrogen atoms were added at their geometrically idea positions and refined isotropically. Crystal data for syn-3i: $\mathrm{C}_{21} \mathrm{H}_{20} \mathrm{~N}_{3} \mathrm{O}_{4} \mathrm{~S}, T$ $=293(2) \mathrm{K}$, monoclinic, space group I2, $a=12.8339$ (4) $\AA, b=8.8764(3) \AA, c=19.2260(6) \AA, \alpha=90$ deg, $\beta=92.145(3) \mathrm{deg}, \gamma=90 \mathrm{deg}, V=2188.67(12) \AA^{3} . \mathrm{Z}=4, d_{\text {calc }}=1.246 \mathrm{~g} / \mathrm{cm}^{3} .19149$ reflections measured, $3803\left[\mathrm{R}_{\text {int }}=0.1235, \mathrm{R}_{\text {sigma }}=0.0556\right], \mathrm{R}_{1}=0.0749, \mathrm{wR}_{2}=0.2090,(I>2 \sigma(I)$, final $), \mathrm{R}_{1}=$ $0.0848, \mathrm{wR}_{2}=0.2209$ (all data), $\mathrm{GOF}=1.061$, and 265 parameters.

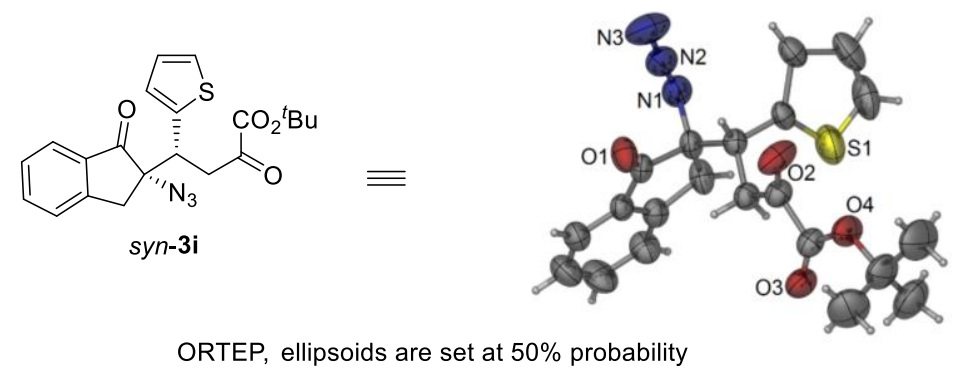

Table S2. Crystal data and structure refinement for syn-3i.

Identification code

Empirical formula

Formula weight

Temperature

Crystal system

Space group

Unit cell dimensions

Volume

Z syn-3i

$\mathrm{C}_{21} \mathrm{H}_{20} \mathrm{~N}_{3} \mathrm{O}_{4} \mathrm{~S}$

410.46

293(2) K

monoclinic

I2

$\mathrm{a}=12.8339(4) \AA \quad \mathrm{a}=90^{\circ}$.

$\mathrm{b}=8.8764(3) \AA \quad \mathrm{b}=92.145(3)^{\circ}$.

$\mathrm{c}=19.2260(6) \AA \quad \mathrm{g}=90^{\circ}$. 2188.67(12) $\AA^{3}$

${ }^{8}$ Supplementary crystallographic data have been deposited at the Cambridge Crystallographic Data Center (CCDC number: 1959344). 
$\rho_{\text {calc }} \mathrm{g} / \mathrm{cm}^{3}$

$\mu / \mathrm{mm}^{-1}$

$\mathrm{F}(000)$

Crystal size $/ \mathrm{mm}^{3}$

Radiation

$2 \Theta$ range for data collection/ ${ }^{\circ}$

Index ranges

Reflections collected

Independent reflections

Data/restraints/parameters

Goodness-of-fit on $\mathrm{F}^{2}$

Final $\mathrm{R}$ indexes $[\mathrm{I}>=2 \sigma(\mathrm{I})]$

Final $\mathrm{R}$ indexes [all data]

Largest diff. peak/hole / e $\AA^{-3}$

Flack parameter
1.246

1.573

860.0

$0.32 \times 0.26 \times 0.18$

$\operatorname{CuK} \alpha(\lambda=1.54184)$

8.144 to 134.044

$-15 \leq \mathrm{h} \leq 15,-10 \leq \mathrm{k} \leq 10,-22 \leq 1 \leq 22$

19149

$3803\left[\mathrm{R}_{\text {int }}=0.1235, \mathrm{R}_{\text {sigma }}=0.0556\right]$

$3803 / 1 / 265$

1.061

$$
\begin{aligned}
& \mathrm{R}_{1}=0.0749, \mathrm{wR}_{2}=0.2090 \\
& \mathrm{R}_{1}=0.0848, \mathrm{wR}_{2}=0.2209 \\
& 0.31 /-0.22 \\
& -0.01(5)
\end{aligned}
$$

Table S3 Fractional Atomic Coordinates $\left(\times 10^{4}\right)$ and Equivalent Isotropic Displacement Parameters $\left(\AA^{2} \times 10^{3}\right)$ for $s y n-3 i$. $U_{\text {eq }}$ is defined as $1 / 3$ of of the trace of the orthogonalised $U_{\text {IJ }}$ tensor.

\begin{tabular}{lrrrr} 
Atom & $\boldsymbol{x}$ & $\boldsymbol{y}$ & \multicolumn{1}{c}{$\boldsymbol{z}(\mathbf{e q})$} \\
$\mathrm{S} 1$ & $1148.3(19)$ & $8068(3)$ & $3473.5(13)$ & $77.3(7)$ \\
C1A & $1148.3(19)$ & $8068(3)$ & $3473.5(13)$ & $77.3(7)$ \\
O1 & $3565(4)$ & $6075(5)$ & $5473.4(19)$ & $77.8(12)$ \\
O2 & $4201(4)$ & $8594(6)$ & $3580(3)$ & $93.9(16)$ \\
O3 & $5628(4)$ & $5734(5)$ & $2897(2)$ & $77.9(12)$ \\
O4 & $5387(3)$ & $8102(6)$ & $2506(2)$ & $82.1(12)$ \\
N1 & $1402(4)$ & $5610(8)$ & $4819(3)$ & $78.9(15)$ \\
N2 & $1388(5)$ & $6775(9)$ & $5144(3)$ & $89.3(17)$ \\
N3 & $1246(7)$ & $7832(12)$ & $5446(5)$ & $134(3)$ \\
C1 & $2888(4)$ & $2572(7)$ & $4740(3)$ & $60.8(13)$ \\
C2 & $2961(6)$ & $1034(8)$ & $4738(3)$ & $78.7(16)$ \\
C3 & $3558(7)$ & $341(9)$ & $5261(5)$ & $96(2)$
\end{tabular}




\begin{tabular}{|c|c|c|c|c|}
\hline $\mathrm{C} 4$ & $4067(6)$ & 1168(9) & $5776(4)$ & $89(2)$ \\
\hline $\mathrm{C} 5$ & 4008(4) & $2714(7)$ & $5781(3)$ & $67.2(14)$ \\
\hline C6 & $3406(4)$ & $3404(6)$ & $5259(2)$ & $54.9(11)$ \\
\hline $\mathrm{C} 7$ & $3214(4)$ & $5004(6)$ & $5153(2)$ & $56.9(11)$ \\
\hline $\mathrm{C} 8$ & $2423(4)$ & $5168(6)$ & $4525(2)$ & $58.0(12)$ \\
\hline C9 & $2284(5)$ & $3577(7)$ & $4235(3)$ & $70.0(15)$ \\
\hline $\mathrm{C} 10$ & $2808(4)$ & $6389(6)$ & $4018(2)$ & $54.0(11)$ \\
\hline C11 & $2043(4)$ & $6715(6)$ & $3425(2)$ & $58.3(12)$ \\
\hline $\mathrm{C} 12$ & $665(6)$ & $7790(11)$ & $2696(5)$ & $100(3)$ \\
\hline C13 & 1119(8) & $6706(12)$ & $2335(4)$ & $107(3)$ \\
\hline $\mathrm{C} 14$ & 2020(3) & $5830(5)$ & 2697.1(17) & $113.7(12)$ \\
\hline S1A & $2020(3)$ & $5830(5)$ & 2697.1(17) & $113.7(12)$ \\
\hline C15 & $3876(4)$ & $5975(6)$ & $3761(3)$ & $59.2(12)$ \\
\hline C16 & $4386(4)$ & $7324(6)$ & $3427(3)$ & $61.2(12)$ \\
\hline $\mathrm{C} 17$ & $5228(4)$ & $6936(7)$ & 2906(3) & $61.1(12)$ \\
\hline C18 & $6214(6)$ & 8091(11) & 1984(4) & $98(2)$ \\
\hline C19 & $6062(9)$ & $9611(14)$ & $1635(6)$ & $143(5)$ \\
\hline $\mathrm{C} 20$ & $6022(8)$ & 6801(16) & $1470(5)$ & $129(4)$ \\
\hline $\mathrm{C} 21$ & $7246(6)$ & $7950(15)$ & $2373(6)$ & $128(4)$ \\
\hline
\end{tabular}

Table S4 Anisotropic Displacement Parameters $\left(\AA^{2} \times 10^{3}\right)$ for $s y n-3 i$. The Anisotropic displacement factor exponent takes the form: $-2 \pi^{2}\left[h^{2} a^{* 2} U_{11}+2 h k a * b * U_{12}+\ldots\right]$.

\begin{tabular}{|c|c|c|c|c|c|}
\hline Atom & $\mathbf{U}_{11}$ & $\mathbf{U}_{22}$ & $\mathbf{U}_{23}$ & $\mathbf{U}_{13}$ & $\mathbf{U}_{12}$ \\
\hline $\mathrm{S} 1$ & $76.6(13)$ & $78.0(17) 76.4(13)$ & $14.8(12)$ & $-10.1(10)$ & $9.0(12)$ \\
\hline $\mathrm{C} 1 \mathrm{~A}$ & $76.6(13)$ & $78.0(17) 76.4(13)$ & $14.8(12)$ & $-10.1(10)$ & $9.0(12)$ \\
\hline $\mathrm{O} 1$ & 108(3) & $63(3) \quad 61(2)$ & $-0.6(19)$ & $-17(2)$ & $-8(2)$ \\
\hline $\mathrm{O} 2$ & $114(3)$ & $65(3) \quad 106(3)$ & $-19(2)$ & $51(3)$ & $-22(2)$ \\
\hline $\mathrm{O} 3$ & $87(3)$ & $78(2)$ & $-2(2)$ & 23.1(19) & $0(2)$ \\
\hline $\mathrm{O} 4$ & $87(3)$ & $79(3) \quad 82(2)$ & $20(2)$ & $31(2)$ & $1(2)$ \\
\hline
\end{tabular}




\begin{tabular}{|c|c|c|c|c|c|c|}
\hline N1 & $62(2)$ & $99(4)$ & $77(3)$ & $15(3)$ & $13(2)$ & $-7(3)$ \\
\hline $\mathrm{N} 2$ & $78(3)$ & $106(5)$ & $86(4)$ & $18(4)$ & $29(3)$ & $11(3)$ \\
\hline N3 & $120(6)$ & $135(7)$ & $151(7)$ & $-20(6)$ & $54(5)$ & $23(5)$ \\
\hline $\mathrm{C} 1$ & $61(2)$ & $63(3)$ & $59(3)$ & $5(2)$ & $3(2)$ & $-10(2)$ \\
\hline $\mathrm{C} 2$ & $94(4)$ & $59(4)$ & $81(4)$ & $-3(3)$ & $-10(3)$ & $-4(3)$ \\
\hline $\mathrm{C} 3$ & $114(5)$ & $57(4)$ & $114(5)$ & $3(4)$ & $-17(4)$ & $9(4)$ \\
\hline $\mathrm{C} 4$ & $90(4)$ & $82(5)$ & $93(4)$ & $11(4)$ & $-14(4)$ & $14(4)$ \\
\hline $\mathrm{C} 5$ & $67(3)$ & $70(4)$ & $63(3)$ & $5(3)$ & $-3(2)$ & $0(3)$ \\
\hline C6 & $58(2)$ & $58(3)$ & $49(2)$ & $8(2)$ & $5.3(19)$ & $-2(2)$ \\
\hline $\mathrm{C} 7$ & $62(2)$ & $64(3)$ & $44(2)$ & $4(2)$ & $0.6(19)$ & $-7(2)$ \\
\hline $\mathrm{C} 8$ & $58(2)$ & $67(3)$ & $49(2)$ & $10(2)$ & $-1.4(19)$ & $-5(2)$ \\
\hline C9 & $87(3)$ & $64(3)$ & $58(3)$ & $11(2)$ & $-15(3)$ & $-20(3)$ \\
\hline $\mathrm{C} 10$ & $60(2)$ & $53(3)$ & $49(2)$ & $1(2)$ & $-0.6(19)$ & $-5(2)$ \\
\hline C11 & $62(2)$ & $57(3)$ & $55(2)$ & $15(2)$ & $-2(2)$ & $-3(2)$ \\
\hline $\mathrm{C} 12$ & $92(4)$ & $94(6)$ & 112(6) & $41(5)$ & $-29(4)$ & $-5(4)$ \\
\hline C13 & $141(7)$ & $102(7)$ & $74(4)$ & $13(4)$ & $-42(4)$ & $-22(6)$ \\
\hline C14 & $138(3)$ & $121(3)$ & $0.0(16)$ & $8.3(18)$ & $-26.3(17)$ & $13(2)$ \\
\hline S1A & $138(3)$ & $121(3)$ & $0.0(16)$ & $8.3(18)$ & $-26.3(17)$ & $13(2)$ \\
\hline C15 & $63(3)$ & $58(3)$ & $57(2)$ & $3(2)$ & $7(2)$ & $-3(2)$ \\
\hline C16 & $68(3)$ & $56(3)$ & $60(3)$ & $-5(2)$ & $8(2)$ & $-5(2)$ \\
\hline C17 & $70(3)$ & $54(3)$ & $60(3)$ & $-8(2)$ & $9(2)$ & $-11(2)$ \\
\hline C18 & $99(5)$ & 113(6) & $86(4)$ & $19(4)$ & $41(4)$ & $-6(4)$ \\
\hline C19 & $166(10)$ & $135(9)$ & $134(7)$ & $58(7)$ & $77(8)$ & $9(7)$ \\
\hline $\mathrm{C} 20$ & $127(7)$ & $174(11)$ & $90(5)$ & $-7(6)$ & $40(5)$ & $1(8)$ \\
\hline $\mathrm{C} 21$ & $80(5)$ & $160(10)$ & $145(8)$ & $13(7)$ & $33(5)$ & $-17(6)$ \\
\hline
\end{tabular}

Table S5 Bond Lengths for syn-3i.

Atom Atom Length/Å

Atom Atom Length/Å 


$\begin{array}{llrlll}\mathrm{S} 1 & \mathrm{C} 11 & 1.666(6) & \mathrm{C} 5 & \mathrm{C} 6 & 1.386(7) \\ \mathrm{S} 1 & \mathrm{C} 12 & 1.616(9) & \mathrm{C} 6 & \mathrm{C} 7 & 1.455(8) \\ \mathrm{C} 1 \mathrm{~A} & \mathrm{C} 11 & 1.666(6) & \mathrm{C} 7 & \mathrm{C} 8 & 1.555(7) \\ \mathrm{C} 1 \mathrm{~A} & \mathrm{C} 12 & 1.616(9) & \mathrm{C} 8 & \mathrm{C} 9 & 1.526(9) \\ \mathrm{O} 1 & \mathrm{C} 7 & 1.210(7) & \mathrm{C} 8 & \mathrm{C} 10 & 1.551(7) \\ \mathrm{O} 2 & \mathrm{C} 16 & 1.191(7) & \mathrm{C} 10 & \mathrm{C} 11 & 1.505(7) \\ \mathrm{O} 3 & \mathrm{C} 17 & 1.184(7) & \mathrm{C} 10 & \mathrm{C} 15 & 1.519(7) \\ \mathrm{O} 4 & \mathrm{C} 17 & 1.310(7) & \mathrm{C} 11 & \mathrm{C} 14 & 1.604(7) \\ \mathrm{O} 4 & \mathrm{C} 18 & 1.488(7) & \mathrm{C} 11 & \mathrm{~S} 1 \mathrm{~A} & 1.604(7) \\ \mathrm{N} 1 & \mathrm{~N} 2 & 1.209(10) & \mathrm{C} 12 & \mathrm{C} 13 & 1.333(14) \\ \mathrm{N} 1 & \mathrm{C} 8 & 1.499(7) & \mathrm{C} 13 & \mathrm{C} 14 & 1.538(11) \\ \mathrm{N} 2 & \mathrm{~N} 3 & 1.121(12) & \mathrm{C} 13 & \mathrm{~S} 1 \mathrm{~A} & 1.538(11) \\ \mathrm{C} 1 & \mathrm{C} 2 & 1.369(9) & \mathrm{C} 15 & \mathrm{C} 16 & 1.519(7) \\ \mathrm{C} 1 & \mathrm{C} 6 & 1.390(7) & \mathrm{C} 16 & \mathrm{C} 17 & 1.541(7) \\ \mathrm{C} 1 & \mathrm{C} 9 & 1.511(8) & \mathrm{C} 18 & \mathrm{C} 19 & 1.515(14) \\ \mathrm{C} 2 & \mathrm{C} 3 & 1.385(10) & \mathrm{C} 18 & \mathrm{C} 20 & 1.525(15) \\ \mathrm{C} 3 & \mathrm{C} 4 & 1.378(11) & \mathrm{C} 18 & \mathrm{C} 21 & 1.503(13) \\ \mathrm{C} 4 & \mathrm{C} 5 & 1.375(10) & & & \\ \mathrm{T} 1 \mathrm{~S} 613 & & & \end{array}$

Table S6 Bond Angles for syn-3i.

\begin{tabular}{|c|c|c|c|c|c|c|c|}
\hline Aton & Atom & Atom & Angle ${ }^{\circ}$ & Atom & Atom & Atom & Angle $/^{\circ}$ \\
\hline $\mathrm{C} 12$ & S1 & $\mathrm{C} 11$ & $94.6(4)$ & $\mathrm{C} 15$ & $\mathrm{C} 10$ & $\mathrm{C} 8$ & $110.4(4)$ \\
\hline $\mathrm{C} 12$ & $\mathrm{C} 1 \mathrm{~A}$ & $\mathrm{C} 11$ & $94.6(4)$ & $\mathrm{C} 10$ & $\mathrm{C} 11$ & S1 & $121.8(4)$ \\
\hline C17 & $\mathrm{O} 4$ & $\mathrm{C} 18$ & $121.4(6)$ & $\mathrm{C} 10$ & $\mathrm{C} 11$ & $\mathrm{C} 1 \mathrm{~A}$ & $121.8(4)$ \\
\hline $\mathrm{N} 2$ & N1 & C8 & $116.6(5)$ & $\mathrm{C} 10$ & C11 & C14 & $123.9(4)$ \\
\hline N3 & $\mathrm{N} 2$ & $\mathrm{~N} 1$ & $171.4(8)$ & $\mathrm{C} 10$ & $\mathrm{C} 11$ & S1A & $123.9(4)$ \\
\hline $\mathrm{C} 2$ & $\mathrm{C} 1$ & $\mathrm{C} 6$ & $120.0(5)$ & C14 & C11 & S1 & $114.3(3)$ \\
\hline $\mathrm{C} 2$ & $\mathrm{C} 1$ & C9 & $128.4(5)$ & S1A & C11 & $\mathrm{C} 1 \mathrm{~A}$ & $114.3(3)$ \\
\hline
\end{tabular}




\begin{tabular}{llllllll}
$\mathrm{C} 6$ & $\mathrm{C} 1$ & $\mathrm{C} 9$ & $111.6(5)$ & $\mathrm{C} 13$ & $\mathrm{C} 12$ & $\mathrm{~S} 1$ & $115.6(6)$ \\
$\mathrm{C} 1$ & $\mathrm{C} 2$ & $\mathrm{C} 3$ & $118.5(7)$ & $\mathrm{C} 13$ & $\mathrm{C} 12$ & $\mathrm{C} 1 \mathrm{~A}$ & $115.6(6)$ \\
$\mathrm{C} 4$ & $\mathrm{C} 3$ & $\mathrm{C} 2$ & $121.3(7)$ & $\mathrm{C} 12$ & $\mathrm{C} 13$ & $\mathrm{C} 14$ & $117.7(6)$ \\
$\mathrm{C} 5$ & $\mathrm{C} 4$ & $\mathrm{C} 3$ & $120.8(7)$ & $\mathrm{C} 12$ & $\mathrm{C} 13$ & $\mathrm{~S} 1 \mathrm{~A}$ & $117.7(6)$ \\
$\mathrm{C} 4$ & $\mathrm{C} 5$ & $\mathrm{C} 6$ & $117.7(6)$ & $\mathrm{C} 13$ & $\mathrm{C} 14$ & $\mathrm{C} 11$ & $97.8(5)$ \\
$\mathrm{C} 1$ & $\mathrm{C} 6$ & $\mathrm{C} 7$ & $110.1(4)$ & $\mathrm{C} 13$ & $\mathrm{~S} 1 \mathrm{~A}$ & $\mathrm{C} 11$ & $97.8(5)$ \\
$\mathrm{C} 5$ & $\mathrm{C} 6$ & $\mathrm{C} 1$ & $121.6(5)$ & $\mathrm{C} 16$ & $\mathrm{C} 15$ & $\mathrm{C} 10$ & $110.8(5)$ \\
$\mathrm{C} 5$ & $\mathrm{C} 6$ & $\mathrm{C} 7$ & $128.3(5)$ & $\mathrm{O} 2$ & $\mathrm{C} 16$ & $\mathrm{C} 15$ & $123.3(5)$ \\
$\mathrm{O} 1$ & $\mathrm{C} 7$ & $\mathrm{C} 6$ & $129.7(5)$ & $\mathrm{O} 2$ & $\mathrm{C} 16$ & $\mathrm{C} 17$ & $121.6(5)$ \\
$\mathrm{O} 1$ & $\mathrm{C} 7$ & $\mathrm{C} 8$ & $122.8(5)$ & $\mathrm{C} 15$ & $\mathrm{C} 16$ & $\mathrm{C} 17$ & $115.0(5)$ \\
$\mathrm{C} 6$ & $\mathrm{C} 7$ & $\mathrm{C} 8$ & $107.6(4)$ & $\mathrm{O} 3$ & $\mathrm{C} 17$ & $\mathrm{O} 4$ & $128.8(5)$ \\
$\mathrm{N} 1$ & $\mathrm{C} 8$ & $\mathrm{C} 7$ & $106.6(4)$ & $\mathrm{O} 3$ & $\mathrm{C} 17$ & $\mathrm{C} 16$ & $121.7(5)$ \\
$\mathrm{N} 1$ & $\mathrm{C} 8$ & $\mathrm{C} 9$ & $106.8(5)$ & $\mathrm{O} 4$ & $\mathrm{C} 17$ & $\mathrm{C} 16$ & $109.5(5)$ \\
$\mathrm{N} 1$ & $\mathrm{C} 8$ & $\mathrm{C} 10$ & $111.0(5)$ & $\mathrm{O} 4$ & $\mathrm{C} 18$ & $\mathrm{C} 19$ & $102.1(7)$ \\
$\mathrm{C} 9$ & $\mathrm{C} 8$ & $\mathrm{C} 7$ & $105.1(4)$ & $\mathrm{O} 4$ & $\mathrm{C} 18$ & $\mathrm{C} 20$ & $109.9(7)$ \\
$\mathrm{C} 9$ & $\mathrm{C} 8$ & $\mathrm{C} 10$ & $116.9(4)$ & $\mathrm{O} 4$ & $\mathrm{C} 18$ & $\mathrm{C} 21$ & $107.5(6)$ \\
$\mathrm{C} 10$ & $\mathrm{C} 8$ & $\mathrm{C} 7$ & $109.8(4)$ & $\mathrm{C} 19$ & $\mathrm{C} 18$ & $\mathrm{C} 20$ & $111.6(8)$ \\
$\mathrm{C} 1$ & $\mathrm{C} 9$ & $\mathrm{C} 8$ & $105.2(4)$ & $\mathrm{C} 21$ & $\mathrm{C} 18$ & $\mathrm{C} 19$ & $113.0(9)$ \\
$\mathrm{C} 11$ & $\mathrm{C} 10$ & $\mathrm{C} 8$ & $113.4(4)$ & $\mathrm{C} 21$ & $\mathrm{C} 18$ & $\mathrm{C} 20$ & $112.2(9)$ \\
$\mathrm{C} 11$ & $\mathrm{C} 10$ & $\mathrm{C} 15$ & $111.7(4)$ & & & & \\
\hline
\end{tabular}

Table S7 Hydrogen Atom Coordinates $\left(\AA \times 10^{4}\right)$ and Isotropic Displacement Parameters $\left(\AA^{2} \times 10^{3}\right)$ for syn-3i.

\begin{tabular}{lrrrr} 
Atom & \multicolumn{1}{c}{$\boldsymbol{x}$} & $\boldsymbol{y}$ & \multicolumn{1}{c}{$\boldsymbol{z}$} & U(eq) \\
H1A & 985.39 & 8741.77 & 3823.36 & 93 \\
H2 & 2617.54 & 466.4 & 4393.5 & 94 \\
H3 & 3616.28 & -703.33 & 5264.53 & 115 \\
H4 & 4455.91 & 674.52 & 6124.73 & 107 \\
H5 & 4359.06 & 3278.57 & 6123.63 & 81
\end{tabular}




\begin{tabular}{|c|c|c|c|c|c|c|}
\hline H9 & & 1909.22 & 3288.69 & & 3833.95 & \\
\hline $\mathrm{H} 10$ & & 2892.73 & 7322.49 & & 4286.56 & \\
\hline H12 & & 106.51 & 8348.7 & & 2512.42 & \\
\hline $\mathrm{H} 12 \mathrm{~A}$ & & 106.51 & 8348.7 & & 2512.42 & \\
\hline H13 & & 898.41 & 6486.73 & & 1879.78 & \\
\hline H13A & & 898.41 & 6486.73 & & 1879.78 & \\
\hline H14 & & 2424.36 & 5036.69 & & 2542.71 & \\
\hline $\mathrm{H} 15 \mathrm{~A}$ & & 4314.6 & 5619.55 & & 4148.79 & \\
\hline H15B & & 3804.48 & 5164.81 & & 3423.97 & \\
\hline H19A & & 5370.77 & 9670.2 & & 1428.83 & \\
\hline H19B & & 6563.01 & 9729.91 & & 1280.85 & \\
\hline H19C & & 6156.72 & 10394.71 & & 1975.83 & \\
\hline $\mathrm{H} 20 \mathrm{~A}$ & & 6196.8 & 5862.28 & & 1693.25 & \\
\hline H20B & & 6447.75 & 6938.93 & & 1075.21 & \\
\hline $\mathrm{H} 20 \mathrm{C}$ & & 5300.43 & 6791 & & 1319.44 & \\
\hline $\mathrm{H} 21 \mathrm{~A}$ & & 7275.99 & 8655.75 & & 2751.95 & \\
\hline $\mathrm{H} 21 \mathrm{~B}$ & & 7798.81 & 8160.18 & & 2064.38 & \\
\hline $\mathrm{H} 21 \mathrm{C}$ & & 7323.58 & 6945.41 & & 2552.33 & \\
\hline 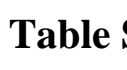 & mic Occupancy & syn-3i. & & & & \\
\hline Atom & Occupancy & Atom & Occupancy & & Atom & Occupancy \\
\hline $\mathrm{S} 1$ & $0.5 \quad \mathrm{C} 1 \mathrm{~A}$ & & & 0.5 & H1A & \\
\hline H12 & $0.5 \mathrm{H} 12 \mathrm{~A}$ & & & 0.5 & H13 & \\
\hline $\mathrm{H} 13 \mathrm{~A}$ & $0.5 \quad \mathrm{C} 14$ & & & 0.5 & H14 & \\
\hline S1A & 0.5 & & & & & \\
\hline
\end{tabular}


Single crystals of $\mathbf{4 b}$ were obtained by slow diffusion of the solution of $\mathbf{4 b}$ in PE/EtOAc at RT. Data intensity of $\mathbf{4 b}^{9}$ was collected using a 'Bruker APEX-II CCD' diffractometer at 293(2) K. Data collection and reduction were done by using Olex2 and the structure was solved with the ShelXS structure solution program using direct methods and refined by full-matrix least-squares on $F^{2}$ with anisotropic displacement parameters for non-H atoms using SHELX-97. Hydrogen atoms were added at their geometrically idea positions and refined isotropically. Crystal data for $4 \mathbf{b}: \mathrm{C}_{24} \mathrm{H}_{24} \mathrm{ClNO}_{3}, T=$ 293(2) K, Monoclinic, P21, $a=13.3465(4) \AA, b=5.7186(2) \AA, c=14.4926(6) \AA, \alpha=90^{\circ}, \beta=$ $104.1320(10)^{\mathrm{o}}, \gamma=90^{\circ}, V=1072.65(7) \AA^{3} . \mathrm{Z}=2, d_{\text {calc }}=1.269 \mathrm{Mg} / \mathrm{m}^{3} .10976$ reflections measured, $4173[\mathrm{R}($ int $)=0.0298], \mathrm{R}_{1}=0.0411, \mathrm{wR}_{2}=0.0919(I>2 \sigma(I)$, final $), \mathrm{R}_{1}=0.0595, \mathrm{wR}_{2}=0.1044$ (all data), $\mathrm{GOF}=1.057$, and 267 parameters.

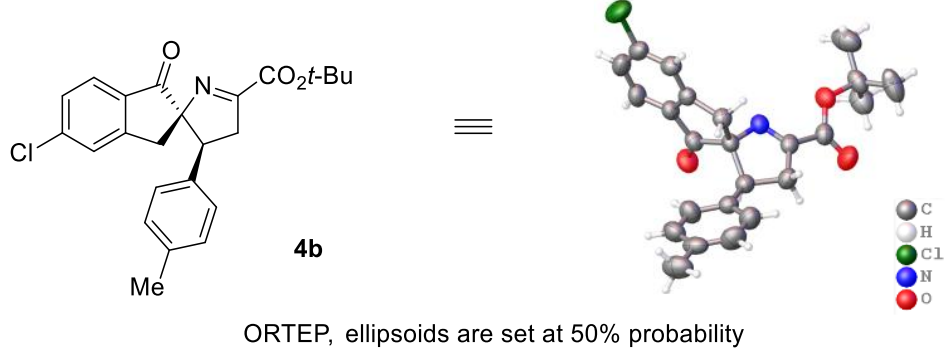

Table S9. Crystal data and structure refinement for $\mathbf{4 b}$.

Identification code

Empirical formula

Formula weight

Temperature

Wavelength

Crystal system

Space group

Unit cell dimensions

Volume

$\mathrm{Z}$

Density (calculated)

Absorption coefficient
$4 \mathrm{~b}$

$\mathrm{C}_{24} \mathrm{H}_{24} \mathrm{ClNO}_{3}$

409.89

293(2) K

$0.71073 \AA$

Monoclinic

$\mathrm{P} 21$

$\mathrm{a}=13.3465(4) \AA$ $\mathrm{a}=90^{\circ}$. $\mathrm{b}=104.1320(10)^{\circ}$.

$\mathrm{b}=5.7186(2) \AA$ $\mathrm{c}=14.4926(6) \AA$ $\mathrm{g}=90^{\circ}$. 1072.65(7) $\AA^{3}$

2

$1.269 \mathrm{Mg} / \mathrm{m}^{3}$

$0.203 \mathrm{~mm}^{-1}$

${ }^{9}$ Supplementary crystallographic data have been deposited at the Cambridge Crystallographic Data Center (CCDC number: 1959303). 
$\mathrm{F}(000)$

Crystal size

Theta range for data collection

Index ranges

Reflections collected

Independent reflections

Completeness to theta $=25.242^{\circ}$

Absorption correction

Max. and min. transmission

Refinement method

Data / restraints / parameters

Goodness-of-fit on $\mathrm{F}^{2}$

Final $\mathrm{R}$ indices [I $>2 \operatorname{sigma}(\mathrm{I})]$

$\mathrm{R}$ indices (all data)

Absolute structure parameter

Extinction coefficient

Largest diff. peak and hole
432

$0.200 \times 0.130 \times 0.070 \mathrm{~mm}^{3}$

2.941 to $25.997^{\circ}$.

$-16<=\mathrm{h}<=16,-7<=\mathrm{k}<=7,-15<=\mathrm{l}<=17$

10976

$4173[\mathrm{R}(\mathrm{int})=0.0298]$

$99.3 \%$

Semi-empirical from equivalents

0.7456 and 0.6585

Full-matrix least-squares on $\mathrm{F}^{2}$

4173 / 1 / 267

1.057

$\mathrm{R}_{1}=0.0411, \mathrm{w}_{2}=0.0919$

$\mathrm{R}_{1}=0.0595, \mathrm{wR}_{2}=0.1044$

$0.01(3)$

$0.043(8)$

0.144 and -0.143 e. $\AA^{-3}$

Table S10. Atomic coordinates $\left(\times 10^{4}\right)$ and equivalent isotropic displacement parameters $\left(\AA^{2} \times 10^{3}\right)$ for 4b. $U(e q)$ is defined as one third of the trace of the orthogonalized $U^{i j}$ tensor.

\begin{tabular}{lcccc}
\hline & $\mathrm{x}$ & $\mathrm{y}$ & $\mathrm{z}$ & $\mathrm{U}(\mathrm{eq})$ \\
\hline $\mathrm{Cl}(1)$ & $7133(1)$ & $-904(2)$ & $8741(1)$ & $100(1)$ \\
$\mathrm{O}(1)$ & $3448(2)$ & $7464(4)$ & $8548(2)$ & $70(1)$ \\
$\mathrm{O}(2)$ & $1344(2)$ & $9267(6)$ & $4694(2)$ & $87(1)$ \\
$\mathrm{O}(3)$ & $3030(2)$ & $8552(4)$ & $4786(2)$ & $63(1)$ \\
$\mathrm{N}(1)$ & $3131(2)$ & $6311(4)$ & $6456(2)$ & $50(1)$ \\
$\mathrm{C}(1)$ & $3638(2)$ & $5708(6)$ & $8153(2)$ & $51(1)$ \\
$\mathrm{C}(2)$ & $4550(2)$ & $4197(6)$ & $8430(2)$ & $51(1)$ \\
$\mathrm{C}(3)$ & $5433(2)$ & $4506(7)$ & $9163(2)$ & $65(1)$ \\
$\mathrm{C}(4)$ & $6214(3)$ & $2896(8)$ & $9249(3)$ & $74(1)$ \\
$\mathrm{C}(5)$ & $6105(3)$ & $1033(7)$ & $8624(3)$ & $64(1)$
\end{tabular}




\begin{tabular}{|c|c|c|c|c|}
\hline$C(6)$ & $5226(3)$ & $675(6)$ & $7916(2)$ & $59(1)$ \\
\hline$C(7)$ & $4438(2)$ & 2297(6) & $7821(2)$ & $49(1)$ \\
\hline$C(8)$ & $3402(2)$ & $2310(6)$ & $7112(2)$ & $52(1)$ \\
\hline $\mathrm{C}(9)$ & $2954(2)$ & $4745(5)$ & $7218(2)$ & $47(1)$ \\
\hline $\mathrm{C}(10)$ & $1781(2)$ & $4947(6)$ & $7155(2)$ & $54(1)$ \\
\hline $\mathrm{C}(11)$ & $1340(2)$ & $5545(7)$ & $6108(2)$ & $63(1)$ \\
\hline $\mathrm{C}(12)$ & $2259(2)$ & $6711(6)$ & $5885(2)$ & $52(1)$ \\
\hline $\mathrm{C}(13)$ & $2157(3)$ & $8325(6)$ & $5050(2)$ & $58(1)$ \\
\hline $\mathrm{C}(14)$ & $3097(3)$ & $10013(6)$ & $3957(2)$ & $67(1)$ \\
\hline$C(15)$ & $2376(4)$ & $9062(9)$ & $3066(3)$ & $102(2)$ \\
\hline$C(16)$ & $2868(3)$ & $12531(7)$ & $4138(3)$ & $86(1)$ \\
\hline $\mathrm{C}(17)$ & $4216(4)$ & $9716(10)$ & $3935(4)$ & $106(2)$ \\
\hline $\mathrm{C}(18)$ & $1313(2)$ & 2951(6) & $7593(2)$ & $56(1)$ \\
\hline $\mathrm{C}(19)$ & $1659(3)$ & $2515(8)$ & $8561(3)$ & $72(1)$ \\
\hline $\mathrm{C}(20)$ & 1231(3) & $749(8)$ & $8988(3)$ & $76(1)$ \\
\hline $\mathrm{C}(21)$ & $442(3)$ & $-634(7)$ & $8488(3)$ & $71(1)$ \\
\hline $\mathrm{C}(22)$ & $106(3)$ & $-206(8)$ & $7532(3)$ & $84(1)$ \\
\hline $\mathrm{C}(23)$ & $529(3)$ & $1557(7)$ & $7087(3)$ & $74(1)$ \\
\hline $\mathrm{C}(24)$ & $-16(3)$ & $-2540(9)$ & $8967(3)$ & $101(2)$ \\
\hline
\end{tabular}

Table S11. Bond lengths $[\AA]$ and angles $\left[{ }^{\circ}\right]$ for $\mathbf{4 b}$.

\begin{tabular}{ll}
\hline $\mathrm{Cl}(1)-\mathrm{C}(5)$ & $1.739(4)$ \\
$\mathrm{O}(1)-\mathrm{C}(1)$ & $1.213(4)$ \\
$\mathrm{O}(2)-\mathrm{C}(13)$ & $1.208(4)$ \\
$\mathrm{O}(3)-\mathrm{C}(13)$ & $1.319(4)$ \\
$\mathrm{O}(3)-\mathrm{C}(14)$ & $1.485(4)$ \\
$\mathrm{N}(1)-\mathrm{C}(12)$ & $1.274(4)$ \\
$\mathrm{N}(1)-\mathrm{C}(9)$ & $1.485(4)$
\end{tabular}




\begin{tabular}{|c|c|}
\hline$C(1)-C(2)$ & $1.466(4)$ \\
\hline $\mathrm{C}(1)-\mathrm{C}(9)$ & $1.540(4)$ \\
\hline$C(2)-C(7)$ & $1.385(4)$ \\
\hline$C(2)-C(3)$ & $1.393(4)$ \\
\hline$C(3)-C(4)$ & $1.373(5)$ \\
\hline $\mathrm{C}(3)-\mathrm{H}(3)$ & 0.9300 \\
\hline$C(4)-C(5)$ & $1.383(5)$ \\
\hline $\mathrm{C}(4)-\mathrm{H}(4)$ & 0.9300 \\
\hline$C(5)-C(6)$ & $1.373(5)$ \\
\hline$C(6)-C(7)$ & $1.384(4)$ \\
\hline $\mathrm{C}(6)-\mathrm{H}(6)$ & 0.9300 \\
\hline$C(7)-C(8)$ & $1.507(4)$ \\
\hline $\mathrm{C}(8)-\mathrm{C}(9)$ & $1.538(4)$ \\
\hline $\mathrm{C}(8)-\mathrm{H}(8 \mathrm{~A})$ & 0.9700 \\
\hline $\mathrm{C}(8)-\mathrm{H}(8 \mathrm{~B})$ & 0.9700 \\
\hline $\mathrm{C}(9)-\mathrm{C}(10)$ & $1.550(4)$ \\
\hline $\mathrm{C}(10)-\mathrm{C}(18)$ & $1.513(4)$ \\
\hline $\mathrm{C}(10)-\mathrm{C}(11)$ & $1.526(4)$ \\
\hline $\mathrm{C}(10)-\mathrm{H}(10)$ & 0.9800 \\
\hline $\mathrm{C}(11)-\mathrm{C}(12)$ & $1.499(5)$ \\
\hline $\mathrm{C}(11)-\mathrm{H}(11 \mathrm{~A})$ & 0.9700 \\
\hline $\mathrm{C}(11)-\mathrm{H}(11 \mathrm{~B})$ & 0.9700 \\
\hline $\mathrm{C}(12)-\mathrm{C}(13)$ & $1.501(4)$ \\
\hline $\mathrm{C}(14)-\mathrm{C}(16)$ & $1.508(5)$ \\
\hline $\mathrm{C}(14)-\mathrm{C}(15)$ & $1.511(6)$ \\
\hline$C(14)-C(17)$ & $1.511(5)$ \\
\hline $\mathrm{C}(15)-\mathrm{H}(15 \mathrm{~A})$ & 0.9600 \\
\hline $\mathrm{C}(15)-\mathrm{H}(15 \mathrm{~B})$ & 0.9600 \\
\hline $\mathrm{C}(15)-\mathrm{H}(15 \mathrm{C})$ & 0.9600 \\
\hline
\end{tabular}




\begin{tabular}{|c|c|}
\hline $\mathrm{C}(16)-\mathrm{H}(16 \mathrm{~A})$ & 0.9600 \\
\hline $\mathrm{C}(16)-\mathrm{H}(16 \mathrm{~B})$ & 0.9600 \\
\hline $\mathrm{C}(16)-\mathrm{H}(16 \mathrm{C})$ & 0.9600 \\
\hline $\mathrm{C}(17)-\mathrm{H}(17 \mathrm{~A})$ & 0.9600 \\
\hline $\mathrm{C}(17)-\mathrm{H}(17 \mathrm{~B})$ & 0.9600 \\
\hline $\mathrm{C}(17)-\mathrm{H}(17 \mathrm{C})$ & 0.9600 \\
\hline$C(18)-C(23)$ & $1.376(5)$ \\
\hline$C(18)-C(19)$ & $1.388(5)$ \\
\hline$C(19)-C(20)$ & $1.379(5)$ \\
\hline $\mathrm{C}(19)-\mathrm{H}(19)$ & 0.9300 \\
\hline$C(20)-C(21)$ & $1.373(6)$ \\
\hline $\mathrm{C}(20)-\mathrm{H}(20)$ & 0.9300 \\
\hline$C(21)-C(22)$ & $1.370(6)$ \\
\hline $\mathrm{C}(21)-\mathrm{C}(24)$ & $1.501(5)$ \\
\hline $\mathrm{C}(22)-\mathrm{C}(23)$ & $1.389(5)$ \\
\hline $\mathrm{C}(22)-\mathrm{H}(22)$ & 0.9300 \\
\hline $\mathrm{C}(23)-\mathrm{H}(23)$ & 0.9300 \\
\hline $\mathrm{C}(24)-\mathrm{H}(24 \mathrm{~A})$ & 0.9600 \\
\hline $\mathrm{C}(24)-\mathrm{H}(24 \mathrm{~B})$ & 0.9600 \\
\hline $\mathrm{C}(24)-\mathrm{H}(24 \mathrm{C})$ & 0.9600 \\
\hline $\mathrm{C}(13)-\mathrm{O}(3)-\mathrm{C}(14)$ & $121.5(3)$ \\
\hline $\mathrm{C}(12)-\mathrm{N}(1)-\mathrm{C}(9)$ & $107.8(2)$ \\
\hline $\mathrm{O}(1)-\mathrm{C}(1)-\mathrm{C}(2)$ & $128.0(3)$ \\
\hline $\mathrm{O}(1)-\mathrm{C}(1)-\mathrm{C}(9)$ & $124.3(3)$ \\
\hline $\mathrm{C}(2)-\mathrm{C}(1)-\mathrm{C}(9)$ & $107.7(3)$ \\
\hline $\mathrm{C}(7)-\mathrm{C}(2)-\mathrm{C}(3)$ & $121.7(3)$ \\
\hline $\mathrm{C}(7)-\mathrm{C}(2)-\mathrm{C}(1)$ & $109.6(3)$ \\
\hline$C(3)-C(2)-C(1)$ & $128.7(3)$ \\
\hline $\mathrm{C}(4)-\mathrm{C}(3)-\mathrm{C}(2)$ & $117.8(4)$ \\
\hline
\end{tabular}




\begin{tabular}{|c|c|}
\hline $\mathrm{C}(4)-\mathrm{C}(3)-\mathrm{H}(3)$ & 121.1 \\
\hline $\mathrm{C}(2)-\mathrm{C}(3)-\mathrm{H}(3)$ & 121.1 \\
\hline$C(3)-C(4)-C(5)$ & $120.1(3)$ \\
\hline $\mathrm{C}(3)-\mathrm{C}(4)-\mathrm{H}(4)$ & 120.0 \\
\hline $\mathrm{C}(5)-\mathrm{C}(4)-\mathrm{H}(4)$ & 120.0 \\
\hline$C(6)-C(5)-C(4)$ & $122.6(3)$ \\
\hline $\mathrm{C}(6)-\mathrm{C}(5)-\mathrm{Cl}(1)$ & $119.2(3)$ \\
\hline $\mathrm{C}(4)-\mathrm{C}(5)-\mathrm{Cl}(1)$ & $118.2(3)$ \\
\hline$C(5)-C(6)-C(7)$ & $117.7(3)$ \\
\hline $\mathrm{C}(5)-\mathrm{C}(6)-\mathrm{H}(6)$ & 121.2 \\
\hline $\mathrm{C}(7)-\mathrm{C}(6)-\mathrm{H}(6)$ & 121.2 \\
\hline$C(6)-C(7)-C(2)$ & $120.1(3)$ \\
\hline$C(6)-C(7)-C(8)$ & $128.3(3)$ \\
\hline$C(2)-C(7)-C(8)$ & $111.6(3)$ \\
\hline$C(7)-C(8)-C(9)$ & $104.5(3)$ \\
\hline $\mathrm{C}(7)-\mathrm{C}(8)-\mathrm{H}(8 \mathrm{~A})$ & 110.8 \\
\hline $\mathrm{C}(9)-\mathrm{C}(8)-\mathrm{H}(8 \mathrm{~A})$ & 110.8 \\
\hline $\mathrm{C}(7)-\mathrm{C}(8)-\mathrm{H}(8 \mathrm{~B})$ & 110.8 \\
\hline $\mathrm{C}(9)-\mathrm{C}(8)-\mathrm{H}(8 \mathrm{~B})$ & 110.8 \\
\hline $\mathrm{H}(8 \mathrm{~A})-\mathrm{C}(8)-\mathrm{H}(8 \mathrm{~B})$ & 108.9 \\
\hline $\mathrm{N}(1)-\mathrm{C}(9)-\mathrm{C}(8)$ & $109.6(2)$ \\
\hline$N(1)-C(9)-C(1)$ & $105.7(2)$ \\
\hline $\mathrm{C}(8)-\mathrm{C}(9)-\mathrm{C}(1)$ & $104.5(2)$ \\
\hline $\mathrm{N}(1)-\mathrm{C}(9)-\mathrm{C}(10)$ & $104.7(2)$ \\
\hline $\mathrm{C}(8)-\mathrm{C}(9)-\mathrm{C}(10)$ & $118.3(2)$ \\
\hline$C(1)-C(9)-C(10)$ & $113.5(2)$ \\
\hline$C(18)-C(10)-C(11)$ & $119.2(3)$ \\
\hline $\mathrm{C}(18)-\mathrm{C}(10)-\mathrm{C}(9)$ & $115.7(2)$ \\
\hline $\mathrm{C}(11)-\mathrm{C}(10)-\mathrm{C}(9)$ & $102.3(2)$ \\
\hline
\end{tabular}




\begin{tabular}{|c|c|}
\hline $\mathrm{C}(18)-\mathrm{C}(10)-\mathrm{H}(10)$ & 106.2 \\
\hline $\mathrm{C}(11)-\mathrm{C}(10)-\mathrm{H}(10)$ & 106.2 \\
\hline $\mathrm{C}(9)-\mathrm{C}(10)-\mathrm{H}(10)$ & 106.2 \\
\hline$C(12)-C(11)-C(10)$ & $100.3(3)$ \\
\hline $\mathrm{C}(12)-\mathrm{C}(11)-\mathrm{H}(11 \mathrm{~A})$ & 111.7 \\
\hline $\mathrm{C}(10)-\mathrm{C}(11)-\mathrm{H}(11 \mathrm{~A})$ & 111.7 \\
\hline $\mathrm{C}(12)-\mathrm{C}(11)-\mathrm{H}(11 \mathrm{~B})$ & 111.7 \\
\hline $\mathrm{C}(10)-\mathrm{C}(11)-\mathrm{H}(11 \mathrm{~B})$ & 111.7 \\
\hline $\mathrm{H}(11 \mathrm{~A})-\mathrm{C}(11)-\mathrm{H}(11 \mathrm{~B})$ & 109.5 \\
\hline $\mathrm{N}(1)-\mathrm{C}(12)-\mathrm{C}(11)$ & $116.5(3)$ \\
\hline $\mathrm{N}(1)-\mathrm{C}(12)-\mathrm{C}(13)$ & $121.5(3)$ \\
\hline $\mathrm{C}(11)-\mathrm{C}(12)-\mathrm{C}(13)$ & $122.0(3)$ \\
\hline $\mathrm{O}(2)-\mathrm{C}(13)-\mathrm{O}(3)$ & $126.7(3)$ \\
\hline $\mathrm{O}(2)-\mathrm{C}(13)-\mathrm{C}(12)$ & $120.9(3)$ \\
\hline $\mathrm{O}(3)-\mathrm{C}(13)-\mathrm{C}(12)$ & $112.5(3)$ \\
\hline $\mathrm{O}(3)-\mathrm{C}(14)-\mathrm{C}(16)$ & $109.9(3)$ \\
\hline $\mathrm{O}(3)-\mathrm{C}(14)-\mathrm{C}(15)$ & $109.7(3)$ \\
\hline$C(16)-C(14)-C(15)$ & $112.3(4)$ \\
\hline $\mathrm{O}(3)-\mathrm{C}(14)-\mathrm{C}(17)$ & $102.1(3)$ \\
\hline$C(16)-C(14)-C(17)$ & $110.8(4)$ \\
\hline $\mathrm{C}(15)-\mathrm{C}(14)-\mathrm{C}(17)$ & $111.6(4)$ \\
\hline $\mathrm{C}(14)-\mathrm{C}(15)-\mathrm{H}(15 \mathrm{~A})$ & 109.5 \\
\hline $\mathrm{C}(14)-\mathrm{C}(15)-\mathrm{H}(15 \mathrm{~B})$ & 109.5 \\
\hline $\mathrm{H}(15 \mathrm{~A})-\mathrm{C}(15)-\mathrm{H}(15 \mathrm{~B})$ & 109.5 \\
\hline $\mathrm{C}(14)-\mathrm{C}(15)-\mathrm{H}(15 \mathrm{C})$ & 109.5 \\
\hline $\mathrm{H}(15 \mathrm{~A})-\mathrm{C}(15)-\mathrm{H}(15 \mathrm{C})$ & 109.5 \\
\hline $\mathrm{H}(15 \mathrm{~B})-\mathrm{C}(15)-\mathrm{H}(15 \mathrm{C})$ & 109.5 \\
\hline $\mathrm{C}(14)-\mathrm{C}(16)-\mathrm{H}(16 \mathrm{~A})$ & 109.5 \\
\hline $\mathrm{C}(14)-\mathrm{C}(16)-\mathrm{H}(16 \mathrm{~B})$ & 109.5 \\
\hline
\end{tabular}




\begin{tabular}{|c|c|}
\hline $\mathrm{H}(16 \mathrm{~A})-\mathrm{C}(16)-\mathrm{H}(16 \mathrm{~B})$ & 109.5 \\
\hline $\mathrm{C}(14)-\mathrm{C}(16)-\mathrm{H}(16 \mathrm{C})$ & 109.5 \\
\hline $\mathrm{H}(16 \mathrm{~A})-\mathrm{C}(16)-\mathrm{H}(16 \mathrm{C})$ & 109.5 \\
\hline $\mathrm{H}(16 \mathrm{~B})-\mathrm{C}(16)-\mathrm{H}(16 \mathrm{C})$ & 109.5 \\
\hline $\mathrm{C}(14)-\mathrm{C}(17)-\mathrm{H}(17 \mathrm{~A})$ & 109.5 \\
\hline $\mathrm{C}(14)-\mathrm{C}(17)-\mathrm{H}(17 \mathrm{~B})$ & 109.5 \\
\hline $\mathrm{H}(17 \mathrm{~A})-\mathrm{C}(17)-\mathrm{H}(17 \mathrm{~B})$ & 109.5 \\
\hline $\mathrm{C}(14)-\mathrm{C}(17)-\mathrm{H}(17 \mathrm{C})$ & 109.5 \\
\hline $\mathrm{H}(17 \mathrm{~A})-\mathrm{C}(17)-\mathrm{H}(17 \mathrm{C})$ & 109.5 \\
\hline $\mathrm{H}(17 \mathrm{~B})-\mathrm{C}(17)-\mathrm{H}(17 \mathrm{C})$ & 109.5 \\
\hline $\mathrm{C}(23)-\mathrm{C}(18)-\mathrm{C}(19)$ & $117.2(3)$ \\
\hline $\mathrm{C}(23)-\mathrm{C}(18)-\mathrm{C}(10)$ & $123.5(3)$ \\
\hline$C(19)-C(18)-C(10)$ & $119.3(3)$ \\
\hline $\mathrm{C}(20)-\mathrm{C}(19)-\mathrm{C}(18)$ & $120.8(4)$ \\
\hline $\mathrm{C}(20)-\mathrm{C}(19)-\mathrm{H}(19)$ & 119.6 \\
\hline $\mathrm{C}(18)-\mathrm{C}(19)-\mathrm{H}(19)$ & 119.6 \\
\hline$C(21)-C(20)-C(19)$ & $122.3(4)$ \\
\hline $\mathrm{C}(21)-\mathrm{C}(20)-\mathrm{H}(20)$ & 118.9 \\
\hline $\mathrm{C}(19)-\mathrm{C}(20)-\mathrm{H}(20)$ & 118.9 \\
\hline $\mathrm{C}(22)-\mathrm{C}(21)-\mathrm{C}(20)$ & $116.8(4)$ \\
\hline $\mathrm{C}(22)-\mathrm{C}(21)-\mathrm{C}(24)$ & $121.8(4)$ \\
\hline$C(20)-C(21)-C(24)$ & $121.4(4)$ \\
\hline$C(21)-C(22)-C(23)$ & $121.9(4)$ \\
\hline $\mathrm{C}(21)-\mathrm{C}(22)-\mathrm{H}(22)$ & 119.0 \\
\hline $\mathrm{C}(23)-\mathrm{C}(22)-\mathrm{H}(22)$ & 119.0 \\
\hline $\mathrm{C}(18)-\mathrm{C}(23)-\mathrm{C}(22)$ & $121.1(4)$ \\
\hline $\mathrm{C}(18)-\mathrm{C}(23)-\mathrm{H}(23)$ & 119.5 \\
\hline $\mathrm{C}(22)-\mathrm{C}(23)-\mathrm{H}(23)$ & 119.5 \\
\hline $\mathrm{C}(21)-\mathrm{C}(24)-\mathrm{H}(24 \mathrm{~A})$ & 109.5 \\
\hline
\end{tabular}


$\mathrm{C}(21)-\mathrm{C}(24)-\mathrm{H}(24 \mathrm{~B}) \quad 109.5$

$\mathrm{H}(24 \mathrm{~A})-\mathrm{C}(24)-\mathrm{H}(24 \mathrm{~B}) \quad 109.5$

C(21)-C(24)-H(24C) 109.5

H(24A)-C(24)-H(24C) 109.5

H(24B)-C(24)-H(24C) 109.5

Symmetry transformations used to generate equivalent atoms:

Table S12. Anisotropic displacement parameters $\left(\AA^{2} \times 10^{3}\right)$ for $4 \mathrm{~b}$. The anisotropic displacement factor exponent takes the form: $-2 \mathrm{p}^{2}\left[\mathrm{~h}^{2} \mathrm{a}^{* 2} \mathrm{U}^{11}+\ldots+2 \mathrm{hk} \mathrm{a}^{*} \mathrm{~b}^{*} \mathrm{U}^{12}\right]$

\begin{tabular}{|c|c|c|c|c|c|c|}
\hline & $\mathrm{U}^{11}$ & $\mathrm{U}^{22}$ & $\mathrm{U}^{33}$ & $\mathrm{U}^{23}$ & $\mathrm{U}^{13}$ & $\mathrm{U}^{12}$ \\
\hline $\mathrm{Cl}(1)$ & $69(1)$ & $112(1)$ & $118(1)$ & $34(1)$ & $21(1)$ & $34(1)$ \\
\hline $\mathrm{O}(1)$ & $88(2)$ & $61(1)$ & $59(1)$ & $-13(1)$ & $17(1)$ & $6(1)$ \\
\hline $\mathrm{O}(2)$ & $71(2)$ & $107(2)$ & $79(2)$ & $34(2)$ & $10(1)$ & $18(2)$ \\
\hline $\mathrm{O}(3)$ & $74(1)$ & $58(1)$ & $59(1)$ & $14(1)$ & $22(1)$ & $10(1)$ \\
\hline $\mathrm{N}(1)$ & $58(2)$ & $47(2)$ & $46(1)$ & $0(1)$ & $14(1)$ & $4(1)$ \\
\hline $\mathrm{C}(1)$ & $63(2)$ & $47(2)$ & $45(2)$ & $1(2)$ & $16(1)$ & $1(2)$ \\
\hline $\mathrm{C}(2)$ & $54(2)$ & $56(2)$ & $44(2)$ & $2(2)$ & $12(1)$ & $-3(2)$ \\
\hline $\mathrm{C}(3)$ & $68(2)$ & $77(2)$ & $47(2)$ & $3(2)$ & $7(2)$ & $-4(2)$ \\
\hline $\mathrm{C}(4)$ & $55(2)$ & $103(3)$ & $58(2)$ & $17(2)$ & $5(2)$ & $-2(2)$ \\
\hline $\mathrm{C}(5)$ & $54(2)$ & $76(3)$ & $64(2)$ & $19(2)$ & $18(2)$ & $10(2)$ \\
\hline $\mathrm{C}(6)$ & $61(2)$ & $55(2)$ & $65(2)$ & $8(2)$ & $20(2)$ & $7(2)$ \\
\hline $\mathrm{C}(7)$ & $51(2)$ & $50(2)$ & $47(2)$ & $8(2)$ & $15(1)$ & $3(2)$ \\
\hline$C(8)$ & $58(2)$ & $46(2)$ & $52(2)$ & $-2(2)$ & $13(1)$ & $2(2)$ \\
\hline$C(9)$ & $54(2)$ & $44(2)$ & $43(2)$ & $1(1)$ & $13(1)$ & $4(1)$ \\
\hline$C(10)$ & $53(2)$ & $58(2)$ & $53(2)$ & $3(2)$ & $15(1)$ & $8(2)$ \\
\hline$C(11)$ & $54(2)$ & $71(2)$ & $60(2)$ & $11(2)$ & $7(2)$ & $5(2)$ \\
\hline$C(12)$ & $58(2)$ & $49(2)$ & $47(2)$ & $-1(1)$ & $10(1)$ & $7(1)$ \\
\hline $\mathrm{C}(13)$ & $66(2)$ & $57(2)$ & $50(2)$ & $2(2)$ & $8(2)$ & $6(2)$ \\
\hline$C(14)$ & $99(3)$ & $55(2)$ & $54(2)$ & $9(2)$ & $29(2)$ & $3(2)$ \\
\hline$C(15)$ & 173(4) & $79(3)$ & $54(2)$ & $3(2)$ & $26(3)$ & $-11(3)$ \\
\hline$C(16)$ & $128(3)$ & $56(2)$ & $77(3)$ & $6(2)$ & $31(2)$ & $6(2)$ \\
\hline$C(17)$ & $124(4)$ & 101(4) & $115(4)$ & $26(3)$ & $71(3)$ & $14(3)$ \\
\hline$C(18)$ & $50(2)$ & $62(2)$ & $59(2)$ & $4(2)$ & $18(2)$ & $8(2)$ \\
\hline
\end{tabular}




$\begin{array}{lllllll}\mathrm{C}(19) & 78(2) & 81(3) & 62(2) & 7(2) & 24(2) & -9(2) \\ \mathrm{C}(20) & 78(2) & 87(3) & 70(2) & 15(2) & 34(2) & 10(2) \\ \mathrm{C}(21) & 63(2) & 68(2) & 94(3) & 16(2) & 42(2) & 13(2) \\ \mathrm{C}(22) & 62(2) & 85(3) & 106(3) & 10(3) & 20(2) & -14(2) \\ \mathrm{C}(23) & 66(2) & 81(3) & 72(2) & 13(2) & 14(2) & -2(2) \\ \mathrm{C}(24) & 97(3) & 87(3) & 135(4) & 27(3) & 62(3) & 2(3)\end{array}$

Table S13. Hydrogen coordinates $\left(\times 10^{4}\right)$ and isotropic displacement parameters $\left(\AA^{2} \times 10^{3}\right)$ for $\mathbf{4 b}$.

\begin{tabular}{|c|c|c|c|c|}
\hline & $\mathrm{x}$ & $\mathrm{y}$ & $\mathrm{Z}$ & $\mathrm{U}(\mathrm{eq})$ \\
\hline $\mathrm{H}(3)$ & 5493 & 5762 & 9580 & 79 \\
\hline $\mathrm{H}(4)$ & 6816 & 3059 & 9729 & 88 \\
\hline $\mathrm{H}(6)$ & 5162 & -612 & 7513 & 71 \\
\hline $\mathrm{H}(8 \mathrm{~A})$ & 2959 & 1091 & 7257 & 63 \\
\hline $\mathrm{H}(8 \mathrm{~B})$ & 3482 & 2078 & 6471 & 63 \\
\hline $\mathrm{H}(10)$ & 1693 & 6350 & 7516 & 65 \\
\hline $\mathrm{H}(11 \mathrm{~A})$ & 1137 & 4153 & 5726 & 76 \\
\hline $\mathrm{H}(11 \mathrm{~B})$ & 756 & 6600 & 6022 & 76 \\
\hline $\mathrm{H}(15 \mathrm{~A})$ & 2505 & 7422 & 3011 & 154 \\
\hline $\mathrm{H}(15 \mathrm{~B})$ & 2492 & 9870 & 2520 & 154 \\
\hline $\mathrm{H}(15 \mathrm{C})$ & 1674 & 9288 & 3100 & 154 \\
\hline $\mathrm{H}(16 \mathrm{~A})$ & 2160 & 12674 & 4169 & 129 \\
\hline $\mathrm{H}(16 \mathrm{~B})$ & 2985 & 13489 & 3631 & 129 \\
\hline $\mathrm{H}(16 \mathrm{C})$ & 3312 & 13031 & 4730 & 129 \\
\hline $\mathrm{H}(17 \mathrm{~A})$ & 4652 & 10268 & 4522 & 159 \\
\hline $\mathrm{H}(17 \mathrm{~B})$ & 4351 & 10600 & 3415 & 159 \\
\hline $\mathrm{H}(17 \mathrm{C})$ & 4355 & 8092 & 3853 & 159 \\
\hline H(19) & 2186 & 3427 & 8927 & 87 \\
\hline $\mathrm{H}(20)$ & 1485 & 485 & 9636 & 91 \\
\hline $\mathrm{H}(22)$ & -422 & -1122 & 7170 & 101 \\
\hline $\mathrm{H}(23)$ & 279 & 1798 & 6437 & 88 \\
\hline $\mathrm{H}(24 \mathrm{~A})$ & 514 & -3636 & 9253 & 151 \\
\hline $\mathrm{H}(24 \mathrm{~B})$ & -546 & -3332 & 8504 & 151 \\
\hline $\mathrm{H}(24 \mathrm{C})$ & -310 & -1873 & 9449 & 151 \\
\hline
\end{tabular}


Table S14. Torsion angles $\left[^{\circ}\right]$ for $\mathbf{4 b}$.

\begin{tabular}{|c|c|}
\hline $\mathrm{O}(1)-\mathrm{C}(1)-\mathrm{C}(2)-\mathrm{C}(7)$ & $176.5(3)$ \\
\hline $\mathrm{C}(9)-\mathrm{C}(1)-\mathrm{C}(2)-\mathrm{C}(7)$ & $-6.2(3)$ \\
\hline $\mathrm{O}(1)-\mathrm{C}(1)-\mathrm{C}(2)-\mathrm{C}(3)$ & $-4.9(5)$ \\
\hline $\mathrm{C}(9)-\mathrm{C}(1)-\mathrm{C}(2)-\mathrm{C}(3)$ & $172.4(3)$ \\
\hline$C(7)-C(2)-C(3)-C(4)$ & $1.9(5)$ \\
\hline$C(1)-C(2)-C(3)-C(4)$ & $-176.5(3)$ \\
\hline $\mathrm{C}(2)-\mathrm{C}(3)-\mathrm{C}(4)-\mathrm{C}(5)$ & $-0.5(5)$ \\
\hline$C(3)-C(4)-C(5)-C(6)$ & $-1.4(5)$ \\
\hline $\mathrm{C}(3)-\mathrm{C}(4)-\mathrm{C}(5)-\mathrm{Cl}(1)$ & $178.0(3)$ \\
\hline $\mathrm{C}(4)-\mathrm{C}(5)-\mathrm{C}(6)-\mathrm{C}(7)$ & $1.7(5)$ \\
\hline $\mathrm{Cl}(1)-\mathrm{C}(5)-\mathrm{C}(6)-\mathrm{C}(7)$ & $-177.6(2)$ \\
\hline$C(5)-C(6)-C(7)-C(2)$ & $-0.2(4)$ \\
\hline$C(5)-C(6)-C(7)-C(8)$ & $-179.7(3)$ \\
\hline$C(3)-C(2)-C(7)-C(6)$ & $-1.6(4)$ \\
\hline$C(1)-C(2)-C(7)-C(6)$ & 177.1(3) \\
\hline $\mathrm{C}(3)-\mathrm{C}(2)-\mathrm{C}(7)-\mathrm{C}(8)$ & $178.0(3)$ \\
\hline $\mathrm{C}(1)-\mathrm{C}(2)-\mathrm{C}(7)-\mathrm{C}(8)$ & $-3.3(3)$ \\
\hline $\mathrm{C}(6)-\mathrm{C}(7)-\mathrm{C}(8)-\mathrm{C}(9)$ & $-169.2(3)$ \\
\hline $\mathrm{C}(2)-\mathrm{C}(7)-\mathrm{C}(8)-\mathrm{C}(9)$ & $11.3(3)$ \\
\hline $\mathrm{C}(12)-\mathrm{N}(1)-\mathrm{C}(9)-\mathrm{C}(8)$ & $110.3(3)$ \\
\hline $\mathrm{C}(12)-\mathrm{N}(1)-\mathrm{C}(9)-\mathrm{C}(1)$ & $-137.6(2)$ \\
\hline $\mathrm{C}(12)-\mathrm{N}(1)-\mathrm{C}(9)-\mathrm{C}(10)$ & $-17.5(3)$ \\
\hline $\mathrm{C}(7)-\mathrm{C}(8)-\mathrm{C}(9)-\mathrm{N}(1)$ & $98.8(3)$ \\
\hline $\mathrm{C}(7)-\mathrm{C}(8)-\mathrm{C}(9)-\mathrm{C}(1)$ & $-14.1(3)$ \\
\hline$C(7)-C(8)-C(9)-C(10)$ & $-141.4(2)$ \\
\hline $\mathrm{O}(1)-\mathrm{C}(1)-\mathrm{C}(9)-\mathrm{N}(1)$ & $74.6(3)$ \\
\hline $\mathrm{C}(2)-\mathrm{C}(1)-\mathrm{C}(9)-\mathrm{N}(1)$ & $-102.9(2)$ \\
\hline $\mathrm{O}(1)-\mathrm{C}(1)-\mathrm{C}(9)-\mathrm{C}(8)$ & $-169.8(3)$ \\
\hline $\mathrm{C}(2)-\mathrm{C}(1)-\mathrm{C}(9)-\mathrm{C}(8)$ & $12.7(3)$ \\
\hline $\mathrm{O}(1)-\mathrm{C}(1)-\mathrm{C}(9)-\mathrm{C}(10)$ & $-39.6(4)$ \\
\hline $\mathrm{C}(2)-\mathrm{C}(1)-\mathrm{C}(9)-\mathrm{C}(10)$ & $143.0(2)$ \\
\hline $\mathrm{N}(1)-\mathrm{C}(9)-\mathrm{C}(10)-\mathrm{C}(18)$ & $159.0(2)$ \\
\hline $\mathrm{C}(8)-\mathrm{C}(9)-\mathrm{C}(10)-\mathrm{C}(18)$ & $36.7(4)$ \\
\hline $\mathrm{C}(1)-\mathrm{C}(9)-\mathrm{C}(10)-\mathrm{C}(18)$ & $-86.2(3)$ \\
\hline
\end{tabular}




\begin{tabular}{|c|c|}
\hline $\mathrm{N}(1)-\mathrm{C}(9)-\mathrm{C}(10)-\mathrm{C}(11)$ & $27.8(3)$ \\
\hline$C(8)-C(9)-C(10)-C(11)$ & $-94.5(3)$ \\
\hline$C(1)-C(9)-C(10)-C(11)$ & $142.6(3)$ \\
\hline $\mathrm{C}(18)-\mathrm{C}(10)-\mathrm{C}(11)-\mathrm{C}(12)$ & $-155.7(3)$ \\
\hline $\mathrm{C}(9)-\mathrm{C}(10)-\mathrm{C}(11)-\mathrm{C}(12)$ & $-26.6(3)$ \\
\hline $\mathrm{C}(9)-\mathrm{N}(1)-\mathrm{C}(12)-\mathrm{C}(11)$ & $-0.8(4)$ \\
\hline $\mathrm{C}(9)-\mathrm{N}(1)-\mathrm{C}(12)-\mathrm{C}(13)$ & $177.4(3)$ \\
\hline $\mathrm{C}(10)-\mathrm{C}(11)-\mathrm{C}(12)-\mathrm{N}(1)$ & $18.6(4)$ \\
\hline $\mathrm{C}(10)-\mathrm{C}(11)-\mathrm{C}(12)-\mathrm{C}(13)$ & $-159.5(3)$ \\
\hline $\mathrm{C}(14)-\mathrm{O}(3)-\mathrm{C}(13)-\mathrm{O}(2)$ & $-2.1(5)$ \\
\hline $\mathrm{C}(14)-\mathrm{O}(3)-\mathrm{C}(13)-\mathrm{C}(12)$ & $178.2(3)$ \\
\hline $\mathrm{N}(1)-\mathrm{C}(12)-\mathrm{C}(13)-\mathrm{O}(2)$ & $-156.8(3)$ \\
\hline $\mathrm{C}(11)-\mathrm{C}(12)-\mathrm{C}(13)-\mathrm{O}(2)$ & $21.3(5)$ \\
\hline $\mathrm{N}(1)-\mathrm{C}(12)-\mathrm{C}(13)-\mathrm{O}(3)$ & $22.9(4)$ \\
\hline $\mathrm{C}(11)-\mathrm{C}(12)-\mathrm{C}(13)-\mathrm{O}(3)$ & $-159.0(3)$ \\
\hline $\mathrm{C}(13)-\mathrm{O}(3)-\mathrm{C}(14)-\mathrm{C}(16)$ & $62.8(4)$ \\
\hline $\mathrm{C}(13)-\mathrm{O}(3)-\mathrm{C}(14)-\mathrm{C}(15)$ & $-61.1(4)$ \\
\hline $\mathrm{C}(13)-\mathrm{O}(3)-\mathrm{C}(14)-\mathrm{C}(17)$ & $-179.6(3)$ \\
\hline $\mathrm{C}(11)-\mathrm{C}(10)-\mathrm{C}(18)-\mathrm{C}(23)$ & $2.8(5)$ \\
\hline $\mathrm{C}(9)-\mathrm{C}(10)-\mathrm{C}(18)-\mathrm{C}(23)$ & $-119.8(3)$ \\
\hline $\mathrm{C}(11)-\mathrm{C}(10)-\mathrm{C}(18)-\mathrm{C}(19)$ & $-175.2(3)$ \\
\hline $\mathrm{C}(9)-\mathrm{C}(10)-\mathrm{C}(18)-\mathrm{C}(19)$ & $62.2(4)$ \\
\hline $\mathrm{C}(23)-\mathrm{C}(18)-\mathrm{C}(19)-\mathrm{C}(20)$ & $0.2(5)$ \\
\hline $\mathrm{C}(10)-\mathrm{C}(18)-\mathrm{C}(19)-\mathrm{C}(20)$ & $178.3(3)$ \\
\hline $\mathrm{C}(18)-\mathrm{C}(19)-\mathrm{C}(20)-\mathrm{C}(21)$ & $-0.9(6)$ \\
\hline $\mathrm{C}(19)-\mathrm{C}(20)-\mathrm{C}(21)-\mathrm{C}(22)$ & $1.1(5)$ \\
\hline$C(19)-C(20)-C(21)-C(24)$ & $-179.7(4)$ \\
\hline $\mathrm{C}(20)-\mathrm{C}(21)-\mathrm{C}(22)-\mathrm{C}(23)$ & $-0.8(6)$ \\
\hline $\mathrm{C}(24)-\mathrm{C}(21)-\mathrm{C}(22)-\mathrm{C}(23)$ & $-180.0(4)$ \\
\hline $\mathrm{C}(19)-\mathrm{C}(18)-\mathrm{C}(23)-\mathrm{C}(22)$ & $0.1(5)$ \\
\hline $\mathrm{C}(10)-\mathrm{C}(18)-\mathrm{C}(23)-\mathrm{C}(22)$ & $-177.9(3)$ \\
\hline $\mathrm{C}(21)-\mathrm{C}(22)-\mathrm{C}(23)-\mathrm{C}(18)$ & $0.2(6)$ \\
\hline
\end{tabular}

Symmetry transformations used to generate equivalent atoms: 
${ }^{1} \mathrm{H}$ NMR of Compound $\mathbf{C 4 a}\left(400 \mathrm{MHz}, \mathrm{CDCl}_{3}\right)$

DPG-DH-45 H $400 \mathrm{MHz}$

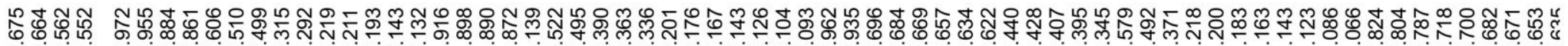

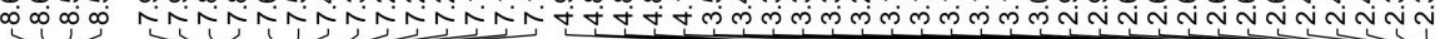

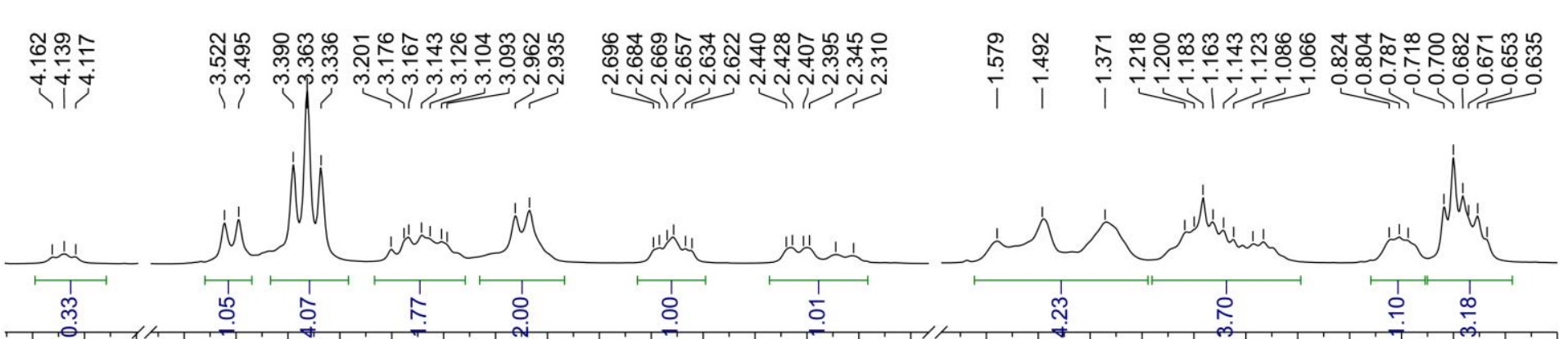

$\begin{array}{lllllllllllllllllllllllllllllll}4.2 & 4.1 & 4.0 & 3.6 & 3.5 & 3.4 & 3.3 & 3.2 & 3.1 & 3.0 & 2.9 & 2.8 & 2.7 & 2.6 & 2.5 & 2.4 & 2.3 & 2.2 & 1.6 & 1.5 & 1.4 & 1.3 & 1.2 & 1.1 & 1.0 & 0.9 & 0.8 & 0.7 & 0.6 & 0.5\end{array}$ $\mathrm{f} 1(\mathrm{ppm})$
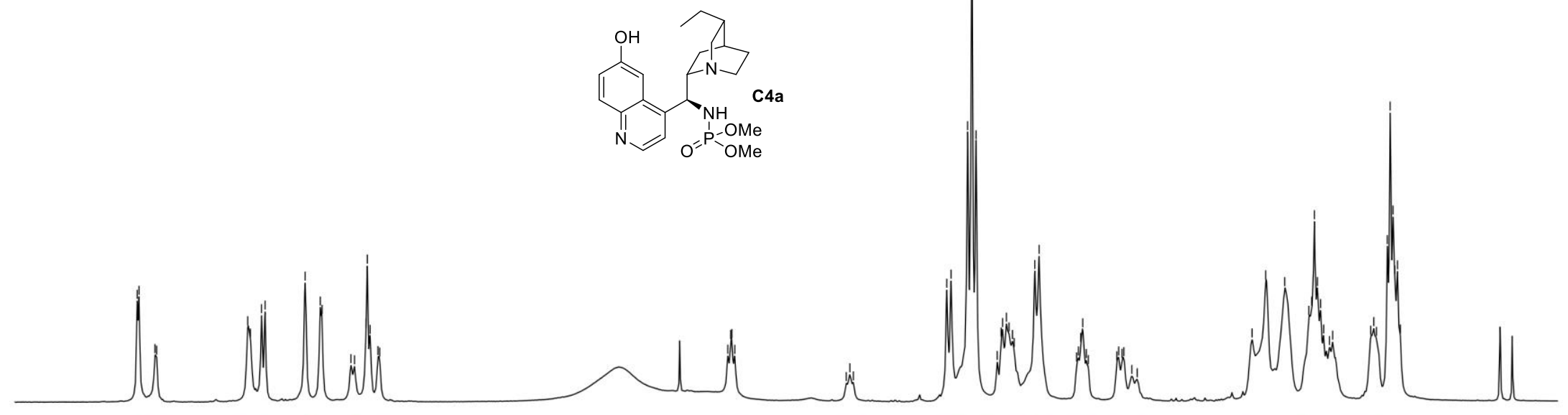

\begin{tabular}{|c|c|c|c|c|c|c|c|c|c|c|c|c|c|c|c|c|c|c|c|}
\hline & 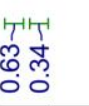 & & $\stackrel{1}{?}$ & 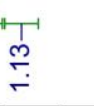 & & & & $\begin{array}{c}T \\
\stackrel{T}{N} \\
0\end{array}$ & & 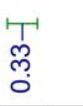 & "ris & : & $\begin{array}{l}\text { 'T } \\
\stackrel{\circ}{\circ}\end{array}$ & $\stackrel{\Gamma}{\stackrel{T}{\circ}}$ & & $\underset{\sim}{\stackrel{N}{\sim}}$ & 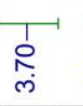 & 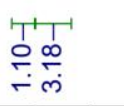 & \\
\hline 9.0 & 8.5 & 8.0 & 7.5 & 7.0 & 6.5 & 6.0 & 5.5 & 5.0 & $\begin{array}{c}4.5 \\
1(\mathrm{ppm})\end{array}$ & 4.0 & 3.5 & 3.0 & & 2.5 & 2.0 & 1.5 & 1.0 & 0.5 & 0.0 \\
\hline
\end{tabular}


${ }^{31} \mathrm{P}$ NMR of Compound C4a (162 MHz, $\mathrm{CDCl}_{3}$ )

DPG-DH-45 P 161.7 MHz

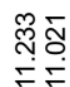

ॠ

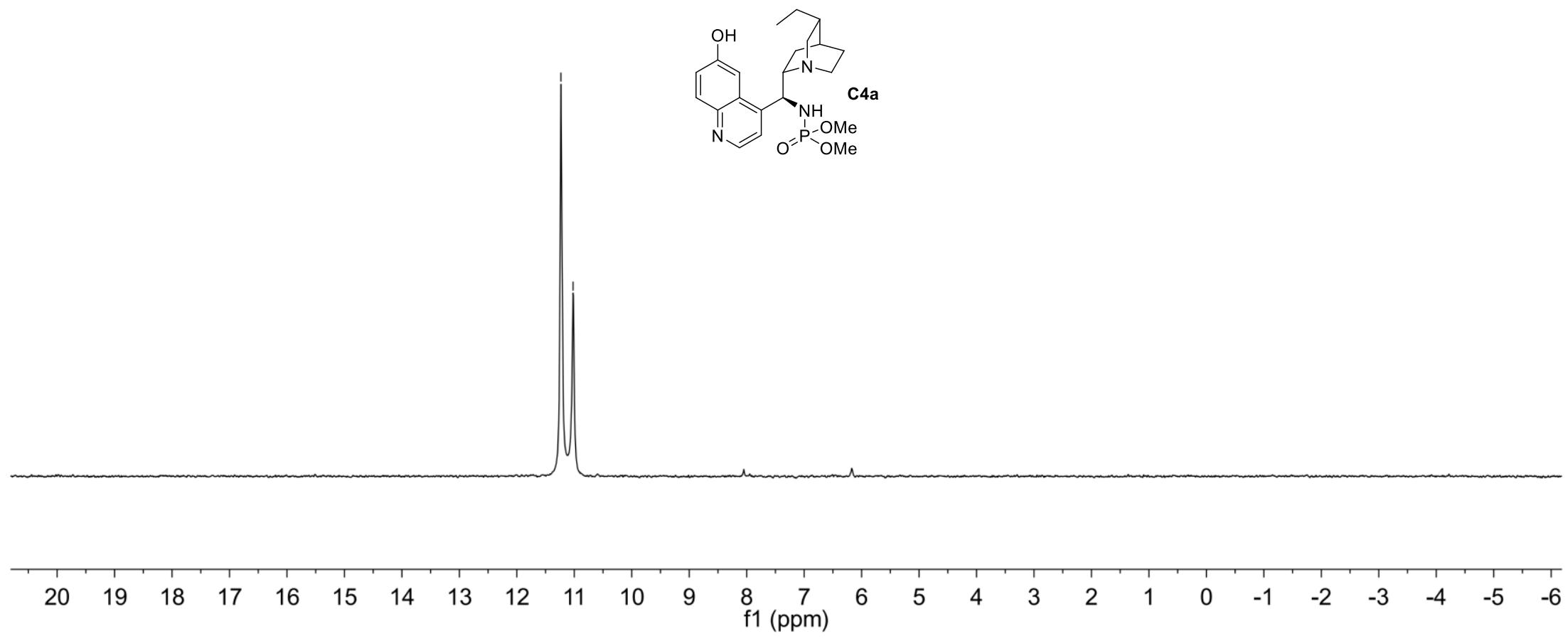


${ }^{13} \mathrm{C}$ NMR of Compound $\mathbf{C 4 a}\left(100 \mathrm{MHz}, \mathrm{CDCl}_{3}\right)$

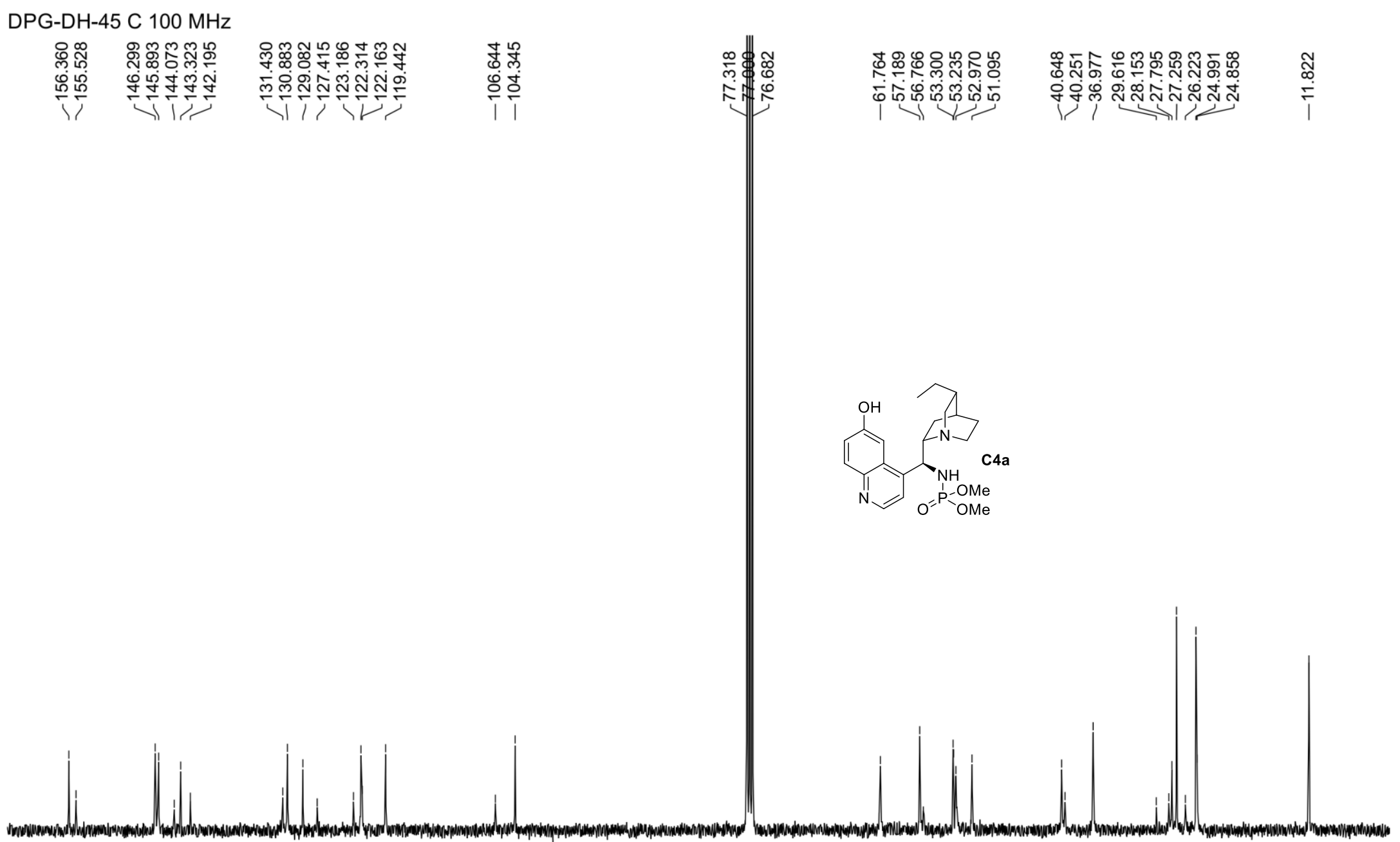

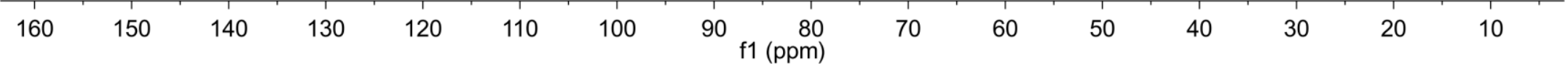


${ }^{1} \mathrm{H}$ NMR of Compound $\mathbf{C 4 b}\left(400 \mathrm{MHz}, \mathrm{CDCl}_{3}\right)$

DPG-DI-66 H $400 \mathrm{MHz}$

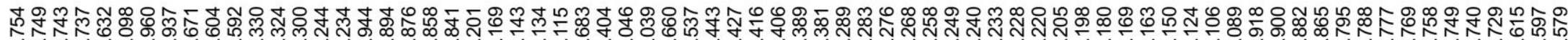

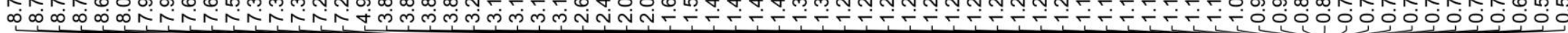

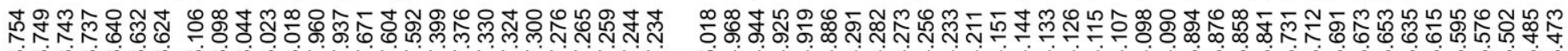

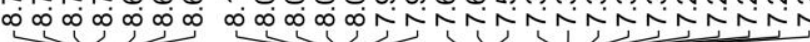

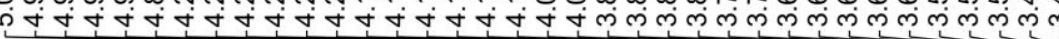

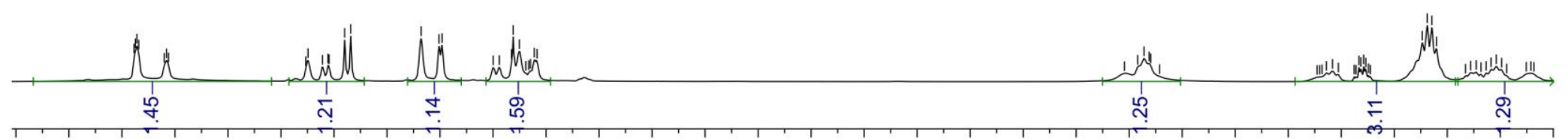

$\begin{array}{lllllllllllllllllllllllllllllllllll}9.2 & 9.0 & 8.8 & 8.6 & 8.4 & 8.2 & 8.0 & 7.8 & 7.6 & 7.4 & 7.2 & 7.0 & 6.8 & 6.6 & 6.4 & 6.2 & 6.0 & 5.8 & 5.6 & 5.4 & 5.2 & 5.0 & 4.8 & 4.6 & 4.4 & 4.2 & 4.0 & 3.8 & 3.6 & 3.4\end{array}$ $\mathrm{f1}(\mathrm{ppm})$

苯

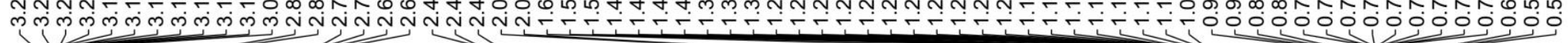

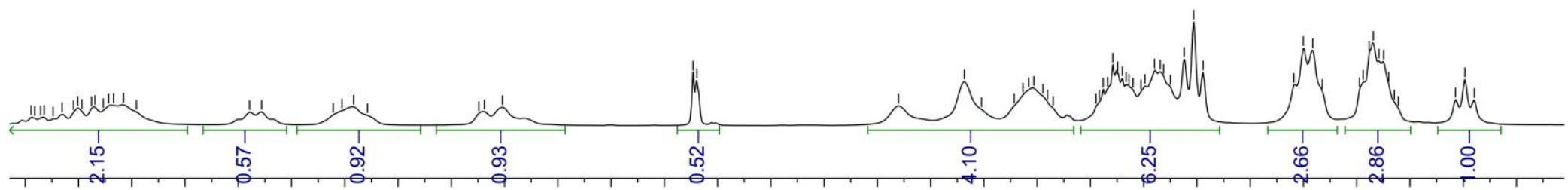

$\begin{array}{lllllllllllllllllllllllllllllll}3.3 & 3.2 & 3.1 & 3.0 & 2.9 & 2.8 & 2.7 & 2.6 & 2.5 & 2.4 & 2.3 & 2.2 & 2.1 & 2.0 & \begin{array}{c}1.9 \\ \mathrm{f} 1\end{array}(\mathrm{ppm}) & 1.7 & 1.6 & 1.5 & 1.4 & 1.3 & 1.2 & 1.1 & 1.0 & 0.9 & 0.8 & 0.7 & 0.6 & 0.5\end{array}$

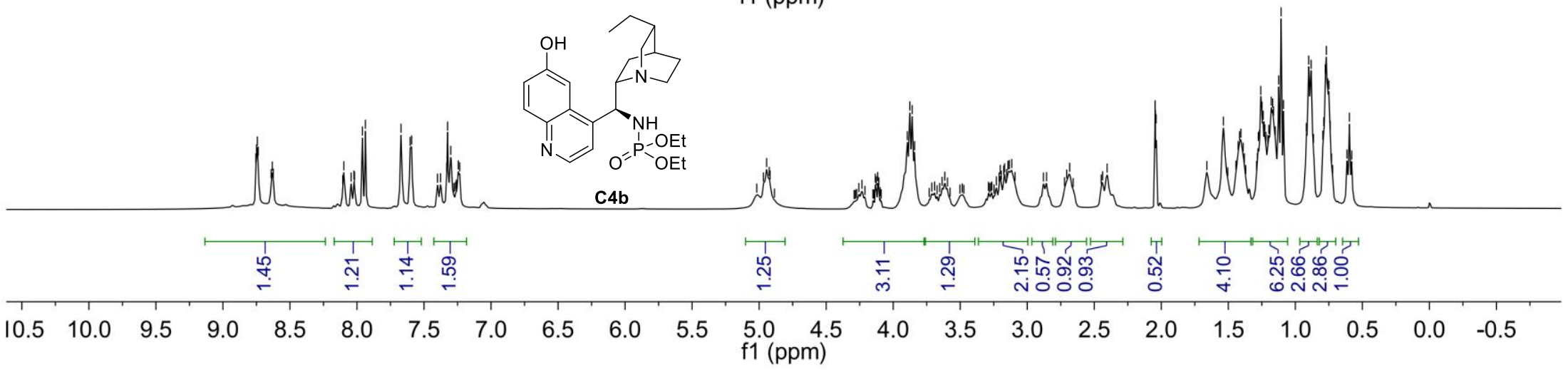


${ }^{31}$ P NMR of Compound C4a (162 MHz, $\left.\mathrm{CDCl}_{3}\right)$

DPG-DI-66 P $162 \mathrm{MHz}$

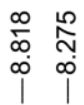

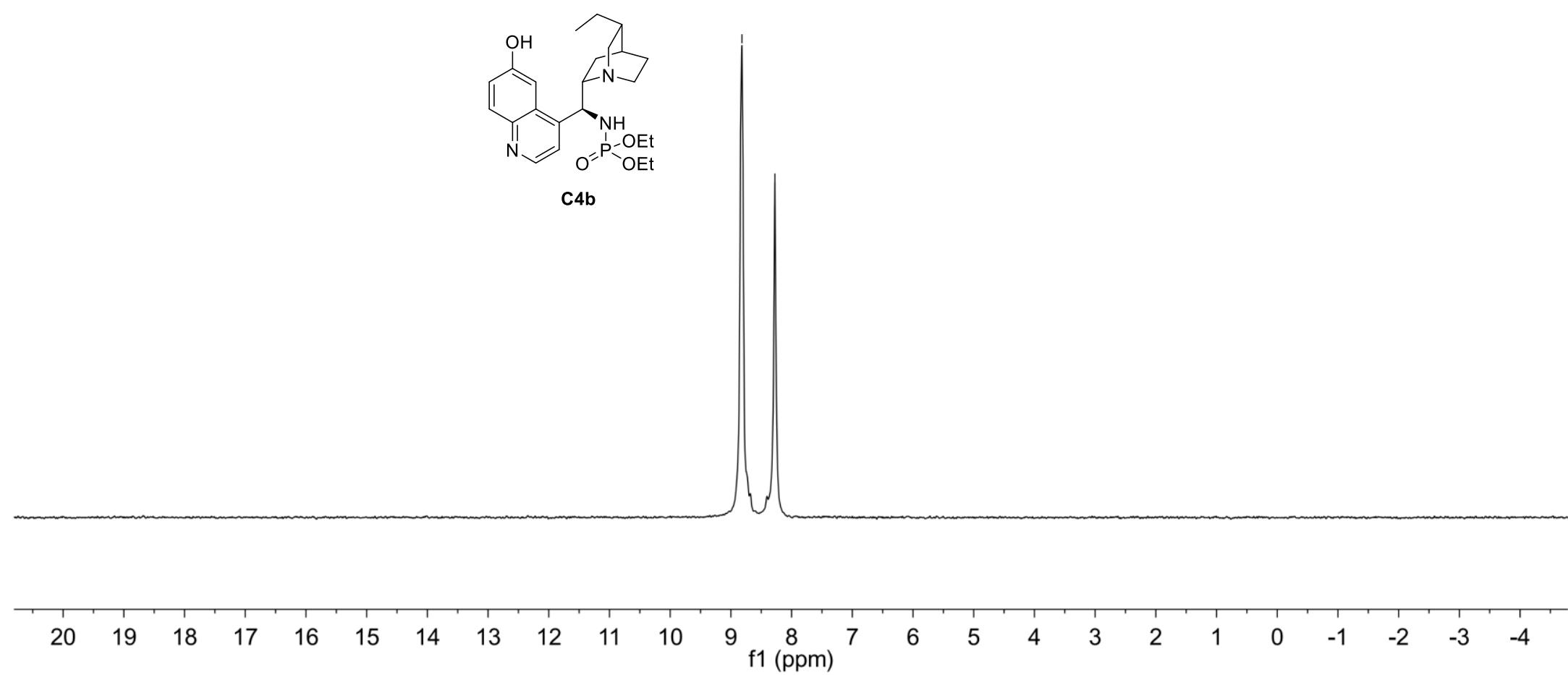


${ }^{13} \mathrm{C}$ NMR of Compound C4b $\left(100 \mathrm{MHz}, \mathrm{CDCl}_{3}\right)$

DPG-DI-66 C $100 \mathrm{MHz}$

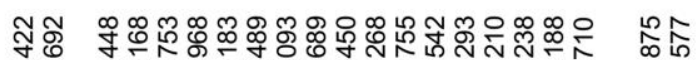

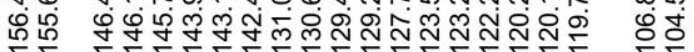

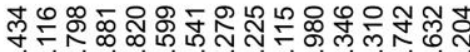

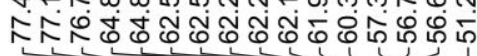

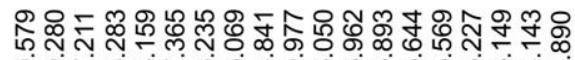

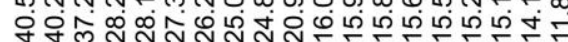

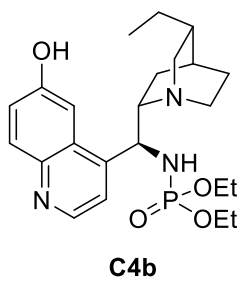

C4b

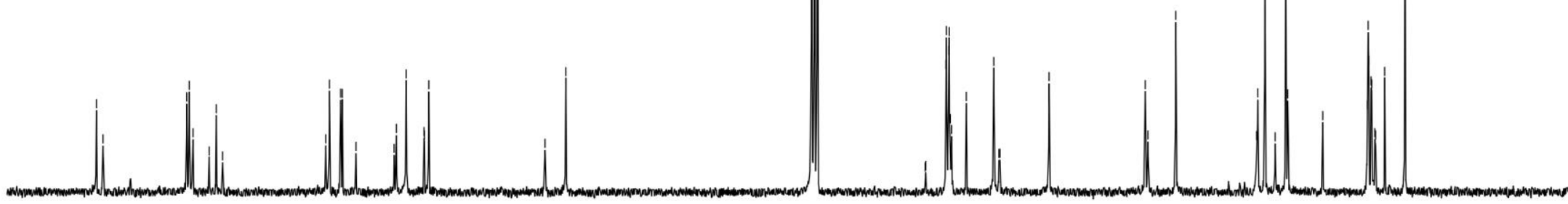

160

150

140

130

120

110

100

90

80

70

60

50

40

30

20

10

0 
${ }^{1} \mathrm{H}$ NMR of Compound $\mathbf{C 4 c}\left(400 \mathrm{MHz}, \mathrm{CDCl}_{3}\right)$

\section{DPG-DH-27 H $400 \mathrm{MHz}$}

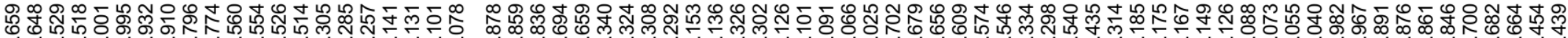

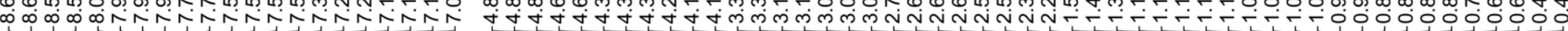

|

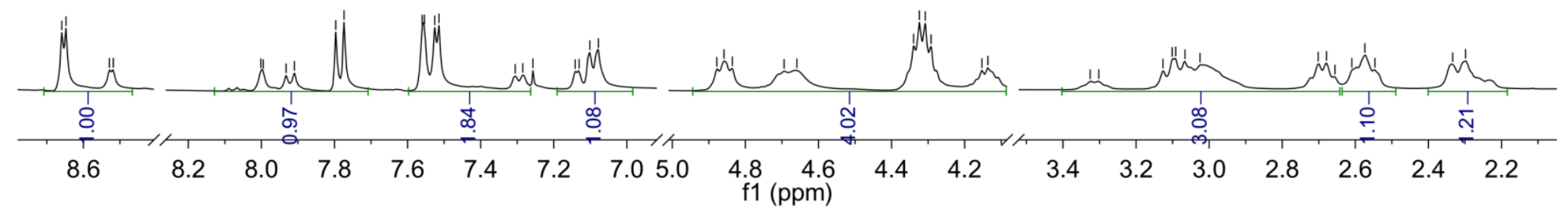

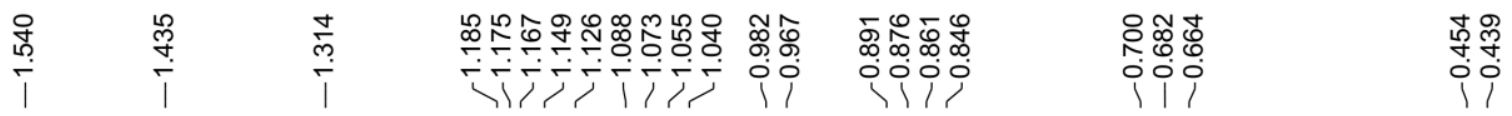
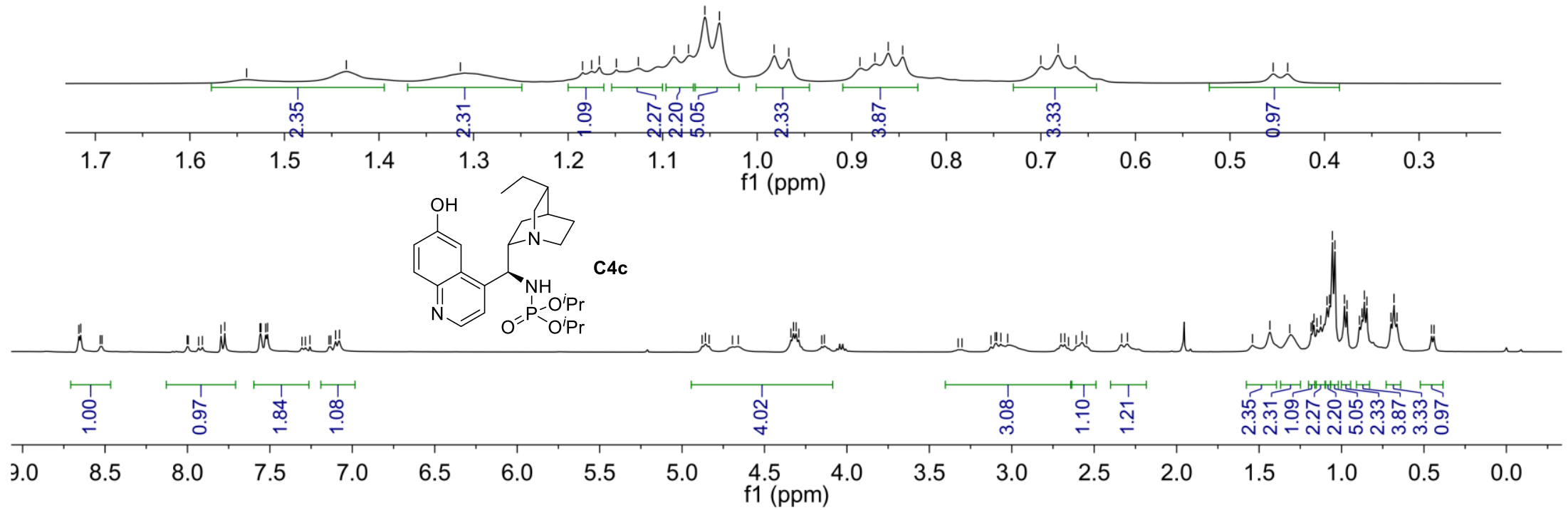
${ }^{31}$ P NMR of Compound $\mathbf{C 4 c}\left(162 \mathrm{MHz}, \mathrm{CDCl}_{3}\right)$

DPG-DH-27 P 161.7 MHz

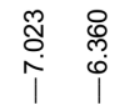

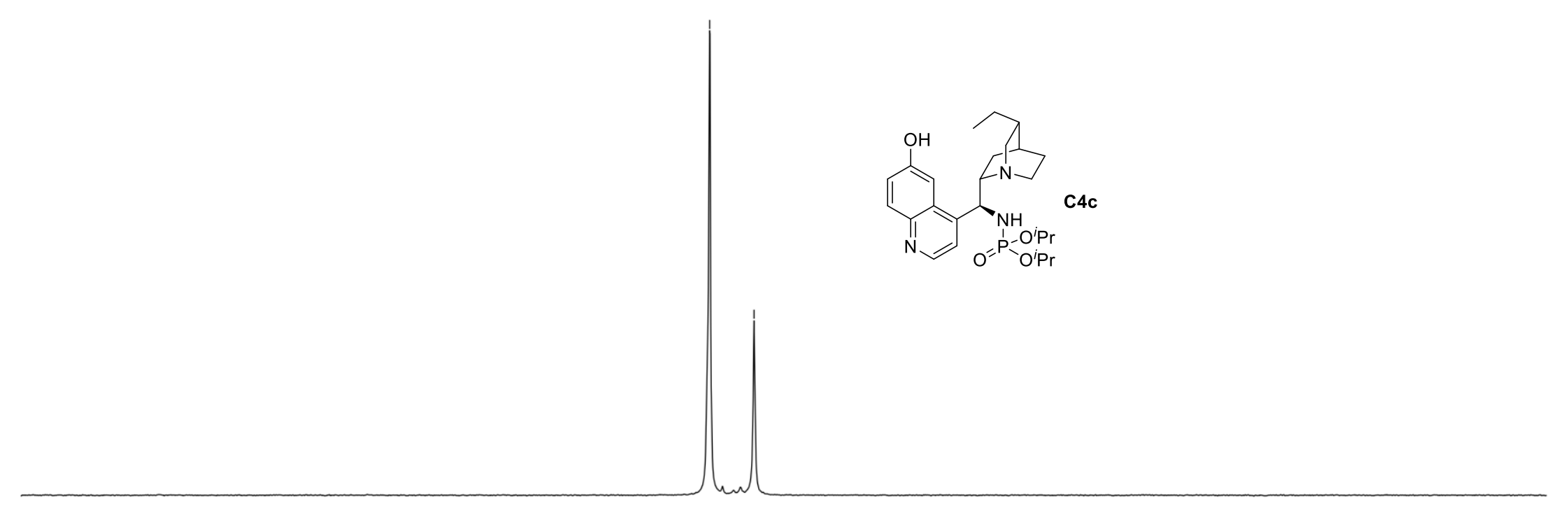

$\begin{array}{lll}17 & 16 & 15\end{array}$

13

12

$11 \quad 10$

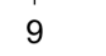

8

$7 \quad \begin{aligned} & 6 \\ & \mathrm{f} 1 \\ & (\mathrm{ppm})\end{aligned}$ 
${ }^{13} \mathrm{C}$ NMR of Compound $\mathbf{C 4 c}\left(100 \mathrm{MHz}, \mathrm{CDCl}_{3}\right)$

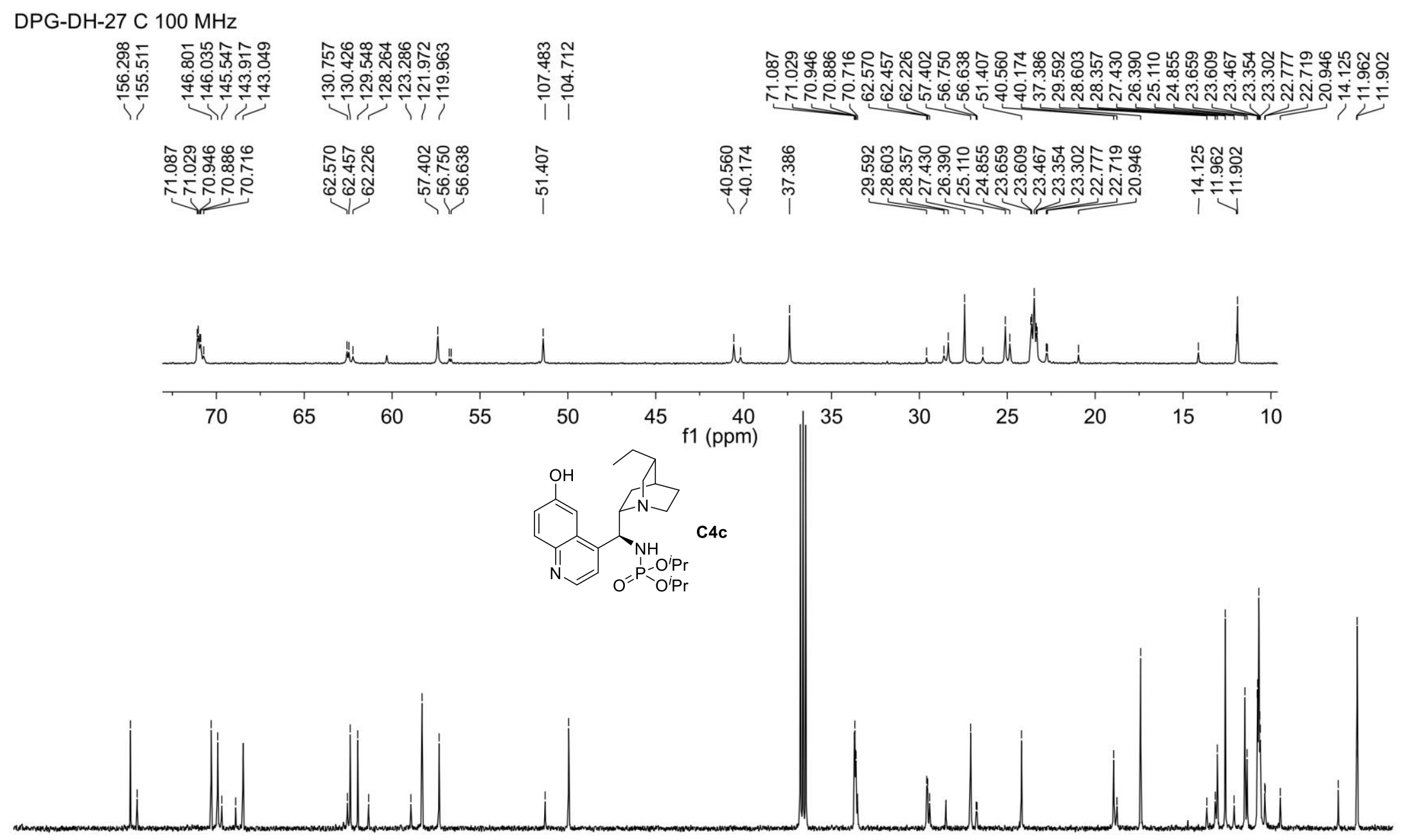

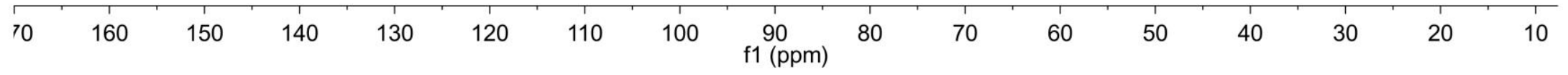




\section{${ }^{1} \mathrm{H}$ NMR of Compound $\mathbf{C 4 d}\left(400 \mathrm{MHz}, \mathrm{CDCl}_{3}\right)$}

DPG-DH-102 H $400 \mathrm{MHz}$

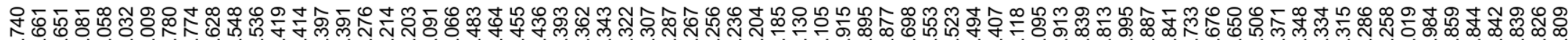

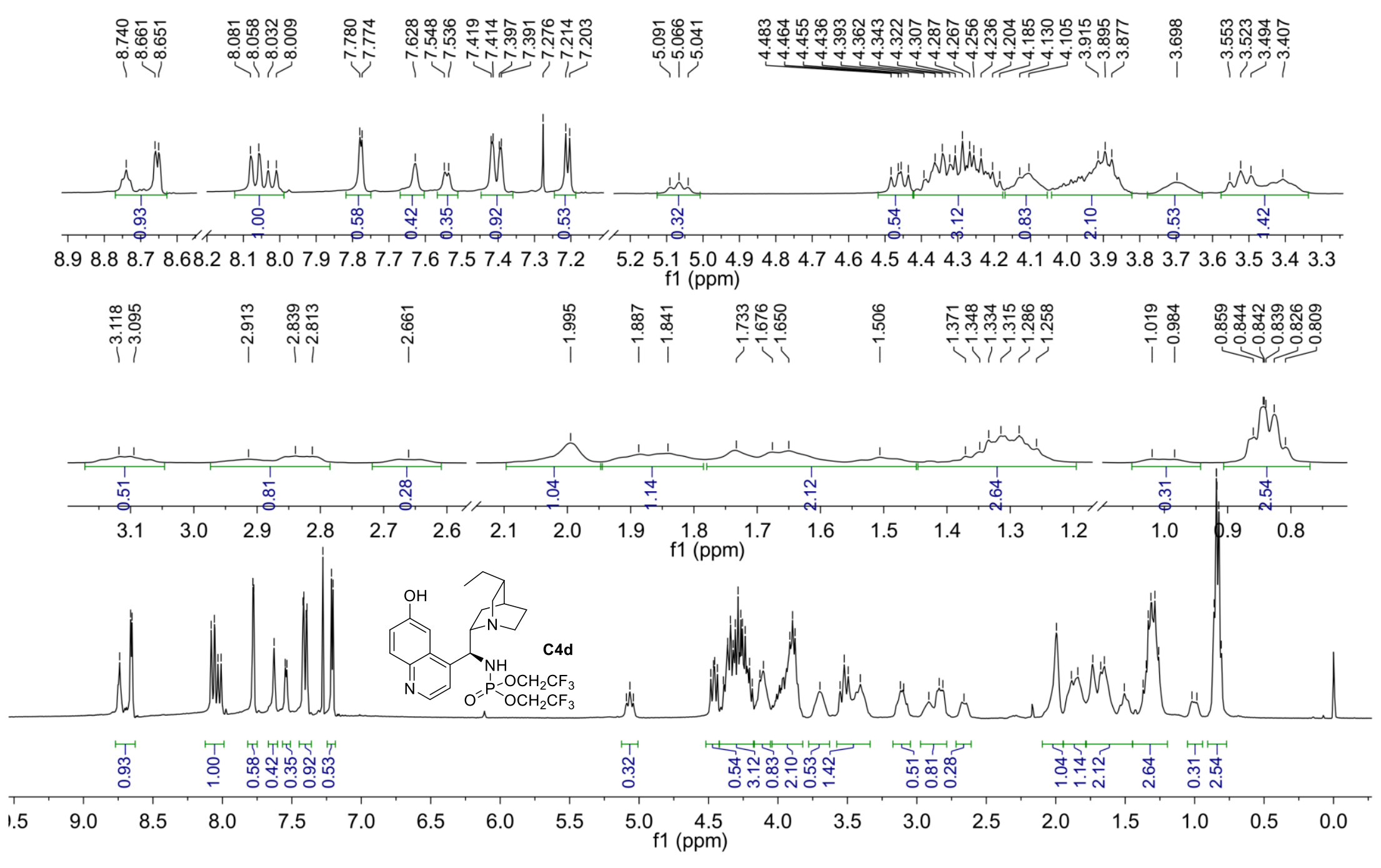


${ }^{19}$ F NMR of Compound $\mathbf{C 4 d}\left(376 \mathrm{MHz}, \mathrm{CDCl}_{3}\right.$ )

DPG-DH-102 F 376 MHz

응ํㅠำ

रำ
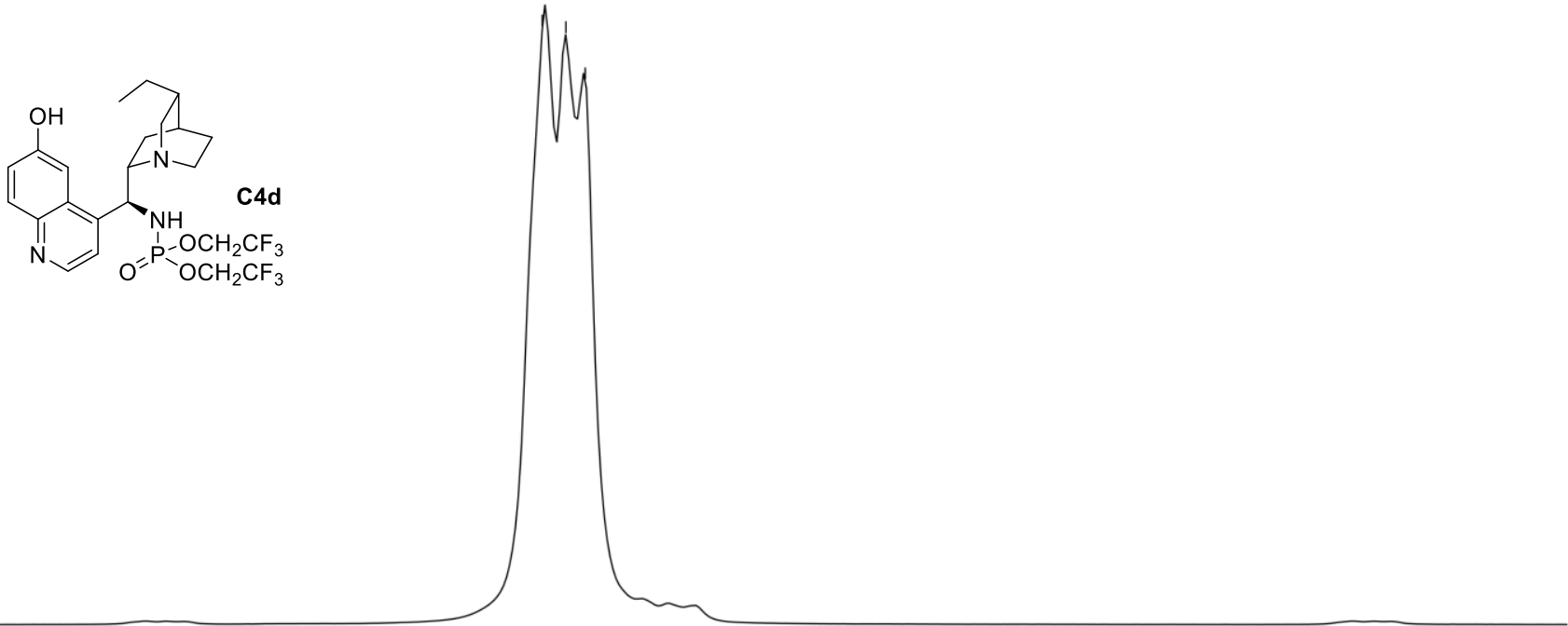

$-75.00$

$-75.10$

$-75.20$

$-75.30$

$-75.40$

$-75.50$ f1 (ppm)

$-75.60$

$-75.70$

$-75.80$

$-75.90$

$-76.00$

$-76.10$ 
${ }^{31} \mathrm{P}$ NMR of Compound C4d (162 MHz, $\left.\mathrm{CDCl}_{3}\right)$

DPG-DH-102 P $162 \mathrm{MHz}$

$\stackrel{\text { }}{\stackrel{\mathrm{O}}{i}}$

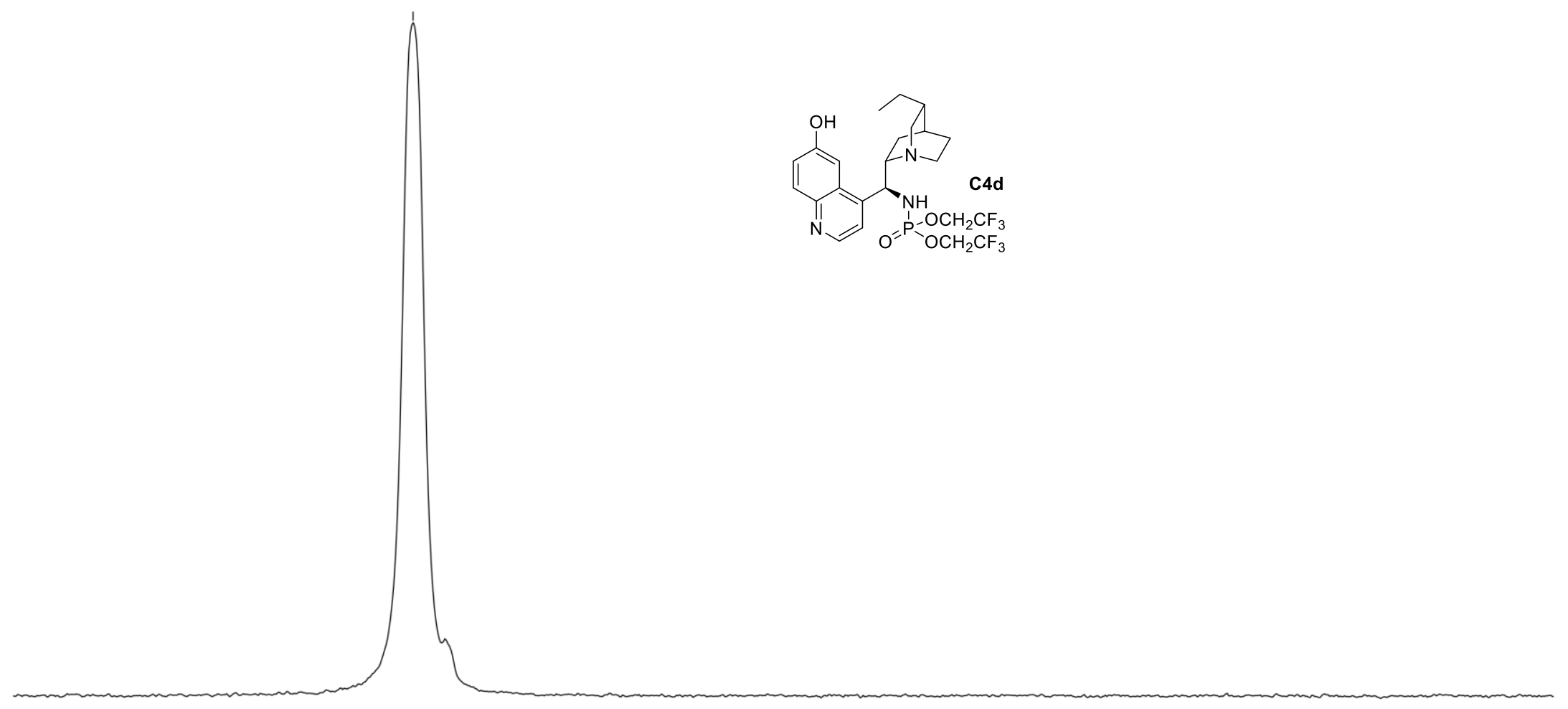

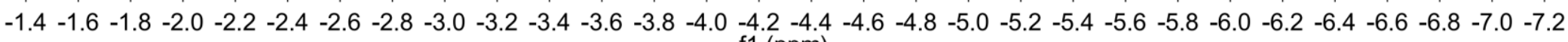
f1 (ppm) 


\section{${ }^{13} \mathrm{C}$ NMR of Compound $\mathbf{C 4 d}\left(125 \mathrm{MHz}, \mathrm{CDCl}_{3}\right)$}

\section{DPG-DH-102 C 125 MHz}

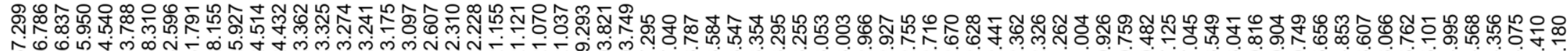
ம்

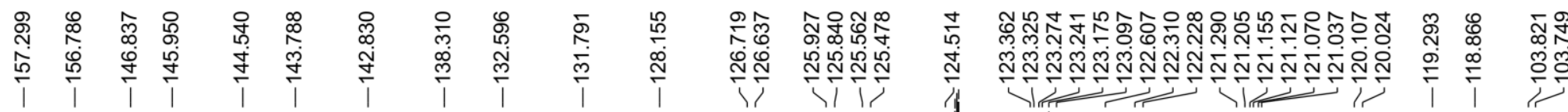

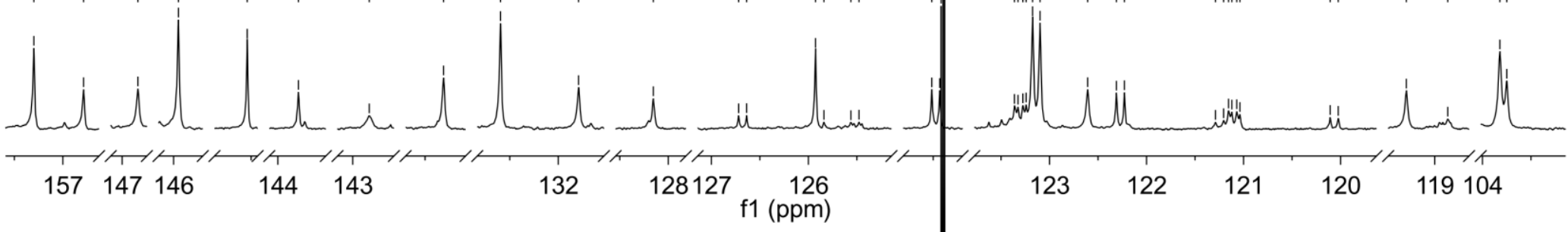

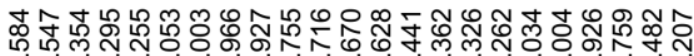

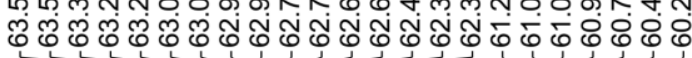

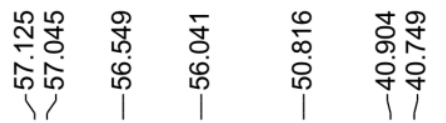

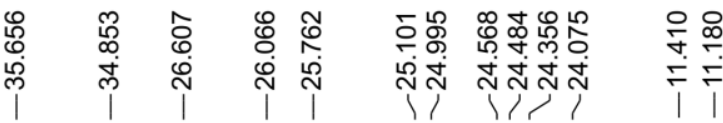
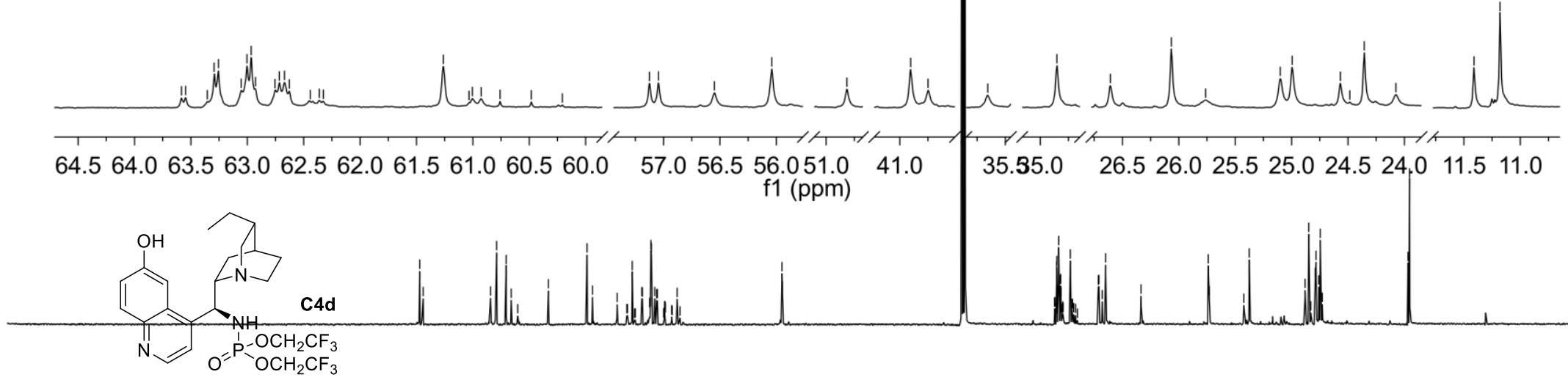
f1 (ppm) 
${ }^{1} \mathrm{H}$ NMR of Compound $\mathbf{C 4 e}\left(400 \mathrm{MHz}, \mathrm{CDCl}_{3}\right)$

\section{DPG-DL-41 H $400 \mathrm{MHz}$}

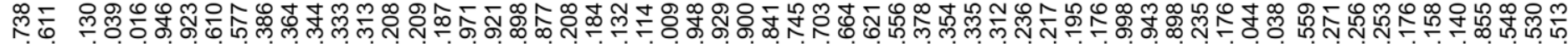

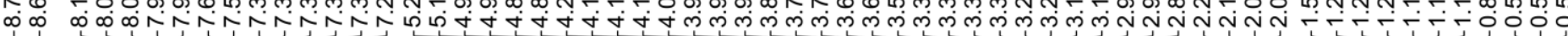
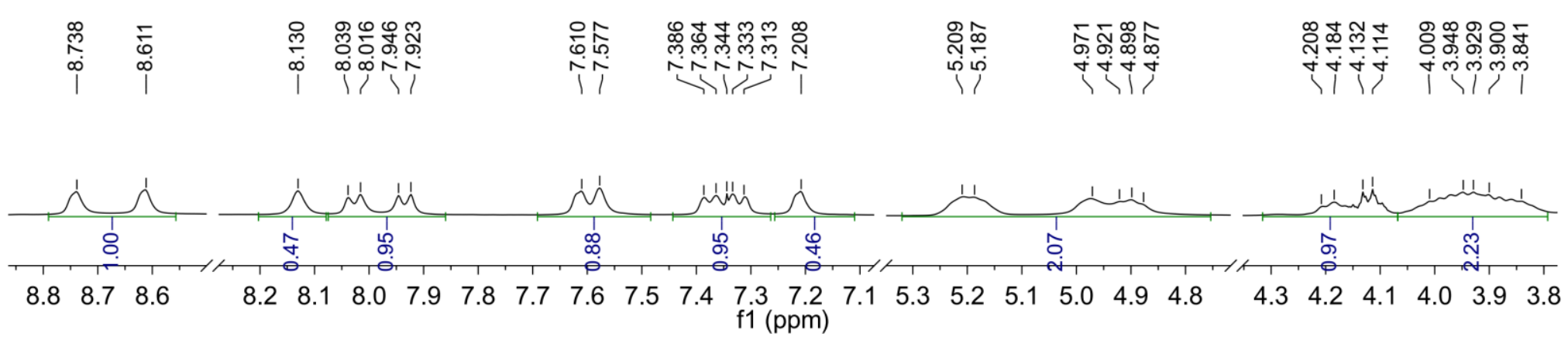

\begin{tabular}{|c|c|c|c|c|}
\hline 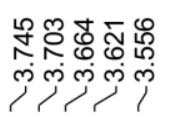 & 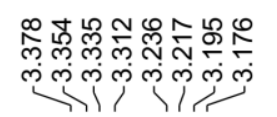 & 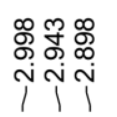 & $\underset{N}{\stackrel{N}{N} \underset{i}{\stackrel{\rho}{\leftarrow}}}$ & $\begin{array}{l}\forall \\
\text { đio } \\
\text { No }\end{array}$ \\
\hline
\end{tabular}
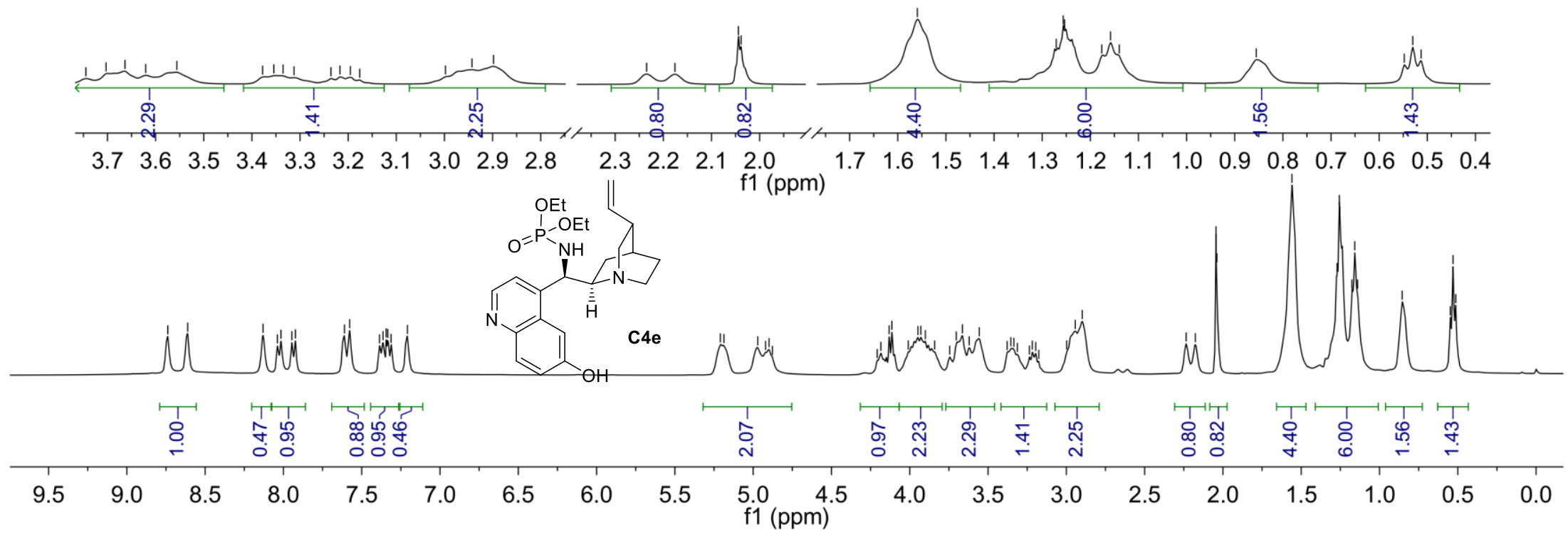
${ }^{31} \mathrm{P}$ NMR of Compound $\mathbf{C 4 e}\left(132 \mathrm{MHz}, \mathrm{CDCl}_{3}\right)$

DPG-DL-41 P 132 MHz

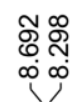

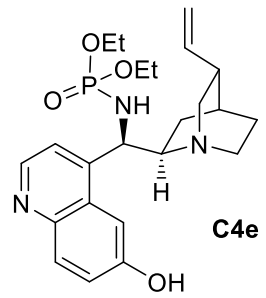

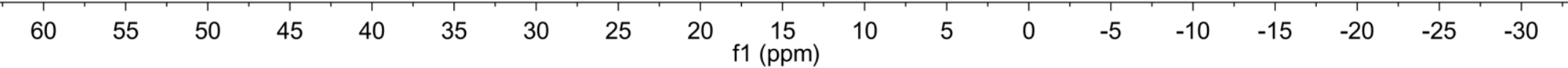


${ }^{13} \mathrm{C}$ NMR of Compound $\mathbf{C 4 e}\left(100 \mathrm{MHz}, \mathrm{CDCl}_{3}\right)$

\section{DPG-DL-41 C $100 \mathrm{MHz}$}

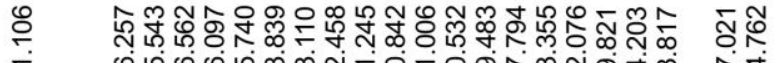

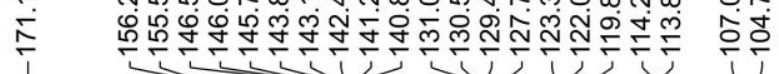

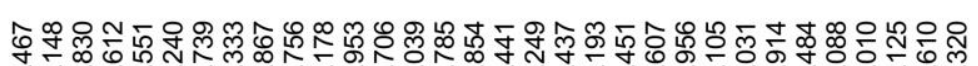

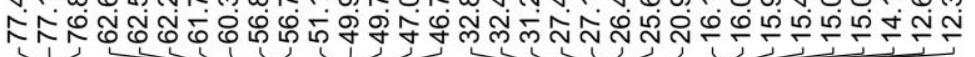

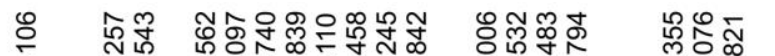

它

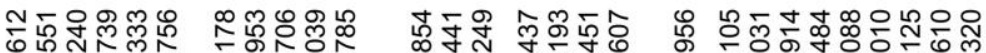

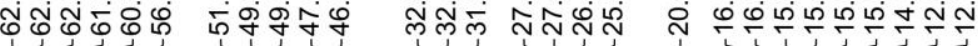
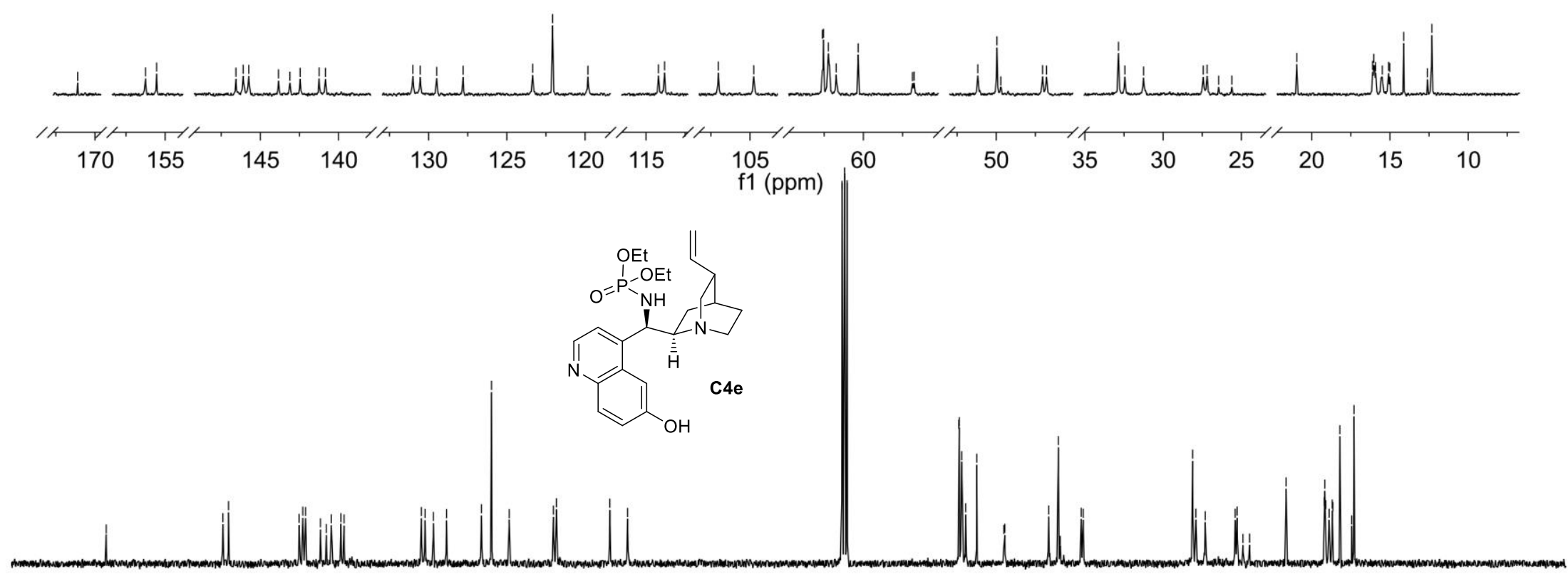

180

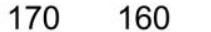

$150 \quad 140 \quad 130$

120

$110 \quad 100$

90 f1 (ppm) 
${ }^{1} \mathrm{H}$ NMR of Compound 1c (300 MHz, $\left.\mathrm{CDCl}_{3}\right)$

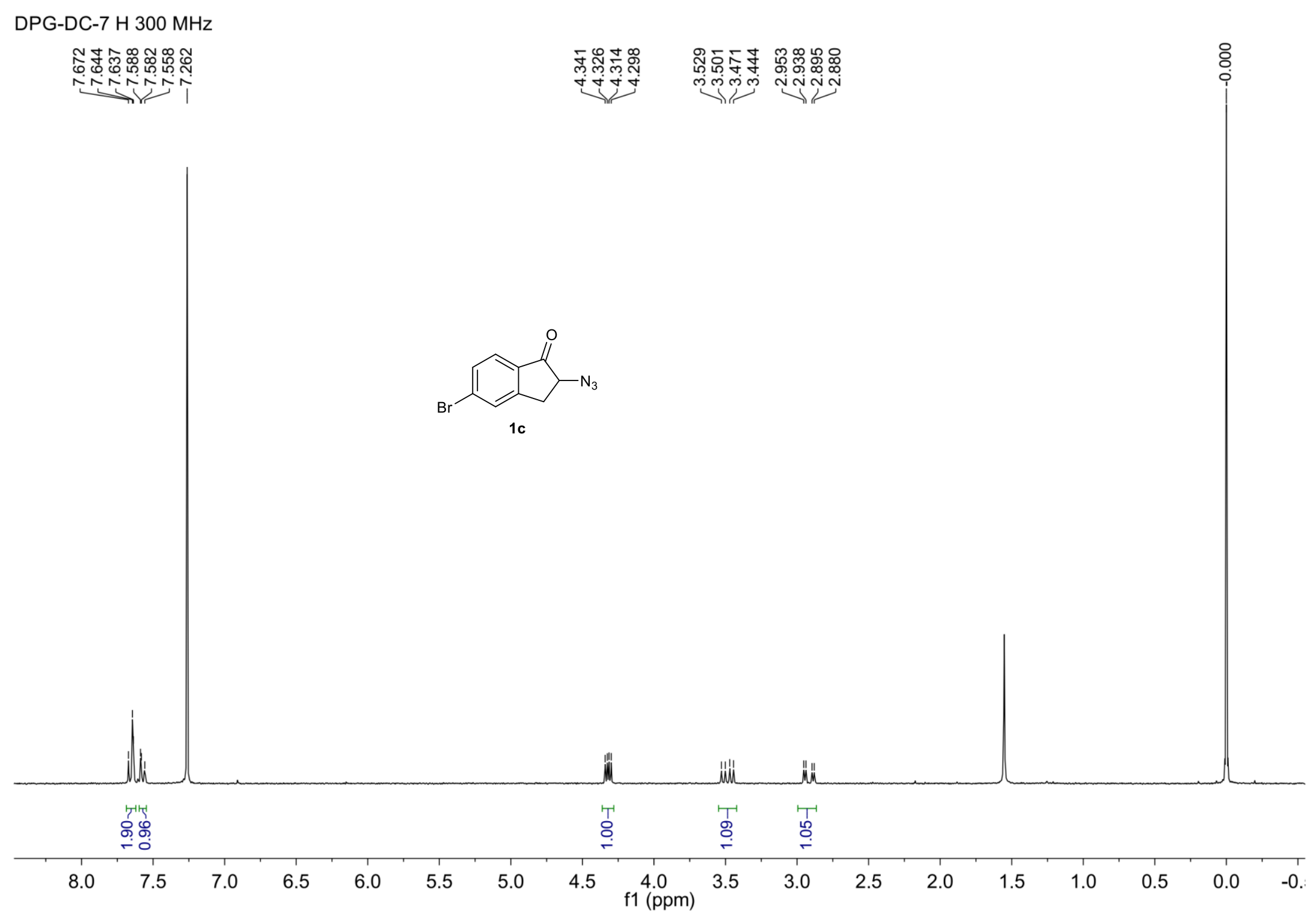


${ }^{13} \mathrm{C}$ NMR of Compound $1 \mathrm{c}\left(100 \mathrm{MHz}, \mathrm{CDCl}_{3}\right)$

dpg-da-112-2 C $100 \mathrm{MHz}$

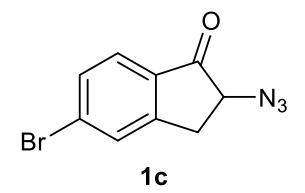

$1 c$

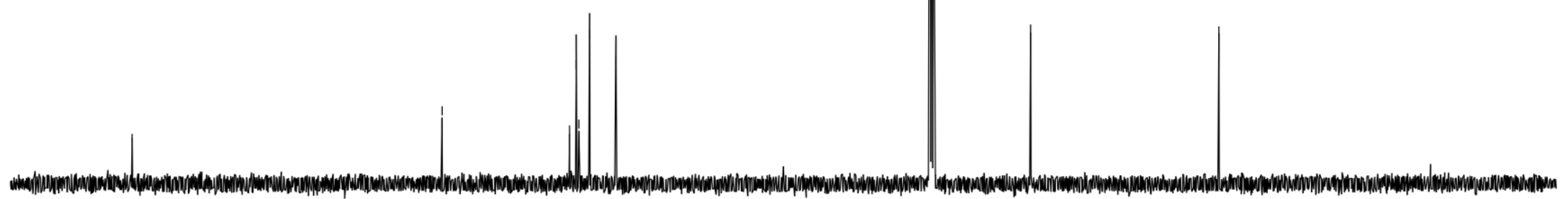

$\begin{array}{llllll}210 & 200 & 190 & 180 & 170 & 160\end{array}$

$\begin{array}{lllll}150 & 140 & 130 & 120 & 110\end{array}$

$110 \quad 100$

80

$\begin{array}{lll}70 & 60 & 50\end{array}$

$40 \quad 30$

20 
${ }^{1} \mathrm{H}$ NMR of Compound $1 e\left(300 \mathrm{MHz}, \mathrm{CDCl}_{3}\right)$

\section{dpg-dd-47 H $300 \mathrm{MHz}$}

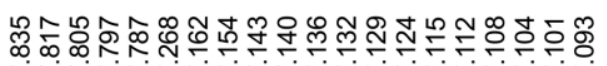

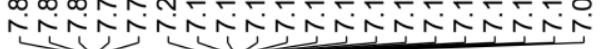

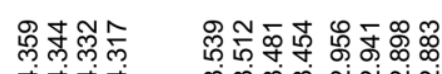

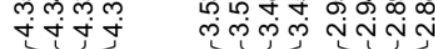

$\stackrel{\text { i }}{\stackrel{5}{6}}$

o
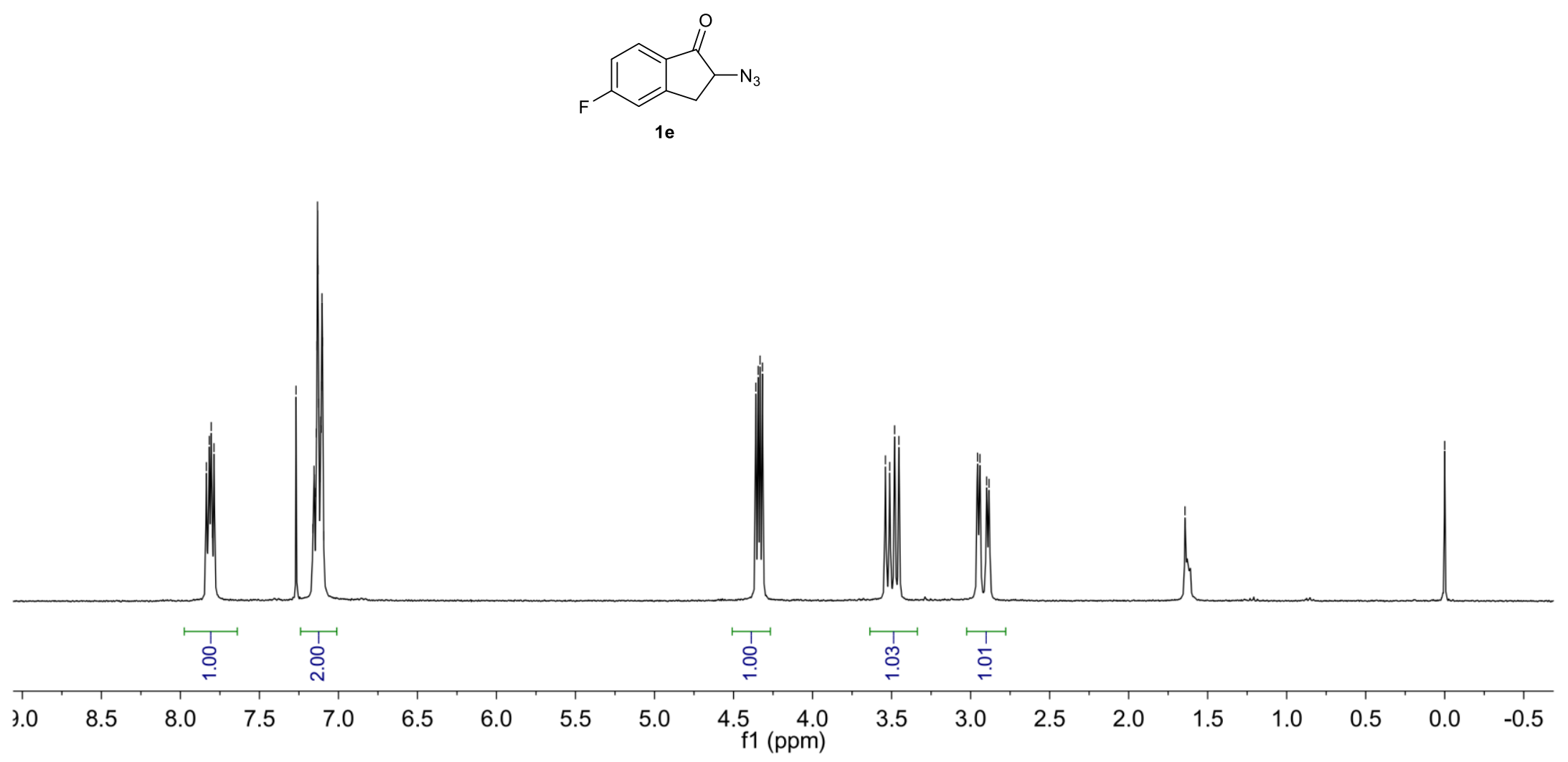
${ }^{19} \mathrm{~F}$ NMR of Compound $1 \mathrm{e}\left(282 \mathrm{MHz}, \mathrm{CDCl}_{3}\right)$

dpg-dd-47 F $282 \mathrm{MHz}$



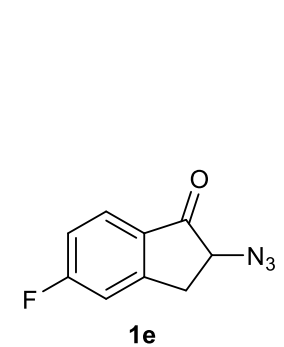

$1 \mathrm{e}$

\begin{tabular}{|c|c|c|c|c|c|c|c|c|c|c|c|c|c|c|c|}
\hline-30 & -40 & -50 & -60 & -70 & -80 & -90 & -100 & $\begin{array}{l}-110 \\
\mathrm{pm})\end{array}$ & -120 & -130 & -140 & -150 & -160 & -170 & -180 \\
\hline
\end{tabular}


${ }^{13} \mathrm{C}$ NMR of Compound 1e (100 MHz, $\left.\mathrm{CDCl}_{3}\right)$

dpg-dd-47 C $100 \mathrm{MHz}$

兽

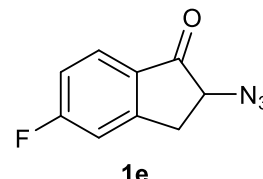

1e

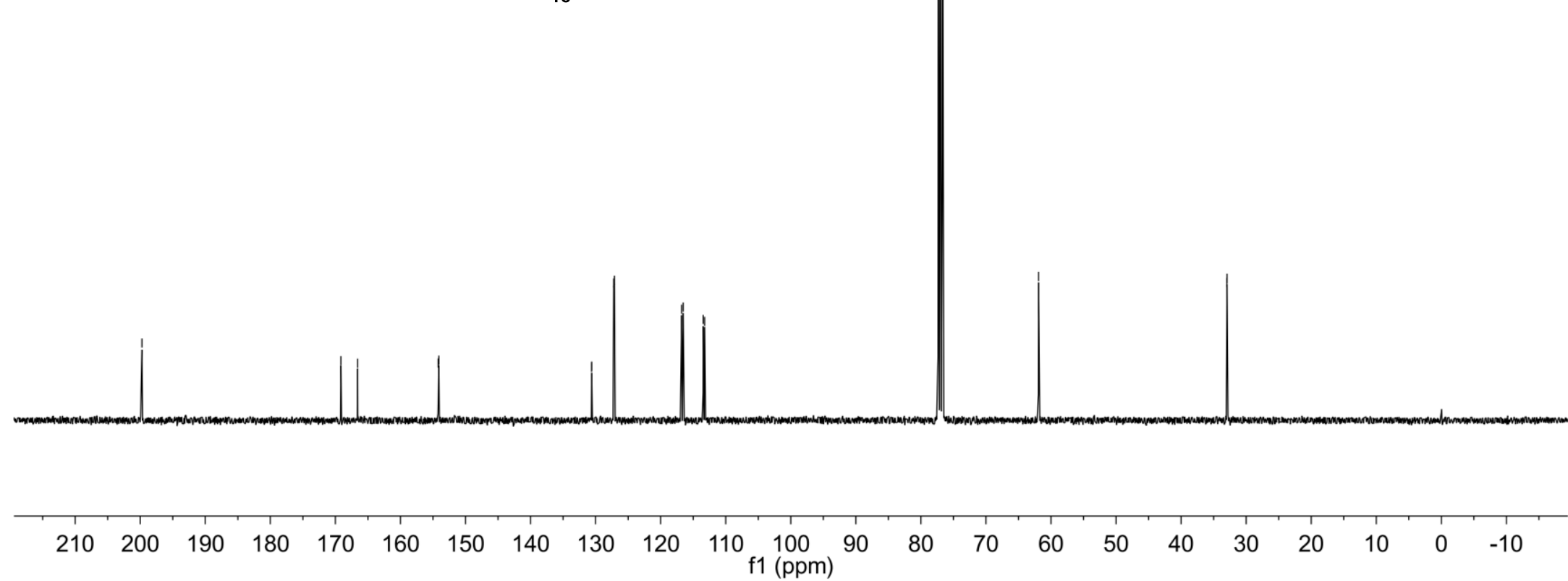


${ }^{1} \mathrm{H}$ NMR of Compound $1 \mathbf{f}\left(400 \mathrm{MHz}, \mathrm{CDCl}_{3}\right)$

DPG-DJ-41 H $400 \mathrm{MHz}$

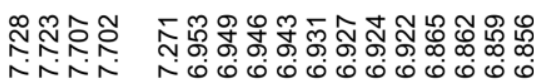

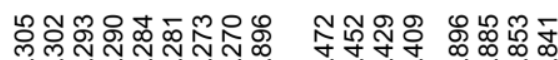

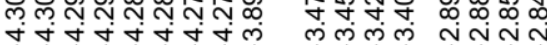

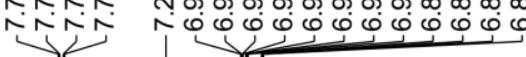

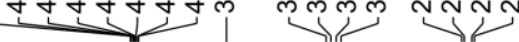

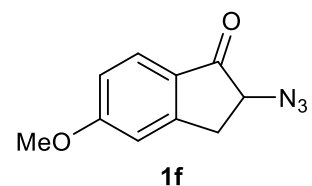

$1 f$

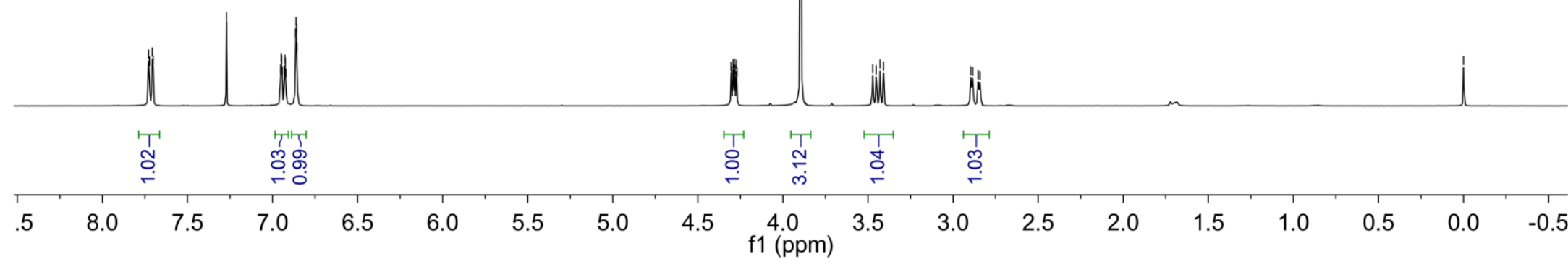


${ }^{13} \mathrm{C}$ NMR of Compound $1 f\left(100 \mathrm{MHz}, \mathrm{CDCl}_{3}\right)$

DPG-DJ-41 C $100 \mathrm{MHz}$

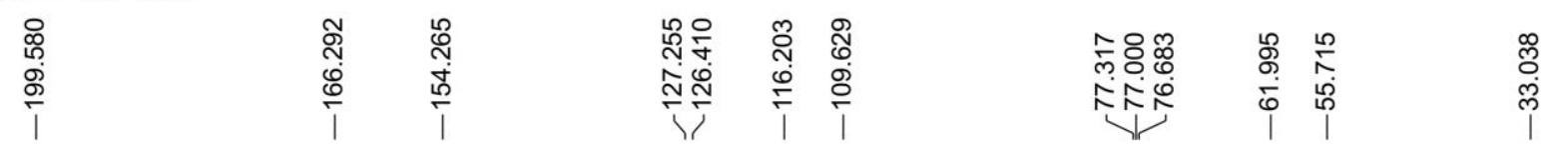

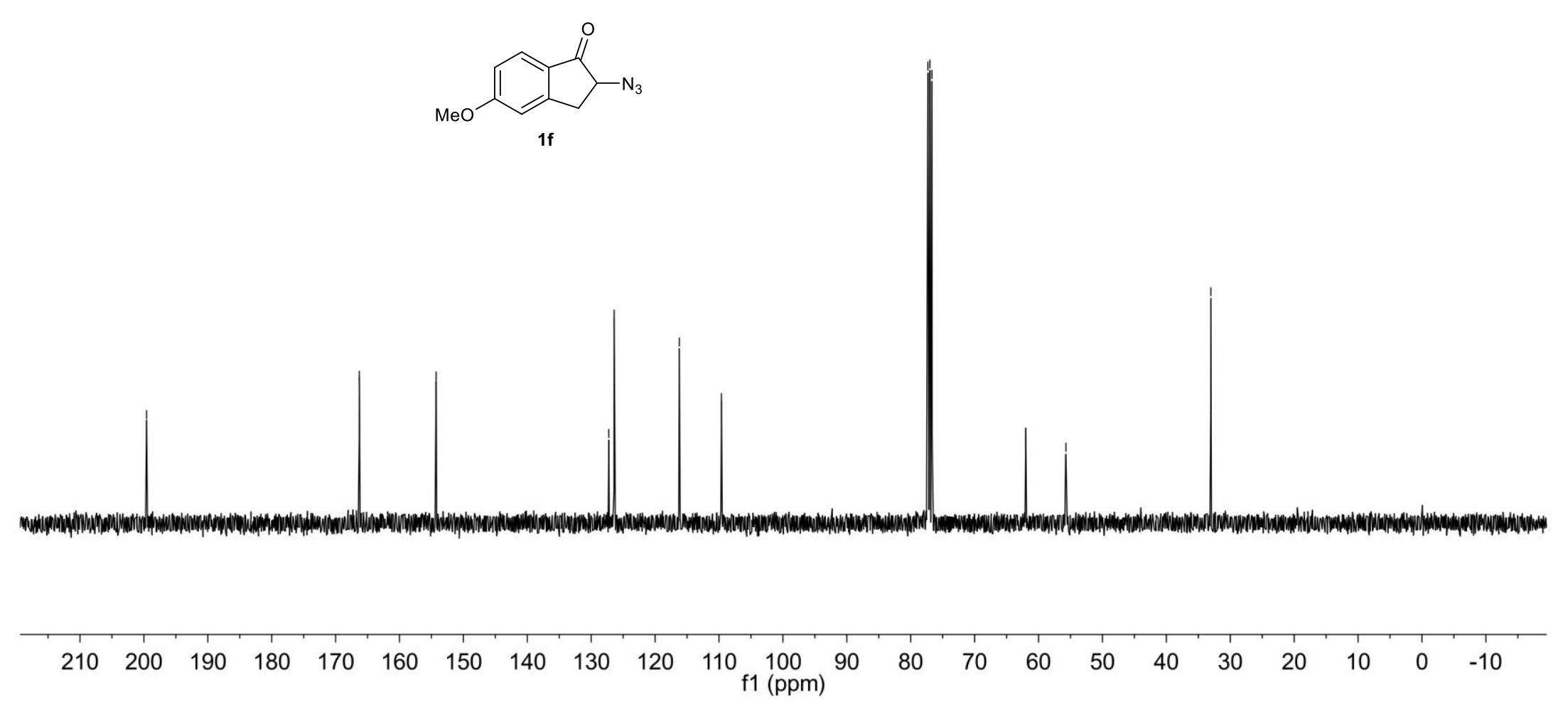


${ }^{1} \mathrm{H}$ NMR of Compound syn-3a (300 MHz, $\mathrm{CDCl}_{3}$ )

\section{DPG-DN-28-1 H $300 \mathrm{MHz}$}

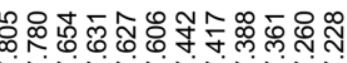

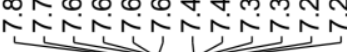

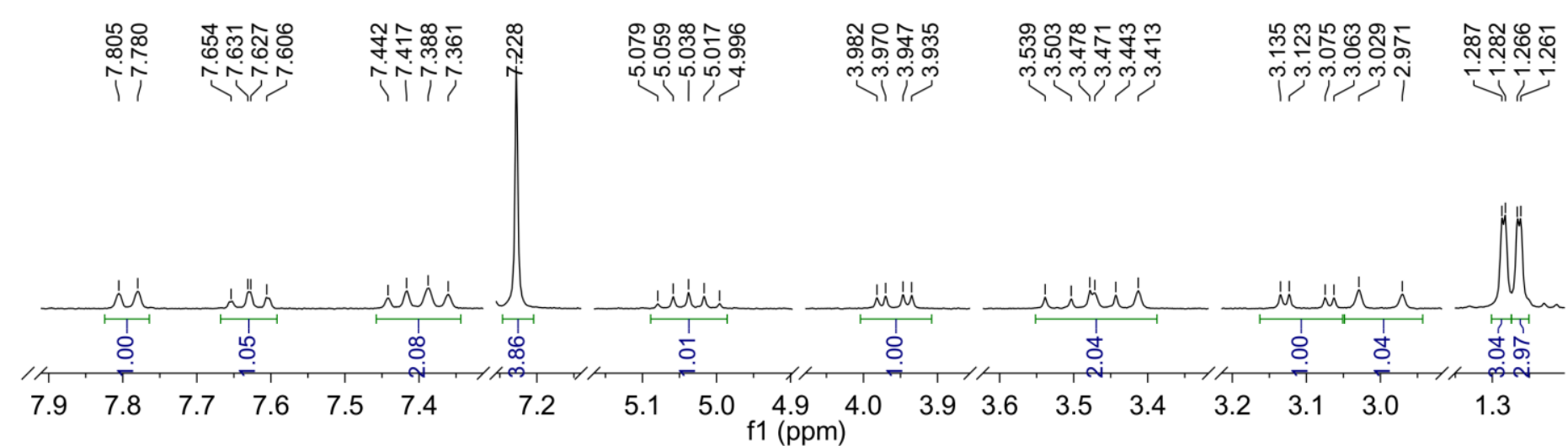

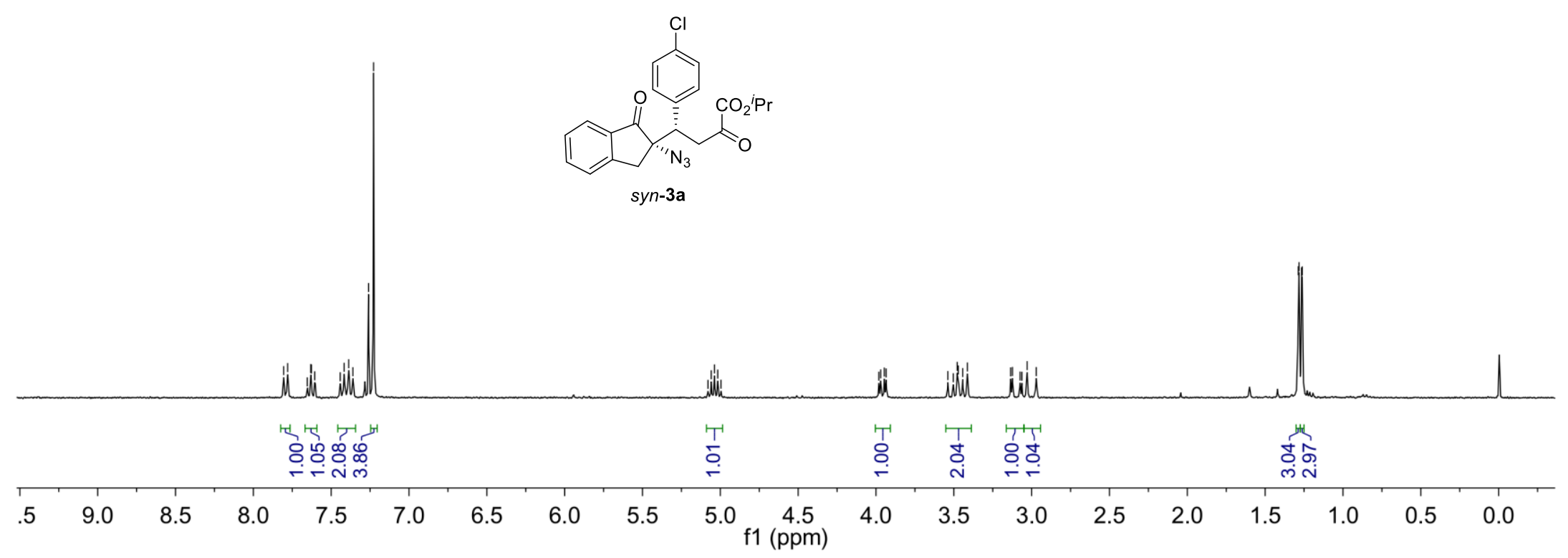


${ }^{13} \mathrm{C}$ NMR of Compound syn-3a $\left(100 \mathrm{MHz}, \mathrm{CDCl}_{3}\right)$

DPG-DN-28-1 C 100 MHz

울 ळ

8.

○

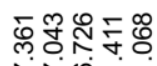

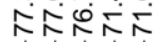

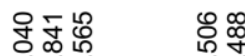

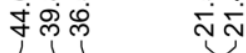
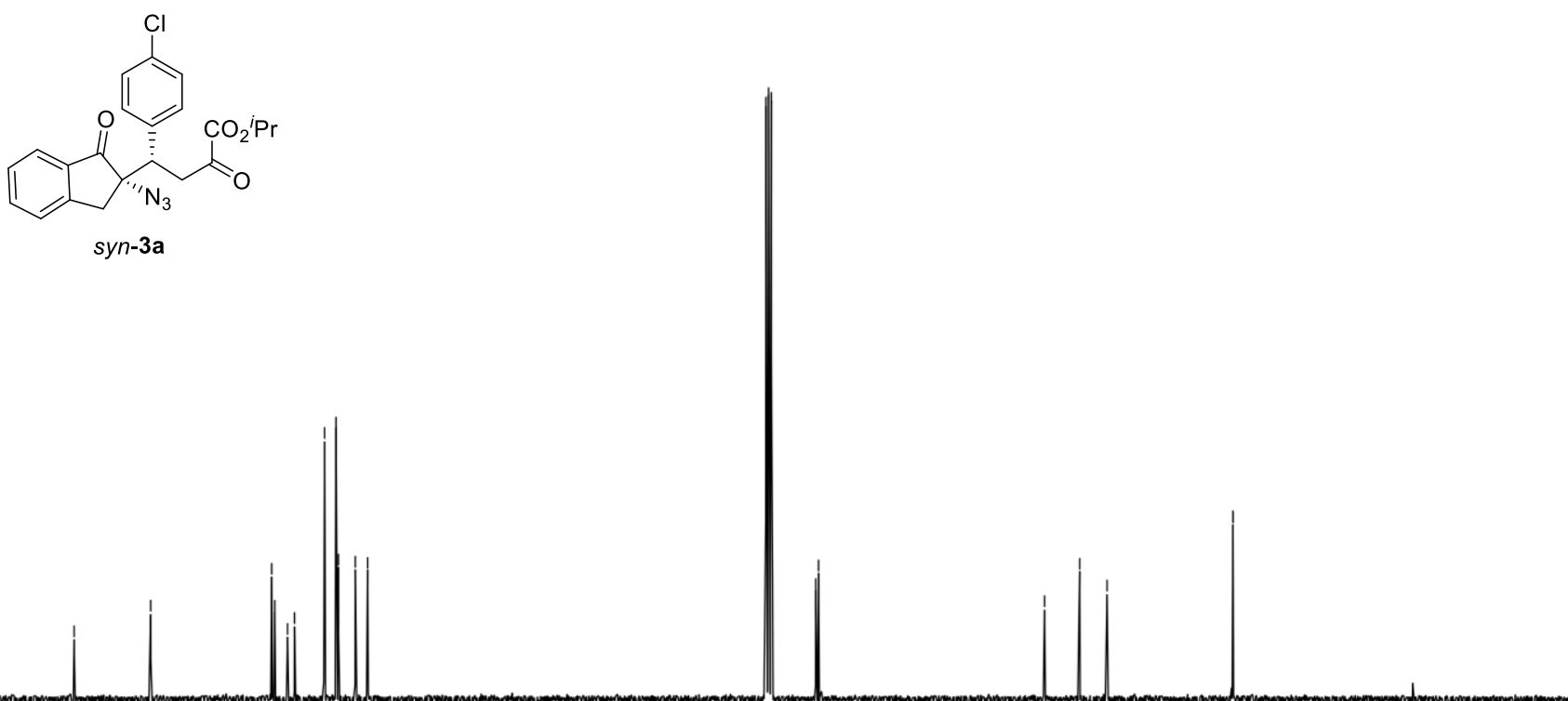

210

$\begin{array}{lllll}200 & 190 & 180 & 170 & 160\end{array}$

$150 \quad 140 \quad 130$

$\begin{array}{lll}120 & 110 \quad 100\end{array}$ f1 (ppm)

80

$70 \quad 60$

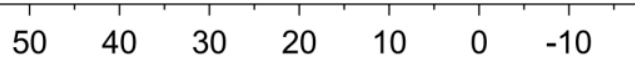


${ }^{1} \mathrm{H}$ NMR of Compound syn-3b $\left(300 \mathrm{MHz}, \mathrm{CDCl}_{3}\right)$

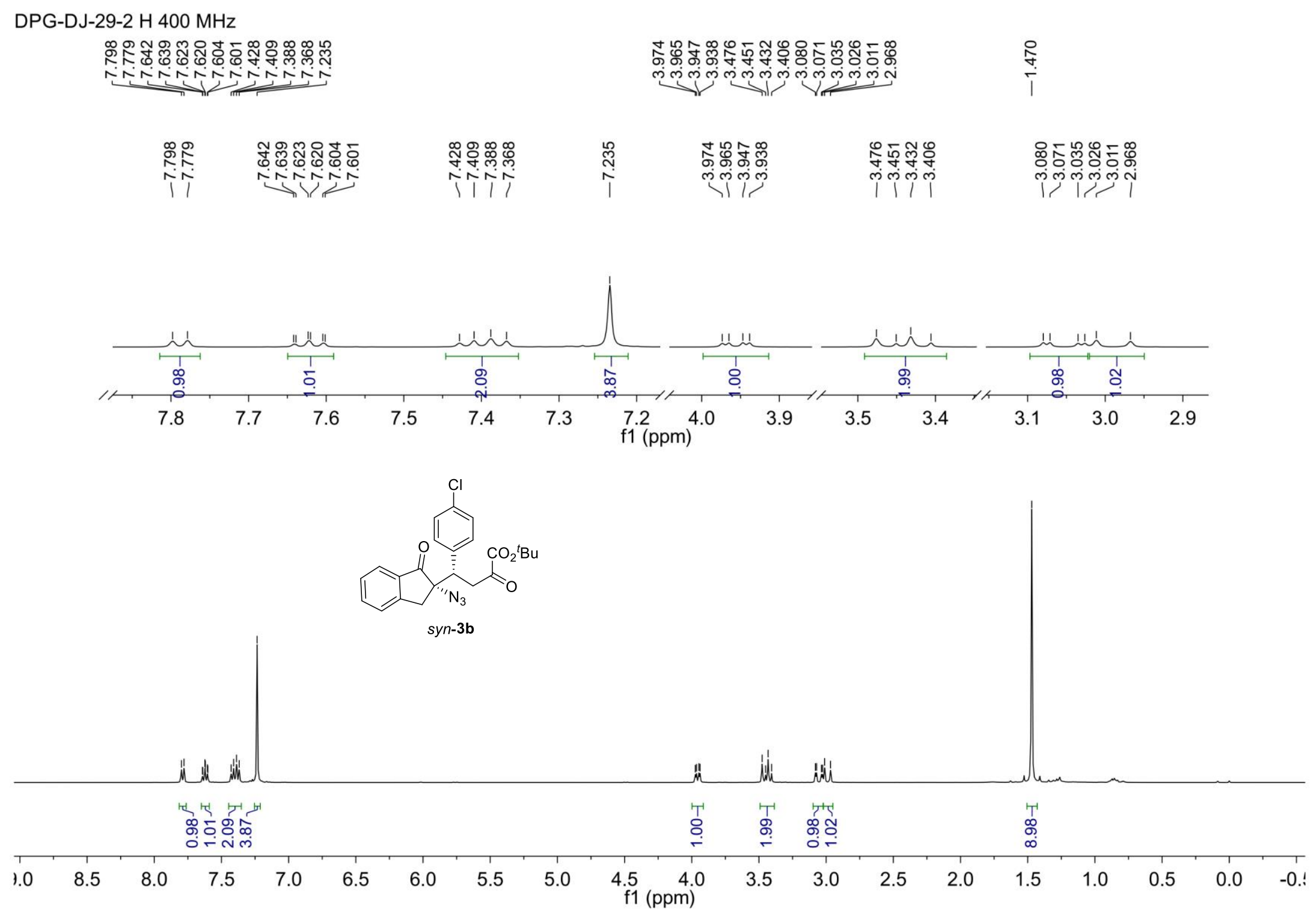


${ }^{13} \mathrm{C}$ NMR of Compound syn-3b $\left(100 \mathrm{MHz}, \mathrm{CDCl}_{3}\right)$

DPG-DJ-29-2 C $100 \mathrm{MHz}$

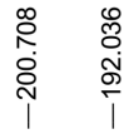

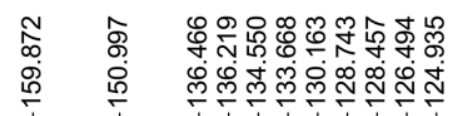

吕

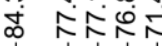

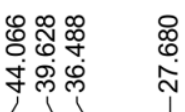
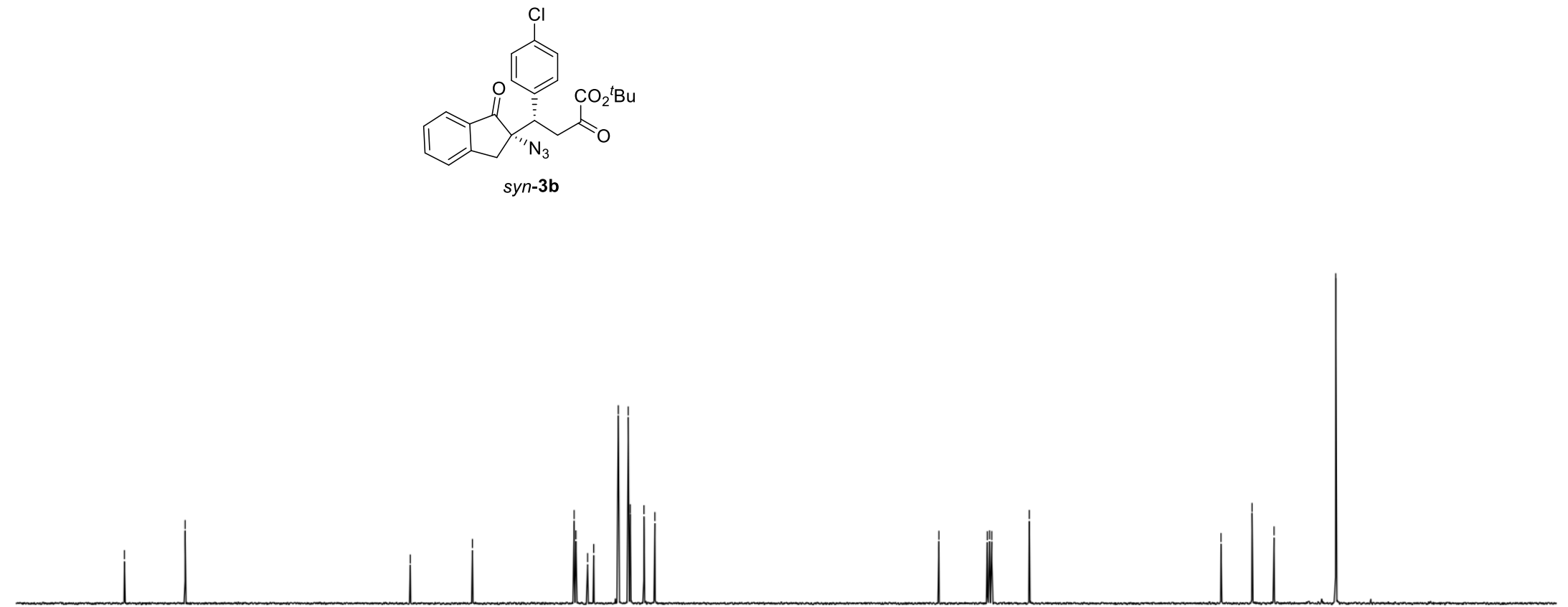

$210 \quad 200 \quad 190$

$\begin{array}{llll}80 & 170 & 160 & 150\end{array}$

$140 \quad 130$

120

110
$\mathrm{f} 1(\mathrm{ppm})$ 
${ }^{1} \mathrm{H}$ NMR of Compound syn-3c $\left(300 \mathrm{MHz}, \mathrm{CDCl}_{3}\right)$
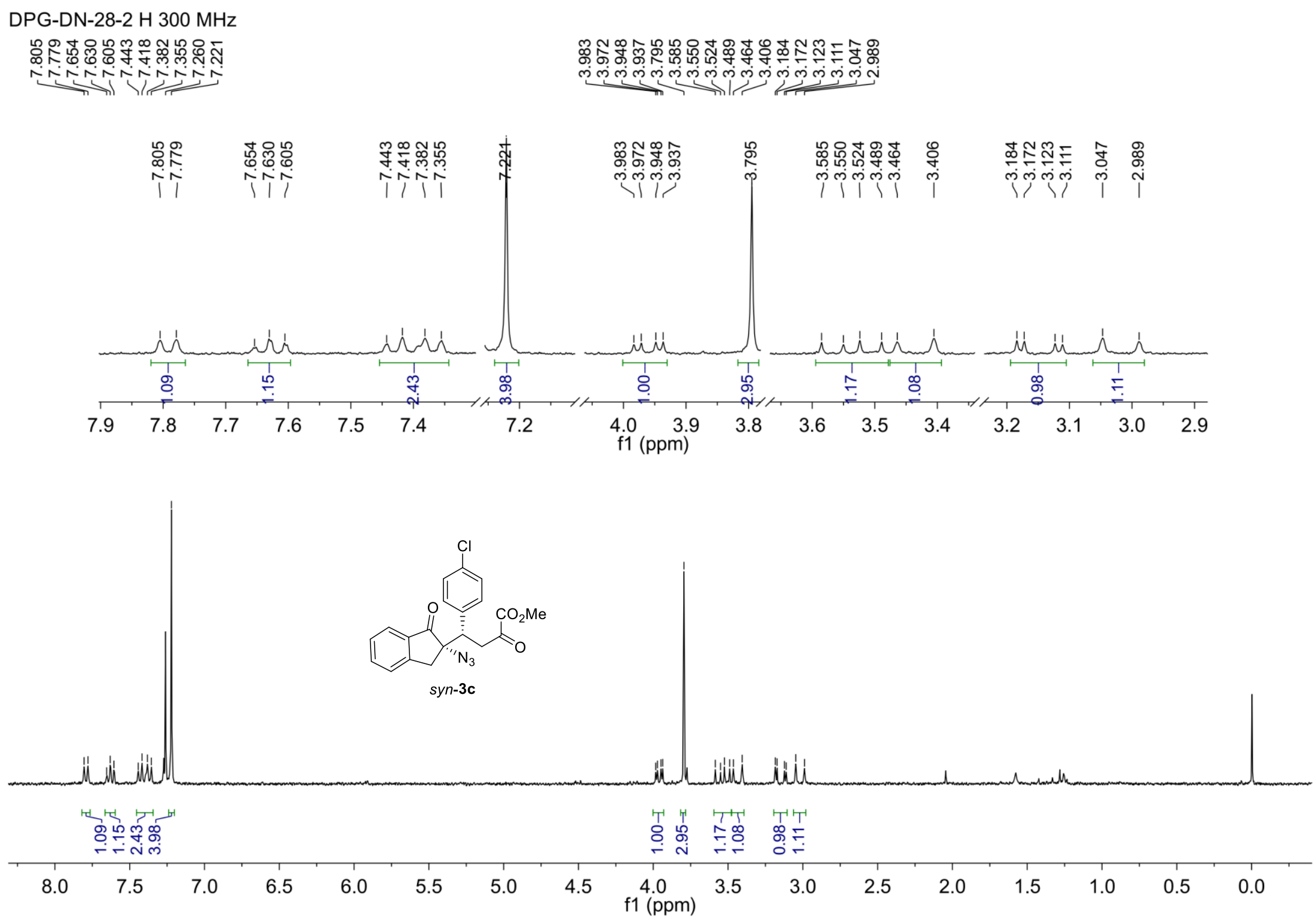
${ }^{13} \mathrm{C}$ NMR of Compound syn-3c $\left(100 \mathrm{MHz}, \mathrm{CDCl}_{3}\right)$

DPG-DN-28-2 C $100 \mathrm{MHz}$

울 ڤ્ల

œ

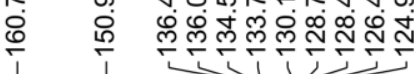

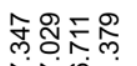

रतिi

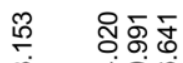

角 迎赤
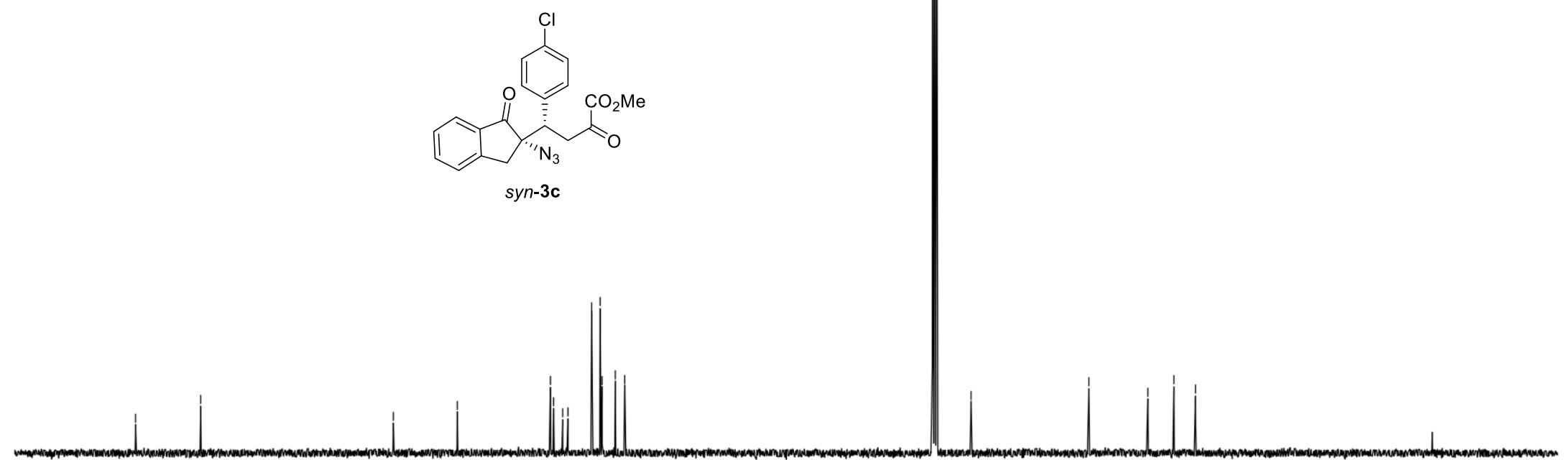

$\begin{array}{lllll}210 & 200 \quad 190 \quad 180 \quad 170 \quad 160\end{array}$

$\begin{array}{llll}150 & 140 \quad 130 \quad 120\end{array}$ $\stackrel{100}{\mathrm{f} 1(\mathrm{ppm})}$

80

$70 \quad 60$

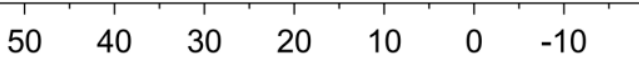


${ }^{1} \mathrm{H}$ NMR of Compound syn-3d $\left(400 \mathrm{MHz}, \mathrm{CDCl}_{3}\right)$

DPG-DJ-80-1 H $400 \mathrm{MHz}$

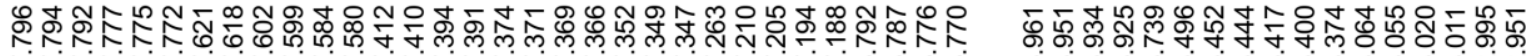

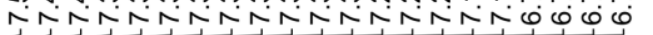

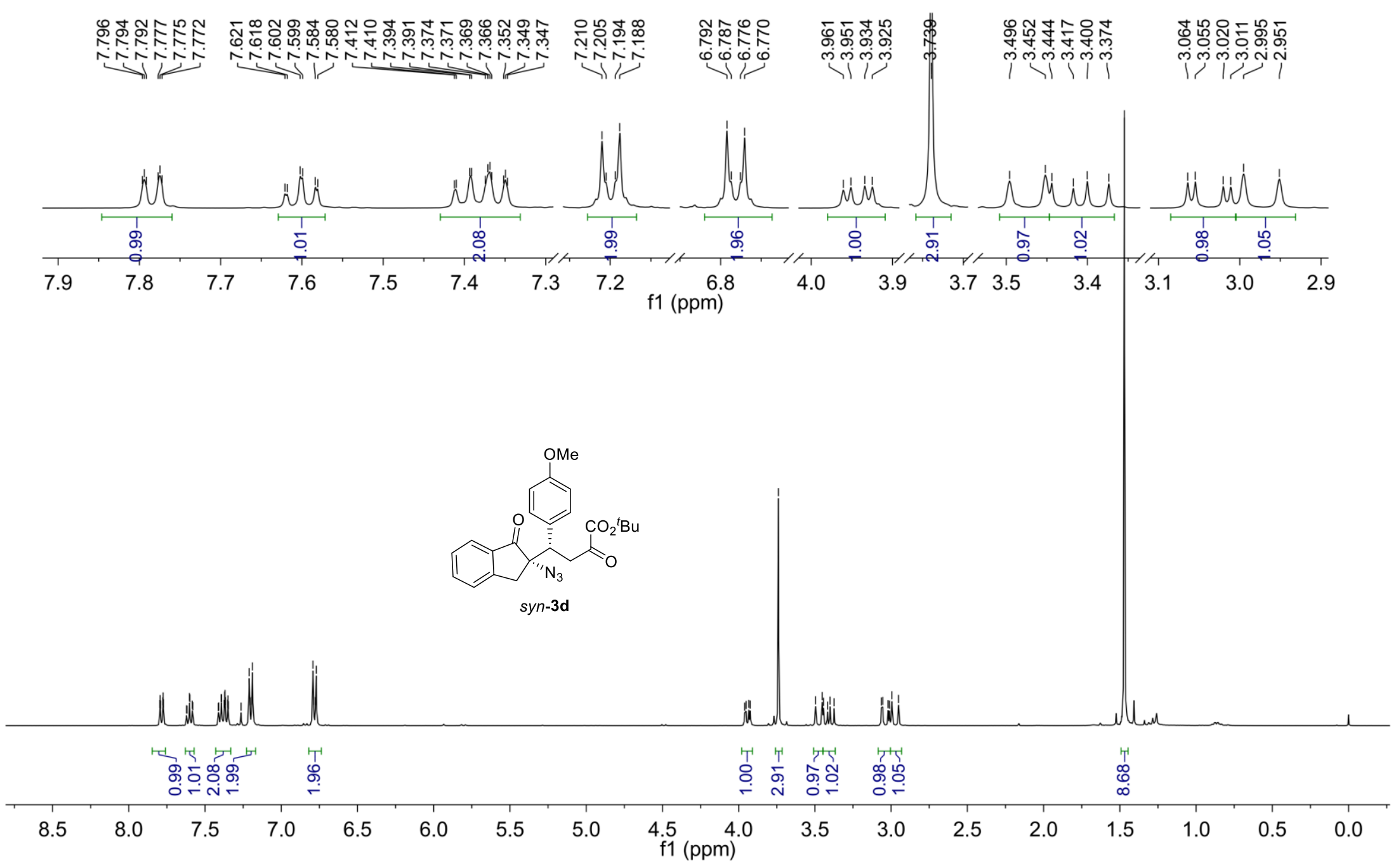


${ }^{13} \mathrm{C}$ NMR of Compound syn-3d $\left(100 \mathrm{MHz}, \mathrm{CDCl}_{3}\right)$

DPG-DJ-80-1 C 100 MHz

ণ্ণั)

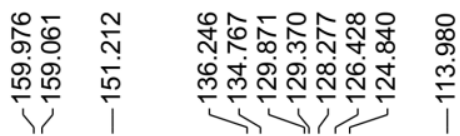

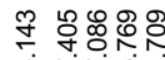

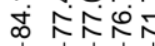

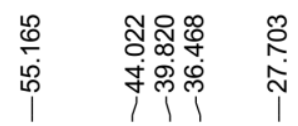

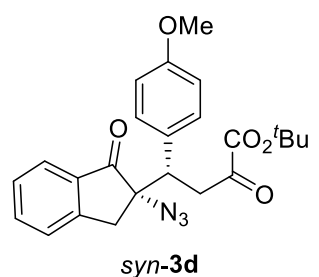

210

$\begin{array}{lllll}200 & 190 & 180 & 170 & 160\end{array}$

$150 \quad 140 \quad 130$

120

$\stackrel{100}{\mathrm{f} 1}(\mathrm{ppm})$

80

$60 \quad 50$

$50 \quad 40$

30

$20 \quad 10$

$0 \quad-10$ 
${ }^{1} \mathrm{H}$ NMR of Compound syn-3e (400 MHz, $\left.\mathrm{CDCl}_{3}\right)$

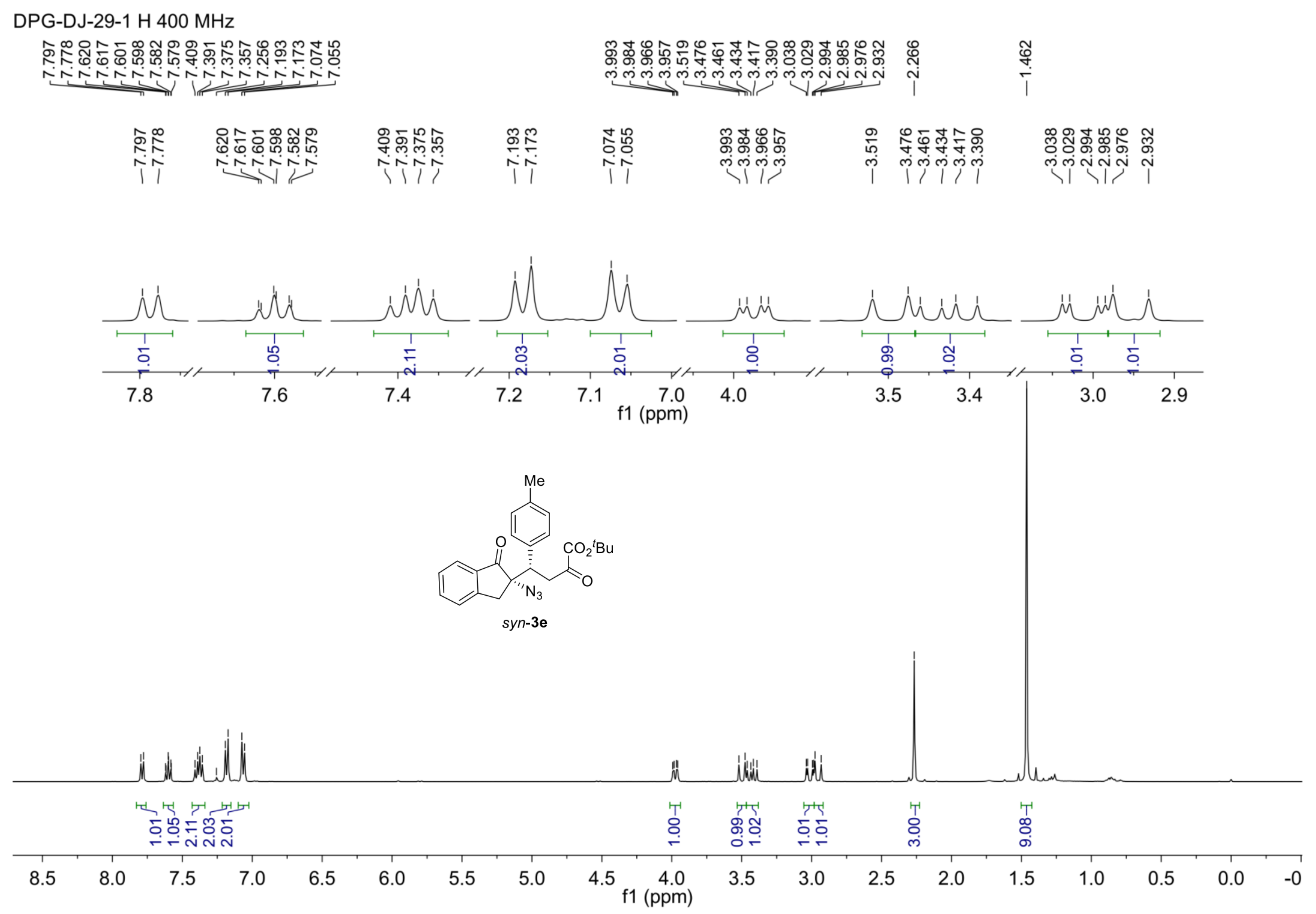


${ }^{13} \mathrm{C}$ NMR of Compound syn-3e (100 MHz, $\left.\mathrm{CDCl}_{3}\right)$
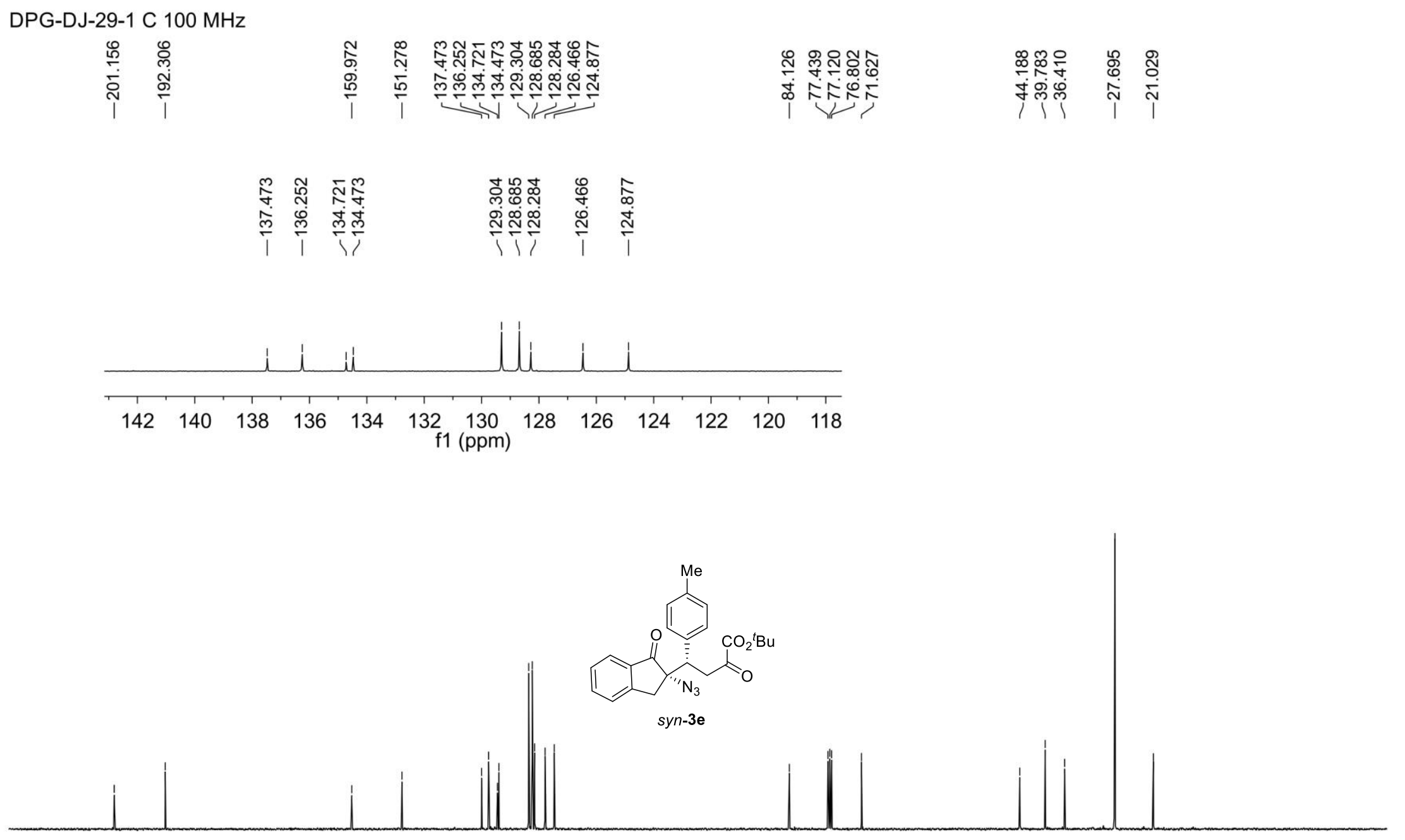

$\begin{array}{lllllllllllllllllllllll}210 & 200 & 190 & 180 & 170 & 160 & 150 & 140 & 130 & 120 & 110 & \begin{array}{c}100 \\ \mathrm{f} 1(\mathrm{ppm})\end{array} & 90 & 80 & 70 & 60 & 50 & 40 & 30 & 20 & 10 & 0 & -10\end{array}$ 
${ }^{1} \mathrm{H}$ NMR of Compound syn-3f $\left(400 \mathrm{MHz}, \mathrm{CDCl}_{3}\right)$

\section{DPG-DJ-80-3 H $400 \mathrm{MHz}$}

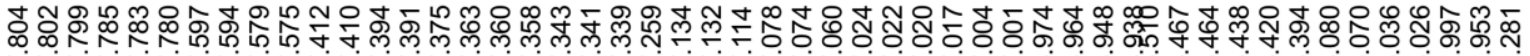

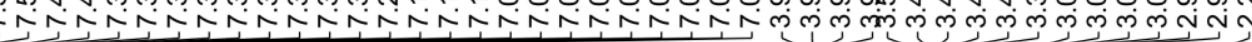

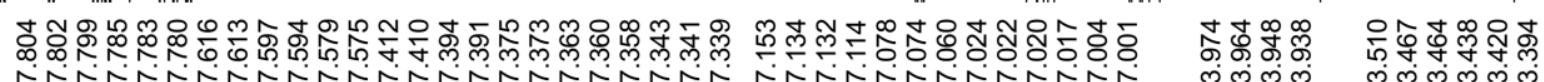

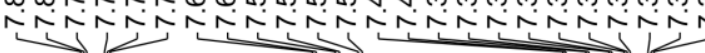

NiñNiñN minm
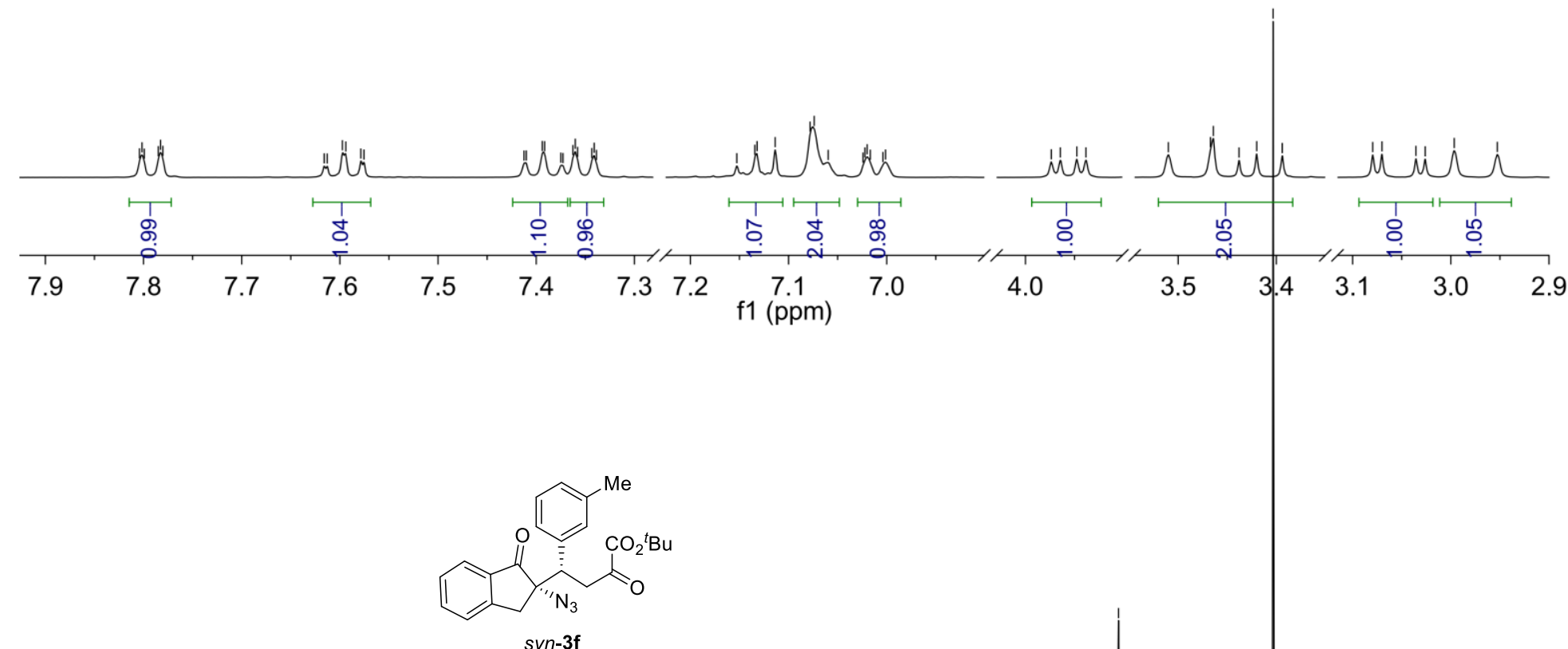

syn-3f

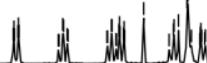
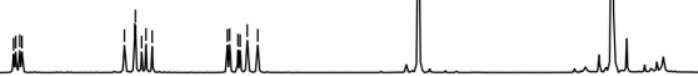

\begin{tabular}{|c|c|c|c|c|c|c|c|c|c|c|c|c|c|c|c|c|c|c|}
\hline & & 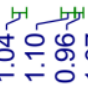 & 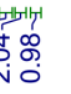 & & & & & & $\begin{array}{l}\text { 'T } \\
\stackrel{8}{\circ}\end{array}$ & 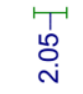 & 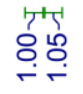 & & & 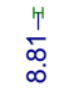 & & & & \\
\hline 8.5 & 8.0 & 7.5 & 7.0 & 6.5 & 6.0 & 5.5 & 5.0 & 4.5 & $\begin{array}{c}4.0 \\
\mathrm{f} 1(\mathrm{ppm})\end{array}$ & 3.5 & 3.0 & 2.5 & 2.0 & 1.5 & 1.0 & 0.5 & 0.0 & -0 . \\
\hline
\end{tabular}


${ }^{13} \mathrm{C}$ NMR of Compound syn-3f $\left(100 \mathrm{MHz}, \mathrm{CDCl}_{3}\right)$

DPG-DJ-80-3 C $100 \mathrm{MHz}$

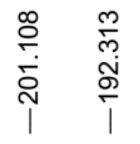

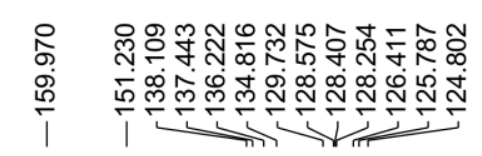

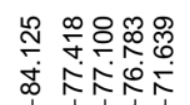

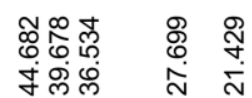

穴
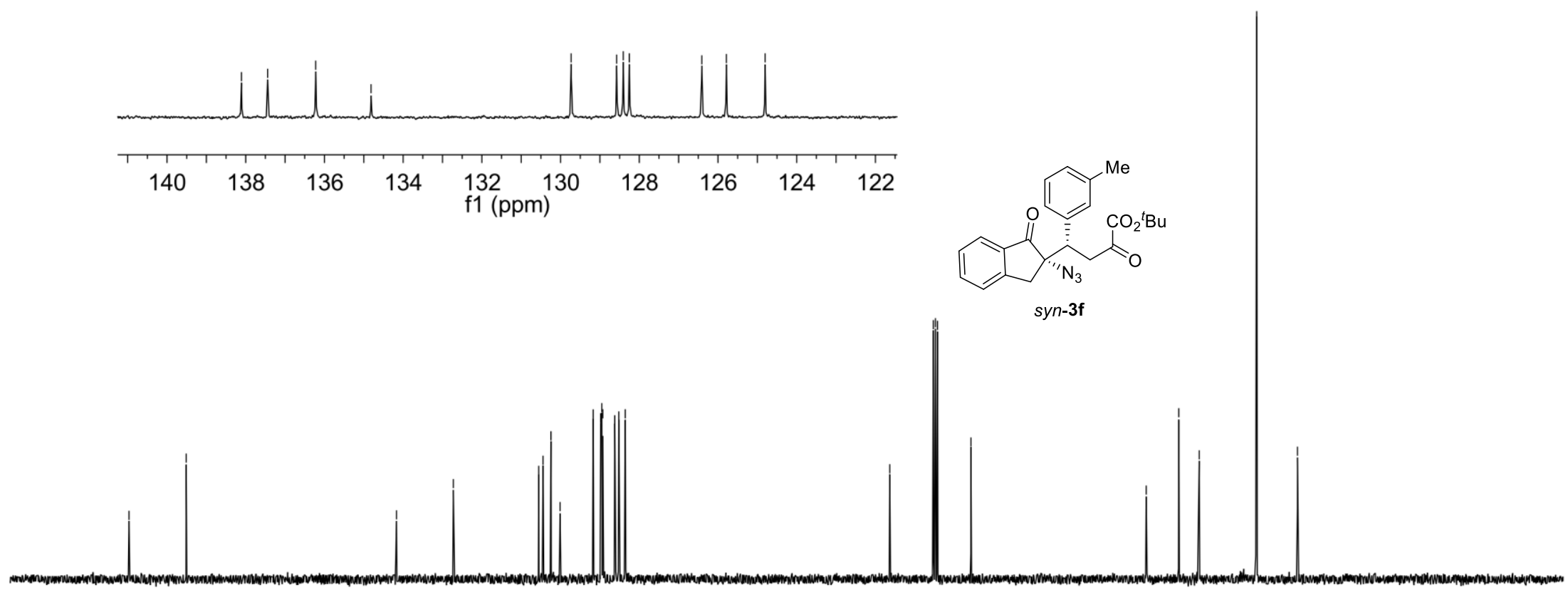

$210 \quad 200 \quad 190$

$\begin{array}{llll}80 & 170 & 160 & 150\end{array}$

$\begin{array}{lllll}130 & 120 & 110 & 100\end{array}$ f1 (ppm)

80

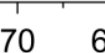

$60 \quad 50$

$40 \quad 30 \quad 20$

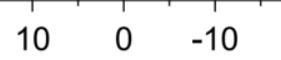


${ }^{1} \mathrm{H}$ NMR of Compound syn-3g (300 MHz, $\left.\mathrm{CDCl}_{3}\right)$

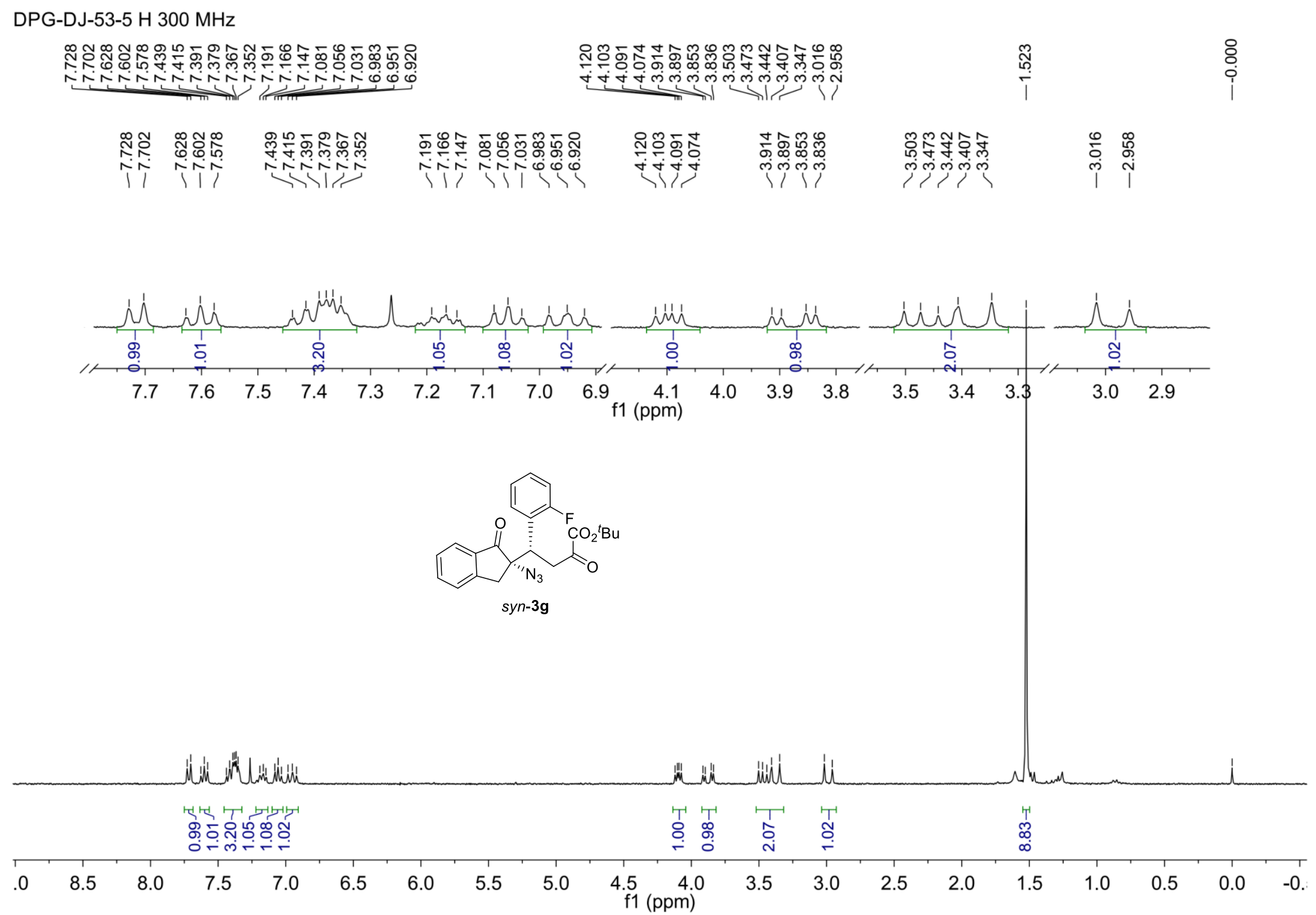


${ }^{19} \mathrm{~F}$ NMR of Compound syn-3g $\left(282 \mathrm{MHz}, \mathrm{CDCl}_{3}\right)$

DPG-DJ-53-5 F $282 \mathrm{MHz}$
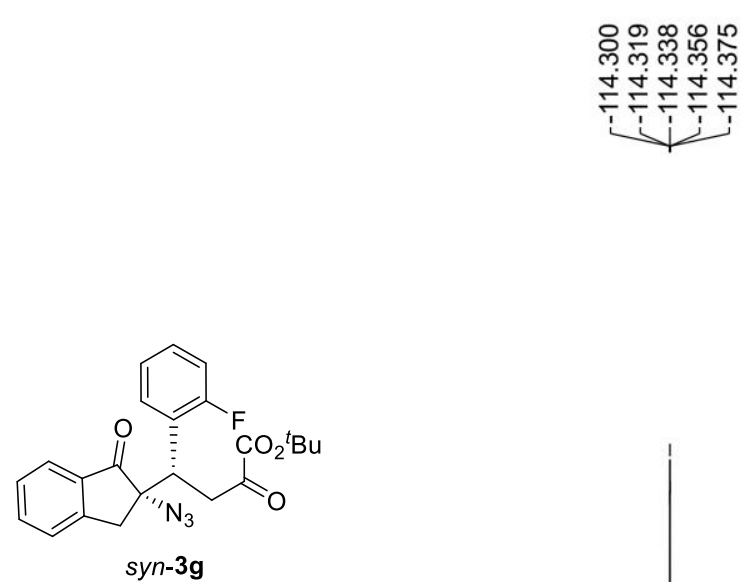

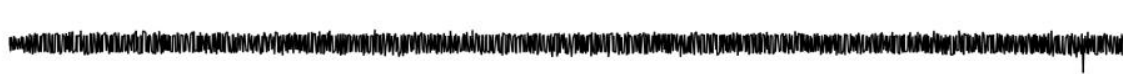

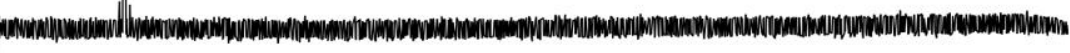

$\begin{array}{lllllllllllllllllllllllllllllllll}10 & 0 & -10 & -20 & -30 & -40 & -50 & -60 & -70 & -80 & -90 & -100 & -110 & -120 & -130 & -140 & -150 & -160 & -170 & -180 & -190 & -200 & -210\end{array}$


${ }^{13} \mathrm{C}$ NMR of Compound syn-3g (125 MHz, $\left.\mathrm{CDCl}_{3}\right)$

dpg-dn-74 C 125MHz

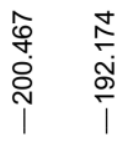

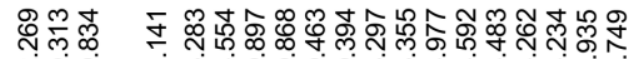

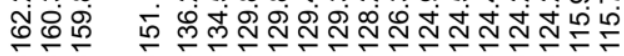

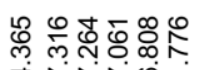

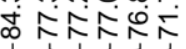

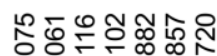

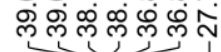

管।

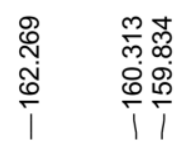

$\frac{\frac{5}{5}}{\frac{5}{5}}$

i⿱

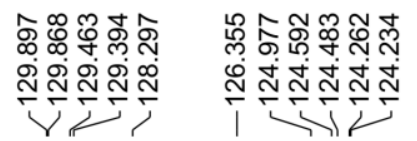

婴昌
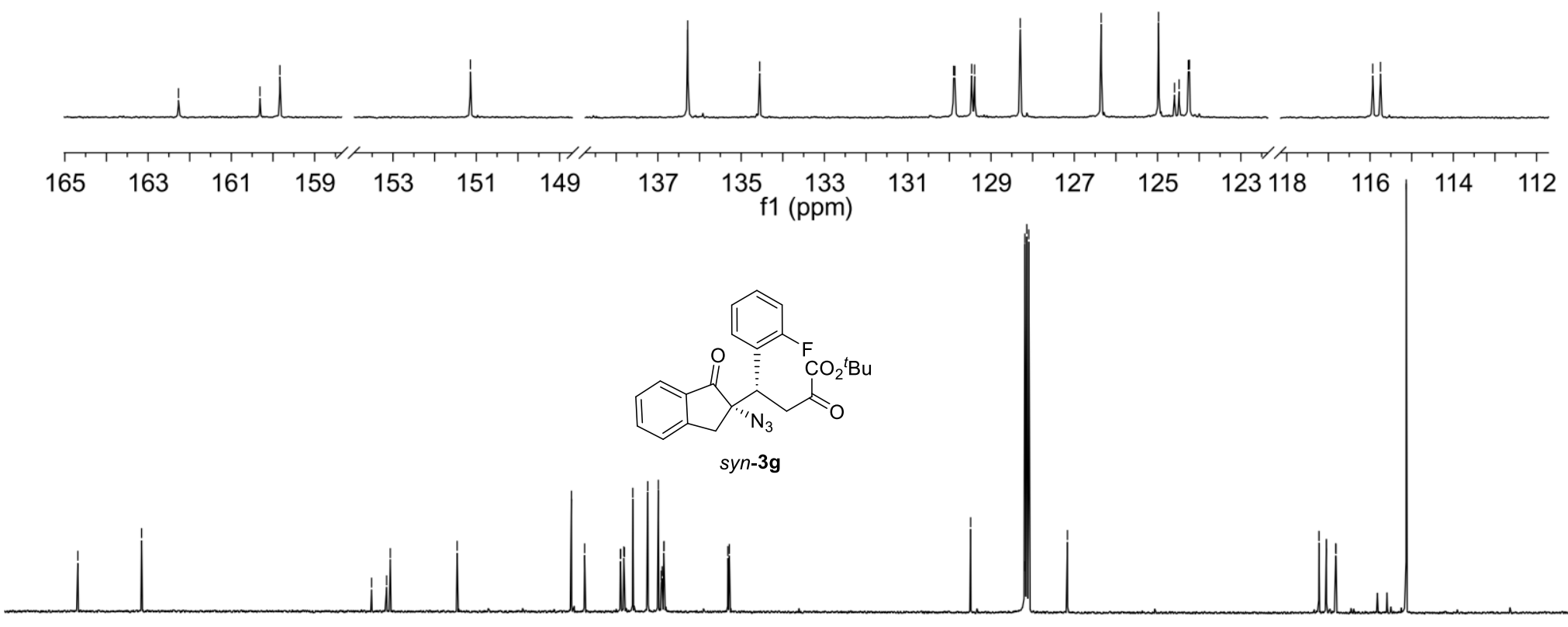

$10 \quad 200$

$190 \quad 180$

$170 \quad 160$

$\begin{array}{llll}150 & 140 & 130 & 120\end{array}$

$\begin{array}{lll}120 & 110 \quad 100\end{array}$

$90 \quad 80$

$70 \quad 60$ f1 $(\mathrm{ppm})$ 
${ }^{1} \mathrm{H}$ NMR of Compound syn-3h $\left(400 \mathrm{MHz}, \mathrm{CDCl}_{3}\right)$

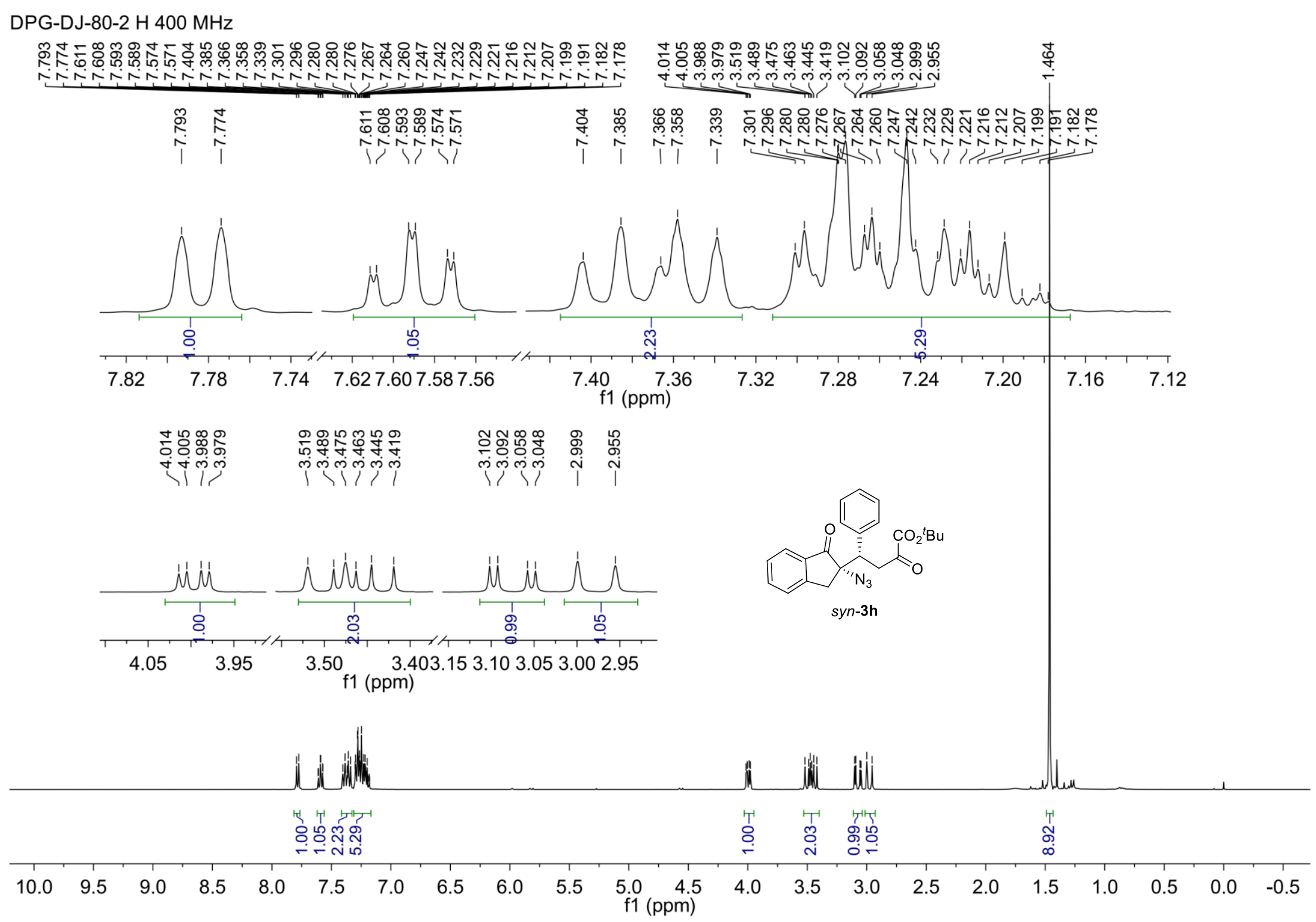


${ }^{13} \mathrm{C}$ NMR of Compound syn-3h $\left(100 \mathrm{MHz}, \mathrm{CDCl}_{3}\right)$
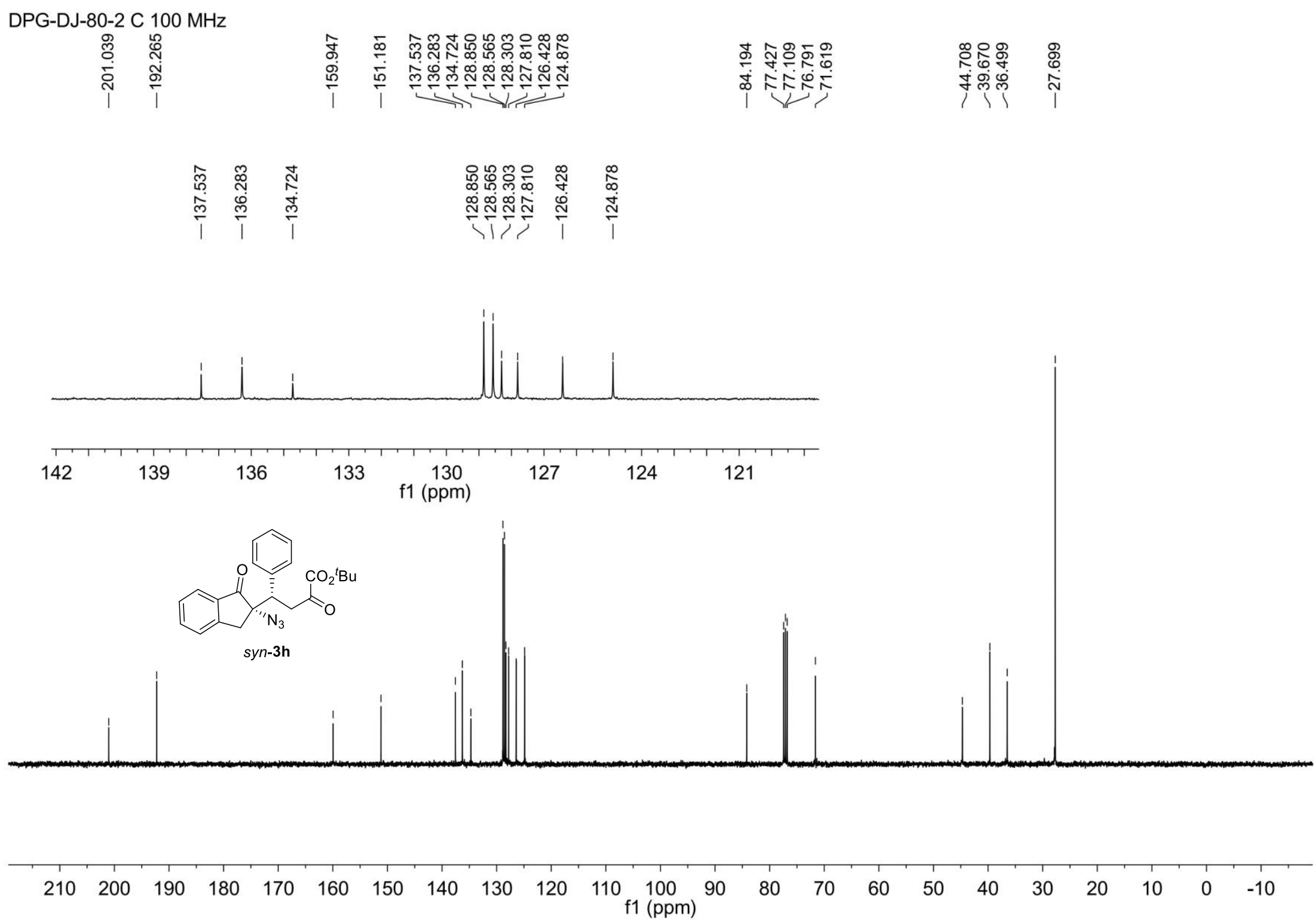
${ }^{1} \mathrm{H}$ NMR of Compound syn-3i (400 MHz, $\left.\mathrm{CDCl}_{3}\right)$

DPG-DJ-53-2 H $400 \mathrm{HMz}$

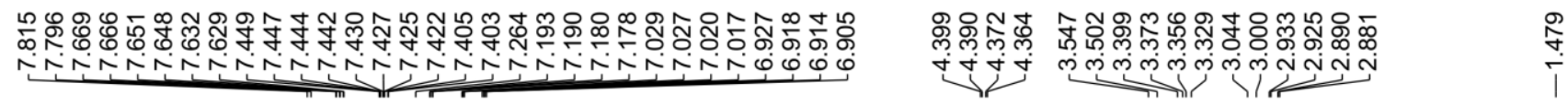
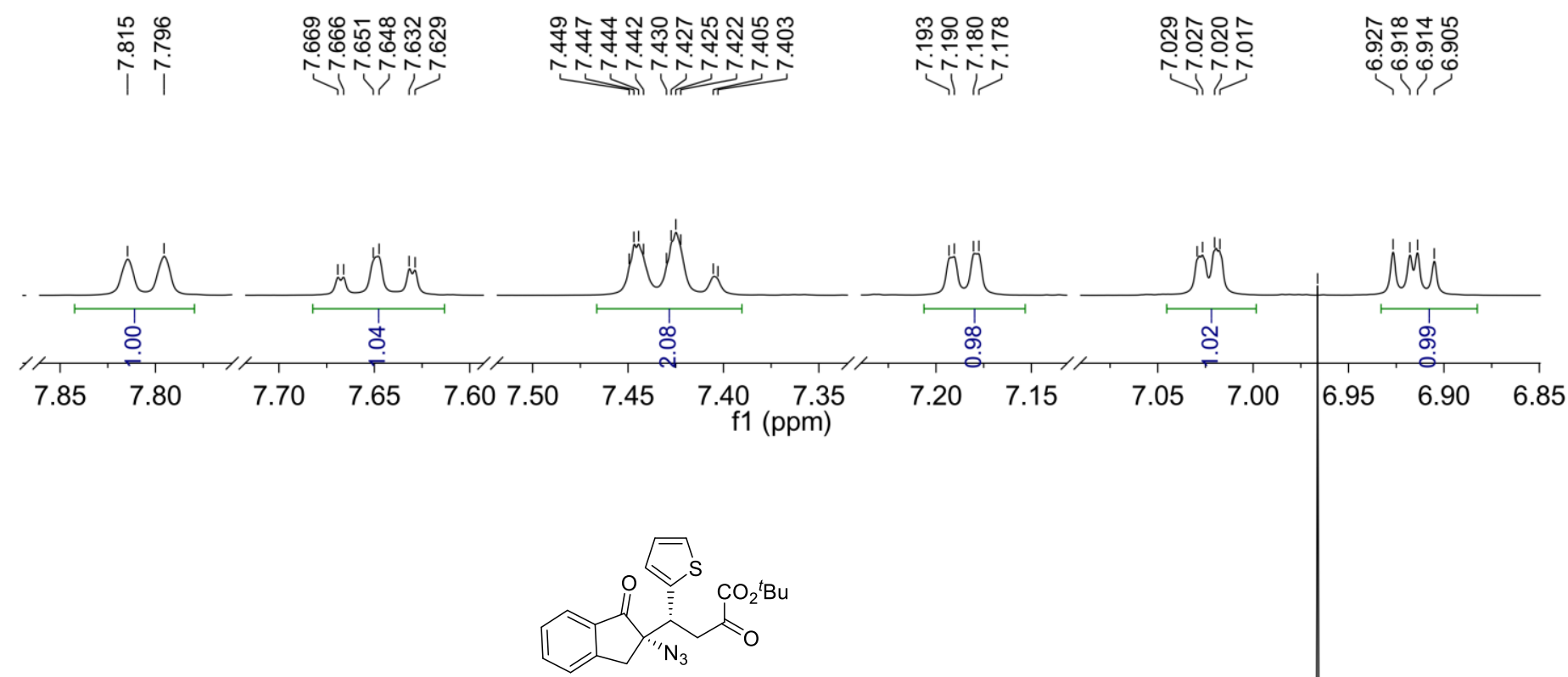

syn-3i

ubill

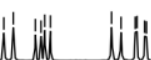

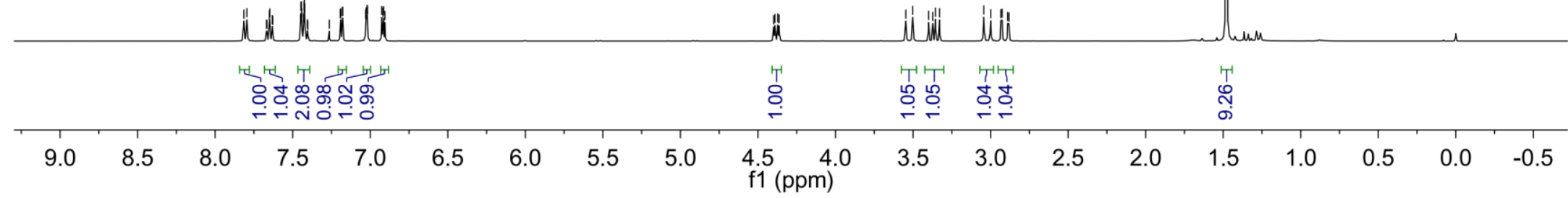


${ }^{13} \mathrm{C}$ NMR of Compound syn-3i $\left(100 \mathrm{MHz}, \mathrm{CDCl}_{3}\right)$

DPG-DJ-53-2 C $100 \mathrm{HMz}$

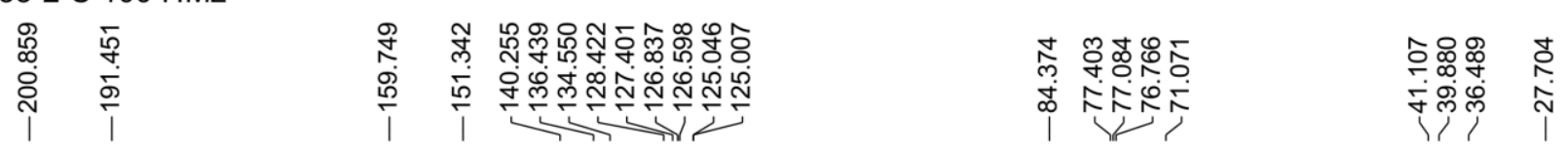
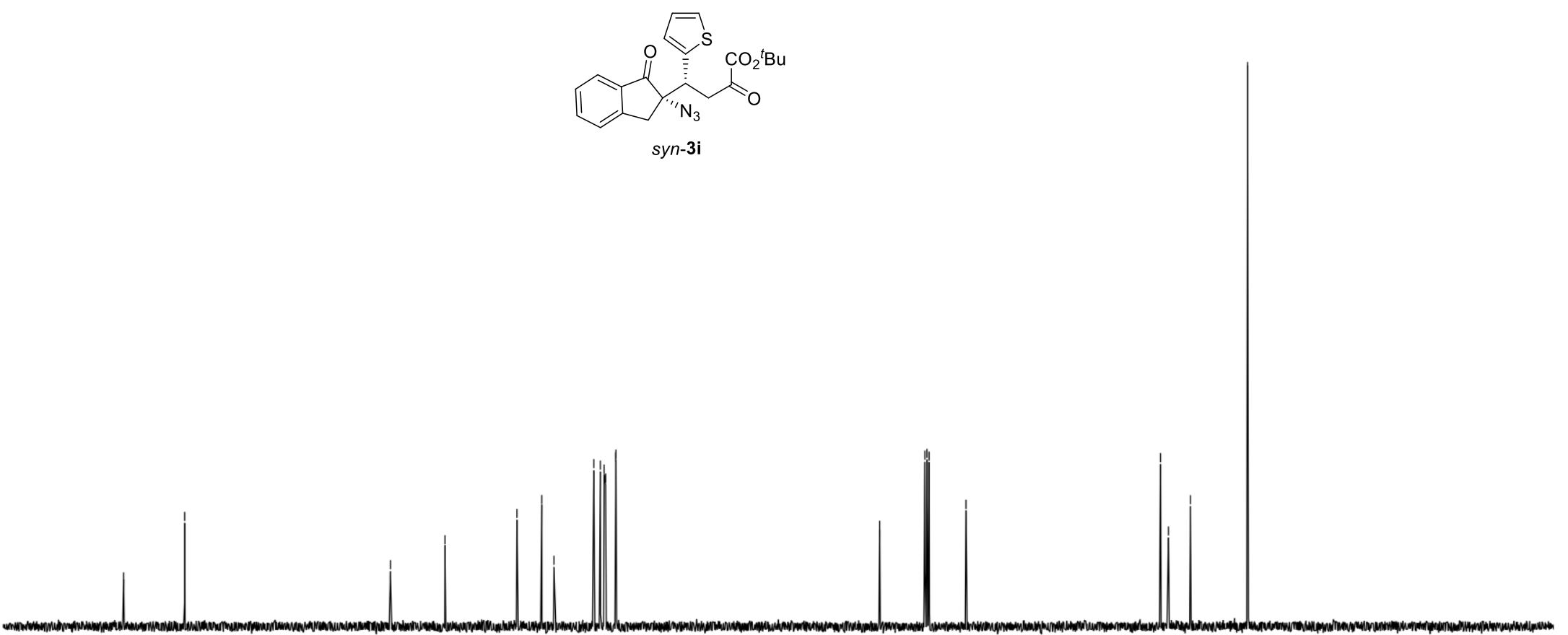

$\begin{array}{lllllllllllllllllllllll}210 & 200 & 190 & 180 & 170 & 160 & 150 & 140 & 130 & 120 & 110 & \begin{array}{l}100 \\ \mathrm{f} 1(\mathrm{ppm})\end{array} & 90 & 80 & 70 & 60 & 50 & 40 & 30 & 20 & 10 & 0 & -10\end{array}$ 
${ }^{1} \mathrm{H}$ NMR of Compound syn-3j (400 MHz, $\mathrm{CDCl}_{3}$ )

\section{DPG-DJ-53-3-A H $400 \mathrm{MHz}$

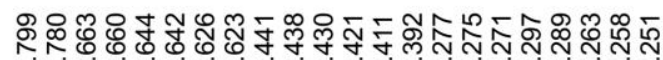

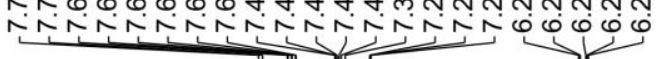

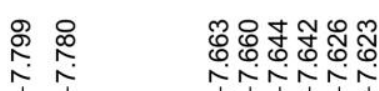

î

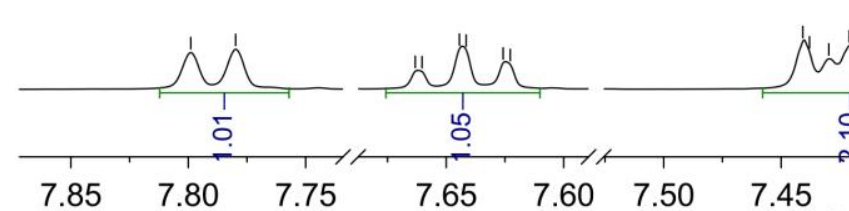

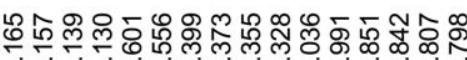
ن̛ं̛

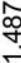

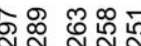

iा il

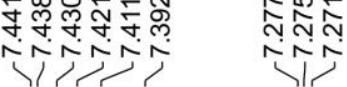

ili

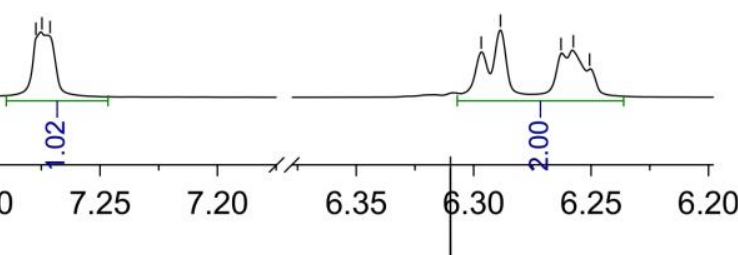

syn-3j

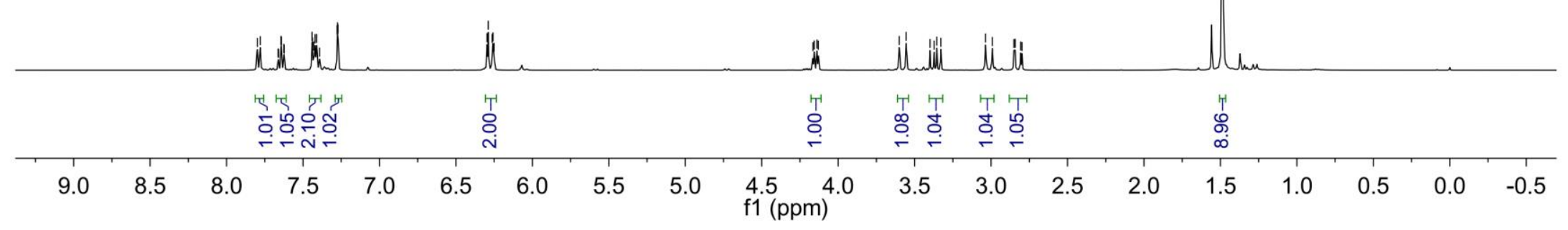


${ }^{13} \mathrm{C}$ NMR of Compound syn-3j (100 MHz, $\left.\mathrm{CDCl}_{3}\right)$

DPG-DJ-53-3-A C $100 \mathrm{MHz}$

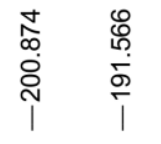

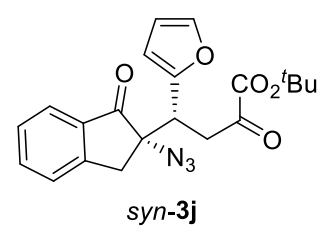

$210 \quad 200$

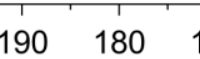

170160

150

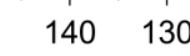

120

$110 \quad 100$

$90 \quad 80$

$70 \quad 60$

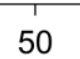

$\begin{array}{lllll}40 & 30 & 20 & 10 & 0\end{array}$ 
${ }^{1} \mathrm{H}$ NMR of Compound syn-3k $\left(400 \mathrm{MHz}, \mathrm{CDCl}_{3}\right)$

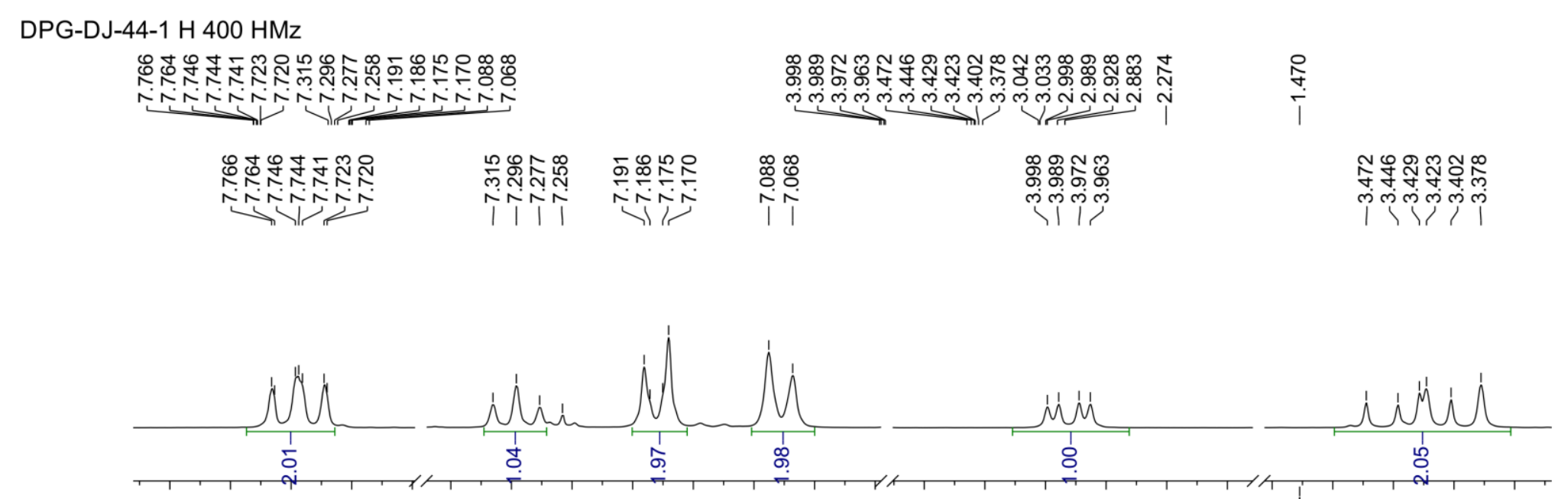

\begin{tabular}{llllllllllllllllll|ll|l|l|l|l|l|l}
7.85 & 7.80 & 7.75 & 7.70 & 7.65 .35 & 7.30 & 7.25 & 7.20 & 7.15 & 7.10 & 7.05 & 7.004 .10 & 4.05 & 4.00 & 3.95 & 3.90 & 3.853 .55 & 3.50 & 3.45 & 3.40 & 3.35
\end{tabular} f1 (ppm)

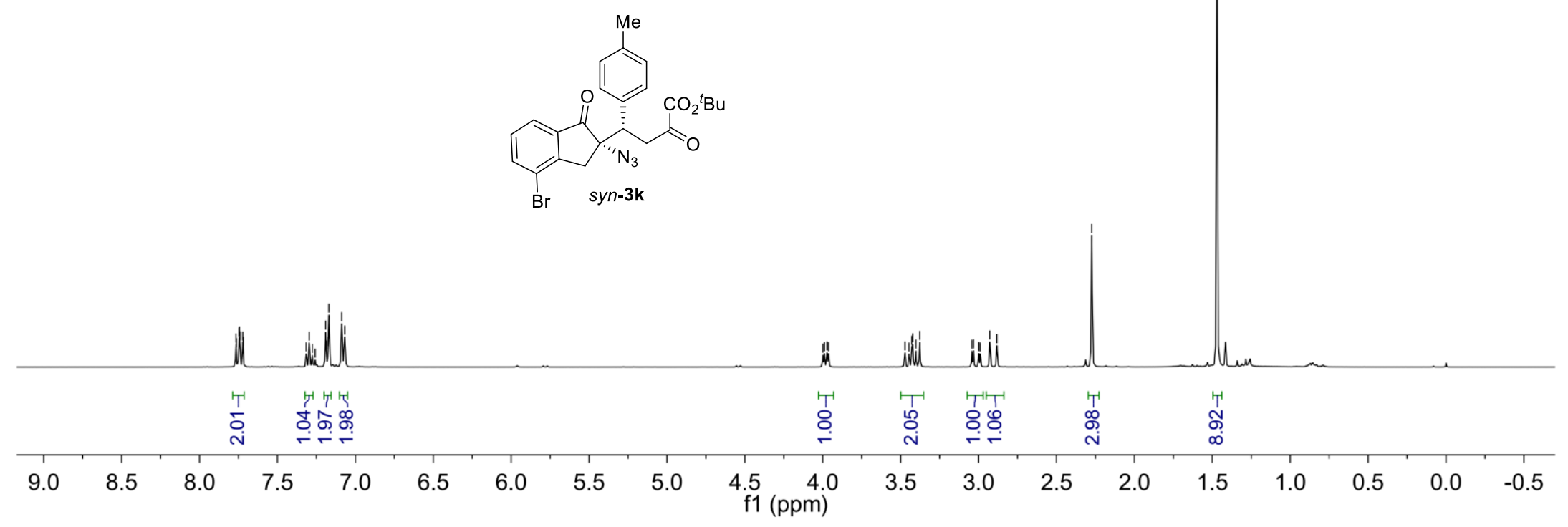


${ }^{13} \mathrm{C}$ NMR of Compound syn-3k $\left(100 \mathrm{MHz}, \mathrm{CDCl}_{3}\right)$

DPG-DJ-44-1 C $100 \mathrm{HMz}$

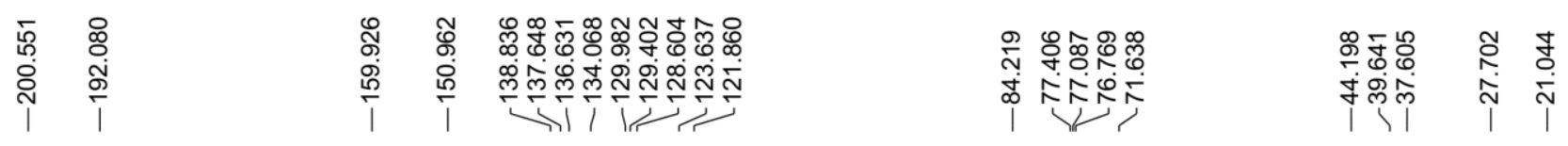
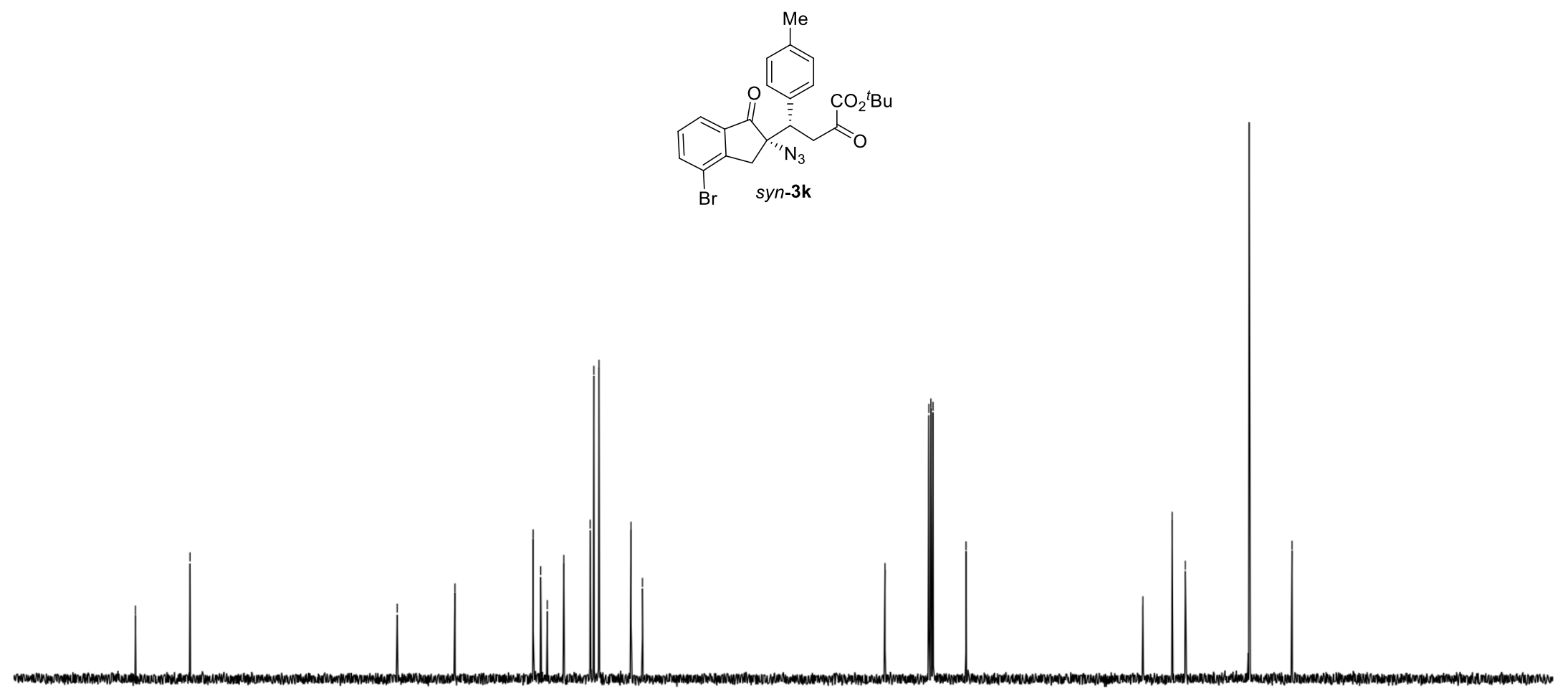

$\begin{array}{lllllllllllllllllllllll}210 & 200 & 190 & 180 & 170 & 160 & 150 & 140 & 130 & 120 & 110 & \begin{array}{l}100 \\ \mathrm{f} 1(\mathrm{ppm})\end{array} & 90 & 80 & 70 & 60 & 50 & 40 & 30 & 20 & 10 & 0 & -10\end{array}$ 
${ }^{1} \mathrm{H}$ NMR of Compound syn-3l (400 MHz, $\mathrm{CDCl}_{3}$ )

\section{DPG-DJ-81-1 H 400 MHz}

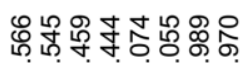

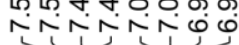

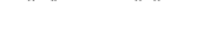

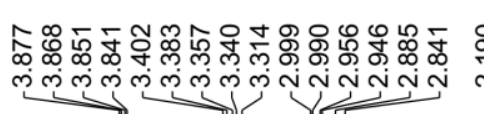
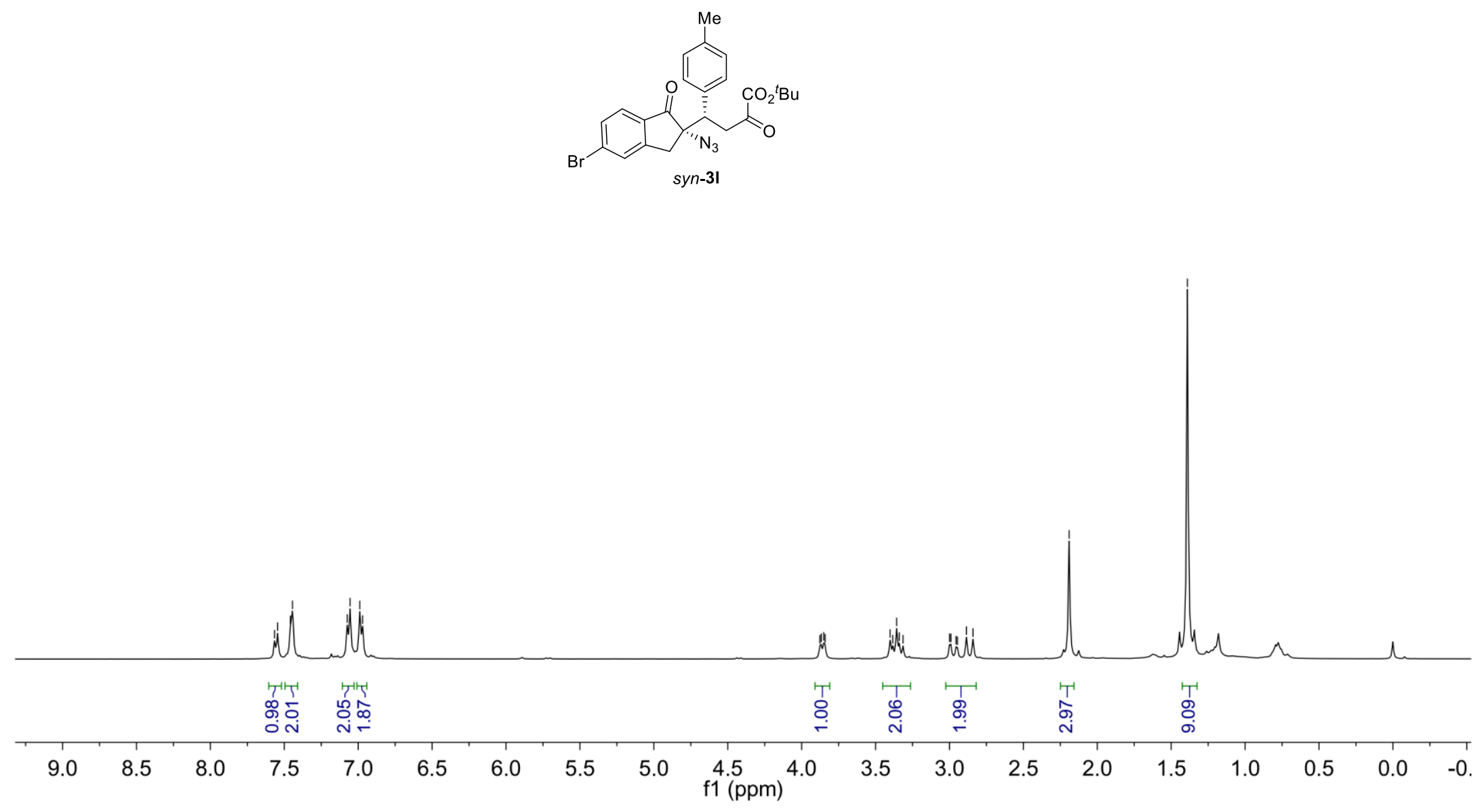
${ }^{13} \mathrm{C}$ NMR of Compound syn-3l (100 MHz, $\left.\mathrm{CDCl}_{3}\right)$

DPG-DJ-81-1 C $100 \mathrm{MHz}$

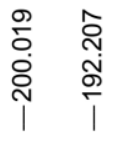

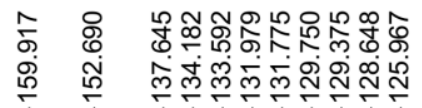

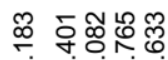

赫定

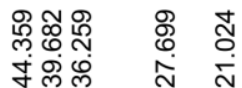
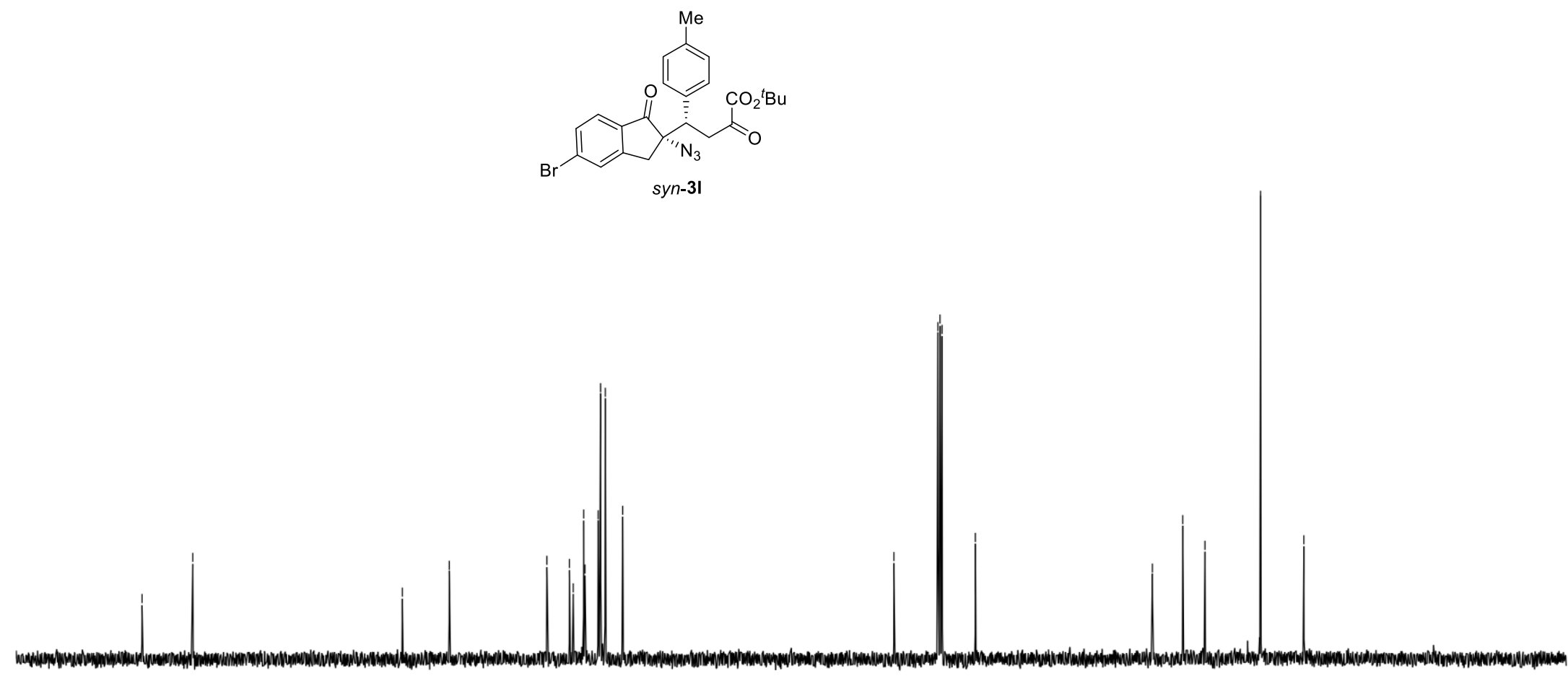

$150 \quad 140 \quad 130$

$\begin{array}{lll}120 & 110 \quad 100\end{array}$ f1 (ppm) 
${ }^{1} \mathrm{H}$ NMR of Compound $(S, S)-\mathbf{3 m}\left(400 \mathrm{MHz}, \mathrm{CDCl}_{3}\right)$

\section{DPG-DJ-38-1 H $400 \mathrm{MHz}$}

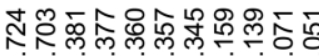

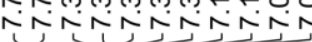

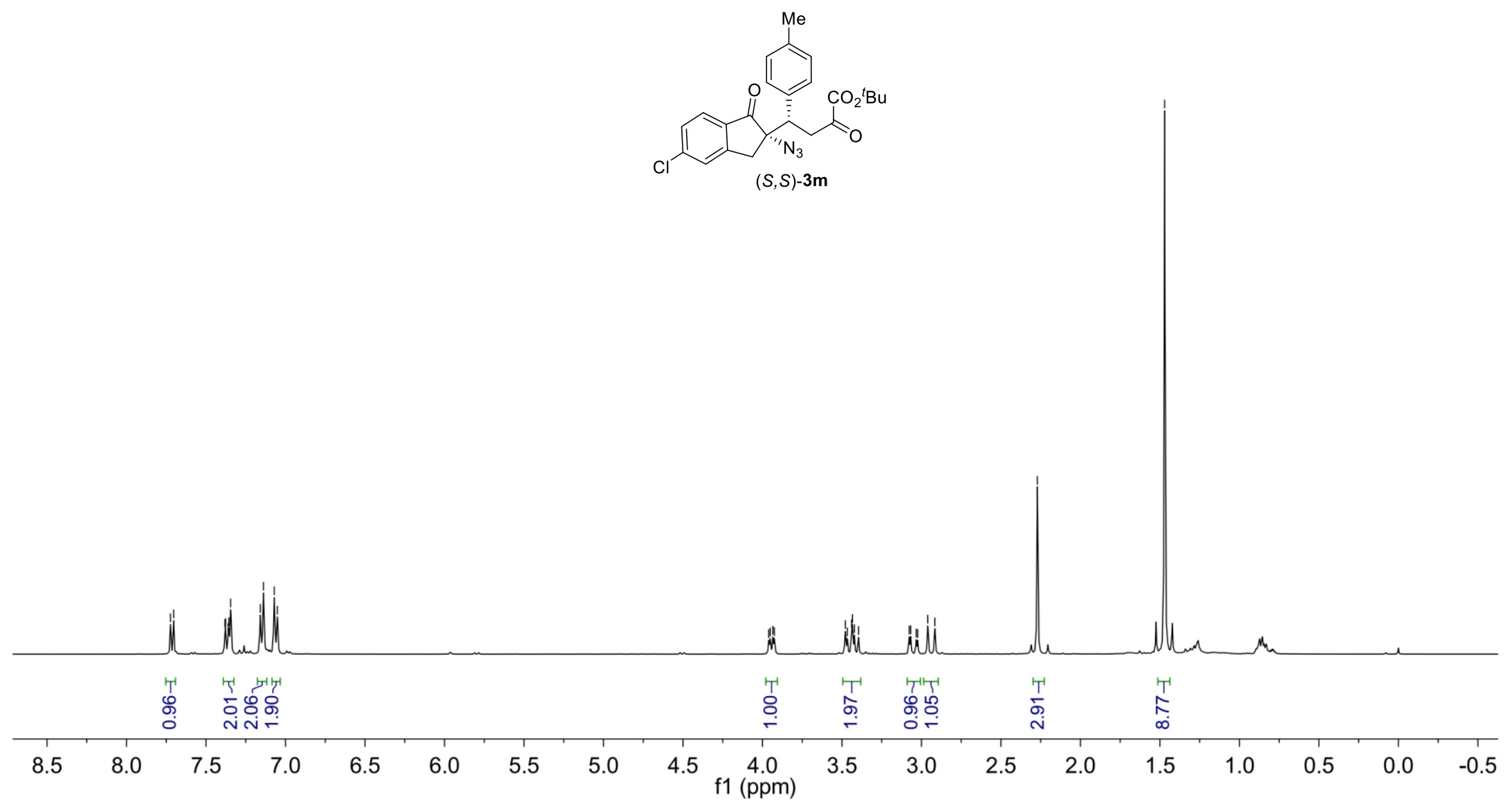

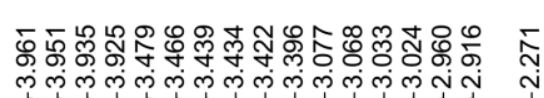


${ }^{13} \mathrm{C}$ NMR of Compound $(S, S)-3 m\left(100 \mathrm{MHz}, \mathrm{CDCl}_{3}\right)$

DPG-DJ-38-1 C $100 \mathrm{MHz}$

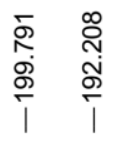

Г कृ

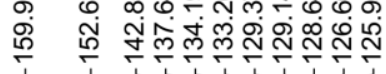

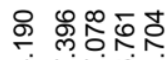

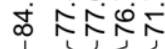

잉్ㅀㅇㅠ \&

उं্्ल Nं
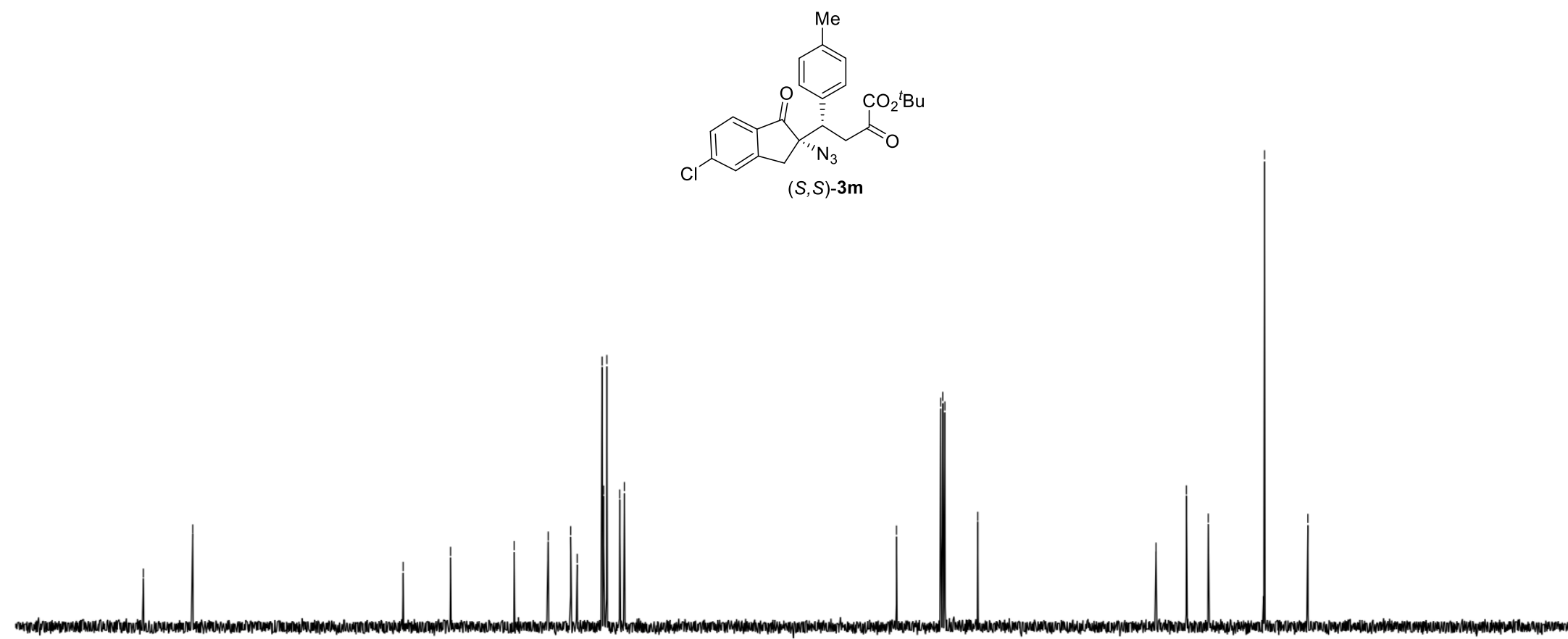

$\begin{array}{llllll}210 & 200 & 190 & 180 & 170 & 160\end{array}$

$\begin{array}{lllll}150 & 140 & 130 & 120\end{array}$

$110 \underset{f 1(p p m)}{100} 90$

80

706

$50 \quad 40 \quad 30$

30

$20 \quad 10$

$0 \quad-10$ 
${ }^{1} \mathrm{H}$ NMR of Compound $(R, R)-\mathbf{3 m}\left(400 \mathrm{MHz}, \mathrm{CDCl}_{3}\right)$

\section{DPG-DN-39 H $400 \mathrm{MHz}$}

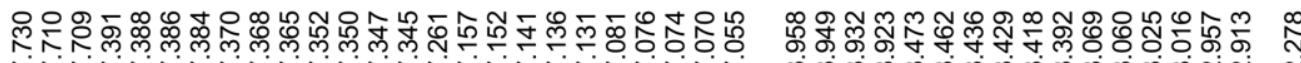

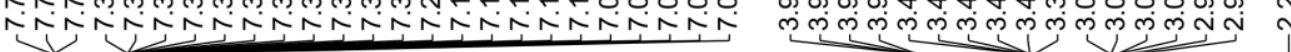

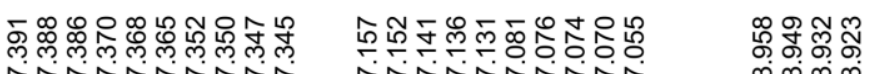

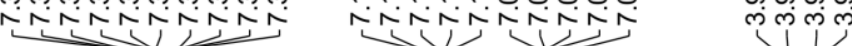

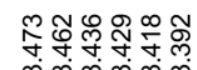

लिलंखिं

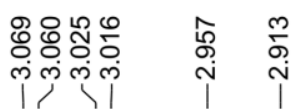

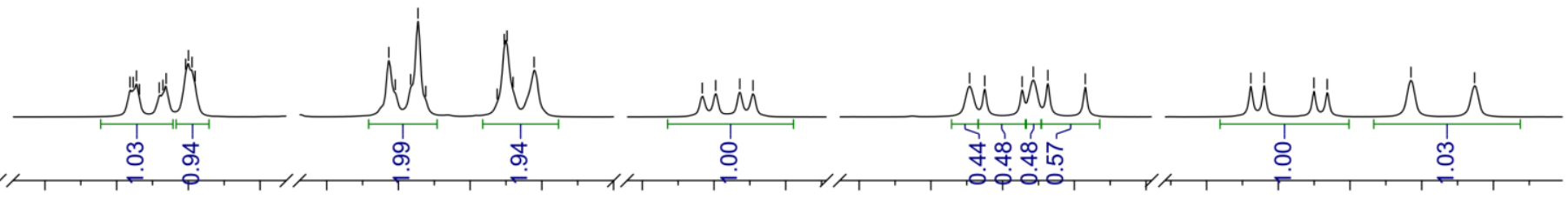

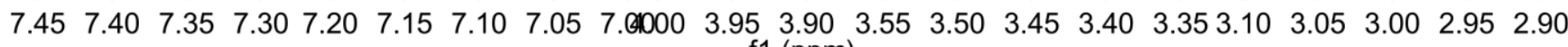
f1 (ppm)
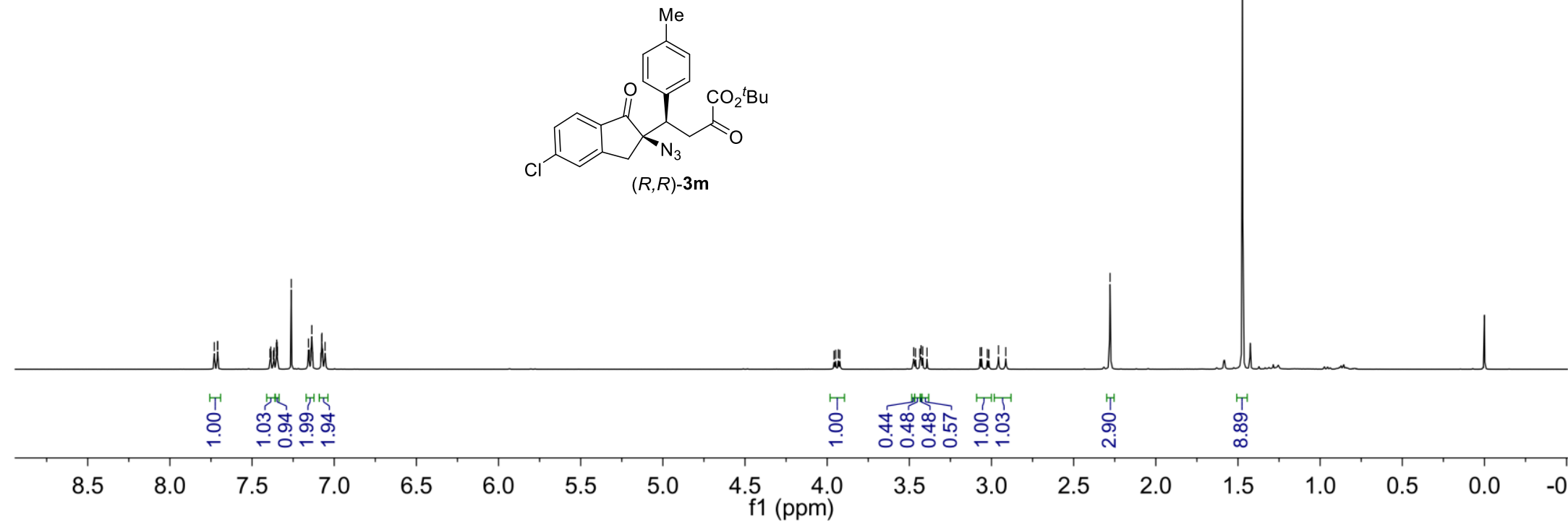
${ }^{13} \mathrm{C}$ NMR of Compound $(R, R)-3 m\left(100 \mathrm{MHz}, \mathrm{CDCl}_{3}\right)$
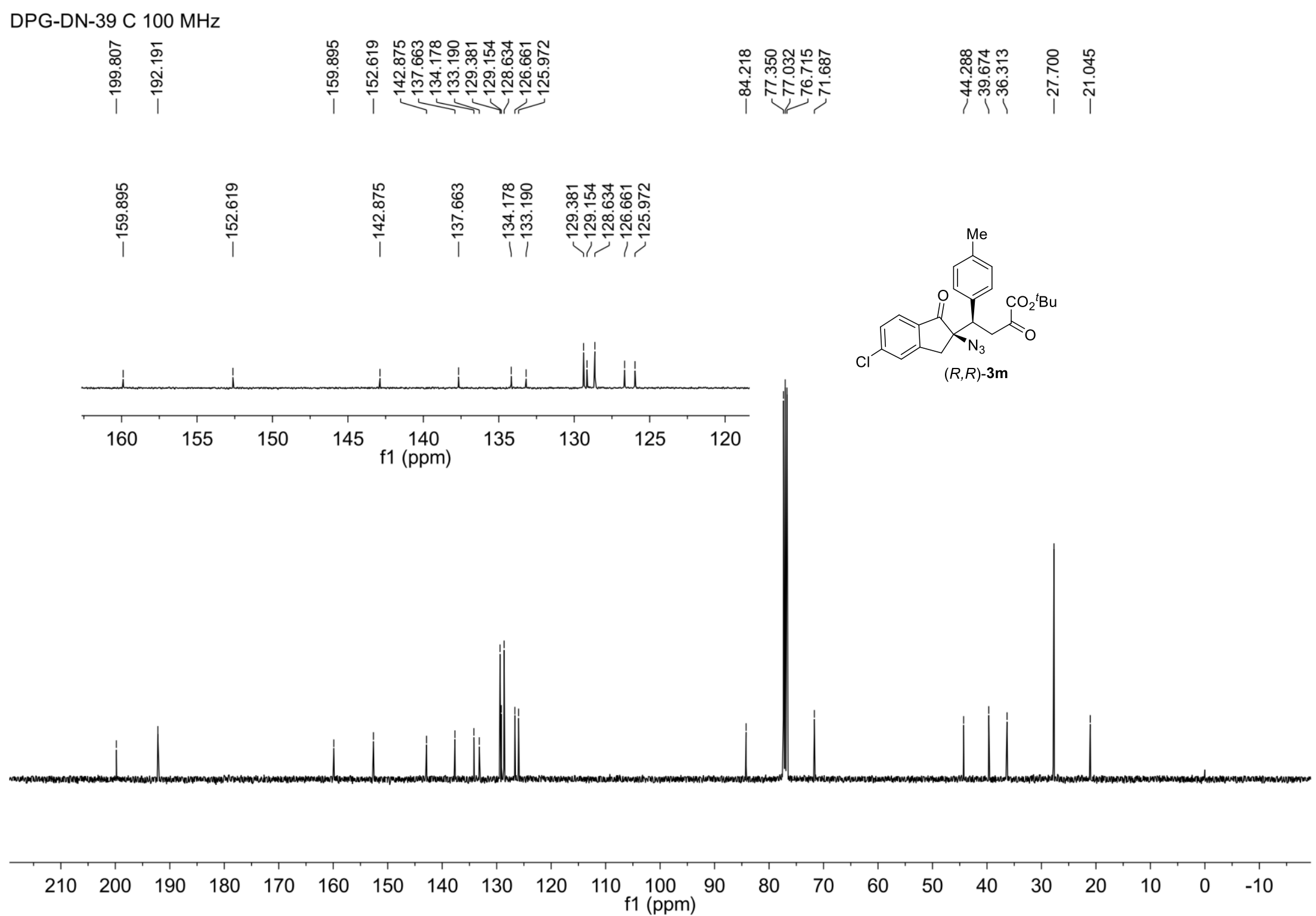
${ }^{1} \mathrm{H}$ NMR of Compound syn-3n $\left(300 \mathrm{MHz}, \mathrm{CDCl}_{3}\right)$

\section{DPG-DJ-44-3 H $300 \mathrm{MHz}$}

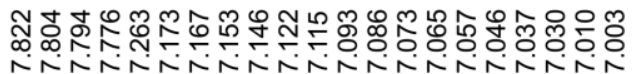

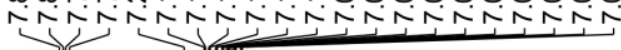

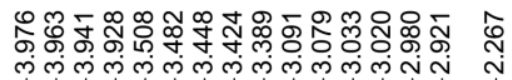

ن

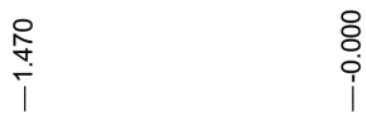

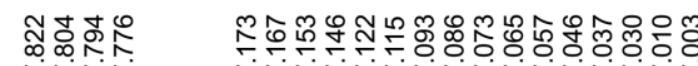 \\ iNit iNiNiNiNiNiN}

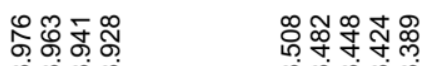

लंखिं

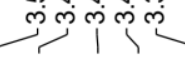

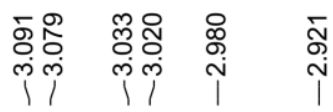

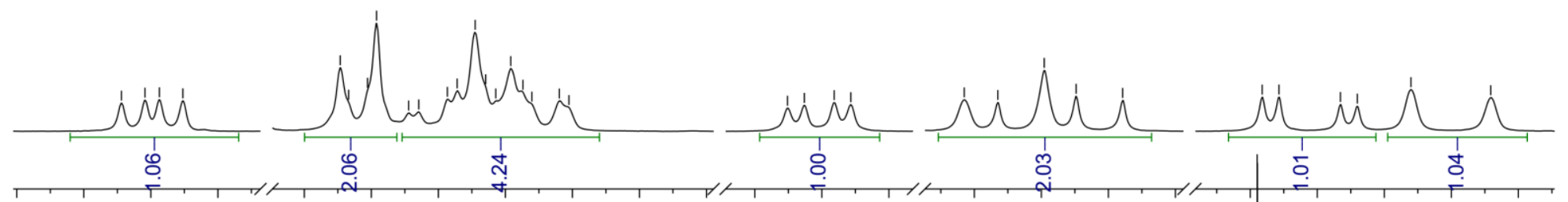

$\begin{array}{lllllllllllllllllllllll}7.90 & 7.85 & 7.80 & 7.75 & 7.20 & 7.15 & 7.10 & 7.05 & 7.00 & 6.95 & 6.904 .00 & 3.95 & 3.90 & 3.50 & 3.45 & 3.40 & 3.35 & 3.10 & 3.05 & 3.00 & 2.95 & 2.90\end{array}$ f1 (ppm)
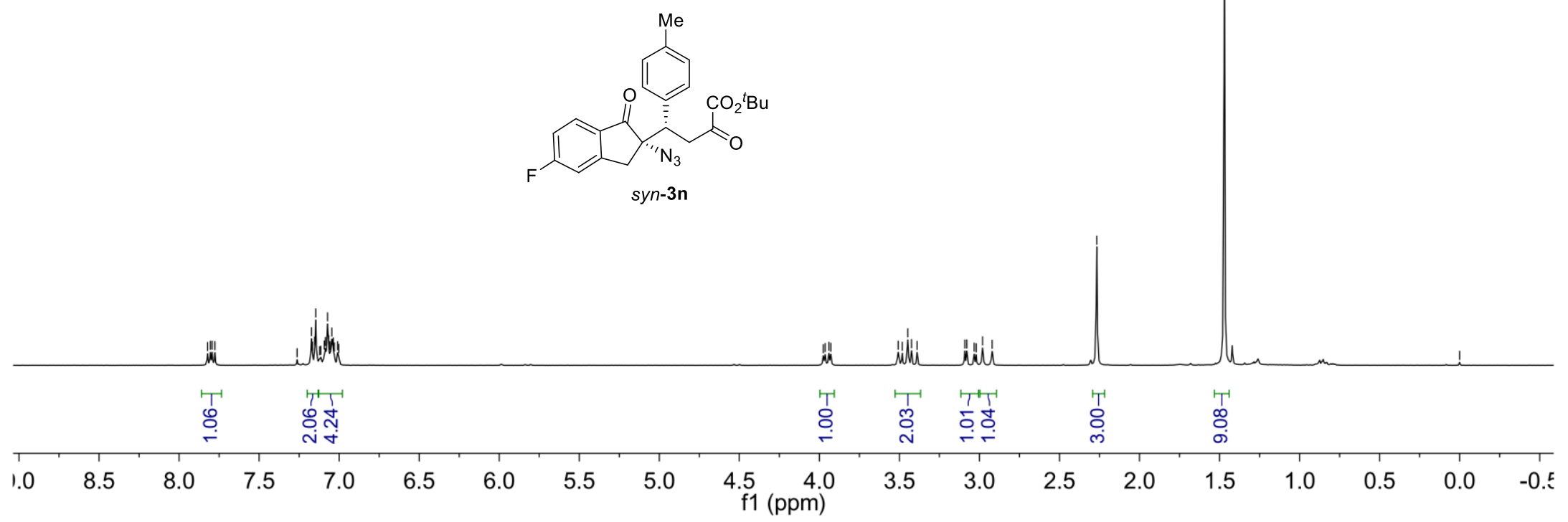
${ }^{19} \mathrm{~F}$ NMR of Compound syn-3n $\left(282 \mathrm{MHz}, \mathrm{CDCl}_{3}\right)$

DPG-DJ-44-3 F 282 MHz

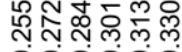

किंक्ष
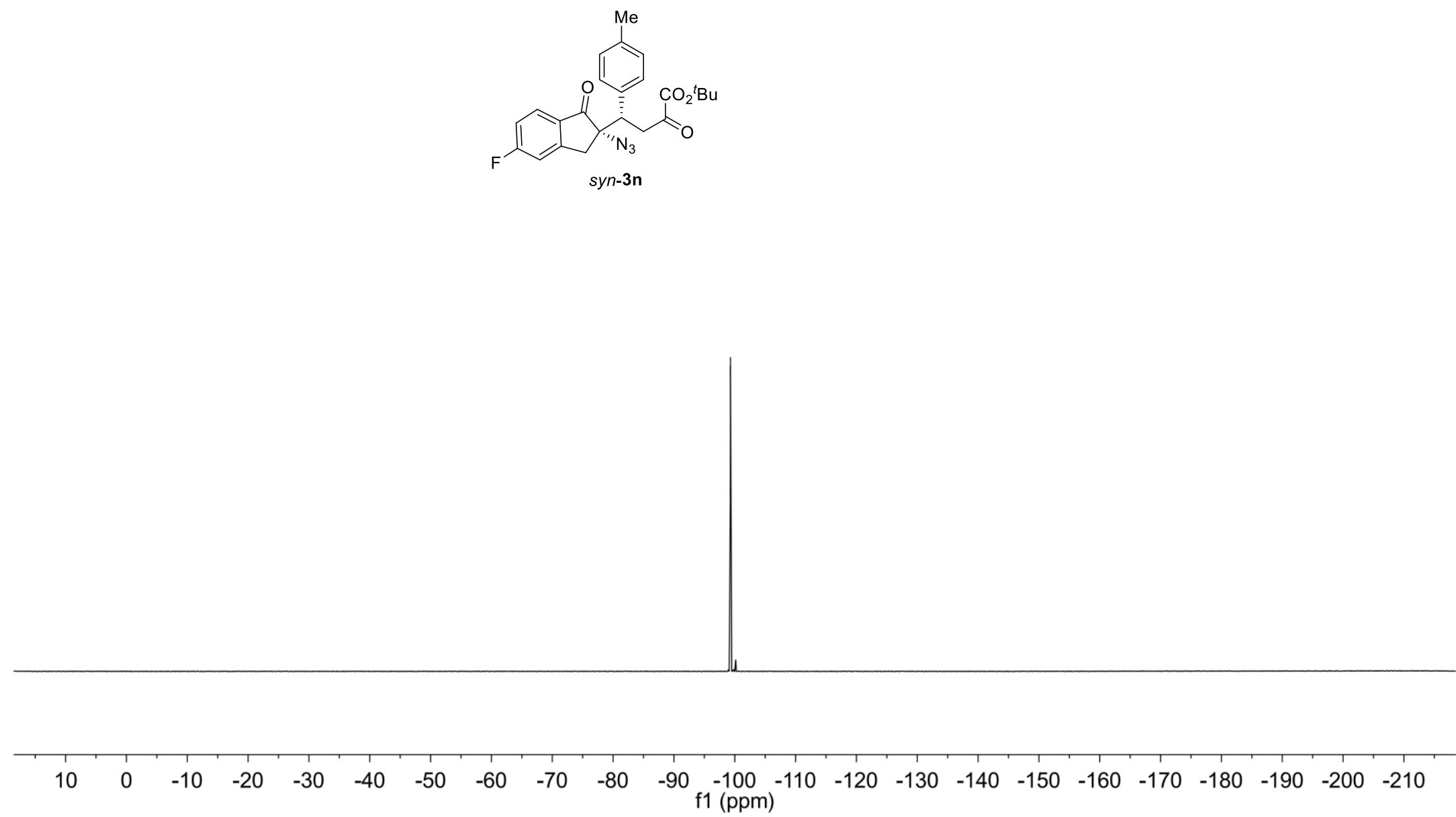
${ }^{13} \mathrm{C}$ NMR of Compound syn-3n $\left(100 \mathrm{MHz}, \mathrm{CDCl}_{3}\right)$

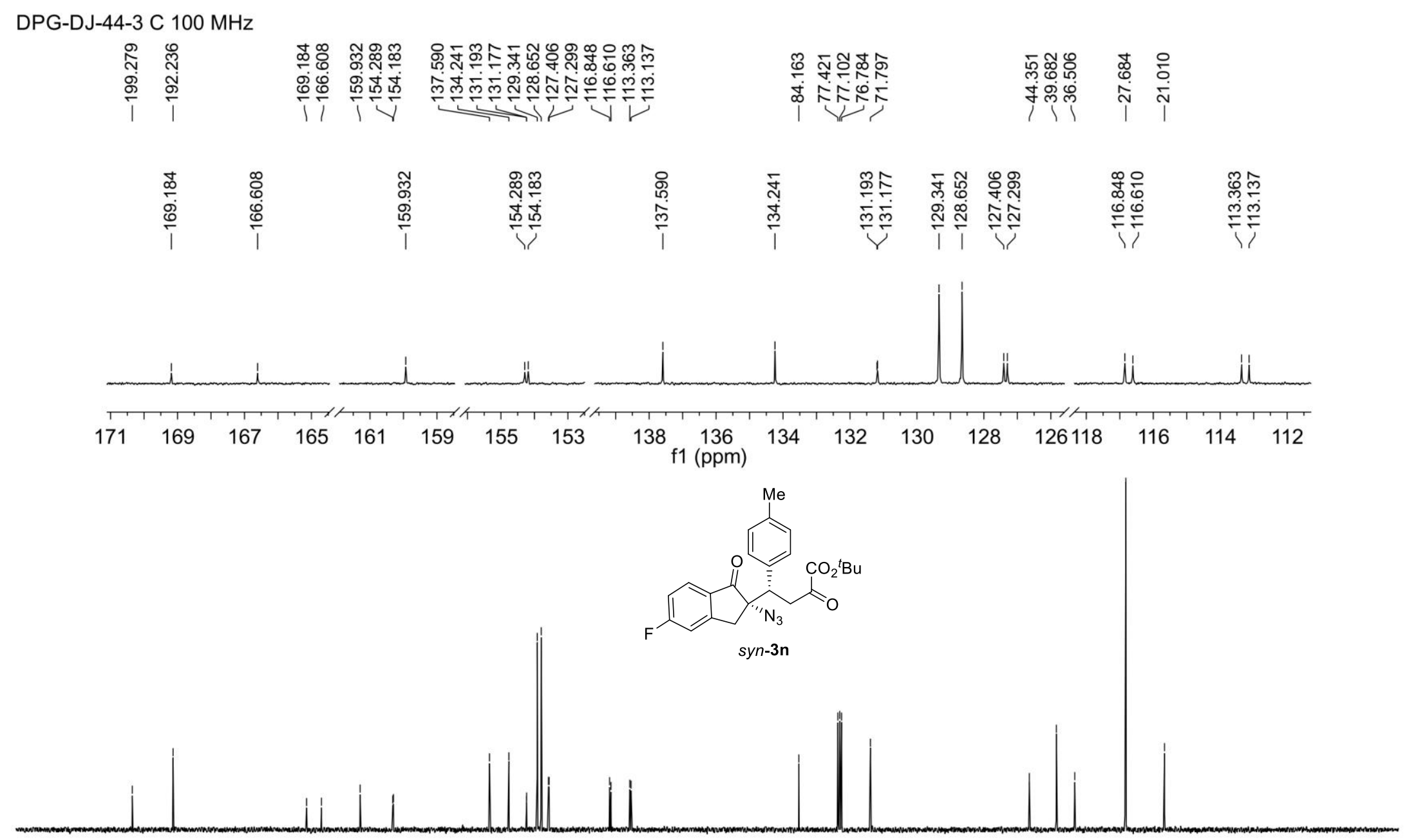

$\begin{array}{lllllllllllllllllllllll}210 & 200 & 190 & 180 & 170 & 160 & 150 & 140 & 130 & 120 & 110 \underset{\mathrm{f} 1(\mathrm{ppm})}{100} & 90 & 80 & 70 & 60 & 50 & 40 & 30 & 20 & 10 & 0 & -10\end{array}$ 
${ }^{1} \mathrm{H}$ NMR of Compound syn-3o (400 MHz, $\left.\mathrm{CDCl}_{3}\right)$

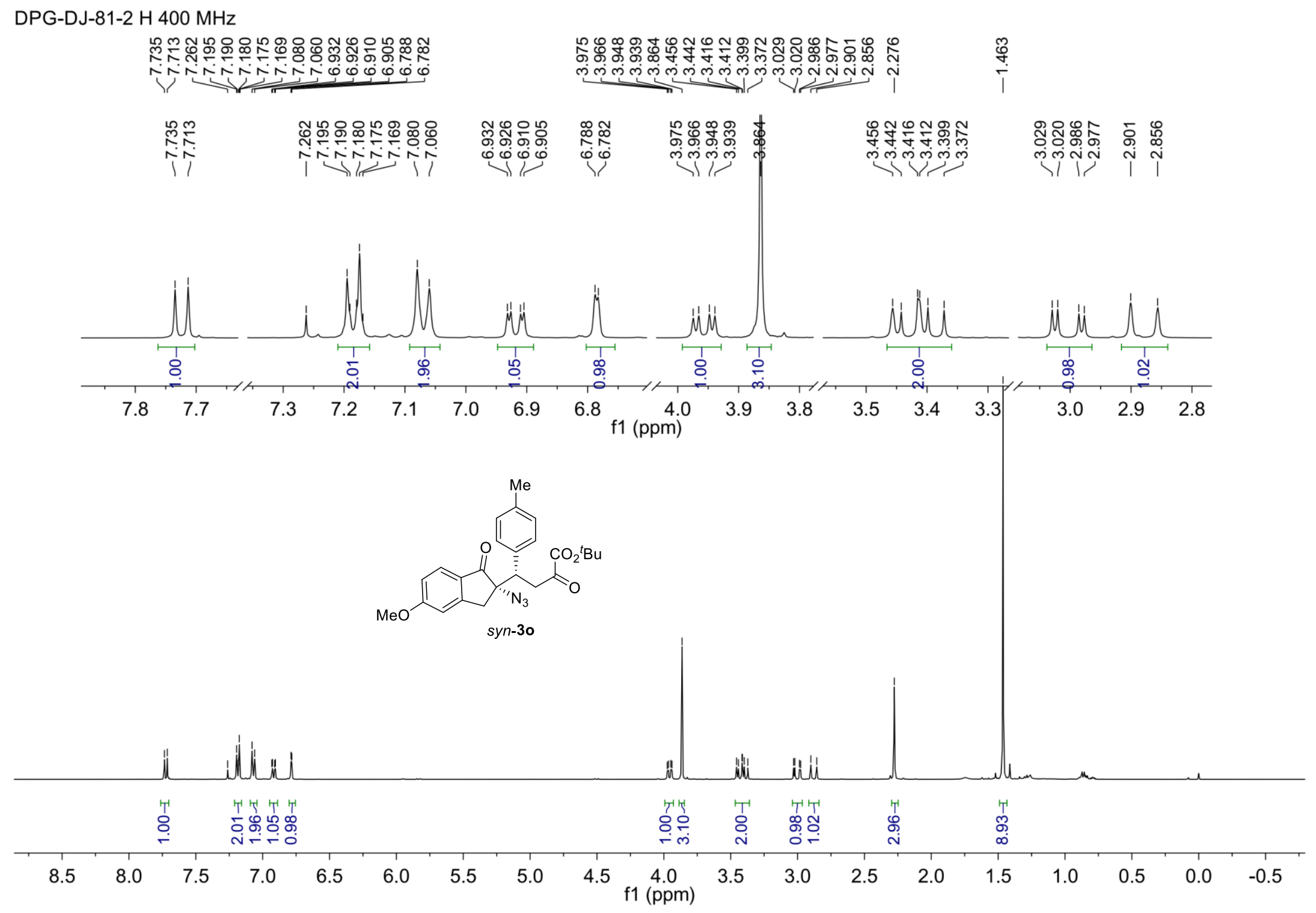


${ }^{13} \mathrm{C}$ NMR of Compound syn-3o $\left(100 \mathrm{MHz}, \mathrm{CDCl}_{3}\right)$

DPG-DJ-81-2 C 100 MHz

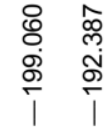
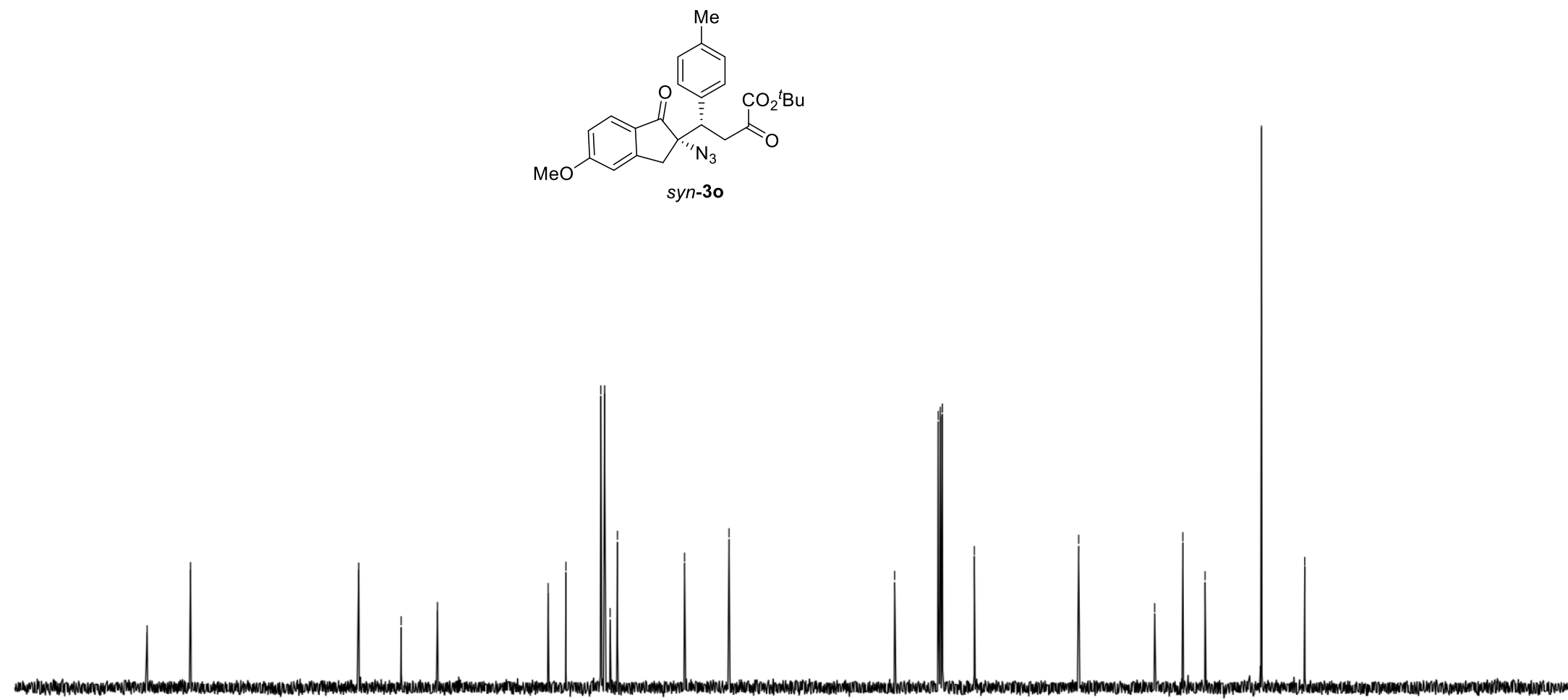

210

$\begin{array}{lllll}200 & 190 & 180 & 170 & 160\end{array}$

$\begin{array}{lll}150 & 140 \quad 130\end{array}$

$120 \quad 110$ $\mathrm{f} 1(\mathrm{ppm})$ 
${ }^{1} \mathrm{H}$ NMR of Compound syn-3p $\left(400 \mathrm{MHz}, \mathrm{CDCl}_{3}\right)$

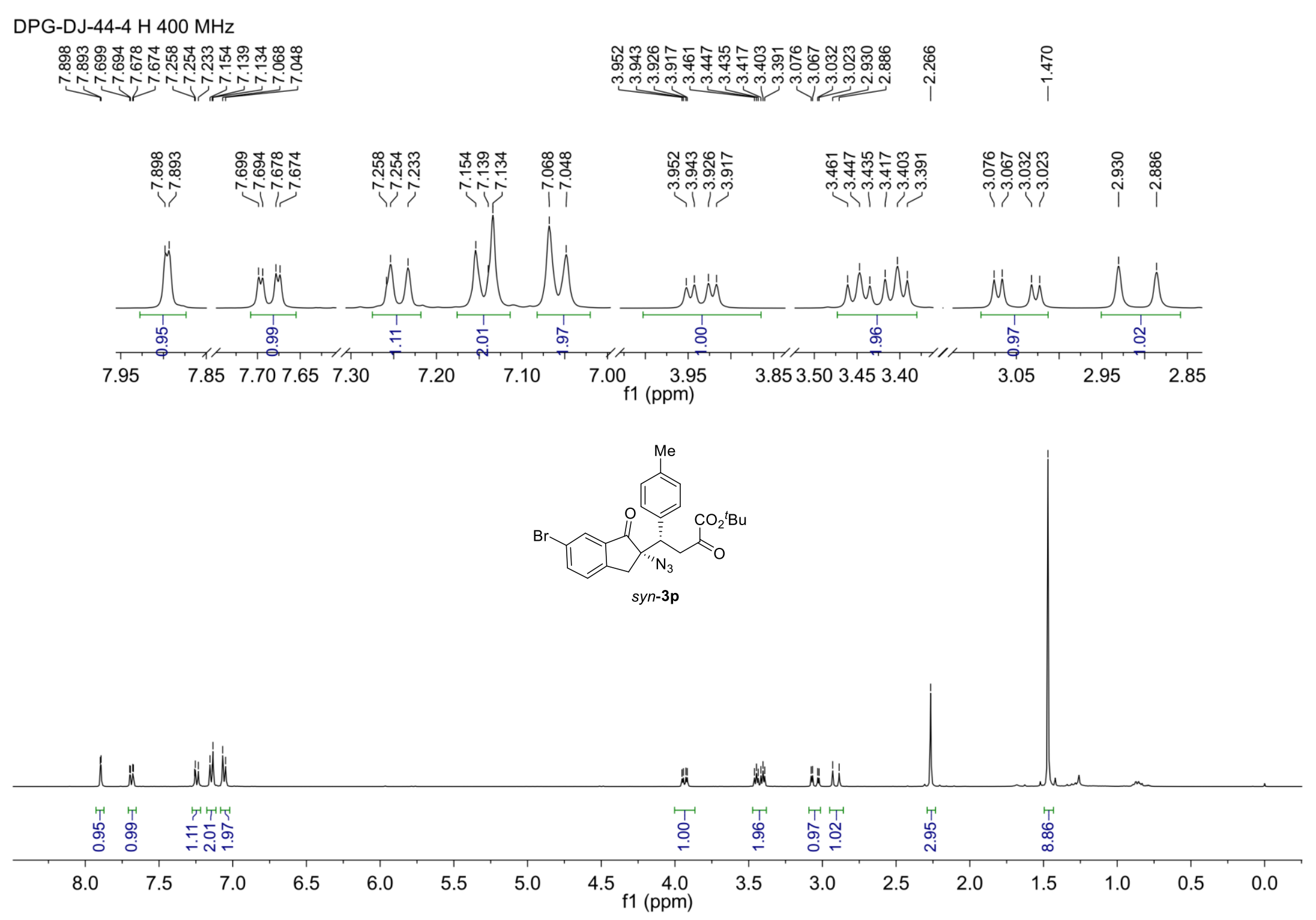


${ }^{13} \mathrm{C}$ NMR of Compound syn-3p (100 MHz, $\left.\mathrm{CDCl}_{3}\right)$

DPG-DJ-44-4 C $100 \mathrm{MHz}$

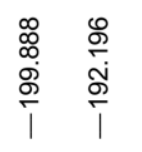

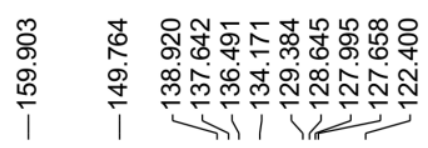

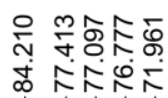

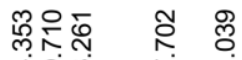

过无 N
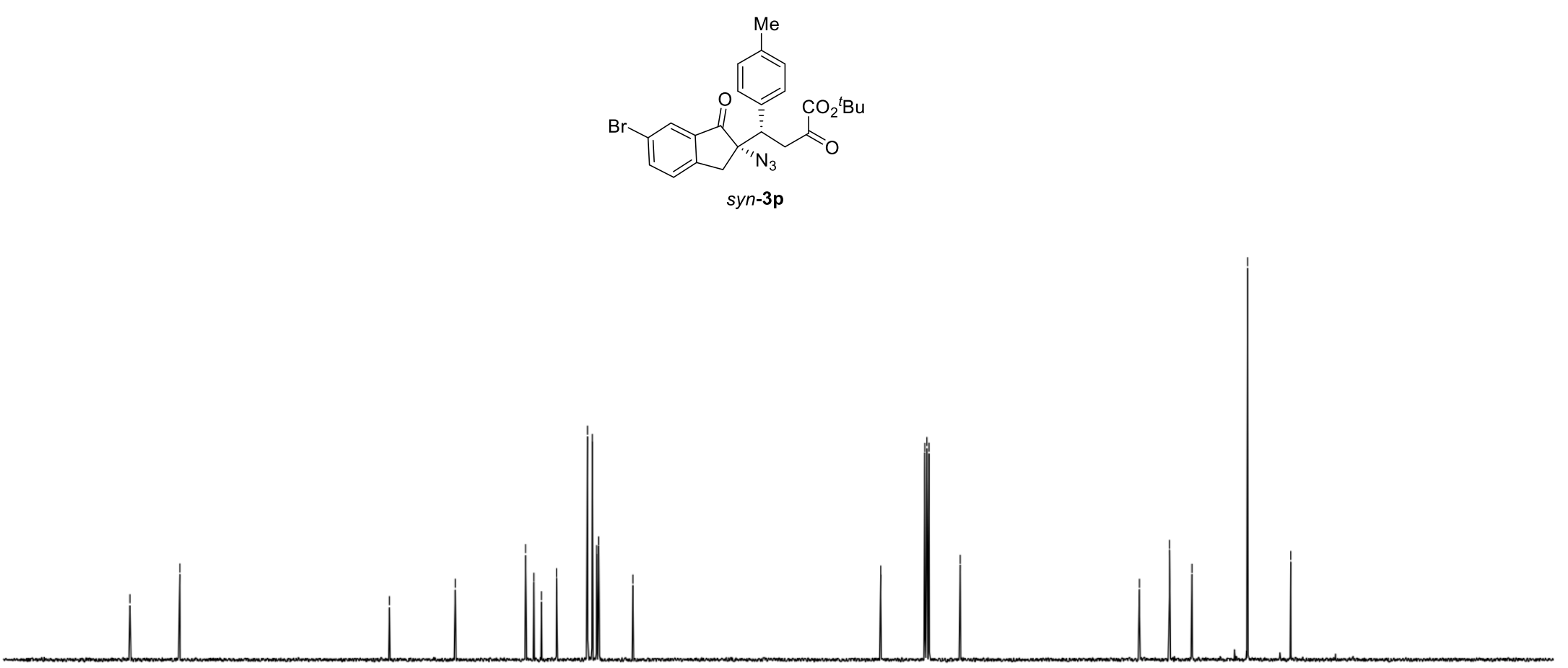

2102

$\begin{array}{lllll}200 & 190 & 180 & 170 & 160\end{array}$

150

$140 \quad 130$

$120 \quad 110 \quad 100$ f1 (ppm) 
${ }^{1} \mathrm{H}$ NMR of Compound syn-3q $\left(400 \mathrm{MHz}, \mathrm{CDCl}_{3}\right)$

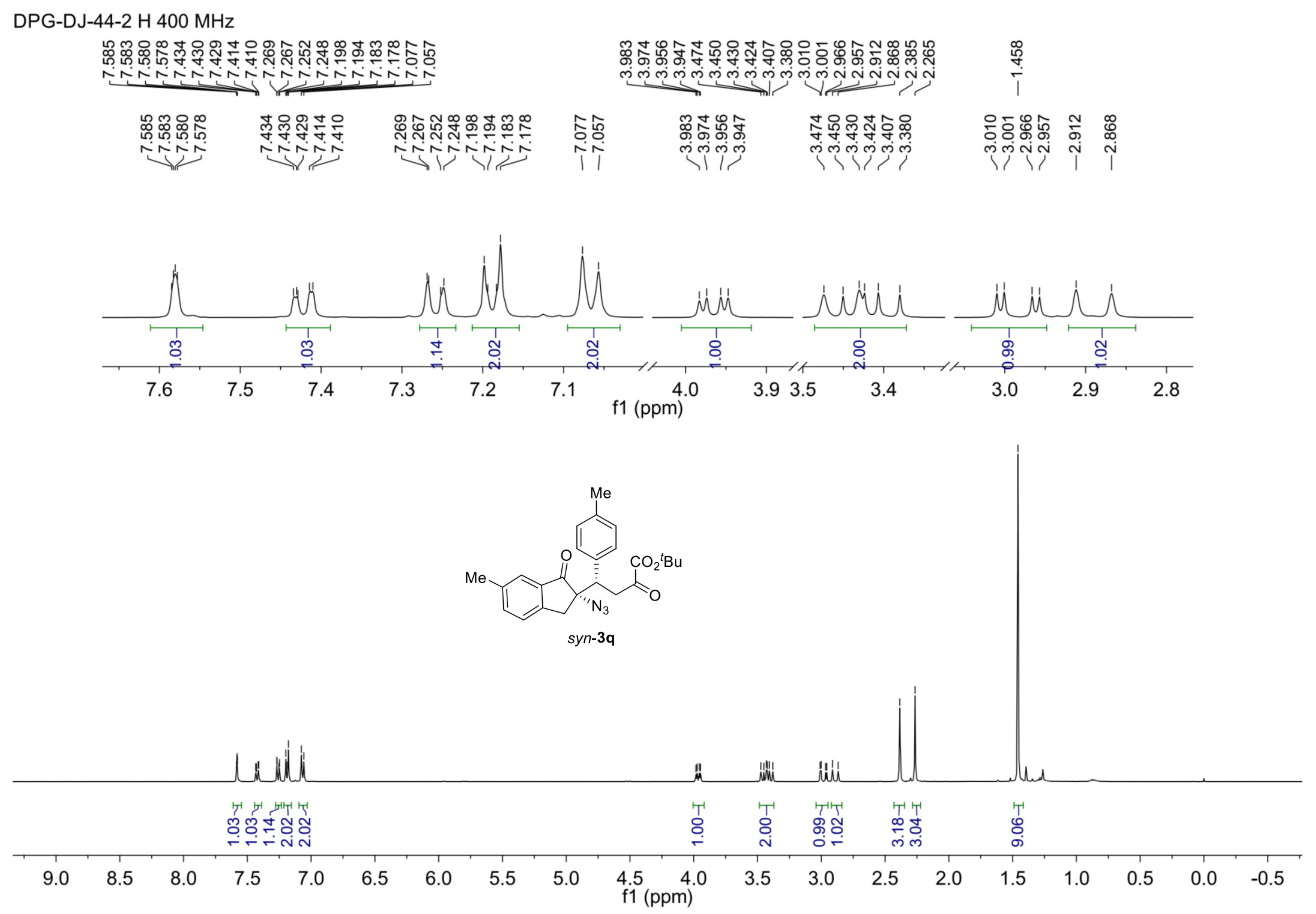


${ }^{13} \mathrm{C}$ NMR of Compound syn-3q $\left(100 \mathrm{MHz}, \mathrm{CDCl}_{3}\right)$

DPG-DJ-44-2 C 100 MHz

$$
\text { ঙั. }
$$

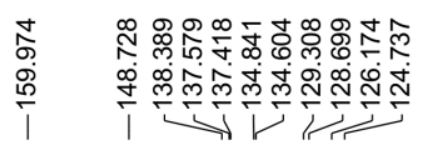

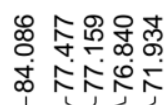

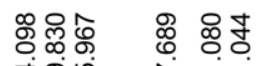

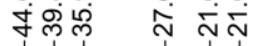
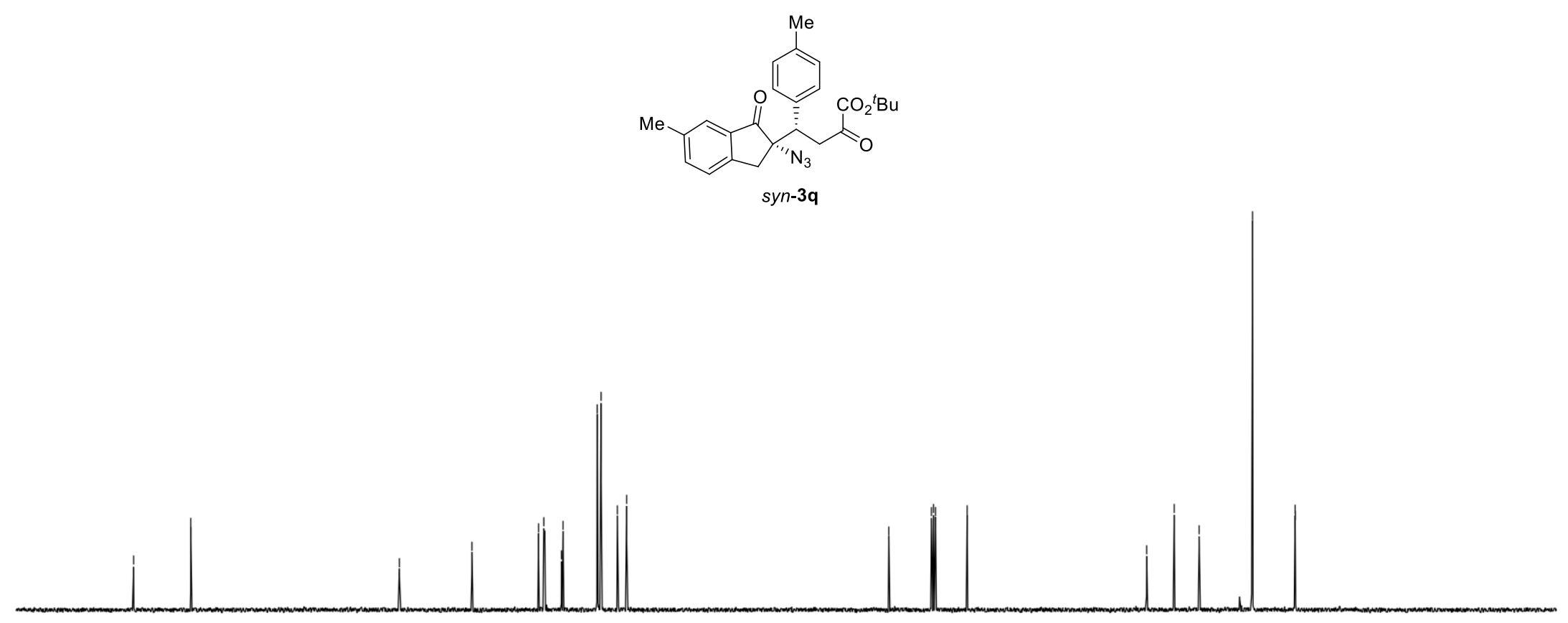

$\begin{array}{lllllllllllllllllllllll}210 & 200 & 190 & 180 & 170 & 160 & 150 & 140 & 130 & 120 & 110 & \begin{array}{l}100 \\ \mathrm{f} 1(\mathrm{ppm})\end{array} & 90 & 80 & 70 & 60 & 50 & 40 & 30 & 20 & 10 & 0 & -10\end{array}$ 
${ }^{1} \mathrm{H}$ NMR of Compound syn-3r $\left(400 \mathrm{MHz}, \mathrm{CDCl}_{3}\right)$

DPG-DJ-81-3 H $400 \mathrm{MHz}$

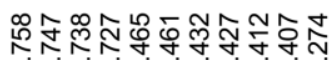

rivivinitivis

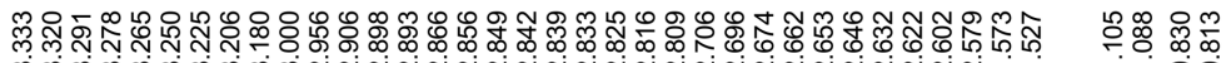

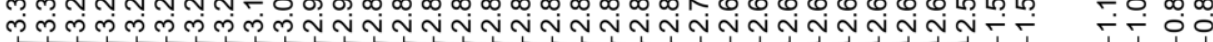
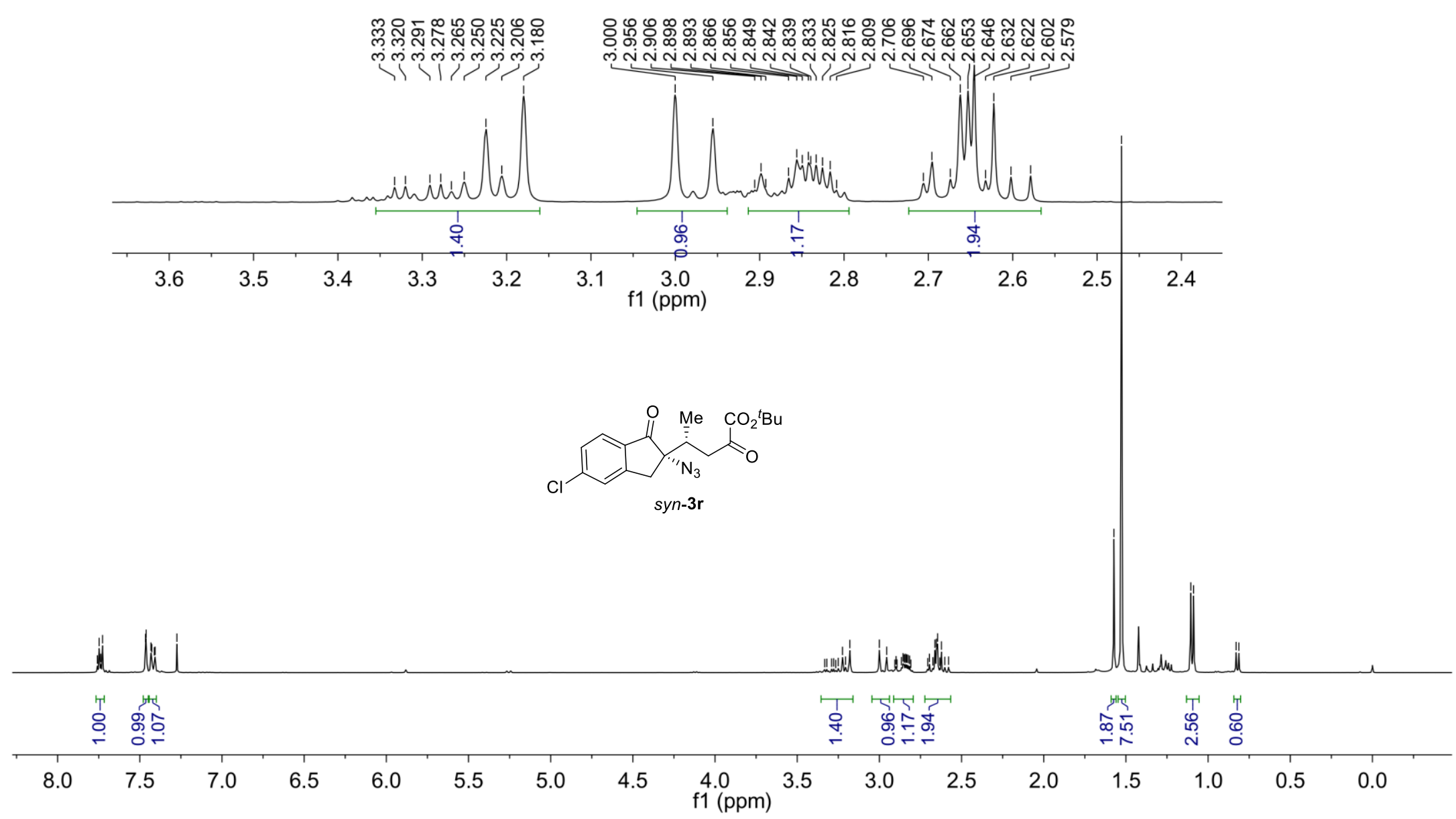
${ }^{13} \mathrm{C}$ NMR of Compound syn-3r $\left(100 \mathrm{MHz}, \mathrm{CDCl}_{3}\right)$
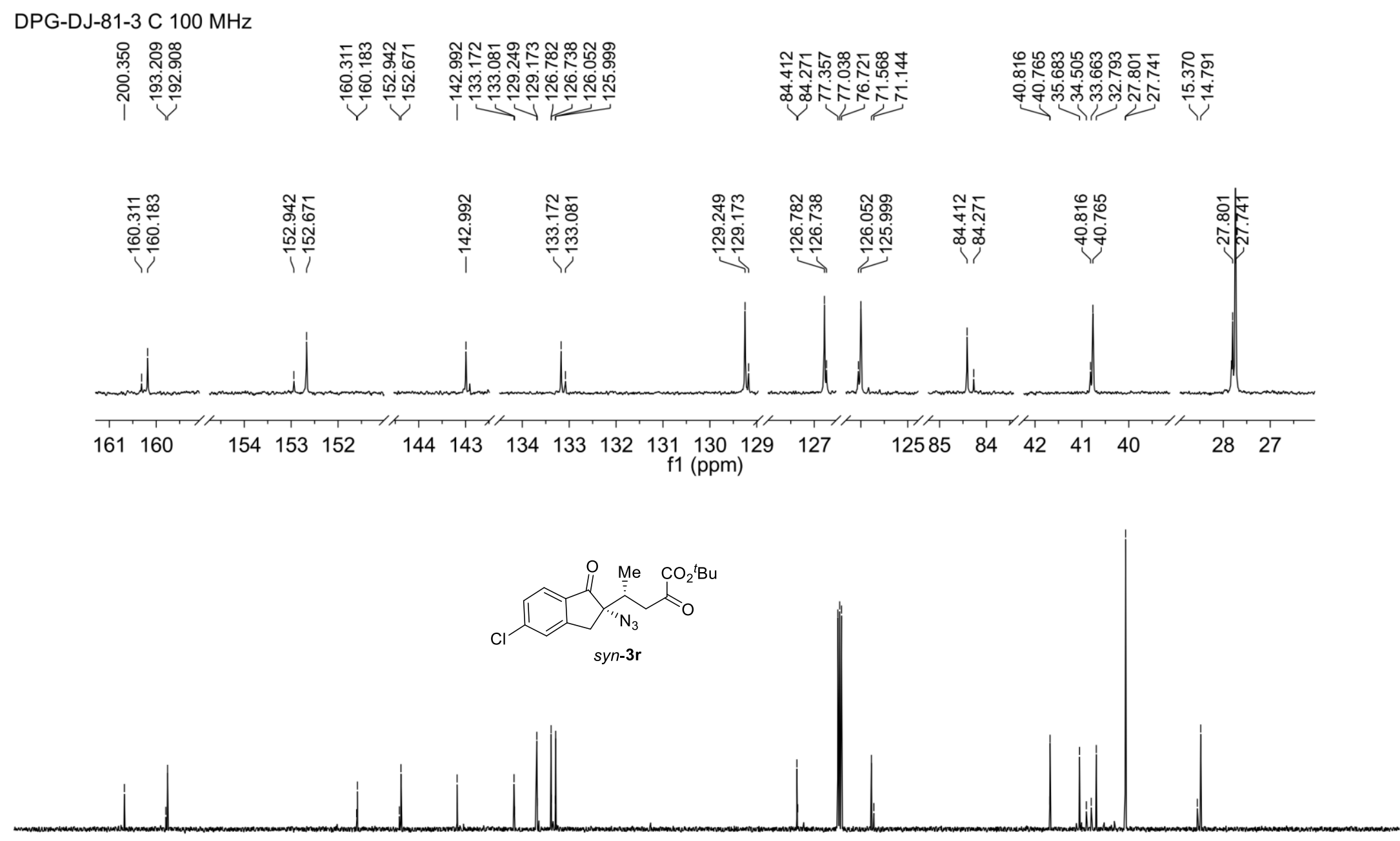

$\begin{array}{lllllllllllllllllllllll}210 & 200 & 190 & 180 & 170 & 160 & 150 & 140 & 130 & 120 & 110 & \begin{array}{c}100 \\ \mathrm{f} 1(\mathrm{ppm})\end{array} & 90 & 80 & 70 & 60 & 50 & 40 & 30 & 20 & 10 & 0 & -10\end{array}$ 
${ }^{1} \mathrm{H} \mathrm{NMR}$ of Compound anti-3a $\left(300 \mathrm{MHz}, \mathrm{CDCl}_{3}\right)$

DPG-DN-28-3 H $300 \mathrm{MHz}$

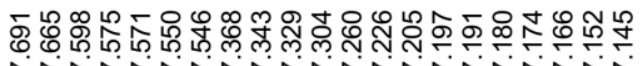

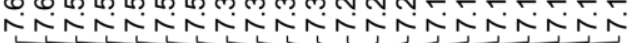

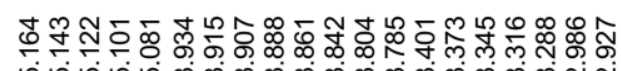

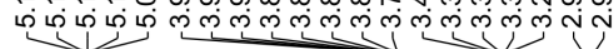

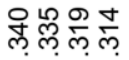

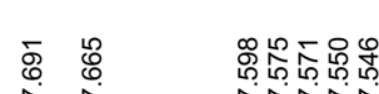

i

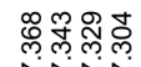

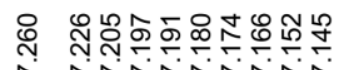

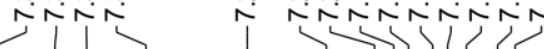
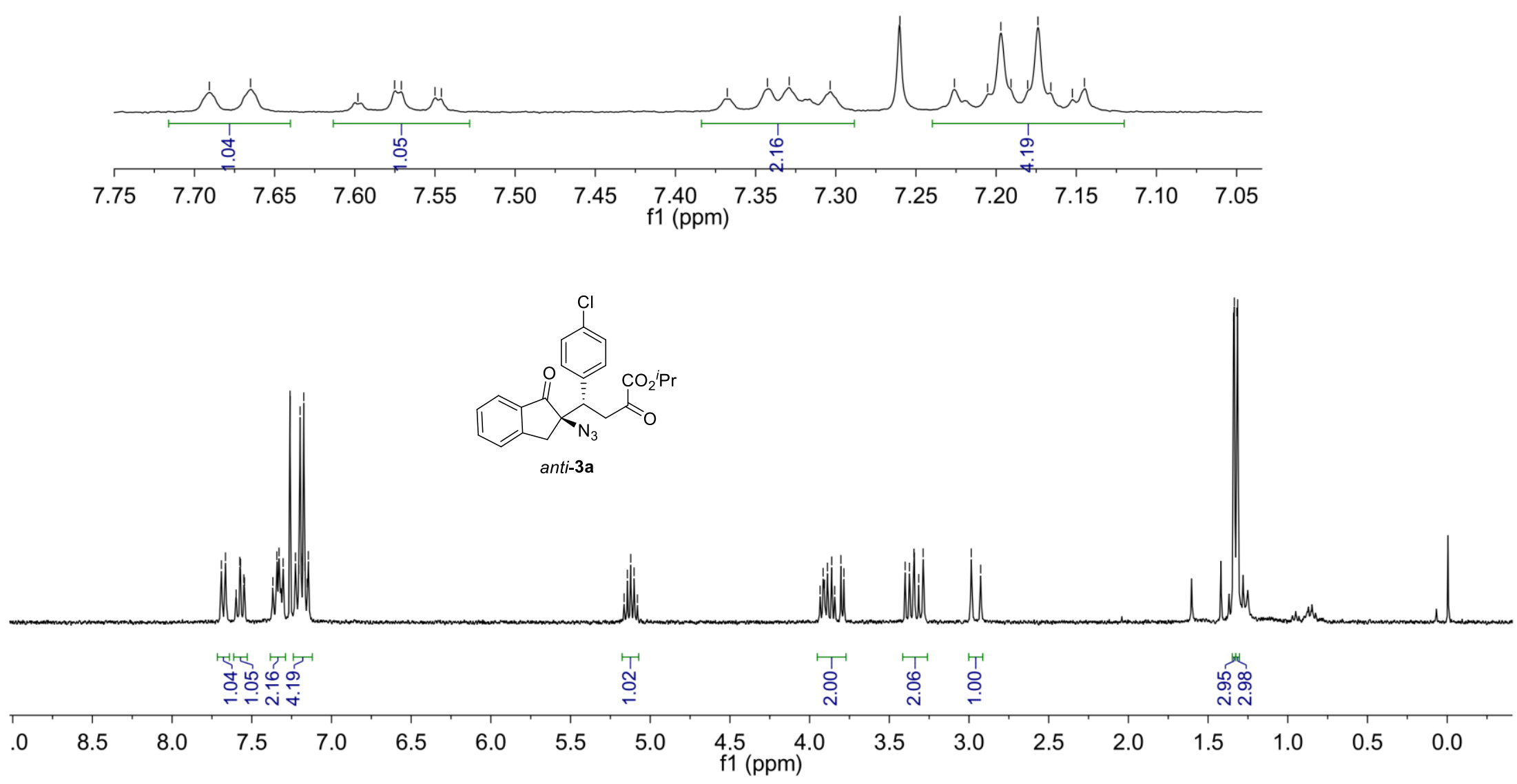
${ }^{13} \mathrm{C}$ NMR of Compound anti-3a $\left(100 \mathrm{MHz}, \mathrm{CDCl}_{3}\right)$

DPG-DN-28-3 C 100 MHz

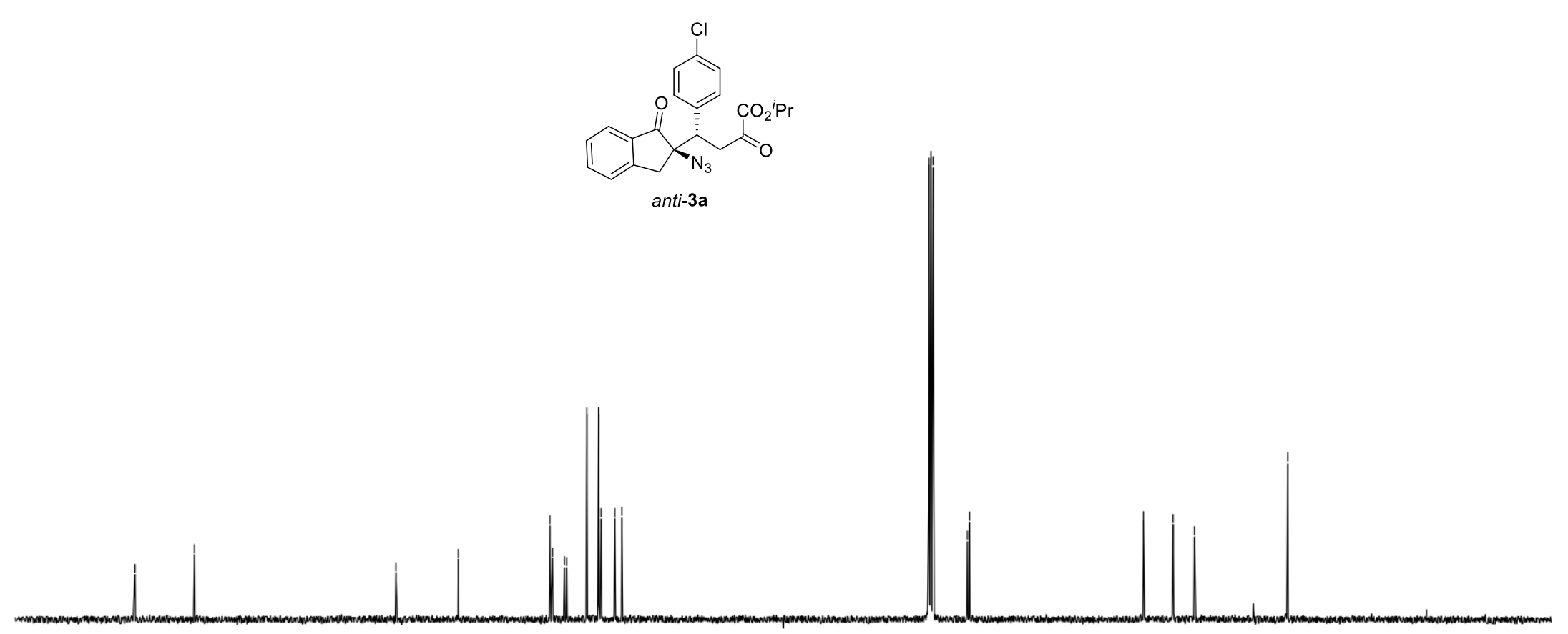


${ }^{1} \mathrm{H} \mathrm{NMR}$ of Compound anti-3b $\left(400 \mathrm{MHz}, \mathrm{CDCl}_{3}\right)$

dpg-dj-132-1-a H

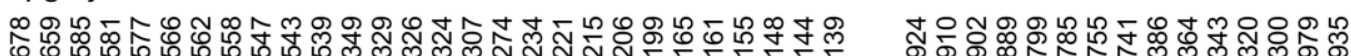

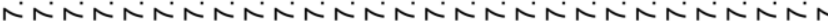

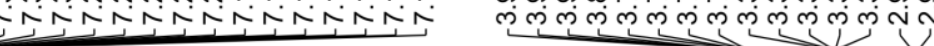
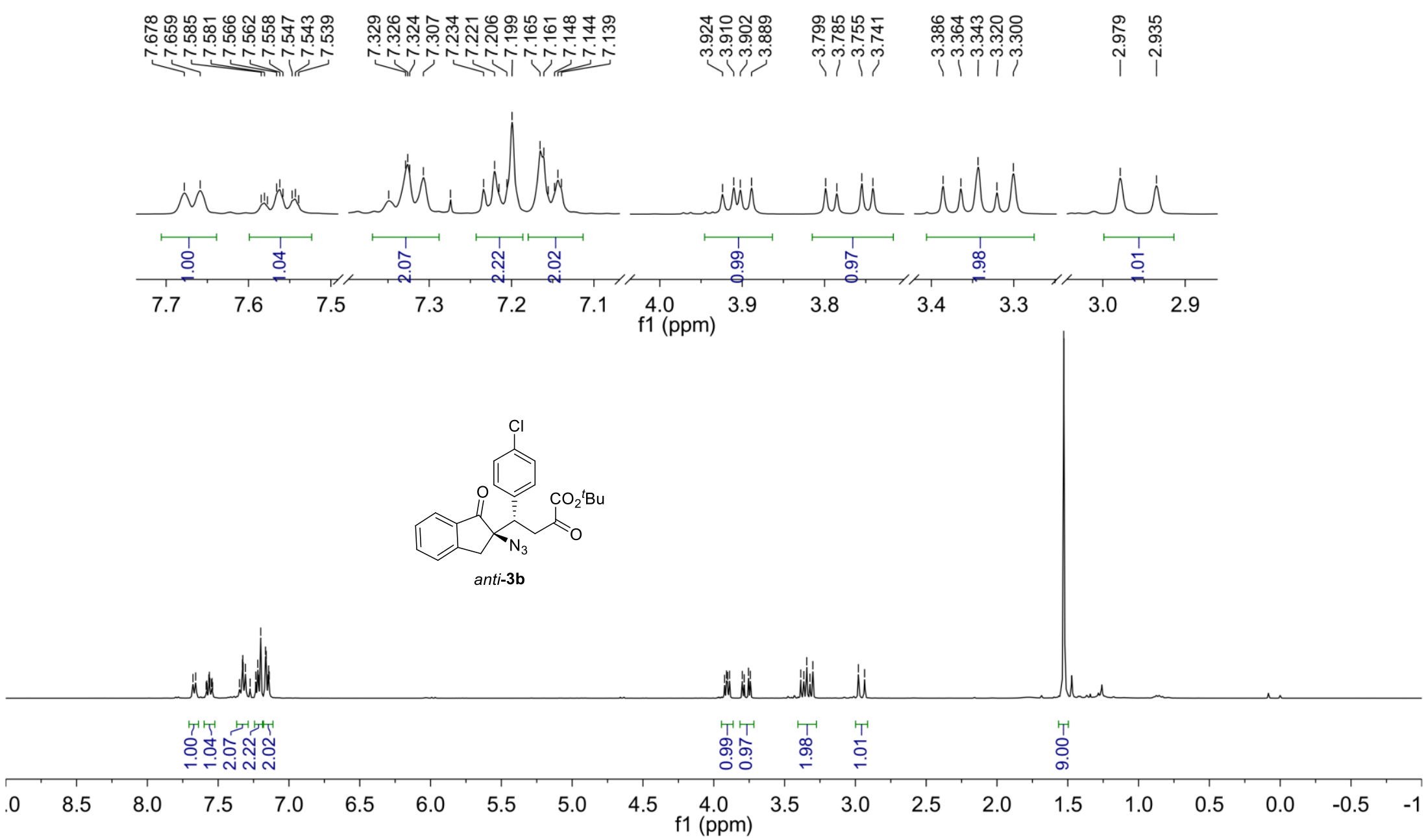
${ }^{13} \mathrm{C}$ NMR of Compound anti-3b (100 MHz, $\left.\mathrm{CDCl}_{3}\right)$

DPG-DJ-132-1 C $100 \mathrm{MHz}$

品

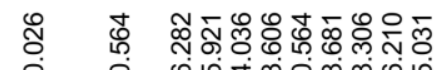

○ :

กุ

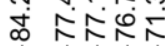

ஜำกิ

भुग्ण त्र
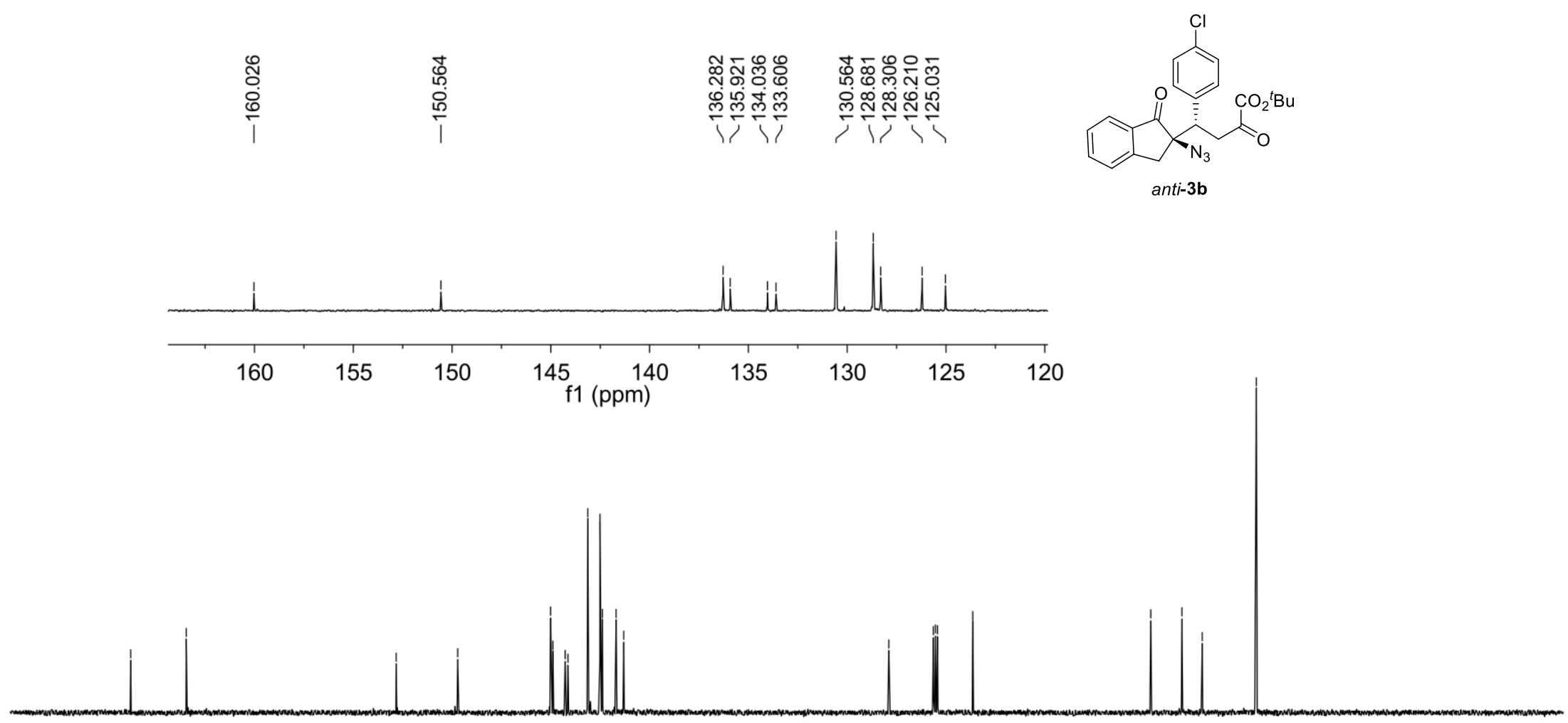

$210 \quad 200-190-18$

$\begin{array}{llll}80 & 170 & 160 & 150\end{array}$

$140 \quad 130$

$120110 \quad 100$ f1 (ppm) 
${ }^{1} \mathrm{H} \mathrm{NMR}$ of Compound anti-3c $\left(300 \mathrm{MHz}, \mathrm{CDCl}_{3}\right)$

\section{DPG-DN-28-4 H $300 \mathrm{MHz}$}

\%

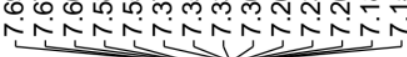

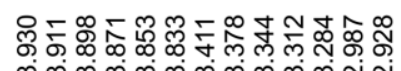

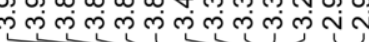
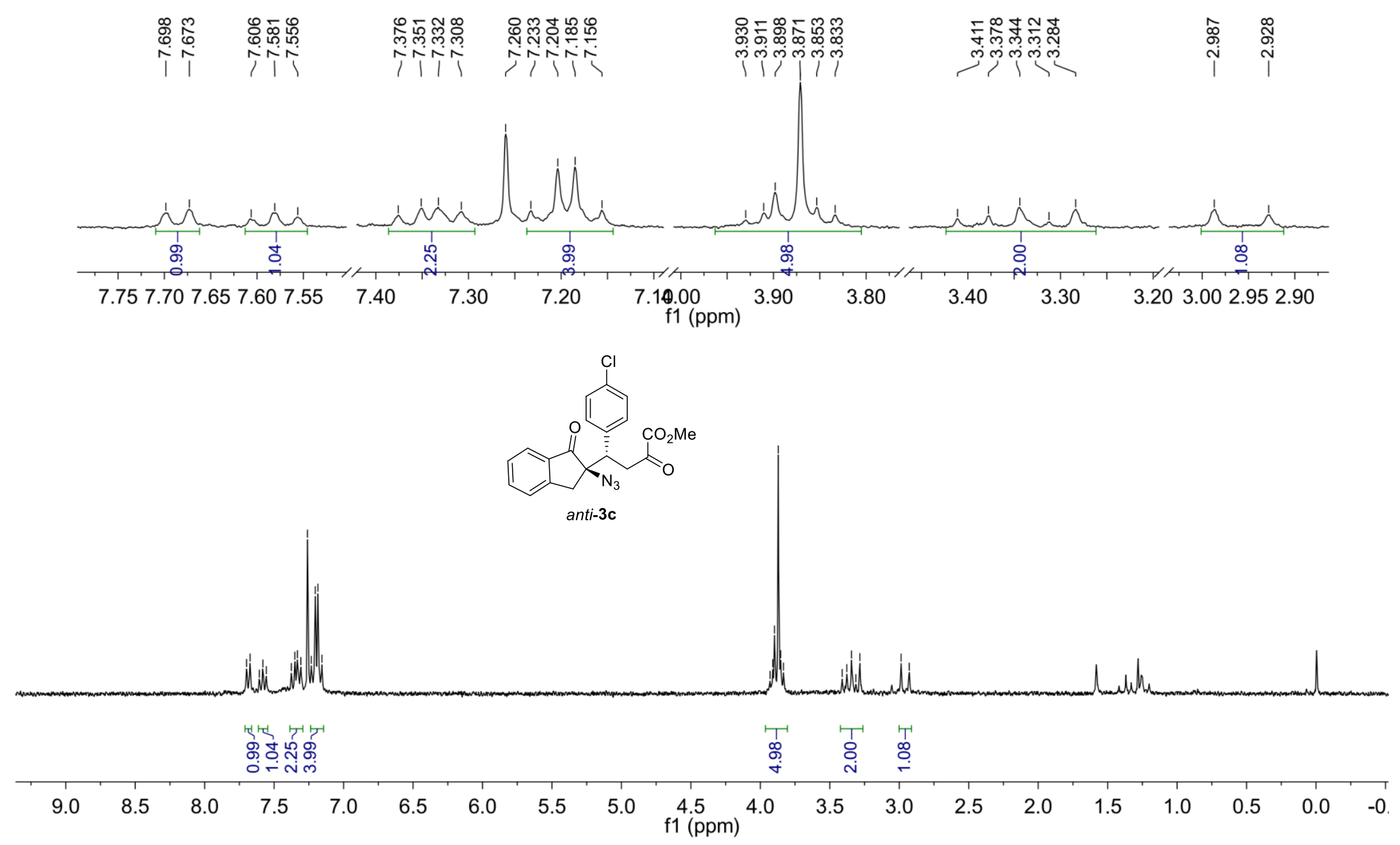
${ }^{13} \mathrm{C}$ NMR of Compound anti-3c $\left(100 \mathrm{MHz}, \mathrm{CDCl}_{3}\right)$

DPG-DN-28-4 C $100 \mathrm{MHz}$

衣 N

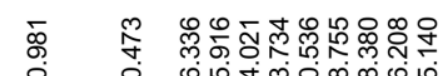

० :

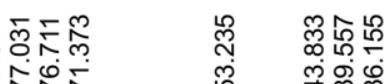

(n)

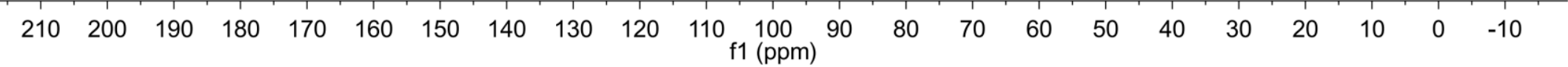


${ }^{1} \mathrm{H} \mathrm{NMR}$ of Compound anti-3d $\left(400 \mathrm{MHz}, \mathrm{CDCl}_{3}\right)$

DPG-DJ-114-3 H 400 MHz

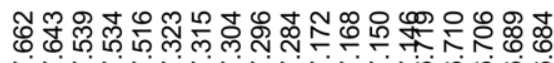

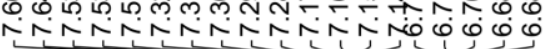

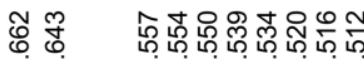

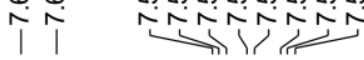

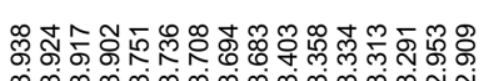

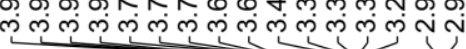

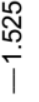

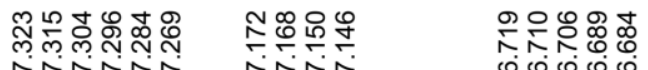

فं

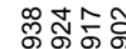

लंग लंग

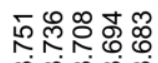

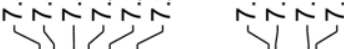

एलंग्रा
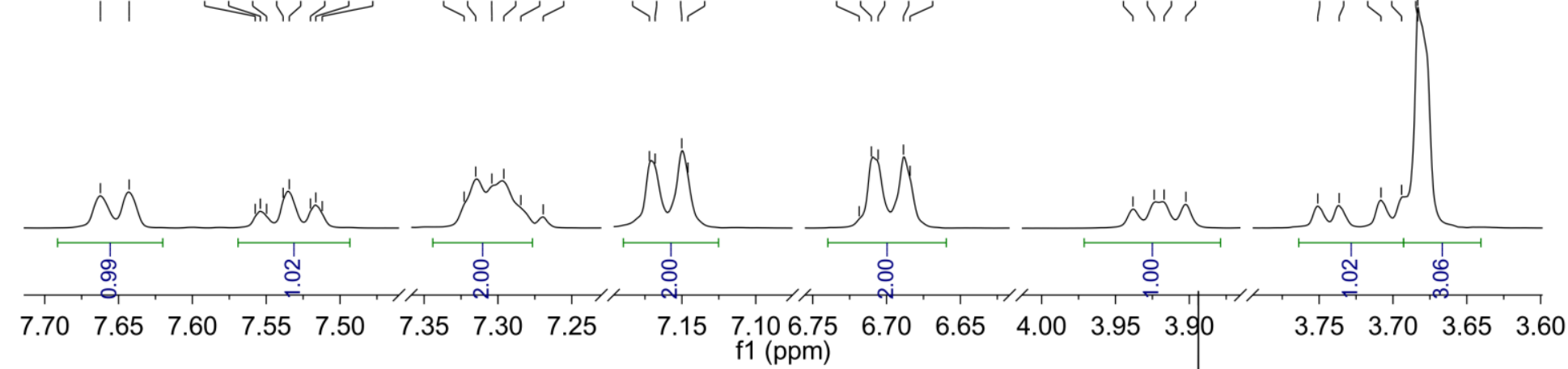

f1 (ppm)

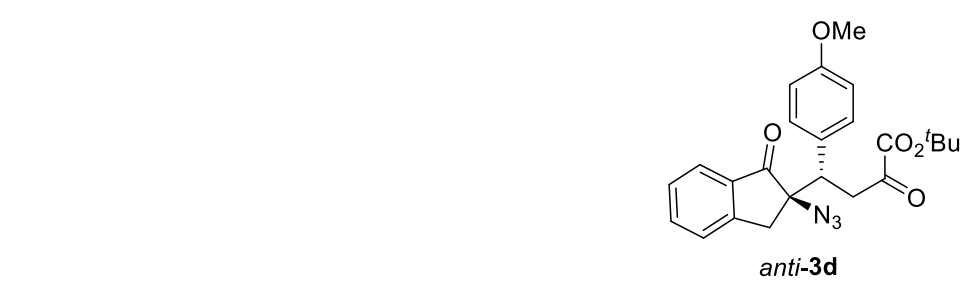

anti-3d

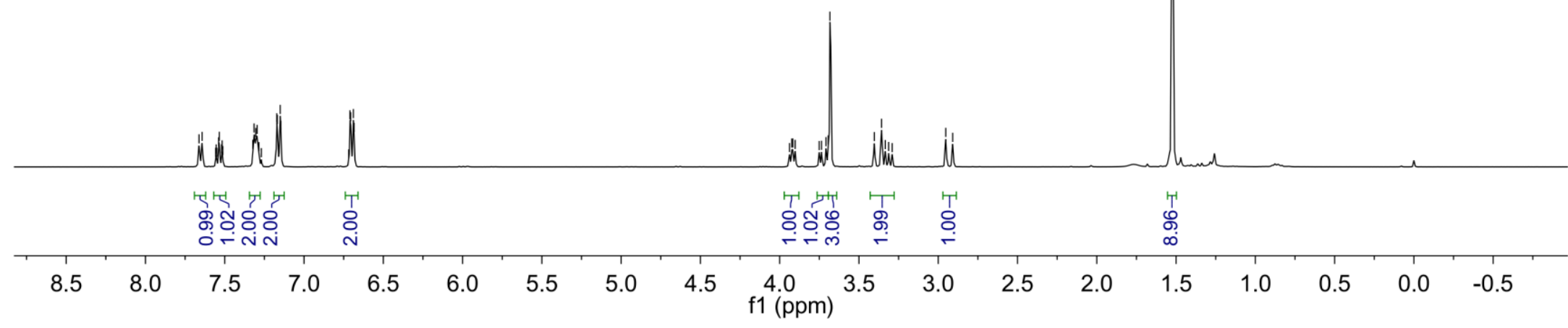


${ }^{13} \mathrm{C}$ NMR of Compound anti-3d $\left(100 \mathrm{MHz}, \mathrm{CDCl}_{3}\right)$

DPG-DJ-114-3 C $100 \mathrm{MHz}$

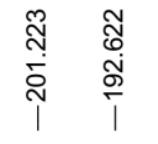

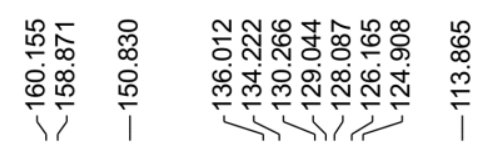

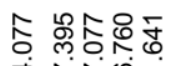

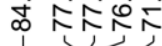

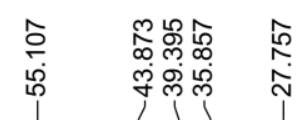

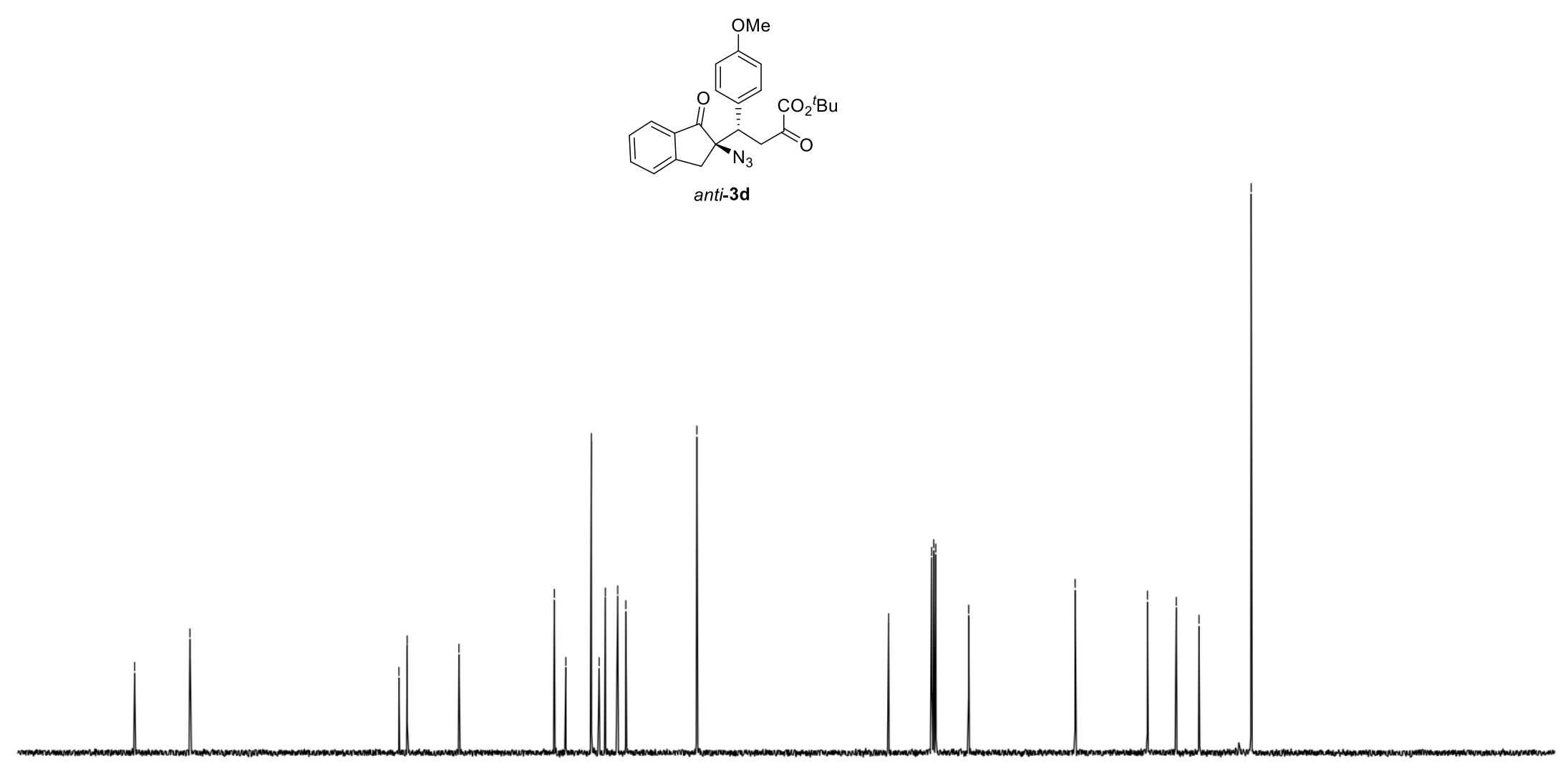

$\begin{array}{llllll}210 & 200 & 190 & 180 & 170 & 160\end{array}$

$\begin{array}{lll}150 & 140 \quad 130\end{array}$

110100 f1 (ppm) 
${ }^{1} \mathrm{H} \mathrm{NMR}$ of Compound anti-3e $\left(400 \mathrm{MHz}, \mathrm{CDCl}_{3}\right)$

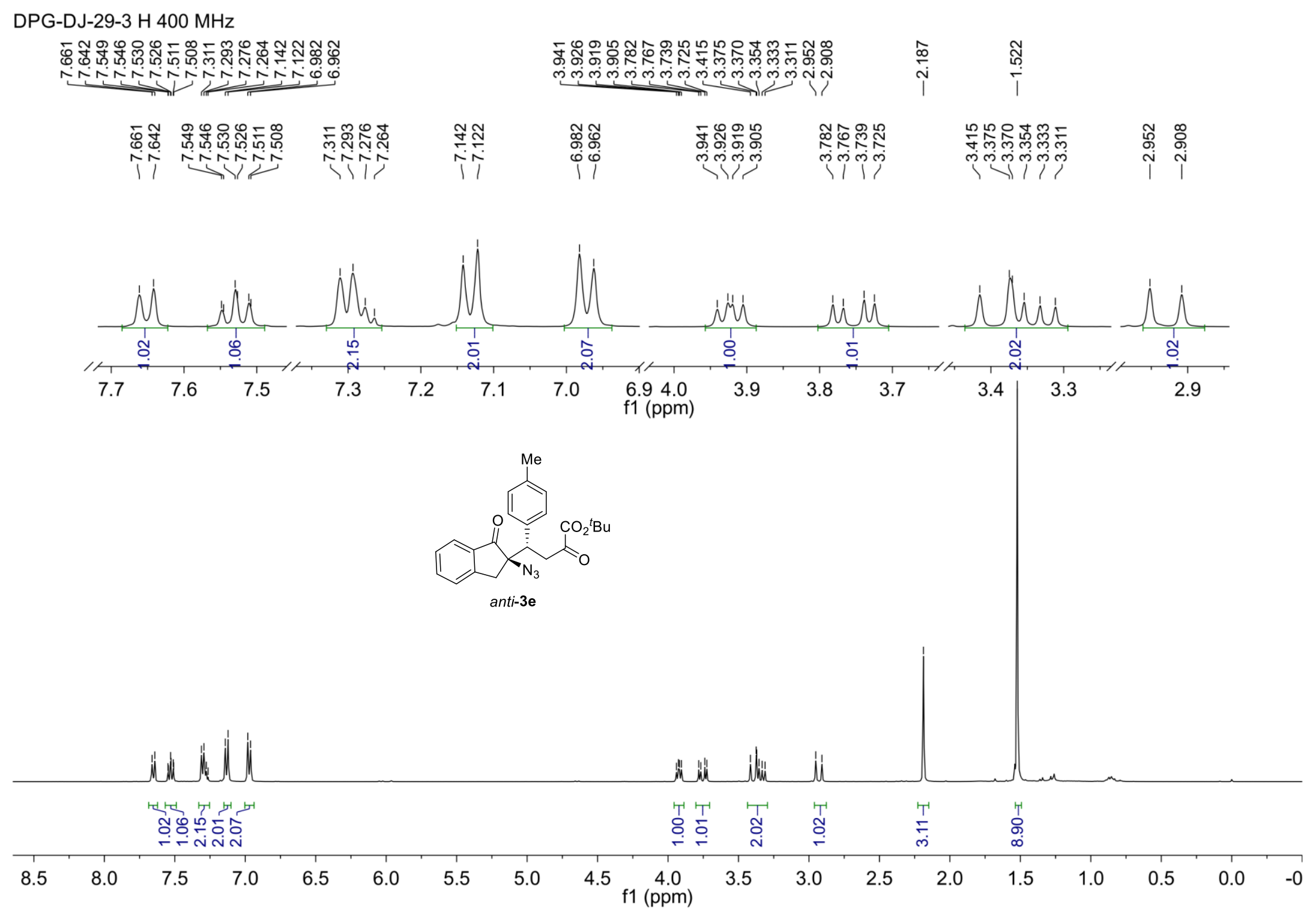


${ }^{13} \mathrm{C}$ NMR of Compound anti-3e $\left(100 \mathrm{MHz}, \mathrm{CDCl}_{3}\right)$

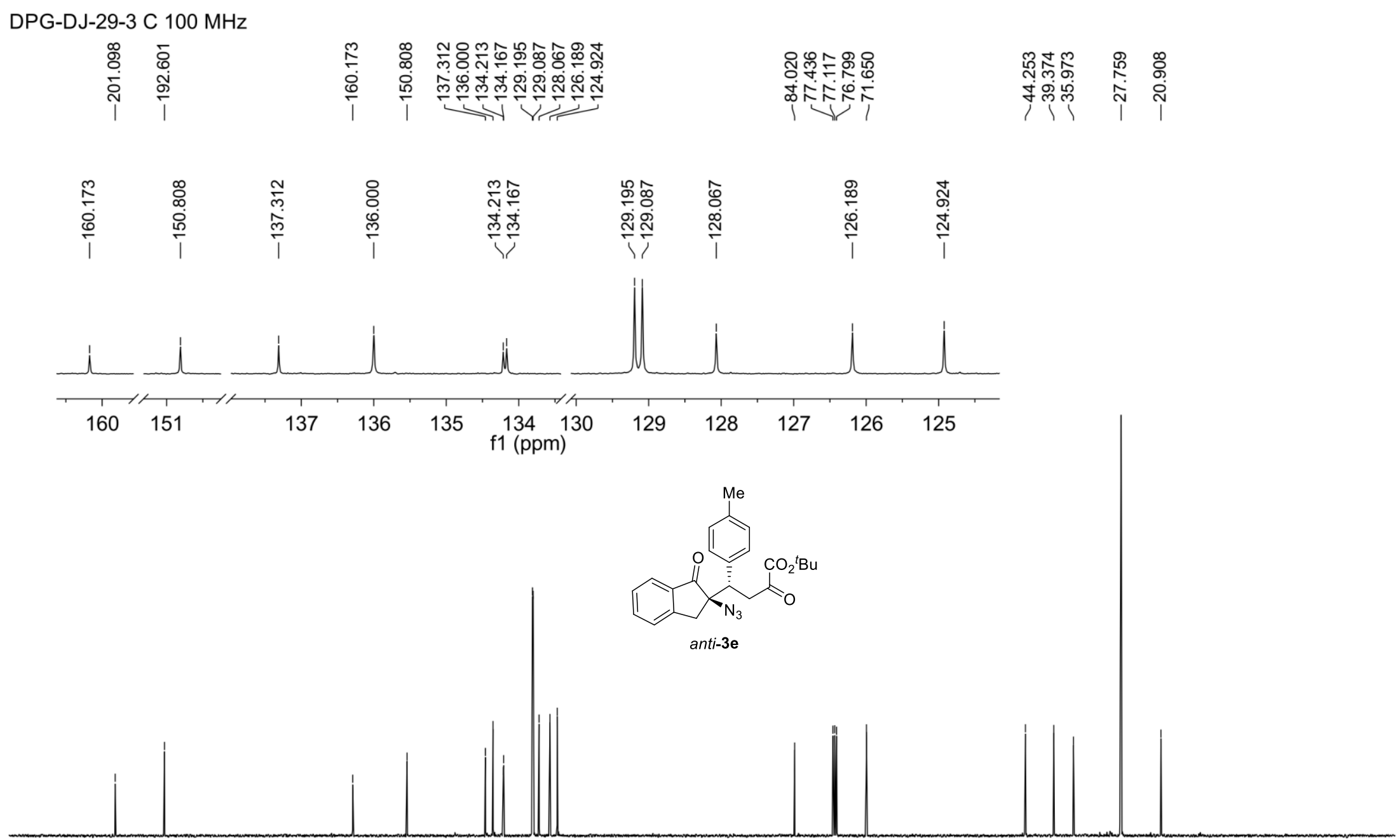

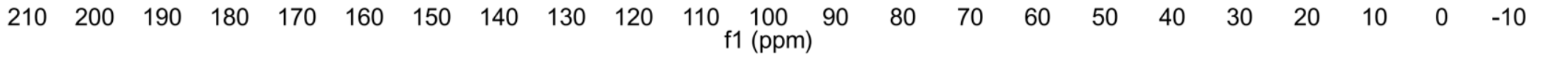


${ }^{1} \mathrm{H}$ NMR of Compound anti-3f (400 MHz, $\left.\mathrm{CDCl}_{3}\right)$

\section{DPG-DJ-114-2 H $400 \mathrm{HMz}$}

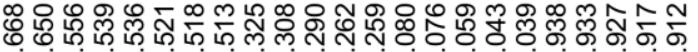

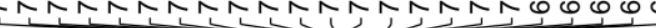

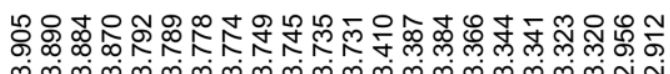

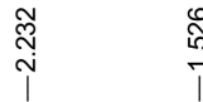

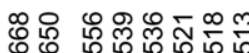

itrivin

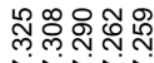

îin

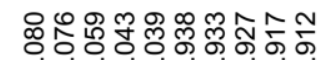

NANTOG00

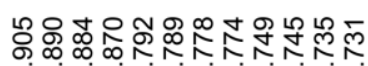

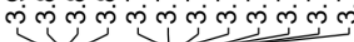

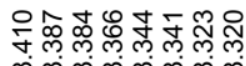

लंलुलंखुल
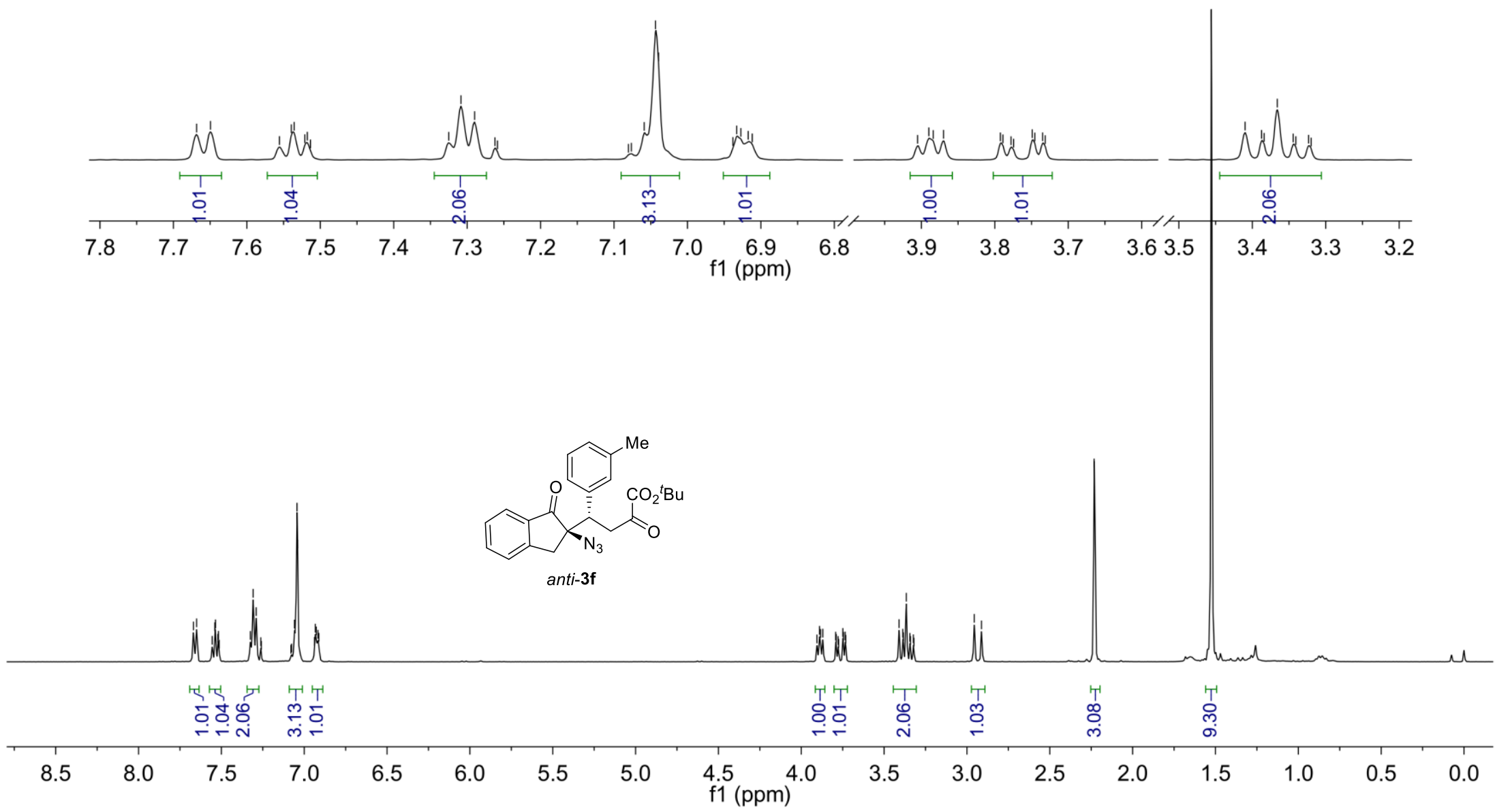
${ }^{13} \mathrm{C}$ NMR of Compound anti-3f $\left(100 \mathrm{MHz}, \mathrm{CDCl}_{3}\right)$

DPG-DJ-114-2 C $100 \mathrm{HMz}$

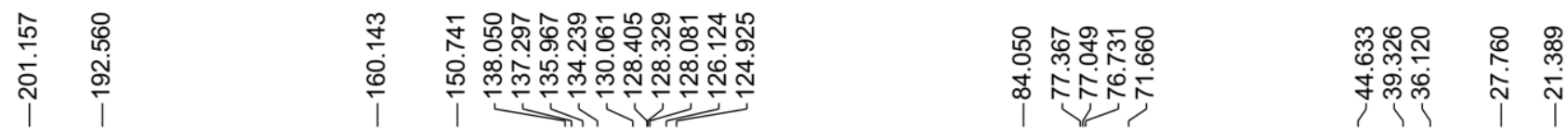

\begin{tabular}{|c|c|c|c|c|}
\hline 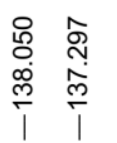 & $\begin{array}{l}\hat{\mathscr{Q}} \\
\stackrel{\rho}{\oplus} \\
\stackrel{m}{\mid}\end{array}$ & 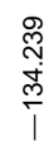 & $\begin{array}{l}\overline{8} \\
\stackrel{9}{\dot{m}}\end{array}$ & 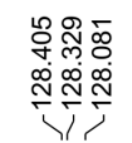 \\
\hline
\end{tabular}

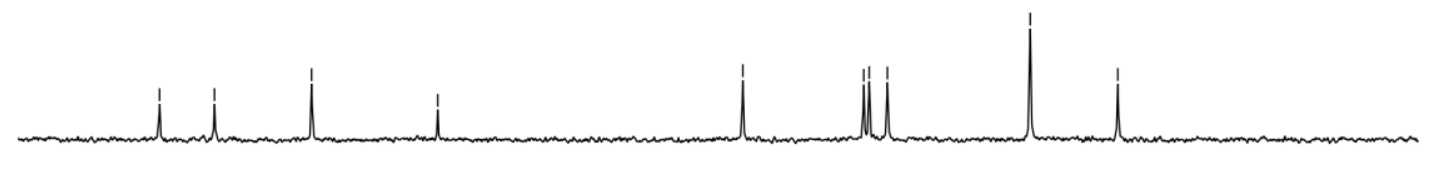

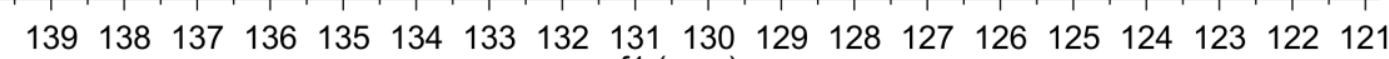
f1 (ppm)

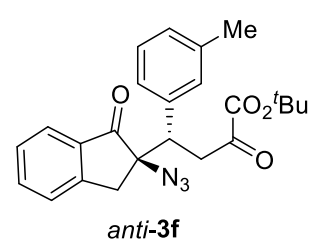

$\begin{array}{lllllll}210 & 200 & 190 & 180 & 170 & 160 & 150\end{array}$

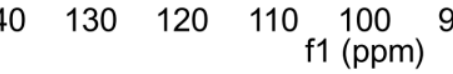

80

$70 \quad 60$

$\begin{array}{llllllllllll}210 & 200 & 190 & 180 & 170 & 160 & 150 & 140 & 130 & 120 & 110 \begin{array}{l}100 \\ \mathrm{f} 1(\mathrm{ppm})\end{array}\end{array}$


${ }^{1} \mathrm{H}$ NMR of Compound anti-3g (300 MHz, $\left.\mathrm{CDCl}_{3}\right)$

\section{DPG-DJ-55-5-A H $300 \mathrm{MHz}$}

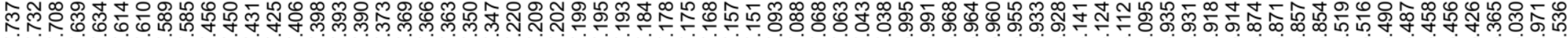

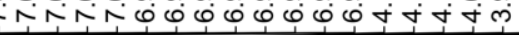

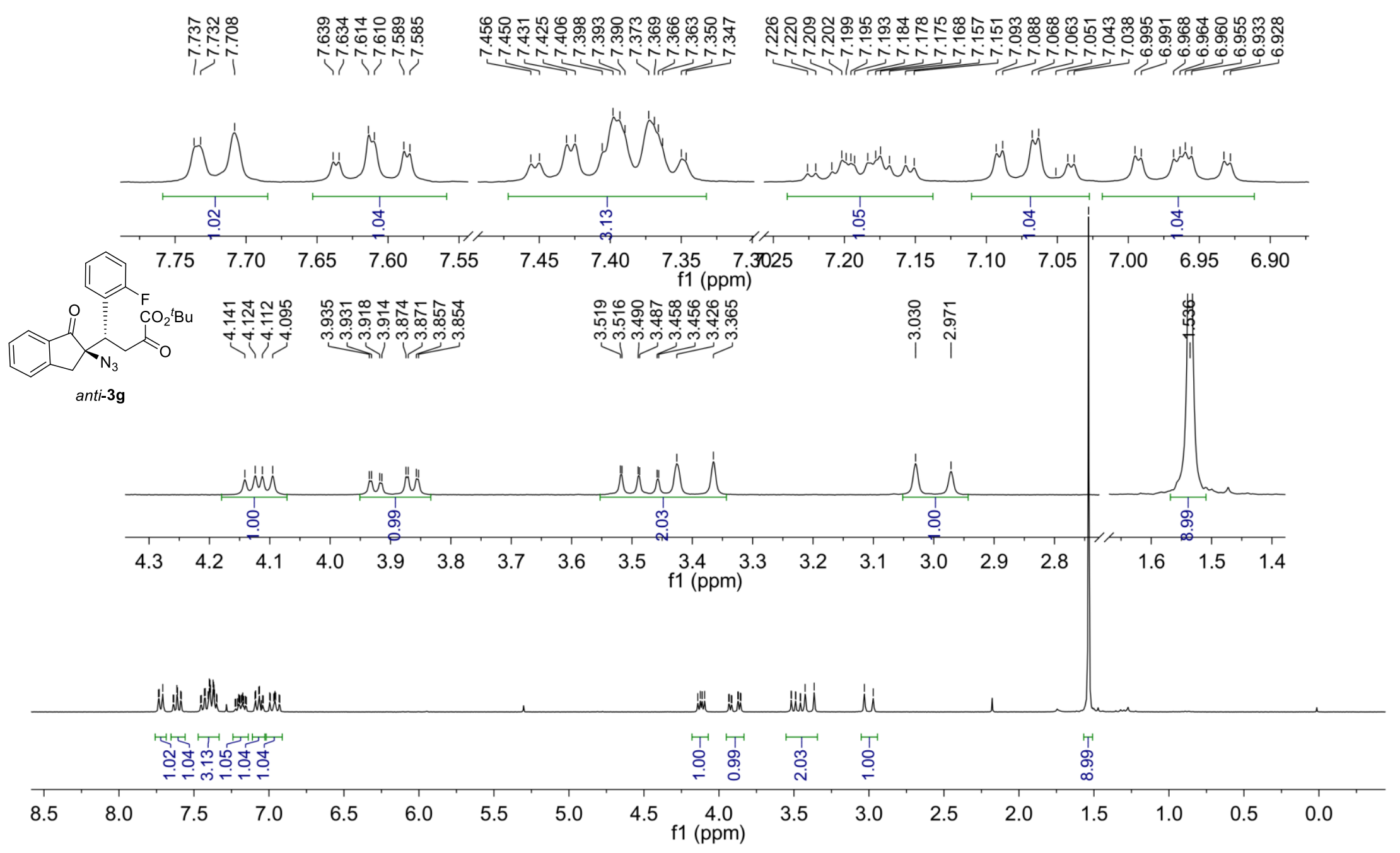


${ }^{19} \mathrm{~F}$ NMR of Compound anti-3g (282 $\left.\mathrm{MHz}, \mathrm{CDCl}_{3}\right)$

DPG-DJ-55-5-A F $282 \mathrm{MHz}$
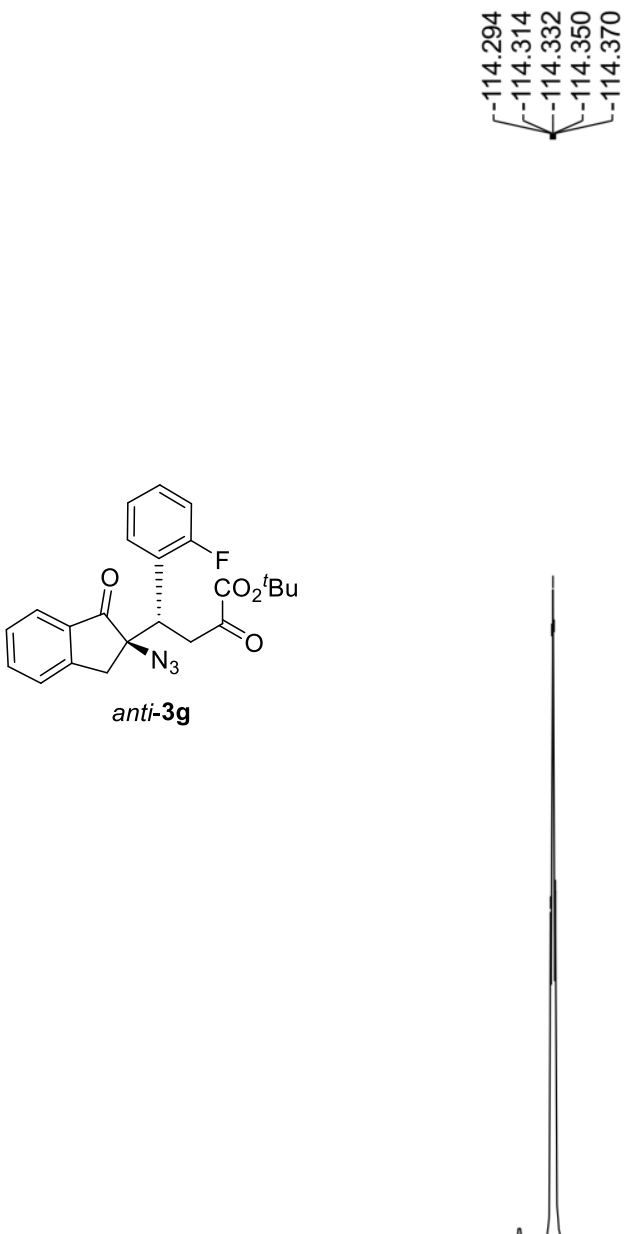

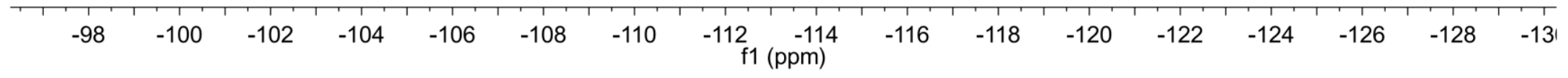


${ }^{13} \mathrm{C}$ NMR of Compound anti-3g $\left(100 \mathrm{MHz}, \mathrm{CDCl}_{3}\right)$

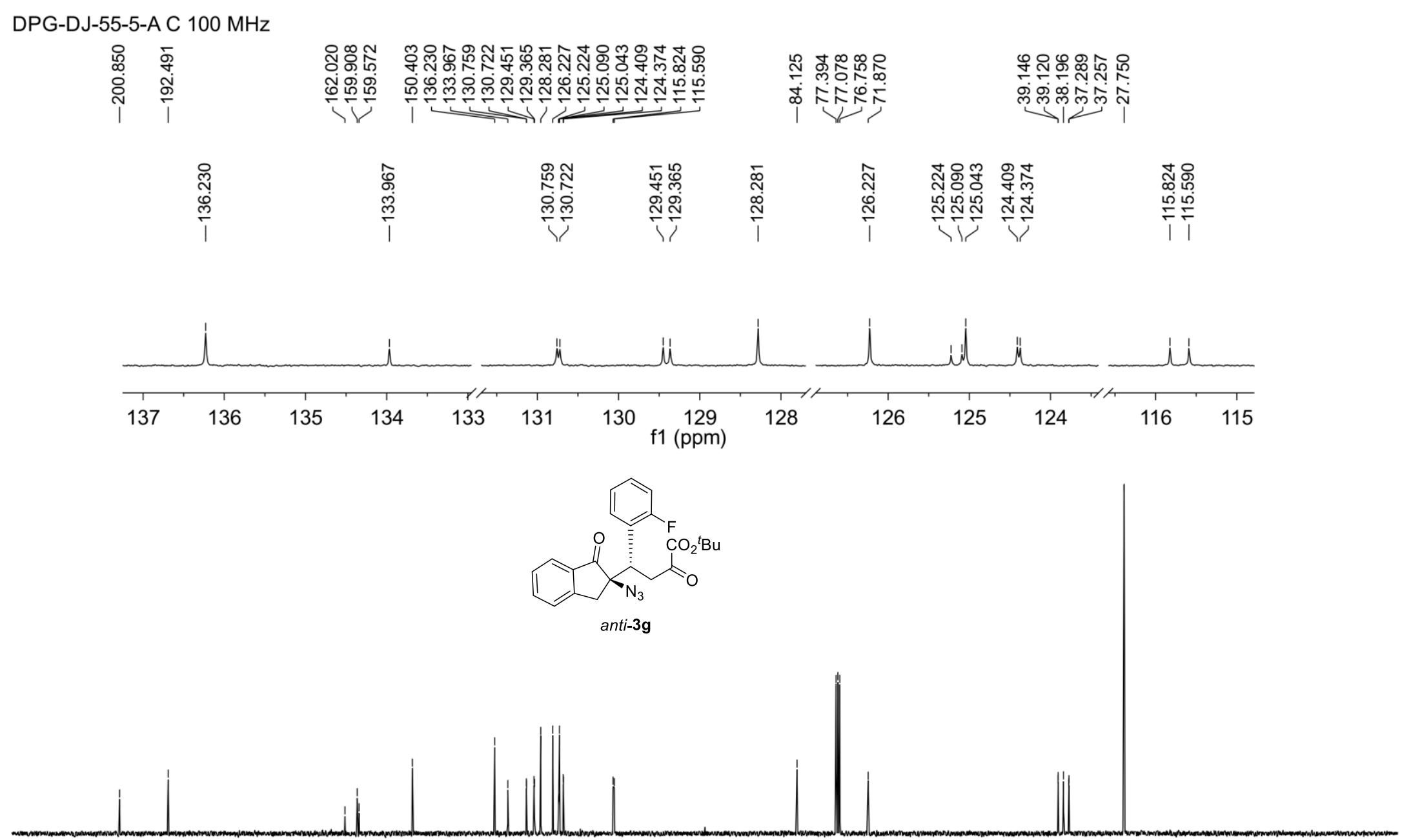

$\begin{array}{lllllllllllllllllllllll}210 & 200 & 190 & 180 & 170 & 160 & 150 & 140 & 130 & 120 & 110 & \begin{array}{c}100 \\ \mathrm{f} 1(\mathrm{ppm})\end{array} & 90 & 80 & 70 & 60 & 50 & 40 & 30 & 20 & 10 & 0 & -10\end{array}$ 
${ }^{1} \mathrm{H}$ NMR of Compound anti-3h $\left(400 \mathrm{MHz}, \mathrm{CDCl}_{3}\right)$

\section{DPG-DJ-114-1 H 400 MHz}

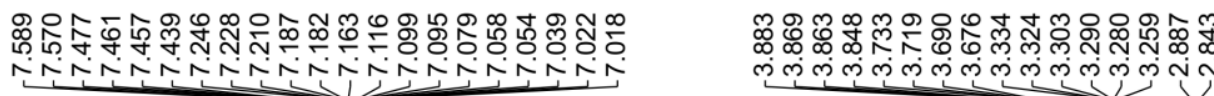

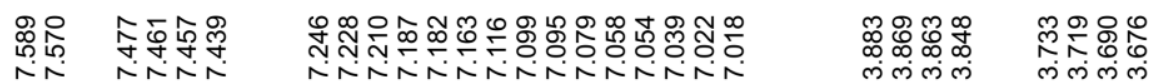

il vinj

लंखिलुक

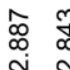

ஸ่
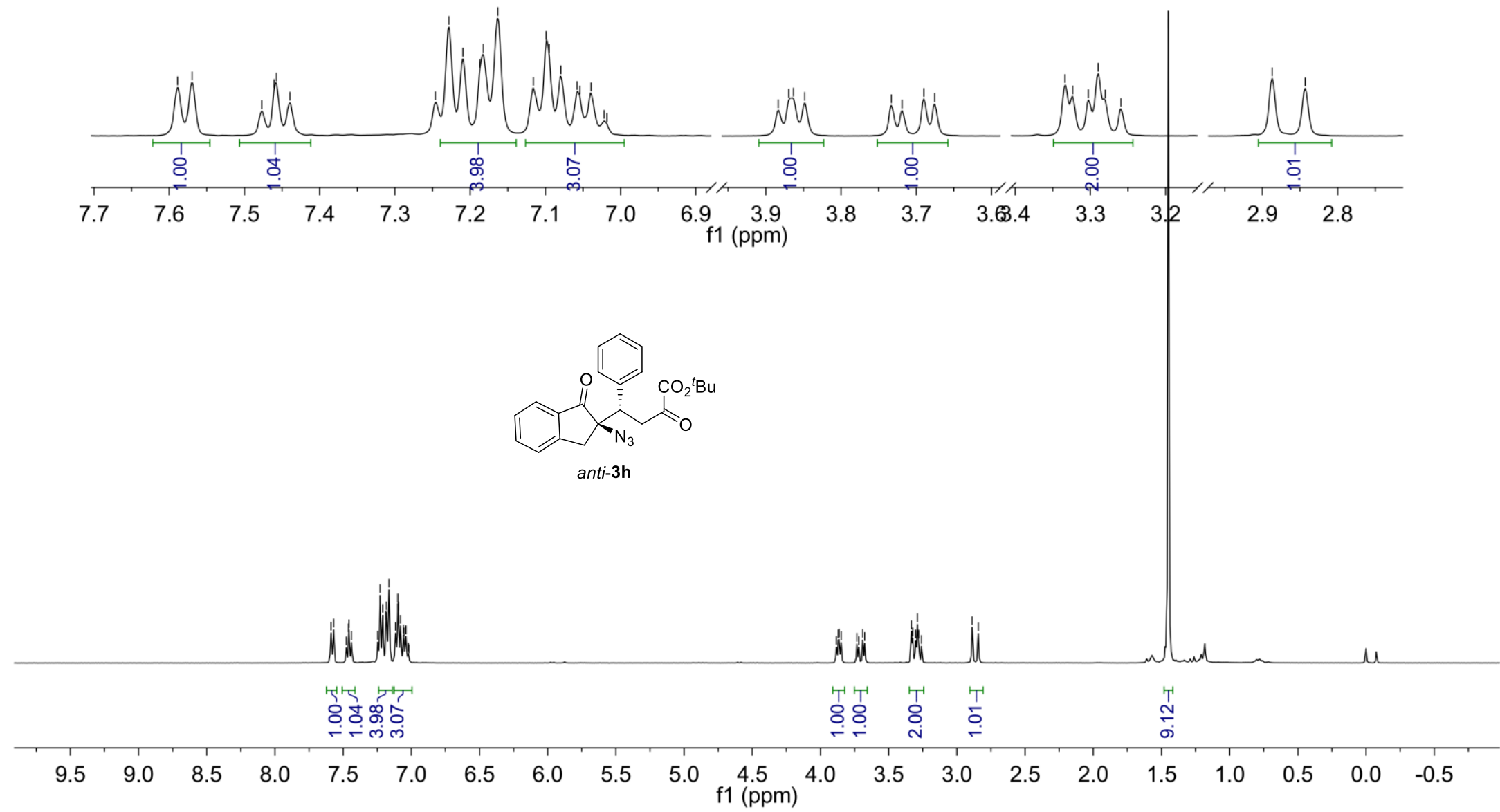
${ }^{13} \mathrm{C}$ NMR of Compound anti-3h $\left(100 \mathrm{MHz}, \mathrm{CDCl}_{3}\right)$

DPG-DJ-114-1 C $100 \mathrm{MHz}$

仓ัল

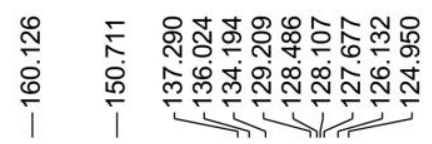

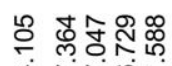

却造

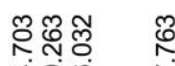

उळ्लं N

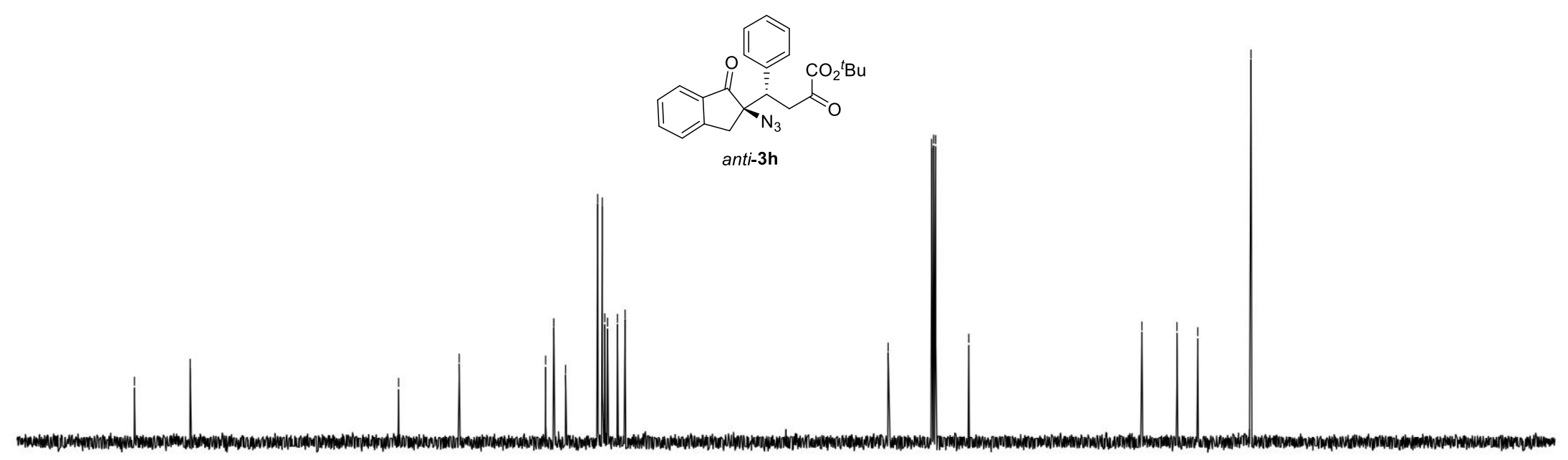

$\begin{array}{lllllllllllllllllllllll}210 & 200 & 190 & 180 & 170 & 160 & 150 & 140 & 130 & 120 & 110 & \begin{array}{l}100 \\ \mathrm{f} 1(\mathrm{ppm})\end{array} & 90 & 80 & 70 & 60 & 50 & 40 & 30 & 20 & 10 & 0 & -10\end{array}$ 
${ }^{1} \mathrm{H} \mathrm{NMR}$ of Compound anti-3i $\left(400 \mathrm{MHz}, \mathrm{CDCl}_{3}\right)$

DPG-DJ-55-2-A H $400 \mathrm{MHz}$

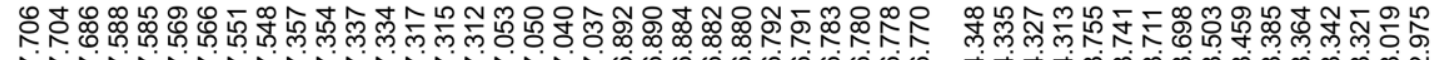

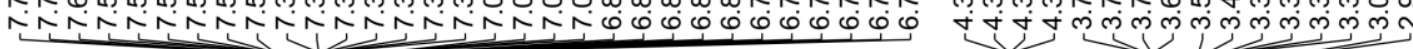

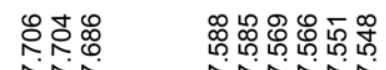

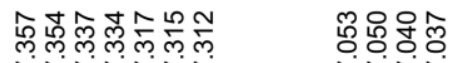

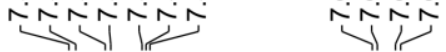

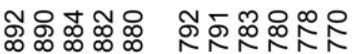

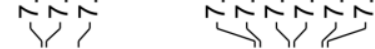

$\rightarrow 11$

0000 0 00000

11
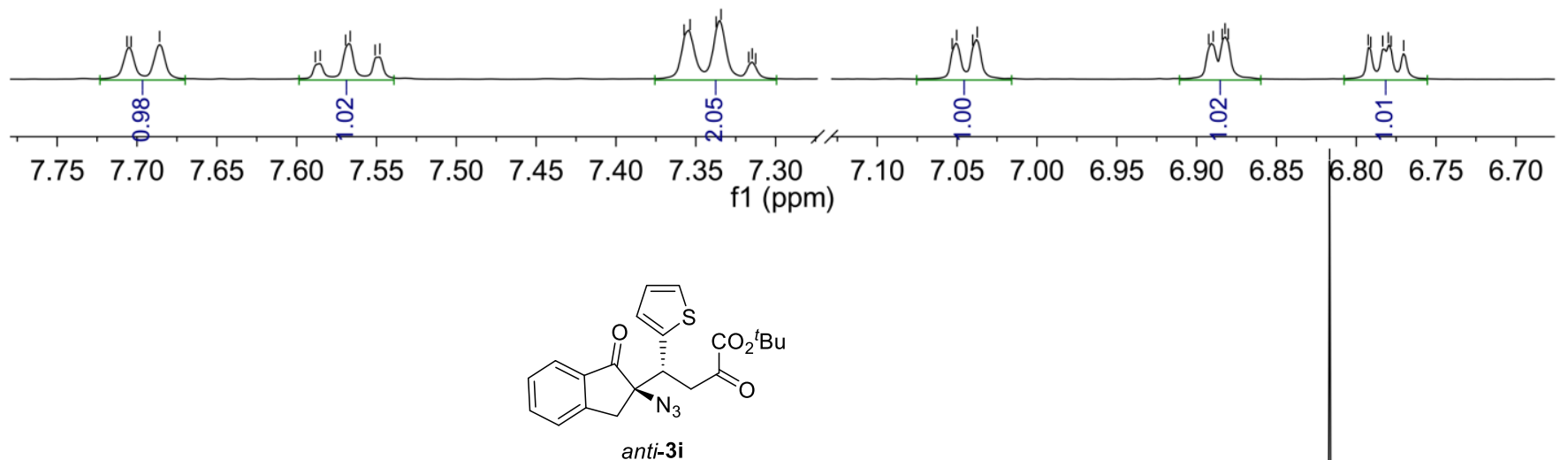

anti-3i

Hith H H in

\begin{tabular}{|c|c|c|c|c|c|c|c|c|c|c|c|c|c|c|c|c|c|c|}
\hline & & 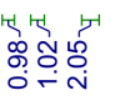 & 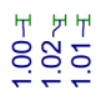 & & & & & $\stackrel{\text { T⿱ }}{\circ}$ & & 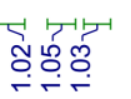 & 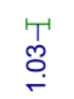 & & & 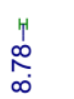 & & & & \\
\hline 8.5 & 8.0 & 7.5 & 7.0 & 6.5 & 6.0 & 5.5 & 5.0 & 4.5 & $\begin{array}{c}4.0 \\
1(\mathrm{ppm})\end{array}$ & 3.5 & 3.0 & 2.5 & 2.0 & 1.5 & 1.0 & 0.5 & 0.0 & -0.5 \\
\hline
\end{tabular}


${ }^{13} \mathrm{C}$ NMR of Compound anti-3i $\left(100 \mathrm{MHz}, \mathrm{CDCl}_{3}\right)$

DPG-DJ-55-2-A C $100 \mathrm{MHz}$

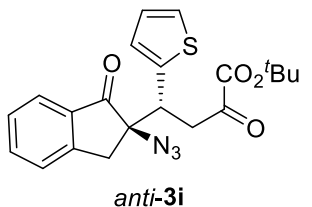


${ }^{1} \mathrm{H}$ NMR of Compound anti-3j $\left(400 \mathrm{MHz}, \mathrm{CDCl}_{3}\right)$

\section{DPG-DJ-55-3-A H $400 \mathrm{MHz}$}

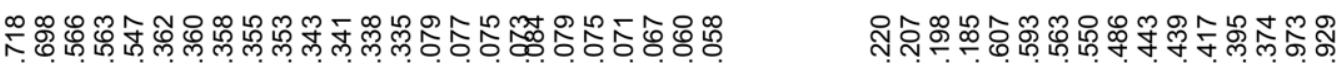

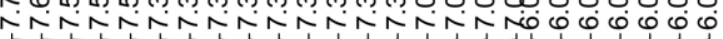

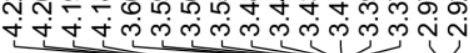

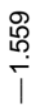
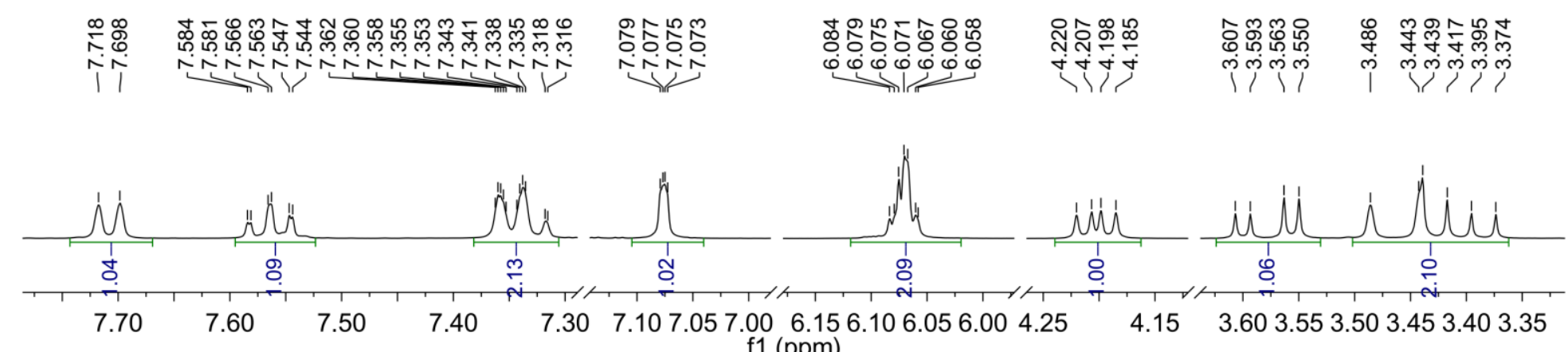

$\mathrm{f1}(\mathrm{ppm})$

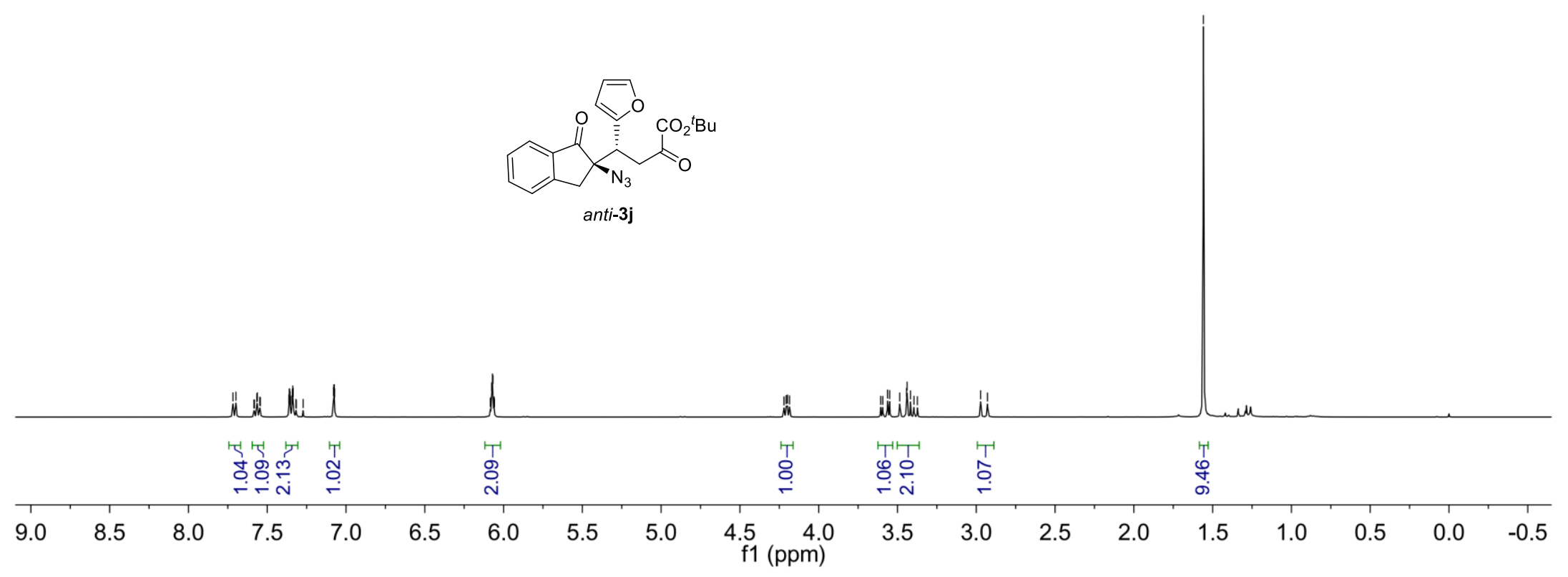


${ }^{13} \mathrm{C}$ NMR of Compound anti-3j $\left(100 \mathrm{MHz}, \mathrm{CDCl}_{3}\right)$

DPG-DJ-55-3-A C $100 \mathrm{MHz}$

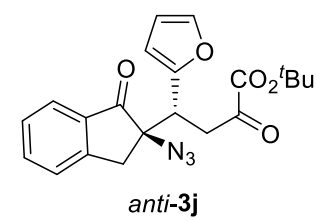


${ }^{1} \mathrm{H} \mathrm{NMR}$ of Compound anti-3k (400 MHz, $\mathrm{CDCl}_{3}$ )

DPG-DJ-112-1 H $400 \mathrm{MHz}$

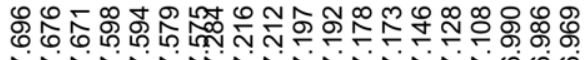

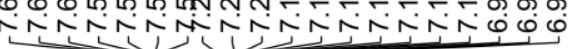

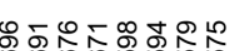

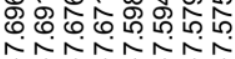

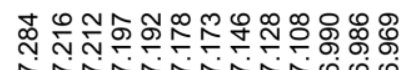

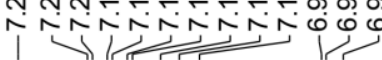
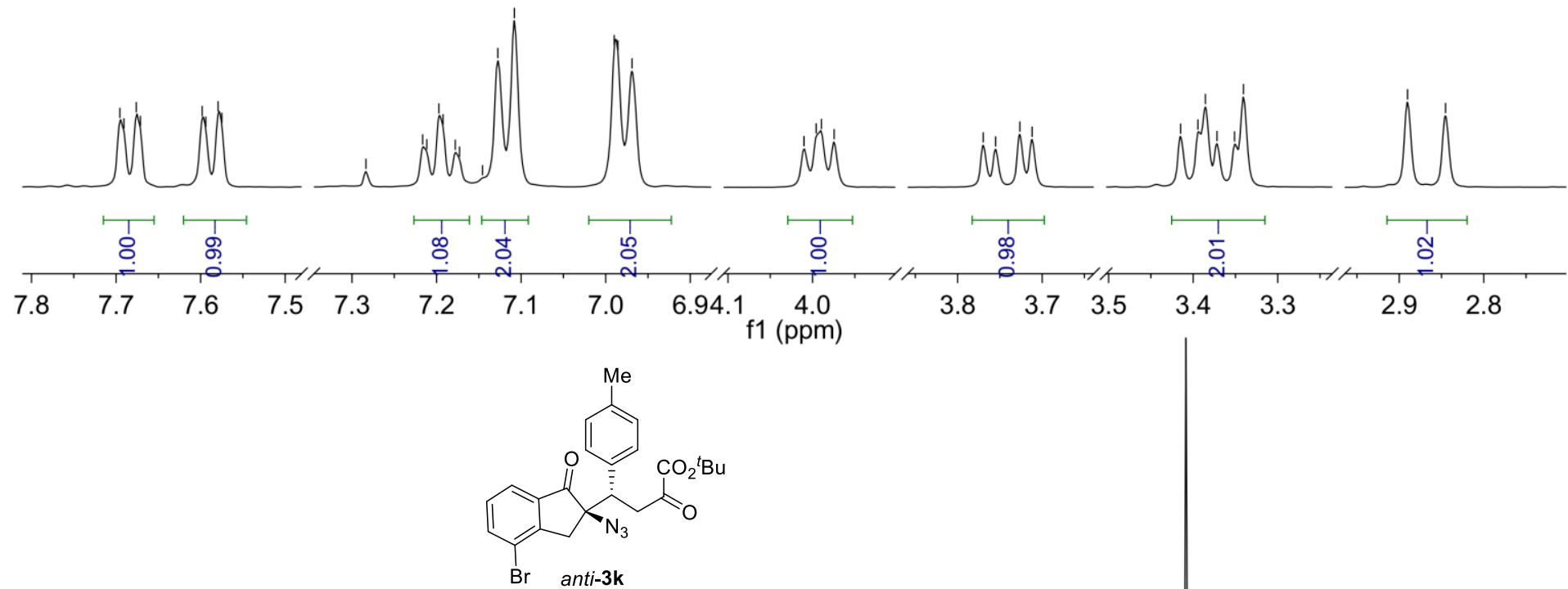

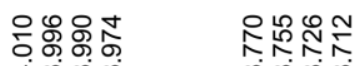

कुंखिय

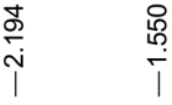

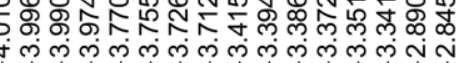

मंल

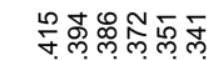

लंखिलाल

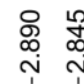

I

f1 (ppm)
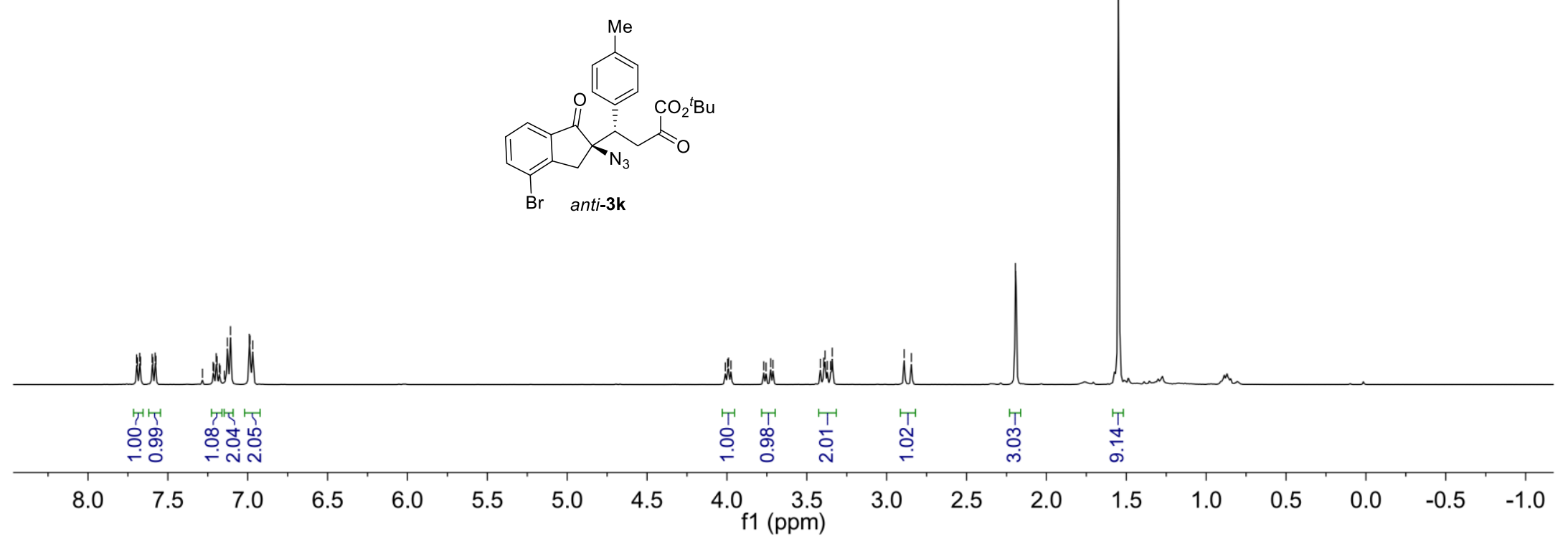
${ }^{13} \mathrm{C}$ NMR of Compound anti-3k $\left(100 \mathrm{MHz}, \mathrm{CDCl}_{3}\right)$

DPG-DJ-112-1 C 100 MHz

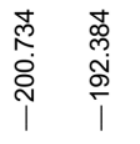

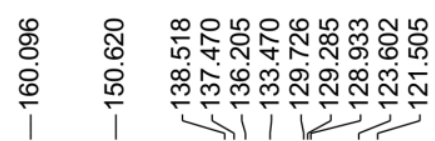

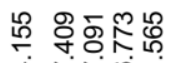

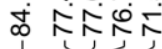

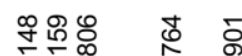

अं্्ल ते ले
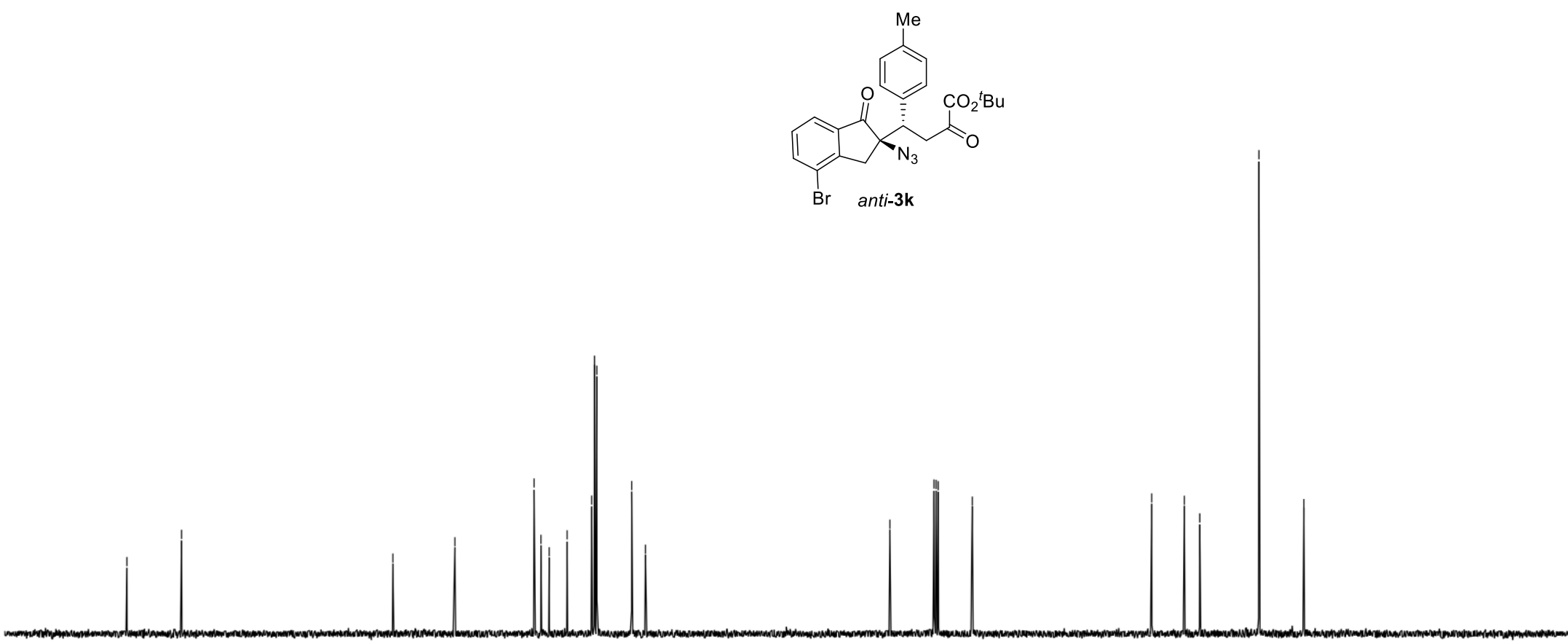

210

$\begin{array}{lllll}200 & 190 & 180 & 170 & 160\end{array}$

$150 \quad 140 \quad 130$

120

$\stackrel{100}{f 1(p p m)} 90$

80

$60 \quad 50$

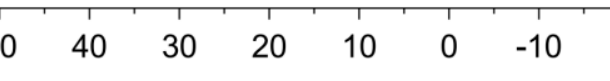


${ }^{1} \mathrm{H}$ NMR of Compound anti-3l (400 MHz, $\left.\mathrm{CDCl}_{3}\right)$
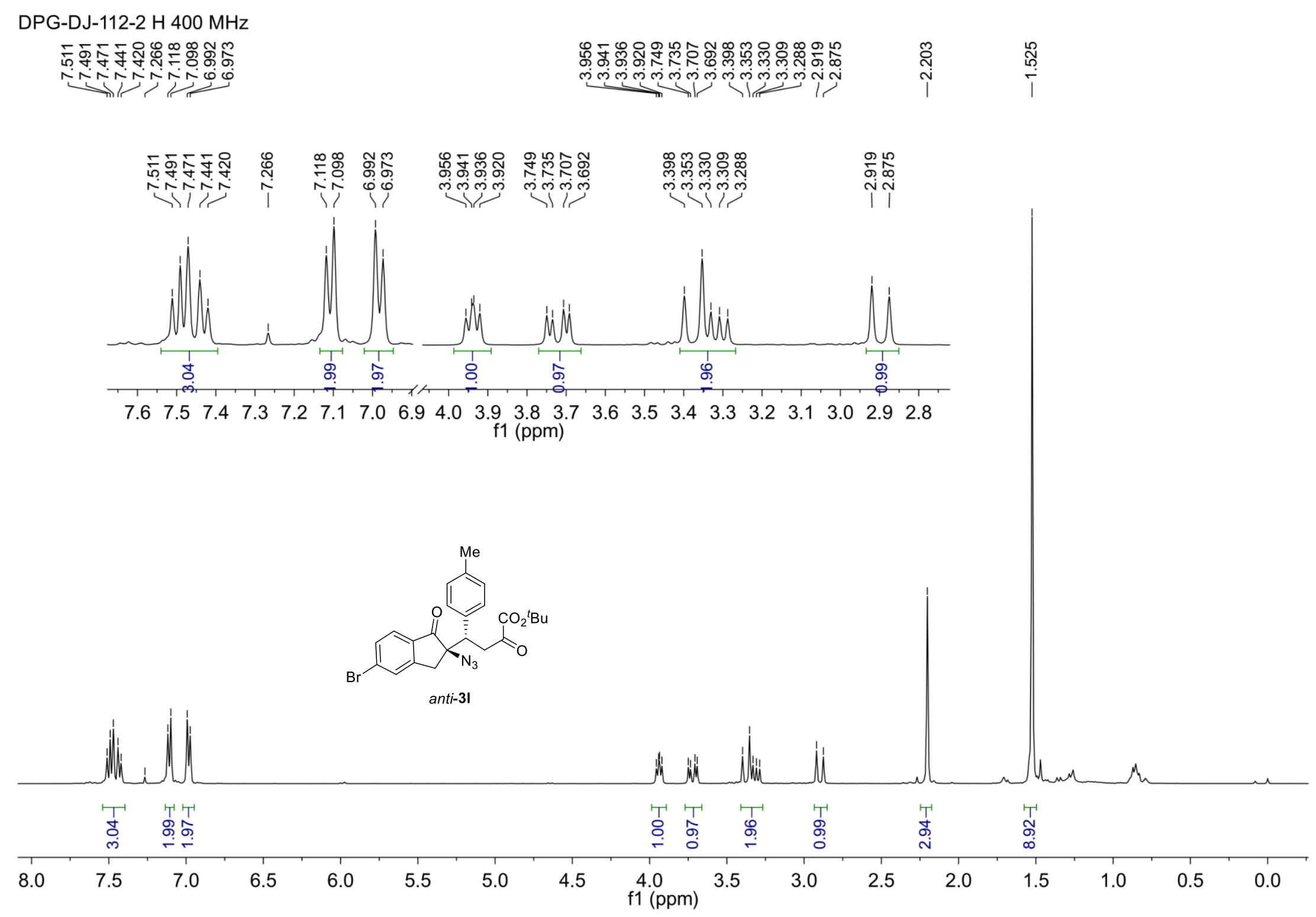
${ }^{13} \mathrm{C}$ NMR of Compound anti-3l $\left(100 \mathrm{MHz}, \mathrm{CDCl}_{3}\right)$

DPG-DJ-112-2 C 100 MHz

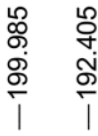

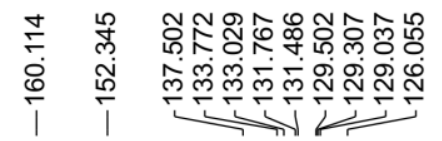

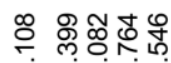

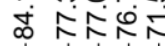

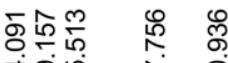

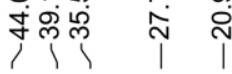

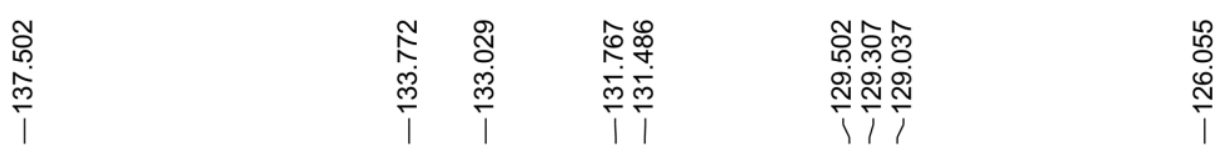
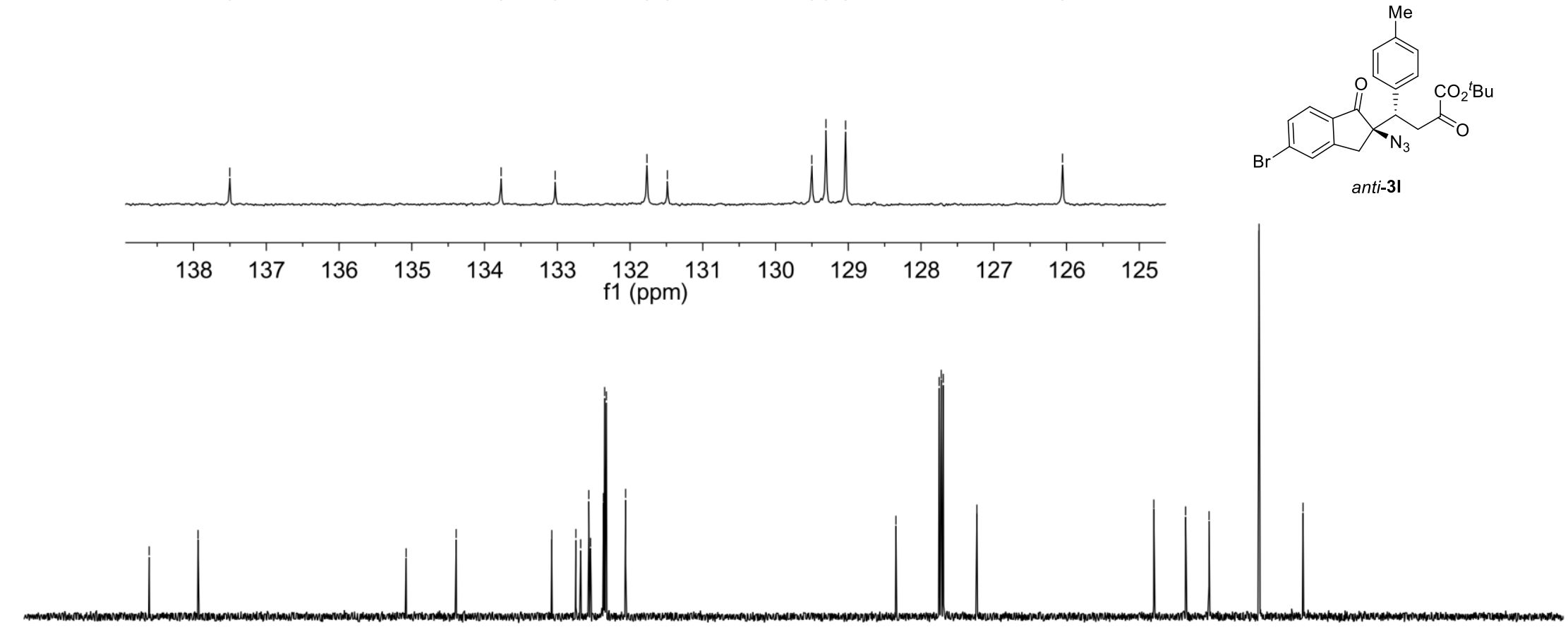

$210 \quad 200 \quad 190$

$\begin{array}{llll}170 & 170 & 160 & 150\end{array}$

$\begin{array}{llll}30 & 120 & 110 & 100\end{array}$ f1 (ppm)

80

$70 \quad 60$

$60 \quad 40$

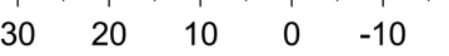


${ }^{1} \mathrm{H}$ NMR of Compound $(R, S)-\mathbf{3 m}\left(400 \mathrm{MHz}, \mathrm{CDCl}_{3}\right)$

\section{DPG-DJ-46-1 H 400 MHz}

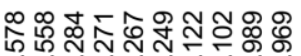

NiNiño

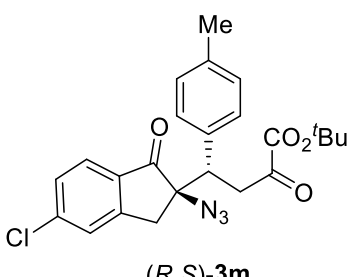

$(R, S)-3 \mathrm{~m}$

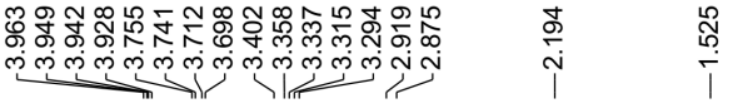

$\mathrm{CO}_{2}{ }^{t} \mathrm{Bu}$

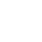

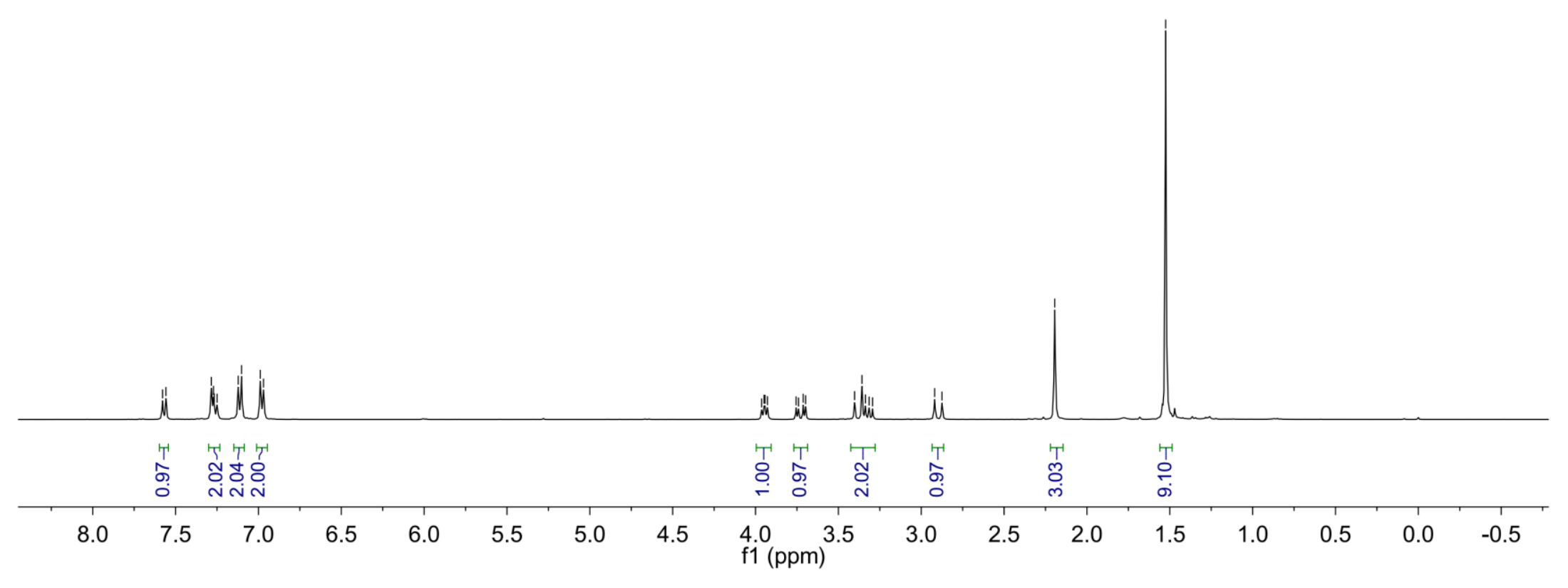


${ }^{13} \mathrm{C}$ NMR of Compound $(R, S)-3 \mathbf{m}\left(100 \mathrm{MHz}, \mathrm{CDCl}_{3}\right)$

DPG-DJ-46-1 C $100 \mathrm{MHz}$

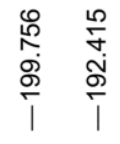

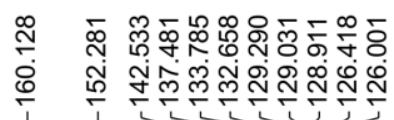

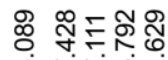

करतिए

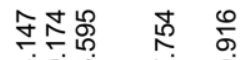

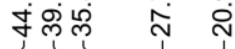

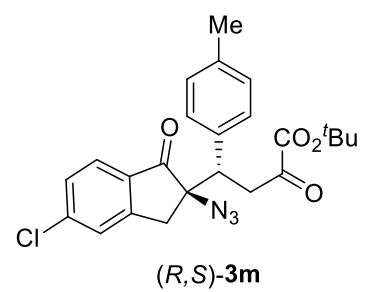

$(R, S)-3 \mathrm{~m}$

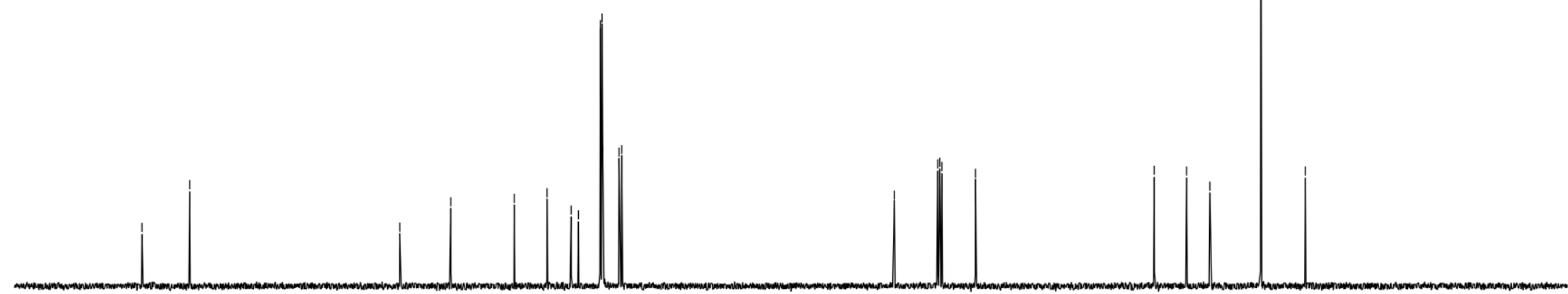

$\begin{array}{lllll}200 & 190 & 180 & 170 & 160\end{array}$

$150 \quad 140 \quad 130$

110100 $\mathrm{f1}(\mathrm{ppm})$

80

$\begin{array}{llll}60 & 50 \quad 40 \quad 30\end{array}$

30

2010

$0 \quad-10$ 
${ }^{1} \mathrm{H}$ NMR of Compound $(S, R)-\mathbf{3 m}\left(400 \mathrm{MHz}, \mathrm{CDCl}_{3}\right)$

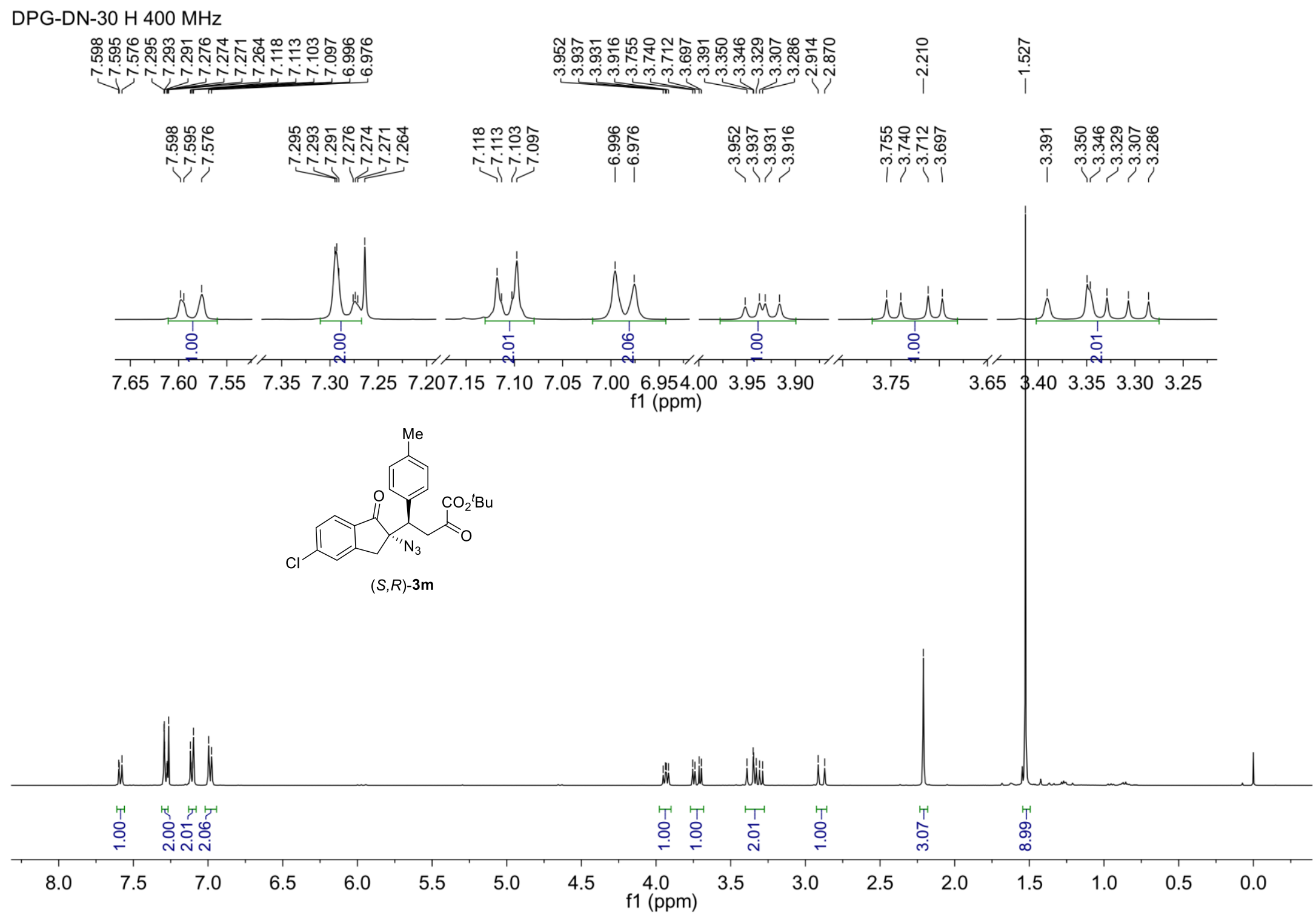


${ }^{13} \mathrm{C}$ NMR of Compound $(S, R)-3 m\left(125 \mathrm{MHz}, \mathrm{CDCl}_{3}\right)$

\section{DPG-DN-30 C $125 \mathrm{MHz}$}

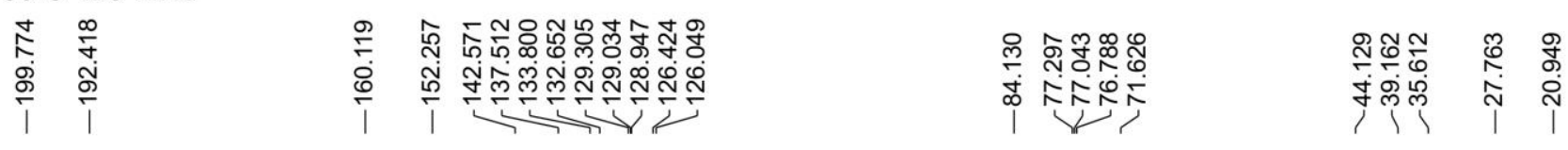

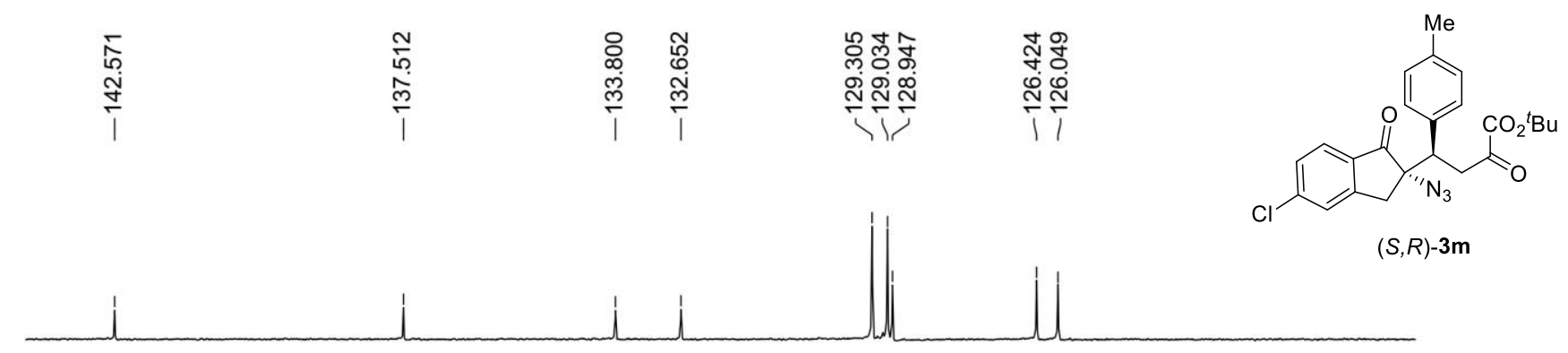

144143142141140139138137136135134133132131130129128127126125124123122121120 f1 (ppm)

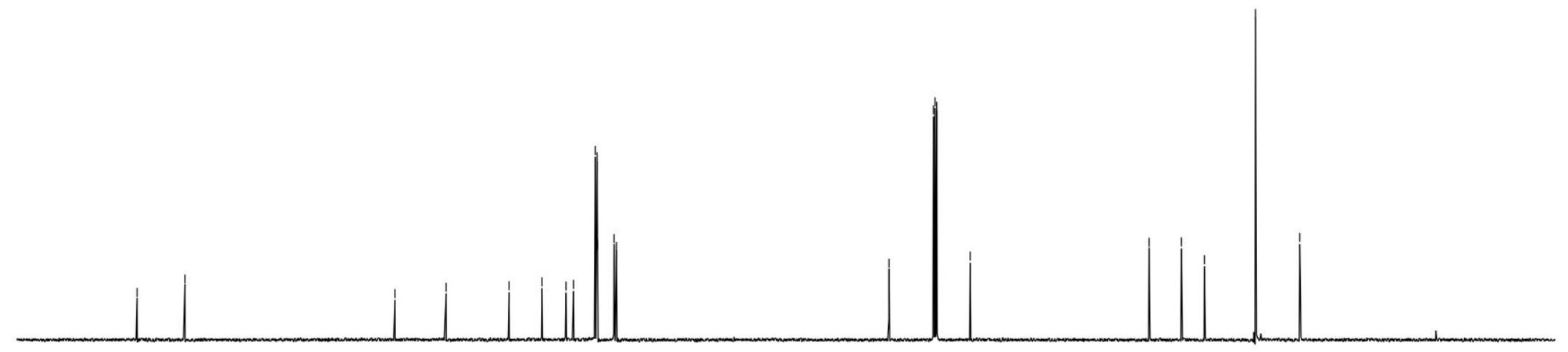

$\begin{array}{lllllllllllllllllllllll}210 & 200 & 190 & 180 & 170 & 160 & 150 & 140 & 130 & 120 & 110 & \begin{array}{l}100 \\ \mathrm{f} 1(\mathrm{ppm})\end{array} & 90 & 80 & 70 & 60 & 50 & 40 & 30 & 20 & 10 & 0 & -10\end{array}$ 
${ }^{1} \mathrm{H} \mathrm{NMR}$ of Compound anti-3n $\left(300 \mathrm{MHz}, \mathrm{CDCl}_{3}\right)$

\section{DPG-DJ-112-3 H $300 \mathrm{MHz}$}

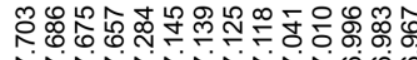

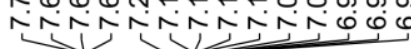

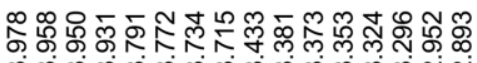

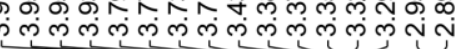

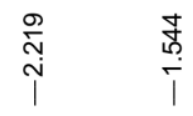

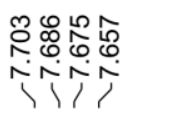

i

둥요용

गंगु

करำ

兽

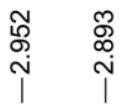
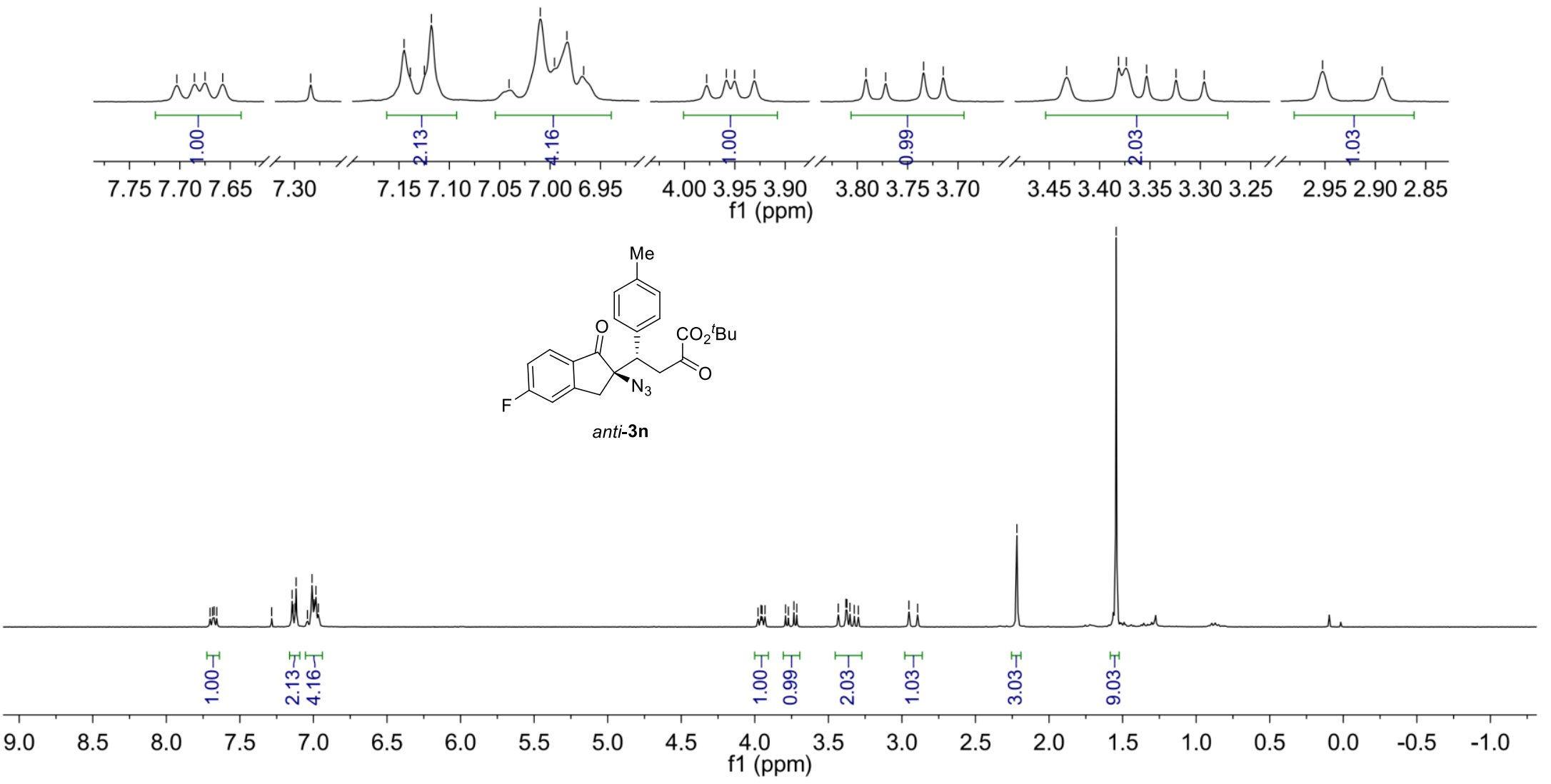

anti-3n $1(\mathrm{ppm})$ 
${ }^{19} \mathrm{~F}$ NMR of Compound anti-3n $\left(282 \mathrm{MHz}, \mathrm{CDCl}_{3}\right)$

DPG-DJ-112-3 F 282 MHz

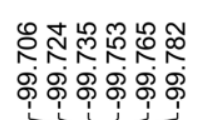

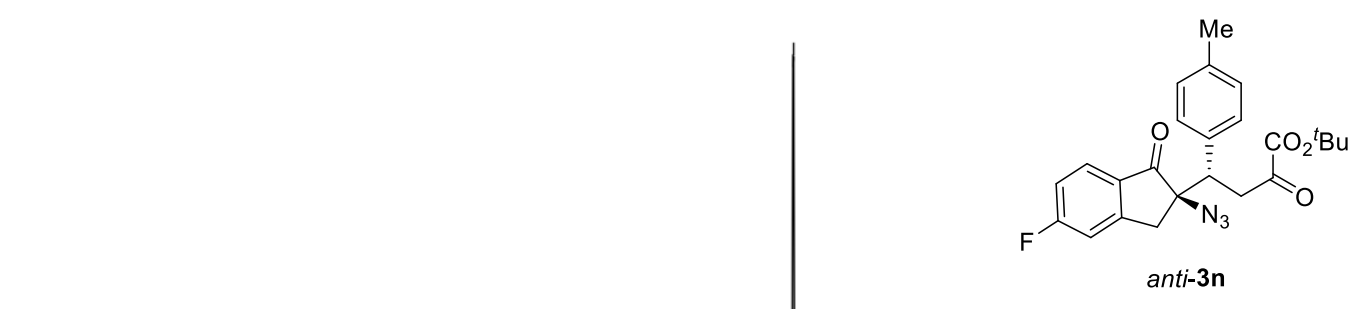

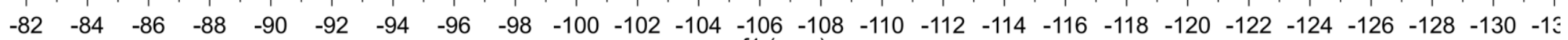
f1 (ppm) 
${ }^{13} \mathrm{C}$ NMR of Compound anti-3n $\left(100 \mathrm{MHz}, \mathrm{CDCl}_{3}\right)$

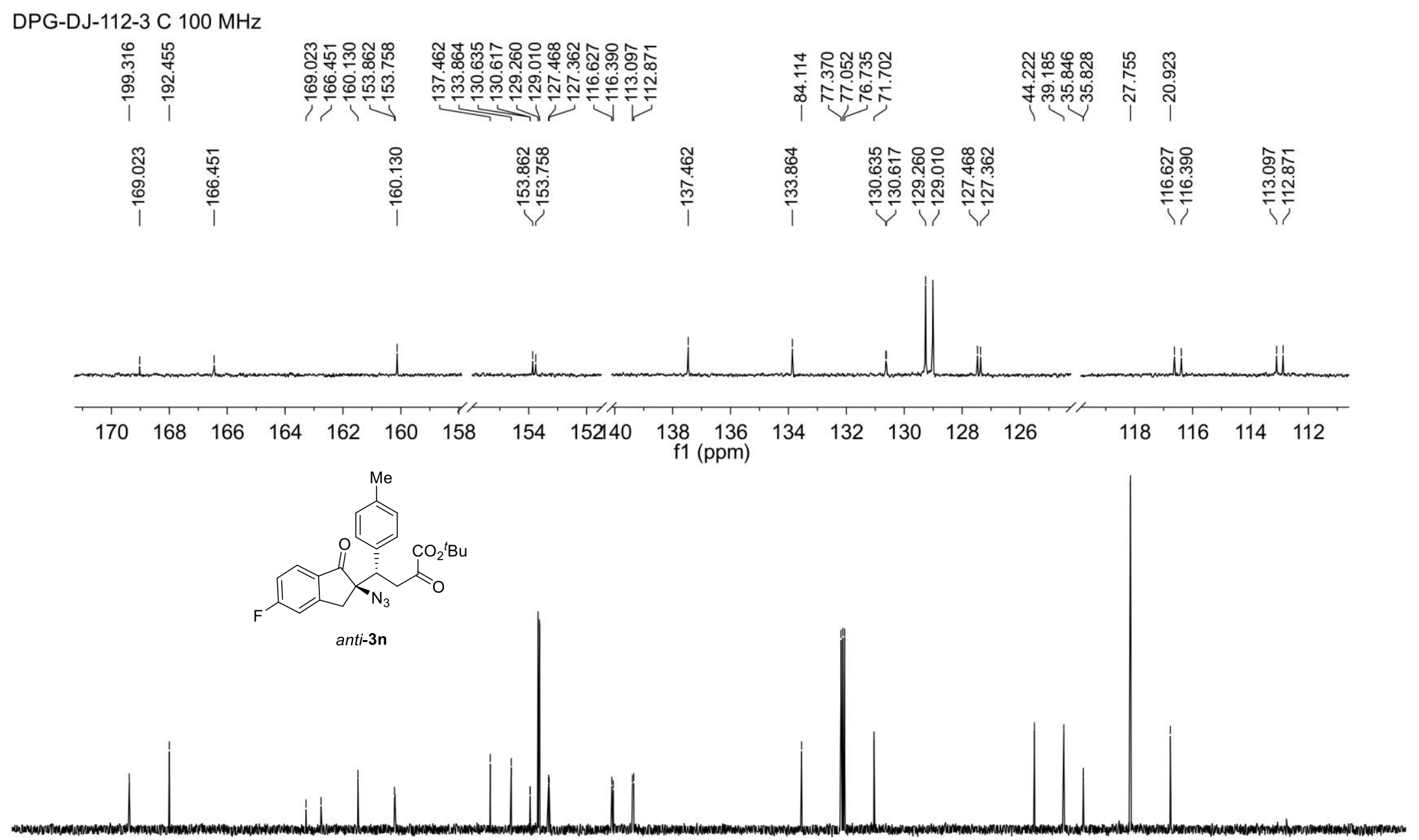

$\begin{array}{lllllllllllllllllllllll}210 & 200 & 190 & 180 & 170 & 160 & 150 & 140 & 130 & 120 & 110 \underset{\mathrm{f} 1 \mathrm{(ppm})}{100} & 90 & 80 & 70 & 60 & 50 & 40 & 30 & 20 & 10 & 0 & -10\end{array}$ 
${ }^{1} \mathrm{H} \mathrm{NMR}$ of Compound anti-3o $\left(400 \mathrm{MHz}, \mathrm{CDCl}_{3}\right)$

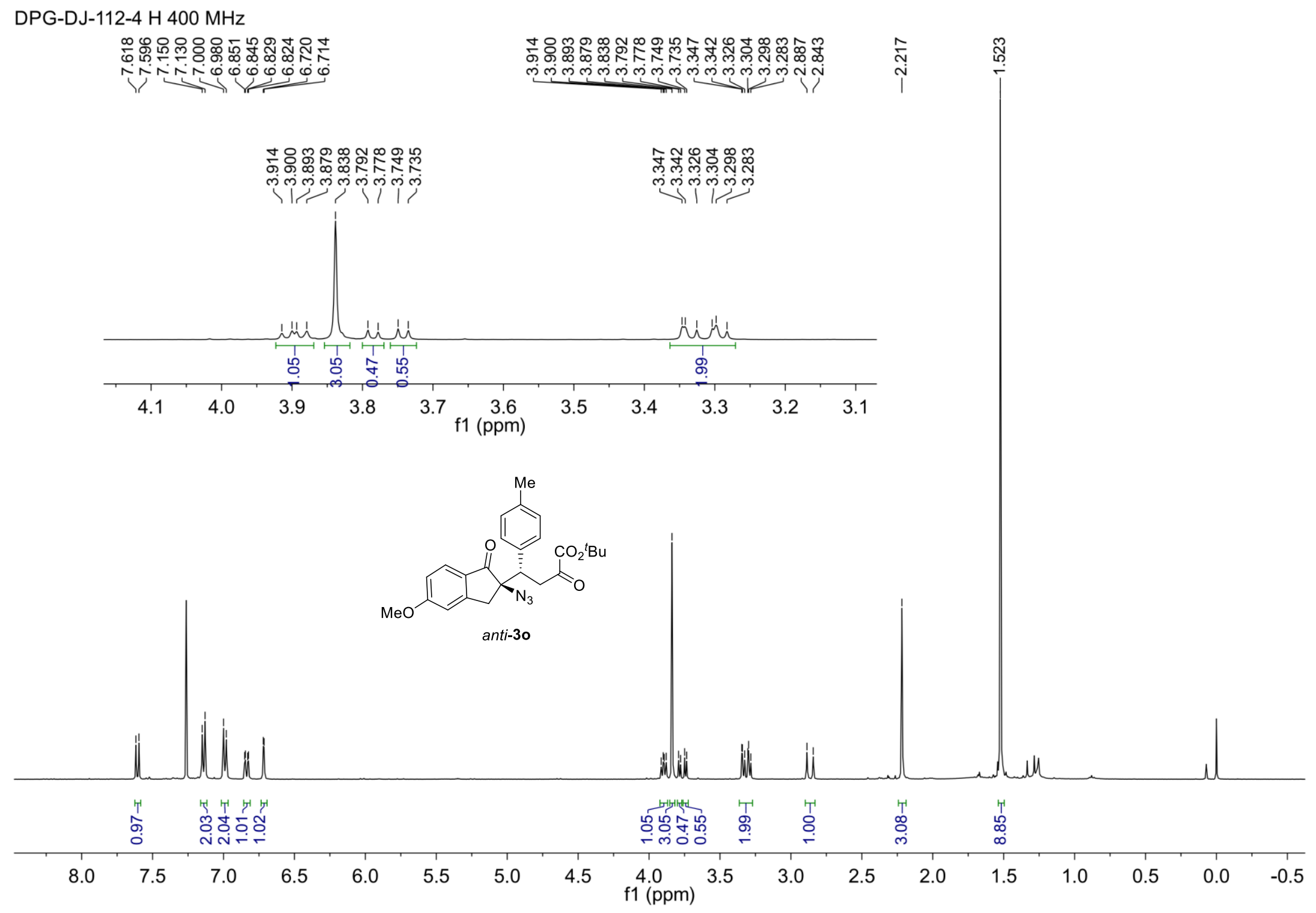


${ }^{13} \mathrm{C}$ NMR of Compound anti-3o $\left(100 \mathrm{MHz}, \mathrm{CDCl}_{3}\right)$

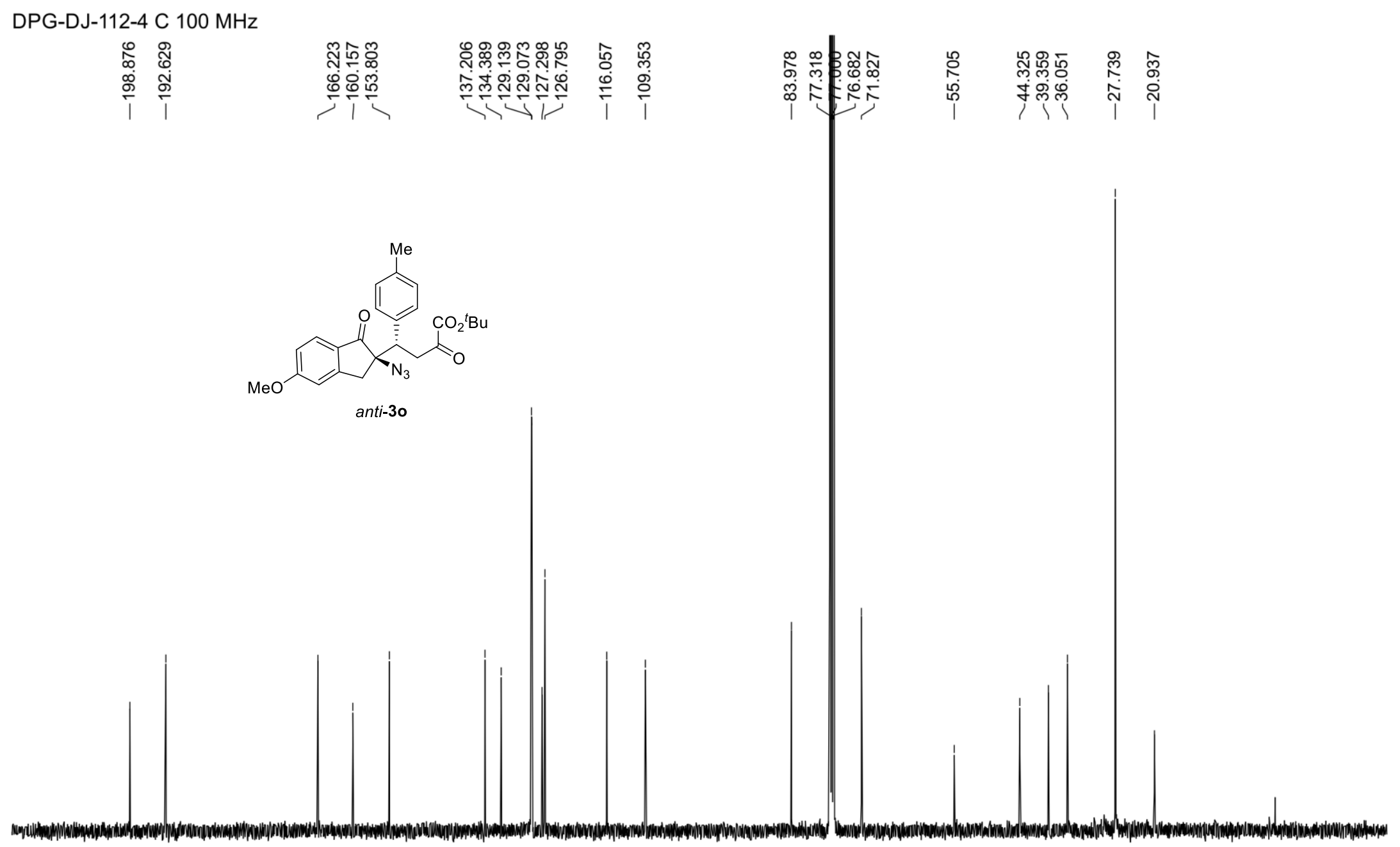

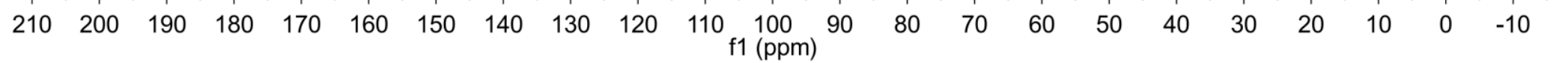


${ }^{1} \mathrm{H}$ NMR of Compound anti-3p $\left(400 \mathrm{MHz}, \mathrm{CDCl}_{3}\right)$

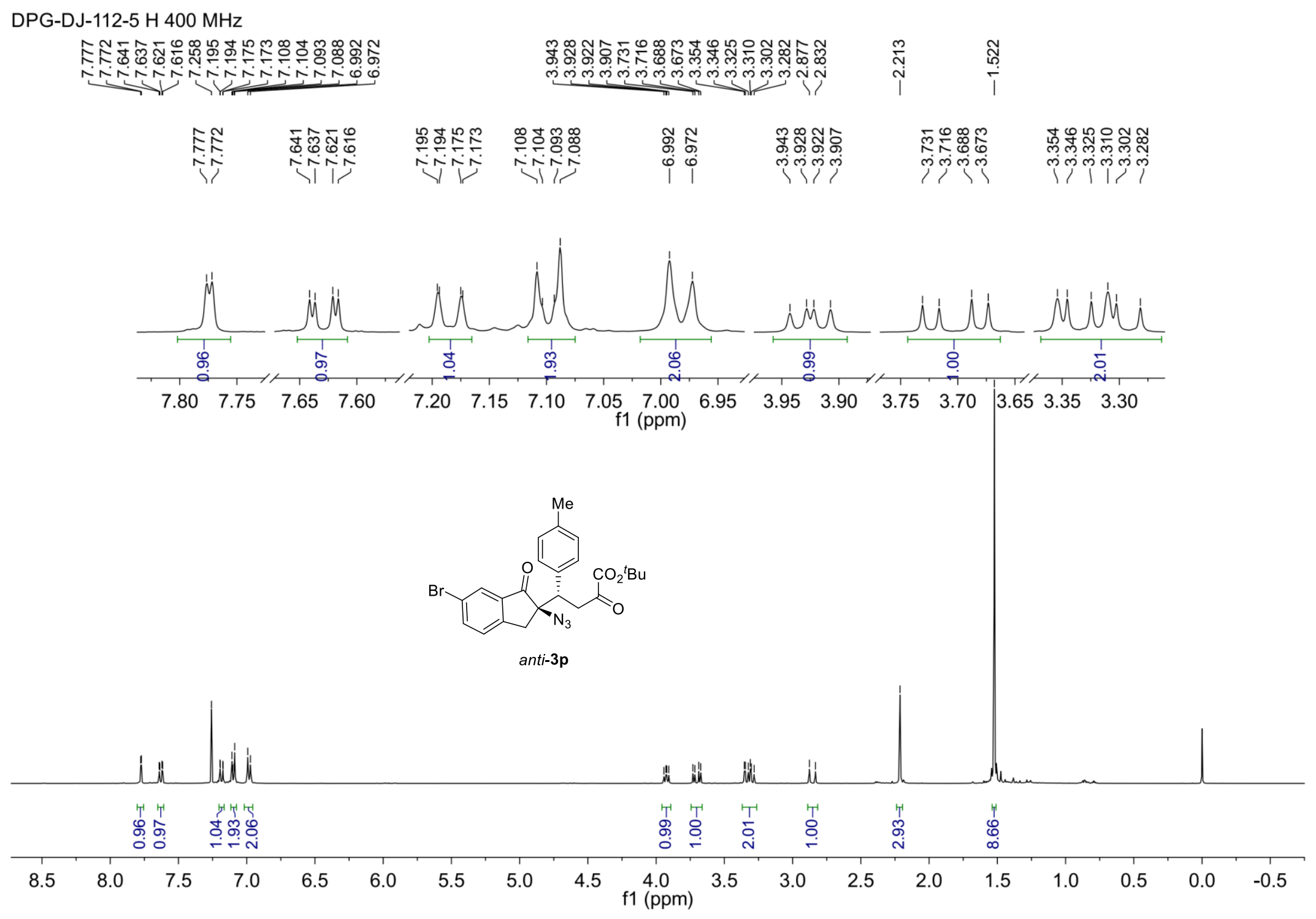


${ }^{13} \mathrm{C}$ NMR of Compound anti-3p (100 MHz, $\left.\mathrm{CDCl}_{3}\right)$

DPG-DJ-112-5 C 100 MHz

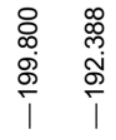

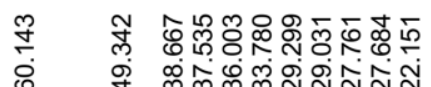

○ $\quad$ ơ

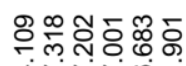

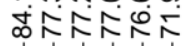

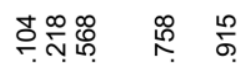

अं்

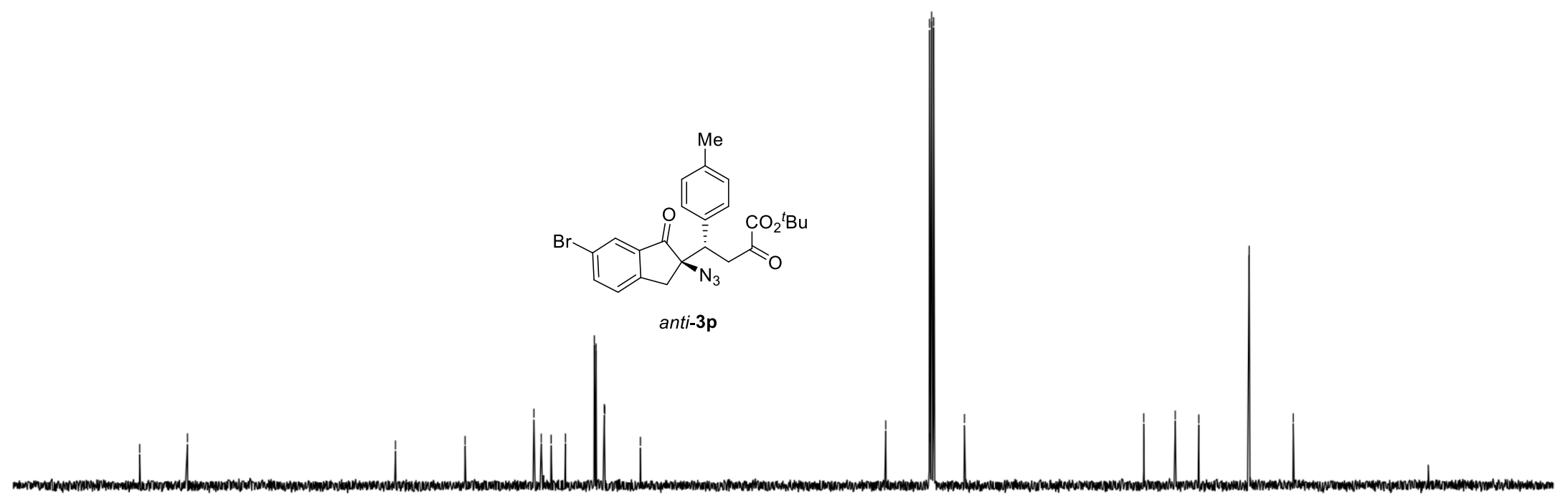

210

$\begin{array}{lllll}200 & 190 & 180 & 170 & 160\end{array}$

$150 \quad 140 \quad 130$

120

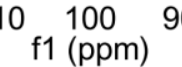

80

$70 \quad 60$ $60 \quad 50$

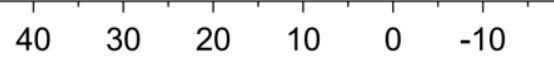


${ }^{1} \mathrm{H} \mathrm{NMR}$ of Compound anti-3q (400 MHz, $\mathrm{CDCl}_{3}$ )

\section{DPG-DJ-46-3 $400 \mathrm{MHz}$}

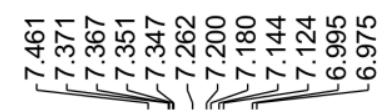

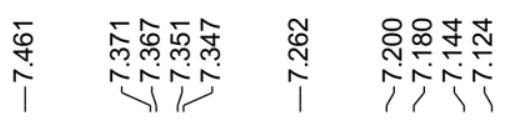

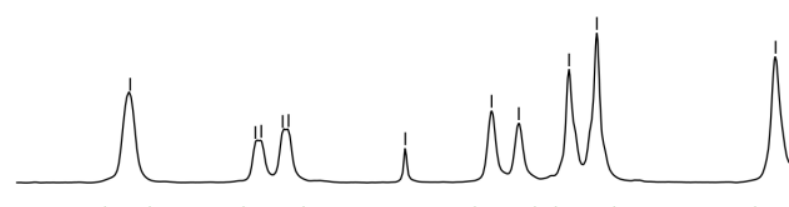

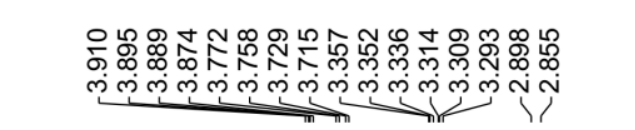

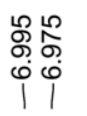

웅요욤뭉 लंime शुं लिखिं

लైల

ก

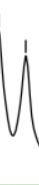

$\stackrel{1}{\infty}$

$7.5 \quad 7.4$

7.3

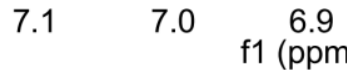

f1 (ppm)
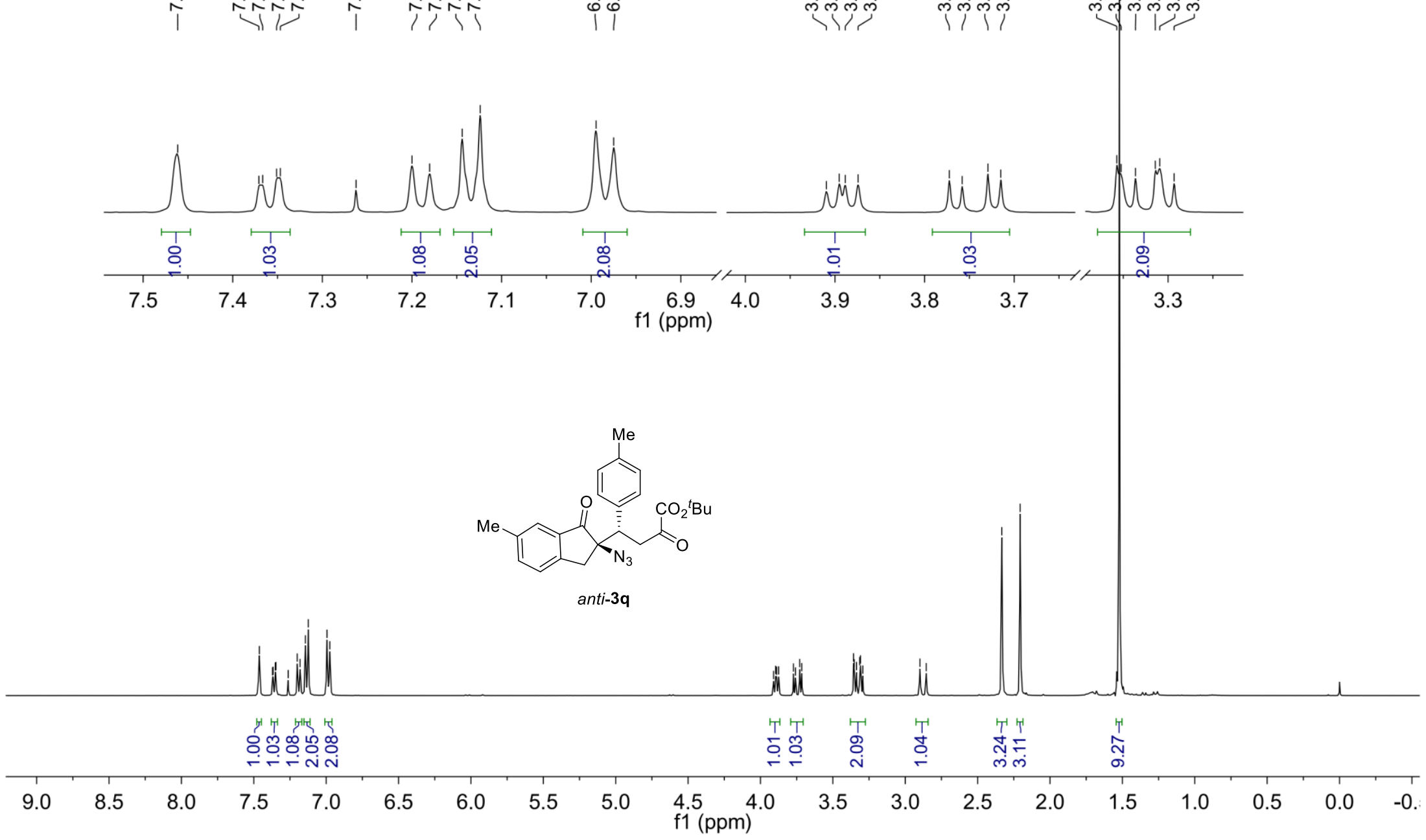
${ }^{13} \mathrm{C}$ NMR of Compound anti-3q $\left(100 \mathrm{MHz}, \mathrm{CDCl}_{3}\right)$

DPG-DJ-46-3 C $100 \mathrm{MHz}$

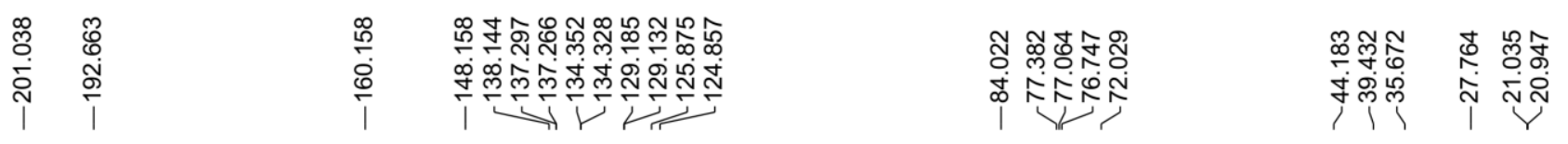
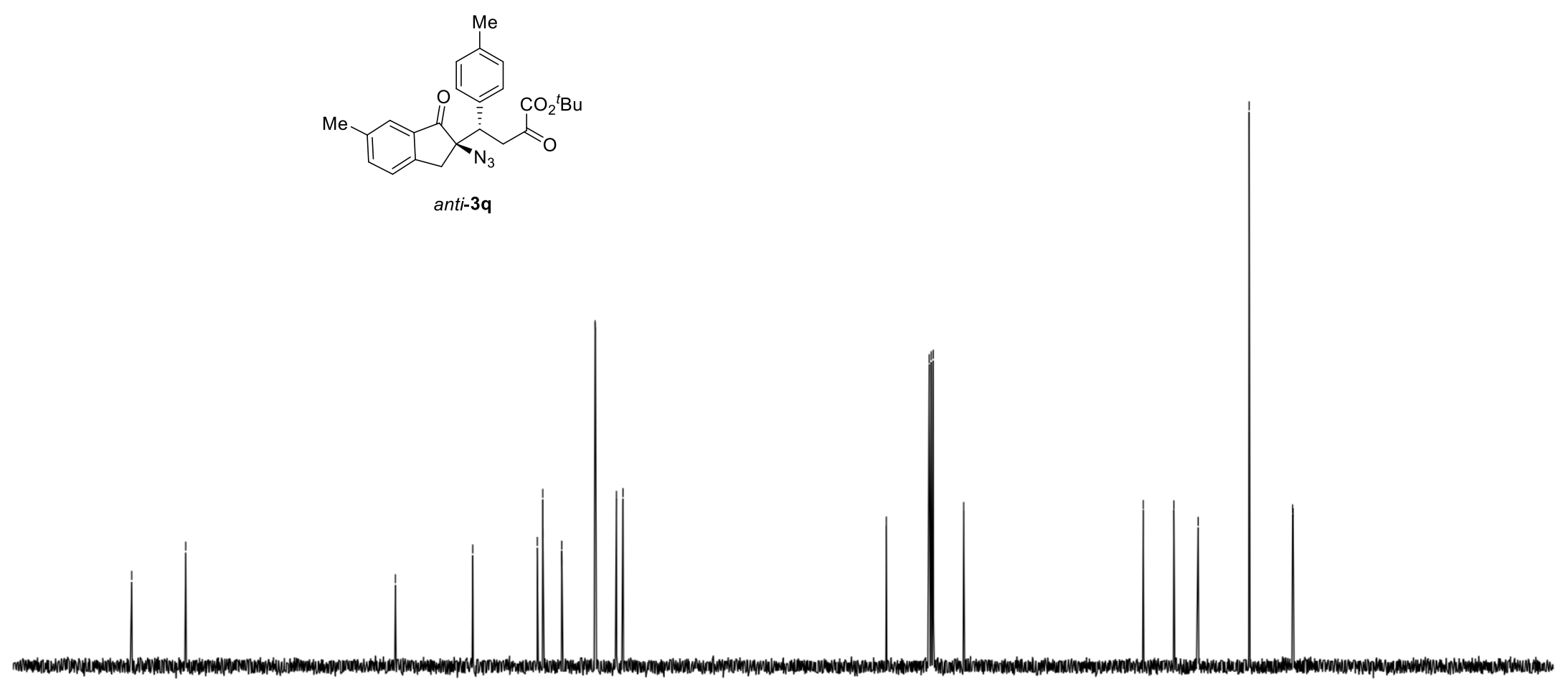

$\begin{array}{llllll}210 & 200 & 190 & 180 & 170 & 160\end{array}$

$150 \quad 140 \quad 130$

$\begin{array}{llll}120 & 110 & 100 & 90\end{array}$ f1 (ppm) 
${ }^{1} \mathrm{H} \mathrm{NMR}$ of Compound anti-3r $\left(400 \mathrm{MHz}, \mathrm{CDCl}_{3}\right)$

\section{DPG-DJ-112-6 H $400 \mathrm{MHz}$}

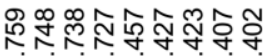

ivivivisin

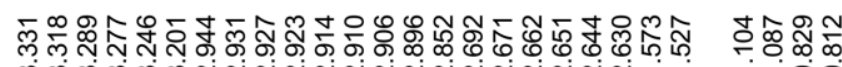

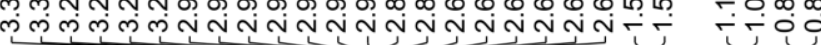

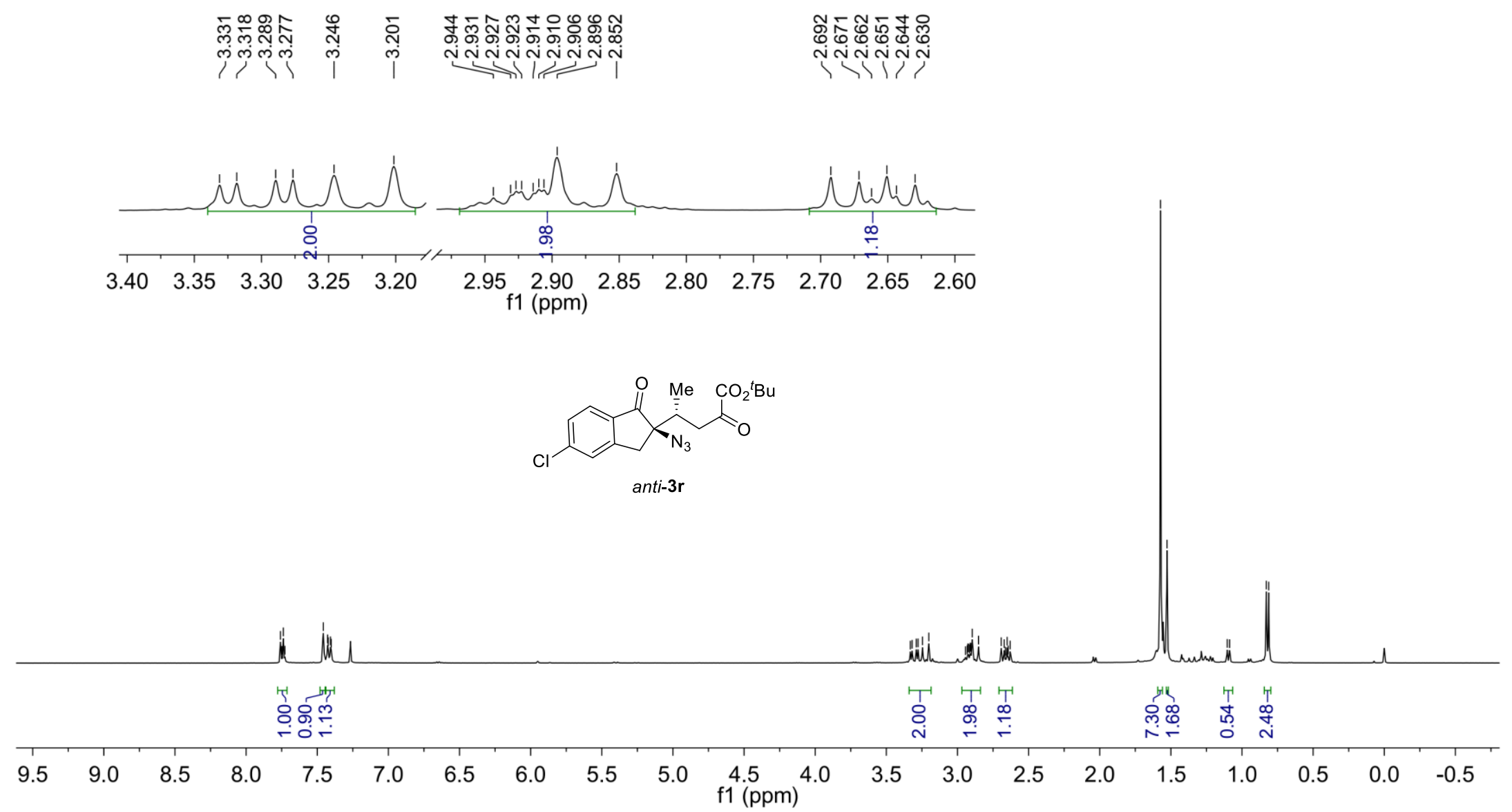


${ }^{13} \mathrm{C}$ NMR of Compound anti-3r $\left(100 \mathrm{MHz}, \mathrm{CDCl}_{3}\right)$

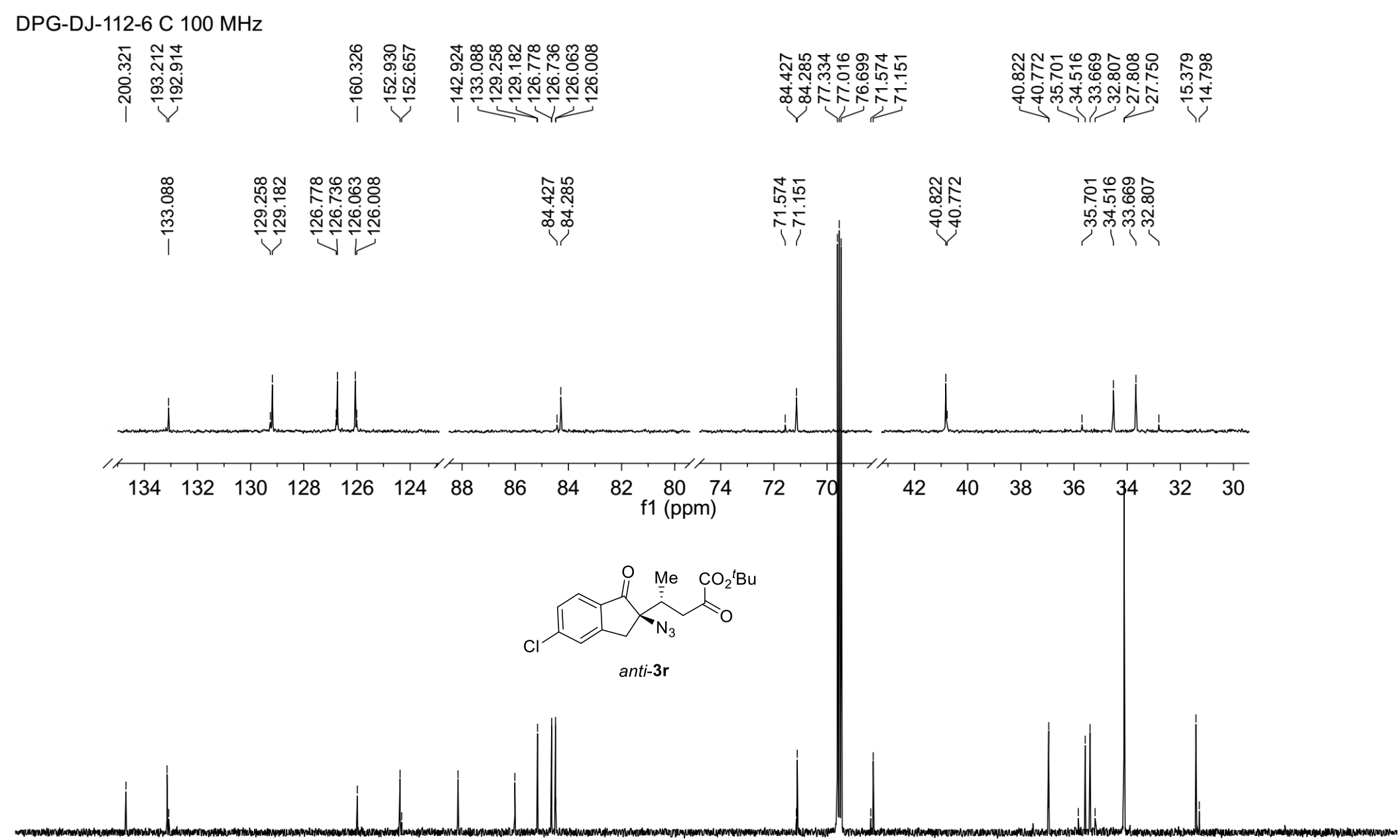

$\begin{array}{llllllllllllllllllllllll}210 & 200 & 190 & 180 & 170 & 160 & 150 & 140 & 130 & 120 & 110 & 100 & 90 & 80 & 70 & 60 & 50 & 40 & 30 & 20 & 10 & 0 & -10 & 10\end{array}$ 
${ }^{1} \mathrm{H}$ NMR of Compound $4 \mathbf{a}\left(400 \mathrm{MHz}, \mathrm{CDCl}_{3}\right)$

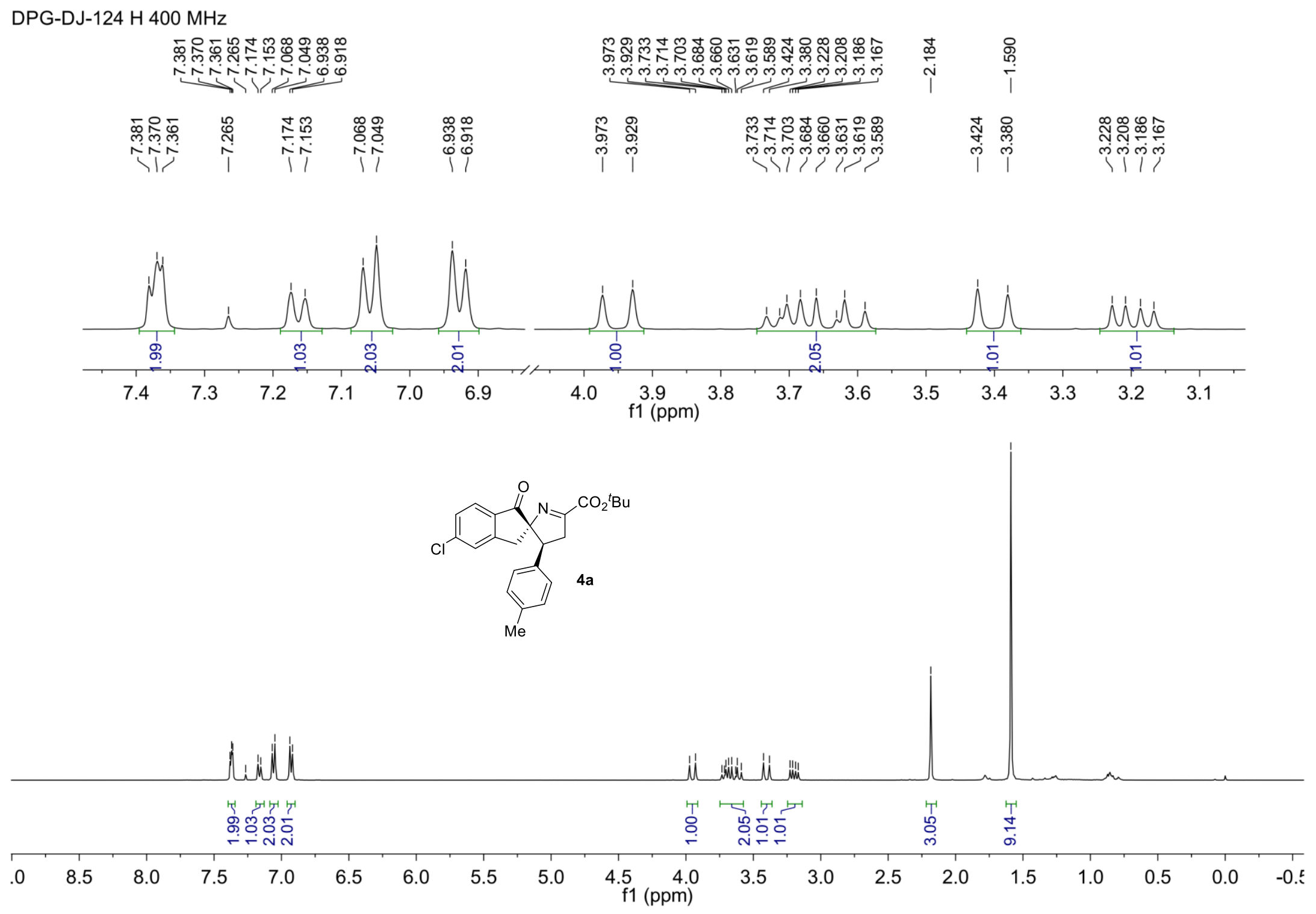


${ }^{13} \mathrm{C}$ NMR of Compound $4 \mathbf{a}\left(100 \mathrm{MHz}, \mathrm{CDCl}_{3}\right)$

DPG-DJ-124 C $100 \mathrm{MHz}$

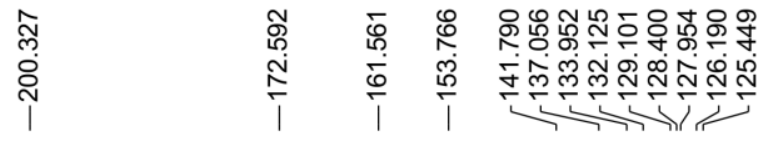

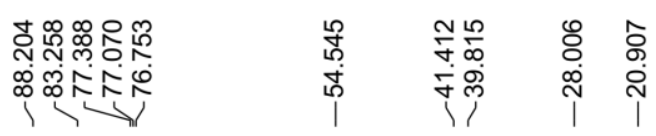

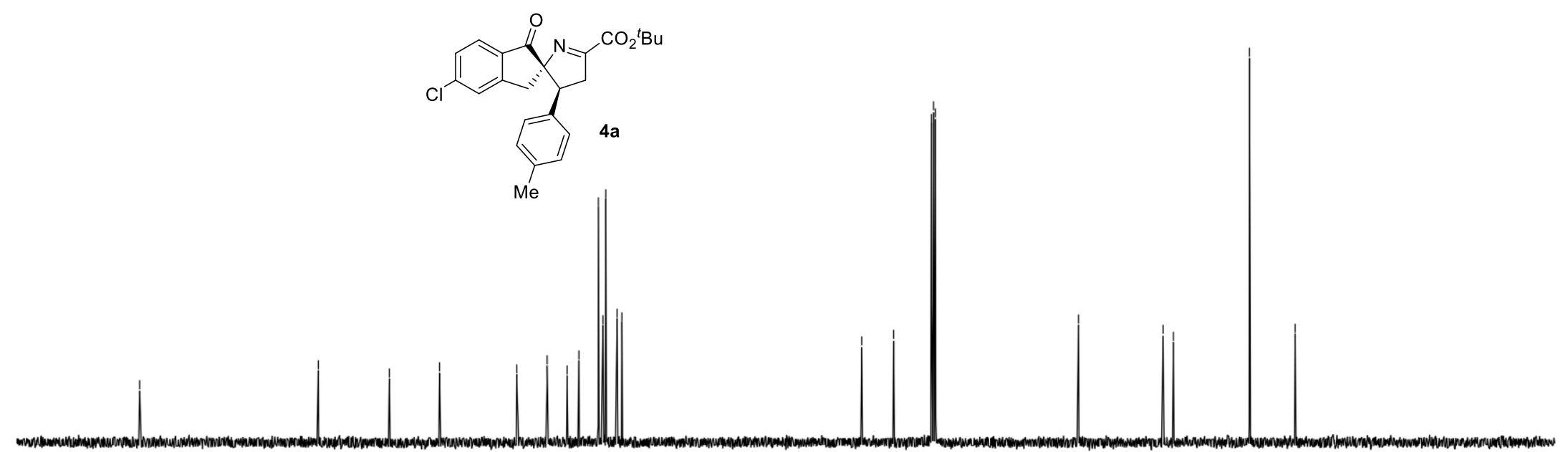

$\begin{array}{lllllllllllllllllllllll}210 & 200 & 190 & 180 & 170 & 160 & 150 & 140 & 130 & 120 & 110 & \begin{array}{l}100 \\ \mathrm{f} 1(\mathrm{ppm})\end{array} & 90 & 80 & 70 & 60 & 50 & 40 & 30 & 20 & 10 & 0 & -10\end{array}$ 
${ }^{1} \mathrm{H}$ NMR of Compound $5 \mathbf{a}\left(400 \mathrm{MHz}, \mathrm{CDCl}_{3}\right)$

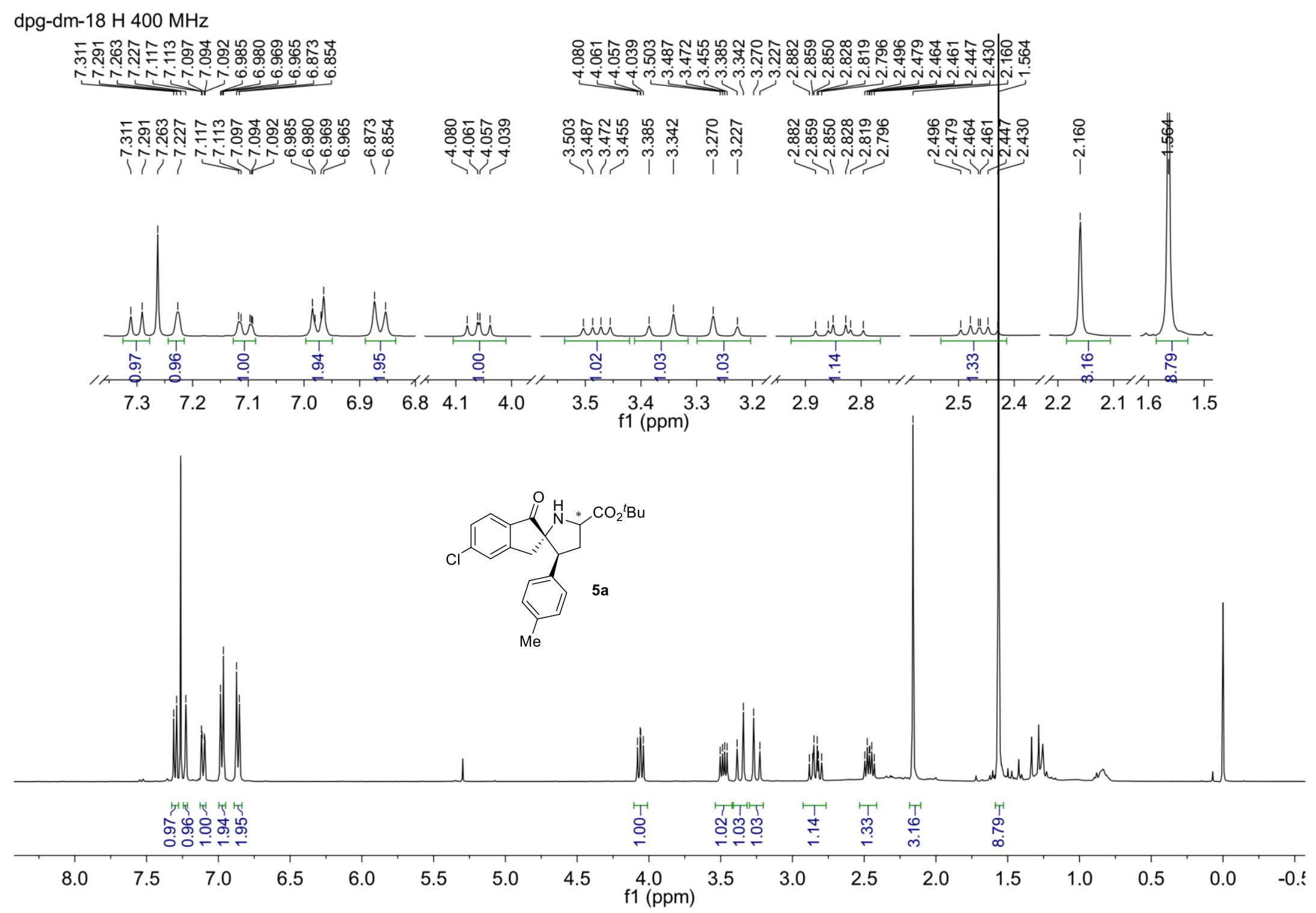


${ }^{13} \mathrm{C}$ NMR of Compound $5 \mathbf{a}\left(100 \mathrm{MHz}, \mathrm{CDCl}_{3}\right)$

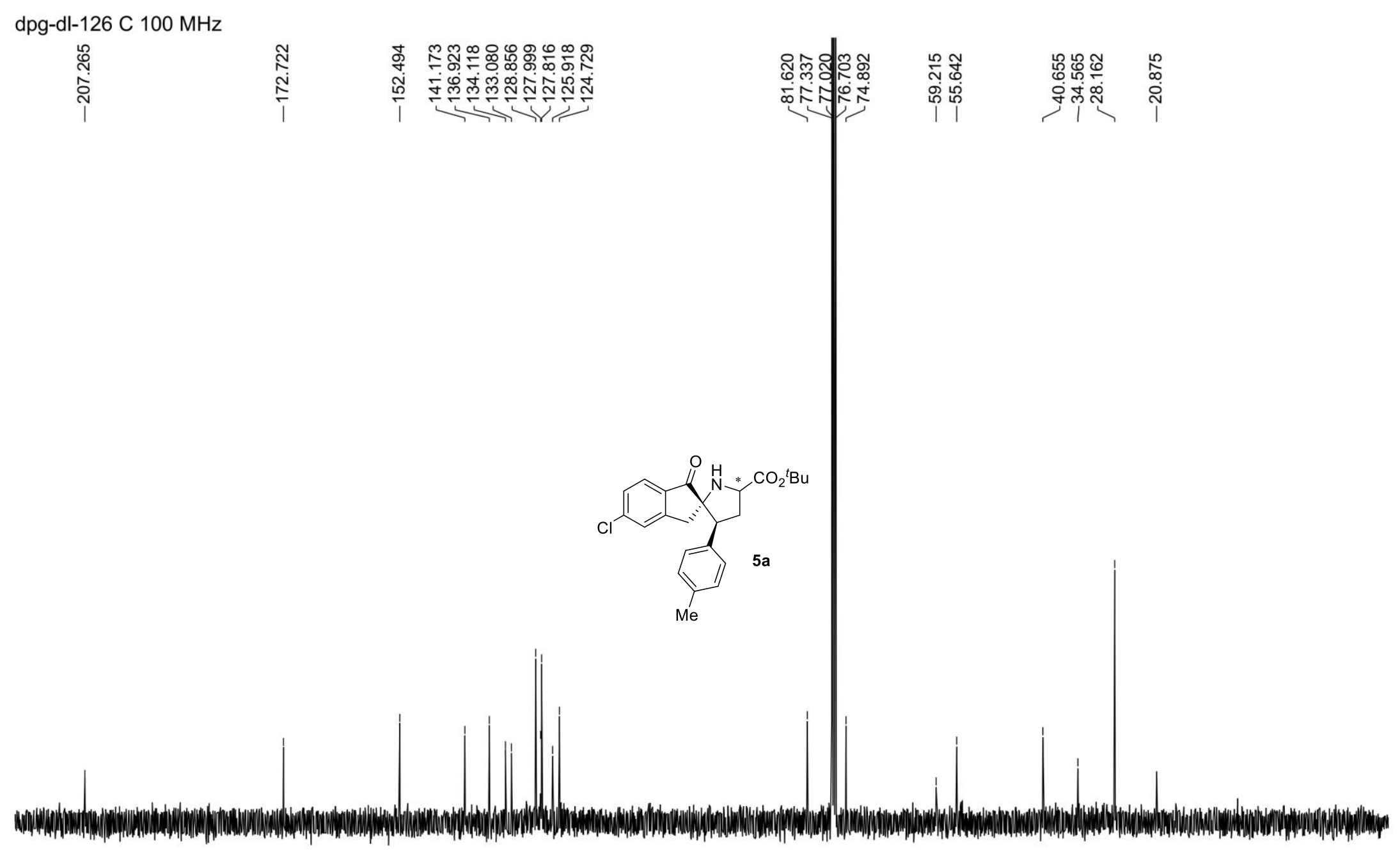

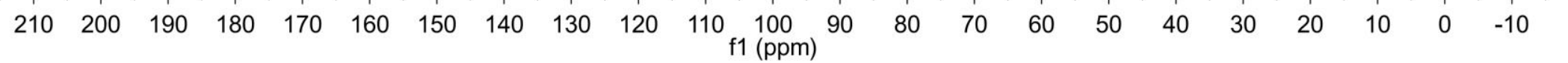


${ }^{1} \mathrm{H}$ NMR of Compound $\mathbf{4 b}\left(300 \mathrm{MHz}, \mathrm{CDCl}_{3}\right)$

\section{DPG-DJ-120 H $300 \mathrm{MHz}$}

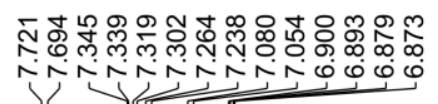

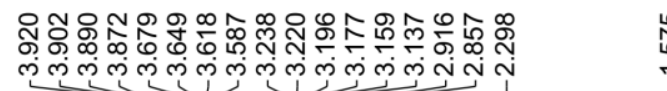

$\frac{1}{\frac{1}{2}}$

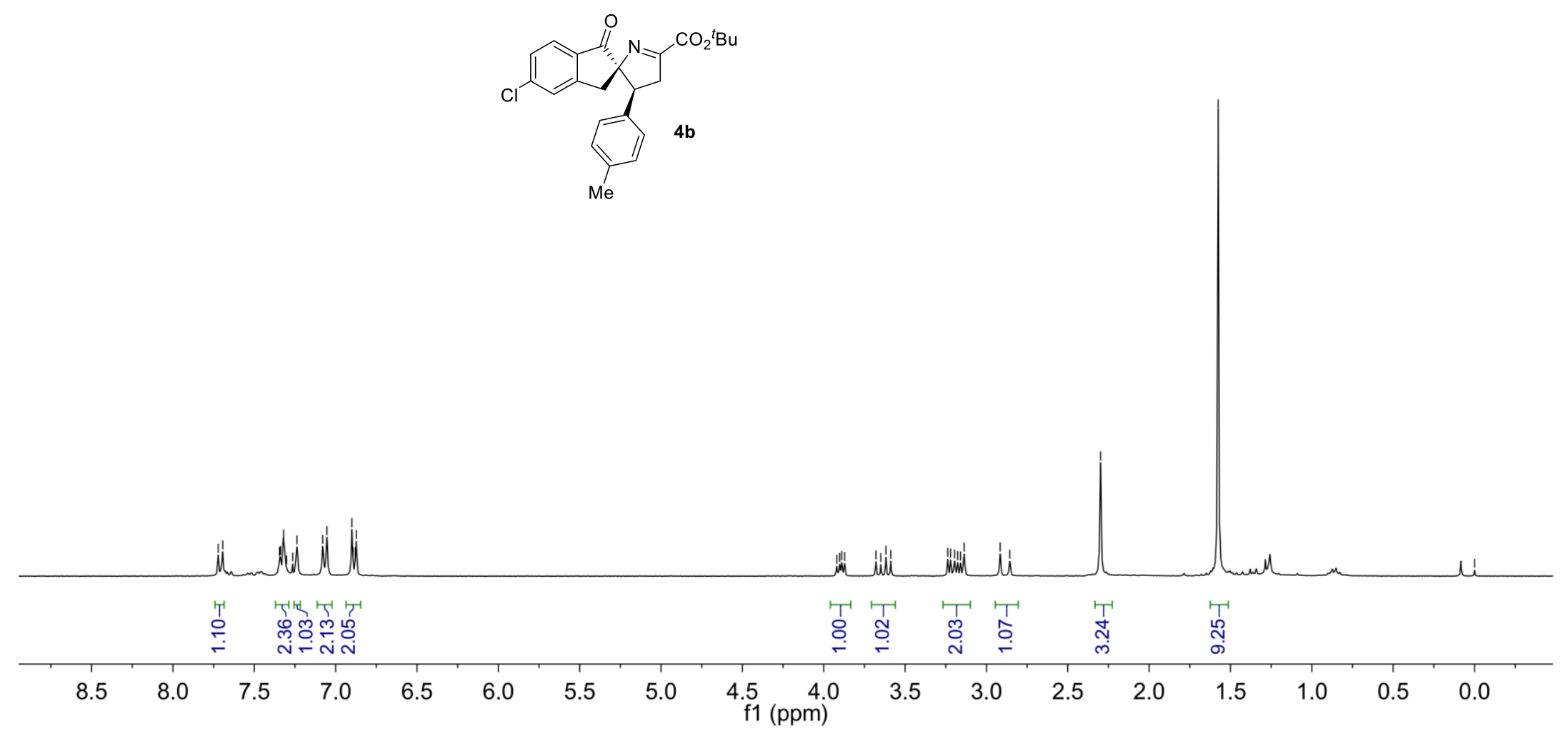


${ }^{13} \mathrm{C} \mathrm{NMR}$ of Compound $\mathbf{4 b}\left(100 \mathrm{MHz}, \mathrm{CDCl}_{3}\right)$

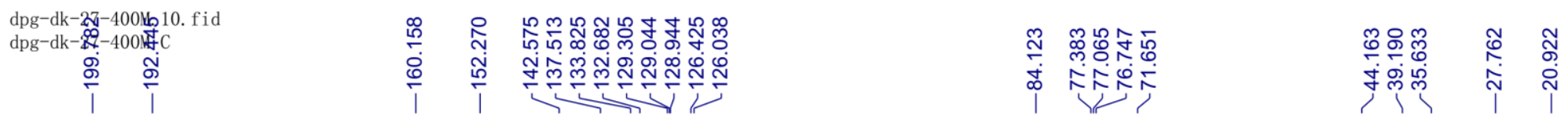

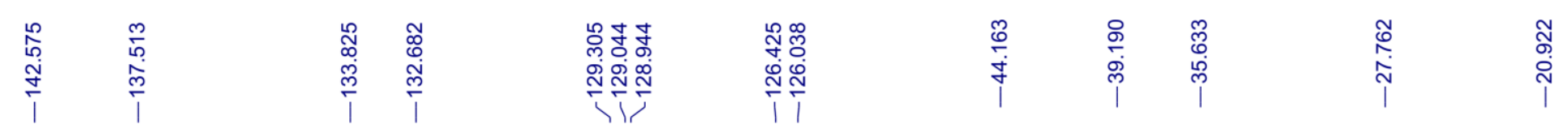
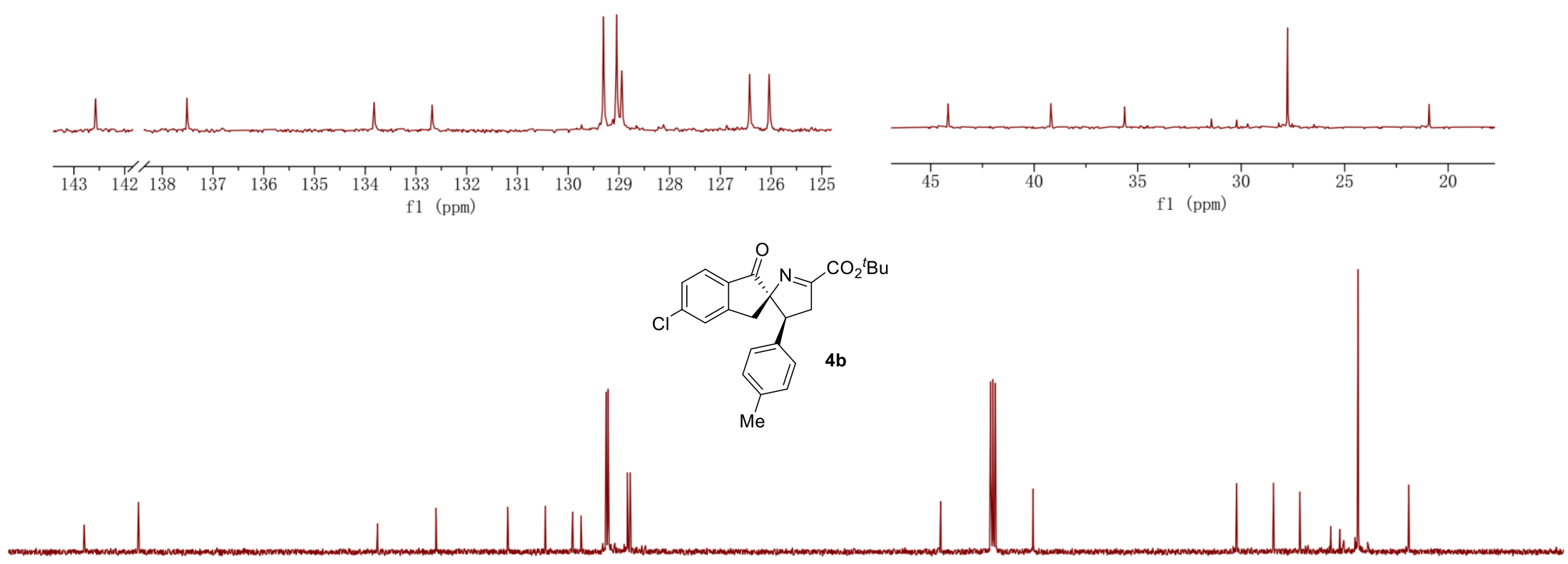

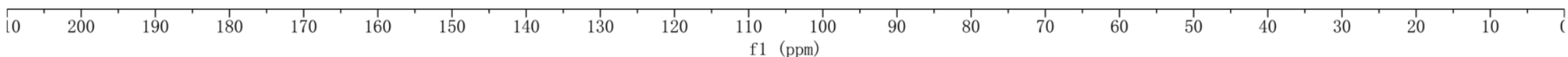


${ }^{1} \mathrm{H}$ NMR of Compound $\mathbf{5 b}\left(400 \mathrm{MHz}, \mathrm{CDCl}_{3}\right)$

\section{DPG-DM-22 H $400 \mathrm{MHz}$}

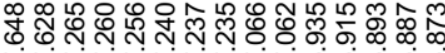

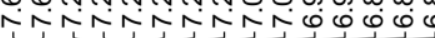

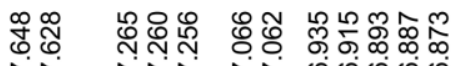

NT Ni

证

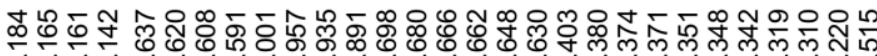

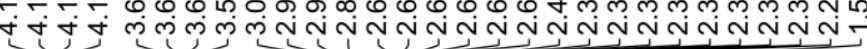
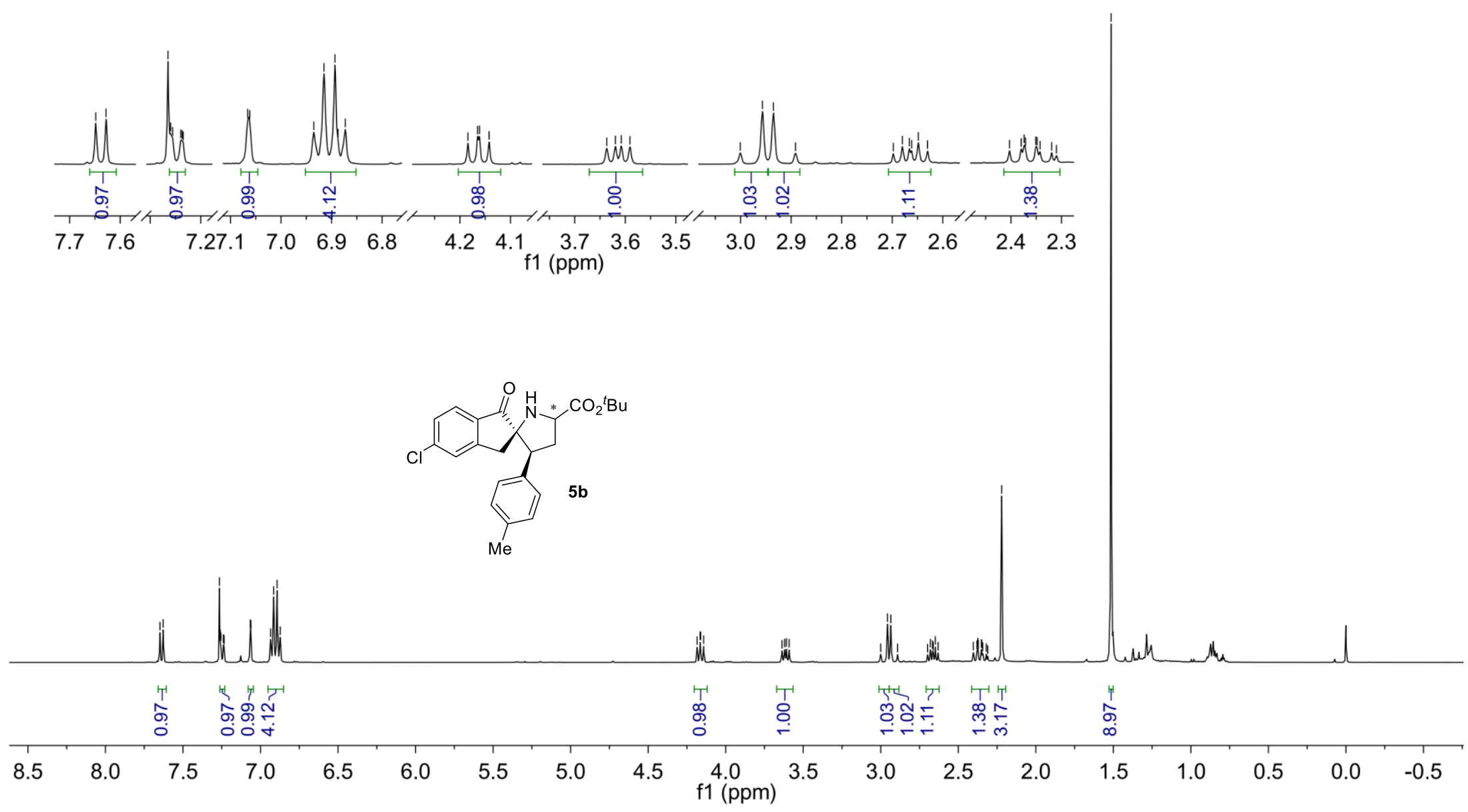
${ }^{13} \mathrm{C}$ NMR of Compound $\mathbf{5 b}\left(100 \mathrm{MHz}, \mathrm{CDCl}_{3}\right)$

DPG-DM-22 C $100 \mathrm{MHz}$

î̀

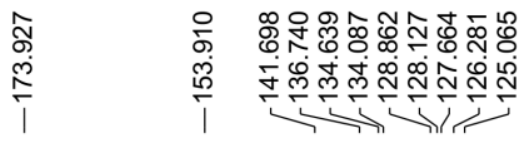

क

苗 ถึ

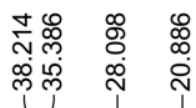

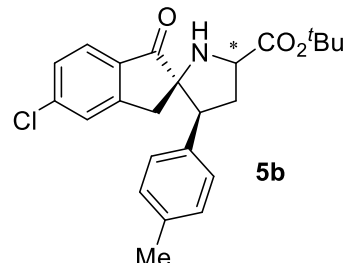

Me

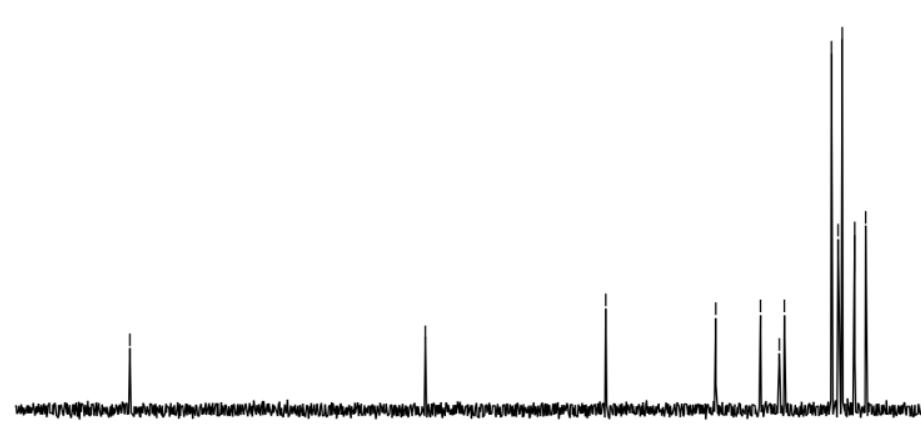

210

$\begin{array}{lllll}200 & 190 & 180 & 170 & 160\end{array}$

150

140130

120

$10 \begin{gathered}100 \\ \mathrm{f} 1\end{gathered}$

80

$70 \quad 60$

50

4030

$20 \quad 10$

(1) 


\section{HPLC spectra}

\section{Analysis Report}

<Sample Information $\begin{array}{l:l}\text { Data Filename } & \text { :dpg-dn-28-1-rac-0ZH-97-3-1.0-230.lcd } \\ \text { Method Filename } & \text { dpg-OZH-95-5-1.0-230 } \mathrm{lcm}\end{array}$

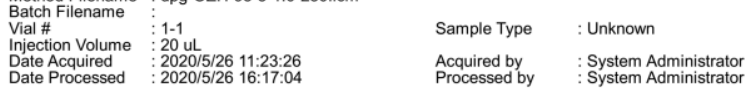

$<$ Chromatogram>
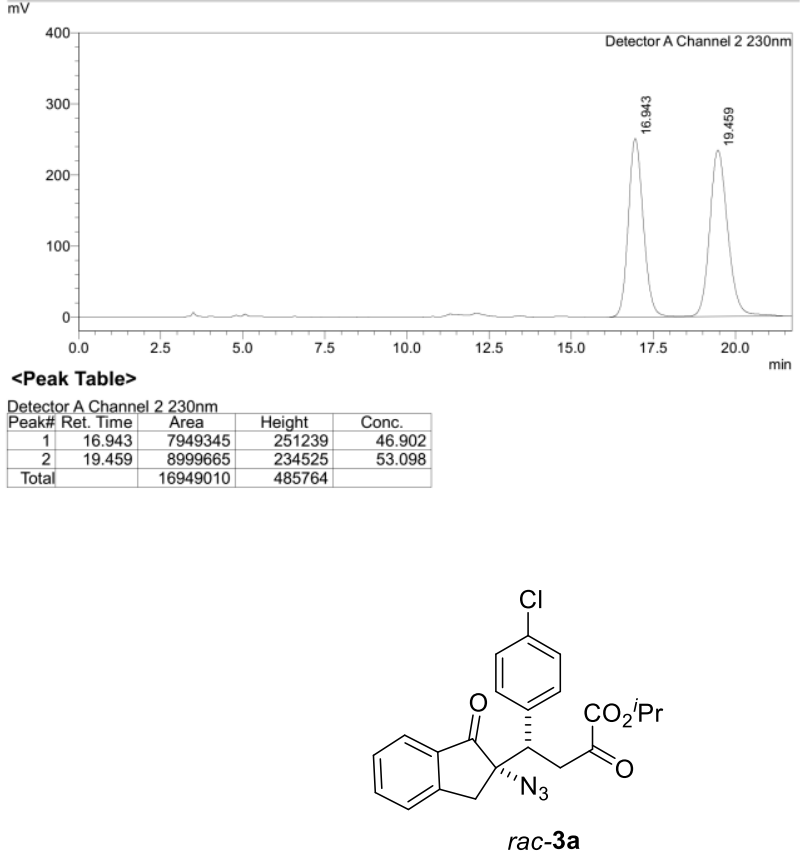

D:|Datalddpgldnldpg-dn-28-1-rac-OZH-97-3-1.0-230.lcd
Analysis Report

<Sample Information>

$\begin{array}{l:l}\text { Data Filename } & \text { dpg-dn-28-1-asy-OZH-97-3-1.-2-230.1cd } \\ \text { Method Filename } & \text { dpg-OZH-95-5-1.0-230.1cm }\end{array}$

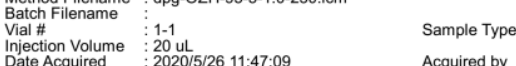

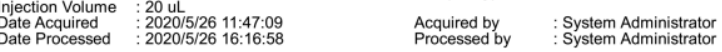
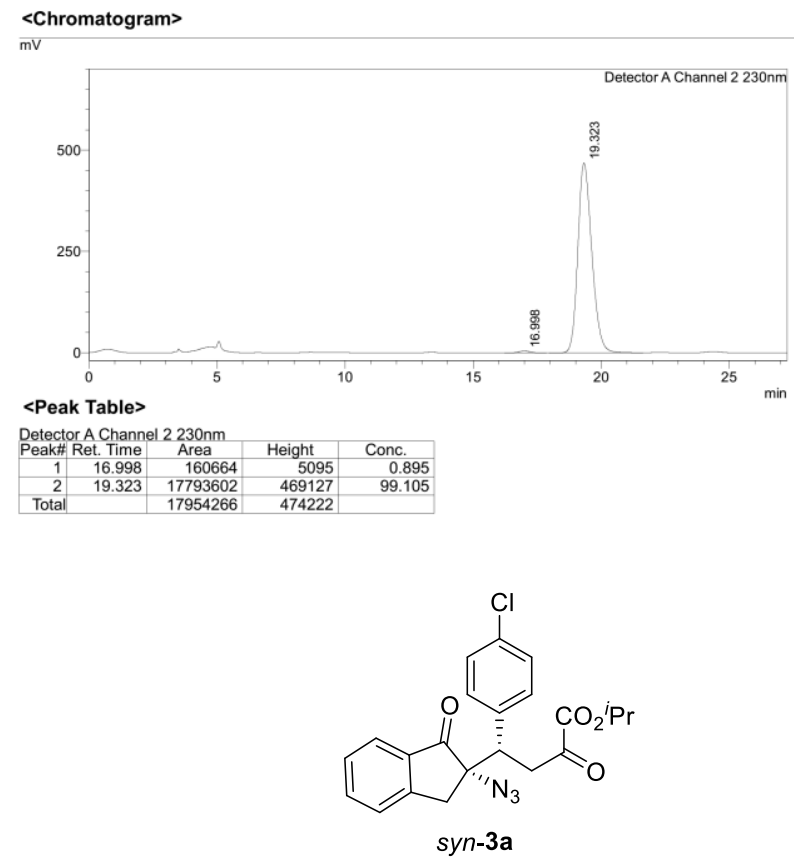

D.1Dataldpglddildpg-dd-28-1-asy-OZH-97-3-1.0-230.1 
Analysis Report

Sample Name dpg-dh-51-1-rac-OZH-97-3-1.0-229

Sample ID
Data Filename dpg-dh-51-1-rac-OZH-97-3-1.0-230.lcd

$\begin{array}{l:ll}\text { Batch Filename } & 1-1 & \text { Sample Type }\end{array}$

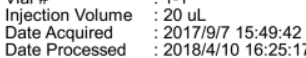

$\begin{array}{ll}\text { Acquired by } & \text { : System Administrator } \\ \text { Processed by } & \text { System Administrator }\end{array}$

$<$ Chromatogram>
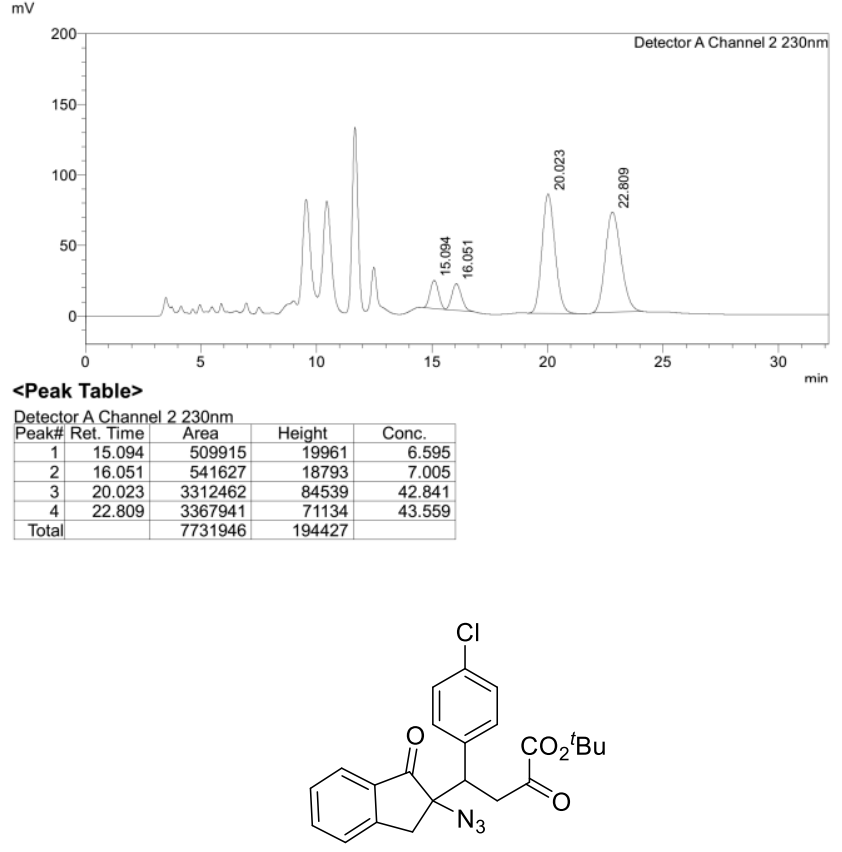

rac-3b

\section{Analysis Report}

$<$ Sample Information

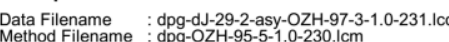

$\begin{array}{l:cc}\text { Batch Filename } & 1-1 & \text { Sample Type }\end{array}$

$<$ Chromatogram>
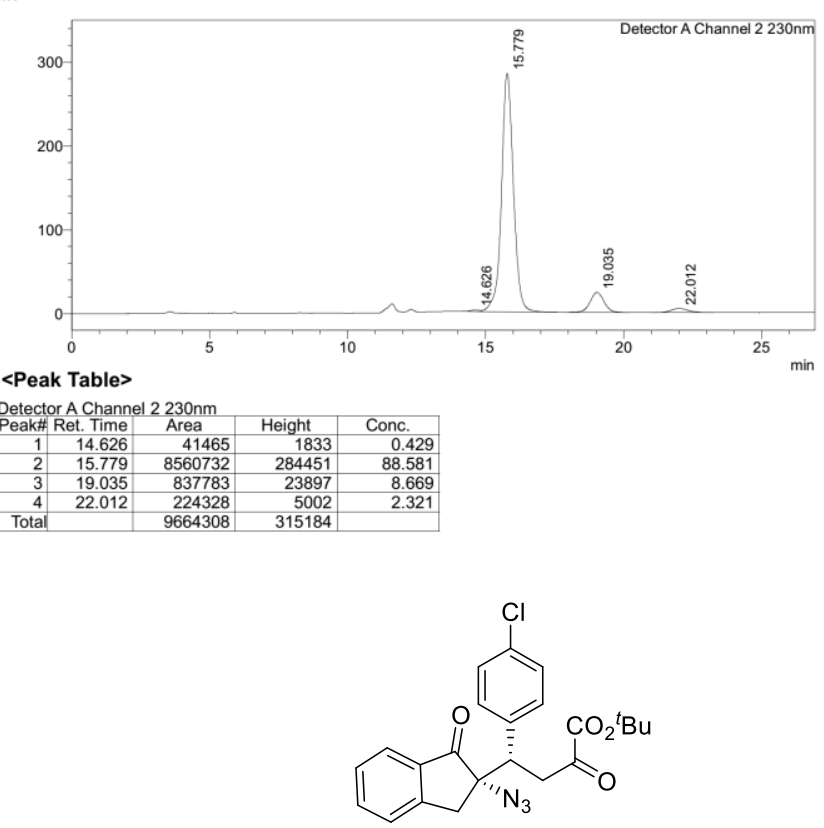

syn-3b 
Asample informalysis Report

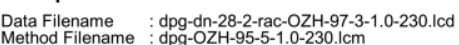

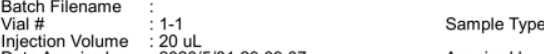

$\begin{array}{lll}\text { lnjection Volume } & 1-1 \\ \text { Date Acquired } & \text { 202015/31 20:09:07 } \\ \text { Date Processed } & : 202015 / 31 \text { 21:42:46 }\end{array}$

Acquired by
Processed by

<Chromatogram>
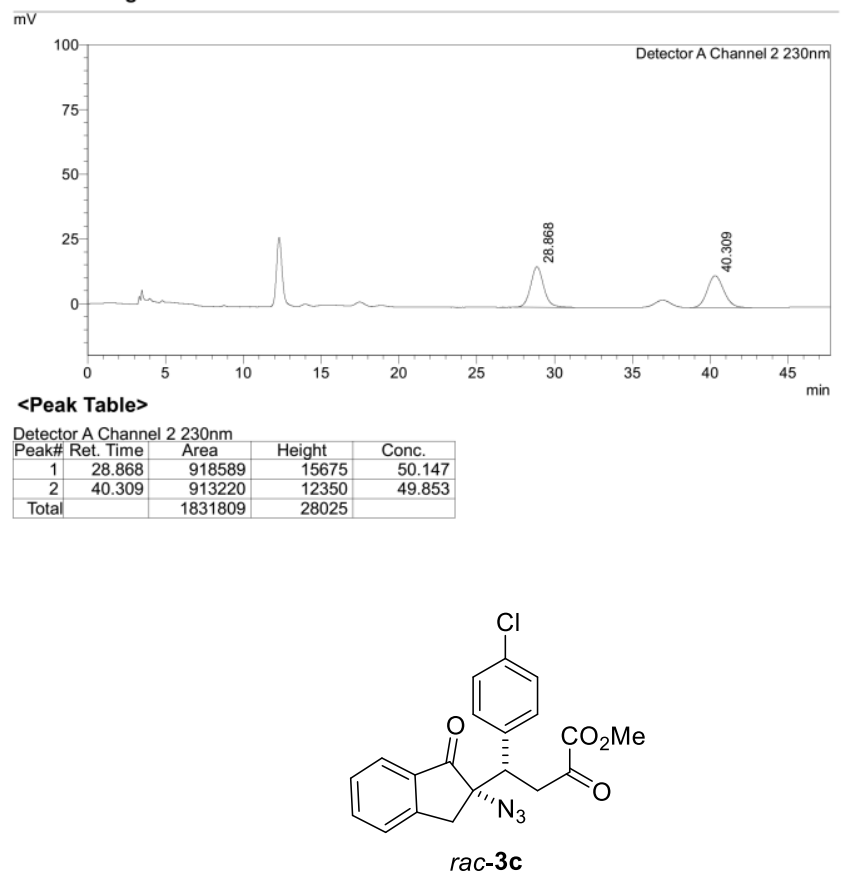

D:IDataldpgldnldpg-dn-28-2-rac-OZH-97-3-1.0-230.Icd

\section{Analysis Report}

<Sample Information

Data Filename $:$ :dpg-dn-28-2-asy-0ZH-97-3-10-230.1c

$\begin{array}{l:cc}\text { Batch Filename } & 1-1 & \text { Sample Type } \\ \text { Vial } & \end{array}$

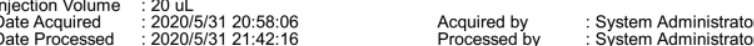

<Chromatogram>
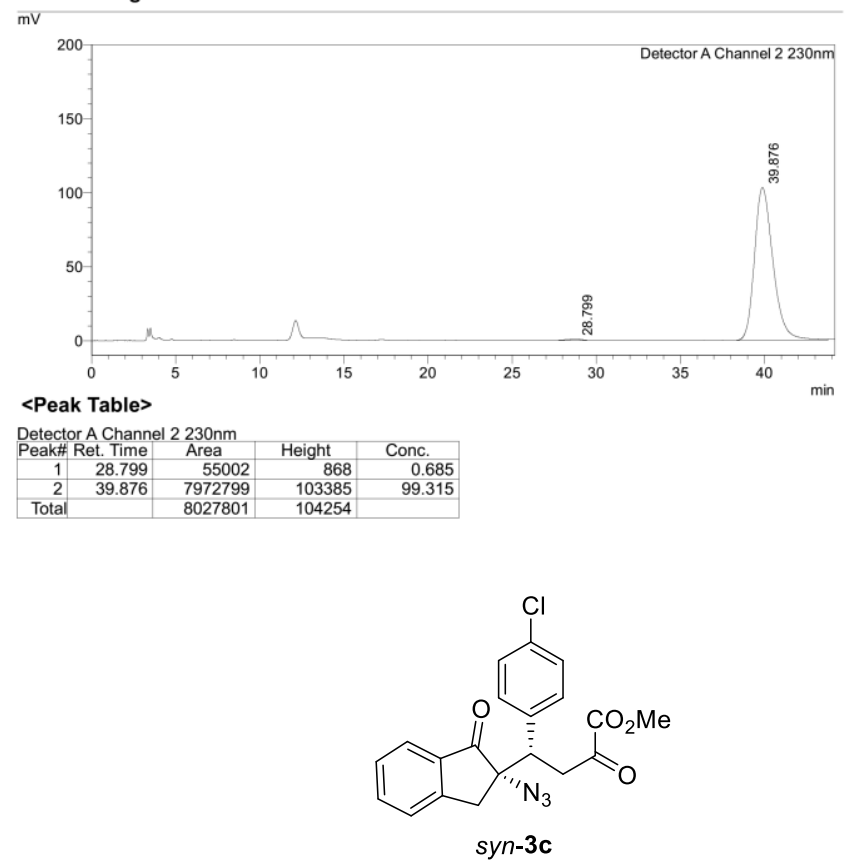

D:|Dataldpgldnldpg-dn-28-2-asy-OZH-97-3-1.0-230.lcd 


\section{Analysis Report}

$<$ Sample Information

Data Filename $:$ dpg-dJ-80-1-RAC-OZH-97-3-1.0-232.lcd

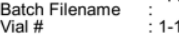

$\begin{array}{l:ll}\text { Vial \# } & 1-1 \\ \text { Injection Volume } & 20 \mathrm{uL} & \text { Sample Type }\end{array}$

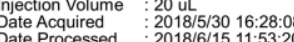

Acquired by
Processed by $\quad$ System Administrator
System Administrator

$<$ Chromatogram>
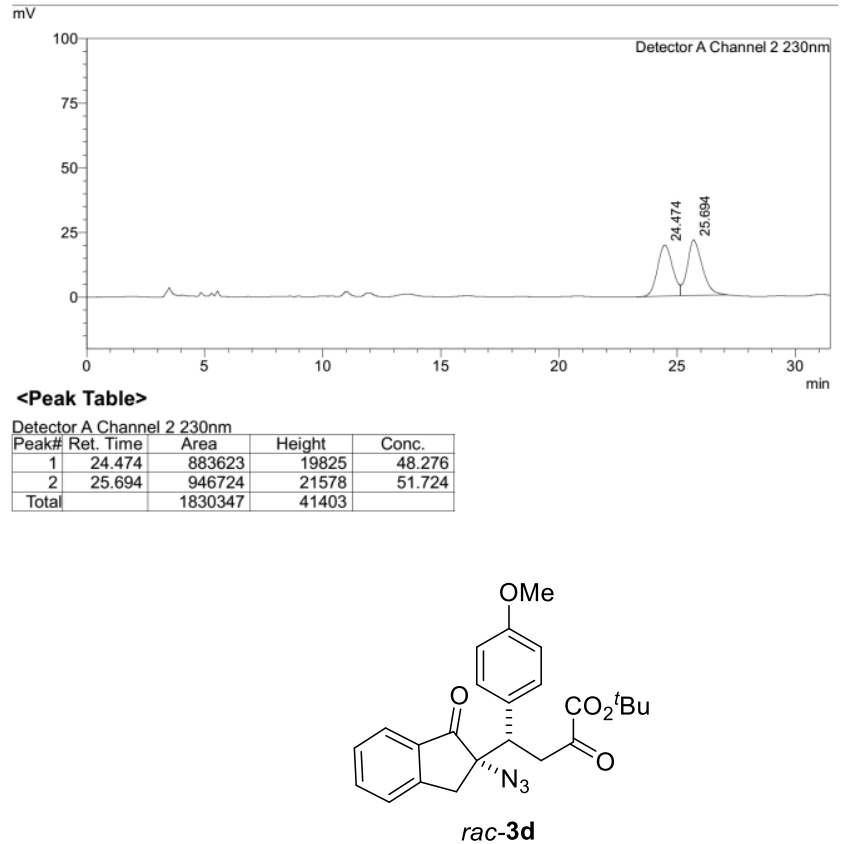

\section{Analysis Report}

<Sample Information>

Oata Filename : dpg-dJ-80-1-ASY-0ZH-97-3-1.0-232.10

$\begin{array}{l:cc}\text { Batch Filename } & 1-1 & \text { Sample Type } \\ \text { Vial } & 1-1 & \end{array}$

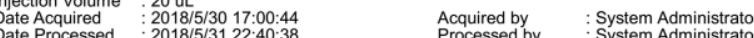

$<$ Chromatogram>
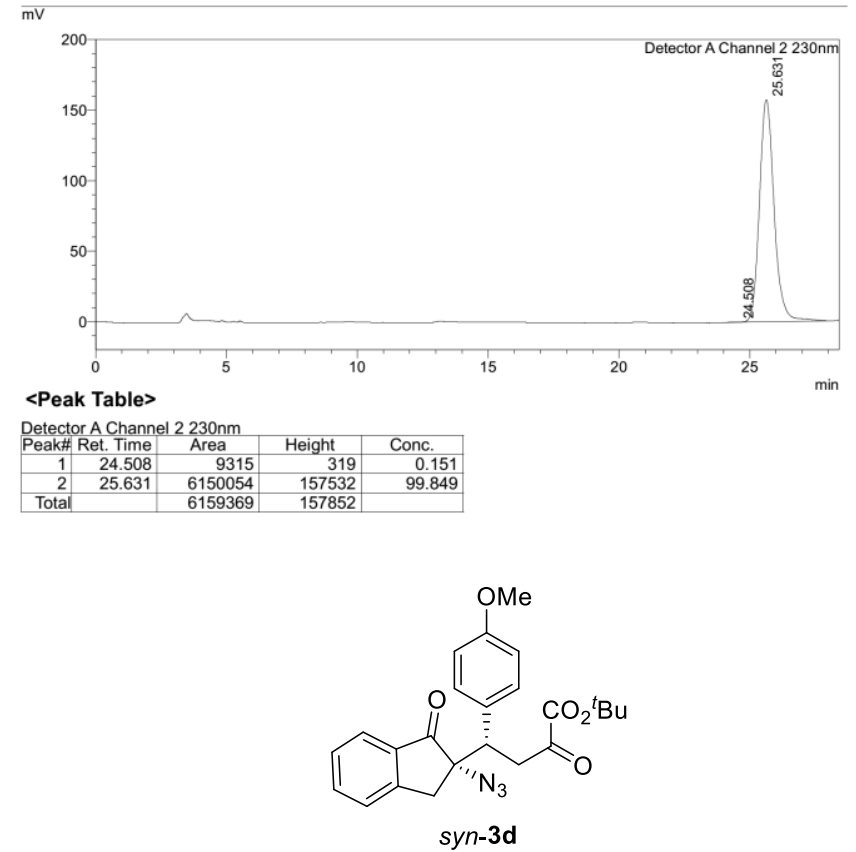


\section{Analysis Report}

$<$ Sample Information

Data Filename : dpg-dJ-29-13-rac-ASH-97-3-1.0-231.lcd

Batch Filename
Vial \#

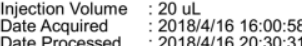

Acquired by
Processed by $\quad$ System Administrator
System Administrator

<Chromatogram>
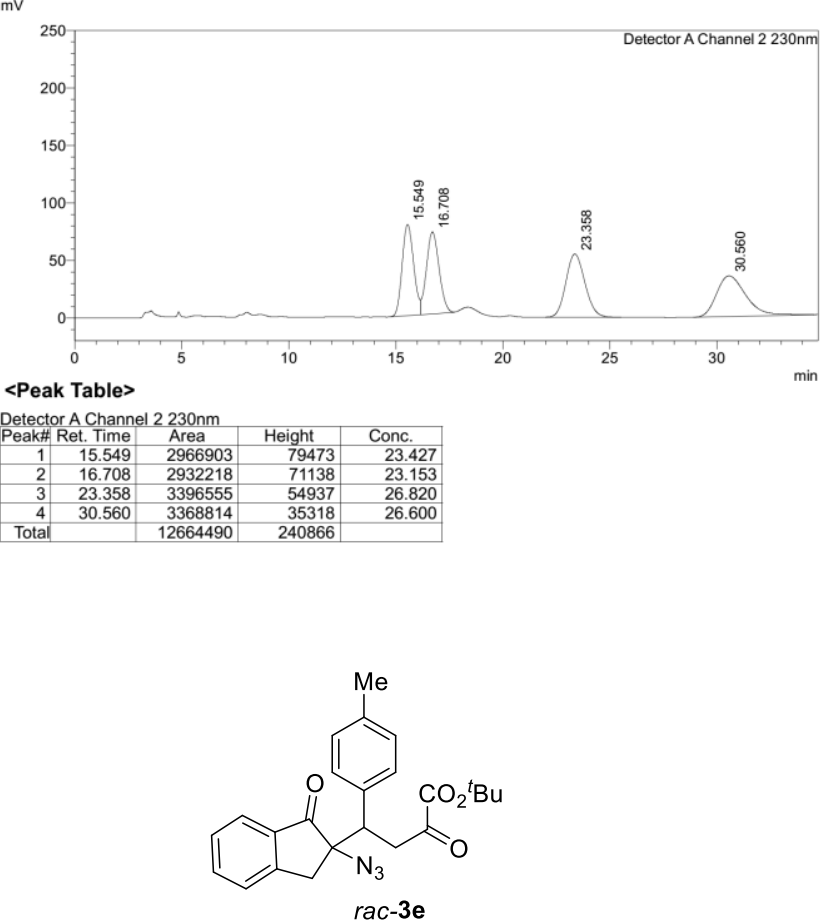

\section{Analysis Report}

<Sample Information>

Data Filename $\quad$ dpg-dJ-29-1-1-ASY-ASH-97-3-1.0-231.lcd

Batch Filename
Viall

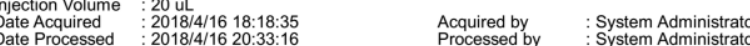

<Chromatogram>
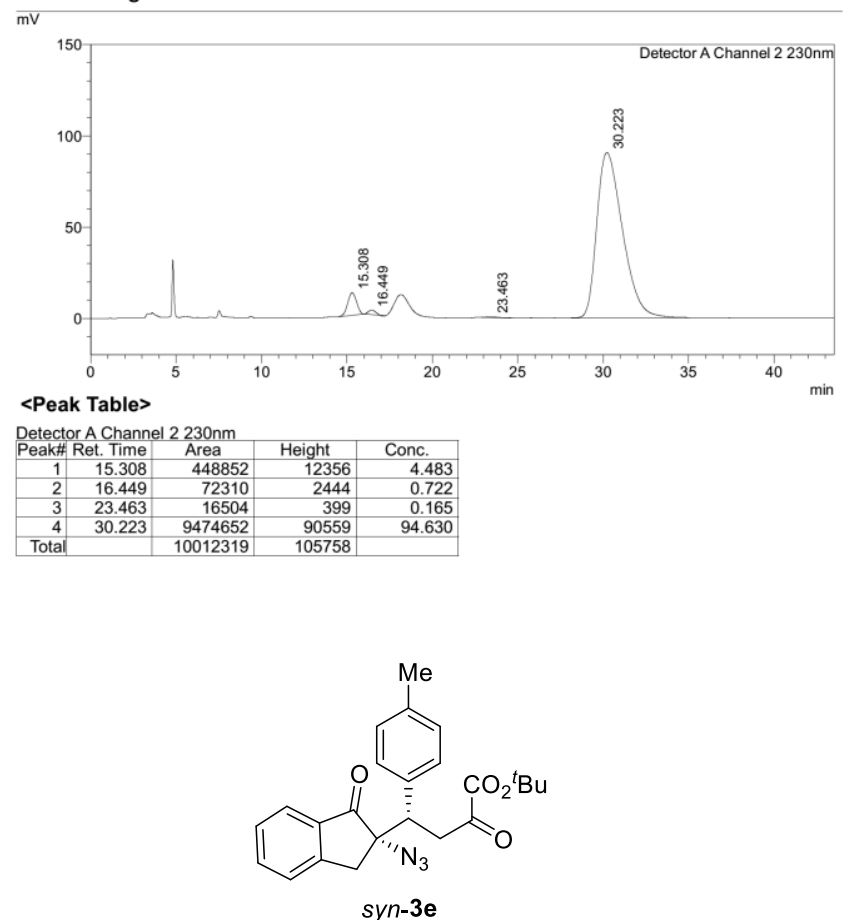
Asample Information Analysis Report

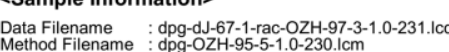

$\begin{array}{l:ll}\text { Batch Filename } & 1-1 & \text { Sample Type }\end{array}$

$\begin{array}{l:l}\text { Injection Volume } & : 20 \mathrm{uL} \\ \text { Date Acquired } & 2018 / 51015: 23: 3 \\ \text { Date Processed } & 2018 / 5 / 1016: 31: 25\end{array}$

: Unknow

$\begin{array}{ll}\text { Acquired by } & \text { : System Administrator } \\ \text { Processed by } & \text { System Administrator }\end{array}$

<Chromatogram>
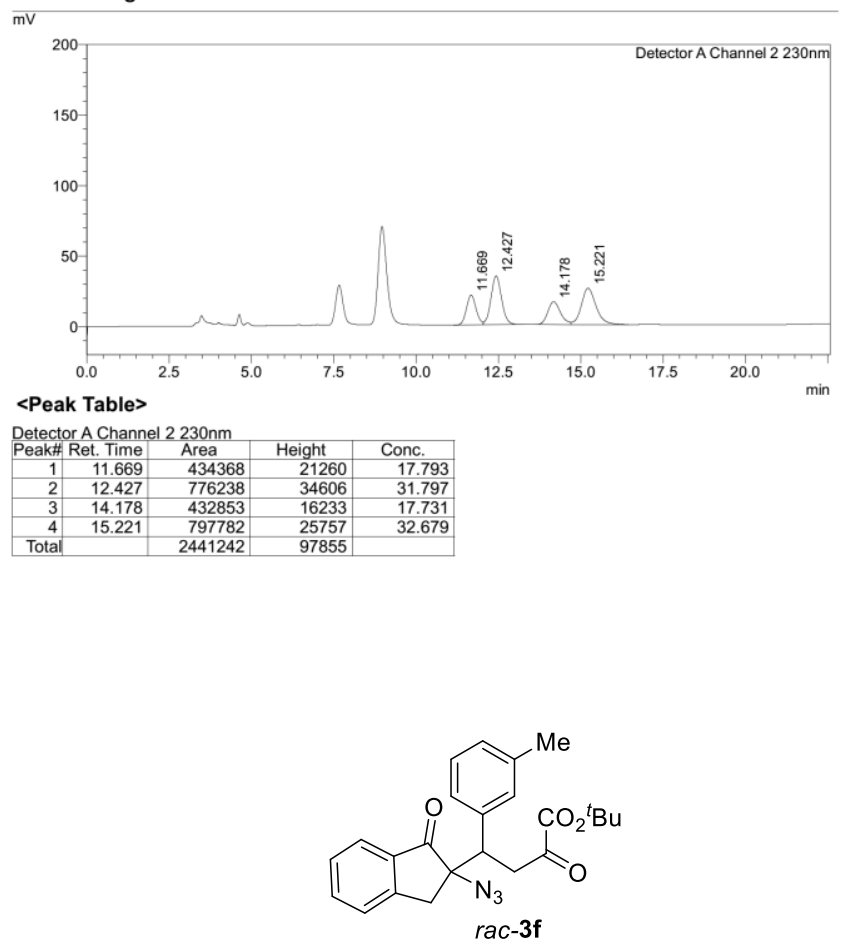

\section{Analysis Report}

$<$ Sample Information

Data Filename : dpg-dJ-65-1-ASY-OZH-97-3-1.0-231.1c

Batch Filename
Vial $t 1-1$

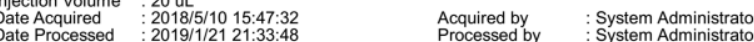

<Chromatogram>
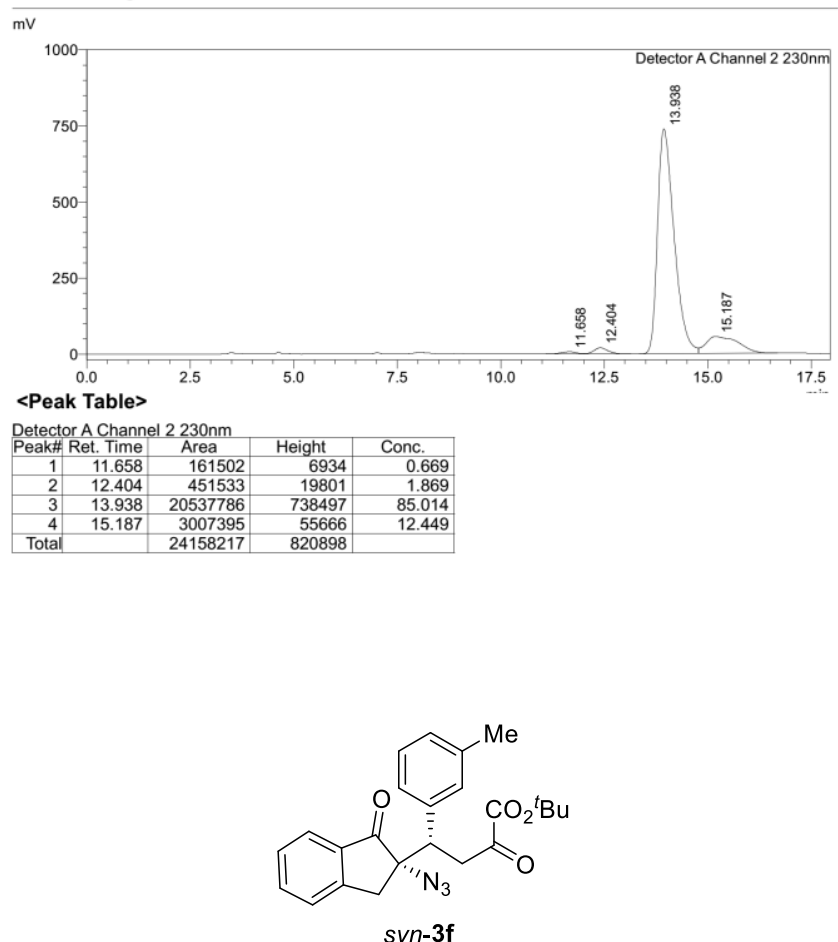


\section{Analysis Report}

<Sample Information>

Data Filename : dpg-dj-53-5b-RAC-ASH-95-5-1.0-230.1c

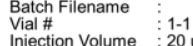

$\begin{array}{lll}\text { Vhjection Volume } & 20 \mathrm{uL} & \\ \text { Date Acquired } & 2020 / 6 / 8 & 16: 21: 45 \\ \text { Date Processed } & : 2020 / 6 / 8 & 16: 44: 20\end{array}$

Sample Type : Unknow

$\begin{array}{ll}\text { Acquired by } & \text { System Administrator } \\ \text { Processed by } & \text { System Administrator }\end{array}$

$<$ Chromatogram>

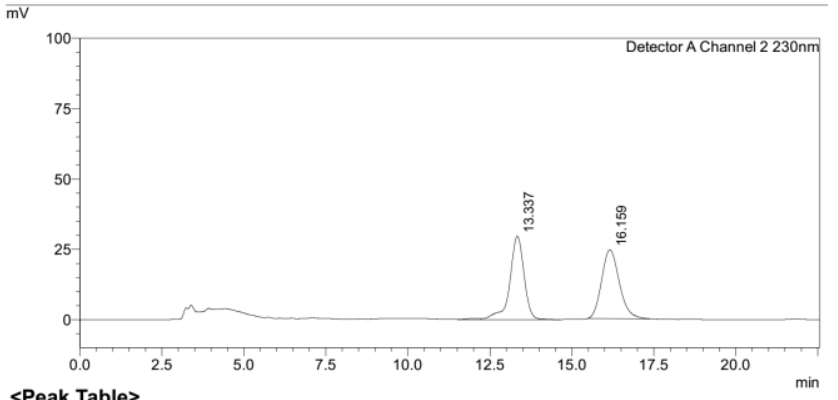

<Peak Table>

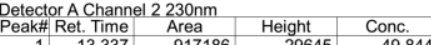

\begin{tabular}{|r|r|r|r|r|}
\hline 1 & 13.337 & 917186 & 29645 & 49.844 \\
\hline 2 & 16.159 & 922913 & 24518 & 50.156 \\
\hline Total & & 1840099 & 54163 & \\
& & & &
\end{tabular}

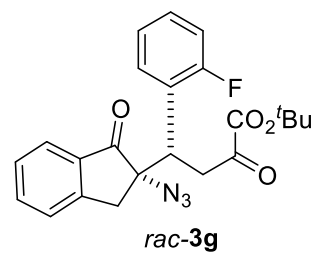

D:IDataldpgIDJddpg-dj-53-5b-RAC-ASH-95-5-1.0-230.llcd

\section{Analysis Report}

<Sample Information>

Data Filename : dpg-di-53-5b-ASY-ASH-95-5-10-230.1c

$\begin{array}{l:cl}\text { Batch Filename } & 1-1 & \text { Sample Type } \\ \text { Vial } & \end{array}$

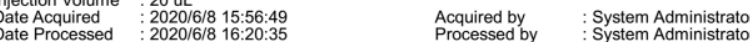

$<$ Chromatogram>
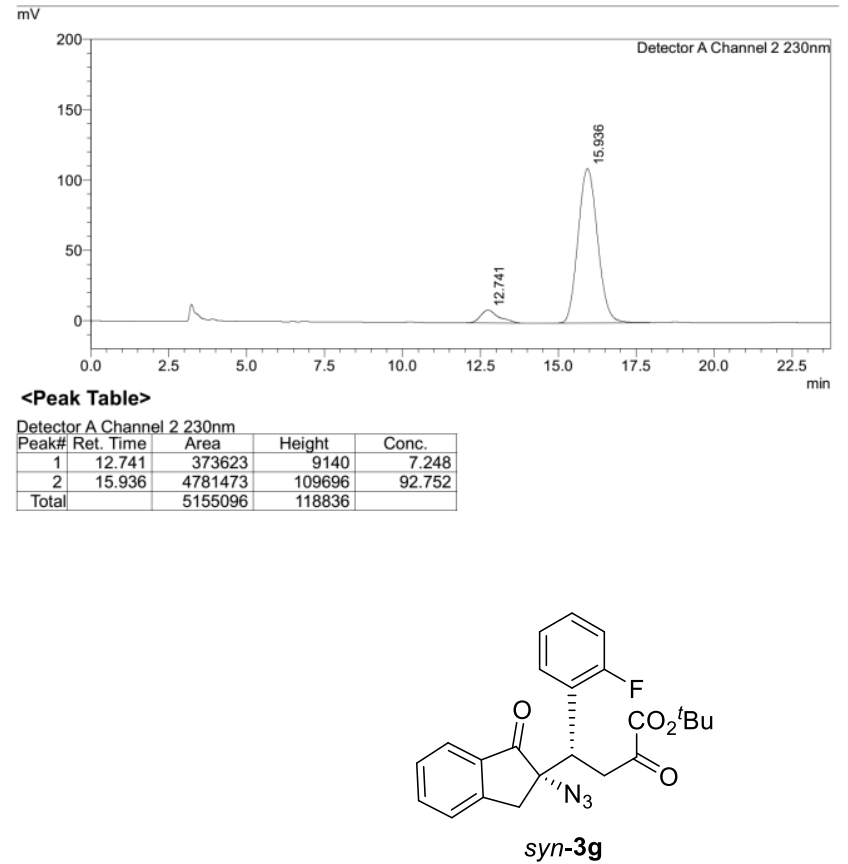

D:IDataldpg|DJIdpg-dj-53-5b-ASY-ASH-95-5-1.0-230.Icd 


\section{Analysis Report}

$<$ Sample Information

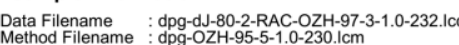

$\begin{array}{l:l}\text { Batch Filename } & \vdots \\ \text { Vial \# } & 1-1\end{array}$

$\begin{array}{l:ll}\text { Vial \# } & 1-1 \\ \text { Injection Volume } & : 20 \mathrm{uL} & \text { Sample Type }\end{array}$

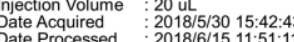

Acquired by
Processed by

<Chromatogram>
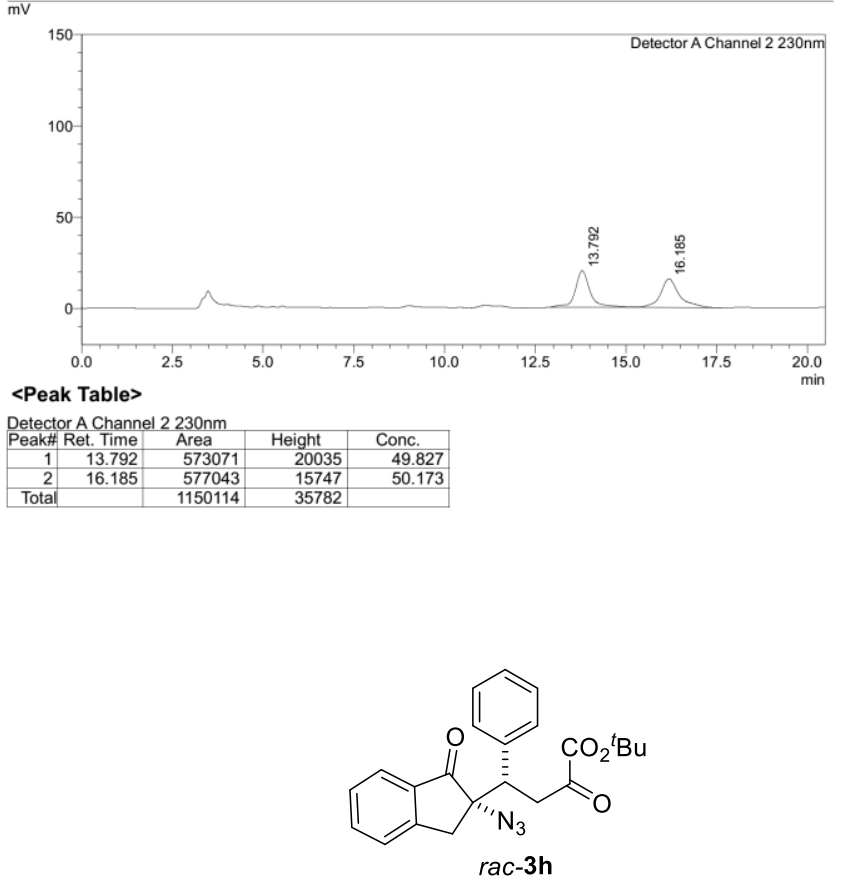

\section{Analysis Report}

<Sample Information

Data Filename : dpg-dJ-80-2-ASY-OZH-97-3-1.0-232.10

$\begin{array}{l:cc}\text { Batch Filename } & 1-1 & \text { Sample Type } \\ \text { Vial } & 1-1\end{array}$

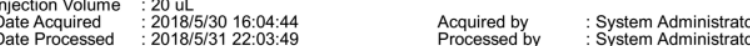

<Chromatogram>
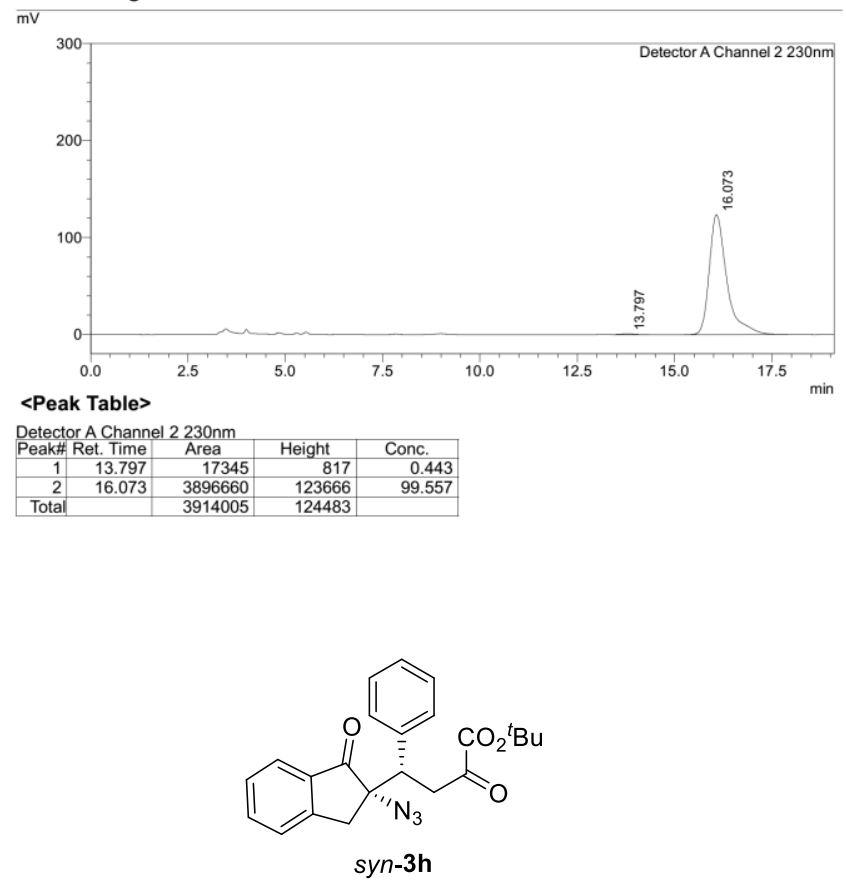
¿sample Information Analysis Report

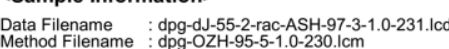

$\begin{array}{l:ll}\text { Batch Filename } & 1-1 \\ \text { Vial } \# \text { Sample Type }\end{array}$

$\begin{array}{l:ll}\text { Injection Volume } & : 21 \mathrm{uL} \\ \text { Date Acquired } & 2018 / 5 / 315: 03: 56\end{array}$

: Unknow

Acquired by
Processed by

<Chromatogram>
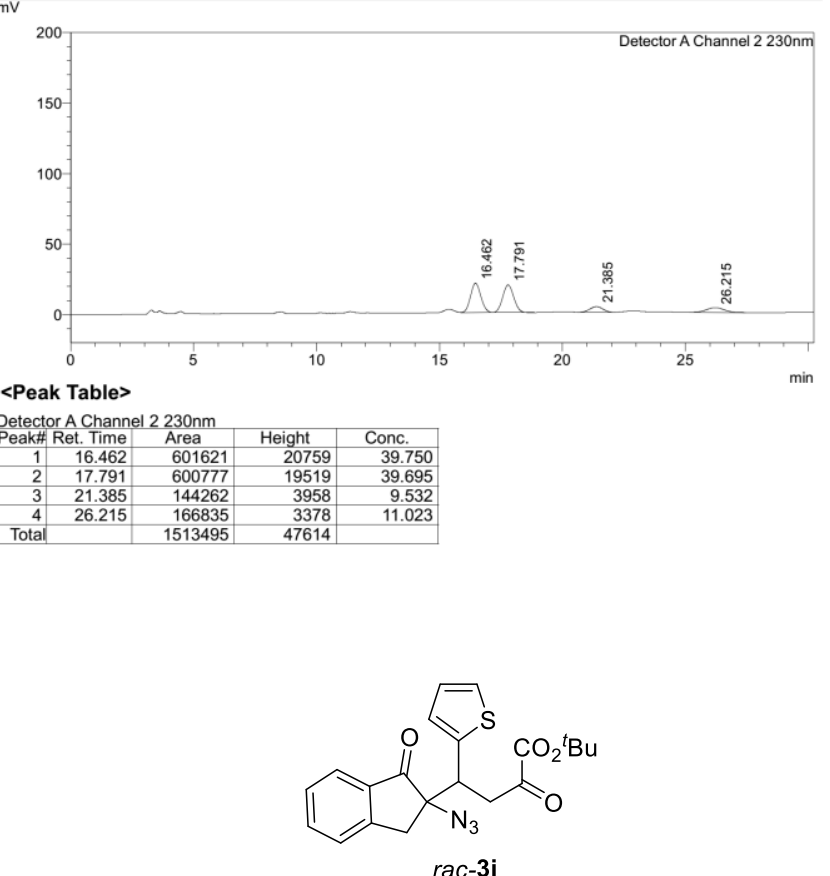

\section{Analysis Report}

$<$ Sample Information>

Data Filename : dpg-dJ-53-2-ASY-ASH-97-3-1.0-231.lcd

Vial Filename $11-1 \quad$ Sample Type

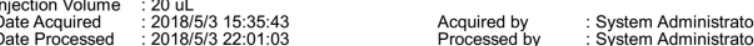

<Chromatogram>
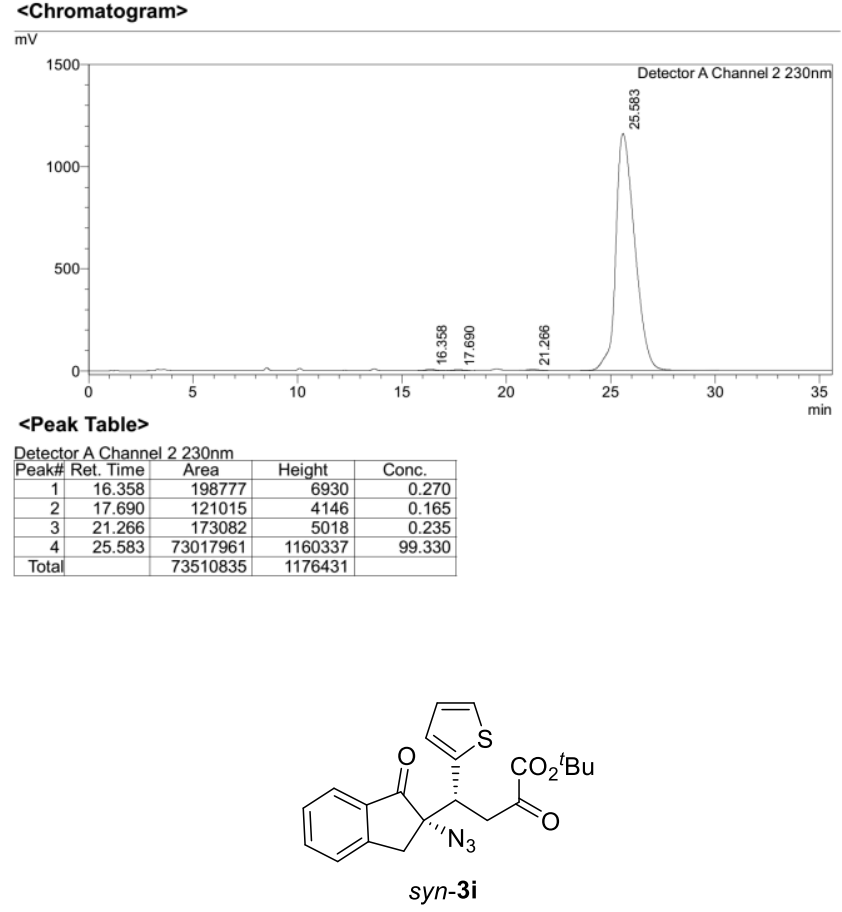
¿sample Information Analysis Report

Data Filename
Method Filename

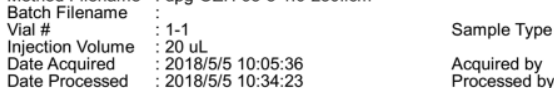

Acquired by
Processed by

<Chromatogram>
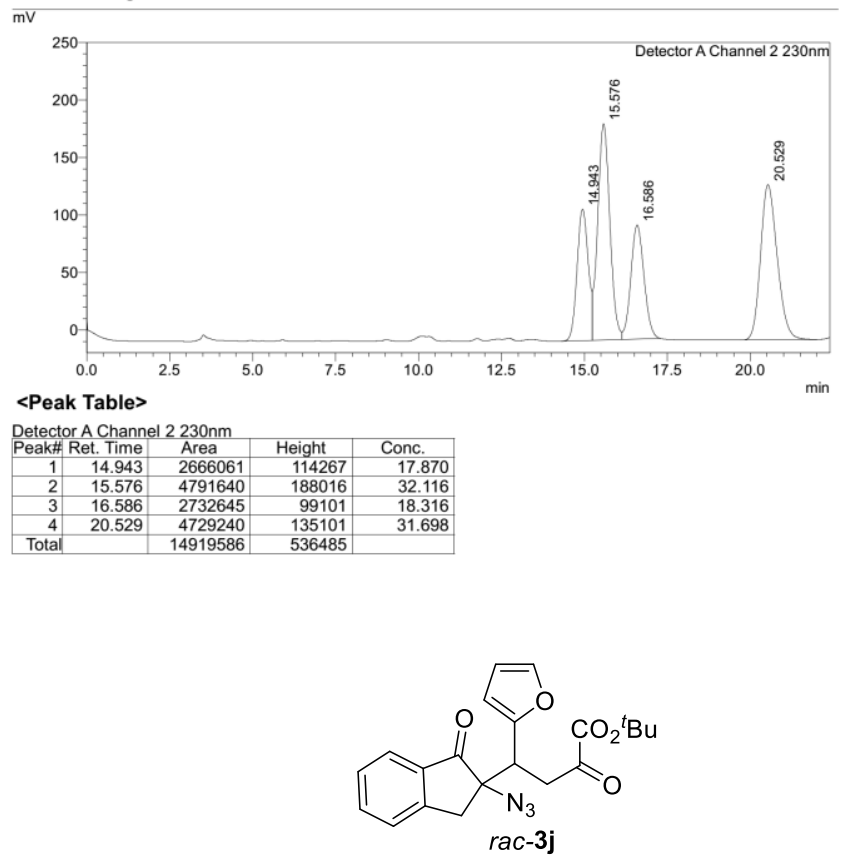

\section{Analysis Report}

<Sample Information

Data Filename $\quad$ dop-dJ-53-3-asy-OZH-97-3-10-232.1cd

$\begin{array}{l:ll}\text { Batch Filename } & 1-1 & \text { Sample Type } \\ \text { Vial } & 1-1\end{array}$

$\begin{array}{llll} & \text { Acquired by } & \text { System Administrator } \\ \text { Date Acquired } & 20101 / 5159: 16: 08 & \text { System Administorator }\end{array}$

<Chromatogram>
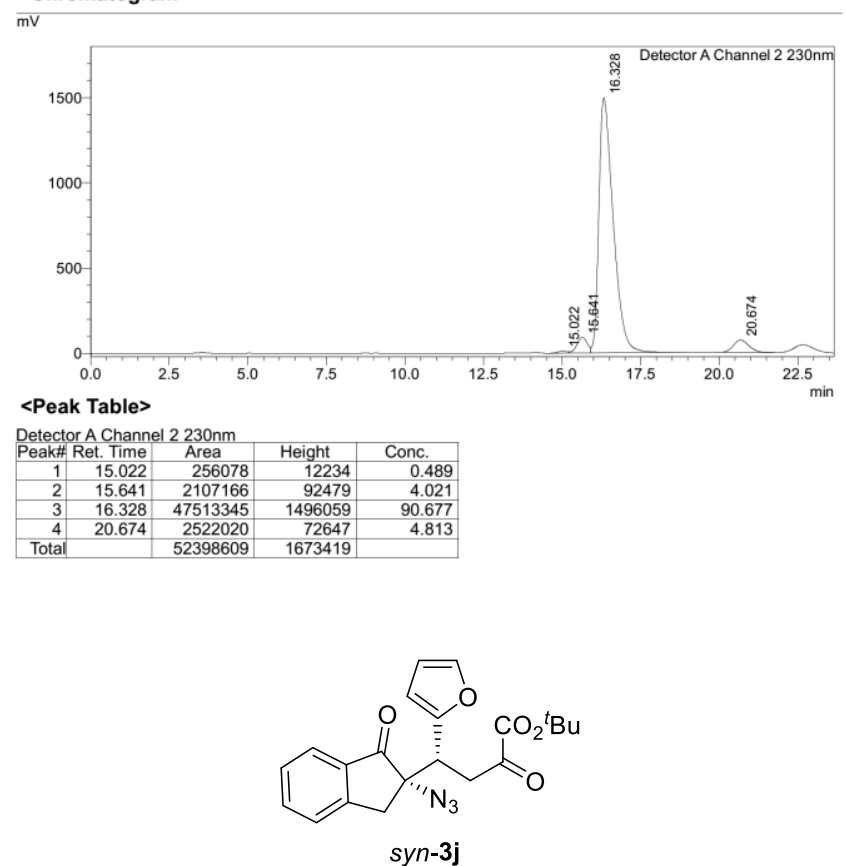


\section{Analysis Report}

<Sample Information>

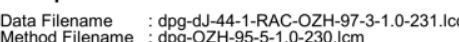

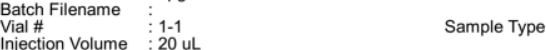

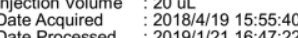

Acquired by
Processed by

<Chromatogram>
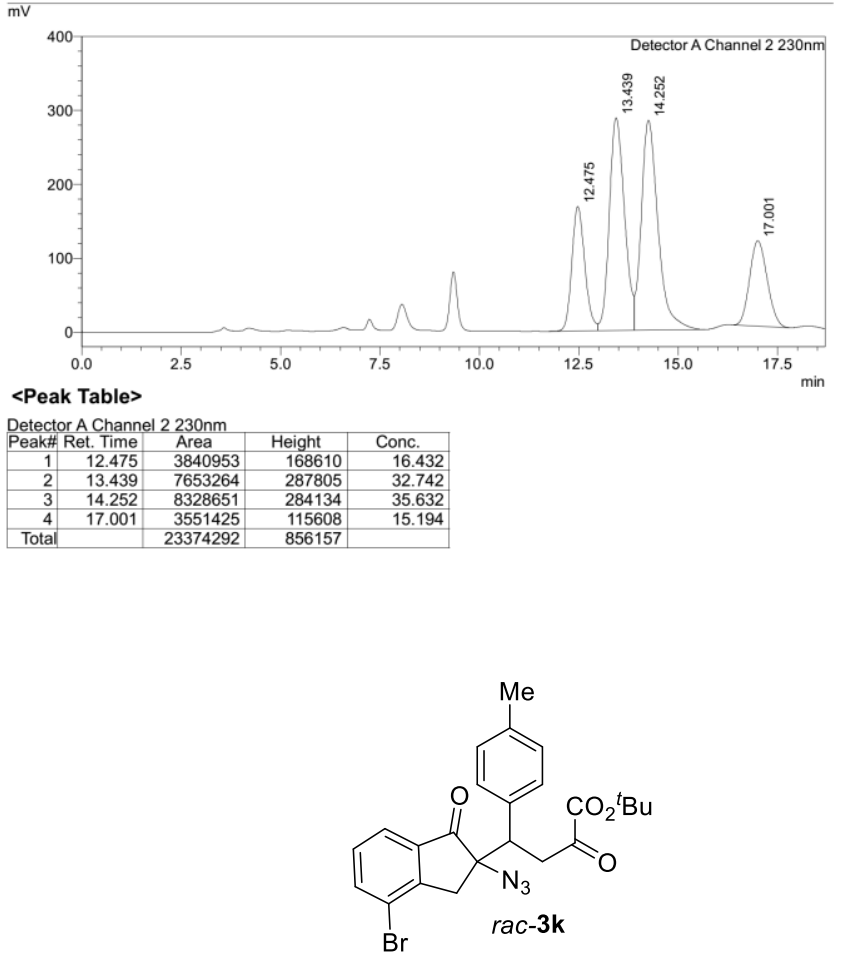

\section{Analysis Report}

<Sample Information

Data Filename : dpg-dJ-44-1-asy-OZH-97-3-1.0-231.lcd

$\begin{array}{l:ll}\text { Batch Filename } & 1-1 & \text { Sample Type } \\ \text { Vial } & 1-1\end{array}$

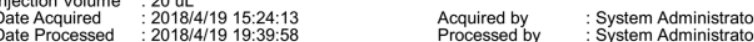

<Chromatogram>
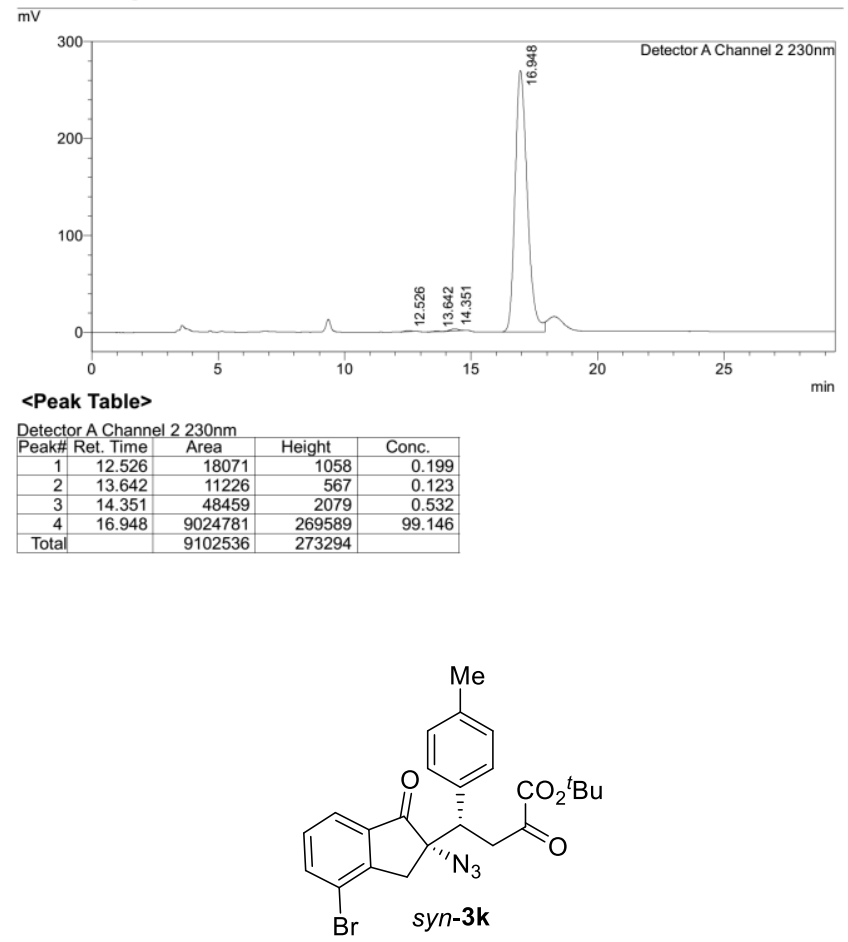


\section{Analysis Report}

<Sample Information

Data Filename
Method Filename

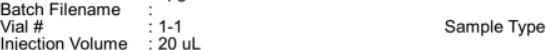

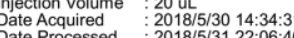

Acquired by
Processed by $\quad$ System Administrator
System Administrator

<Chromatogram>
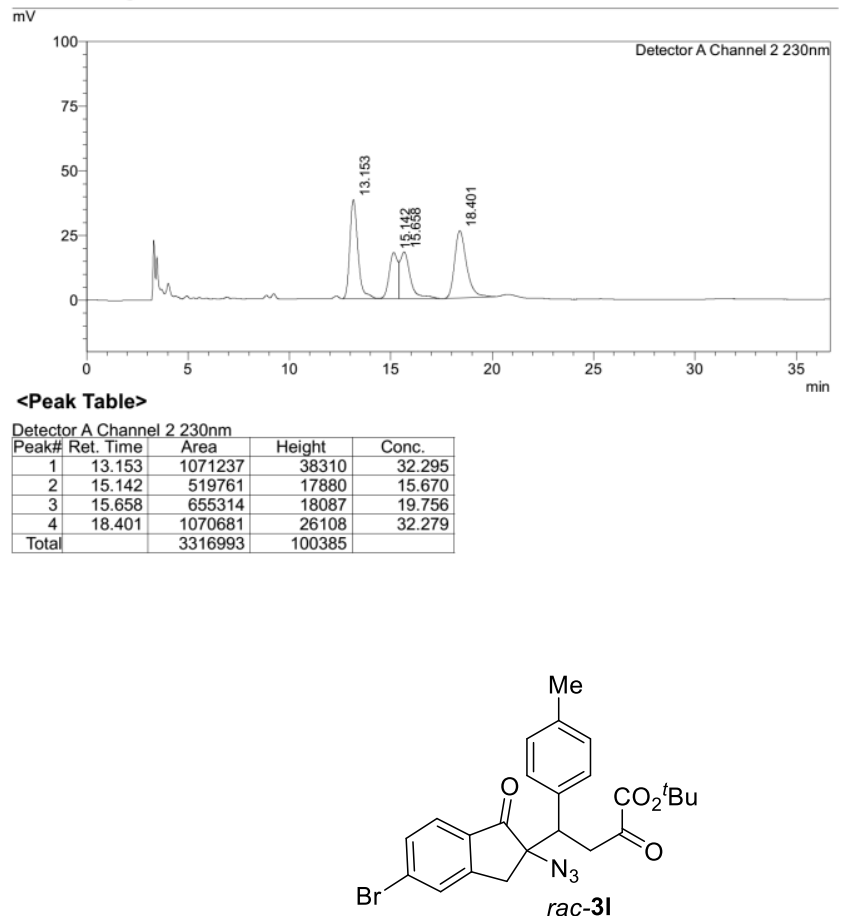

\section{Analysis Report}

<Sample Information

Data Filename : dpg-dJ-81-1-ASY-OZH-97-3-1.0-232.10

$\begin{array}{lll}\text { Batch Filename } & 1-1 & \text { Sample Type }\end{array}$

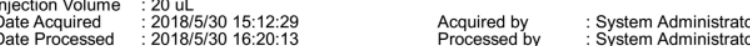

\section{$<$ Chromatogram>}

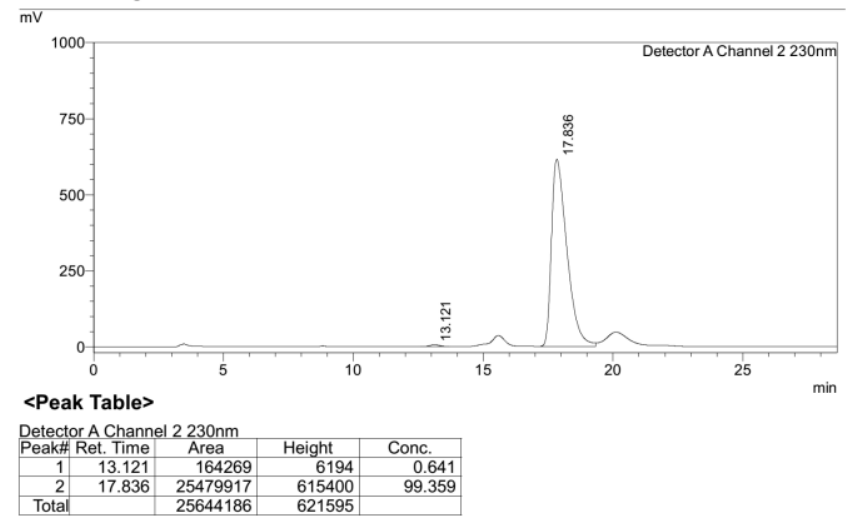

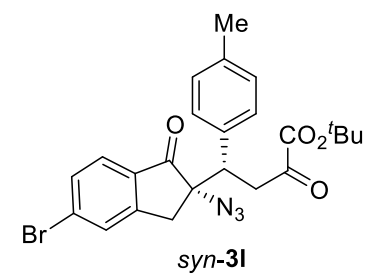


Asample Information Analysis Report

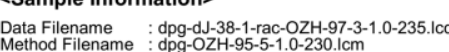

Batch Filename
Vial $\#$ Sample Type

$\begin{array}{l:l}\text { Injection Volume } & : 20 \mathrm{uL} \\ \text { Date Acquired } & 2018 / 4 / 917: 13: 18 \\ \text { Date Processed } & 2018 / 4 / 920: 44: 19\end{array}$

Sample Type : Unknow

$\begin{array}{ll}\text { Acquired by } & \text { : System Administrator } \\ \text { Processed by } & \text { System Administrator }\end{array}$

<Chromatogram>
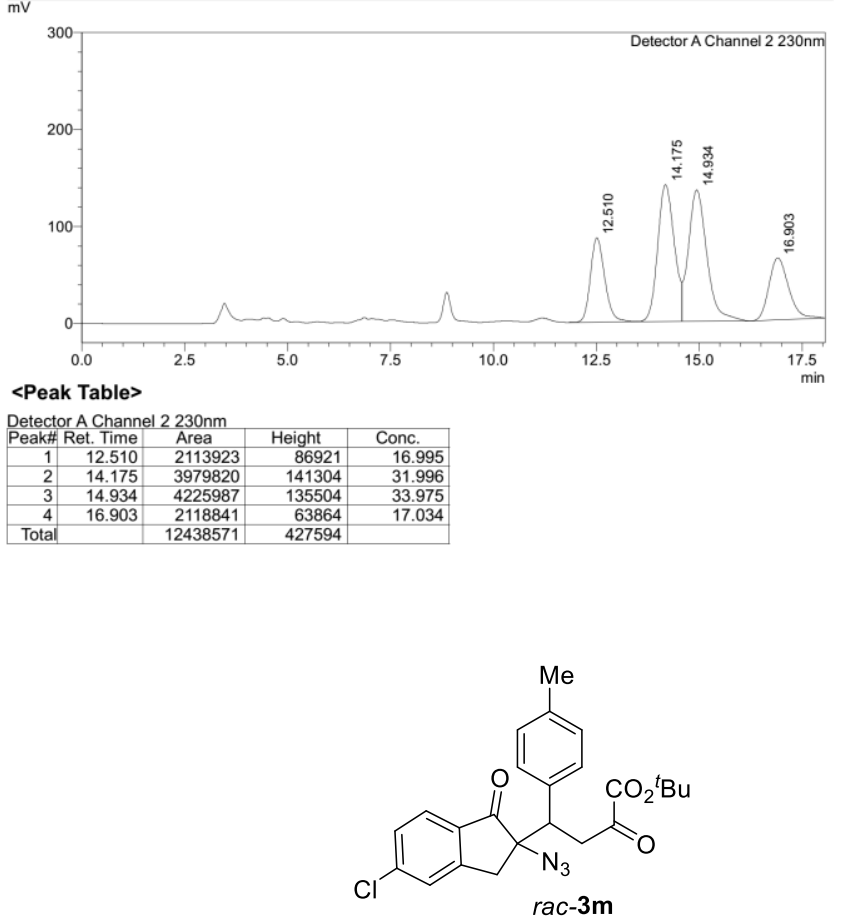

\section{Analysis Report}

$<$ Sample Information

Data Filename : dpg-dJ-38-1-asy-OZH-97-3-1.0-235.lcd

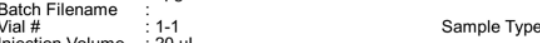

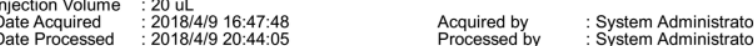

<Chromatogram>

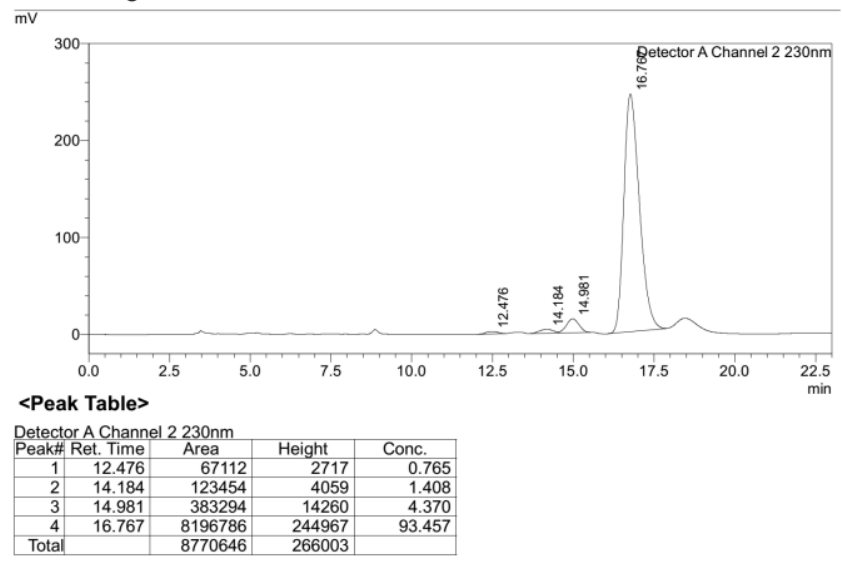

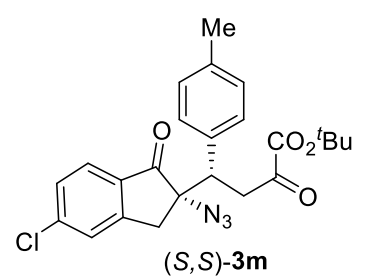


Analysis Report

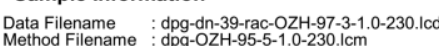

Sample Type - Unkno

Injection Volume : 20 uL

$\begin{array}{lll}\text { lnjection Volume } & 1-1 \\ \text { Date Acquired } & \text { 2020/5/31 16:28:28 } \\ \text { Date Processed } & : 2020 / 5 / 31 \text { 21:15:44 }\end{array}$

Acquired by
Processed by

$<$ Chromatogram>
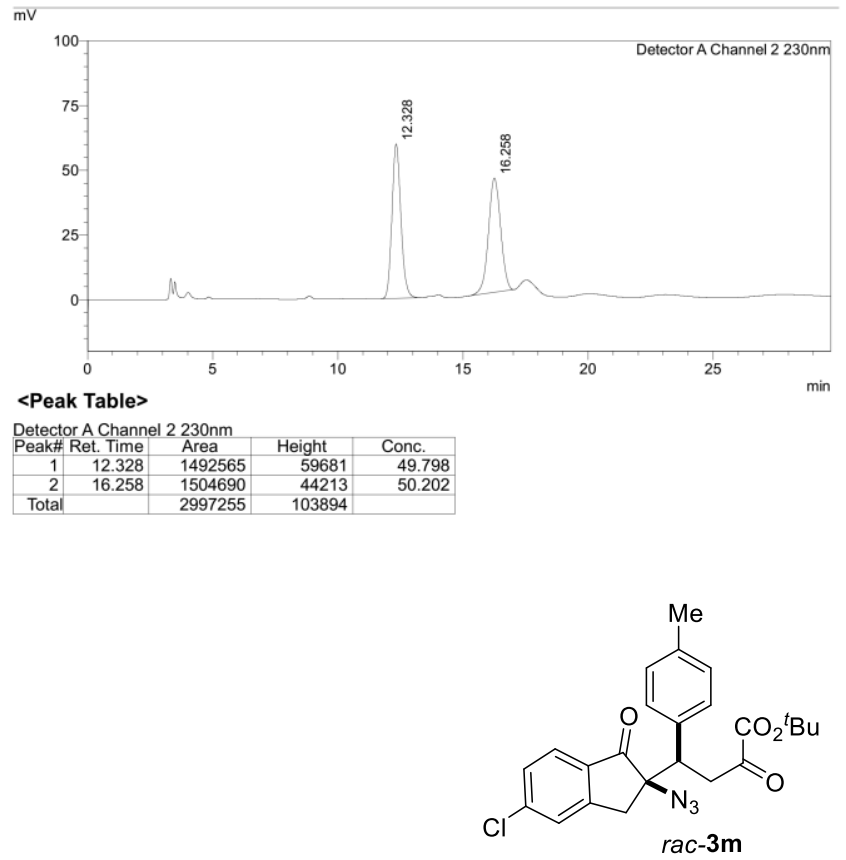

D:|Dataldpgldnldpg-dn-39-rac-OZH-97-3-1.0-230.Jcd

\section{Analysis Report}

<Sample Information>

Data Filename : dpg-dn-39-1-asy-0ZH-97-3-10-230.1cd

$\begin{array}{l:c}\text { Batch Filename } & 1-1 \\ \text { Vial \# Sample Type }\end{array}$

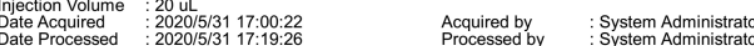

<Chromatogram>
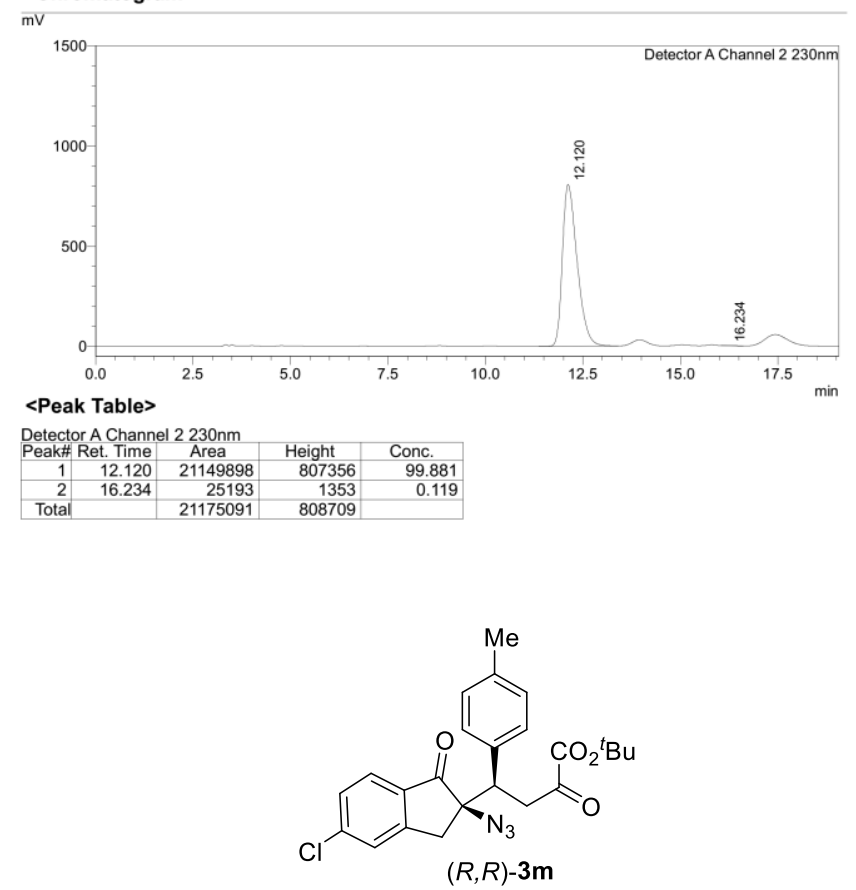

D:|Dataldpgldnldpg-dn-39-1-asy-0ZH-97-3-1. 0-230 icd 


\section{Analysis Report}

<Sample Information

Data Filename
Method Filename : dpg-d-d-44-3-rac-0ZH-97-3-1.0-231.10
dpg-OZH-95-5-1.0-230 Icm

$\begin{array}{l:l}\text { Batch Filename } & \vdots 1-1 \\ \text { Vial \# }\end{array}$

$\begin{array}{l:l}\text { Injection Volume } & 21 \mathrm{LL} \\ \text { Date Acquired } & 2018 / 5 / 514: 42: 06 \\ \text { Date Processed } & 2018 / 5 / 515: 09: 40\end{array}$

Sample Type

Acquired by
Processed by

System Admininstrator
System Administrator

<Chromatogram>
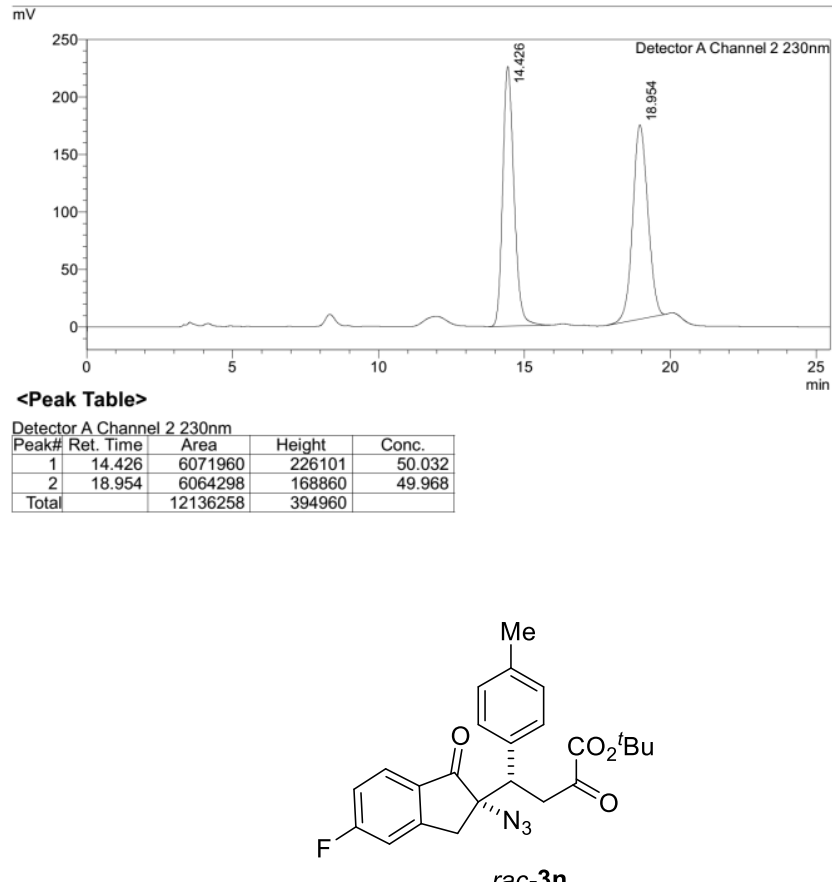

rac-3n

\section{Analysis Report}

<Sample Information

Data Filename $:$ dpg-dJ-44-3-asy-0ZH-97-3-1.0-231.1cd

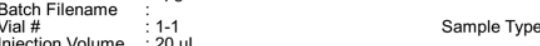

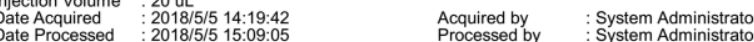

<Chromatogram>
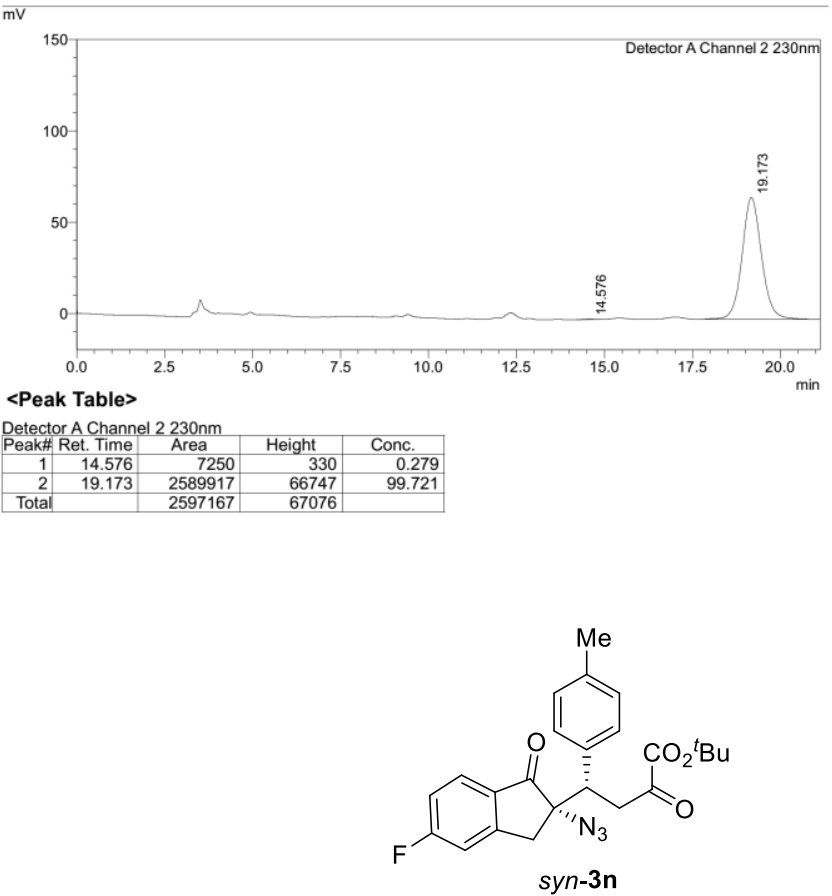
¿sample Information Analysis Report

$\begin{array}{lll}\text { Data Filename } & \text { :dpg-dJ-81-2-rac-OZH-90-10-1.0-231.lcd } \\ \text { Method Filename } & \text { dpg-OZH-95-5-1.0-230.Icm }\end{array}$

$\begin{array}{l:ll}\text { Batch Filename } & 1-1 & \text { Sial } \# \text { Sample Type }\end{array}$

$\begin{array}{l:l}\text { Injection Volume } & 20 \mathrm{uL} \\ \text { Date Acquired } & 2018 / 72014: 57: 37 \\ \text { Date Processed } & : 20187720 \text { 15:37:52 }\end{array}$

Acquired by
Processed by

<Chromatogram>
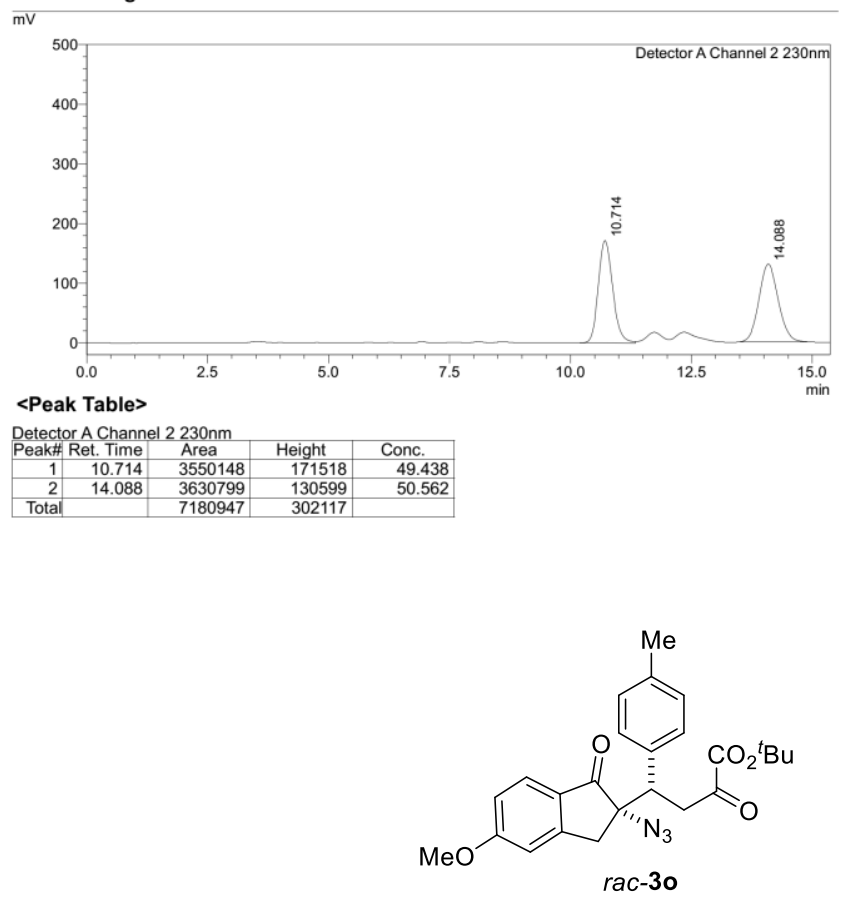

\section{Analysis Report}

<Sample Information>

Data Filename : dpg-dJ-81-2-asy-OZH-90-10-1.0-231.lcd

Vial Filename $11-1 \quad$ Sample Type

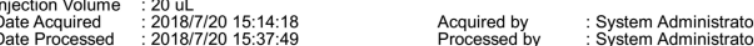

<Chromatogram>

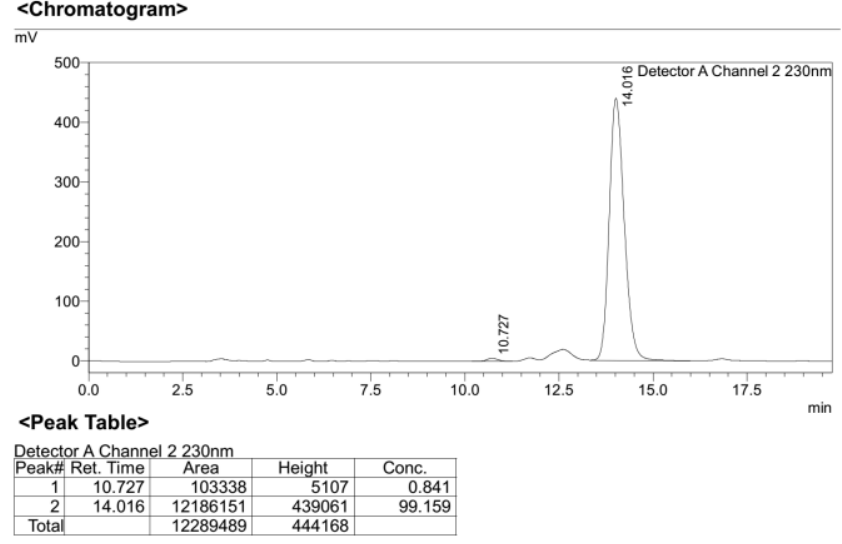

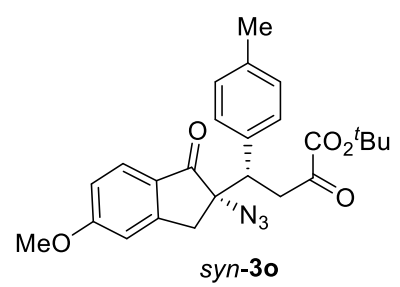




\section{Analysis Report}

<Sample Information>

Data Filename : dpg-dJ-44-4-rac-ASH-95-5-1.0-231.lcd

$\begin{array}{l:l}\text { Batch Filename } & \vdots \\ \text { Vial \# } & 1-1 \\ \text { lnijection Volume } & : 20\end{array}$

$\begin{array}{l:l}\text { Injection Volume } & 200 \mathrm{LL} \\ \text { Date Acquired } & 202015 / 2316: 41: 5 \\ \text { Date Processed } & 202015 / 2321: 10: 1\end{array}$

Sample Type : Unknow

$\begin{array}{ll}\text { Acquired by } & \text { : System Administrator } \\ \text { Processed by } & \text { System Administrator }\end{array}$

<Chromatogram>
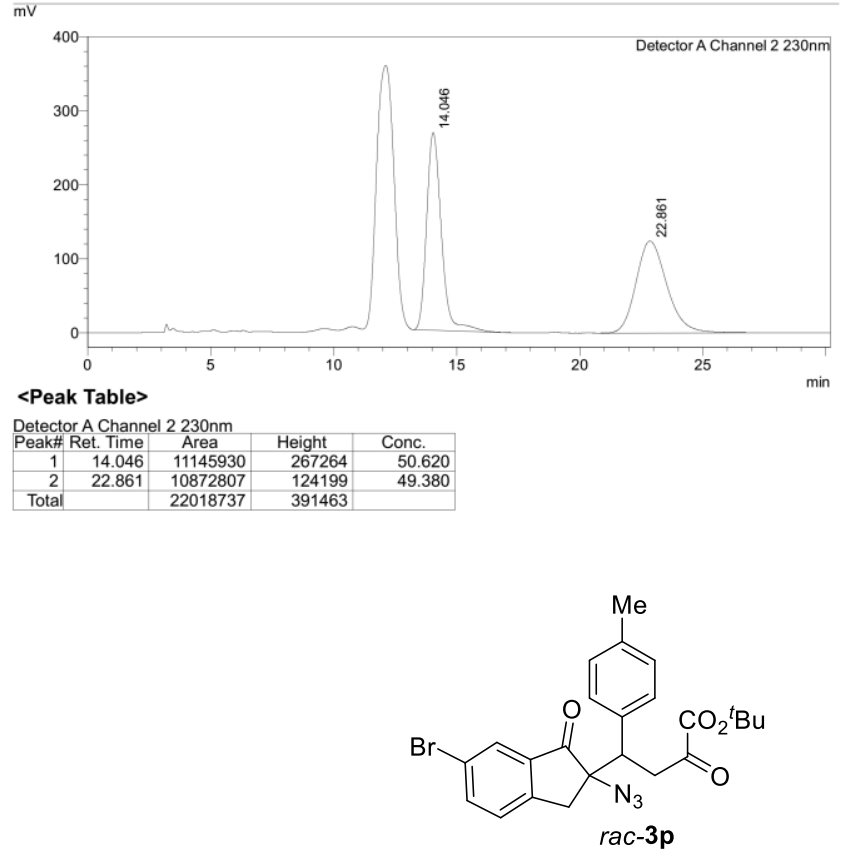

\section{Analysis Report}

<Sample Information

Data Filename : : dpg-dJ-44-4-asy-ASH-95-5-1.0-231.1co

$\begin{array}{l:cc}\text { Batch Filename } & 1-1 & \text { Sample Type }\end{array}$

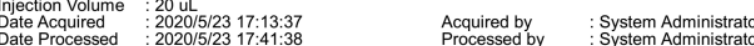

<Chromatogram>
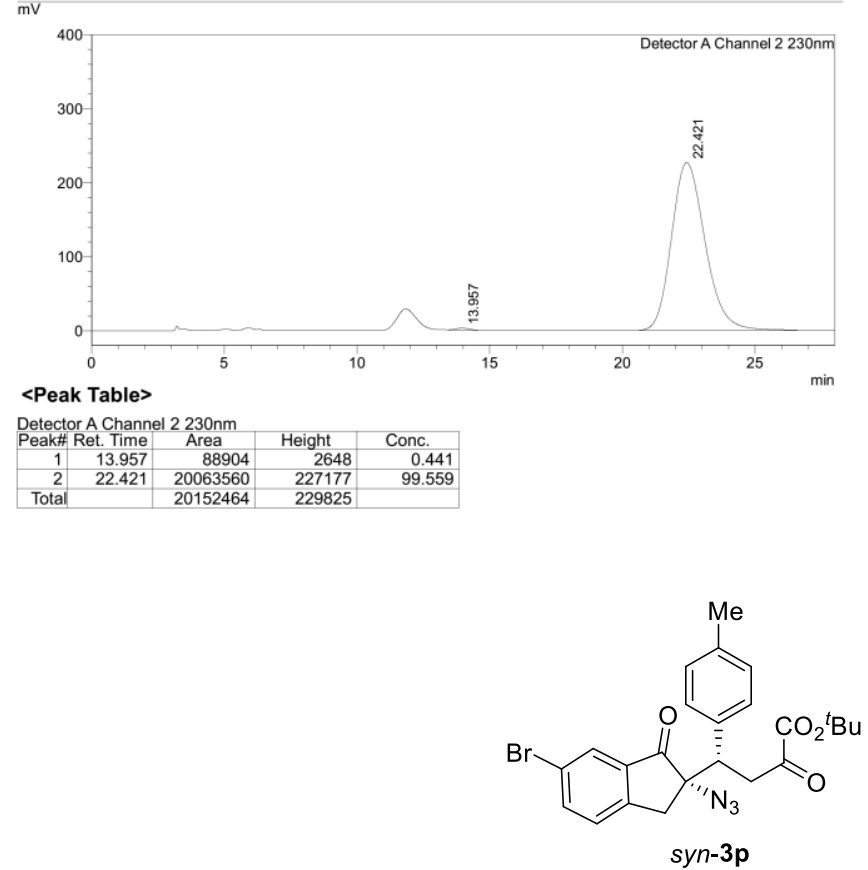


\section{Analysis Report}

$<$ Sample Information

$\begin{array}{l:l}\text { Data Filename } & \text { dpg-d-2-28-2-rac-ASH-97-3-1.0-231.10 } \\ \text { Method Filename } & \text { dpg-OZH-95-5-1.0-230.1cm }\end{array}$

Batch Filename
Vial $\#$ Sample Type

$\begin{array}{l:l}\text { Injection Volume } & : 20 \mathrm{uL} \\ \text { Date Acquired } & 2018 / 4 / 921: 15: 15 \\ \text { Date Processed } & 2018 / 4 / 923: 11: 21\end{array}$

: Unknow

Acquired by
Processed by

<Chromatogram>

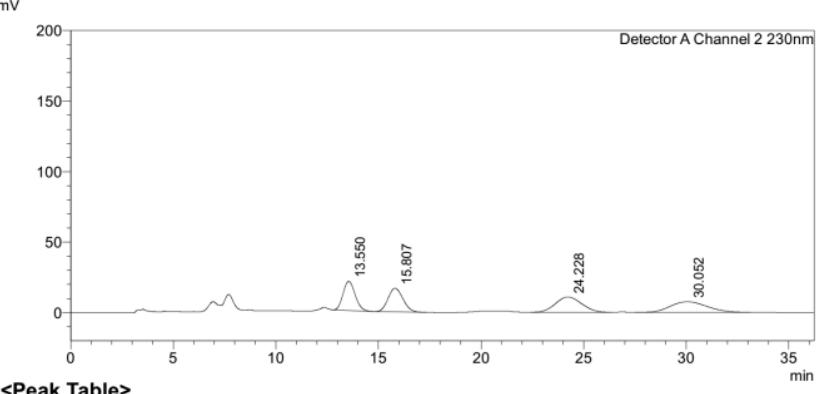

<Peak Table>

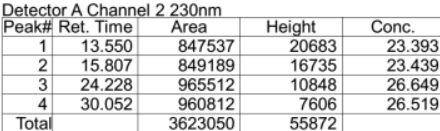

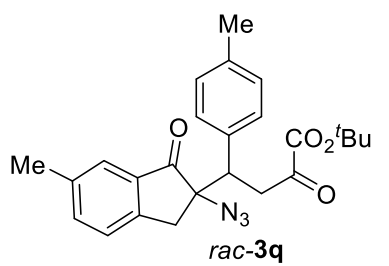

\section{Analysis Report}

<Sample Information>

Oata Filename : dpg-dJ-31-2-ASY-ASH-97-3-1.0-231.10

$\begin{array}{l:ll}\text { Batch Filename } & 1-1 & \text { Sample Type } \\ \text { Vial } & 1-1\end{array}$

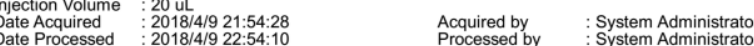

<Chromatogram>

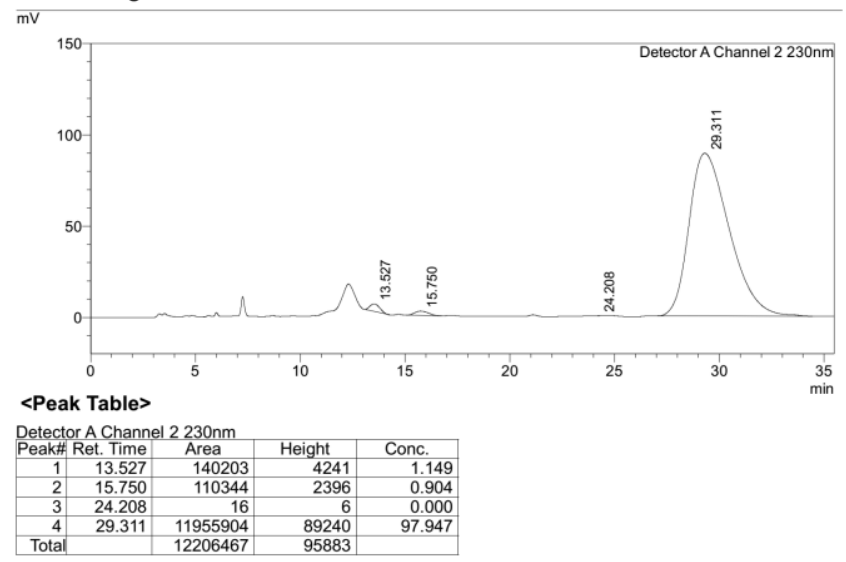

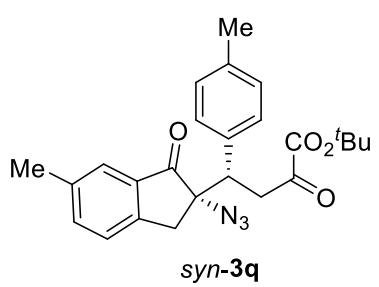


¿sample Information Analysis Report

Data Filename : dpg-dJ-112-6-RAC-OZH-97-3-1.0-231.lcd

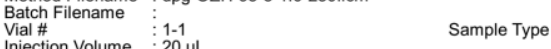

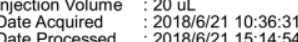

$\begin{array}{ll}\text { Acquired by } & \text { : System Administrator } \\ \text { Processed by } & \text { System Administrator }\end{array}$

<Chromatogram>

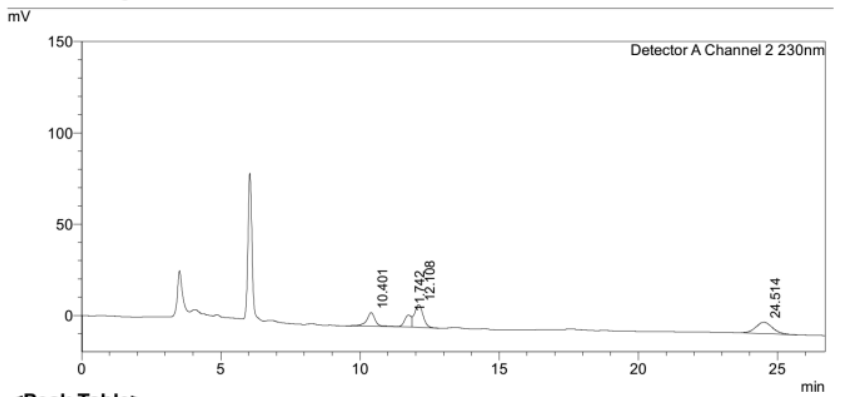

<Peak Table>

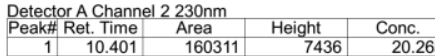

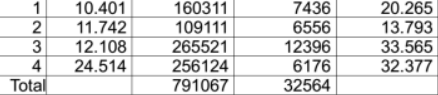

\section{Analysis Report}

<Sample Information

Data Filename : dpg-dJ-81-3-ass-0ZH-97-3-1.0-231.10

$\begin{array}{l:ll}\text { Batch Filename } & 1-1 & \text { Sample Type } \\ \text { Vial } & 1-1\end{array}$

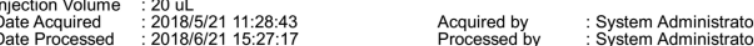

<Chromatogram>
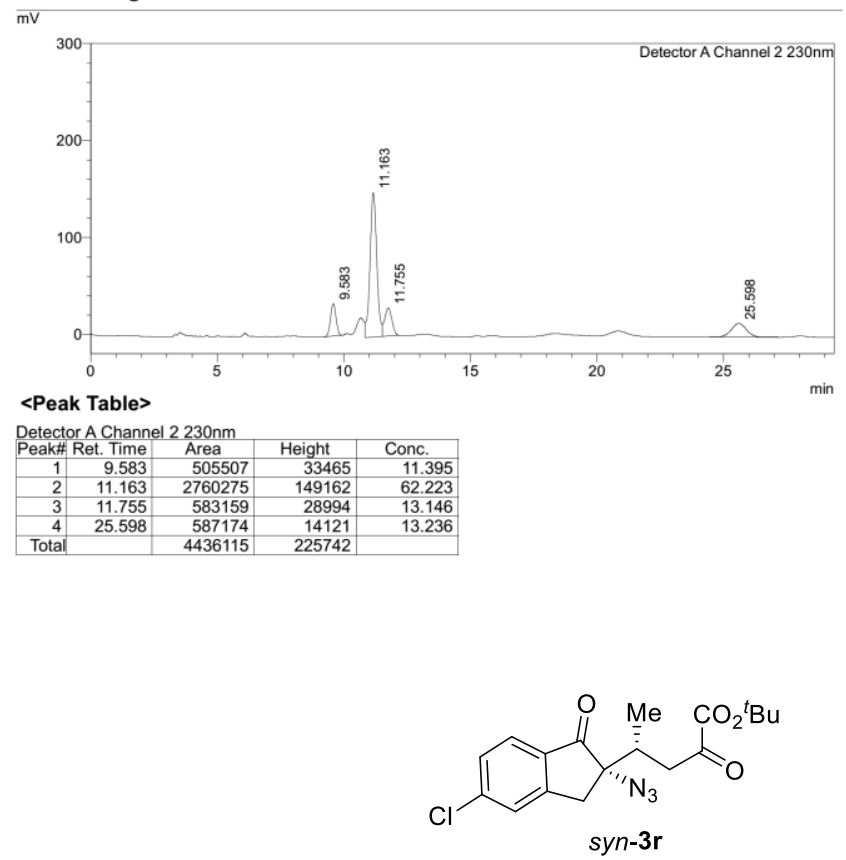
Asample Information Ansis Report

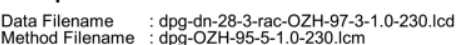

(ample

$\begin{array}{l:ll}\text { Vial \# } & 1-1 \\ \text { Injection Volume } & : 20 \\ & \text { Date Processed } & : 2025\end{array}$

$\begin{array}{lll}\text { Injection Volume } & 20 \mathrm{uL} \\ \text { Date Acquired } & 2020 / 5 / 26 & 10: 12: 46 \\ \text { Date Processed } & : 2020 / 5 / 26 \text { 11:47:44 }\end{array}$

Sample Type : Unknow

$\begin{array}{ll}\text { Acquired by } & \text { : System Administrator } \\ \text { Processed by } & \text { System Administrator }\end{array}$

<Chromatogram>
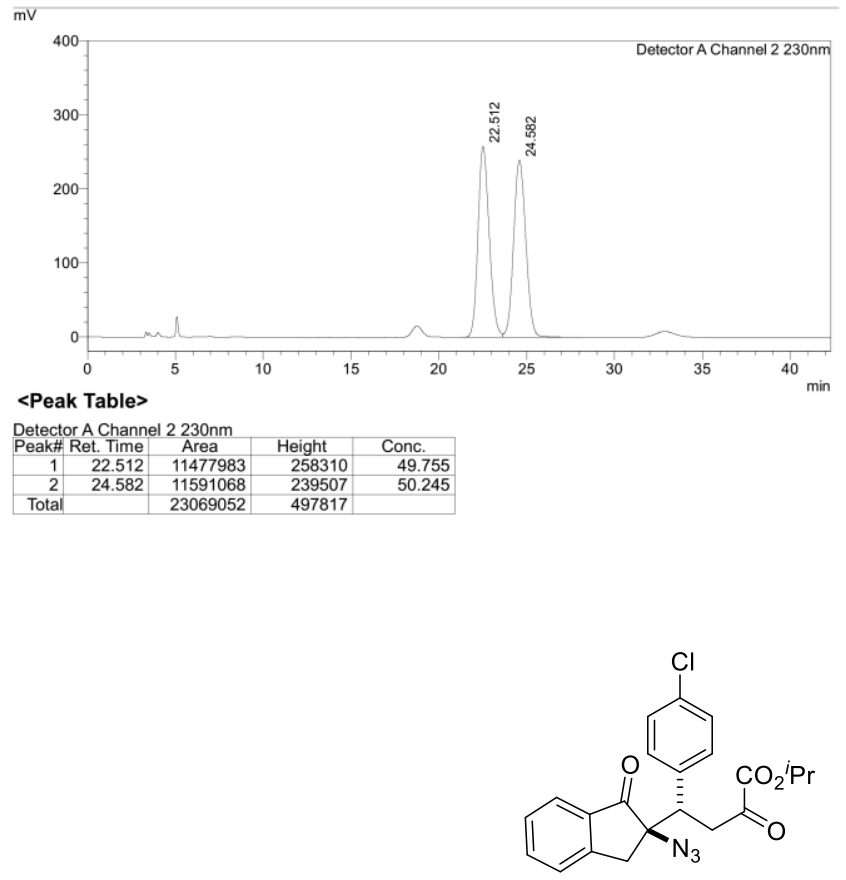

rac-3a

D:IDataldpgldnidpg-dn-28-3-rac-0ZH-97-3-10-230. Icd

\section{Analysis Report}

<Sample Information

$\begin{array}{lll}\text { Data Filename } & \text { dpg-dn-28-3-asy-OZH-97-3-1.0-230.lcd } \\ \text { Method Filename } & \text { dpg-OZH-95-5-1.0-230.lcm }\end{array}$

$\begin{array}{l:cc}\text { Batch Filename } & 1-1 & \text { Sample Type } \\ \text { Vial } & \end{array}$

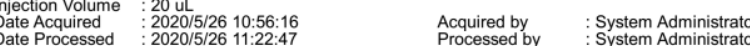

<Chromatogram>
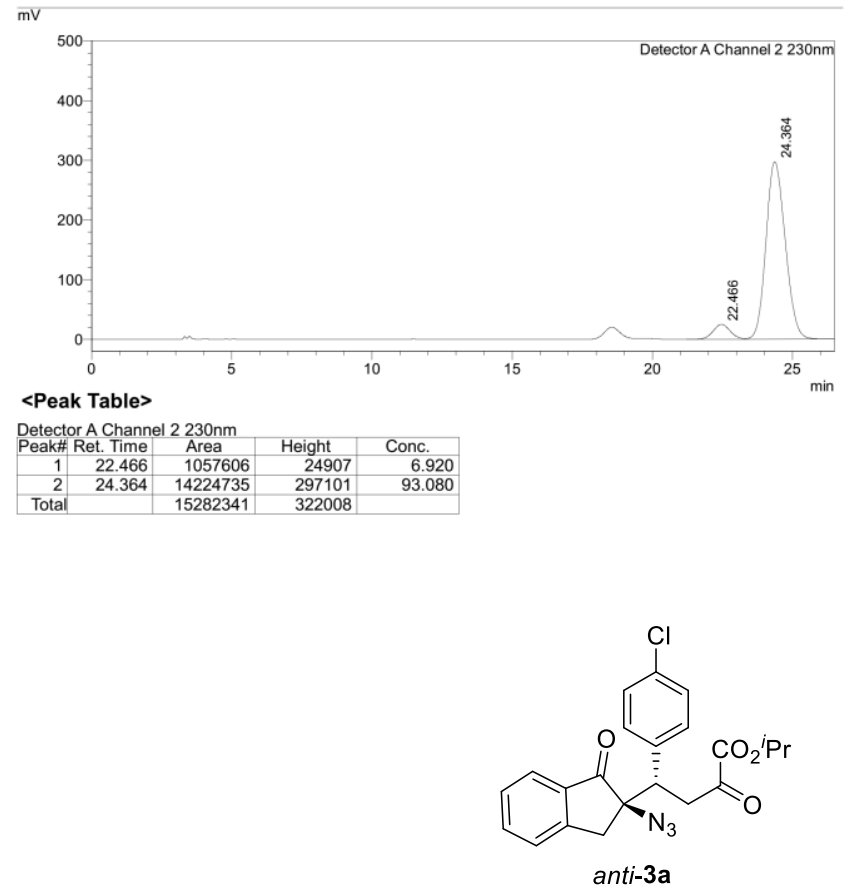

D:IDataldpglddidpg-dn-28-3-asy-0ZH-97-3-1.0-230 Icd 
¿sample Information Analysis Report

$\begin{array}{lll}\text { Data Filename } & \text { :dpg-dJ-132-1-1-rac-OZH-97-3-1.0-232.lcd } \\ \text { Method Filename } & \text { dpg-OZH-95-5-1.0-230. } \mathrm{lcm}\end{array}$

$\begin{array}{l:ll}\text { Batch Filename } & 1-1 & \text { Sial } \# \text { Sample Type }\end{array}$

$\begin{array}{l:l}\text { Injection Volume } & \text { 20 ul } \\ \text { Date Acquired } & 2018 / 6 / 2922: 05: 11 \\ \text { Date Processed } & : 2018 / 6 / 29 \\ 22: 54: 18\end{array}$

Acquired by
Processed by

<Chromatogram>
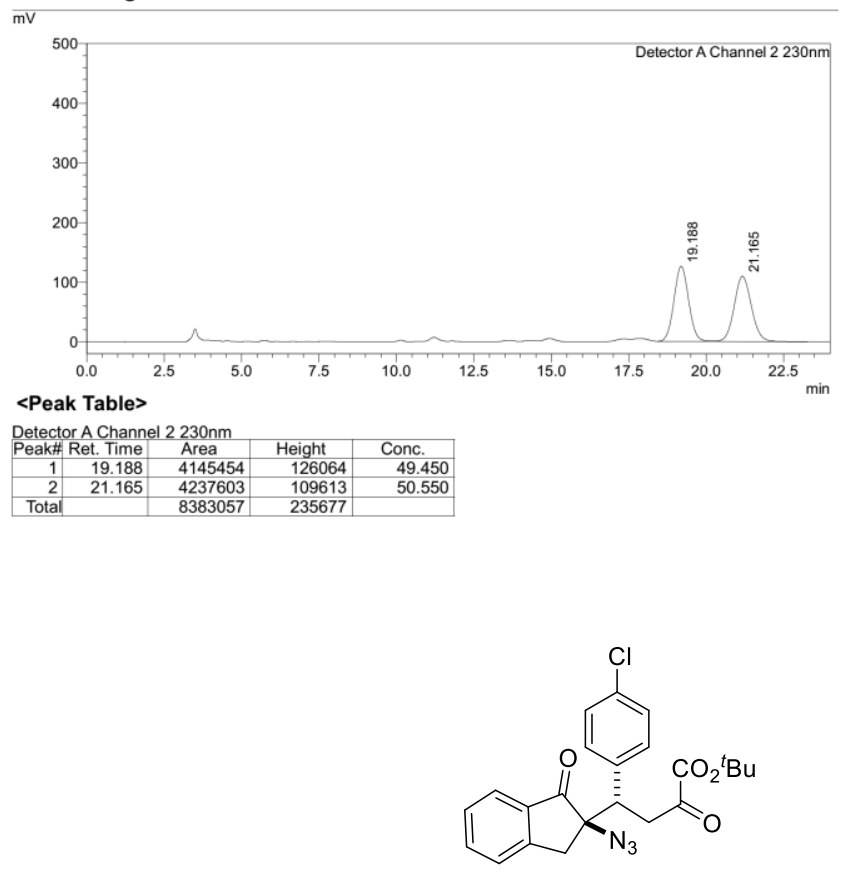

rac-3b

\section{Analysis Report}

<Sample Information

Data Filename : dpg-dJ-132-1-asy-OZH-97-3-1.0-232.lc

$\begin{array}{l:ll}\text { Batch Filename } & 1-1 & \text { Sample Type } \\ \text { Vial } & 1-1\end{array}$

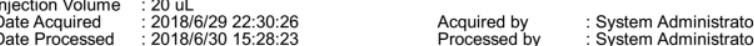

<Chromatogram>
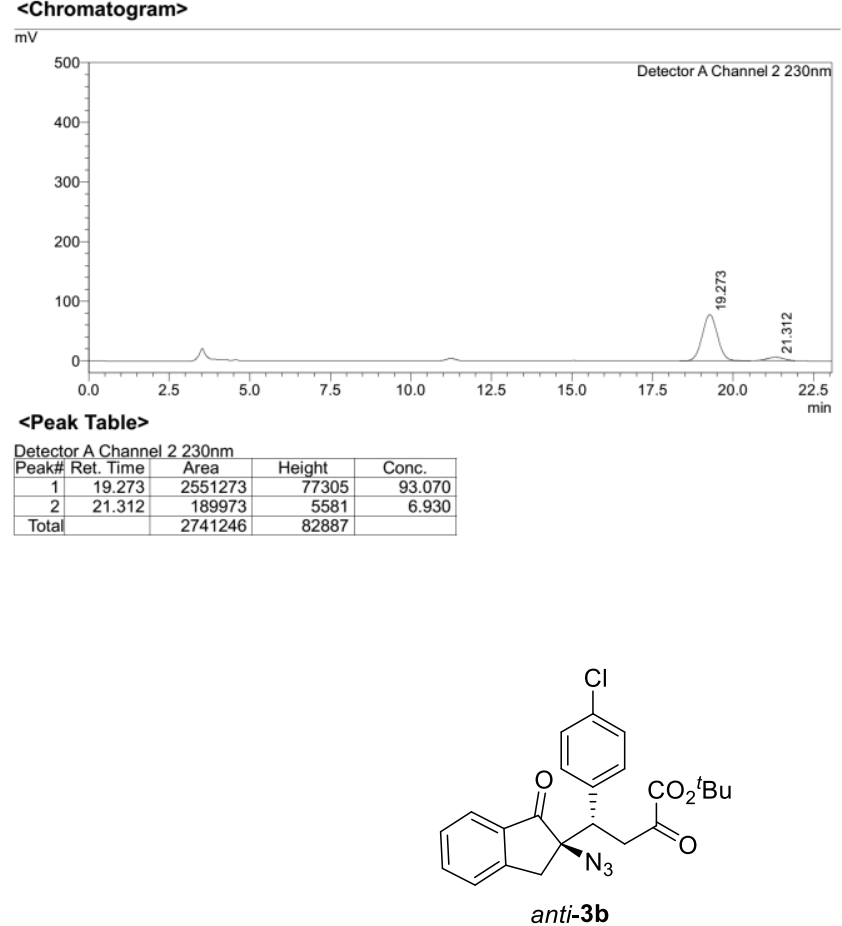
ssample Information Analysis Report

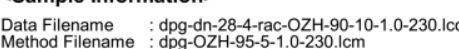

Sample Type

Vial \#
Injection Volume

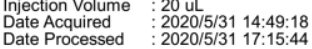

Sample Type : Unknow

$\begin{array}{ll}\text { Acquired by } & \text { : System Administrator } \\ \text { Processed by } & \text { System Administrator }\end{array}$

<Chromatogram>
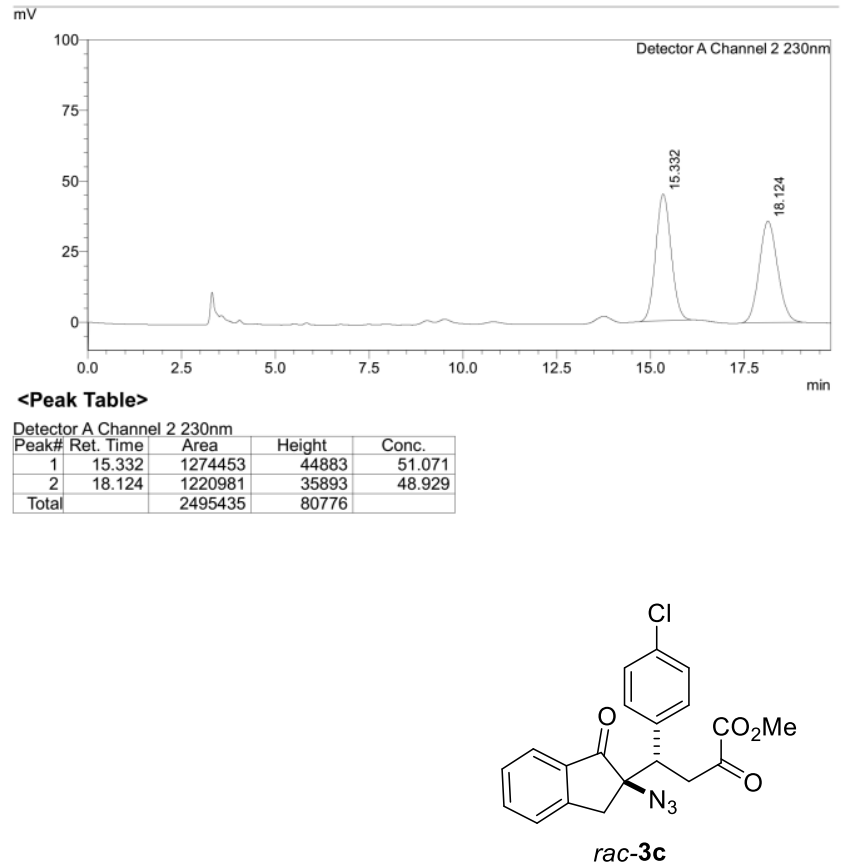

D:Dataldpglddidpg-dn-28-4-rac-OZH-90-10-1.0-230.lcd

\section{Analysis Report}

<Sample Information>

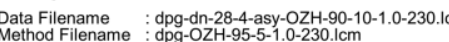

$\begin{array}{l:cc}\text { Batch Filename } & 1-1 & \text { Sample Type } \\ \text { Vial } & \end{array}$

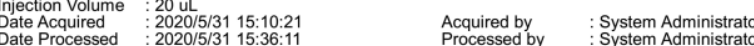

<Chromatogram>
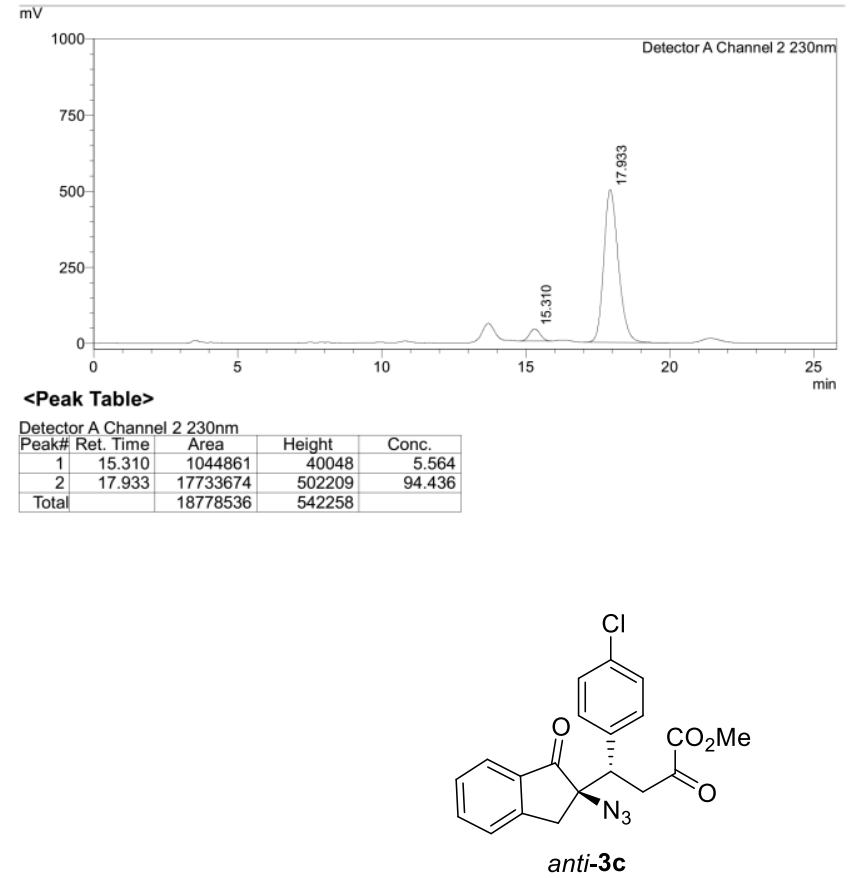

D:IDataldpgldnldpg-dn-28-4-asy-OZH-90-10-1.0-230.lcd 


\section{Analysis Report}

$<$ Sample Information>

Data Filename : dpg-dJ-114-3-RAC-OZH-90-10-1.0-232.1cd

$\begin{array}{l:ll}\text { Batch Filename } & 1-1 & \text { Sample Type }\end{array}$

$\begin{array}{l:l}\text { Injection Volume } & 20 \mathrm{uL} \\ \text { Date Acquired } & 2018 / 6 / 1515: 40: 08 \\ \text { Date Processed } & : 2019 / 1 / 2121: 21: 58\end{array}$

Acquired by
Processed by

<Chromatogram>
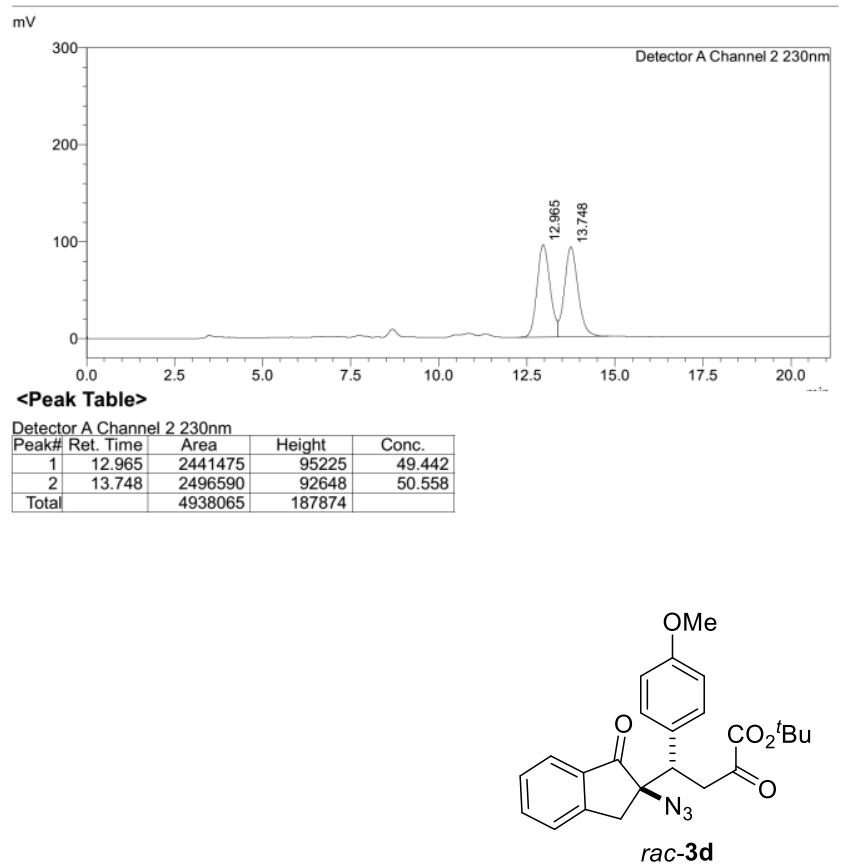

\section{Analysis Report}

$<$ Sample Information

Data Filename : dpg-dJ-114-3-ASY-OZH-90-10-1.0-232.1cd

$\begin{array}{l:ll}\text { Batch Filename } & 1-1 & \text { Sample Type } \\ \text { Vial } & 1-1\end{array}$

$\begin{array}{llll} & \text { Acquired by } & \text { System Administrator } \\ \text { Date Acquired } & 2018 / 6 / 15 & 16: 02: 32 & \text { System Administato }\end{array}$

$<$ Chromatogram>
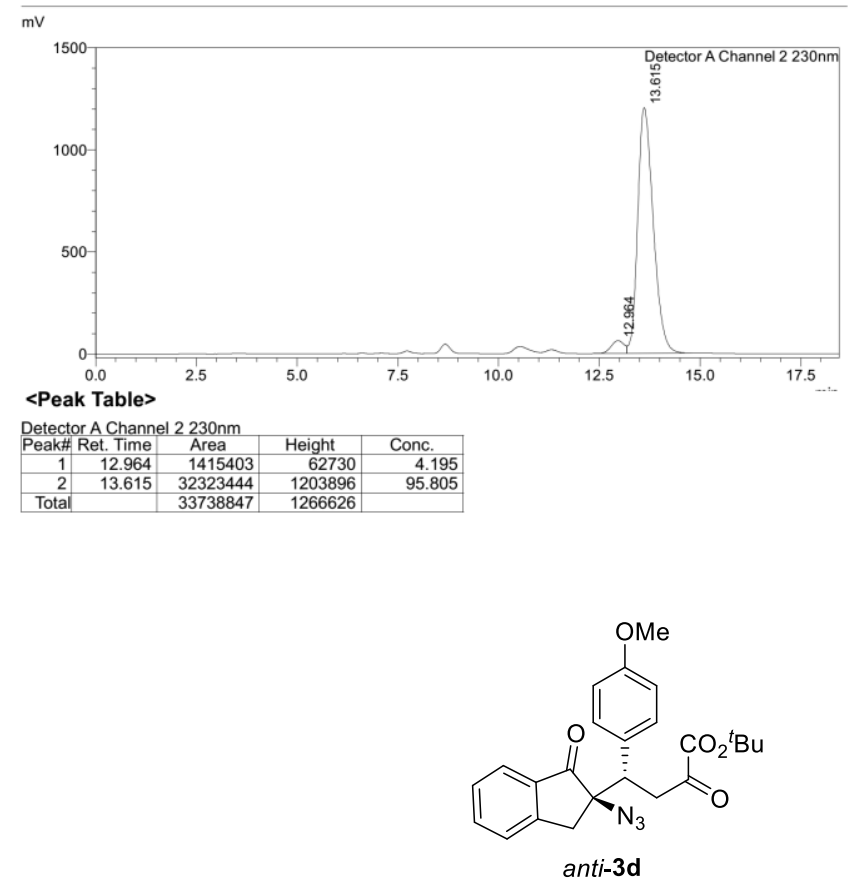


\section{Analysis Report}

$<$ Sample Information>

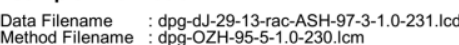

Batch Filename
Vial $\#$ :

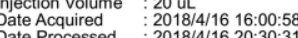

$\begin{array}{ll}\text { Acquired by } & \text { : System Administrator } \\ \text { Processed by } & \text { System Administrator }\end{array}$

<Chromatogram>

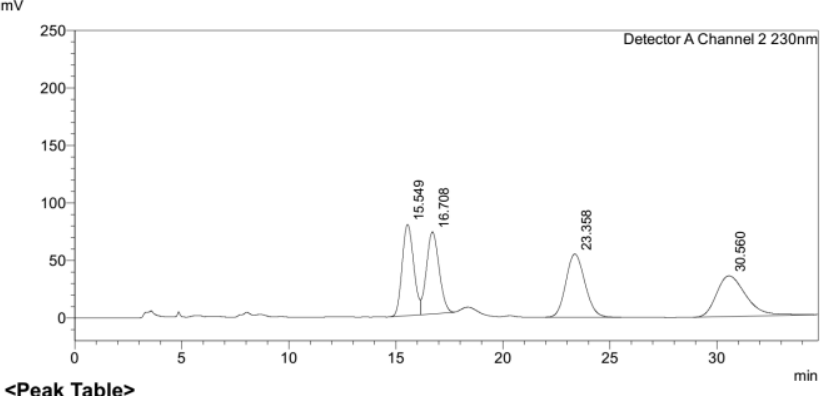

<Peak Table>

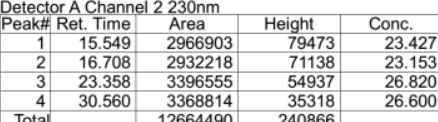

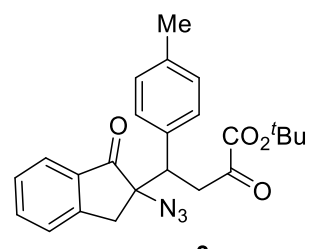

rac-3e

\section{Analysis Report}

<Sample Information

Oata Filename : dpg-dJ-29-3-ASY-ASH-97-3-1.0-231.10

Batch Filename
Vial $t 1-1$

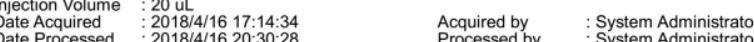

<Chromatogram>
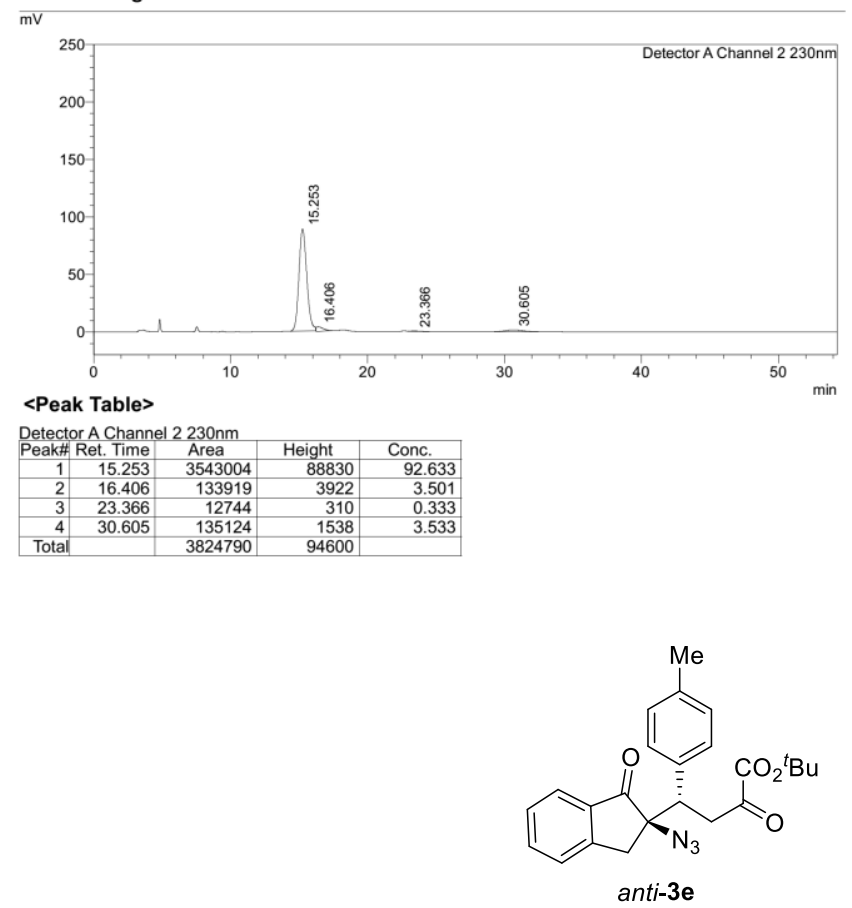


\section{Analysis Report}

$<$ Sample Information

$\begin{array}{ll}\text { Data Filename } & \text { dpg-dJ-114-2-rac-OZH-97-3-1.0-231.lcd } \\ \text { Method Filename } & \text { dpg-OZZ-95-5-1.0-230. } 1 \mathrm{~cm}\end{array}$

$\begin{array}{l:ll}\text { Batch Filename } & 1-1 & \text { Sample Type } \\ \text { Vial } \# \text { i }\end{array}$

$\begin{array}{l:l}\text { Injection Volume } & 20 \mathrm{uL} \\ \text { Date Acquired } & 2018 / 6 / 1315: 17: 18 \\ \text { Date Processed } & : 2019 / 1 / 21 \text { 17: 17:32 }\end{array}$

: Unknown

Acquired by
Processed by

<Chromatogram>
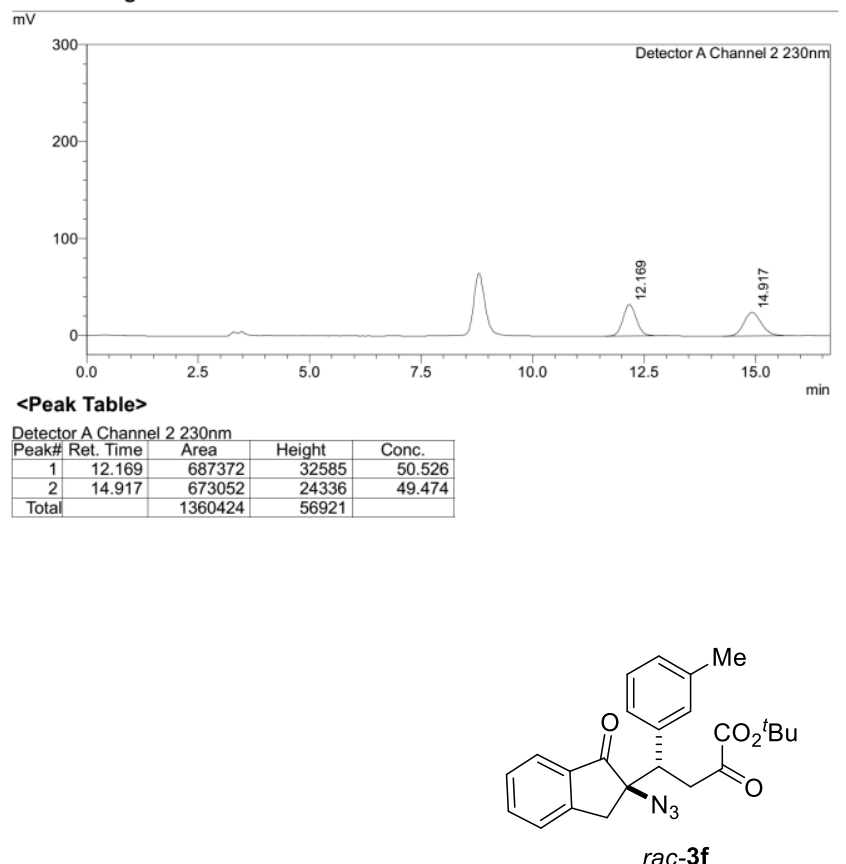

\section{Analysis Report}

<Sample Information

Oata Filename : dpg-dJ-114-2-asy-OZH-97-3-1.0-231.lc

$\begin{array}{l:ll}\text { Batch Filename } & 1-1 & \text { Sample Type } \\ \text { Vial } & \end{array}$

$\begin{array}{llll} & \text { Acquired by } & \text { System Administrator } \\ \text { Date Acquired } & 2018 / 6 / 133 & 15: 35: 17 & \text { System Administato }\end{array}$

<Chromatogram>
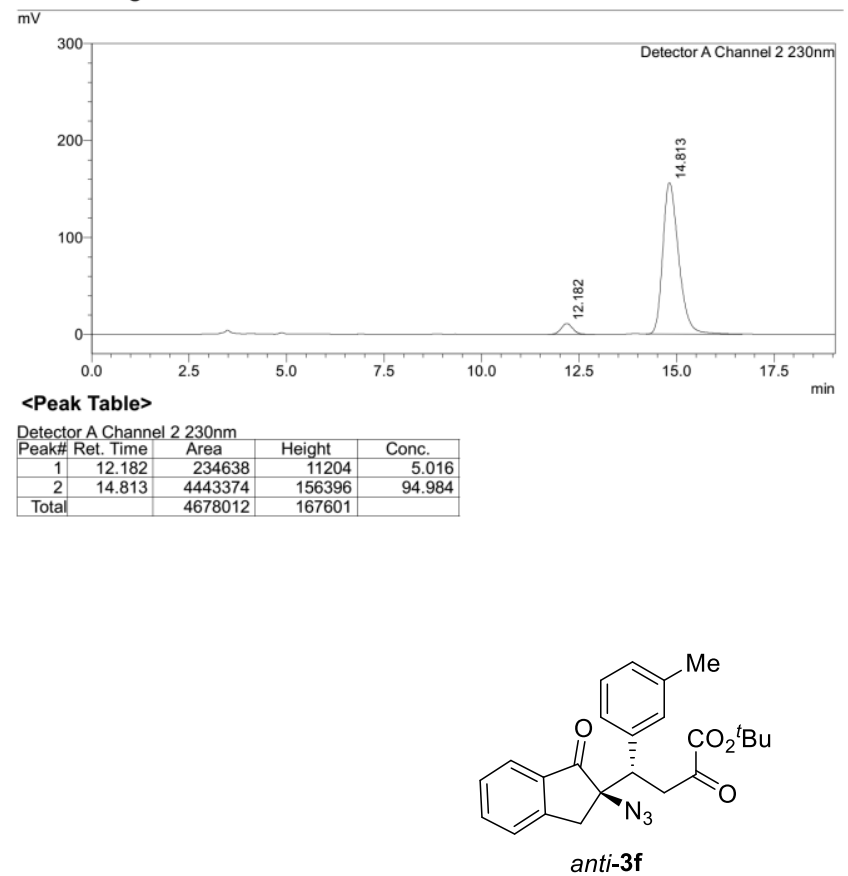
¿sample Information Analysis Report

$\begin{array}{lll}\text { Data Filename } & \text { :dpg-dJ-125-1-1-rac-OZH-97-3-1.0-231.lcd } \\ \text { Method Filename } & \text { dpg-OZH-95-5-1.0-230. } 1 \mathrm{~cm}\end{array}$

$\begin{array}{l:ll}\text { Batch Filename } & 1-1 \\ \text { Vial } \# \text { Sample Type }\end{array}$

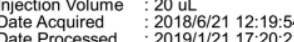

$\begin{array}{ll}\text { Acquired by } & \text { : System Administrator } \\ \text { Processed by } & \text { System Administrator }\end{array}$

<Chromatogram>
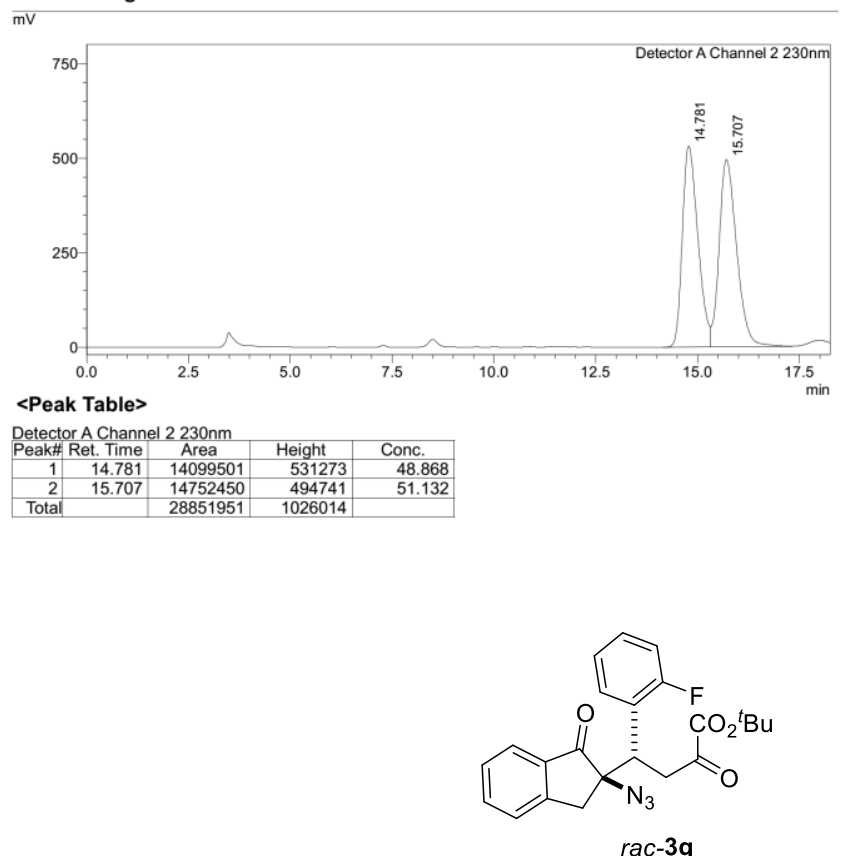

\section{Analysis Report}

$<$ Sample Information

Data Filename : dpg-dJ-125-1-asy-OZH-97-3-1.0-231.1c

$\begin{array}{l:cc}\text { Batch Filename } & 1-1 & \text { Sample Type } \\ \text { Vial } & \end{array}$

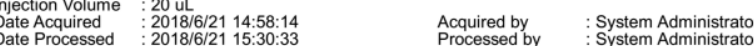

<Chromatogram>
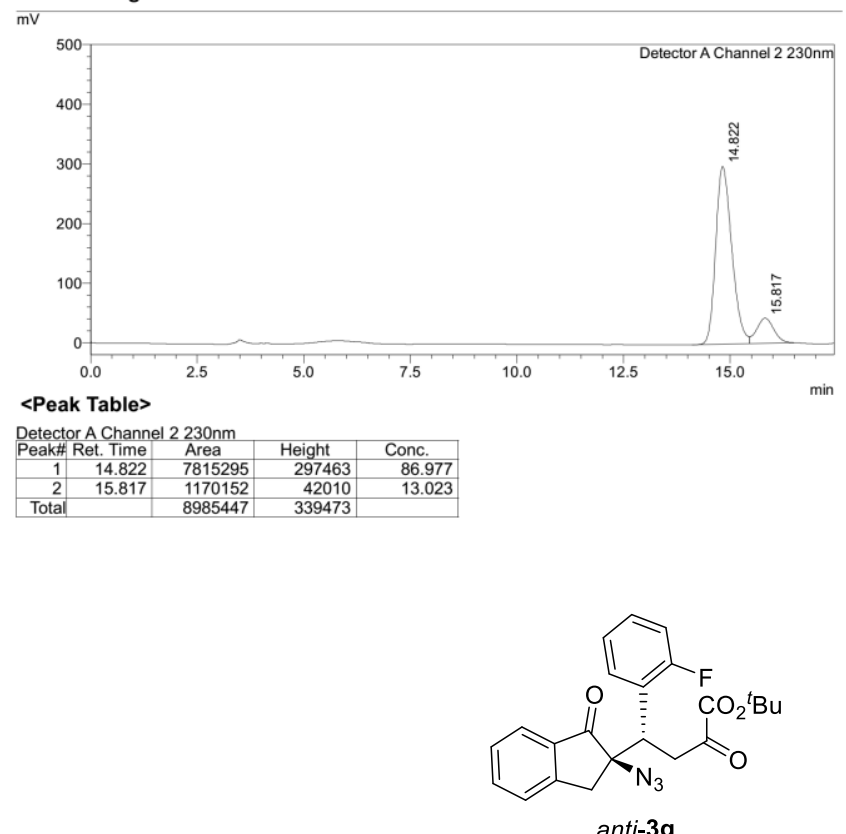


\section{Analysis Report}

<Sample Information>

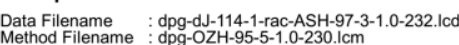

$\begin{array}{l:ll}\text { Batch Filename } & 1-1 & \text { Sample Type }\end{array}$

\begin{tabular}{l:l} 
Injection Volume & 20 uL \\
Date Acquired & $2018 / 6 / 159: 05: 13$ \\
\hline
\end{tabular}

: Unknown

Acquired by
Processed by

<Chromatogram>
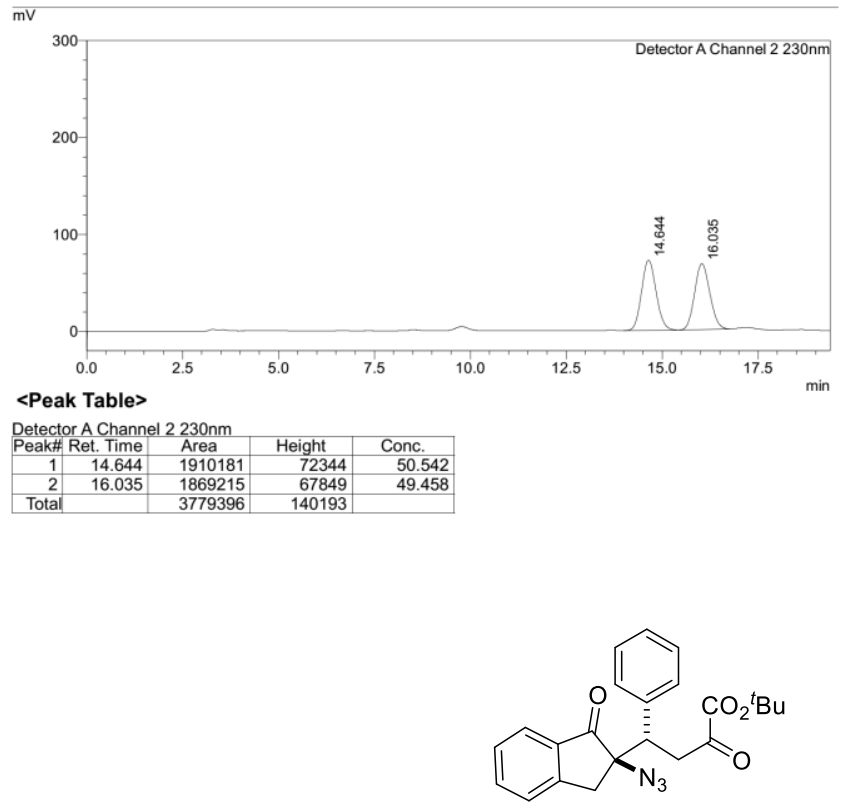

rac-3h

\section{Analysis Report}

<Sample Information>

Data Filename : dpg-dJ-114-1-ASY-ASH-97-3-10-232.1cd

$\begin{array}{l:ll}\text { Batch Filename } & 1-1 & \text { Sample Type } \\ \text { Vial } & \end{array}$

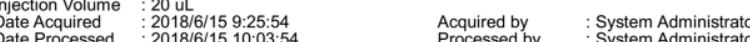

$<$ Chromatogram>
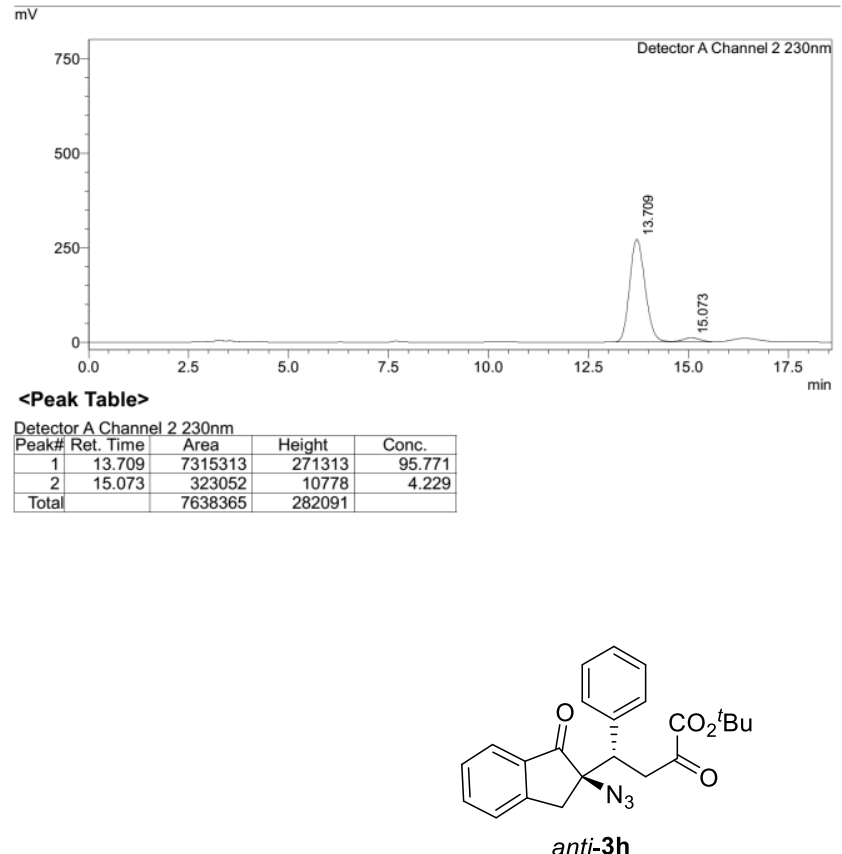
Asample Information Analysis Report

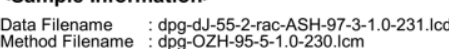

$\begin{array}{l:ll}\text { Batch Filename } & 1-1 & \text { Sample Type } \\ \text { Vial } & 1\end{array}$

$\begin{array}{l:l}\text { Injection Volume } & : 20 \mathrm{uL} \\ \text { Date Acquired } & 2018 / 5 / 315: 03: 56 \\ \text { Date Processed } & 2018 / 5 / 316: 45: 20\end{array}$

: Unknow

Acquired by
Processed by

<Chromatogram>
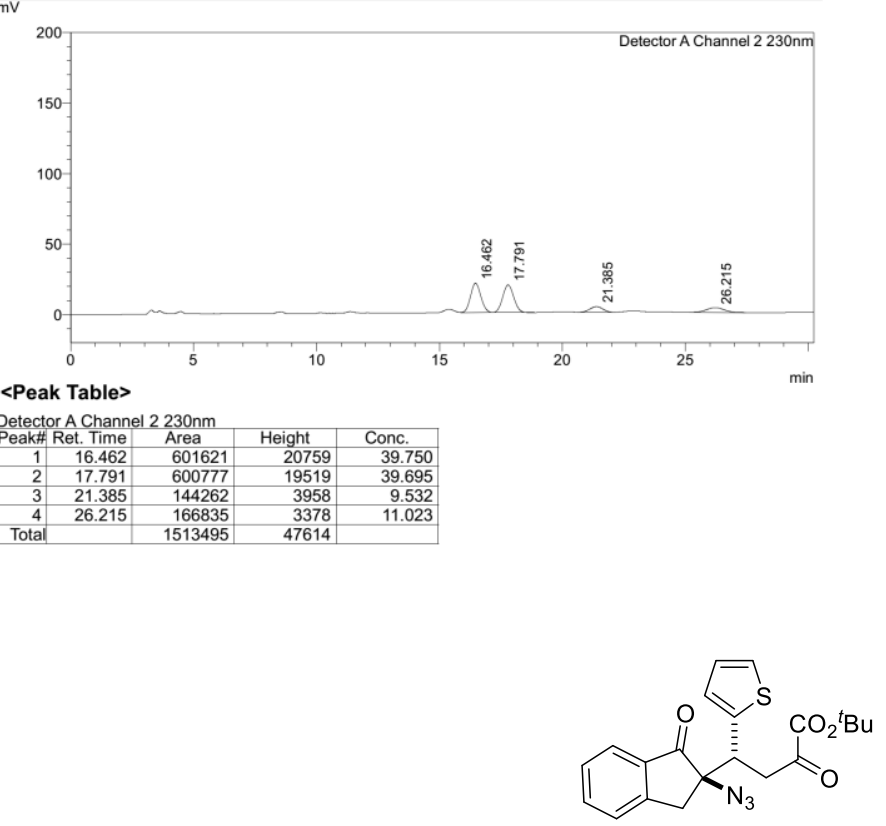

rac-3i

\section{Analysis Report}

<Sample Information

Data Filename : dpg-dJ-55-2-ASY-ASH-97-3-1.0-231.lcd

$\begin{array}{l:ll}\text { Batch Filename } & 1-1 & \text { Sample Type } \\ \text { Vial } & 1-1\end{array}$

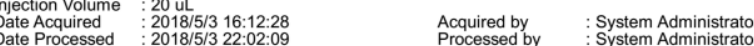

<Chromatogram>
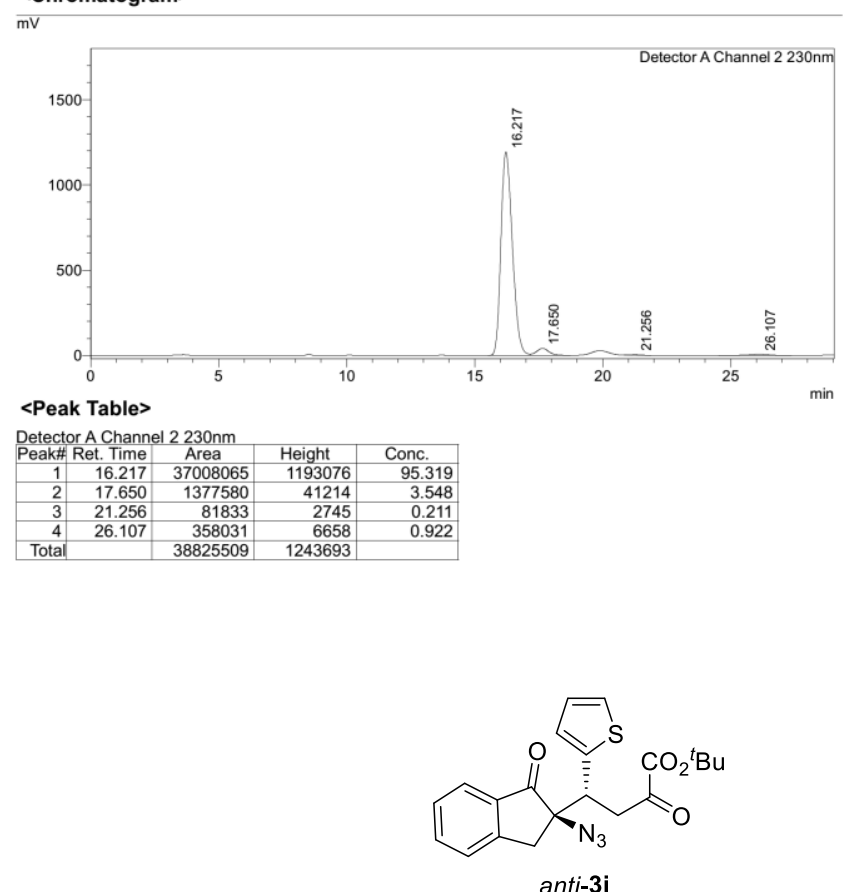
¿sample Information Analysis Report

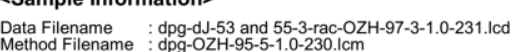

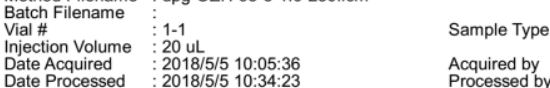

Acquired by
Processed by

<Chromatogram>
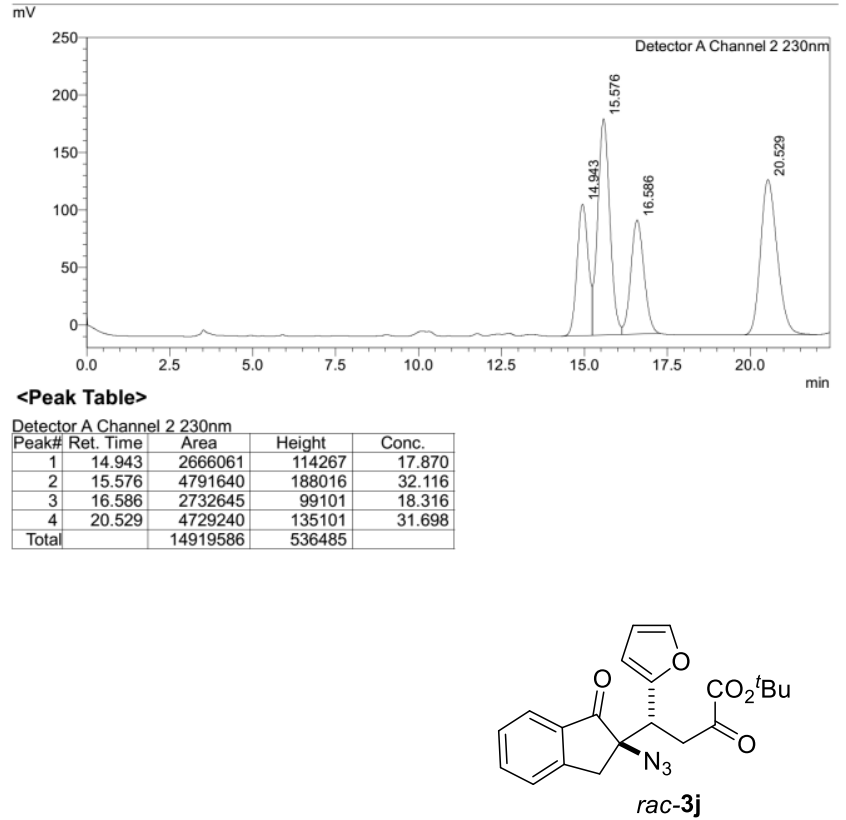

\section{Analysis Report}

<Sample Information

Data Filename : dpg-dJ-55-3-asy-OZH-97-3-1.0-232.lcd

$\begin{array}{l:ll}\text { Batch Filename } & 1-1 & \text { Sample Type } \\ \text { Vial } & 1-1\end{array}$

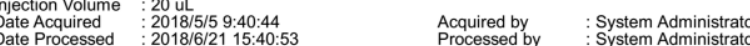

<Chromatogram>
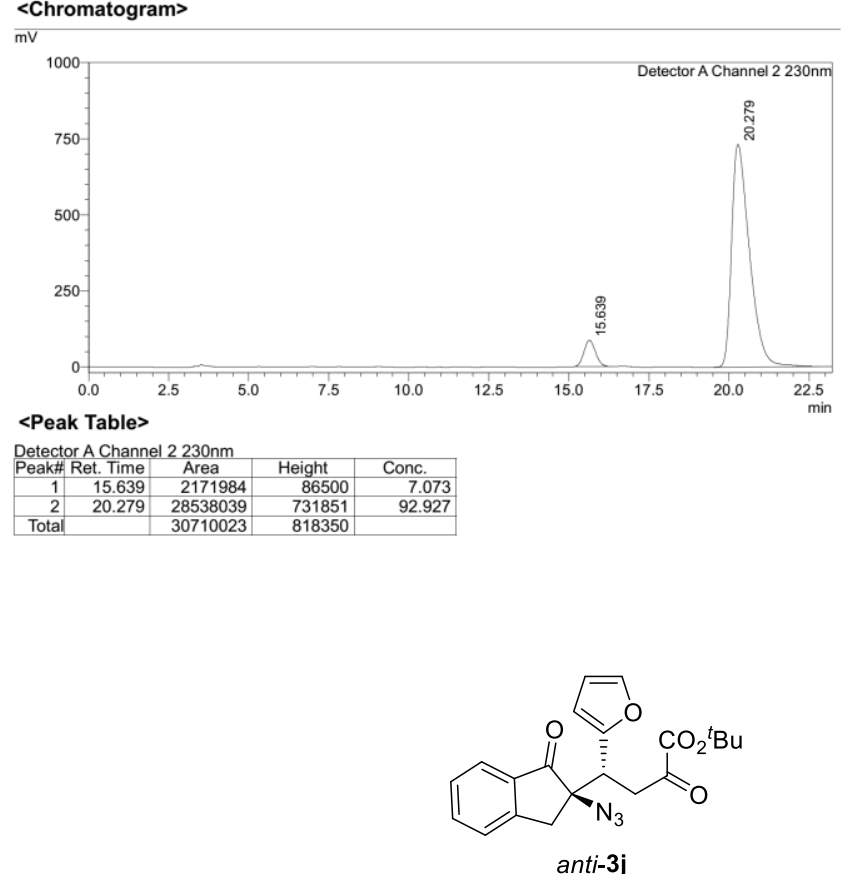
¿sample Information Analysis Report

$\begin{array}{ll}\text { Data Filename } & \text { :dpg-dJ-112-1-1-rac-OZH-97-3-1.0-231.1cd } \\ \text { Method Filename } & \text { dpg-OZH-95-5-1.0-230. } 1 \mathrm{~cm}\end{array}$

$\begin{array}{l:ll}\text { Batch Filename } & 1-1 \\ \text { Vial } \# \text { Sample Type }\end{array}$

$\begin{array}{l:l}\text { Injection Volume } & 20 \mathrm{uL} \\ \text { Date Acquired } & 2018 / 1 / 1310: 35: 36 \\ \text { Date Processed } & : 2018 / 6 / 13 \text { 14:54:14 }\end{array}$

Acquired by
Processed by

<Chromatogram>
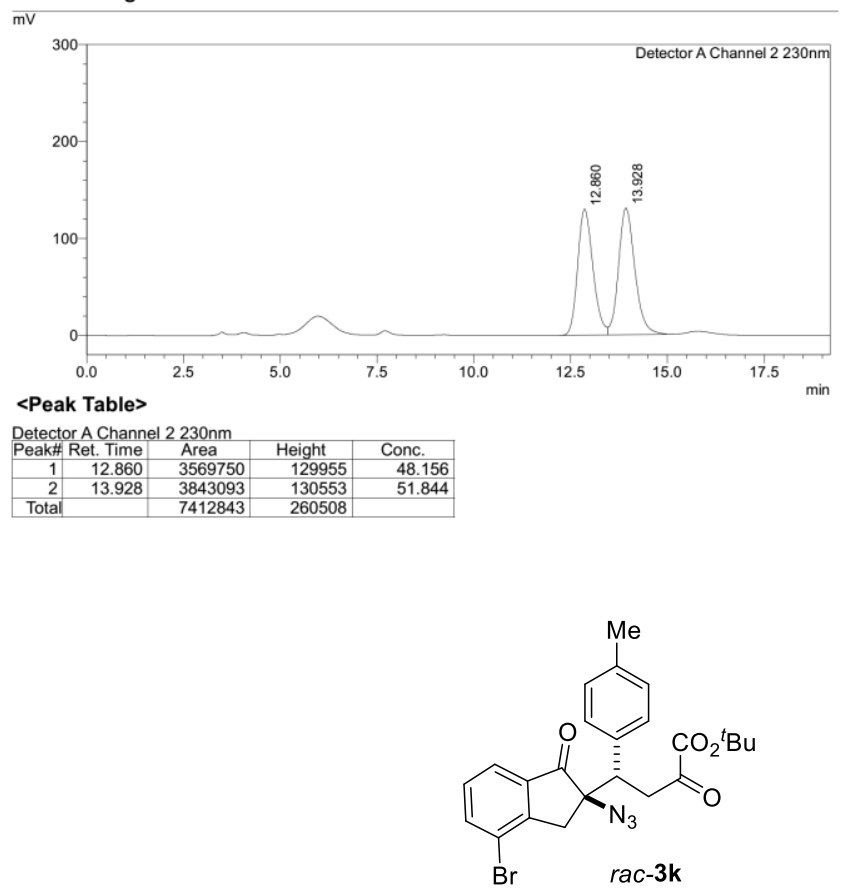

\section{Analysis Report}

<Sample Information>

Data Filename : dpg-dJ-112-1-asy-OZH-97-3-1.0-231.lod

$\begin{array}{l:cc}\text { Batch Filename } & 1-1 & \text { Sample Type }\end{array}$

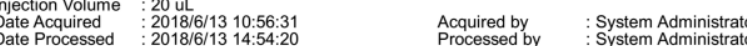

<Chromatogram>
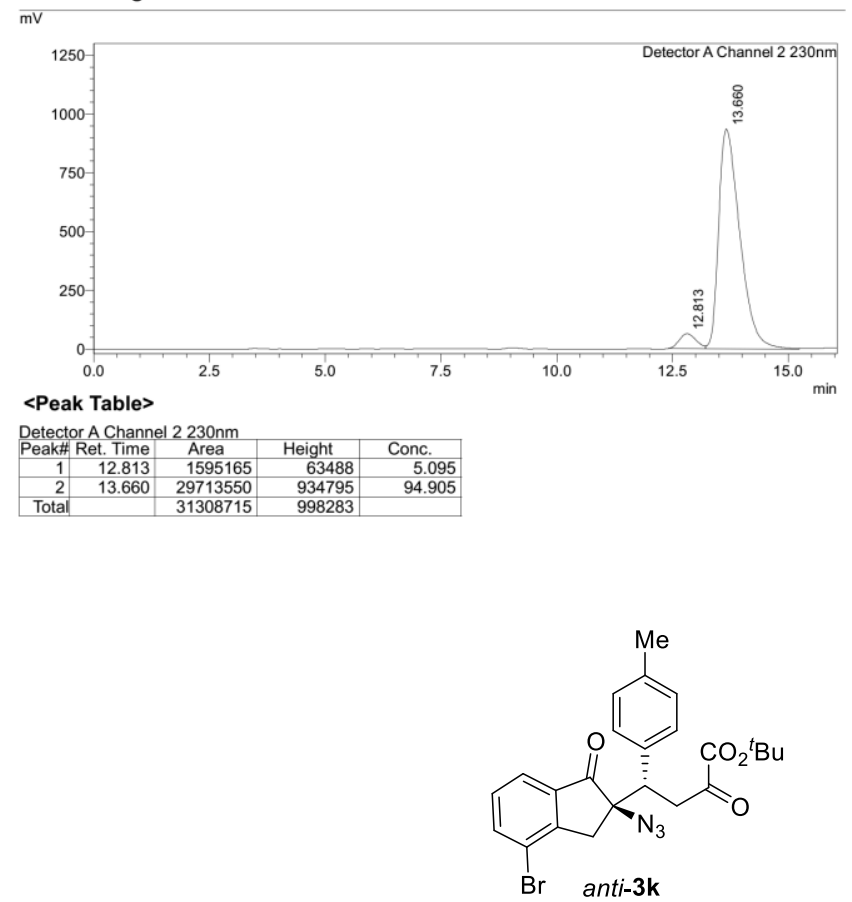


\section{Analysis Report}

$<$ Sample Information>

Data Filename : dpg-dJ-112-2-RAC-ASH-97-3-1.0-232.10

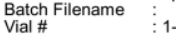

$\begin{array}{l:cc}\text { Vial \# } & 1-1 \\ \text { Injection Volume } & 20 \mathrm{uL} & \text { Sample Type }\end{array}$

$\begin{array}{l:l}\text { Injection Volume } & 20 \mathrm{uL} \\ \text { Date Acquired } & 2018 / 61510: 05: 18 \\ \text { Date Processed } & 20186 / 15 \text { 11:01:51 }\end{array}$

Acquired by
Processed by

<Chromatogram>

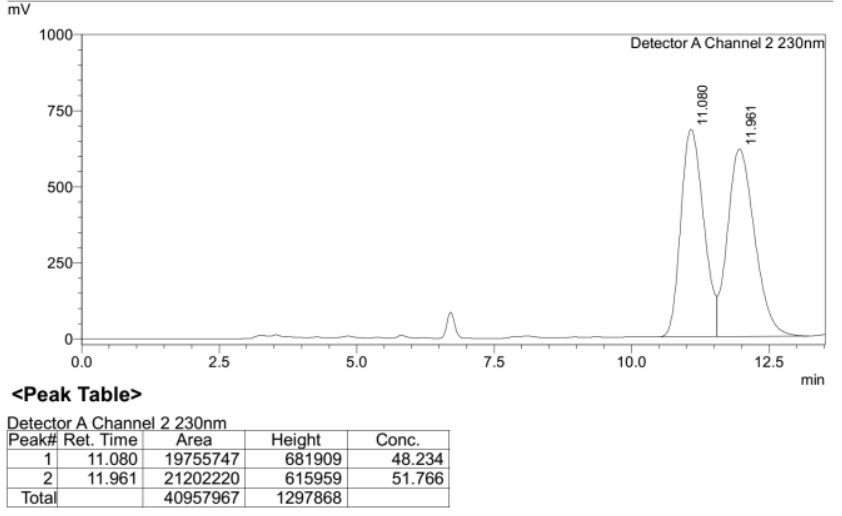

\section{Analysis Report}

<Sample Information>

Data Filename : dpg-d-112-2-ASY-ASH-97-3-1.0-232.ldd

$\begin{array}{l:cc}\text { Batch Filename } & 1-1 & \text { Sample Type } \\ \text { Vial } & 1-1\end{array}$

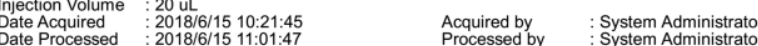

<Chromatogram>
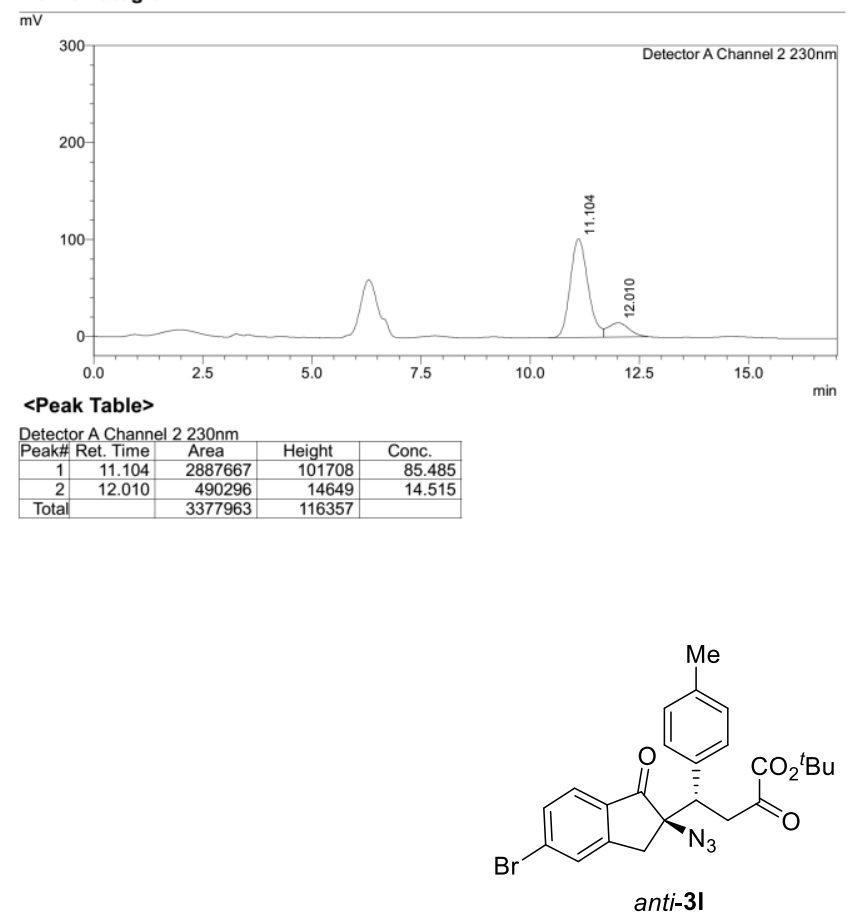


\section{Analysis Report}

<Sample Information>

Data Filename : dpg-dn-73-rac-0ZH-97-3-1.0-235.lc

$\begin{array}{l:c}\text { Batch f rlename } & 1-1 \\ \text { Vial \# } \\ \text { Injection Volume }\end{array}$

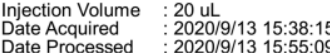

Sample Type

Acquired by
Processed by

$<$ Chromatogram>

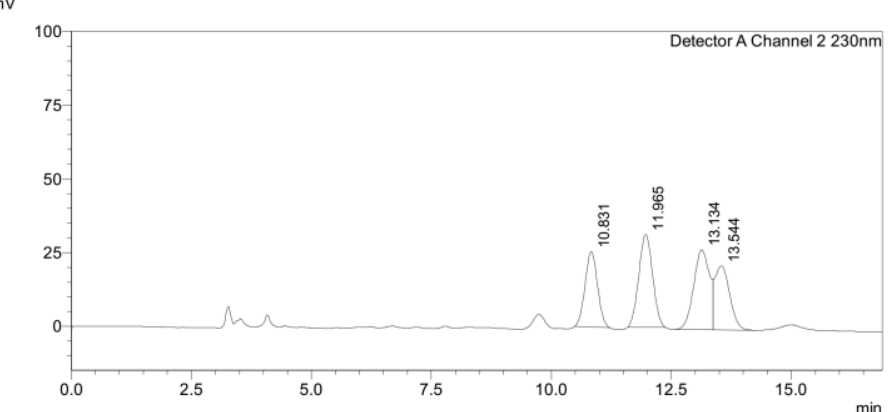

<Peak Table>

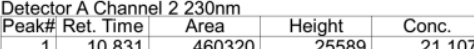

\begin{tabular}{|r|r|r|r|r|}
\hline 1 & 10.831 & 460320 & 25589 & 21.107 \\
\hline 2 & 11.965 & 628023 & 31410 & 28.796 \\
\hline 3 & 13.134 & 624409 & 27059 & 28.612 \\
\hline 4 & 13.544 & 468562 & 21684 & 21.465 \\
\hline
\end{tabular}

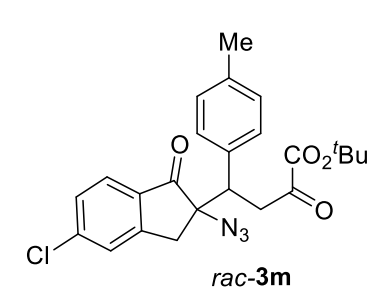

\section{Analysis Report}

<Sample Information>

Data Filename : dpg-dJ-46-1-ASY-OZH-97-3-1.0-234.lcd

Vial \#
Injection Volume
Date $A$ :

Sample Type

: Unknown

\begin{tabular}{ll}
$2020 / 9 / 13$ & $16: 13: 39$ \\
\hline
\end{tabular}

Acquired by
Processed by

System Administrator

$<$ Chromatogram>
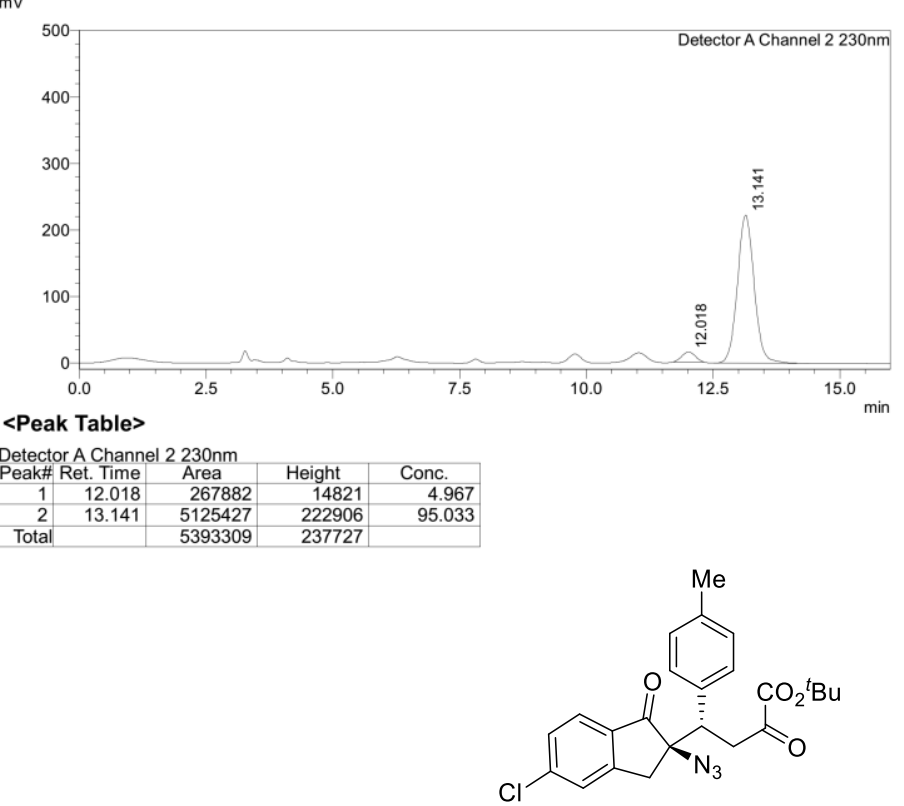

$(R, S)-3 \mathrm{~m}$ 


\section{Analysis Report}

<Sample Information>

Data Filename : dpg-dn-73-rac-0ZH-97-3-1.0-235.lc

$\begin{array}{l:l}\text { Vial \# } \\ \text { Injection Volume } & \vdots 1-1 \\ \text { In } & 20 \mathrm{uL}\end{array}$

Sample Type

$\begin{array}{ll}\text { Date Acquired } & \text { 20 } \\ \text { Date Processed } & \text { 2020/9/13 15:38:15 } \\ \text { Dat } & \text { 2020/13 15:55:09 }\end{array}$

Acquired by
Processed by

Unknown

$<$ Chromatogram $>$

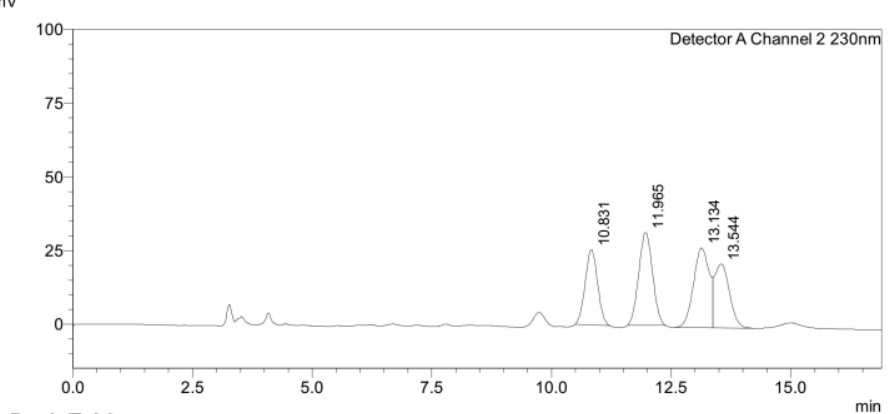

\section{<Peak Table>}

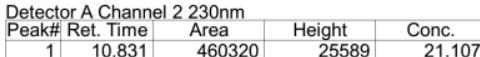

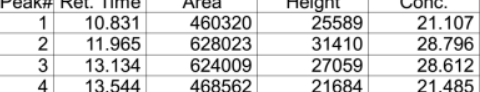

\begin{tabular}{r|r|r|r|r|r|}
\hline 4 & 13.544 & 468562 & 27059 & 28.612 \\
\hline Total & & 2180915 & 105741 & 21.485 \\
\hline
\end{tabular}

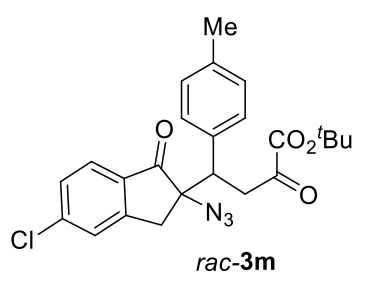

D:IDataldpgldnldpg-dn-73-rac-OZH-97-3-1.0-235.Icd

\section{Analysis Report}

<Sample Information>

Data Filename :dpg-dn-73-asy-0ZH-97-3-1.0-235.lcd

Vial\#

Inijection Volume
Date Acquired

$\begin{array}{ll}1-1 & \text { Sample Type } \\ 200 \mathrm{uL} & \\ 2020 / 9 / 1315: 56: 57 & \text { Acquired by } \\ 2020 / 913316: 12: 09 & \text { Processed by }\end{array}$

Unknown

:2020/9/13 16:12:09

System Administrator
System Administrator

<Chromatogram>

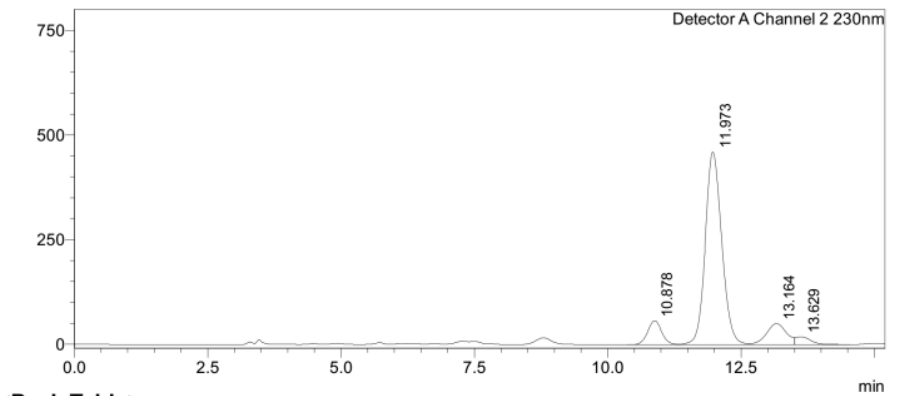

<Peak Table>

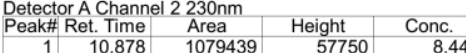
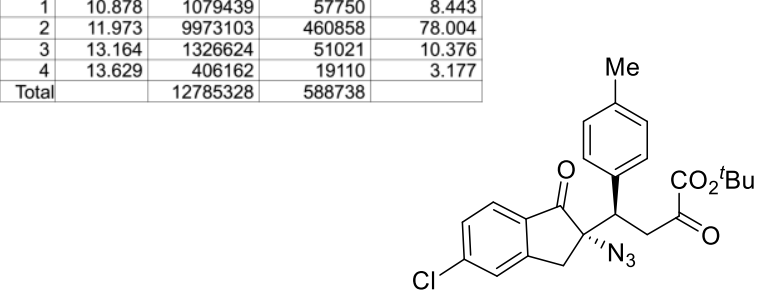

$(S, R)-\mathbf{3 m}$ 


\section{Analysis Report \\ <Sample Information>}

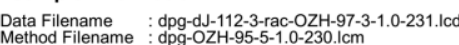

Batch Filename
Vial $\#$ Sample Type

$\begin{array}{l:l}\text { Injection Volume } & 20 \mathrm{uL} \\ \text { Date Acquired } & 2018 / 6 / 1311: 13: 20 \\ \text { Date Processed } & : 2019 / 1 / 21 \text { 17: 13:59 }\end{array}$

mple Type : Unknow

Acquired by
Processed by

<Chromatogram>
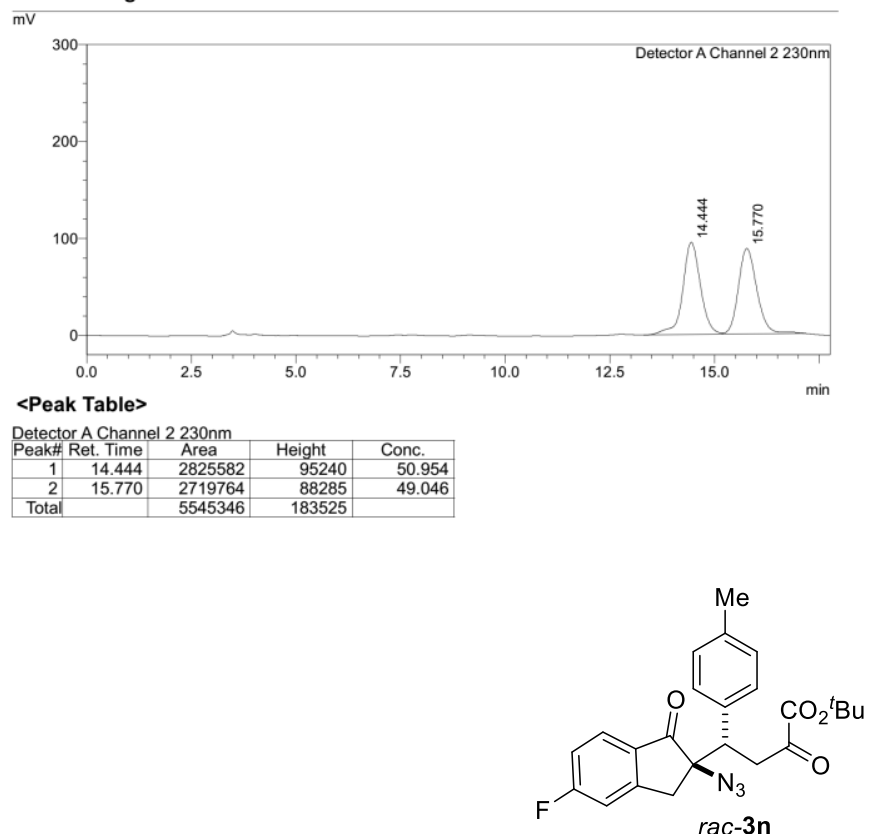

\section{Analysis Report}

<Sample Information>

Data Filename : dpg-dJ-112-3-asy-OZH-97-3-1.0-231.led

$\begin{array}{l:ll}\text { Batch Filename } & 1-1 & \text { Sample Type } \\ \text { Vial } & \end{array}$

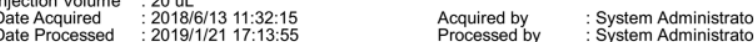

<Chromatogram>
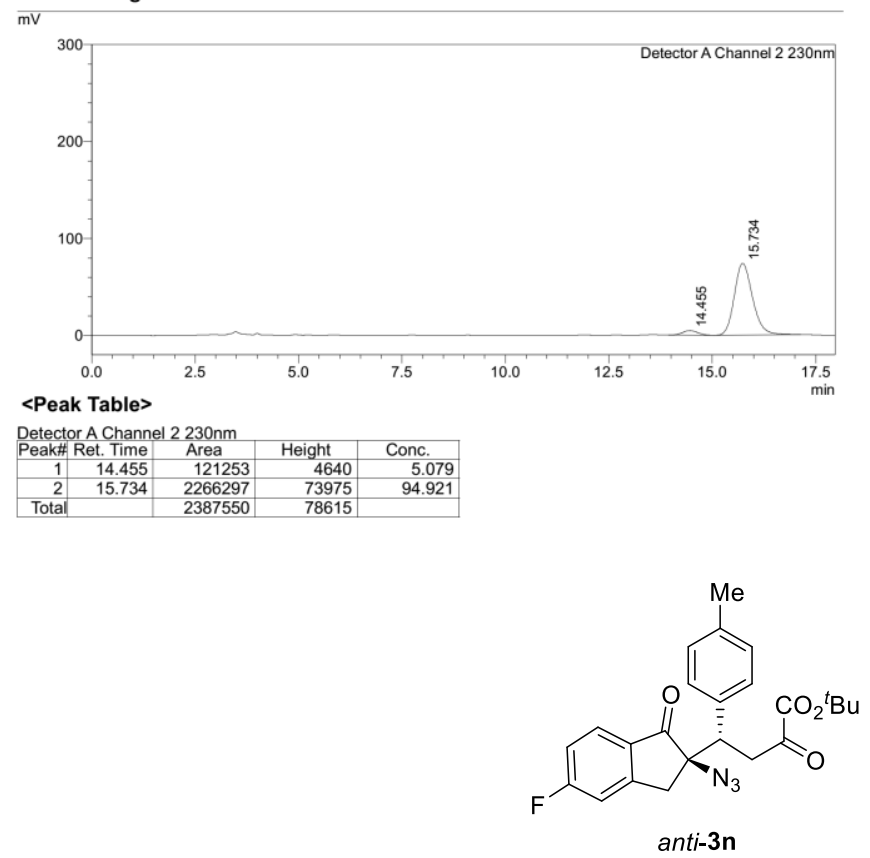


\section{Analysis Report}

$<$ Sample Information>

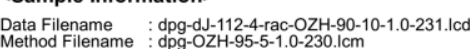

$\begin{array}{l:cc}\text { Batch Filename } & 1-1 & \text { Sample Type }\end{array}$

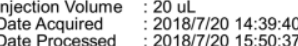

Acquired by
Processed by

<Chromatogram>
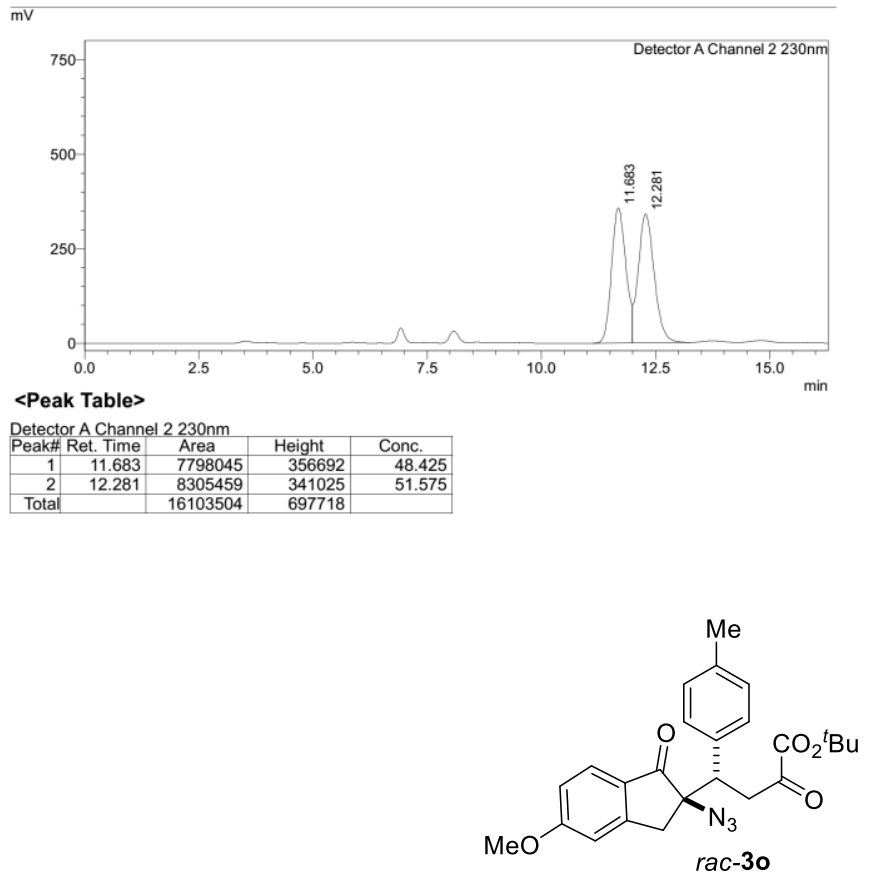

\section{Analysis Report}

$<$ Sample Information

Data Filename : dpg-d-112-4-asy-OZH-90-10-1.0-231.lcd

$\begin{array}{l:ll}\text { Batch Filename } & 1-1 & \text { Sample Type }\end{array}$

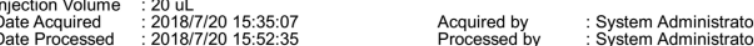

<Chromatogram>
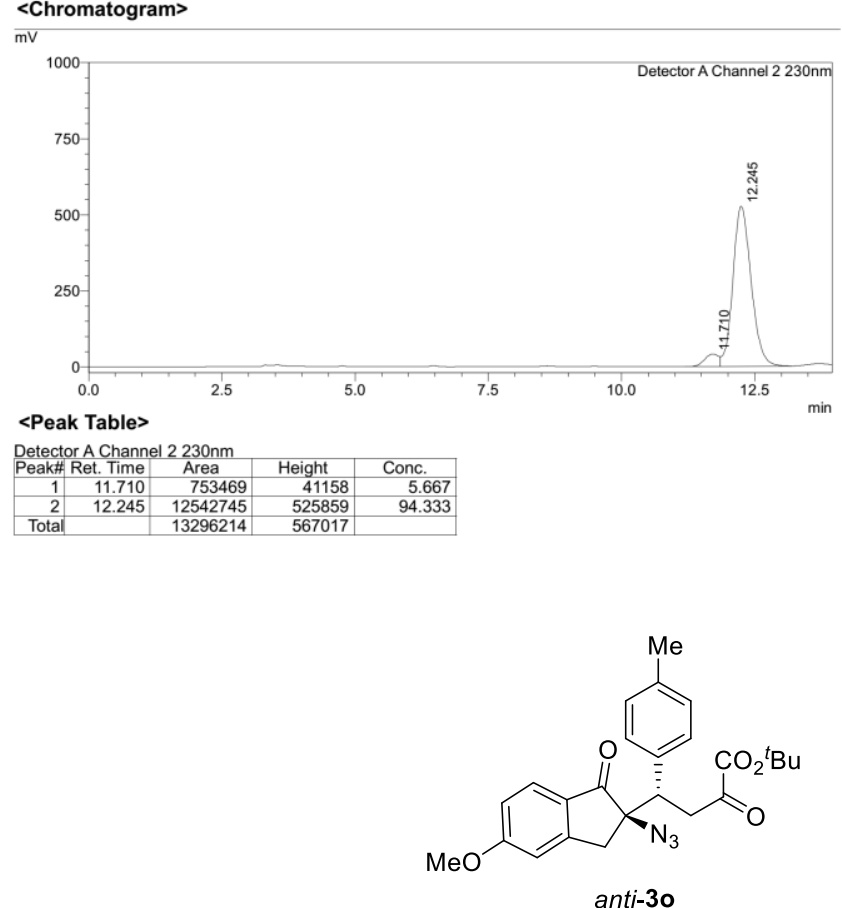


\section{Analysis Report}

$<$ Sample Information

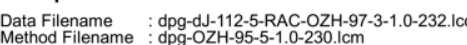

$\begin{array}{l:cc}\text { Batch Filename } & 1-1 & \text { Sample Type } \\ \text { Vial \# } & \text { Injection Volume } & 20 \mathrm{uL}\end{array}$

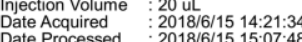

Acquired by
Processed by

<Chromatogram>

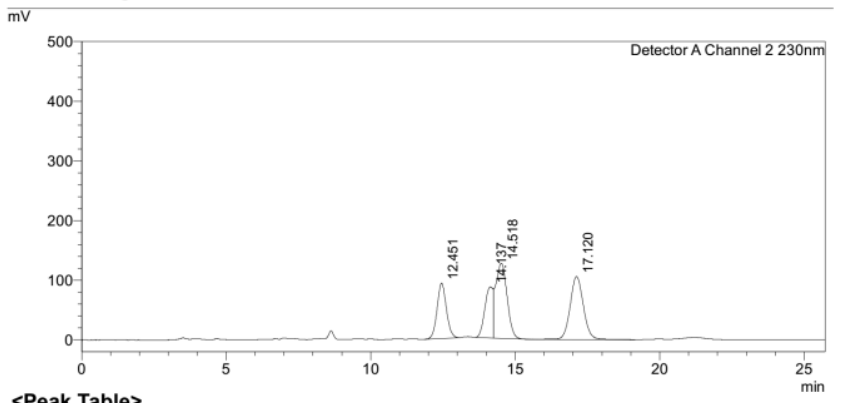

<Peak Table>

Detector A Channel $2230 \mathrm{~nm}$

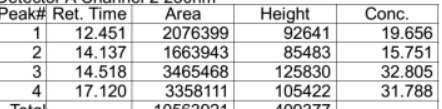

\begin{tabular}{rr|r|r|r|}
4 & 14.518 & 3465468 & 125830 & 32.805 \\
\hline Total & 17.120 & 3358111 & 105422 & 31.788 \\
\hline
\end{tabular}

\section{Analysis Report}

$<$ Sample Information

Data Filename : dpg-d-112-5-ASY-OZH-97-3-1.0-232.ldd

$\begin{array}{l:cc}\text { Batch Filename } & 1-1 & \text { Sample Type } \\ \text { Vial } & \end{array}$

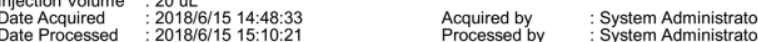

<Chromatogram>
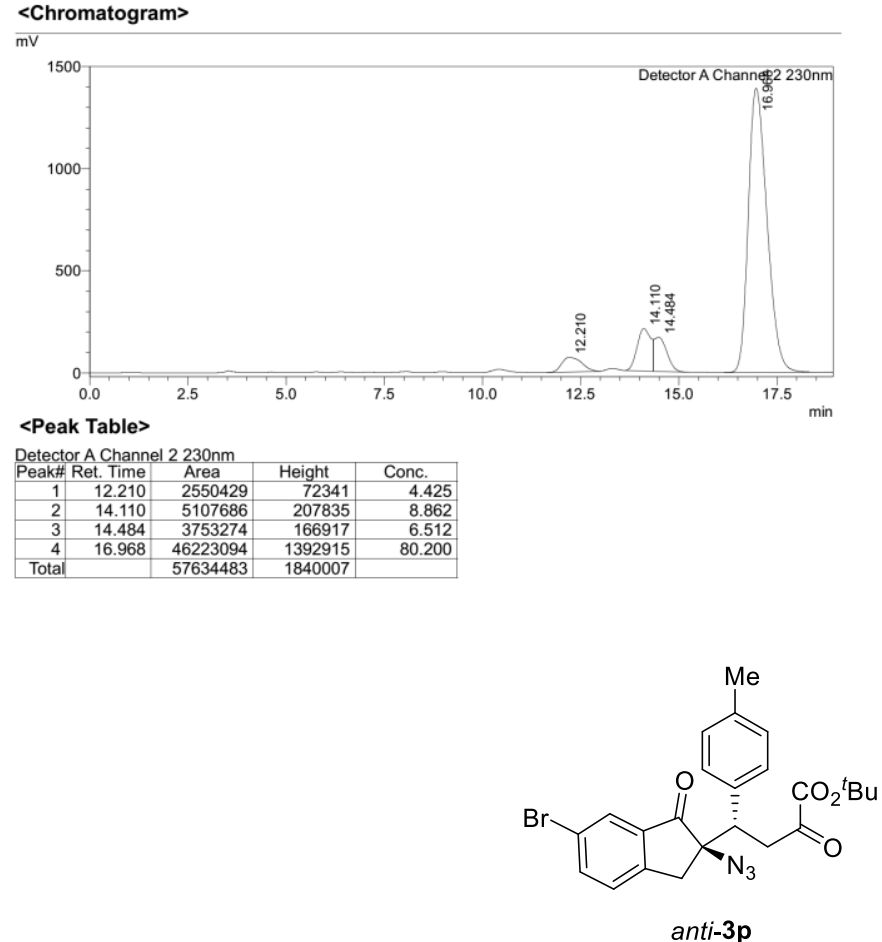


\section{Analysis Report}

<Sample Information>

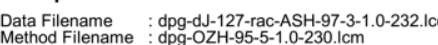

$\begin{array}{l:ll}\text { Batch Filename } & 1-1 \\ \text { Vial } \# \text { Sample Type }\end{array}$

\begin{tabular}{l:l} 
Injection Volume & $20 \mathrm{ul}$ \\
Date Acquired & $2018 / 6259: 54: 21$ \\
Date Processed & $2019 / 1 / 21$ \\
\hline
\end{tabular}

$\begin{array}{ll}\text { Acquired by } & \text { : System Administrator } \\ \text { Processed by } & \text { System Administrator }\end{array}$

<Chromatogram>
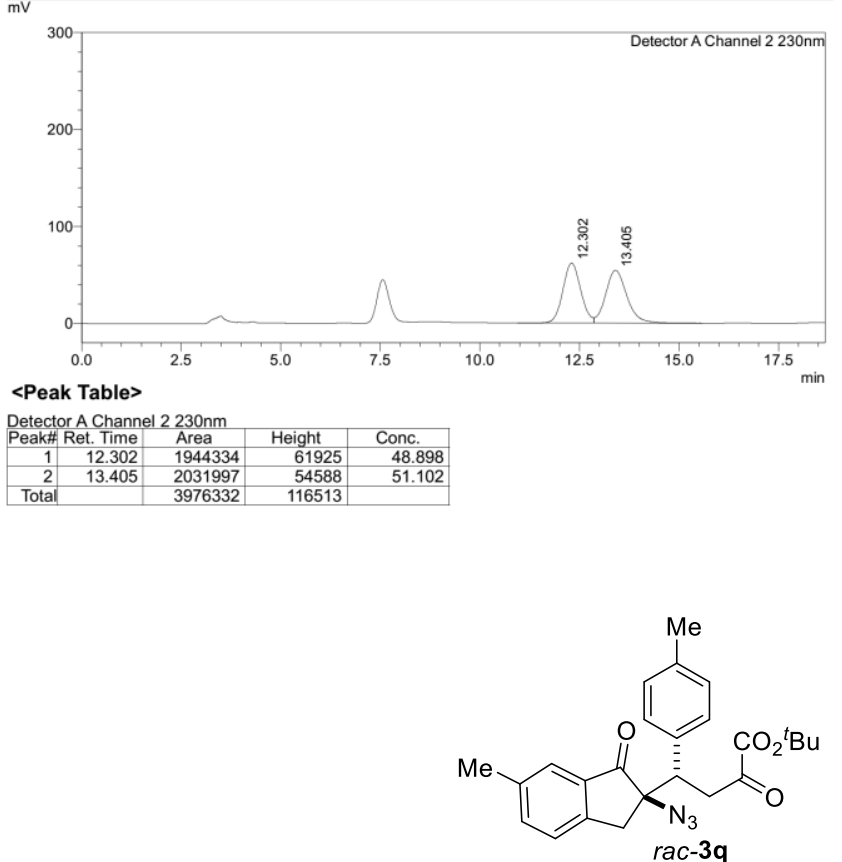

\section{Analysis Report}

<Sample Information>

Data Filename : dpg-dJ-127-ASY-ASH-97-3-10-232.1cd

$\begin{array}{l:cc}\text { Batch Filename } & 1-1 & \text { Sample Type } \\ \text { Vial } & 1-1\end{array}$

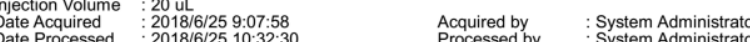

<Chromatogram>
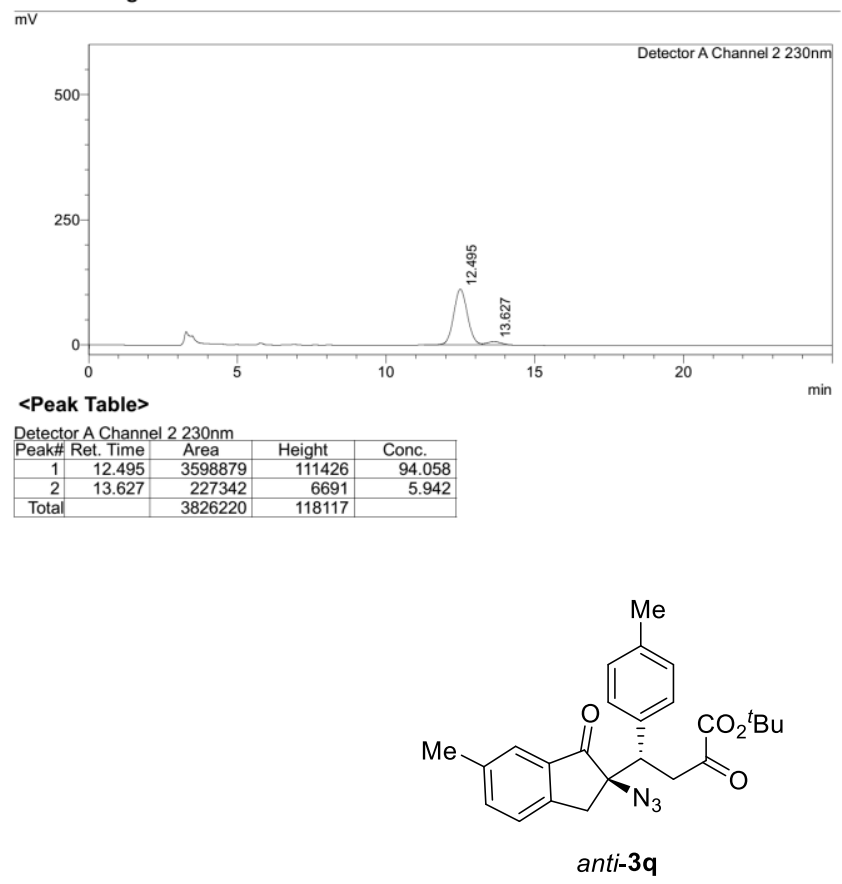


\section{Analysis Report}

$<$ Sample Information>

Data Filename
Method Filename

$\begin{array}{l:cc}\text { Batch Filename } & 1-1 & \text { Sample Type } \\ \text { Vial \# } & \text { Injection Volume } & 20 \mathrm{uL}\end{array}$

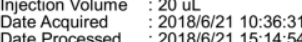

Acquired by
Processed by

<Chromatogram>

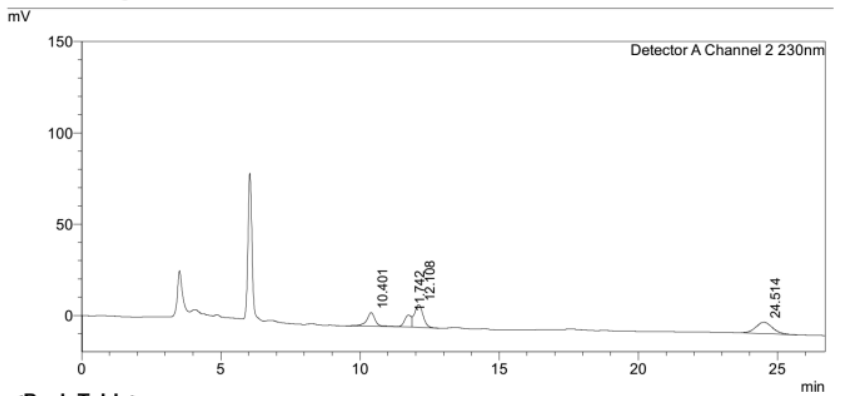

<Peak Table>

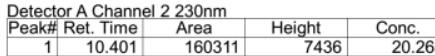

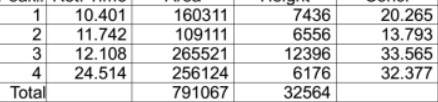

\section{Analysis Report}

$<$ Sample Information

Data Filename : dpg-dJ-112-6-1-asy-OZH-97-3-1.0-231.1cd

$\begin{array}{l:ll}\text { Batch Filename } & \vdots 1-1 & \text { Sample Type } \\ \text { Vial } & \end{array}$

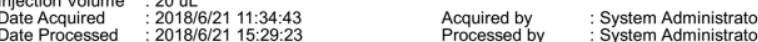

$<$ Chromatogram>
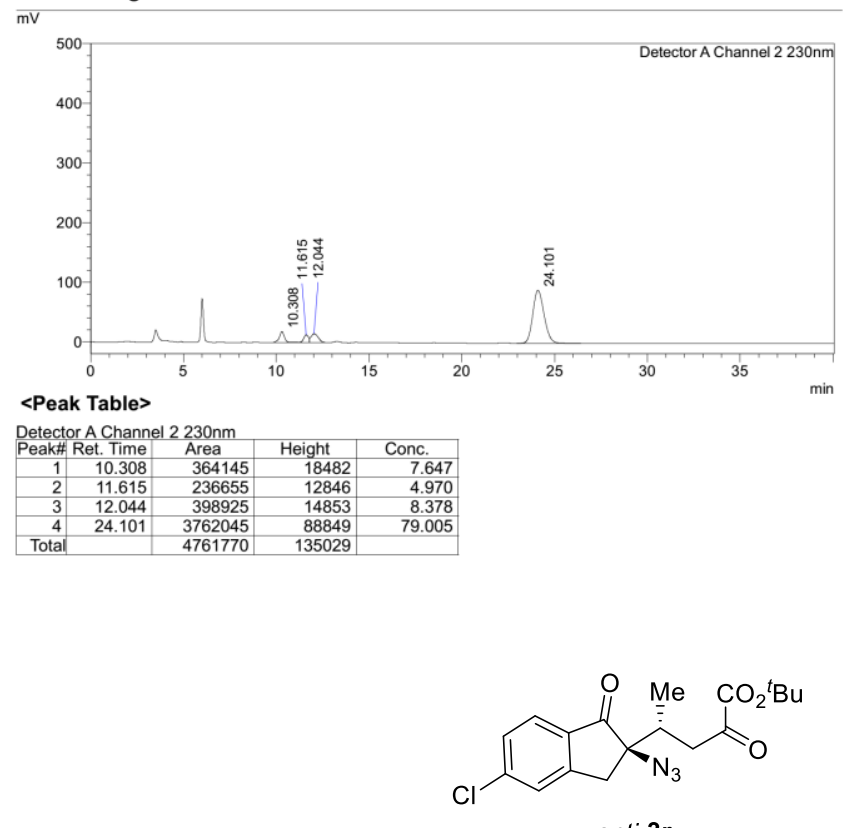

anti-3r 


\section{Analysis Report}

$<$ Sample Information>

Data Filename : dpg-dJ-124-RAC-OZH-90-10-1.0-232.1c

$\begin{array}{l:cc}\text { Batch Filename } & 1-1 & \text { Sample Type } \\ \text { Vial I } & 1\end{array}$

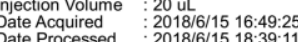

Acquired by
Processed by $\quad$ System Administrator
System Administrator

<Chromatogram>
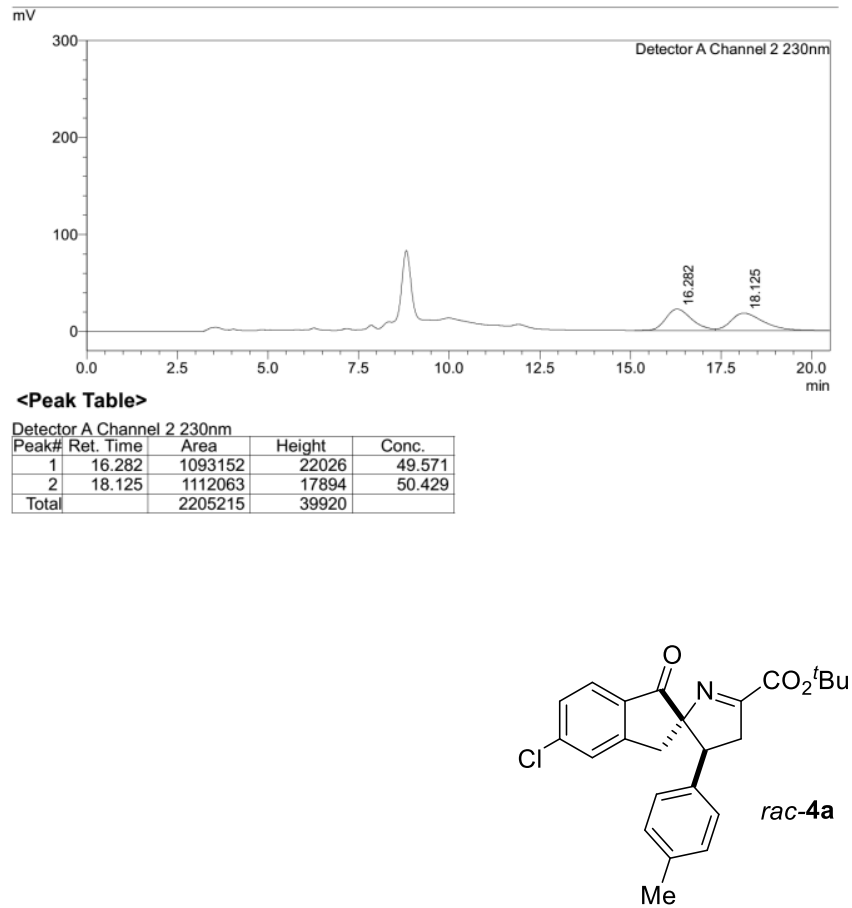

\section{Analysis Report}

$<$ Sample Information

Data Filename : dpg-dJ-124-ASY-OZH-90-10-1.0-232.10

$\begin{array}{l:cc}\text { Batch Filename } & 1-1 & \text { Sample Type } \\ \text { Vial } & \end{array}$

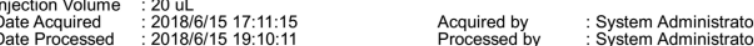

<Chromatogram>
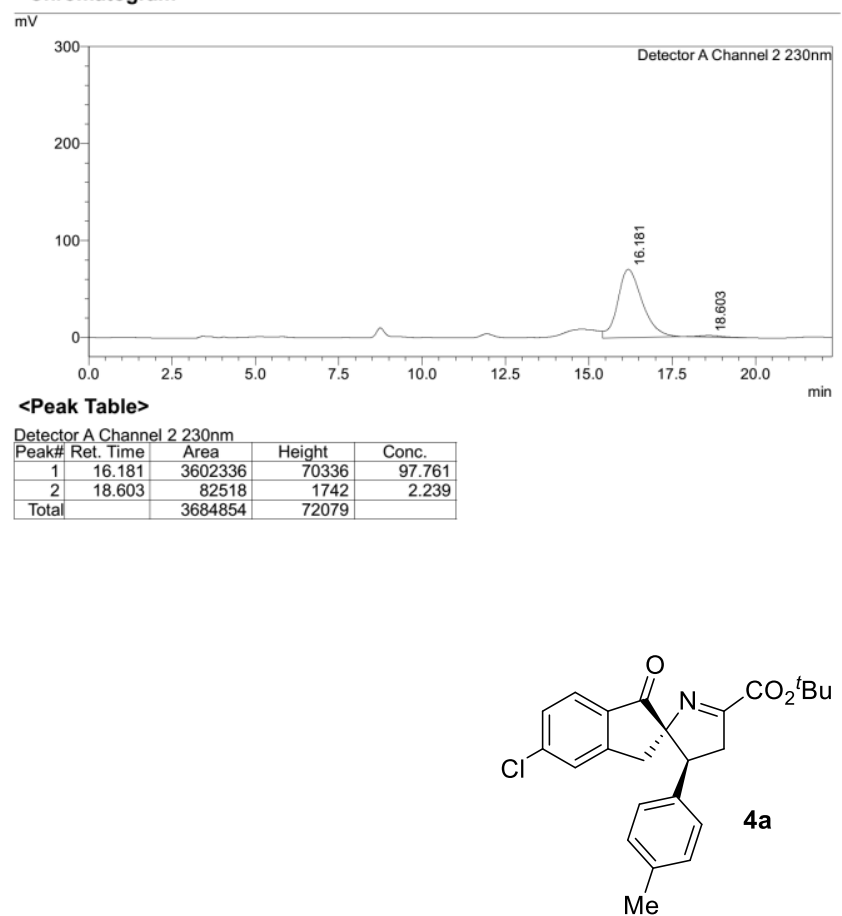


\section{Analysis Report}

<Sample Information>

Data Filename
Method Filename

Batch Filename
Vial I

$\begin{array}{lll}\text { Mnjection Volume } & 120 \mathrm{uL} & \text { Acquired by } \\ \text { Date Acquired } & 20186 / 169: 15: 51 & \end{array}$

Unknown

: System Administrator
System Administrator

<Chromatogram>
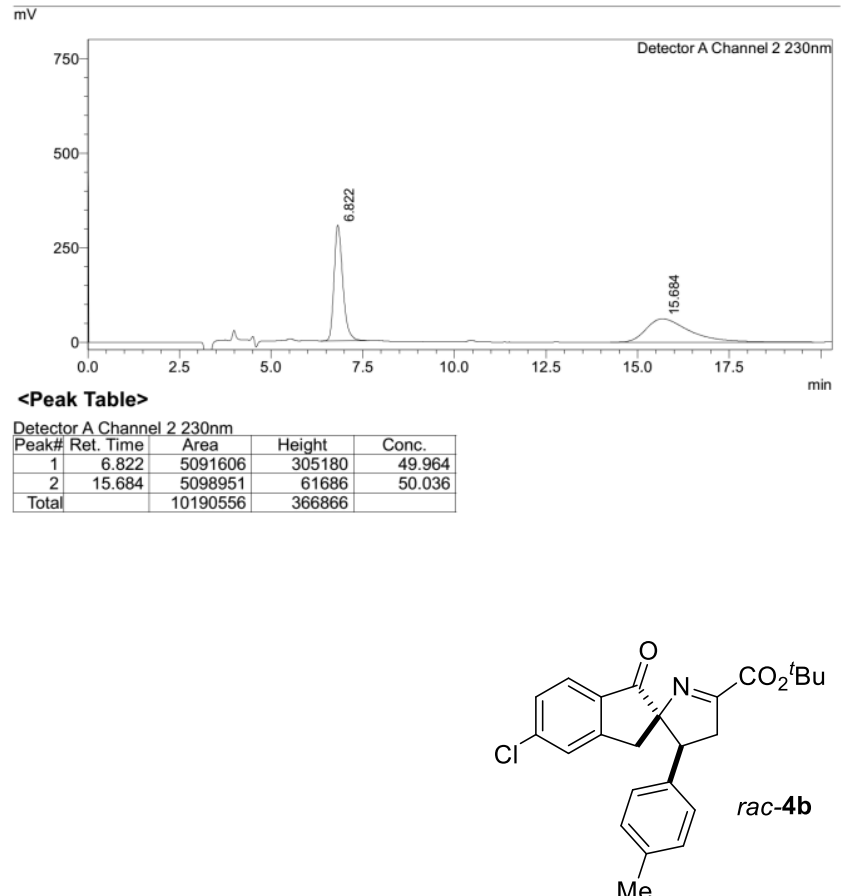

\section{Analysis Report}

<Sample Information>

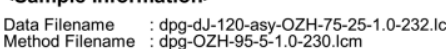

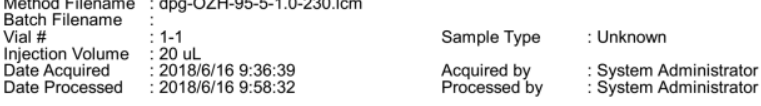

$<$ Chromatogram>
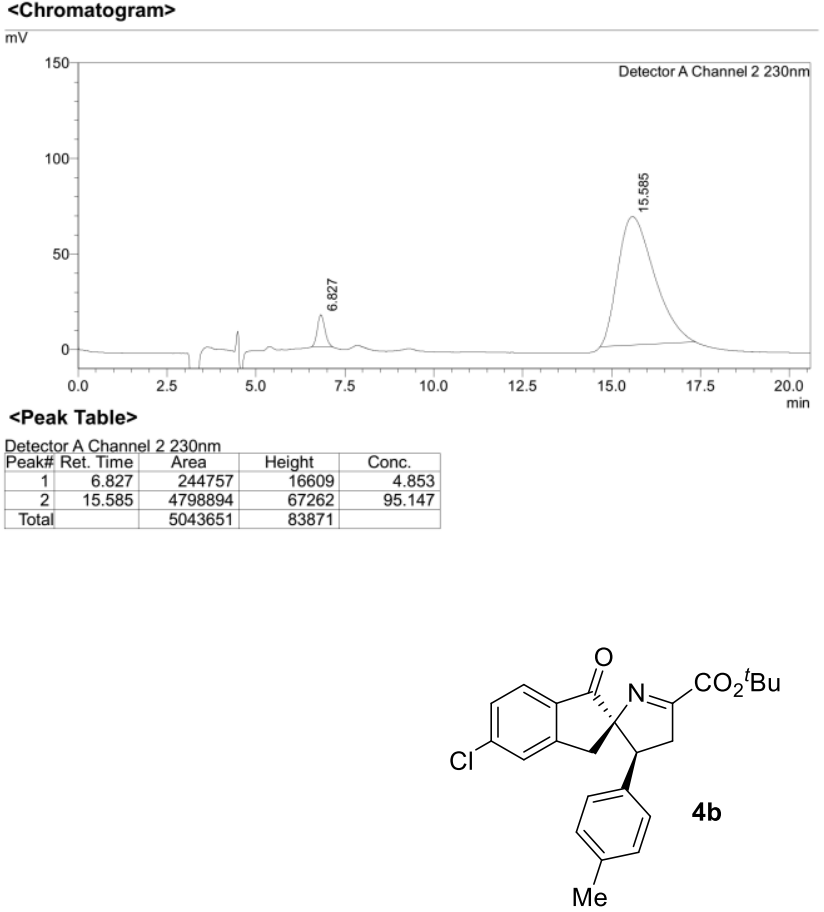
Asample Information> Analysis Report

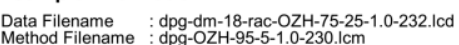

Batch Filename
Vial $\mathbb{H}$ :
1-1.

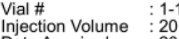

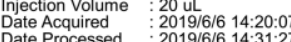

Sample Type : Unknow

Acauired by
Processed by

<Chromatogram>
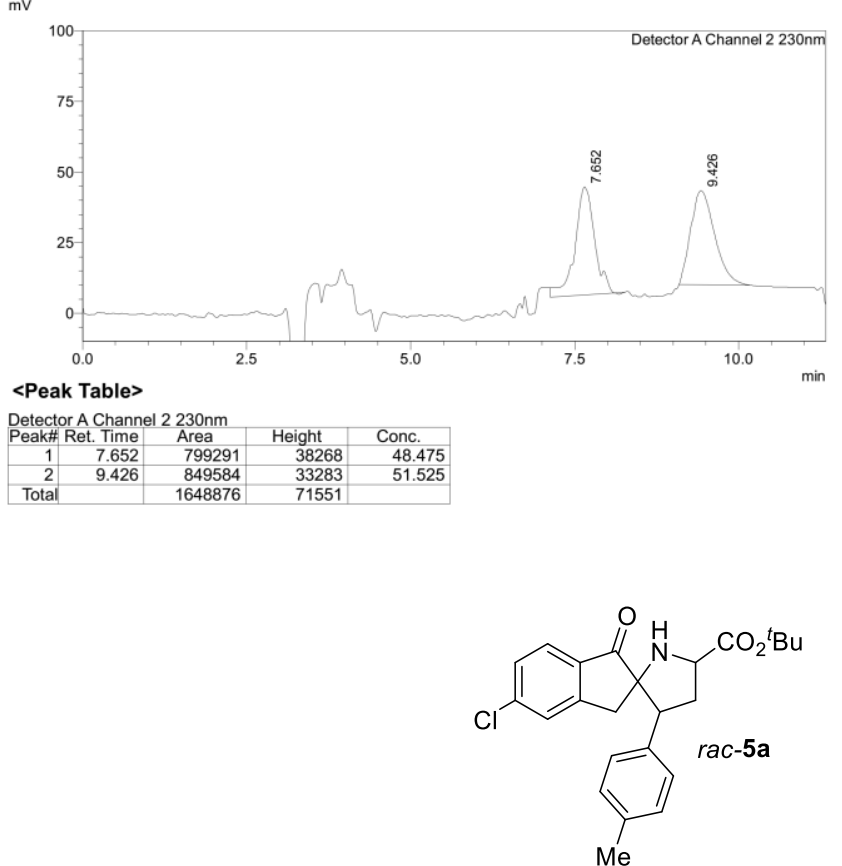

D:IDataldpgldmldpg-dm-18-rac-OZH-75-25-10-232 Icd

\section{Analysis Report}

$<$ Sample Information

Data Filename $:$ :dpg-dm-18-asy-OZH-75-25-10-232.1cd

$\begin{array}{l:cc}\text { Batch Filename } & 1-1 & \text { Sample Type } \\ \text { Vial } & \end{array}$

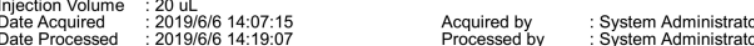

<Chromatogram>
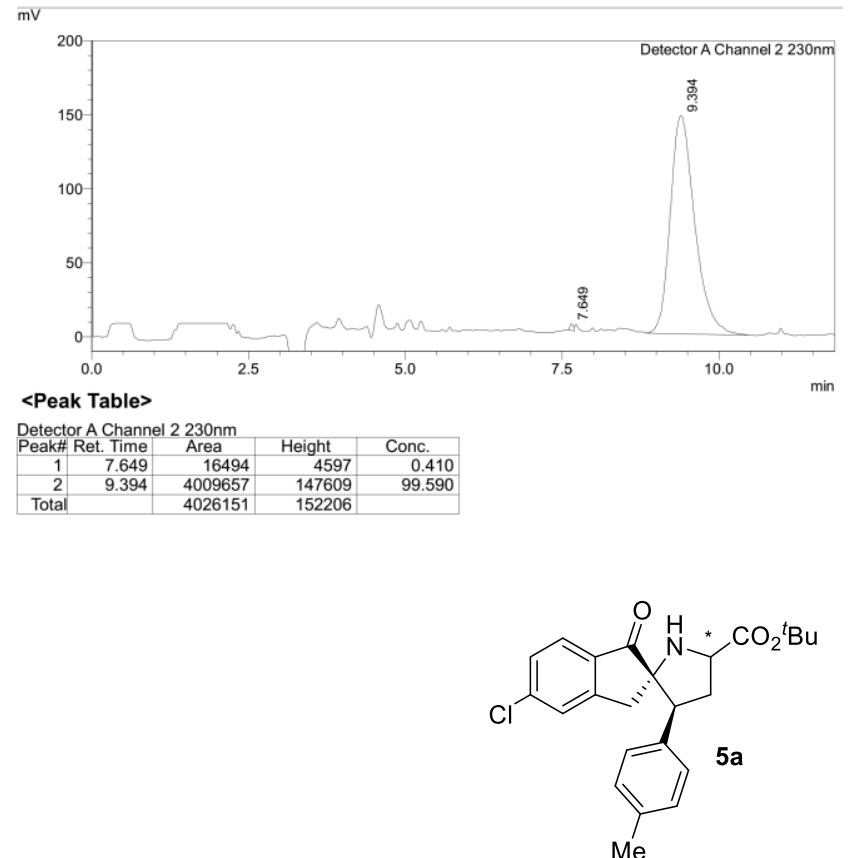

D:Dataldpgldmldpg-dm-18-asy-OZH-75-25-1.0-232.lcd 
Analysis Report

Sample Name $\cdot d p g-d m-22-r a c-o d H-97-3-0.5-23$

Dample ID

$\begin{array}{l:c}\text { Batch Filename } & 1-1 \\ \text { Vial \# } & 1-1\end{array}$

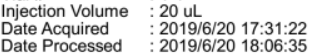

$<$ Chromatogram>
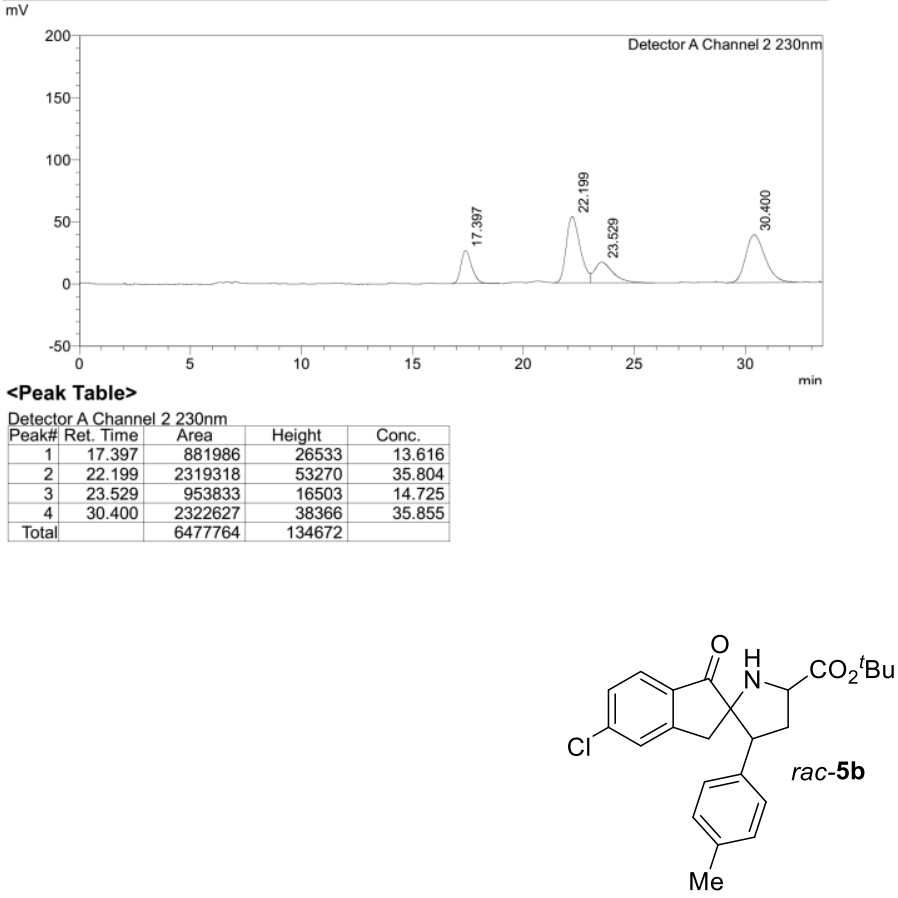

D:Dataldpgldmldpg-dm-22-rac-odH-97-3-0.5-232.Icd

\section{Analysis Report}

<Sample Information>

$\begin{array}{ll}\text { Data Filename } & \text { dpg-dm-22-asy-odH-97-3-0.5-232.lcd } \\ \text { Method Filename } & \text { dog-0.5-230.cm }\end{array}$

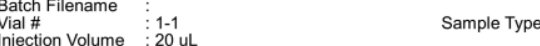

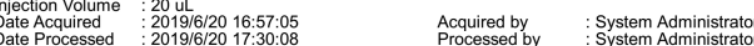

<Chromatogram>
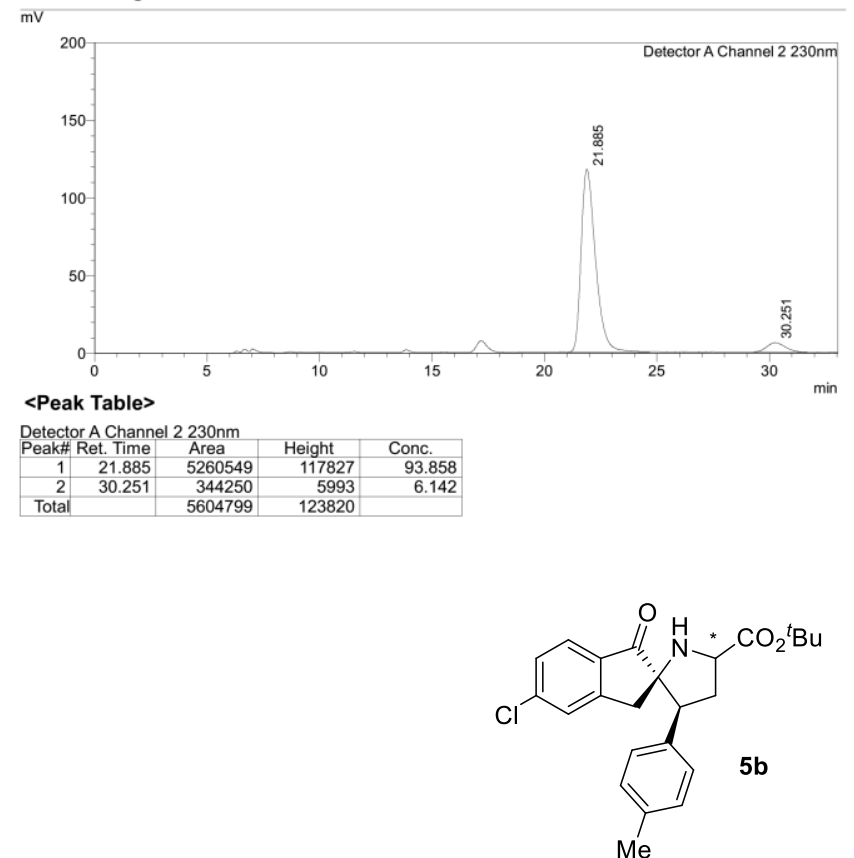

D: Dataldpgldmldpg-dm-22-asy-odH-97-3-0.5-232.Icd 


\section{Typical references for catalytic asymmetric diastereodivergent reactions}

(1) Selected examples by varying chiral ligand of the metal complex: (a) Yan, X.-X.; Peng, Q.; Li, Q.; Zhang, K.; Yao, J.; Hou, X.-L.; Wu, Y.-D. Highly Diastereoselective Switchable Enantioselective Mannich Reaction of Glycine Derivatives with Imines. J. Am. Chem. Soc. 2008, 130, 14362-14363. (b) Luparia, M.; Oliveira, M. T.; Audisio, D.; Frébault, F.; Goddard, R.; Maulide, N. Catalytic Asymmetric Diastereodivergent Deracemization. Angew. Chem., Int. Ed. 2011, 50, 12631-12635. (c) Hao, X.; Lin, L.; Tan, F.; Yin, C.; Liu, X.; Feng, X. Ligand Control of Diastereodivergency in Asymmetric Inverse Electron Demand Diels-Alder Reaction. ACS Catal. 2015, 5, 6052-6056. (d) Teng, H.-L.; Luo, Y.; Nishiura, M.; Hou, Z. Diastereodivergent Asymmetric Carboamination/Annulation of Cyclopropenes with Aminoalkenes by Chiral Lanthanum Catalysts. J. Am. Chem. Soc. 2017, 139, 16506-16509. (e) Itoh, T.; Kanzaki, Y.; Shimizu, Y.; Kanai, M. Copper(I)-Catalyzed Enantio- and Diastereodivergent Borylative Coupling of Styrenes and Imines. Angew. Chem., Int. $E d$. 2018, 57, 8265-8269. (f) Pluta, R.; Kumagai, N.; Shibasaki, M. Direct Catalytic Asymmetric Aldol Reaction of $\alpha$-Alkoxyamides to $\alpha$-Fluorinated Ketones. Angew. Chem., Int. Ed. 2019, 58, 2459-2463. Selected examples by varying metal of chiral metal catalysis: (g) Evans, D. A.; MacMillan, D. W. C.; Campos, K. R. $C_{2}$-Symmetric Tin(II) Complexes as Chiral Lewis Acids. Catalytic Enantioselective anti-Aldol Additions of Enolsilanes to Glyoxylate and Pyruvate Esters. J. Am. Chem. Soc. 1997, 119, 10859-10860. (h) Nojiri, A.; Kumagai, N.; Shibasaki, M. Linking Structural Dynamics and Functional Diversity in Asymmetric Catalysis. J. Am. Chem. Soc. 2009, 131, 3779-3784. (i) Lv, J.; Zhang, L.; Luo, S.; Cheng, J.-P. Switchable Diastereoselectivity in Enatioselective [4+2] Cycloadditions with Simple Olefins by Asymmetric Binary Acid Catalysis. Angew. Chem., Int. Ed. 2013, 52, 9786-9790.

(2) Selected examples by organocatalysis: (a) Mitsumori, S.; Zhang, H.; Cheong, P. H.-Y.; Houk, K. N.; Tanaka, F.; Barbas III, C. F. Direct Asymmetric anti-Mannich-Type Reactions Catalyzed by a Designed Amino Acid. J. Am. Chem. Soc. 2006, 128, 1040-1041. (b) Feng, X.; Zhou, Z.; Zhou, R.; Zhou, Q.-Q.; Dong, L.; Chen, Y.-C. Stereodivergence in Amine-Catalyzed Regioselective [4+2] Cycloadditions of $\beta$-Substituted Cyclic Enones and Polyconjugated Malononitriles. J. Am. Chem. Soc. 2012, 134, 19942-19947. (c) Li, X.; Lu, M.; Dong, Y.; Wu, W.; Qian, Q.; Ye, J.; Dixon, D. J. Diastereodivergent Organocatalytic Asymmetric Vinylogous Michael Reactions. Nat. Commun. 2014, 5, 4479-4487. (d) Uraguchi, D.; Yoshioka, K.; Ooi, T. Complete Diastereodivergence in Asymmetric 1,6-Addition Reactions Enabled by Minimal Modification of a Chiral Catalyst. Nat. Commun. 2017, 8 , 14793-14802. (e) Kan, S. B. J.; Maruyama, H.; Akakura, M.; Kano, T.; Maruoka, K. Catalyst-Controlled, Enantioselective, and Diastereodivergent Conjugate Addition of Aldehydes to Electron-Deficient Olefins. Angew. Chem., Int. Ed. 2017, 56, 9487-9491. (f) Zhang, L.; Yuan, H.; Lin, W.; Cheng, Y.; Li, P.; Li, W. Catalyst-Controlled Diastereodivergent Construction of Vicinal Sulfur-Functionalized Quaternary and Tertiary Stereocenters. Org. Lett. 2018, 20, 4970-4974.

(3) Selected examples for cooperative catalysis: (a) Jiang, J.; Xu, H.-D.; Xi, J.-B.; Ren, B.-Y.; Lv, F.-P.; Guo, X.; Jiang, L.-Q.; Zhang, Z.-Y.; Hu, W.-H. Diastereoselectively Switchable Enantioselective Trapping of Carbamate Ammonium Ylides with Imines. J. Am. Chem. Soc. 2011, 133, 8428-8431. (b) Krautwald, S.; Sarlah, D.; Schafroth, M. A.; Carreira, E. M. Enantio- and Diastereodivergent Dual Catalysis: $\alpha$-Allylation of Branched Aldehydes. Science 
2013, 340, 1065-1068. (c) Krautwald, S.; Schafroth, M. A.; Sarlah, D.; Carreira, E. M. Stereodivergent $\alpha$-Allylation of Linear Aldehydes with Dual Iridium and Amine Catalysis. J. Am. Chem. Soc. 2014, 136, 3020-3023. (d) Rana, N. K.; Huang, H.; Zhao, J. C.-G. Highly Diastereodivergent Synthesis of Tetrasubstituted Cyclohexanes Catalyzed by Modularly Designed Organocatalysts. Angew. Chem., Int. Ed. 2014, 53, 7619-7623. (e) Huo, X.; He, R.; Zhang, X.; Zhang, W. An Ir/Zn Dual Catalysis for Enantio- and Diastereodivergent $\alpha$-Allylation of $\alpha$-Hydroxyketones. J. Am. Chem. Soc. 2016, 138, 11093-11906. (f) Jiang, X.; Beiger, J. J.; Hartwig, J. F. Stereodivergent Allylic Substitutions with Aryl Acetic Acid Esters by Synergistic Iridium and Lewis Base Catalysis. J. Am. Chem. Soc. 2017, 139, 87-90. (g) Cruz, F. A.; Dong, V. M. Stereodivergent Coupling of Aldehydes and Alkynes via Synergistic Catalysis Using Rh and Jacobsen's Amine. J. Am. Chem. Soc. 2017, 139, 1029-1032. (h) Wei, L.; Zhu, Q.; Xu, S.-M.; Chang, X.; Wang, C.-J. Stereodivergent Synthesis of $\alpha, \alpha$-Disubstituted $\alpha$-Amino Acids via Synergistic Cu/Ir Catalysis. J. Am. Chem. Soc. 2018, 140, 1508-1513. (i) Huo, X.; Zhang, J.; Fu, J.; He, R.; Zhang, W. Ir/Cu Dual Catalysis: Enantio- and Diastereodivergent Access to $\alpha$, $\alpha$-Disubstituted $\alpha$-Amino Acids Bearing Vicinal Stereocenters. J. Am. Chem. Soc. 2018, 140, 2080-2084. (j) Zhang, M.-M.; Wang, Y.-N.; Wang, B.-C.; Chen, X.-W.; Lu, L.-Q.; Xiao, W.-J. Synergetic Iridium and Amine Catalysis Enables Asymmetric [4+2] Cycloadditions of Vinyl Aminoalcohols with Carbonyls. Nat. Commun. 2019, 10, 2716-2724. (k) He, Z.-T.; Jiang, X.; Hartwig, J. F. Stereodivergent Construction of Tertiary Fluorides in Vicinal Stereogenic Pairs by Allylic Substitution with Iridium and Copper Catalysts. J. Am. Chem. Soc. 2019, 141, 13066-13073. For tandem catalysis: (1) Huang, Y.; Walji, A. M.; Larsen, C. H.; MacMillan, D. W. C. Enantioselective Organo-Cascade Catalysis. J. Am. Chem. Soc. 2005, 127, 15051-15053. (m) Shi, S.-L.; Wong, Z. L. Buchwald, S. L. Copper-Catalysed Enantioselective Stereodivergent Synthesis of Amino Alcohols. Nature 2016, 532, 353-356. (n) Trost, B. M.; Hung, C.-I.; Saget, T.; Gnanamani, E. Branched Aldehydes as Linchpins for the Enantioselective and Stereodivergent Synthesis of 1,3-Aminoalcohols Featuring a Quaternary Stereocentre. Nat. Catal. 2018, 1, 523-530.

(4) For selected examples by varying temperature: (a) Huang, Z.-Z.; Kang, Y.-B.; Zhou, J.; Ye, M.-C.; Tang, Y. Diastereoselectivity-Switchable and Highly Enantioselective 1,3-Dipolar Cycloaddition of Nitrones to Alkylidene Malonates. Org. Lett. 2004, 6, 1677-1679. By varying additives: (b) Tian, X.; Cassani, C.; Liu, Y.; Moran, A.; Urakawa, A.; Galzerano, P.; Arceo, E.; Melchiorre, P. Diastereodivergent Asymmetric Sulfa-Michael Additions of $\alpha$-Branched Enones Using a Single Chiral Organic Catalyst. J. Am. Chem. Soc. 2011, 133, 17934-17941. By varying solvents: (c) Wu, X.; Chen, Z.; Bai, Y.-B.; Dong, V. M. Diastereodivergent Construction of Bicyclic $\gamma$-Lactones via Enantioselective Ketone Hydroacylation. J. Am. Chem. Soc. 2016, 138, 12013-12016. (d) Cheng, Q.; Zhang, F.; Cai, Y.; Guo, Y.-L.; You, S.-L. Stereodivergent Synthesis of Tetrahydrofuroindoles through Pd-Catalyzed Asymmetric Dearomative Formal [3+2] Cycloaddition. Angew. Chem., Int. Ed. 2018, 57, 2134-2138. 